\title{
IN SITU BIOENGINEERING OF ARTERIAL VEIN GRAFTS
}

by

Mohammed Salim El-Kurdi

Bachelor of Science, Saint Francis Xavier University, 2000

Bachelor of Engineering, Technical University of Nova Scotia, 2000

Submitted to the Graduate Faculty of

Swanson School of Engineering in partial fulfillment

of the requirements for the degree of

Doctor of Philosophy

University of Pittsburgh 


\title{
UNIVERSITY OF PITTSBURGH \\ SWANSON SCHOOL OF ENGINEERING
}

This dissertation was presented

by

\author{
Mohammed S. El-Kurdi
}

It was defended on

April 11, 2008

and approved by

Sanjeev G. Shroff, Professor, Departments of Surgery and BioEngineering

Edith Tzeng, Assistant Professor, Department of Surgery

Jeffery S. Vipperman, Associate Professor, Departments of Mechanical Engineering and BioEngineering

William R. Wagner, Professor, Surgery, Departments of Chemical Engineering and BioEngineering

Chuanyue Wu, Professor, Department of Pathology

Dissertation Director: David A. Vorp, Associate Professor, Departments of Surgery and BioEngineering 
Copyright (C) by Mohammed S. El-Kurdi

2008 


\title{
IN SITU BIOENGINEERING OF ARTERIAL VEIN GRAFTS
}

\author{
Mohammed S. El-Kurdi, Ph.D. \\ University of Pittsburgh, 2008
}

The autogenous saphenous vein remains the graft of choice for both coronary $(500,000$ annually in the US) and peripheral (80,000 annually) arterial bypass procedures. Failure of arterial vein grafts (AVGs) remains a major problem, and patients with failed grafts will die or require reoperation. Intimal hyperplasia (IH) accounts for $20 \%$ to $40 \%$ of all AVG failures. It is believed that this adverse pathological response by AVGs is largely due to their abrupt exposure to the significantly elevated circumferential wall stress (CWS) associated with the arterial system. We believe that if an AVG is given an ample opportunity to adapt and remodel to the stresses of its new environment, cellular injury may be reduced, thus limiting the initiating mechanisms of IH. The goal of this work was to develop a new mechanical conditioning paradigm, in the form of a peri-adventitially placed, biodegradable polymer wrap, to safely and functionally "arterialize" AVGs in situ. The polymer wrap was tuned so that as it degraded over a desired period of time, the mechanical support offered by it was reduced and the vein was exposed to gradually increasing levels of CWS in situ.

To investigate the effects of mechanical conditioning on AVGs, we utilized both our well established, validated ex vivo vascular perfusion system (EVPS) as well as an appropriate preclinical animal model. The "engineering" component of this bioengineering study was to enhance our EVPS capabilities. Enhancements were made in the form of rigorous mathematical modeling, via subspace system identification, and automatic feedback control, via proportional 
integral and derivative control, of the arterial CWS and shear stress waveform generation capabilities of the EVPS. Pairs of freshly harvested porcine internal jugular veins (PIJVs) were perfused ex vivo under several biomechanical conditions. The acute hyperplastic response of PIJVs abruptly exposed to arterial hemodynamic conditions was compared to PIJVs perfused under normal venous conditions. In an attempt to attenuate this acute hyperplastic response, an ex vivo mechanical conditioning paradigm was imposed onto the PIJVs both via manual adjustment of EVPS parameters and via an adventitially placed tuned electrospun biodegradable polymer wrap. Early markers of IH were evaluated post-perfusion, and they included vascular smooth muscle cell apoptosis, proliferation, and phenotypic modulation. Quantification of these markers via immunohistochemical techniques provided the foundation for the final stage of this work. To assess the efficacy of the tuned electrospun biodegradable polymer wrap in attenuating the development of intimal hyperplasia in AVGs, a series of preclinical studies was performed in a pig model.

PIJVs abruptly exposed to arterial levels of CWS showed a significant increase in apoptosis and in the number of synthetic smooth muscle cells, as well as a decrease in proliferation. Mechanical conditioning, via both manual adjustment of the EVPS parameters and placement of the biodegradable adventitial wrap, appeared to have beneficial effects on the acute hyperplastic response of PIJVs perfused ex vivo. The beneficial effects of the adventitially placed polymer wrap was also observed in vivo, however the results did not achieve significance over unwrapped controls.

Future work should be aimed at enhancing the beneficial effects of the electrospun biodegradable polymer wrap by incorporating the delivery of drugs and/or stem cells in addition to the delivery of structural support to AVGs. 
TABLE OF CONTENTS

PREFACE XXXIII

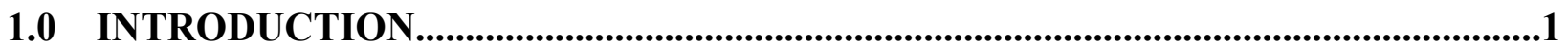

1.1 THE ROLE OF BIOMECHANICS IN THE DEVELOPMENT OF INTIMAL

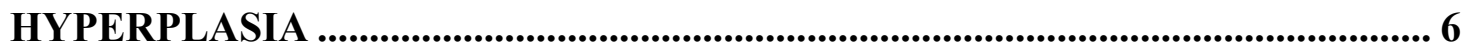

1.2 MOLECULAR AND CELLULAR PROCESSES ASSOCIATED WITH INTIMAL HYPERPLASIA .......................................................................................... 7

1.2.1 Phenotypic Modulation ........................................................................................... 8

1.2.2 De-adhesion and Migration.................................................................................... 10

1.2.3 Proliferation...................................................................................................... 14

1.2.4 Remodeling ..................................................................................................... 15

1.3 CHANGES IN MECHANICAL PROPERTIES OF VEIN GRAFTS DUE TO ARTERIALIZATION...................................................................................... 16

1.4 PREVIOUS ATTEMPTS TO WRAP ARTERIAL VEIN GRAFTS ..................... 18

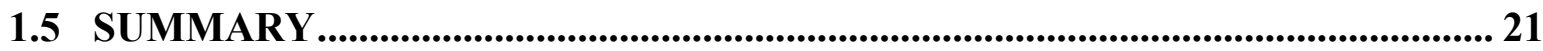

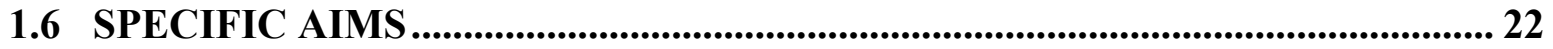

$2.0 \quad E X$ VIVO VASCULAR PERFUSION SYSTEM.........................................................26

2.1 HISTORY OF EX VIVO VASCULAR PERFUSION................................................ 26

2.2 LIMITATIONS OF PREVIOUS SYSTEM DESIGNS ............................................. 28 
2.3 TECHNICAL IMPROVEMENTS TO CURRENT EVPS

2.3.1 Detailed Description of EVPS Flow Circuit Components....................... 29

2.3.2 Custom Designed EVPS Components.................................................. 32

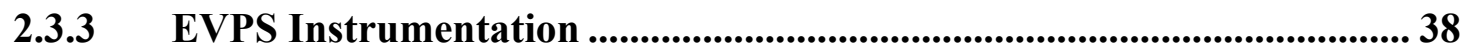

2.4 SUBSPACE SYSTEM IDENTIFICATION OF EVPS........................................ 42

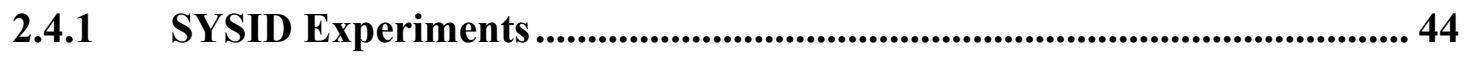

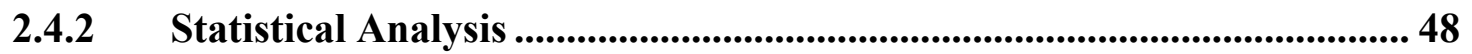

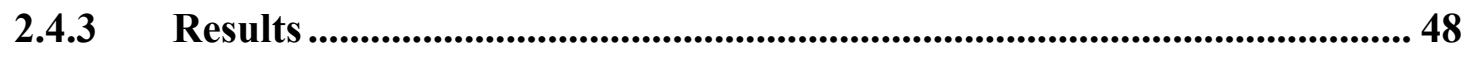

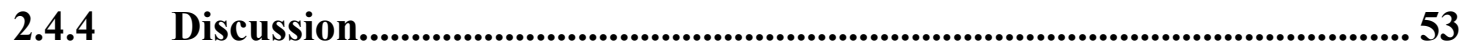

2.4.5 Conclusions ............................................................................................. 54

2.5 PROPORTIONAL INTEGRAL AND DERIVATIVE CONTROL OF CIRCUMFERENTIAL WALL STRESS AND LUMENAL SHEAR STRESS WITHIN VEINS IMPLANTED INTO THE EVPS........................................ 55

2.5.1 Rationale for Control System Architecture............................................. 55

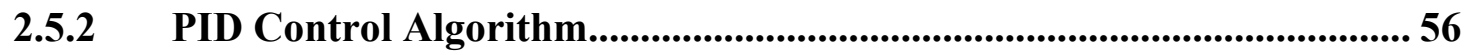

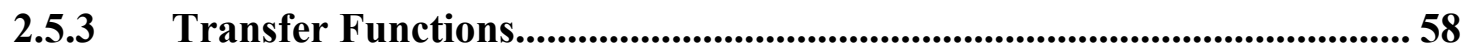

2.5.4 Mid-vein-wall CWS Calculation for Controller........................................ 59

2.5.5 Lumenal Shear Stress Calculation for Controller ................................... 60

2.5.6 CWS and Shear Stress Control Signal Calculations ............................ 61

2.5.7 PID Controller Performance Experiments............................................... 62

2.5.8 Implemented Safety Precautions .................................................... 63

2.5.9 Biological Validation of EVPS ............................................................. 64

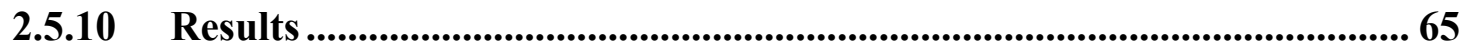

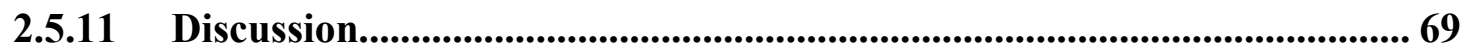


2.5.12 Conclusions ............................................................................................. 71

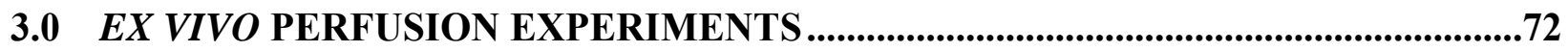

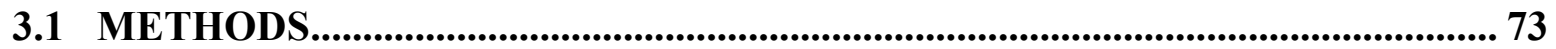

3.1.1 Tissue Harvest and Transport ..................................................................... 73

3.1.2 Perivascular Placement of Electrospun Biodegradable Polymer Wrap 75

3.1.3 Ex vivo Perfusion Conditions .................................................................... 77

3.1.4 Vasomotor Challenge Experiments.................................................... 89

3.1.5 Compliance and $\beta$-stiffness Measurements ........................................... 90

3.1.6 Post-perfusion Tissue Processing................................................................. 91

3.1.7 Biological Analyses.......................................................................................... 92

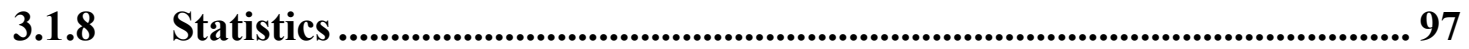

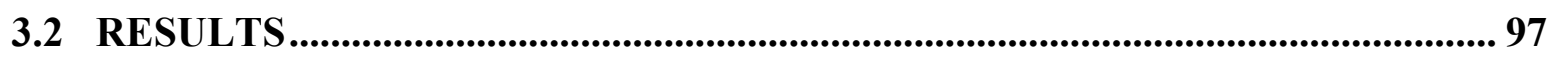

3.2.1 Ex vivo Biomechanical Conditioning Perfusion Experiments ................. 97

3.2.2 ART vs. wART Diameter Profiles ..................................................... 99

3.2.3 CWS Profiles ............................................................................... 100

3.2.4 Vasomotor Challenge Results ................................................................... 101

Compliance and $\beta$-stiffness ......................................................................... 105

3.2.6 Biological Analyses..................................................................... 106

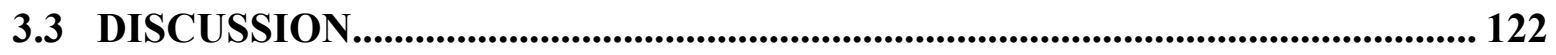

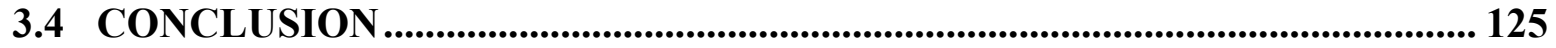

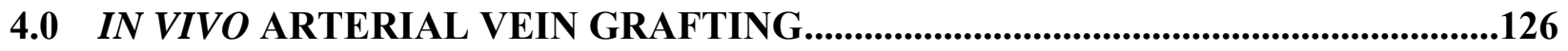

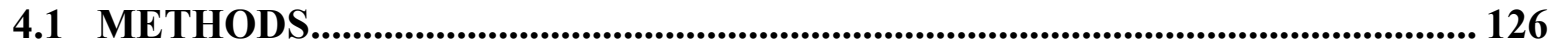


4.1.1 Unilateral Porcine Carotid Interposition Grafting................................. 128

4.1.2 Fluoroscopic Angiography ................................................................ 133

4.1.3 Post-explant Tissue Processing ......................................................... 133

4.1.4 Histological Measurements of IH .............................................................. 133

4.1.5 Scanning Electron Microscopy .............................................................. 134

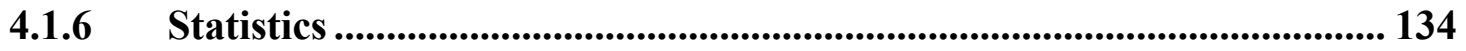

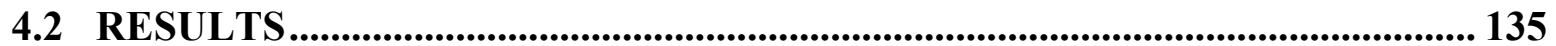

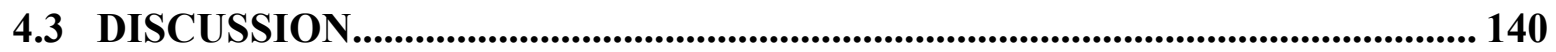

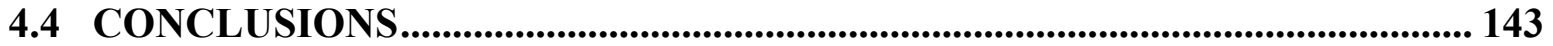

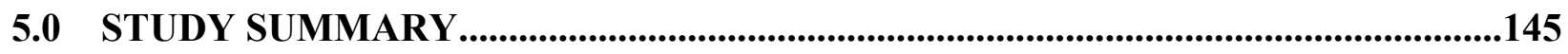

5.1 SUMMARY OF PERTINENT FINDINGS ................................................... 145

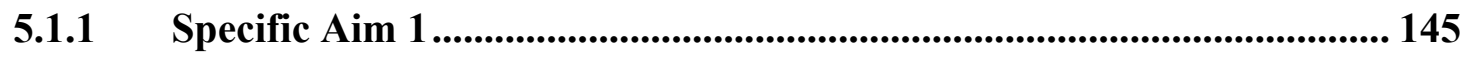

5.1.2 Specific Aim 2

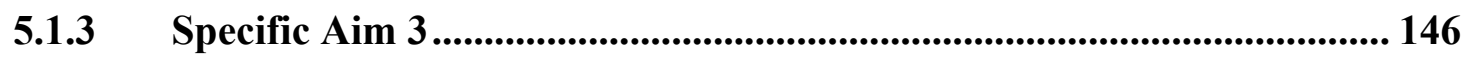

5.1.4 Specific Aim 4 ............................................................................. 147

5.2 SUMMARY OF ACCOMPLISHMENTS ................................................... 147

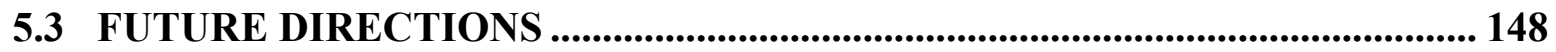

APPENDIX A: CUSTOM BUILT EVPS COMPONENT DRAWINGS ..............................150

APPENDIX B: SYSID MATLAB ${ }^{\circledR}$ CODE ......................................................................202

APPENDIX C: PID CONTROLLER MATLAB ${ }^{\circledR}$ CODE ....................................................220

APPENDIX D: EX VIVO PERFUSION EXPERIMENTAL RECORDS ...........................246

APPENDIX E: BIOLOGICAL ANALYSES PROTOCOLS .........................................268

APPENDIX F: IMMUNOHISTOCHEMISTRY DATA ...............................................309

APPENDIX G: IN VIVO EXPERIMENTAL DATA .....................................................353 
APPENDIX H: TROUBLESHOOTING MOLECULAR BIOLOGICAL ANALYSES ....365

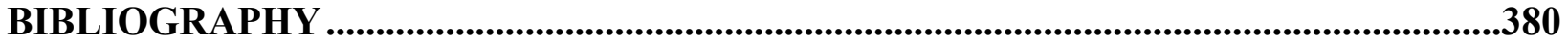




\section{LIST OF TABLES}

Table 1.1 The chronological events leading to vein graft intimal hyperplasia adapted from Mitra et al. [1] . Please note: ECM, extracellular matrix; SMC, smooth muscle cell................... 2

Table 1.2 Summary of and rationale for the chosen endpoints in this study.............................. 22

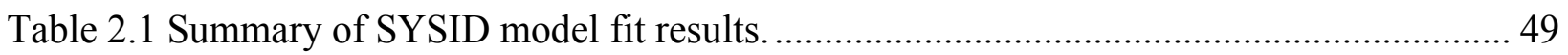

Table 3.1 Summary of polymer tuning parameter combinations. ............................................... 76

Table 3.2 Summary of all ex vivo vascular perfusion experiments that were performed and the respective endpoints that were analyzed. Note that VMC: vasomotor challenge; PCNA: proliferating cell nuclear antigen; TUNEL: terminal deoxynucleotidyl transferasemediated dUTP-biotin in situ nick end labeling; GOLGI: Golgi Complex; H\&E: hematoxylin and eosin; MTC: Masson's trichrome; MPC: Movat's pentachrome; PSR: picrosirius red; SEM: scanning electron microscopy; and TEM: transmission electron microscopy. An " $\mathrm{X}$ " indicates that the analysis was performed.

Table 4.1 Summary of all in vivo AVG experiments that were performed and the respective endpoints that were analyzed. Note that H\&E: hematoxylin and eosin; MPC: Movat's pentachrome; and SEM: scanning electron microscopy. An " $\mathrm{X}$ " indicates that the analysis

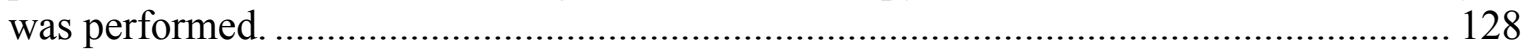

Table D. 1 Perfusion experiment record for VEN vs. ART experiment performed on $04 / 23 / 2003$.

Table D. 2 Perfusion experiment record for VEN vs. ART experiment performed on $05 / 07 / 2003$. 248

Table D. 3 Perfusion experiment record for VEN vs. ART experiment performed on $05 / 24 / 2006$

Table D. 4 Perfusion experiment record for VEN vs. ART experiment performed on $09 / 14 / 2006$. .250

Table D. 5 Perfusion experiment record for VEN vs. ART experiment performed on $10 / 09 / 2004$ 
Table D. 6 Perfusion experiment record for VEN vs. ART experiment performed on

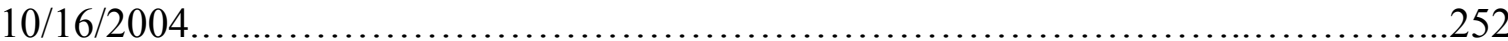

Table D. 7 Mean values of percent constriction in response to epinephrine for all the acute ex vivo vasomotor challenge experiments.... .265

Table D. 8 Microsoft Excel output for student's t-test performed on the percent constriction data from the acute ex vivo vasomotor challenge experiments. 266

Table D. 9 Mean values of percent dilation in response to sodium nitroprusside for all the acute ex vivo vasomotor challenge experiments 266

Table D. 10 Microsoft Excel output for student's t-test performed on the percent dilation data from the acute ex vivo vasomotor challenge experiments. 267

Table F. 1 Mean values of percent TUNEL positive cells for all ex vivo experiments. .322

Table F. 2 Microsoft Excel output for student's t-test performed on the mean percent TUNEL positive cells from 24 hour VEN vs. ART ex vivo experiments.

Table F. 3 Microsoft Excel output for student's t-test performed on the mean percent TUNEL positive cells from 24 hour ART vs. cART ex vivo experiments.

Table F. 4 Microsoft Excel output for student's t-test performed on the mean percent TUNEL positive cells from 72 hour ART vs. cART ex vivo experiments 323

Table F. 5 Microsoft Excel output for student's t-test performed on the mean percent TUNEL positive cells from 24 hour ART vs. WART ex vivo experiments.

Table F. 6 Mean values of percent PCNA positive cells for all ex vivo experiments..... .336

Table F. 7 Microsoft Excel output for student's t-test performed on the mean percent PCNA positive cells from 24 hour VEN vs. ART ex vivo experiments

Table F. 8 Microsoft Excel output for student's t-test performed on the mean percent PCNA positive cells from 24 hour ART vs. cART ex vivo experiments.

Table F. 9 Microsoft Excel output for student's t-test performed on the mean percent PCNA positive cells from 72 hour ART vs. cART ex vivo experiments

Table F. 10 Microsoft Excel output for student's t-test performed on the mean percent PCNA positive cells from 24 hour ART vs. WART ex vivo experiments. 338

Table F. 11 Mean values of percent Golgi complex positive cells for all ex vivo experiments..350 
Table F. 12 Microsoft Excel output for student's t-test performed on the mean percent Golgi complex positive cells from 24 hour VEN vs. ART ex vivo experiments......................................................... 350

Table F. 13 Microsoft Excel output for student's t-test performed on the mean percent Golgi complex positive cells from 24 hour ART vs. cART ex vivo

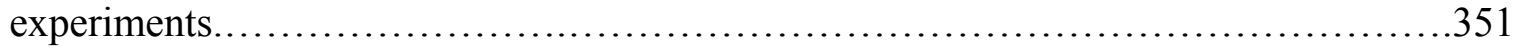

Table F. 14 Microsoft Excel output for student's t-test performed on the mean percent Golgi complex positive cells from 72 hour ART vs. cART ex vivo

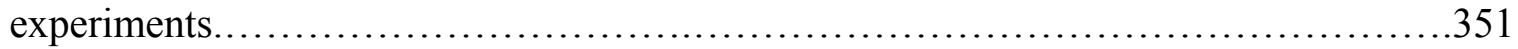

Table F. 15 Microsoft Excel output for student's t-test performed on the mean percent Golgi complex positive cells from 24 hour ART vs. wART ex vivo

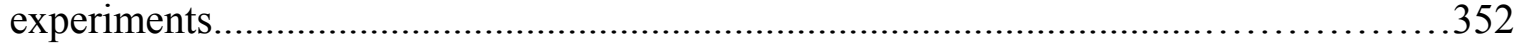

Table G. 1 Mean intimal-to-medial thickness ratios from morphometric analysis of MPC stained

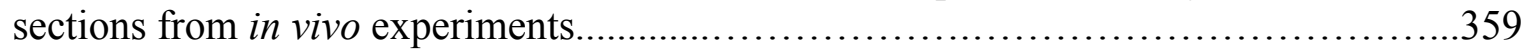

Table G. 2 Microsoft Excel output for student's t-test performed on the mean intimal-to-medial thickness ratio data from the in vivo experiments. 


\section{LIST OF FIGURES}

Figure 1.1 Schematic of intimal hyperplasia progression. Please note: IEL, internal elastic lamina; SMCs, smooth muscle cells. Image adapted from Robbins Pathologic Basis of Disease, 1999 [2].

Figure 1.2 Schematic showing the localization of Tenascin-C (TN-C), thrombospondin-1,2 (TSP), secreted protein acidic and rich in cysteine (SPARC), Mitogen inducible gene 2 (Mig-2) and integrin linked kinase (ILK). Please note: ECM, extracellular matrix; $\alpha$ and $\beta$, integrins. Image adapted from www.cellmigration.org/res_resource_misc.htm........ 12

Figure 1.3 Pressure vs. diameter response of a porcine internal jugular vein segment. 18

Figure 2.1 Schematic of the closed-loop perfusion/organ culture system. The loop is composed of a Biomedicus centrifugal pump that provides pulsatile pressure and flow (A), a heat exchanger (D), a tissue-housing chamber (C), proximal (B1) and distal (B2) pressure transducers, a variable resistance valve $(\mathrm{E})$, flow probe $(\mathrm{F})$, collection reservoir $(\mathrm{G})$, and vessel bypass $(\mathrm{H})$. Components not shown include, adventitial bath loop, He-Ne laser micrometer, and data acquisition system. See Labadie (1996) et al. for more detail [3]. 27

Figure 2.2 Schematic of one loop of the paired perfusion system. Note that there are two separate closed loops: the perfusate loop and the adventitial bathing loop. The components comprising both loops are: (1) perfusate reservoir; (2) metal heat exchanger tubes inside water baths; (3) roller pumps; (4) pulse dampener; (5) one-way valve; (6) piston-cylinder device; (7) vessel cannulae; (8) pressure transducers; (9) tissue housing chamber; (10) inline thermistors; (11) self-sealing media sampling ports; (12) ultrasonic flow transducer; (13) needle-valve flow resistor; and (14) overflow recirculation line... 32

Figure 2.3 Earlier generation paired ex vivo vascular perfusion system. 33

Figure 2.4 Modifications made to render our earlier generation ex vivo vascular perfusion system more compact so that it fits within a laminar flow hood. Note the stacked vessel housing chambers and single laser micrometer, reducing the size of the two independent flow loops

Figure 2.5 Photograph of the components within the closed loop ex vivo perfusion system. The bulk flow was generated using a Masterflex L/S computerized roller pump (a). A pulsedampener (b) was added downstream to remove the very high frequency perturbations generated by the roller pump. Higher frequency components of the physiologic arterial 
pressure and flowrate waveforms were generated using a custom built piston/cylinder device (c). A one way valve (d), was placed between the pulse dampener and the piston to inhibit the flow or pressure from the piston to be dissipated by the pulse dampener. A fluid reservoir (e) completes the closed loop system.

Figure 2.6 A close-up photograph of the piston/cylinder assembly. The components are held together with a custom built frame (a). The voice-coil (b) is coupled to a shaft (c) that translates linearly within the bearing (d). At the end of the shaft, a piston head (e) is connected to a water tight rolling diaphragm (f) that allows the piston head to have frictionless motion within the cylinder $(\mathrm{g})$. The voice-coil is driven by analog signals sent to its own servo- amplifier (h). Also seen are the pressure monitor (i) and the tubing flowmeter $(\mathrm{j})$ used to process and transmit the pressure and flow transducer signals, respectively, to the data acquisition system. 36

Figure 2.7 Blood gas and temperature control measurements made within our EVPS for several $(\mathrm{N}=18) 24$ hour ex vivo perfusion experiments. The panels on the left are perfusate measurements and the panels on the right are bathing media measurements. Please note that measurements were recorded hourly. 40

Figure 2.8 Physiologic arterial pressure (A) and flowrate (B) waveforms recorded from the aorta of a $45 \mathrm{Kg}$ pig using a $150 \mathrm{~Hz}$ sampling frequency 44

Figure 2.9 (A) The step and impulse responses suggest that $H_{R P, p}$ has very slow, over-damped and open-loop-stable poles. (B) The frequency response suggests that $H_{R P, p}$ is low frequency and has a bandwidth of approximately $0.1 \mathrm{~Hz}$. (C) The step and impulse responses suggest that $\mathrm{H}_{\mathrm{RPP}, \mathrm{p}}$ appears to have much faster, slightly under-damped, but open-loop-stable poles. (D) The frequency response suggests that $\mathrm{H}_{\mathrm{RPP}, \mathrm{p}}$ has a band pass

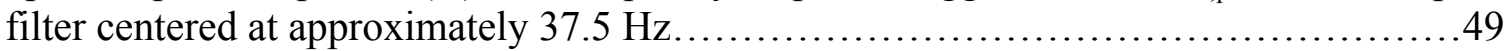

Figure 2.10 (A) The step and impulse responses show that $\mathrm{H}_{\mathrm{RP}, \mathrm{f}}$ has over-damped and open-loopstable poles. (B) The frequency response suggests that $\mathrm{H}_{\mathrm{RP}, \mathrm{f}}$ has a bandwidth of approximately $2 \mathrm{~Hz}$ but also responds well from 18-30 Hz. (C) The step and impulse responses show that $\mathrm{H}_{\mathrm{RPP}, \mathrm{f}}$ appears to have slightly under-damped, but open-loop-stable poles. (D) The frequency response suggests that $\mathrm{H}_{\mathrm{RPP}, \mathrm{f}}$ has a bandwidth of approximately $15 \mathrm{~Hz}$ that is centered about $22 \mathrm{~Hz}$, with good response also observed in the vicinity of 70 Hz. 52

Figure 2.11 (A) Arterial pressure waveform that was used to calculate the circumferential wall stress profile (B). (C) Arterial flowrate waveform that was used to calculate the shear stress profile (D). 62

Figure 2.12 Several representative waveforms from the controller performance experiments. The left panel shows the measured and set CWS waveforms, and the right panel shows the measured and set shear stress waveforms. 66 
Figure 2.13 Leak simulation experiment results. As desired, in the event of a leak the piston stops and the roller pump slows to supply a minimal flowrate of approximately 20 $\mathrm{ml} / \mathrm{min}$.

Figure 2.14 The top three panels show representative SEM images of the lumen of baseline control (BASE), "venous" 48 hour perfused control (VEN), and "arterial" 48 hour perfused (ART) PIJV segments. Note the cobblestone appearance of an intact endothelial cell layer. The second row of panels show representative microstructure and live nuclei via $H \& E$ staining of each group (200x magnification). The third row of panels show representative live (green) and dead (red) cells within each tissue group (200x magnification). Note that there does not appear to be an increased level of necrosis in perfused tissue when compared to BASE control tissue. The bottom three panels show representative TUNEL assay images of tissue from the same 48 hour perfusion experiment (400x magnification under immersion oil). Note that there does not appear to be an increased level of apoptosis in perfused tissue when compared to BASE. In all panels the arrow designates the vessel lumen. 68

Figure 3.1 Harvested PIJV segment within custom designed in vivo-length clamp and immersed in media within the transport box. 74

Figure 3.2 Schematic depicting the paired VEN vs. ART ex vivo perfusion experiments.......... 79

Figure 3.3 Schematic depicting the paired ART vs. cART ex vivo perfusion experiments. ........ 82

Figure 3.4 Schematic depicting the paired ART vs. wART ex vivo perfusion experiments. ....... 83

Figure 3.5 Schematic showing a cross-sectional view of the vein /wrap complex. Note W: electrospun polymer wrap; V: vein segment; $P_{i}$ : intraluminal pressure; $P_{2}$ : interfacial pressure; $P_{o}$ : atmospheric pressure.

Figure 3.6 Schematic of post perfusion PIJV segment processing for endpoint analysis. Lengths given represent unloaded vessel resting lengths. 91

Figure 3.7 Representative mean pressure, outer diameter, and CWS vs. time profiles from a 24 hour ART vs. cART experiment. . 98

Figure 3.8 Representative mean pressure, outer diameter, and CWS vs. time profiles from a 72 hour ART vs. cART experiment.

Figure 3.9 Normalized outer diameter response profiles of PIJVs for both sham and spun PIJVs. Both spun (wART) and sham control PIJVs were perfused under ART conditions of $120 / 80 \mathrm{mmHg}$ pressure and $100 \mathrm{ml} / \mathrm{min}$ mean flowrate. Note that the normalized diameter of the spun veins $(\mathrm{N}=7)$ is dramatically reduced when compared to sham controls $(\mathrm{N}=5)$. Pressurized outer diameter $(\mathrm{ODp})$ was normalized to unpressurized outer diameter (ODup) and data is shown as mean \pm standard error of the mean. 101 
Figure 3.10 CWS vs. time results from 24 hour ex vivo perfusions of electrospun polymer wrapped PIJV segments for each combination in Table 3.1. The lower dashed horizontal line indicates the mean CWS level measured in an unwrapped vein under venous conditions (CWSo $\sim 25 \mathrm{KPa}$ ), and the middle dashed horizontal line indicates the mean CWS in a coronary artery $(\sim 120 \mathrm{KPa})$ [4]. The upper dashed line represents the mean CWS measured in an unwrapped vein (sham control) under ART conditions. In the legend, ET stands for electrospinning time. All CWS values were normalized to $\mathrm{CWS}_{\mathrm{o}}$. The data are presented as mean \pm standard error of the mean. 103

Figure 3.11 Representative vasomotor challenge results obtained using epinephrine (EPI) and sodium nitroprusside (SNP) to stimulate both a spun and a sham control PIJV segment. Please note that SNP was administered immediately upon observing a natural relaxation of the tissue post-stimulation with EPI. That is, SNP was administered at different times for the sham and spun PIJVs, depending on when the natural relaxation of the tissue (post stimulation with EPI) was observed. Outer diameter measurements of each PIJV segment over the duration of the experiments were normalized to the baseline outer diameter which was measured prior to administration of the first dose of EPI. 104

Figure 3.12 Results from vasomotor challenge experiments $(\mathrm{N}=4)$. There appears to be no significant difference in the level of contraction or dilation between the sham control and spun PIJVs. The data are presented as mean \pm standard error of the mean. 105

Figure 3.13 Results from the compliance and $\beta$-stiffness calculations for both sham (A \& C) and spun (B \& D) PIJVs over 24 hours. The data are presented as mean \pm standard error of

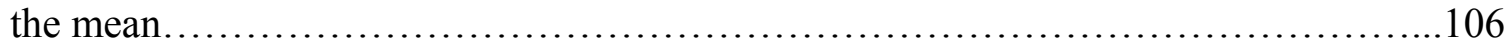

Figure 3.14 (A) shows a low magnification SEM image of the PIJV segment with the electrospun polymer deposited onto its adventitial surface. (B) is an SEM image (taken at 500x magnification) of the adventitial surface of the PIJV after the polymer wrap was applied. Note the high porosity of the polymer wrap. (C) is an SEM image (taken at 500x magnification) showing the attachment of the polymer wrap to the vein. (D) is an SEM image (taken at 500x magnification of the luminal surface of the vein and shows a continuous endothelium layer which appears to have remained intact. 107

Figure 3.15 Representative hematoxylin and eosin (A,B) and Masson's trichrome images (C,D) for both before perfusion and after wrapping procedure $(\mathrm{A}, \mathrm{C})$ and after 24 hours of ex vivo perfusion $(\mathrm{B}, \mathrm{D})$. Note the uniform thickness of the polymer wrap prior to perfusion, and the absence of the polymer wrap in the post-perfusion images. The single-headed arrow indicates the vessel lumen. The double-headed arrow in (A) and (C) indicates the thickness of the polymer wrap, which was not detectable in (B) or (D). 109

Figure 3.16 Representative birefringence images of vein sections stained with picrosirius red. The experimental conditions are defined as: Venous (VEN) conditions of $20 \mathrm{mmHg}$ pressure and $20 \mathrm{ml} / \mathrm{min}$ flowrate; pulsatile arterial (ART) conditions of 120/80 $\mathrm{mmHg}$ pressure and $100 \mathrm{ml} / \mathrm{min}$ mean flowrate; and wrapped arterial (wART) conditions where 
the wrapped vein segments were perfused under ART conditions for 24 hours ex vivo. The arrow indicates the vessel lumen.

Figure 3.17 Representative Movat's pentachrome staining of vein tissue sections. In each image collagen stains yellow, elastin and nuclei stain black, and muscle stains red. The red staining in the adventitial side of the wART sections is unspecific staining of culture media proteins that become entrapped within the polymer during ex vivo perfusion experiments. The arrow indicates the vessel lumen.. 111

Figure 3.18 Left: Representative image of Live/Dead ${ }^{\mathrm{TM}}$ stained tissue section from a spun PIJV. Right: Quantified Live/Dead ${ }^{\text {TM }}$ results to assess the level of necrosis in PIJVs after electrospinning, and after 18 and 92 hours of post-electrospinning static culture. The data shown was for a single experiment, and the error bars result from the 10 fields of view that were analyzed per PIJV segment. The data are presented as mean \pm standard error of the mean. 112

Figure 3.19 Representative immunohistochemistry images from the fluorescent based TUNEL analysis. The top two panels are from a 24-hour VEN (A) vs. ART (B) experiment. The next two panels are from a 24-hour ART (C) vs. cART (D) experiment. The third row of panels are from a 72-hour ART (E) vs. cART (F) experiment. The bottom two panels are from a 24-hour ART $(\mathrm{G})$ vs. wART $(\mathrm{H})$ experiment. The arrows indicate apoptotic cells. L indicates the PIJV lumen. 114

Figure 3.20 Quantified immunohistochemistry results from fluorescent based TUNEL analysis to assess the percentage of apoptotic cells within PIJVs from all the ex vivo vascular perfusion experiments. The data are presented as mean \pm standard error of the mean. 115

Figure 3.21 Representative immunohistochemistry images from the HRP/ABC based PCNA analysis. The top two panels are from a 24-hour VEN (A) vs. ART (B) experiment. The next two panels are from a 24-hour ART (C) vs. cART (D) experiment. The third row of panels are from a 72-hour ART (E) vs. cART (F) experiment. The bottom two panels are from a 24-hour ART $(\mathrm{G})$ vs. wART $(\mathrm{H})$ experiment. The arrows indicate proliferating cells. L indicates the PIJV lumen. 117

Figure 3.22 Quantified immunohistochemistry results from HRP/ABC based PCNA expression analysis to assess the percentage of proliferating cells within PIJVs from all the ex vivo vascular perfusion experiments. The data are presented as mean \pm standard error of the mean. 118

Figure 3.23 Representative immunohistochemistry images from the HRP/ABC based Golgi complex analysis. The top two panels are from a 24-hour VEN (A) vs. ART (B) experiment. The next two panels are from a 24-hour ART (C) vs. cART (D) experiment. The third row of panels are from a 72-hour ART (E) vs. cART (F) experiment. The bottom two panels are from a 24-hour ART (G) vs. wART (H) experiment. The arrows indicate positively stained cells. L indicates the PIJV lumen. 120 
Figure 3.24 Quantified immunohistochemistry results from HRP/ABC based Golgi complex expression analysis to assess the percentage cells staining positive for Golgi complex within PIJVs from all the ex vivo vascular perfusion experiments. The data are presented as mean \pm standard error of the mean. 121

Figure 3.25 Transmission electron microscopy (TEM) images of sections prepared from vein segments perfused, ex vivo, for 24 hours under (A) venous (VEN) conditions of 20 $\mathrm{mmHg}$ pressure and $20 \mathrm{ml} / \mathrm{min}$ flowrate, (B) pulsatile arterial (ART) conditions of $120 / 80 \mathrm{mmHg}$ pressure and $100 \mathrm{ml} / \mathrm{min}$ mean flowrate, and (C) conditioned arterial (cART) conditions where the pressure and flowrate were ramped in equal increments, gradually over 24 hours, from VEN to ART conditions. Note the random orientation of the actin cytoskeleton in (A) and (C) and the aligned orientation of the fibers in (B). Images were taken at a magnification of $60,000 \mathrm{x}$.

Figure 4.1 Schematic depicting the un-paired spun vs. sham AVG in vivo experiments. 127

Figure 4.2 Photographs taken within the animal facility. A) Hand-washing "scrub" area. B) Operating room equipped for two surgeries. C) Alejandro Nieponice, MD (left) and I performing one of the AVG implants.

Figure 4.3 Photograph showing a cannulated PIJV segment within the custom clamp immediately upon harvest. 130

Figure 4.4 Photograph showing a cannulated PIJV segment within (A) the electrospinning device and (B) within the custom clamp immediately post-electrospinning. 131

Figure 4.5 Photograph showing a spun cannulated PIJV segment just prior to being implanted as an AVG. 131

Figure 4.6 A) Wrapped PIJV segment during the electrospinning process. B) Wrapped PIJV implanted as a carotid interposition graft. C) Unwrapped PIJV graft. Note that the wrapped PIJV (B) does not expand under arterial pressure as does the unwrapped vein (C). 136

Figure 4.7 Fluoroscopic angiography images from both spun and sham AVGs. 138

Figure 4.8 Representative MPC staining images that were used for morphometric measurements of IH. Note: $\mathrm{T}_{\mathrm{I}}=$ intimal thickness; $\mathrm{T}_{\mathrm{M}}=$ medial thickness. 139

Figure 4.9 Summary of quantified results from morphometric measurements of IH. $\mathrm{P}<0.05$ was considered statistically significant. Note only a trend towards statistical significance was observed. 139

Figure 4.10 Low magnification (30x) SEM images from two in vivo experiments where the AVGs were not occluded. A and B were from an experiment where the grafts were fully patent. C and D are from an experiment where the grafts were only partially occluded. 
These images show the anastomotic interface between the vein graft and the carotid artery.

Figure A. 1 Solidworks technical drawing of vertical plate for piston/cylinder device frame.

Figure A. 2 Solidworks technical drawing of horizontal plate for piston/cylinder device frame 152

Figure A. 3 Solidworks technical drawing of isometric view of horizontal plate for piston/cylinder device frame. 153

Figure A. 4 Solidworks technical drawing of pillow cushion support for piston/cylinder device frame. 154

Figure A. 5 Solidworks technical drawing of piston plate for piston/cylinder device frame 155

Figure A. 6 Solidworks technical drawing of piston rod for piston/cylinder device .156

Figure A. 7 Solidworks technical drawing of piston head for piston/cylinder device. 157

Figure A. 8 Solidworks technical drawing of the piston cylinder mount for piston/cylinder device. 158

Figure A. 9 Solidworks technical drawing of right side view of vessel tee assembly 159

Figure A. 10 Solidworks technical drawing of top view of vessel tee

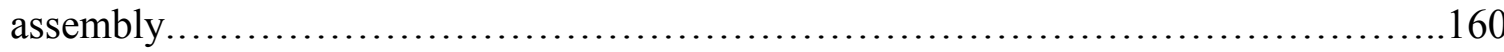

Figure A. 11 Solidworks technical drawing of vessel tee assembly. .161

Figure A. 12 Solidworks technical drawing of isometric view of tissue housing chamber assembly. 162

Figure A. 13 Solidworks technical drawing of exploded isometric view of tissue housing chamber assembly.

Figure A. 14 Solidworks technical drawing of right side view of tissue housing chamber assembly .164

Figure A. 15 Solidworks technical drawing of top view of tissue housing chamber assembly. 165 
Figure A. 16 Solidworks technical drawing of right side view (window) of tissue housing chamber assembly. 166

Figure A. 17 Solidworks technical drawing of top view (window) of tissue housing chamber assembly.

Figure A. 18 Solidworks technical drawing of back view of tissue housing chamber assembly .168

Figure A. 19 Solidworks technical drawing of isometric view of tissue housing chamber assembly. .169

Figure A. 20 Solidworks technical drawing of lid for tissue housing chamber assembly............................................................. 170

Figure A. 21 Solidworks technical drawing of lid for tissue housing chamber assembly..... 171

Figure A. 22 Solidworks technical drawing of spool-block for tissue housing chamber assembly..... 172

Figure A. 23 Solidworks technical drawing of peg for tissue housing chamber assembly............................................................. 173

Figure A. 24 Solidworks technical drawing of spool-block nut for tissue housing chamber assembly. .174

Figure A. 25 Solidworks technical drawing of front spool for tissue housing chamber assembly..... 175

Figure A. 26 Solidworks technical drawing of spool-rod for tissue housing chamber assembly..... 176

Figure A. 27 Solidworks technical drawing of right side view of in vivo length blood vessel clamp assembly.

Figure A. 28 Solidworks technical drawing of right side view (detail) of in vivo length blood vessel clamp assembly.................................................. 178

Figure A. 29 Solidworks technical drawing of front view of in vivo length blood vessel clamp assembly.

Figure A. 30 Solidworks technical drawing of top view of in vivo length blood vessel clamp assembly... 180 
Figure A. 31 Solidworks technical drawing of back view of in vivo length blood vessel clamp assembly..... 181

Figure A. 32 Solidworks technical drawing of isometric view of in vivo length blood vessel clamp assembly

Figure A. 33 Solidworks technical drawing of front view of slider for in vivo length blood vessel clamp assembly.....

Figure A. 34 Solidworks technical drawing of top view of slider for in vivo length blood vessel clamp assembly...... 184

Figure A. 35 Solidworks technical drawing of isometric view of slider for in vivo length blood vessel clamp assembly......................................................... 185

Figure A. 36 Solidworks technical drawing of right side view of guide-rail for transport box assembly...... 186

Figure A. 37 Solidworks technical drawing of front view of guide-rail for transport box assembly. 187

Figure A. 38 Solidworks technical drawing of top view of guide-rail for transport box assembly 188

Figure A. 39 Solidworks technical drawing of isometric view of guide-rail for transport box assembly. 189

Figure A. 40 Solidworks technical drawing of isometric view of transport box assembly. 190

Figure A. 41 Solidworks technical drawing of right side view of lid for transport box assembly.....

Figure A. 42 Solidworks technical drawing of top view of lid for transport box assembly. 192

Figure A. 43 Solidworks technical drawing of isometric view of lid for transport box assembly..... 193

Figure A. 44 Solidworks technical drawing of right side view of top for transport box assembly. 194

Figure A. 45 Solidworks technical drawing of top view of top for transport box assembly..... 195

Figure A. 46 Solidworks technical drawing of isometric view of top for transport box assembly...... 196 
Figure A. 47 Solidworks technical drawing of top view of middle for transport box assembly

Figure A. 48 Solidworks technical drawing of isometric view of middle for transport box assembly 198

Figure A. 49 Solidworks technical drawing of right side view of bottom for transport box

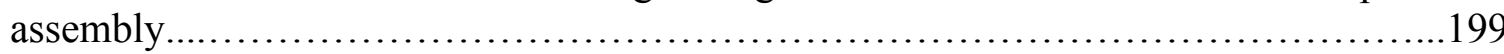

Figure A. 50 Solidworks technical drawing of top view of bottom for transport box

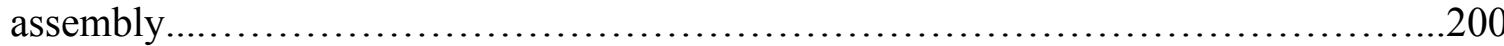

Figure A. 51 Solidworks technical drawing of isometric view of bottom for transport box assembly 201

Figure D. 1 Mean intraluminal pressure, outer diameter, and CWS profiles for 24 hour ART vs. cART experiment performed on 10/22/2004..... .253

Figure D. 2 Mean intraluminal pressure, outer diameter, and CWS profiles for 24 hour ART vs. cART experiment performed on 11/01/2004. 254

Figure D. 3 Mean intraluminal pressure, outer diameter, and CWS profiles for 24 hour ART vs. cART experiment performed on 11/11/2004. .255

Figure D. 4 Mean intraluminal pressure, outer diameter, and CWS profiles for 24 hour ART vs. cART experiment performed on 09/21/2006. 256

Figure D. 5 Mean intraluminal pressure, outer diameter, and CWS profiles for 24 hour ART vs. cART experiment performed on 10/16/2006. 257

Figure D. 6 Mean intraluminal pressure, outer diameter, and CWS profiles for 72 hour ART vs. cART experiment performed on $02 / 16 / 2007$. .258

Figure D. 7 Mean intraluminal pressure, outer diameter, and CWS profiles for 72 hour ART vs. cART experiment performed on 03/07/2007. 259

Figure D. 8 Mean intraluminal pressure, outer diameter, and CWS profiles for 72 hour ART vs. cART experiment performed on $03 / 15 / 2007$ 260

Figure D. 9 Mean intraluminal pressure, outer diameter, and CWS profiles for 72 hour ART vs. cART experiment performed on 04/02/2007. .261

Figure D. 10 Vasomotor challenge outer diameter profiles for acute experiment performed on $03 / 22 / 2007$ 262 
Figure D. 11 Vasomotor challenge outer diameter profiles for acute experiment performed on $05 / 17 / 2007$ 263

Figure D. 12 Vasomotor challenge outer diameter profiles for acute experiment performed on $05 / 23 / 2007$ 264

Figure D. 13 Vasomotor challenge outer diameter profiles for acute experiment performed on 06/07/2007. 265

Figure E. 1 Diagram of the Mini-PROTEAN 3 cell from Bio-Rad. Image adapted from the MiniPROTEAN 3 Cell Instruction Manual. 297

Figure E. 2 Diagram of Mini Trans-Blot Electrophoretic Transfer Cell from Bio-Rad. Image adapted from the Mini-PROTEAN 3 Cell Instruction Manual. 301

Figure E. 3 Diagram of the assembly of the gel transfer cassettes. Image adapted from the MiniPROTEAN 3 Cell Instruction Manual. 302

Figure F. 1 Representative Live/Dead ${ }^{\mathrm{TM}}$ images from all three conditions (92 hour timepoint) of the experiment to assess tissue necrosis in electrospun PIJVs. Note: blue, nuclei; green, live cells; red, necrotic cells. All images were taken at 100x magnification. 310

Figure F. 2Representative TUNEL images from 24 hour VEN vs. ART experiment performed on 04/23/2003. Nuclei are stained blue and TUNEL positive cells are stained red. 311

Figure F. 3 Representative TUNEL images from 24 hour VEN vs. ART experiment performed on 05/07/2003. Nuclei are stained blue and TUNEL positive cells are stained red........311

Figure F. 4 Representative TUNEL images from 24 hour VEN vs. ART experiment performed on 10/09/2004. Nuclei are stained blue and TUNEL positive cells are stained red 312

Figure F. 5 Representative TUNEL images from 24 hour VEN vs. ART experiment performed on 10/16/2004. Nuclei are stained blue and TUNEL positive cells are stained red........312

Figure F. 6 Representative TUNEL images from 24 hour VEN vs. ART experiment performed on $05 / 24 / 2006$. Nuclei are stained blue and TUNEL positive cells are stained red........313

Figure F. 7 Representative TUNEL images from 24 hour VEN vs. ART experiment performed on 09/14/2006. Nuclei are stained blue and TUNEL positive cells are stained red........313

Figure F. 8 Representative TUNEL images from 24 hour ART vs. cART experiment performed on 10/22/2004. Nuclei are stained blue and TUNEL positive cells are stained red........314

Figure F.9 Representative TUNEL images from 24 hour ART vs. cART experiment performed on 11/01/2004. Nuclei are stained blue and TUNEL positive cells are stained red 314 
Figure F. 10 Representative TUNEL images from 24 hour ART vs. cART experiment performed on 11/11/2004. Nuclei are stained blue and TUNEL positive cells are stained red.

Figure F. 11 Representative TUNEL images from 24 hour ART vs. cART experiment performed on 09/21/2006. Nuclei are stained blue and TUNEL positive cells are stained red..... 315

Figure F. 12 Representative TUNEL images from 24 hour ART vs. cART experiment performed on 10/16/2006. Nuclei are stained blue and TUNEL positive cells are stained red. 316

Figure F. 13 Representative TUNEL images from 72 hour ART vs. cART experiment performed on 02/16/2007. Nuclei are stained blue and TUNEL positive cells are stained red 316

Figure F. 14 Representative TUNEL images from 72 hour ART vs. cART experiment performed on 03/07/2007. Nuclei are stained blue and TUNEL positive cells are stained red. 317

Figure F. 15 Representative TUNEL images from 72 hour ART vs. cART experiment performed on 03/15/2007. Nuclei are stained blue and TUNEL positive cells are stained red. 317

Figure F. 16 Representative TUNEL images from 72 hour ART vs. cART experiment performed on 04/02/2007. Nuclei are stained blue and TUNEL positive cells are stained red...... 318

Figure F. 17 Representative TUNEL images from 72 hour ART vs. cART experiment performed on 04/26/2007. Nuclei are stained blue and TUNEL positive cells are stained red..... 318

Figure F. 18 Representative TUNEL images from 24 hour ART vs. wART experiment performed on 10/23/2006. Nuclei are stained blue and TUNEL positive cells are stained red. 319

Figure F. 19 Representative TUNEL images from 24 hour ART vs. wART experiment performed on 10/30/2006. Nuclei are stained blue and TUNEL positive cells are stained red..... 319

Figure F. 20 Representative TUNEL images from 24 hour ART vs. wART experiment performed on 11/06/2006. Nuclei are stained blue and TUNEL positive cells are stained red. 
Figure F. 20 Representative TUNEL images from 24 hour ART vs. wART experiment performed on 11/06/2006. Nuclei are stained blue and TUNEL positive cells are stained red. 320

Figure F. 22 Representative TUNEL images from 24 hour ART vs. wART experiment performed on 11/20/2006. Nuclei are stained blue and TUNEL positive cells are stained red. 321

Figure F. 23 Representative TUNEL images from 24 hour ART vs. wART experiment performed on 11/27/2006. Nuclei are stained blue and TUNEL positive cells are stained red. 321

Figure F. 24 Representative PCNA images from 24 hour VEN vs. ART experiment performed on 04/23/2003. Nuclei are stained blue and PCNA positive cells are stained brown...... 325

Figure F. 25 Representative PCNA images from 24 hour VEN vs. ART experiment performed on 05/07/2003. Nuclei are stained blue and PCNA positive cells are stained brown...... .325

Figure F. 25 Representative PCNA images from 24 hour VEN vs. ART experiment performed on 05/07/2003. Nuclei are stained blue and PCNA positive cells are stained brown 326

Figure F. 27 Representative PCNA images from 24 hour VEN vs. ART experiment performed on 10/16/2004. Nuclei are stained blue and PCNA positive cells are stained brown...... .326

Figure F. 28 Representative PCNA images from 24 hour VEN vs. ART experiment performed on 05/24/2006. Nuclei are stained blue and PCNA positive cells are stained brown .327

Figure F. 29 Representative PCNA images from 24 hour VEN vs. ART experiment performed on 09/14/2006. Nuclei are stained blue and PCNA positive cells are stained brown...... 327

Figure F. 30 Representative PCNA images from 24 hour ART vs. cART experiment performed on 10/22/2004. Nuclei are stained blue and PCNA positive cells are stained brown 328

Figure F. 31 Representative PCNA images from 24 hour ART vs. cART experiment performed on 11/01/2004. Nuclei are stained blue and PCNA positive cells are stained brown 328 
Figure F. 32 Representative PCNA images from 24 hour ART vs. cART experiment performed on 11/11/2004. Nuclei are stained blue and PCNA positive cells are stained brown...... 329

Figure F. 33 Representative PCNA images from 24 hour ART vs. cART experiment performed on 09/21/2006. Nuclei are stained blue and PCNA positive cells are stained brown...... 329

Figure F. 34 Representative PCNA images from 24 hour ART vs. cART experiment performed on 10/16/2006. Nuclei are stained blue and PCNA positive cells are stained brown. 330

Figure F. 35 Representative PCNA images from 72 hour ART vs. cART experiment performed on 02/16/2007. Nuclei are stained blue and PCNA positive cells are stained brown....... 330

Figure F. 36 Representative PCNA images from 72 hour ART vs. cART experiment performed on 03/07/2007. Nuclei are stained blue and PCNA positive cells are stained brown...... 331

Figure F. 37 Representative PCNA images from 72 hour ART vs. cART experiment performed on 03/15/2007. Nuclei are stained blue and PCNA positive cells are stained brown 331

Figure F. 38 Representative PCNA images from 72 hour ART vs. cART experiment performed on 04/02/2007. Nuclei are stained blue and PCNA positive cells are stained brown...... 332

Figure F. 39 Representative PCNA images from 72 hour ART vs. cART experiment performed on 04/26/2007. Nuclei are stained blue and PCNA positive cells are stained brown. .332

Figure F. 40 Representative PCNA images from 24 hour ART vs. wART experiment performed on 10/23/2006. Nuclei are stained blue and PCNA positive cells are stained brown. 333

Figure F. 41 Representative PCNA images from 24 hour ART vs. wART experiment performed on 10/30/2006. Nuclei are stained blue and PCNA positive cells are stained brown...... 333

Figure F. 42 Representative PCNA images from 24 hour ART vs. wART experiment performed on 11/06/2006. Nuclei are stained blue and PCNA positive cells are stained brown...... 334 
Figure F. 43 Representative PCNA images from 24 hour ART vs. wART experiment performed on 11/13/2006. Nuclei are stained blue and PCNA positive cells are stained brown...... .334

Figure F. 44 Representative PCNA images from 24 hour ART vs. wART experiment performed on 11/20/2006. Nuclei are stained blue and PCNA positive cells are stained brown....... 335

Figure F. 45 Representative PCNA images from 24 hour ART vs. wART experiment performed on 11/27/2006. Nuclei are stained blue and PCNA positive cells are stained brown. 335

Figure F. 46 Representative Golgi complex images from 24 hour VEN vs. ART experiment performed on 04/23/2003. Nuclei are stained blue and Golgi complex positive cells are stained brown 339

Figure F. 47 Representative Golgi complex images from 24 hour VEN vs. ART experiment performed on 05/07/2003. Nuclei are stained blue and Golgi complex positive cells are stained brown 339

Figure F. 48 Representative Golgi complex images from 24 hour VEN vs. ART experiment performed on 10/09/2004. Nuclei are stained blue and Golgi complex positive cells are stained brown. 340

Figure F. 49 Representative Golgi complex images from 24 hour VEN vs. ART experiment performed on 10/16/2004. Nuclei are stained blue and Golgi complex positive cells are stained brown .340

Figure F. 50 Representative Golgi complex images from 24 hour VEN vs. ART experiment performed on 05/24/2006. Nuclei are stained blue and Golgi complex positive cells are stained brown 341

Figure F. 51 Representative Golgi complex images from 24 hour VEN vs. ART experiment performed on 09/14/2006. Nuclei are stained blue and Golgi complex positive cells are stained brown 341

Figure F. 52 Representative Golgi complex images from 24 hour ART vs. cART experiment performed on 10/22/2004. Nuclei are stained blue and Golgi complex positive cells are stained brown 342

Figure F. 53 Representative Golgi complex images from 24 hour ART vs. cART experiment performed on 11/01/2004. Nuclei are stained blue and Golgi complex positive cells are stained brown. 342 
Figure F. 54 Representative Golgi complex images from 24 hour ART vs. cART experiment performed on 11/11/2004. Nuclei are stained blue and Golgi complex positive cells are stained brown .343

Figure F. 55 Representative Golgi complex images from 24 hour ART vs. cART experiment performed on 09/21/2006. Nuclei are stained blue and Golgi complex positive cells are stained brown 343

Figure F. 56 Representative Golgi complex images from 24 hour ART vs. cART experiment performed on 10/16/2006. Nuclei are stained blue and Golgi complex positive cells are stained brown 344

Figure F. 57 Representative Golgi complex images from 72 hour ART vs. cART experiment performed on 02/16/2007. Nuclei are stained blue and Golgi complex positive cells are stained brown. 344

Figure F. 58 Representative Golgi complex images from 72 hour ART vs. cART experiment performed on 03/07/2007. Nuclei are stained blue and Golgi complex positive cells are stained brown 345

Figure F. 59 Representative Golgi complex images from 72 hour ART vs. cART experiment performed on 03/15/2007. Nuclei are stained blue and Golgi complex positive cells are stained brown 345

Figure F. 60 Representative Golgi complex images from 72 hour ART vs. cART experiment performed on 04/02/2007. Nuclei are stained blue and Golgi complex positive cells are stained brown .346

Figure F. 61 Representative Golgi complex images from 72 hour ART vs. cART experiment performed on 04/26/2007. Nuclei are stained blue and Golgi complex positive cells are stained brown 346

Figure F. 62 Representative Golgi complex images from 24 hour ART vs. wART experiment performed on 10/23/2006. Nuclei are stained blue and Golgi complex positive cells are stained brown 347

Figure F. 63 Representative Golgi complex images from 24 hour ART vs. wART experiment performed on 10/30/2006. Nuclei are stained blue and Golgi complex positive cells are stained brown 347

Figure F. 64 Representative Golgi complex images from 24 hour ART vs. wART experiment performed on 11/06/2006. Nuclei are stained blue and Golgi complex positive cells are stained brown. 348 
Figure F. 65 Representative Golgi complex images from 24 hour ART vs. wART experiment performed on 11/13/2006. Nuclei are stained blue and Golgi complex positive cells are stained brown. 348

Figure F. 66 Representative Golgi complex images from 24 hour ART vs. wART experiment performed on 11/20/2006. Nuclei are stained blue and Golgi complex positive cells are stained brown 349

Figure F. 67 Representative Golgi complex images from 24 hour ART vs. wART experiment performed on 11/27/2006. Nuclei are stained blue and Golgi complex positive cells are stained brown. 349

Figure G. 1 Image of both sham and spun AVGs implanted as carotid interposition grafts on $06 / 11 / 2007$. Scale bar is $1 \mathrm{~cm}$. 354

Figure G. 2 Image of both sham and spun AVGs implanted as carotid interposition grafts on $06 / 26 / 2007$. Scale bar is $1 \mathrm{~cm}$. 354

Figure G. 3 Image of both sham and spun AVGs implanted as carotid interposition grafts on $06 / 27 / 2007$. Scale bar is $1 \mathrm{~cm}$ 355

Figure G. 4 Image of both sham and spun AVGs implanted as carotid interposition grafts on $06 / 28 / 2007$. Scale bar is $1 \mathrm{~cm}$. 355

Figure G. 5 Representative image of MPC stained section from sham AVG implanted as carotid interposition graft on 12/04/2006. .356

Figure G. 6 Representative images of MPC stained sections from both sham and spun AVGs implanted as carotid interposition grafts on 12/05/2006. .356

Figure G. 7 Representative images of MPC stained sections from both sham and spun AVGs implanted as carotid interposition grafts on 12/06/2006.

Figure G. 8 Representative images of MPC stained sections from both sham and spun AVGs implanted as carotid interposition grafts on 06/11/2007.

Figure G. 9 Representative image of MPC stained sections from sham AVG implanted as carotid interposition graft on $06 / 26 / 2007$

Figure G. 10 Representative images of MPC stained sections from both sham and spun AVGs implanted as carotid interposition grafts on $06 / 27 / 2007$...... .358

Figure G. 11 Low magnification SEM image of sham AVG implanted as a carotid interposition graft on $06 / 11 / 2007$ 360 
Figure G. 12 Low magnification SEM image of spun AVG implanted as a carotid interposition

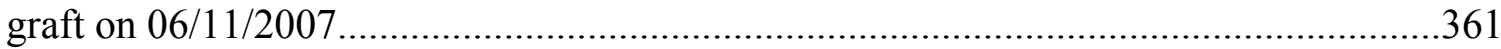

Figure G. 13 Low magnification SEM image of sham AVG implanted as a carotid interposition

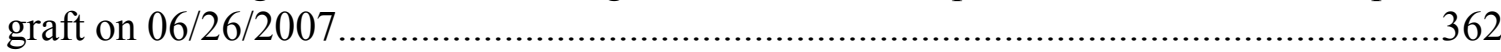

Figure G. 14 Low magnification SEM image of spun AVG implanted as a carotid interposition graft on $06 / 26 / 2007$. .363

Figure G. 15 Low magnification SEM image of spun AVG implanted as a carotid interposition graft on $06 / 28 / 2007$ 364

Figure H. 1 Flowchart depicting the molecular biological analyses that were either successfully evaluated, or attempted but discontinued because troubleshooting them was beyond the scope of this dissertation 366

Figure H. 2 Representative immunohistochemistry images taken from a VEN vs. ART 24 hour experiment. In all images, red represents positive staining, and the nuclei label blue. Images were taken at 400x magnification under immersion oil. In all panels the white arrow designates the vessel lumen, and the red arrow indicates the observed autofluorescence. 367

Figure H. 3 Fluorescent microscopy images of PIJV tissue perfused ex vivo under ART conditions for 24 hours stained with two dilutions of mouse-anti-human primary PCNA antibody. Note: PD, primary delete; DAPI, nuclear stain; Alexa-488, donkey-anti-mouse secondary antibody. The red arrow indicates the observed autofluorescence 368

Figure H. 4 Fluorescent microscopy images of PIJV tissue perfused ex vivo under cART conditions for 24 hours stained with two dilutions of mouse-anti-human primary PCNA antibody. Note: PD, primary delete; DAPI, nuclear stain; Alexa-488, donkey-anti-mouse secondary antibody. The red arrow indicates the observed autofluorescence. 368

Figure H. 5 Fluorescent microscopy images of PIJV tissue: PFA Baseline, porcine femoral artery fixed immediately after harvest; PIJV Baseline, fixed immediately after harvest; and either perfused ex vivo under ART or cART conditions for 24 hours stained with mouseanti-human primary ILK antibody. Note: PD, primary delete; DAPI, nuclear stain; 488, donkey-anti-mouse secondary antibody. The red arrow indicates the observed autofluorescence 369

Figure H. 6 Fluorescent microscopy images of PIJV tissue: PFA Baseline, porcine femoral artery fixed immediately after harvest; PIJV Baseline, fixed immediately after harvest; and either perfused ex vivo under ART or cART conditions for 24 hours stained with mouseanti-human primary Mig-2 antibody. Note: PD, primary delete; DAPI, nuclear stain; 488, donkey-anti-mouse secondary antibody. The red arrow indicates the observed autofluorescence. 
Figure H. 7 HRP/ABC based IHC images of PIJV tissue perfused ex vivo for 24 hours either under ART or cART conditions and stained with ILK and Mig-2 mouse-anti-human primary antibodies. Unspecific binding of primary antibodies results in diffuse brown staining throughout the tissue.... 371

Figure H. $8 \mathrm{HRP} / \mathrm{ABC}$ based IHC images of PIJV tissue perfused ex vivo for 24 hours either under VEN or ART conditions and stained with a SPARC rabbit-anti-human primary antibody. The panels on the left are primary deletes. Unspecific binding of primary antibodies results in diffuse brown staining throughout the tissue. 372

Figure H. 9 Representative western blots performed on total protein isolates from various ex vivo vascular perfusion experiments. The western blots shown were probed for ILK (A), Mig2 (B), SPARC (C), and TN-C (D). Both chemiluminescent (A \& C) and alkalinephosphatase (B \& D) detection techniques were attempted. Note the extra bands, both above and below the correct respective molecular weights, detected in each blot for each protein of interest..... 374

Figure H. $10 \mathrm{Gel}$ representation of capillary electrophoresis result from a representative sample. The two major bands represent the $28 \mathrm{~s}$ and $18 \mathrm{~s}$ ribosomal subunits. Note the clean bands with little or no degradation. 375

Figure H. 11 Transmission electron microscopy (TEM) images of sections prepared from vein segments perfused, ex vivo, for 24 hours under venous VEN vs. ART conditions. The top two panels are from an experiment performed on 05/07/2003. The bottom two panels are from an experiment performed on 10/09/2004. Images were taken at a magnification of $30,000 x$

Figure H. 12 Transmission electron microscopy (TEM) images of sections prepared from vein segments perfused, ex vivo, for 24 hours under venous VEN vs. ART conditions. The images are from an experiment performed on 10/16/2004. Images were taken at a magnification of $30,000 \mathrm{x}$ .378

Figure H. 13 Transmission electron microscopy (TEM) images of sections prepared from vein segments perfused, ex vivo, for 24 hours under venous ART vs. cART conditions. The top two panels are from an experiment performed on 10/22/2004. The bottom two panels are from an experiment performed on 11/11/2004. Images were taken at a magnification of $30,000 x$. 


\section{PREFACE}

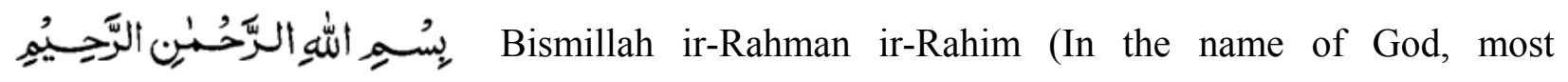
gracious, most merciful). The completion of this doctoral dissertation would not have been possible without all the help and support of my family, friends, and colleagues. I would like to begin by thanking my family. My wonderful son Isaac has truly been my source of strength and motivation in pursuing my Ph.D. Since he was born, he has provided me with more sense of purpose than any man could ever need. I would like to thank my mother for always being there for me with an encouraging word to get me through the rough times, and also for instilling in me the importance of education. She is truly an amazing woman. I would next like to thank my father (god rest his soul) for his encouragement, and for providing me with a great work ethic. He was the hardest working man that I ever knew. Finally, my two loving sisters have always been there whenever I needed anything, and words alone cannot describe my appreciation for their kindness.

I am truly blessed with great friends who have all influenced my life in their own ways, and must be ackowledged. My best friend Mr. Robert Glencross (god rest his soul) died at such a young age, and didn't get to see me finish my Ph.D. He was as close as a brother, and his family (Troy, Bob, Rick, John, Lynne, Karen, Betty and Dick) were like my second family. Since moving to Pittsburgh over eight years ago, I was fortunate to have developed some great 
friendships, the greatest of which was with Mr. Jack Dummar. He and I have had some very interesting discussions over the years about anything and everything. Although not a scientist by training, his thirst for knowledge and the questions he raised about my work were always insightfull and challenging.

Since beginning my tenure in the Vorp Laboratory over 8 years ago, I have had the pleasure to interact with some fantastic people. I would first of all like to thank my mentor Dr. David Vorp who took a chance on a young man from Nova Scotia by hiring me to manage his lab immediately after I finished my bachelor degrees, and then again by offering me the opportunity of a lifetime to do my Ph.D. with him. He was a very wise mentor, always giving me the guidance that I needed to navigate my way into the field of biomedical research. His rigorous training program has provided me with a very strong foundation to build upon in pursuing an academic career. I would like to personally thank all my lab mates, past and present. Special thanks must be given to: Don Severyn, Stephanie Kute, Tim Maul, Scott Van Epps, Alex Neiponice, Doug Chew, Deborah Cleary, Lorenzo Soletti, Brian Morelli, Wei He, Melissa Morgan, and last but certainly not least Roberta Melick. I could not have been blessed with a finer group of people to call my colleagues.

I would like to express a very special thank you to Dr. Harvey Borovetz for his continued help and support in so many ways over the years. He is truly a wonderful role-model for all of his students. I would also very much like to thank Lynette Spataro and Joan Williamson for ensuring that my immigration and departmental paperwork were always in order, and for generally making my life easier! The Bioengineering Department at the University of Pittsburgh was truly a fantastic place to complete my graduate education, and I am very fortunate to have had such a unique learning experience. 
The following dissertation represents the efforts of approximately the last six years of my life. I hope that readers find here some useful piece of information that may somehow help them in their own endavors, or that this work at least be a warning to them of what not to do! 


\subsection{INTRODUCTION}

Coronary artery disease, leading to myocardial infarction and ischemia, is currently the number one cause of morbidity and mortality worldwide. Current treatment alternatives consist of percutaneous transluminal angioplasty, stenting, and coronary artery bypass grafting (CABG). CABG can be carried out using either arterial or venous conduits and is the most effective and most widely used treatment to combat coronary arterial stenosis, with nearly 500,000 procedures being performed annually in the United States alone [5]. In addition there are approximately 80,000 lower extremity bypass surgeries performed annually [6]. The venous conduit used for bypass procedures is most frequently the autogenous saphenous vein and remains the graft of choice for $95 \%$ of surgeons performing these bypass procedures [7,8]. According to the American Heart Association, in 2006 there were 427,000 bypass procedures performed in 249,000 patients [5]. The long term outcome of these procedures is limited due to occlusion of the graft vessel or anastomotic site as a result of intimal hyperplasia (IH), which can occur over a timeframe of months to years (Table 1.1).

Development of successful small diameter synthetic or tissue engineered vascular grafts has yet to be accomplished and use of arterial grafts (internal mammary, radial, or gastroepiploic arteries, for example) is limited by the short size, small diameter and availability of these vessels. Therefore, vein segments, such as the saphenous vein, are widely used as arterial grafts. Despite their wide use, failure of arterial vein grafts (AVGs) remains a major problem [9]: 12\% to 27\% of AVGs become occluded in the first year with a subsequent annual occlusive rate of $2 \%$ to $4 \%$ [6,10-12]. Patients with failed AVGs will die or require re-operation. 
Table 1.1 The chronological events leading to vein graft intimal hyperplasia adapted from Mitra et al. [1]. Please note: ECM, extracellular matrix; SMC, smooth muscle cell.

Graft insertion causes haemodynamic changes

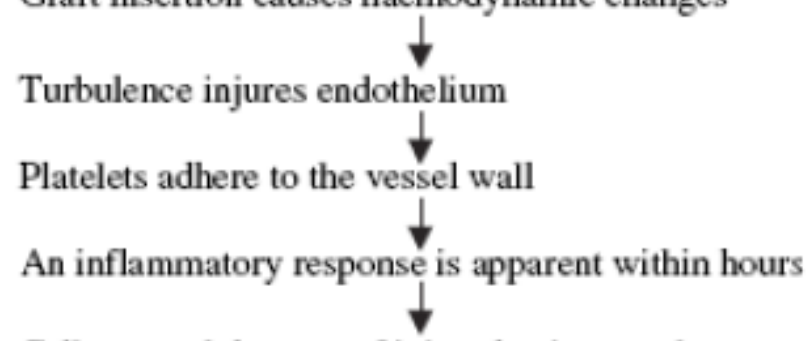

Cells around the area of injury begin to undergo apoptosis within

$1 \mathrm{~h}$ of injury; apoptosis peaks at approximately $6 \mathrm{~h}$

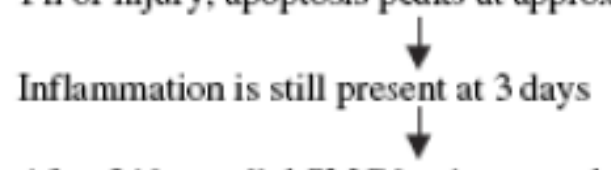

After $24 \mathrm{~h}$, medial SMC begin to synthesize DNA; cell proliferation

increases 20 - to 30 - fold

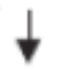

Endothelium begins to regenerate from the edges of the

injury zone

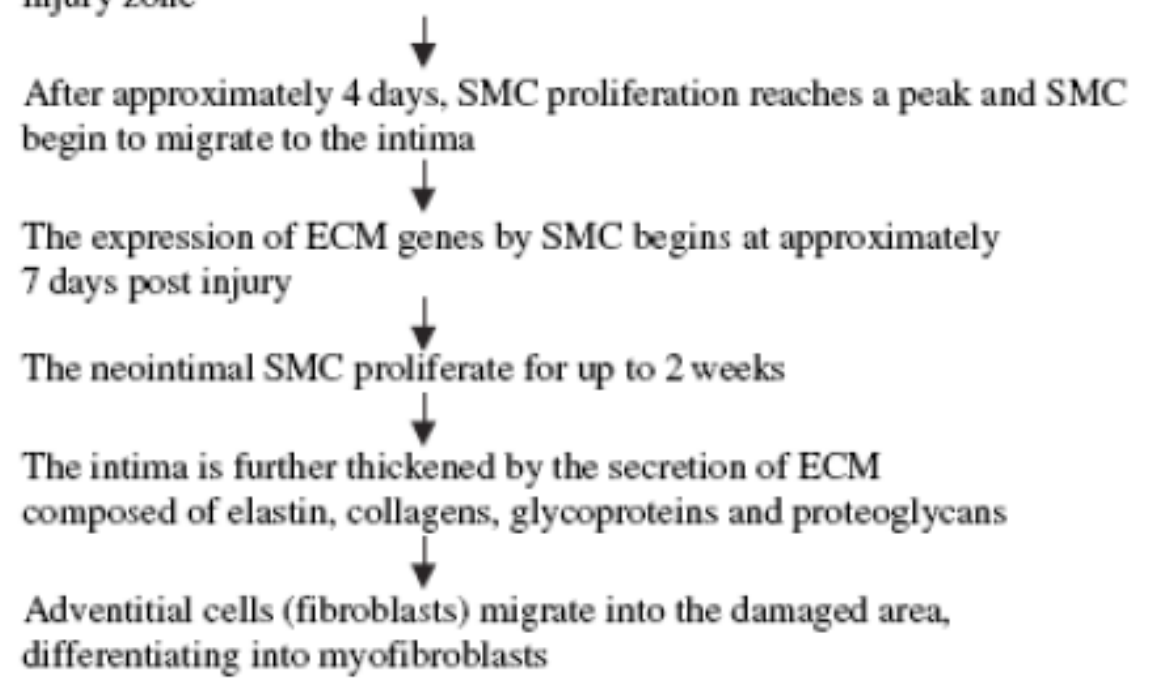

IH accounts for $20 \%$ to $40 \%$ of all AVG failures within the first 5 years [13-17]. Several studies have determined that $\mathrm{IH}$ develops, to some extent, in all mature AVGs and this is 
regarded by many as an unavoidable response of the vein to grafting [18-26]. IH is characterized by phenotypic modulation, followed by de-adhesion and migration of medial and adventitial smooth muscle cells (SMCs) and myofibroblasts into the intima where they proliferate. In many cases, this response can lead to stenosis and diminished blood flow through the graft. It is thought that IH may be initiated by the abrupt exposure of the veins to the dynamic mechanical environment of the arterial circulation [27-37].

Vein segments transposed to the arterial circulation for use as bypass grafts are exposed to increased blood flow and intraluminal pressure [38], and as shown previously by our laboratory, cyclic wall motion (including bending, twisting and stretching) due to their attachment to the beating heart in the case of CABGs [39]. Since veins are much thinner walled and more fragile than arteries, they experience significantly greater stresses in the arterial circuit than those to which they are accustomed in the venous circuit. Indeed, Liu and Fung showed that the average circumferential wall stress (CWS) in an AVG immediately upon reestablishing arterial flow could be 140-fold that in a vein under normal circumstances [30]. This dramatic increase in CWS is due to the AVG being distended to its maximum diameter under arterial pressure. The tissue responds to this perceived injury by thickening, which is thought to be an attempt to return the stress to venous levels. However, this response is uncontrolled and can over-compensate, leading to stenosis instead of the desired thickening or "arterialization" of the vein segment.

It has been suggested that the hyperplastic response by AVGs is a direct result of a "cellular shock" that occurs as a result of their abrupt exposure to the arterial biomechanical environment [27-37]. Preventing acute distension of AVGs by adding an external structural support (or sheath) has seemingly improved the patency of vein grafts [26,31,40-55], for 
example. However, due to one or more fundamental limitations, these previous approaches have not resulted in a clinically viable means for improving AVG patency. All of these previous approaches utilized adventitially placed wraps/sheaths that were biodurable, and/or loose-fitting. The methodology presented here improves upon previous work in an effort to make such a treatment alternative more clinically viable by addressing these limitations. It is our belief that the adverse hyperplastic response by AVGs may instead be reduced or eliminated by more gradually exposing them to the arterial biomechanical environment. That is, if a vein is given an ample opportunity to adapt and remodel to the stresses of its new environment, cellular injury may be reduced, thus limiting the initiating mechanisms of IH. Attempts to inhibit or counter-act AVG failure mechanisms may be facilitated by the placement of a biodegradable polymer wrap on the adventitial surface. Such a wrap could be used as a means to gradually impose arterial levels of CWS to the AVG, thus avoiding the "cellular shock" associated with IH. Such a wrap would require the following characteristics:

i) The biodegradation rate of the wrap must be tunable

ii) The wrap must be tight-fitting in order to prevent distension under arterial pressure

iii) The wrap must not affect tissue viability or functionality

One possible means to achieve the placement of a biodegradable polymer wrap onto an AVG is through electrospinning immediately following harvest and just prior to implantation. The process of electrospinning involves the delivery of an electrically charged polymer solution through a nozzle onto an oppositely charged mandrel [56]. The fibrous polymer accumulates and aligns depending on the shape and movement of the mandrel. To our knowledge there have been no previous reports of electrospinning a polymer onto living tubular tissues. 
Clearly, developing a reliable means to prevent the early events of the IH process would contribute to improvements in the outcome of arterial bypass procedures. Therefore, the longterm goal of this work is to develop a new paradigm that would utilize mechanical conditioning, in the form of an adventitially-placed electrospun biodegradable polymer wrap, to enable an AVG to arterialize after implantation by attenuating the hyperplastic response. The biodegradable polymer wrap, which acts essentially as a temporary "girdle", is tunable so that the degradation rate can be controlled and thus the mechanical support to the vein manipulated as desired [57]. That is, as the polymer degrades over a desired period of time the mechanical support offered by it is reduced over that period and the AVG would be exposed to gradually increasing levels of CWS. This new approach could be used as a peri-surgical tool for the modification of vein segments intended for use as AVGs. In its perceived ultimate form, this "adaptive modification" would be performed by treating the vein at bedside, immediately after saphenectomy and just prior to the arterial bypass surgery. For example, after the saphenous vein is harvested, and while the surgeon is exposing the surgical site, the polymer wrap would be electrospun onto the vein just prior to it being used for the bypass procedure.

Another potential application for the electrospun biodegradable polymer wrap is a vehicle for the delivery of other modes of support to AVGs. While modification of the mechanical environment of an AVG over time could itself improve patency, the proposed work could also result in a paradigm shift in the delivery of not only mechanical but also molecular (genes), biochemical (drugs), and/or biological (cellular) support to AVGs. By tuning an electrospun polymer wrap to degrade at a desired rate, the rate of delivery of these support modalities could be tuned. We believe that mechanical, biochemical, and biological conditioning, via the tunable 
electrospun biodegradable polymer wrap used here, could enhance AVG patency. By providing this support to an AVG, we may be able to facilitate arterialization.

\subsection{THE ROLE OF BIOMECHANICS IN THE DEVELOPMENT OF INTIMAL HYPERPLASIA}

$\mathrm{IH}$ is defined by an increase in the thickness of the inner layer of a blood vessel, typically as a result of an increased number and/or size of cells in the intima, followed by deposition of massive amounts of ECM by these cells. The cells contributing to this response are predominantly SMCs of medial and adventitial origin. IH occurs both physiologically during development as in the closure of the ductus arteriosus [58], and pathologically as a result of vascular injury $[8,22,24,26,48,53,54,59-79]$. It is thought that AVG IH may be initiated by the abrupt exposure of the veins to the dynamic mechanical environment of the arterial circulation [80]. However, while increased levels of CWS has been shown to promote IH formation [31,81], increased levels of shear stress tend to modulate it [31,77,81-85]. These two biomechanical factors, seemingly causing opposing hyperplastic responses by AVGs, were carefully explored by Dobrin et al., who showed that the increased circumferential stretch plays a more significant role in promoting intimal thickening than the increased shear stress does in preventing it [80]. In another study that motivates this work, Zwolak et al. suggested a regulatory role for biomechanical wall stress in the arterialization of AVGs [37]. Jiang et al. demonstrated that increased wall shear stress, in the absence of an increase in wall tension, reduced the hyperplastic response in AVGs [84]. The in vivo work by Liu et al. has shown that 
by reducing the level of CWS in AVGs, via placement of a permanent polytetrafluoroethylene sheath, the hyperplastic response can be reduced $[40,47,48]$.

It is clear from these previous studies that the biomechanical environment of an AVG plays a significant role in the development of IH. In particular, CWS appears to regulate the formation of $\mathrm{IH}$, and controlling this was the focus of the approach described in this study.

\subsection{MOLECULAR AND CELLULAR PROCESSES ASSOCIATED WITH INTIMAL HYPERPLASIA}

Once injury is perceived by a vein, the hyperplastic response is set into motion and can be described by five distinct but interrelated cell processes: 1) Phenotypic modulation of adventitial and medial SMCs from a contractile and quiescent state with low proliferative potential to a synthetic state with high proliferative potential [71,86-99]; 2) De-adhesion of SMCs or alteration of focal adhesions with other cells and the ECM; 3) Migration of SMCs from the outer layers through the basement membrane to the intima, which requires selective reassembling of focal adhesions that allow the cell to "walk" along the ECM [1,100-111]; 4) Proliferation [98,112125]; and 5) Remodeling of the tissue, reflecting the changes in ECM composition caused by the synthetic SMCs secreting collagen, elastin, fibronectin, etc. $[24,107,113,126-131]$, as well as matrix degrading enzymes such as the various matrix metalloproteinases (MMPs) [24,105,132146]. In order to inhibit the initiating events of $\mathrm{AVG} I H$, it is probable that one must take into account each of these five processes. A schematic depicting the chain of events associated with IH is shown in Figure 1.1. 


\subsubsection{Phenotypic Modulation}

Modulation of SMC phenotype is a prominent feature in the pathogenesis of $\mathrm{IH}$ [71,92,94,97,147-149]. Plaques abundant with modified SMCs have been found in the intima as early as the second week after grafting [150]. Fully differentiated adult SMCs demonstrate low turnover as demonstrated by low proliferation and apoptosis rates [93,151]. However, 48 hours after arterial injury, $15-40 \%$ of SMCs are mitotic [152]. This abrupt shift in functionality is

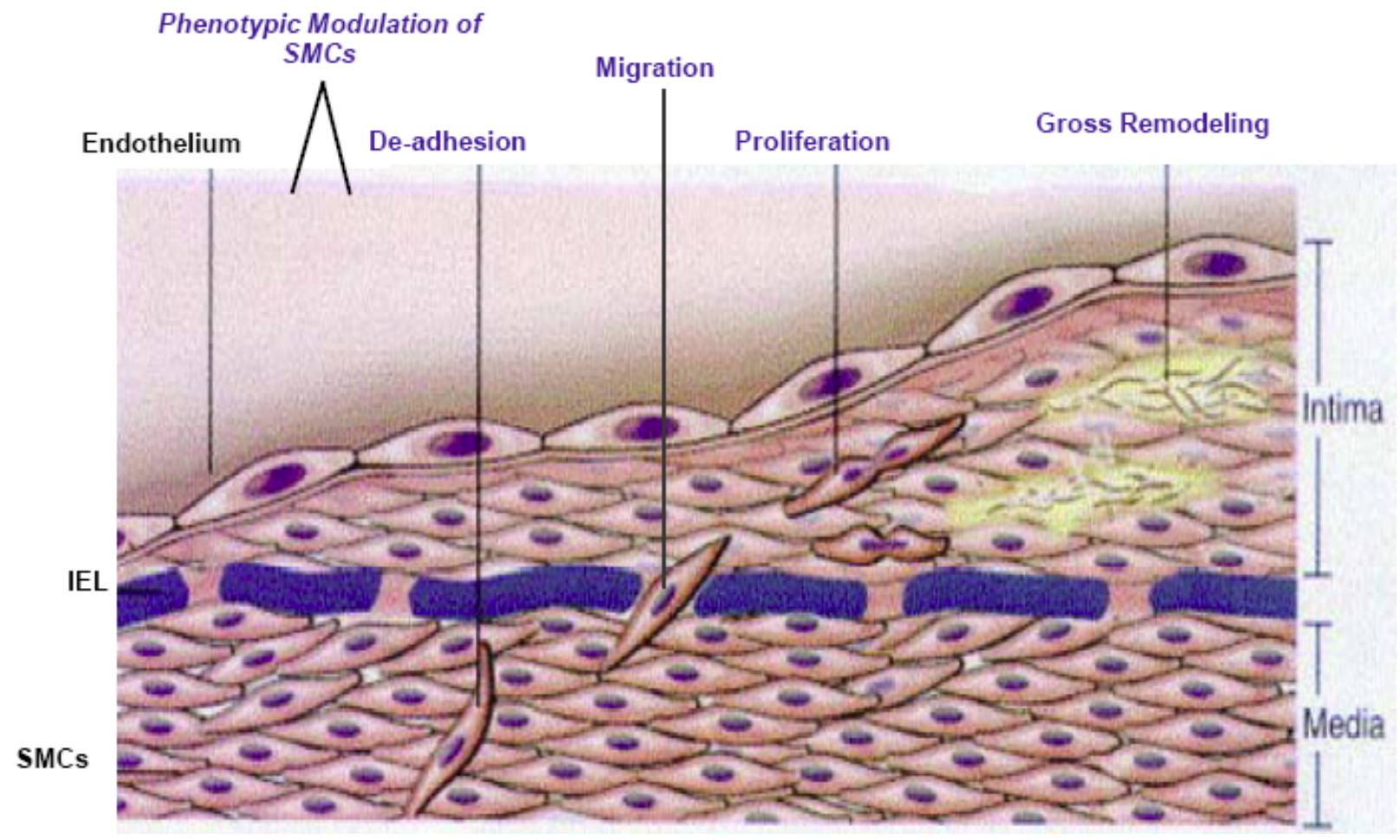

Figure 1.1 Schematic of intimal hyperplasia progression. Please note: IEL, internal elastic lamina; SMCs, smooth muscle cells. Image adapted from Robbins Pathologic Basis of Disease, 1999 [2]. 
related to the fact that SMCs can exist in a spectrum of phenotypes, spanning from fully synthetic to fully contractile. Synthetic SMCs respond to regulatory signals and cytokines, and are capable of ECM turnover as well as growth factor production [95,153-155]. On the other hand, contractile SMCs respond to vasomotor signals and control vessel tone [95,130,156-160]. AVGs exhibit neointimal formation within the first two months by the migration and proliferation of synthetic SMCs [131] and by subsequent, sustained ECM accumulation, including type I collagen production, in the prolonged presence of the de-differentiated type SMCs.

The phenotypic state of SMCs is regulated at least in part by mechanical forces, as demonstrated by the observation that cyclic stretch induces a substrate-dependent modulation of proliferation and h-caldesmon expression in vitro [161]. In vivo studies have also shown the importance of mechanical injury on the phenotype of SMCs. Balloon inflation injury to the media was shown to promote ECM synthesis by SMCs as well as to decrease alpha actin content [162]. Several reports have shown that neointimal SMCs of veins transposed to the arterial circulation are phenotypically altered $[71,131,163]$, supporting the notion that the change from the venous to the arterial environment triggers phenotypic alteration. Further evidence comes from ex vivo organ culture studies where, for example, cyclic stretch was found to be necessary to maintain the contractile function of SMCs in cultured rat portal veins [164]. Goldman et al. exposed rat vena cava to arterial pressures [165], which led to a large increase in medial circumferential strain and a concomitant reduction in the SMC filamentous actin coverage. Clearly, the changes in the mechanical environment related to vein grafting can lead to phenotypic alterations of the mural SMCs, possibly contributing to the development of IH. 
Indicators of a synthetic phenotype include the presence of increased quantities of Golgi complex and rough endoplasmic reticulum (ER) [156,166,167], and decreased quantities of filamentous actin [165]. A contractile phenotype is demonstrated by the presence of an intact contractile apparatus indicated by the expression of contractile proteins such as smoothelin, $\mathrm{h}$ caldesmon, smooth muscle myosin heavy chain, and large quantities of filamentous actin $[165,167-169]$.

The Golgi complex is found in the cytoplasmic matrix of both plant and animal cells. It consists of intertwining vesicles continuous with the ER. The membranes forming this organelle are always smooth. The Golgi complex is also sometimes termed the dictyosome. The two main functions of the Golgi complex within eukaryotic cells are the in secretory activities of the cell, as well as in the synthesis of glycoproteins. Larger quantities of Golgi complex have been observed in phenotypically altered neointimal vascular smooth muscle cells $[97,170]$.

\subsubsection{De-adhesion and Migration}

Cellular de-adhesion is one of the earliest responses in the IH cascade. This process refers to an alteration in a cell's adhesion to the ECM from a state of strong adherence, with focal adhesions and stress fibers, to a state of weaker adherence, characterized by a restructuring of focal adhesions and stress fibers while maintaining a spread cell shape [151]. SMC de-adhesion will of course allow SMC migration and proliferation which will contribute to neointima formation [171].

While there are many important proteins involved in the regulation of cellular adhesion, we focused our attention on matricellular proteins, which function as adaptors and modulators of cell matrix interactions $[172,173]$, and intracellular adhesion proteins, which have been shown to 
localize to cellular focal adhesion sites $[174,175]$. Tenascin C (TN-C), thrombospondin 1,2 (TSP), and secreted protein acidic and rich in cysteine (SPARC) are matricellular proteins that exhibit highly regulated expression during development and cellular injury [176]. Mitogen inducible gene 2 (Mig-2) and integrin linked kinase (ILK) are intracellular proteins involved in cellular shape modulation [174,175] and integrin mediated signal transduction [177], respectively. The actions of TN-C, TSP, and SPARC on the cytoskeleton and focal adhesions are basically indistinguishable $[178,179]$. However, these three proteins each have unique receptors and have similar but separate signaling pathways to produce a state of intermediate adhesion, which is a precursor to cell migration [176]. Mig-2 and ILK have also been implicated in cellular adhesion [174,175]. Specifically, Mig-2 has been shown to participate in the connection between cell matrix adhesions and the actin cytoskeleton as well as to modulate cell shape [175]. Recent studies have indicated that ILK serves as a mediator in integrin mediated signal transduction [180]. Furthermore, both Mig-2 and ILK are required for maintaining focal adhesions $[174,175]$. By examining the changes in the levels of TN-C, TSP, SPARC, Mig-2, and ILK, we believe that we will be able to make conclusions about the state of adhesion of SMCs within the vein segments. A schematic showing the intracellular localization of TN-C, TSP, SPARC, Mig-2 and ILK is shown in Figure 1.2. 


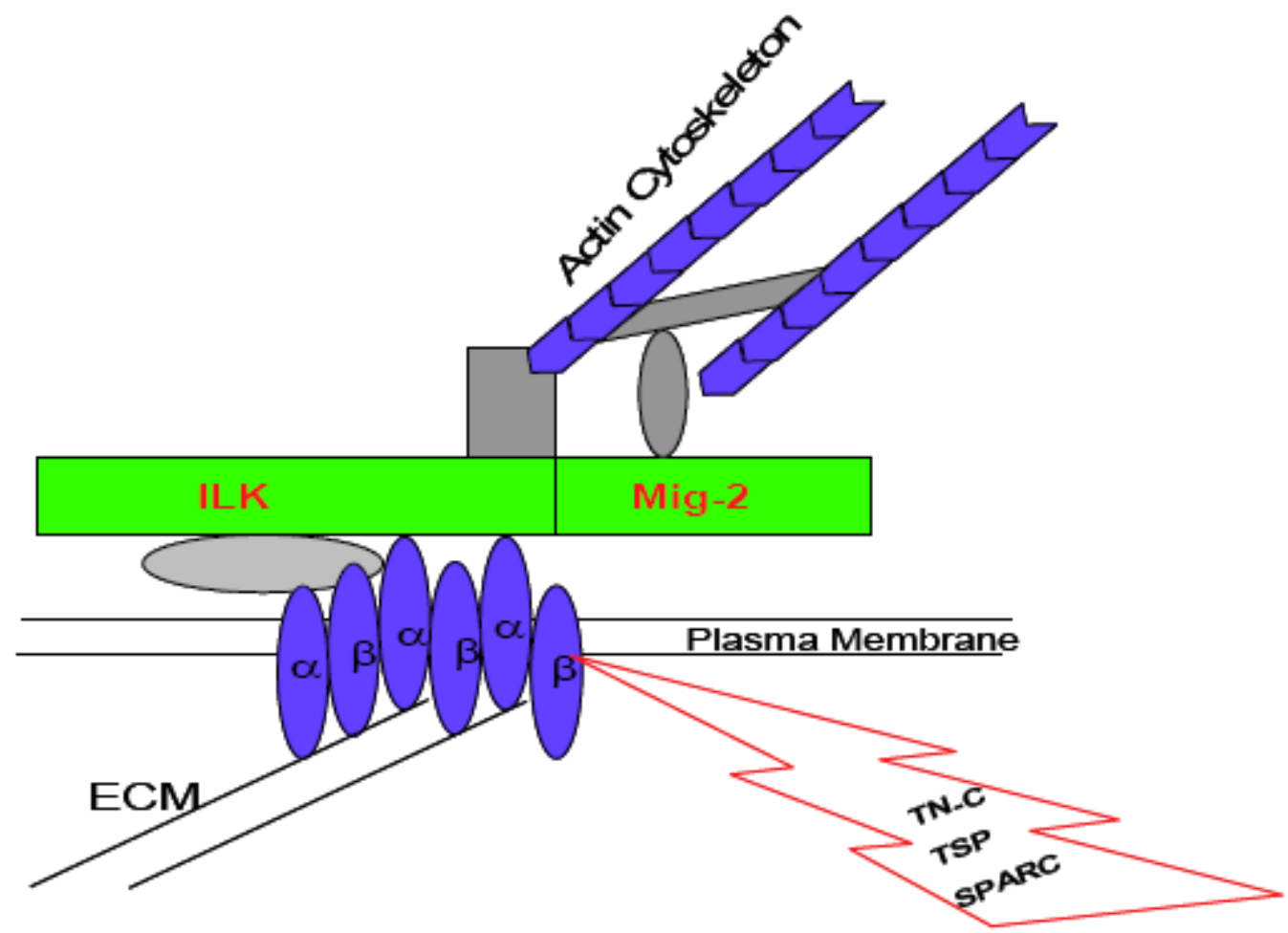

Figure 1.2 Schematic showing the localization of Tenascin-C (TN-C), thrombospondin-1,2 (TSP), secreted protein acidic and rich in cysteine (SPARC), Mitogen inducible gene 2 (Mig-2) and integrin linked kinase (ILK). Please note: ECM, extracellular matrix; $\alpha$ and $\beta$, integrins. Image adapted from www.cellmigration.org/res resource misc.htm.

A prerequisite for SMC migration in vivo is degradation of surrounding matrix proteins. Matrix metalloproteinases (specifically, MMP-1, MMP-2, and MMP-9) can selectively degrade various components of the vascular ECM [137,181-183]. MMPs have been shown to be critical for the development of arterial lesions by regulating SMC migration. The balance between MMPs, their activator (MT-1 MMP) [184], and their inhibitors (specifically, TIMP-1, TIMP-2, TIMP-3, and TIMP-4) determines the level of ECM degradation [143]. Numerous studies have shown that MMPs and TIMPs play a significant role in the early stages of IH in response to 
altered hemodynamics and vascular injury [185-187]. For example, after 6 hours of ex vivo perfusion with arterial hemodynamics, expression of MMP-2 and MMP-9 was increased in human saphenous veins [142]. Other organ culture studies of human saphenous vein have shown increased production of MMP-9 and increased activation of MMP-2 $[145,182,188]$ under arterial conditions. Broad spectrum MMP inhibitors such as simvastatin have been shown to inhibit neointima formation in this model $[182,189]$.

Mechanical forces can influence SMC de-adhesion and migration by directly regulating the above factors. For example, MMP-1 expression is increased in venous SMCs exposed to pulse pressure compared to static controls [190], while MMP-2 mRNA levels are increased in mouse SMCs exposed to cyclic stretch [191]. In cultured SMCs from human saphenous vein, MMP-2 and MMP-9 transcript and protein levels increased when exposed to uniaxial stationary strain, but decreased when exposed to uniaxial cyclic strain [192]. Cyclic strain of fibroblasts has been shown to increase MT-1 MMP levels [193] and decrease TIMP-1 levels [194]. In addition, SMC migration was shown to be regulated by shear stress induced EC signaling [195198]. Mechanical forces can influence SMC de-adhesion and migration by directly regulating the above factors. In this study, we provide novel preliminary data that demonstrates for the first time that Mig-2 and ILK are mechanically regulated in veins that are subjected to arterial conditions, suggesting that these molecules affecting SMC de-adhesion and subsequent migration can be mechanically influenced. In addition, SMC migration was shown to be regulated by shear stress induced EC signaling [103,195-199]. 


\subsubsection{Proliferation}

$\mathrm{IH}$ has been shown to be associated with increases in SMC proliferation $[15,37,62,76,98,112,122,123,200-202]$ and both increases and decreases in apoptosis $[24,108,124,203-208]$. It may seem counter-intuitive that an increase in intimal apoptosis is associated with $\mathrm{IH}$, a condition associated with increased cell numbers. However, it must be kept in mind that increases in cell number is but a singular event in the balance that regulates IH. That is, though there may be an absolute increase in apoptosis, a greater increase in cell proliferation would result in a net increase in cell number. For these reasons, it is important to evaluate both sides of the balance (i.e., both promoting and inhibiting factors) when assessing proliferation.

Proliferating cell nuclear antigen (PCNA) and terminal deoxynucleotidyl transferasemediated dUTP-biotin in situ nick end labeling (TUNEL) have been used to label proliferating and apoptotic cells, respectively, within intact AVGs, both in vivo [209], and in vitro [210]. Cell proliferation and apoptosis are simultaneous processes that occur within the adventitia and media of the vein during the first week following grafting, however this balance is thereafter disrupted with proliferation rates increasing over rates of apoptosis [209]. The level of proliferation within the media and neointima of stenosed aortocoronary bypass grafts excised upon re-operation has been shown to be significantly higher than non-stenosed controls [211].

Increased wall stress has been associated with AVG IH [37,145,205,212], and this may be a direct result of a mechanical regulation of SMC proliferation, and apoptosis. For example, venous SMCs have been shown to increase their proliferation compared to arterial SMCs when exposed to arterial levels of cyclic stretch [212,213]. Liu et al. showed via bromodeoxyuridine staining and TUNEL analysis that mechanical stretch due to arterial hemodynamics induces cell 
death, which possibly mediates subsequent cell proliferation in a rat AVG model [108]. Predel et al. showed that pulsatile stretch stimulates SMC proliferation in saphenous veins, but not internal mammary arteries, and may contribute to venous bypass graft disease [212]. When veins are transposed to the arterial circulation they undergo an increase of luminal shear stress in addition to intramural stress. Indeed it has been shown that a combination of increased shear stress and cyclic stretch imposed on cultured SMCs activates PDGF receptor alpha [214].

Several growth factors have been implicated as key components in the hyperplastic response of vein grafts. Transforming growth factor beta (TGF- $\beta$ ) appears to be of particular importance [121-125]. For example, Wolf et al. demonstrated that systemic administration of antibodies against TGF- $\beta$ significantly reduced the development of IH in a rat model [215]. Platelet derived growth factor (PDGF) and basic fibroblast growth factor (bFGF) also appear to be primary factors involved in IH associated SMC proliferation [216,217]. For example, PDGF causes a dose dependent proliferation response in cultured SMCs [218], while TGF- $\beta$ inhibits proliferation [219]. bFGF released from dead and damaged cells of autologous vein grafts promotes SMC proliferation [220]. mRNA levels of PDGF transcripts as well as numbers of proliferating cells were found to be highest in the neointima of porcine vein grafts [221]. While growth factors clearly play a role in IH, MMPs have also been shown to be critical for the development of arterial lesions by regulating SMC proliferation [183,222], while TIMPs have been shown to promote apoptosis of SMC [223].

\subsubsection{Remodeling}

Vascular remodeling typically refers to a change in the morphology or microstructure of a blood vessel in response to changes in the biomechanical environment [80,224]. It is believed that this 
occurs as an attempt by the tissue to restore biomechanical homeostasis (i.e., to return to normal levels of shear and wall stress). In the case of AVGs, IH is a pathological form of remodeling that includes increased intimal thickness caused by SMC migration and proliferation $[15,37,200]$, increased intimal apoptosis $[24,203,204,207,208]$, sclerosis of the intima and media due to increased ECM deposition, and hypertrophy of the medial and adventitial SMCs [30].

Vascular cells produce the ECM components such as collagen and elastin. The phenotypic modulation of SMCs associated with vein grafting has been shown to alter ECM synthesis characterized by increasing collagen type I and elastin production [130,131]. Veins used as arterial bypass grafts undergo an alteration of their ECM components [225], which can result in a loss of lumenal area and eventual occlusion [15,226]. An alteration in matrix synthesis directly leads to increased collagen content in the hyperplastic neointima during the first week after injury resulting from balloon angioplasty [227]. In addition, AVGs that undergo this hyperplastic remodeling exhibit decreased compliance as compared to fresh veins [228], which can contribute to their failure $[229,230]$.

\subsection{CHANGES IN MECHANICAL PROPERTIES OF VEIN GRAFTS DUE TO ARTERIALIZATION}

When grafted into an arterial environment in vivo, veins remodel in response to the new mechanical environment, thereby changing their mechanical properties and potentially impacting their patency as bypass grafts. Gusic et al. suggested that the mechanical environment effects changes in vessel size, as well as the nature of the remodeling, which contribute to altering vein mechanical properties [231]. 
The degree of AVG distension is directly related to its mechanical properties, which, in turn, is related to patency rates according to Davies et al. [229,230], who reported lower patency rates of less compliant AVGs in peripheral bypass surgery. This reduced patency has been largely attributed to compliance mismatch between the AVG and the native artery to which it is grafted [18,232,233]. Veins are inherently less compliant than arteries [234] and become even less compliant upon abruptly exposed arterialization [235]. It appears as though change in AVG compliance is an important predictor of AVG failure.

In the arterial pressure range an AVG is essentially a rigid tube due to the degree of its over distension [236]. To confirm this we performed a pressure ramping experiment on a freshly excised PIJV segment. The results of this experiment are shown in Figure 1.3. It can be seen that the vein reaches maximum distension at approximately $30 \mathrm{mmHg}$. Consequently, at arterial levels of pressure a vein is very stiff and behaves like a rigid tube with no radial excursions in response to pulsatile arterial pressure. We hope to counteract this phenomenon by providing temporary external structural support with an elastic biodegradable adventitial wrap. 


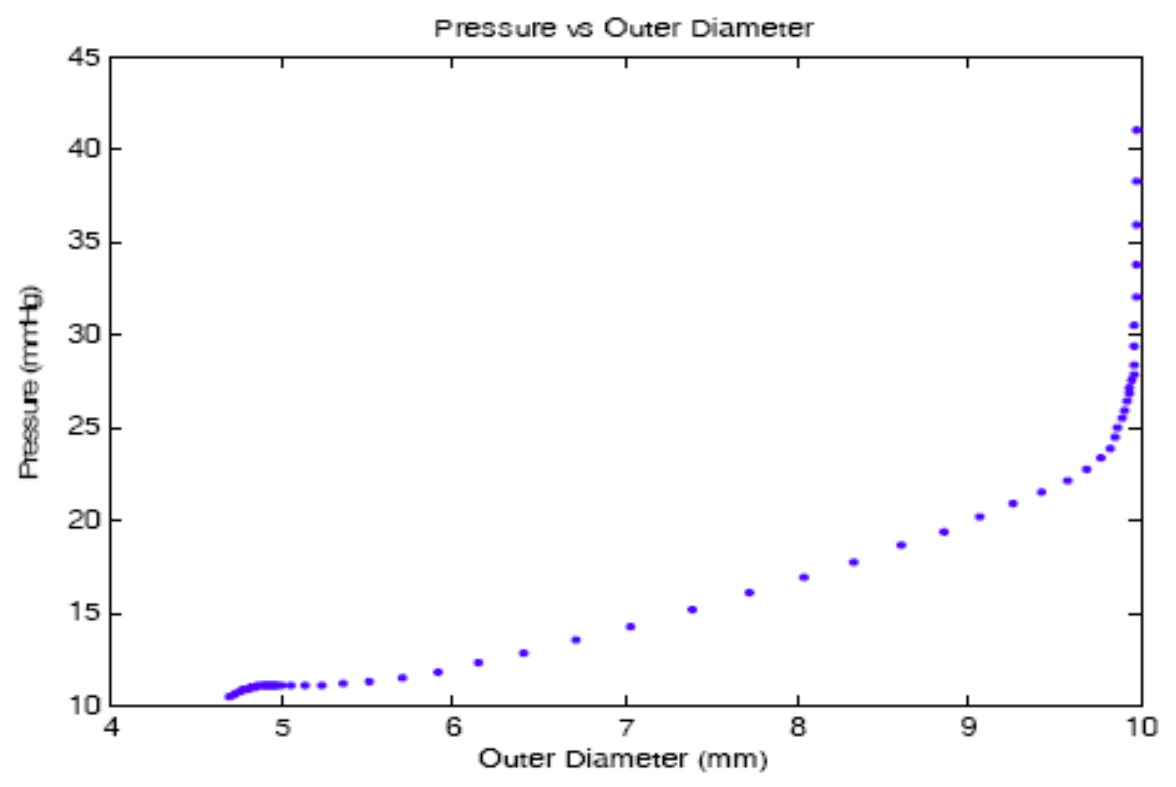

Figure 1.3 Pressure vs. diameter response of a porcine internal jugular vein segment.

\subsection{PREVIOUS ATTEMPTS TO WRAP ARTERIAL VEIN GRAFTS}

The concept of perivascular placement of a wrap to deliver support to AVGs is not new. However, we believe that all the previous approaches had rate-limiting barriers to clinical translation, and that our approach using an electrospun biodegradable polymer addresses these limitations.

The use of an external sheath around vein grafts was first described by Parsonnet et al. [50]. They showed that the sheath prevented dilatation, that it was well accepted by the host tissue, and that there was no difference in the tensile strength between supported and nonsupported vessels [50]. Karayannacos et al. showed reduced thrombosis and sub-endothelial 
proliferation in AVGs with both loose and tight fitting Dacron mesh sheaths compared with unsupported control grafts [44]. Mehta et al. demonstrated that placement of an external, macroporous, nonrestrictive, polyester stent reduces neointima formation in porcine vein grafts [49]. More recently, polytetrafluoroethylene sheaths were used to permanently restrict AVGs from expansion under arterial pressure and this led to reduced IH formation in a pig model [47]. Clinical translation of permanent mechanical support to AVGs has not yet been reported, most likely due to the unfavorable inflammatory response to biodurable synthetic materials in vascular applications [237,238]. This limitation motivated Vijayan et al. and Jeremy et al. to use a polyglactin based biodegradable sheath to reduce IH in AVGs [43,51,52]. The noted beneficial effects included enhanced neo-vasa-vasorum development over unwrapped controls [51]. However, these biodegradable sheaths were loose-fitting and allowed the AVGs to expand to their maximum diameters under arterial pressure, and thus did not offer mechanical support against the increased level of CWS. Prior to the approach used by Vijayan et al. [51,52] and Jeremy et al. [43], Huynh et al. used a temporary external collagen tube support to reduce IH formation in rabbit vein grafts [42]. These collagen tubes were also non-restrictive, and no mention of the degradation kinetics was reported [42]. It has been reported, however, that electrospun cross-linked collagen degrades very rapidly in an aqueous solution [239] and hence the structural support offered to AVGs by sheaths made of collagen alone may be too temporary to be effective over the long-term. An external AVG sheath developed by Liao et al. was designed to degrade at a desired rate in order to transfer CWS to an AVG gradually over time [55]. Poly lactic-co glycolic acid sheets were prefabricated into tubes by wrapping around a Teflon rod, and therefore are not customizable to each AVG [55]. That is, as with previous approaches the Liao et al. approach allows expansion of an AVG under arterial pressure before 
delivering any mechanical support. The degradation kinetics and resulting CWS vs. time profile in the sheaths, not in the mid-AVG-wall as described here, were reported [55]. Our approach addresses the two major limitations associated with the previous work described above, specifically with respect to biodurable and/or non-restrictive external sheaths.

Delivery of mechanical support to AVGs is but one possibility for an adventitial wrap. Other applications could be as a vehicle for the local delivery of biochemicals, drugs, genes, or cells. Kanjickal et al. used a poly(ethylene glycol) hydrogel for sustained local delivery of cyclosporine to AVGs, and successfully reduced anastomotic IH development [240]. In another study, Cagiannos et al. used a polytetrafluoroethylene sheath to locally deliver rapamycin (sirolimus) to AVGs, and effectively reduced anastomotic IH in a pig model [241]. More recently, Kohler et al. used a biodegradable mesh to deliver paclitaxel to effectively reduce IH at the graft-vein anastomosis in a sheep model of dialysis access [46]. Such activities could theoretically be incorporated using the electrospun polymer wrap technique, with the potential to control the delivery rate to some extent by tuning the degradation rate of the electrospun polymer wrap.

To our knowledge, delivery of cells via a biodegradable AVG wrap/sheath has not been previously reported and hence this possible future application of the adventitial wrap would be novel. The polymer that was used here has been characterized [57], and successfully microintegrated with viable SMCs [242], and would lend itself to this potential future application. 


\subsection{SUMMARY}

The autogenous saphenous vein remains the graft of choice for both coronary (500,000 annually) and peripheral (80,000 annually) arterial bypass procedures. Failure of AVGs remains a major problem, and patients with failed grafts will die or require re-operation. IH accounts for $20 \%$ to $40 \%$ of all AVG failures. It is believed that IH is triggered by abrupt exposure of AVGs to the harsh new biomechanical environment of the arterial circulation and the elevated levels of CWS associated with the arterial system (140-fold increase compared to native venous conditions). The working hypothesis of the current study is that the IH response may be reduced or eliminated by more gradually exposing AVGs to arterial levels of CWS. That is, if an AVG is given an ample opportunity to adapt and remodel to the stresses of its new environment, cellular injury may be reduced, thus limiting the initiating mechanisms of IH. Clearly, developing a reliable means to prevent the early events of the $\mathrm{IH}$ process would contribute significantly to improvements in the clinical outcome of arterial bypass procedures. Therefore, the long-term goal of this work is to develop a new mechanical conditioning paradigm, in the form of a periadventitially placed, biodegradable polymer wrap, to safely and functionally "arterialize" AVGs in situ.

Several of the molecular signals outlined in Section 1.2, and the rationale for selecting them as endpoints for this study, are summarized in Table $\mathbf{1 . 2}$. 
Table 1.2 Summary of and rationale for the chosen endpoints in this study.

\begin{tabular}{|c|c|l|}
\hline $\begin{array}{c}\text { Proposed } \\
\text { endpoints in this study }\end{array}$ & Role in IH & Rationale supported by the literature \\
\hline Golgi Complex & $\begin{array}{c}\text { Phenotypic modulation } \\
\text { Protein Synthesis }\end{array}$ & $\begin{array}{l}\text { Increased quantities in synthetic vs. } \\
\text { contractile SMCs [156,166,167] }\end{array}$ \\
\hline PCNA & Proliferation & $\begin{array}{l}\text { Increased cell proliferation in abruptly- } \\
\text { exposed AVGs [209,210]. }\end{array}$ \\
\hline TUNEL & Apoptosis & $\begin{array}{l}\text { Altered apoptosis in abruptly-exposed } \\
\text { AVGs [206,243] }\end{array}$ \\
\hline Compliance & Clinical Performance & $\begin{array}{l}\text { Important predictor of AVG patency } \\
\text { [229]. Compliance decreases in abruptly } \\
\text { exposed arterialized AVGs, thereby } \\
\text { increasing compliance mismatch [235] } \\
\text { Important predictor of AVG patency } \\
\text { [229]. Stiffness increases in abruptly } \\
\text { exposed arterialized AVGs and could } \\
\text { contribute to reduced clinical } \\
\text { performance [234,235] }\end{array}$ \\
\hline Cliffness & Clinical Performance \\
\hline
\end{tabular}

\subsection{SPECIFIC AIMS}

The research described above characterizing the mechanopathobiology of IH in AVGs led to the formation of the following two hypotheses:

Hypothesis 1: Compared to an acute, abrupt exposure of veins to pulsatile arterial levels of CWS, a gradual exposure results in a reduced hyperplastic response. 
Hypothesis 2: A biodegradable polymer wrap can be placed peri-adventitially on AVGs and tuned to provide, in situ, the mid-AVG-wall CWS vs. time profile (henceforth referred to as "CWS profile") necessary to achieve a reduced hyperplastic response in preclinical models.

To address these hypotheses, both our laboratory's well established, validated ex vivo vascular perfusion system (EVPS) as well as appropriate animal models were utilized. Specifically, our aims were as follows:

Specific Aim 1: Design and construct a closed-loop paired EVPS with the capacity to simulate and maintain, using feedback control, either physiologic arterial CWS or shear stress waveforms. Related to this:

Specific Aim 1.i: Develop the optimum state-space (SS) and transfer function (TF) representations of the pressure and flowrate generation capabilities within our EVPS using subspace system identification (SYSID).

Specific Aim 1.ii: Develop a proportional, integral and derivative (PID) control system for regulating the physiologic CWS or shear stress waveforms imposed on vessels implanted into our EVPS.

Specific Aim 2: Establish a CWS profile necessary to achieve a reduced acute hyperplastic response by freshly-excised vein segments perfused ex vivo under incrementally-imposed compared to abruptly-exposed arterial conditions.

Specific Aim 3: Tune the biodegradation rate of an electrospun polymer adventitial wrap so as to achieve a desired CWS profile in wrapped AVGs exposed to arterial conditions.

Specific Aim 4: Evaluate the mitigating effect of the adventitial wrap from Specific Aim 3 on the hyperplasic response of AVGs implanted as carotid interposition grafts in a preclinical model. 
The research project detailed in this dissertation was derived from four distinct ongoing areas of research in Dr. Vorp's laboratory: i) development of a well-controlled EVPS; ii) investigation of the mechanopathobiological response of intact vascular tissue to well controlled biomechanical stimuli; iii) histological studies of vascular tissues; and iv) in vivo vein grafting experiments to study IH. The portion of the work devoted to development of a new generation EVPS (Specific Aim 1) consisted of first re-designing the old bench-top system so that it could be placed into a laminar flow hood in order to maximize sterility for longer term experiments. Next, accurate modeling of the pressure and flowrate generation capabilities within the EVPS (Specific Aim 1.i) was achieved. Once the mathematical models of the EVPS were obtained, individual PID controllers for CWS and lumenal shear stress were developed (Specific Aim 1.ii). The development of the new generation EVPS was performed in tandem with the ex vivo vascular perfusion experiments of Specific Aims 2 and 3. These ex vivo perfusion experiments were performed using the previous generation EVPS. Manual adjustment of the EVPS parameters allowed us to generate two CWS profiles. These two profiles were used in an attempt to determine the beneficial effects, in relation to the acute hyperplastic response by AVGs, of gradual vs. abrupt exposure to arterial biomechanical conditions (Specific Aim 2). The next step was to try and mimic the CWS profile, achieved manually in Specific Aim 2, via tuning the biodegradation rate of an adventitially placed electrospun polymer wrap (Specific Aim 3). The final stage of the work presented in this dissertation was to evaluate the beneficial effects, vis á vis IH development, of a tuned biodegradable electrospun polymer wrap using a pig model of peripheral arterial bypass grafting (Specific Aim 4).

The goal of this study was to show that gradual vs. abrupt exposure of AVGs to arterial levels of CWS attenuates their hyperplastic response. A major hurdle to overcome in this work 
was to find a means to achieve this "conditioning" paradigm in situ. The answer to this need was to use a tunable electrospun biodegradable polymer wrap to provide temporary mechanical support to veins intended for use as AVGs. Although the in vivo vein grafting experiments, performed as part of this study, were purely "proof of concept" in nature, our results provide a framework to build upon in developing this approach. We used the tunability of the wrap degradation rate to provide temporary mechanical support to AVGs, however, delivery of biochemical (drugs) and biological (cellular) support are two additional applications that could be achieved using the same approach. Delivery of these modes of support to AVGs may facilitate arterialization instead of the inevitable stenosis due to IH.

While the original research plan included a rigorous evaluation of all the $\mathrm{IH}$ related endpoints listed in this section, quantification of the protein and gene expression levels for filamentous actin, smoothelin, h-caldesmon, smooth muscle myosin heavy chain, TN-C, TSP, SPARC, Mig-2, ILK, Golgi complex, MMPs, TIMPs, TGF- $\beta$, PDGF, bFGF, collagen, and elastin proved to be experimentally difficult and troubleshooting those experimental techniques was beyond the scope of this work. A description of the experimental difficulties, the preliminary findings, and recommendations to overcome those obstacles can be found in Appendix D. The rest of the dissertation will focus specifically on the endpoints outlined in

\section{Table 1.2.}




\subsection{EX VIVO VASCULAR PERFUSION SYSTEM}

Most of what is known about vascular mechanopathobiological responses has been derived from either cell culture or in vivo models. However, two-dimensional cell culture models may not be effective for extending predictions on cell behavior to more physiologic three-dimensional cellular environments. Similarly, animal models introduce unpredictable biomechanical stresses. For example, deformations of a vein graft due to an animals' natural movement may influence the cellular responses of interest within the tissue. Another disadvantage of in vivo vein graft models is the effects caused by unpredictable neurohormonal stimuli. For these reasons we believe that a precisely controlled EVPS offers the advantage of being able to measure intact vascular tissue responses without concern for confounding experimental conditions.

\subsection{HISTORY OF EX VIVO VASCULAR PERFUSION}

The precise simulation of arterial biomechanical conditions is of great importance in the field of vascular research. Our laboratory's well established, validated EVPS has been used for over two decades to study the mechanopathobiological responses of intact blood vessel segments to accurately simulated physiologic biomechanical signals [3,39,244-249]. The EVPS used in this study has gone through several iterations to improve its functionality and is described in detail below. 
The concept of ex vivo perfusion was originally investigated in the 1930s by aviator Charles Lindbergh in collaboration with vascular surgeon and Nobel laureate Dr. Alexis Carrel. Their system was designed to examine whether or not, and for how long, organs (including blood vessels) could be maintained outside the in vivo environment [250]. The EVPS used in this study has gone through several design iterations to improve its functionality and diversity [3,39,244-247]. The previous generation EVPS, upon which the current design was based, is shown schematically in Figure 2.1.

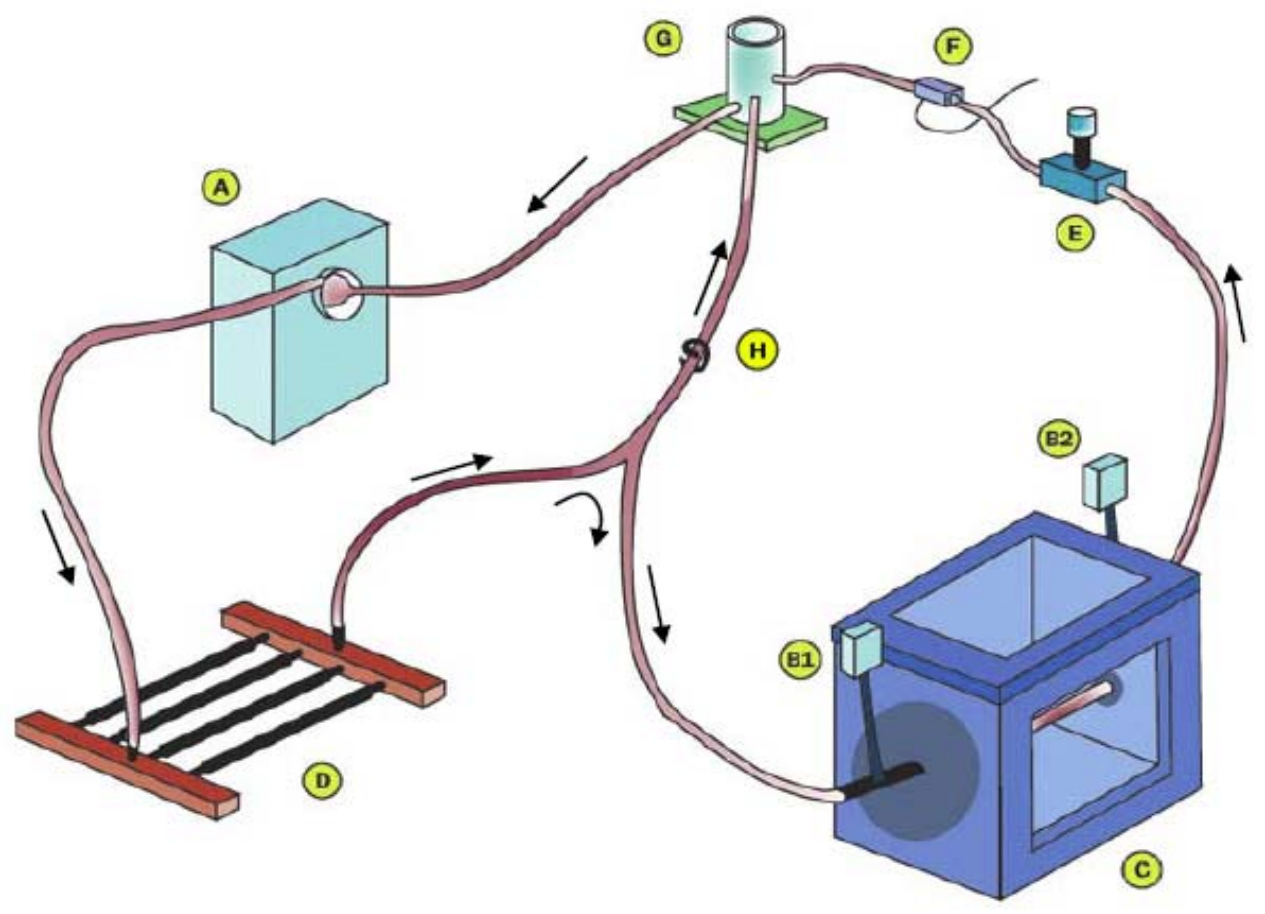

Figure 2.1 Schematic of the closed-loop perfusion/organ culture system. The loop is composed of a Biomedicus centrifugal pump that provides pulsatile pressure and flow (A), a heat exchanger (D), a tissue-housing chamber (C), proximal (B1) and distal (B2) pressure transducers, a variable resistance valve (E), flow probe (F), collection reservoir $(\mathrm{G})$, and vessel bypass $(\mathrm{H})$. Components not shown include, adventitial bath loop, He-Ne laser micrometer, and data acquisition system. See Labadie (1996) et al. for more detail [3]. 
Several other groups have designed specialized EVPSs for studying biochemical responses of blood vessel segments to imposed biomechanical stresses. One of the earliest systems was one designed by Badimon et al. [251]. Their system could generate physiologic arterial levels of shear stress and shear rate and was used to assess thrombogenicity related endpoints [251-255]. Other systems have been reported which were designed without the ability to accurately simulate the higher frequency content of the physiologic arterial pressure or flowrate waveforms. They instead were designed to generate certain desired static [256-260] or oscillatory $[81,142,261-270]$ signals for the purpose of estimating the biomechanical properties of blood vessel segments.

\subsection{LIMITATIONS OF PREVIOUS SYSTEM DESIGNS}

This study will address the limitations identified by our laboratory through years of experience in using the previous generation EVPS designed by Labadie et al. [3]. First and foremost, the system existed in a "bench-top" configuration, which meant that when the tissue housing chamber or perfusate reservoir were opened, the culture media inside was exposed to room air. We found that this was the major source of contamination for long-term perfusion experiments since both the tissue chamber and reservoir quite often were opened in order to add media to the bathing and perfusate loops, respectively, to fix leaks that developed from an implanted blood vessel, to take media samples for blood gas measurements and to make temperature

measurements. Secondly, a catastrophic event that necessitated supervised perfusion experiments was the development of a leak within an implanted blood vessel. This has occurred 
several hours into an experiment and without warning. Response to a leak had to be immediate since the finite volume of perfusate rapidly emptied into the tissue housing chamber.

\subsection{TECHNICAL IMPROVEMENTS TO CURRENT EVPS}

Four significant modifications were implemented within the latest generation of our EVPS: 1) Miniaturization for placement within a class II biosafety cabinet; 2) implementation of safety precautions that allowed the EVPS to be left unattended for short periods of time; 3) implementation of self-sealing sampling ports and inline thermistors for measuring the blood gasses and temperature, respectively, of both the perfusate and bathing media; and 4) development of a PID algorithm to achieve feedback control over the CWS or luminal shear stress waveforms imposed on implanted vascular segments. Having precise control over the

CWS and shear stress generation by our EVPS could help to facilitate the measurement of pathobiological-related molecular responses to physiologic vascular biomechanical stimuli.

\subsubsection{Detailed Description of EVPS Flow Circuit Components}

The EVPS consisted of two separate perfusion circuits to facilitate paired experiments. A schematic of one of the perfusion circuits can be seen in

Figure 2.2 and a photograph of both circuits is shown in Figure 2.4. Also, certain components are shown in Figure 2.5. Improvements were made to the previous generation EVPS (seen in Figure 2.3) in order to create a self-contained bioreactor to minimize user 
intervention (and hence reduce chances of contamination). This included development of online control of physiologic arterial pressure and flowrate within the system. The bulk flow was generated using a Masterflex L/S computerized roller pump (Model 7550-30, Cole Parmer Instrument Co., Bunker CT;

Figure 2.2(3) and Figure 2.5(a)). A pulse dampener (Cole Parmer Instrument Co., Bunker CT;

Figure 2.2(4) and Figure 2.5(b)) was added downstream to remove the high frequency perturbations generated by the roller pump. Higher frequency components of the physiologic arterial pressure and flowrate waveforms were generated using a custom-built piston/cylinder device (Figure 2.5(c)). Figure 2.6 is a close-up photograph of this device the components of which are held together with a custom-built frame (Figure A. 1 to Figure A. 5 in Appendix A and Figure 2.6(a)). A voice coil (Model LA25-42-000A, BEI Kimco Magnetics Division Co., Vista, CA; Figure 2.6(b)) acted as a linear actuator and was coupled to a custom built stainless steel shaft (Figure A.6 in Appendix A and Figure 2.6(c)). The shaft was supported by a linear bearing (Model NP-4, Nook Industries, Cleveland, OH; Figure 2.6(d)). At the end of the shaft, a small black disk was attached and acted as the piston head (Figure A. 7 in Appendix A and Figure 2.6(e)). The piston head was then attached to a rolling diaphragm (Model 4-100-(81)-81C-B-J, Bellofram Co., Newell, WV; Figure 2.6(f)) that provided a water-tight seal as well as facilitated frictionless motion of the piston head within the cylinder (Figure A. 8 in Appendix A and Figure 2.6(g)). 
A one-way valve (Model V24300VG, Star Micronics Inc.; Figure 2.5(d)), was placed between the pulse dampener and the piston to inhibit the flow or pressure from the piston to be dissipated by the pulse dampener. Based on the accepted standard equation [271] relating entrance length, diameter, and Reynolds number (length/diameter $=0.06 *$ Reynolds number , using physiologic Reynolds number $<200$ ), stainless steel cannulae were designed $30 \mathrm{~cm}$ in length (Figure A. 9 to Figure A. 11 in Appendix A and Figure 2.4) to ensure fully developed flow to the implanted blood vessel segments. Previous calculations by Brant et al. have demonstrated that this length is sufficient to achieve fully developed flow in a previous generation EVPS [244]. Ports mounted on the cannulae allow for proximal and distal measurement of perfusate pressure. The mean systemic resistance was adjusted using an inline needle valve flow resistor (Model EW-06393-80, Cole Parmer Instrument Co., Bunker, CT) placed downstream of the distal cannula just before the fluid reservoir (Figure 2.5(e)) which completed the closed-loop system. 


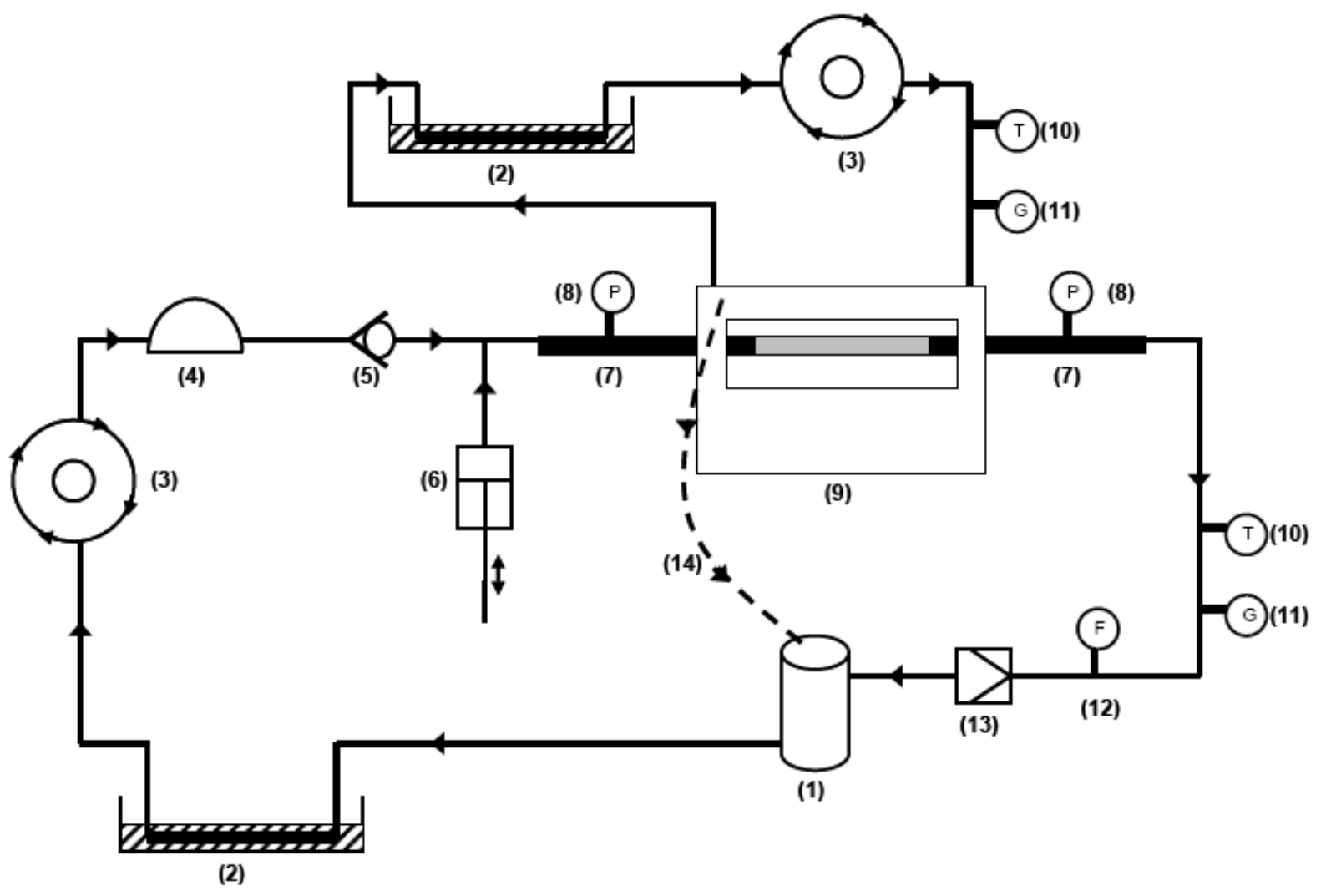

Figure 2.2 Schematic of one loop of the paired perfusion system. Note that there are two separate closed loops: the perfusate loop and the adventitial bathing loop. The components comprising both loops are: (1) perfusate reservoir; (2) metal heat exchanger tubes inside water baths; (3) roller pumps; (4) pulse dampener; (5) one-way valve; (6) piston-cylinder device; (7) vessel cannulae; (8) pressure transducers; (9) tissue housing chamber; (10) inline thermistors; (11) self-sealing media sampling ports; (12) ultrasonic flow transducer; (13) needle-valve flow resistor; and (14) overflow recirculation line.

\subsubsection{Custom Designed EVPS Components}

Several components of the EVPS were custom designed and machined. This section will describe the design of these components. Technical drawings of all custom built parts are provided in Appendix A. 


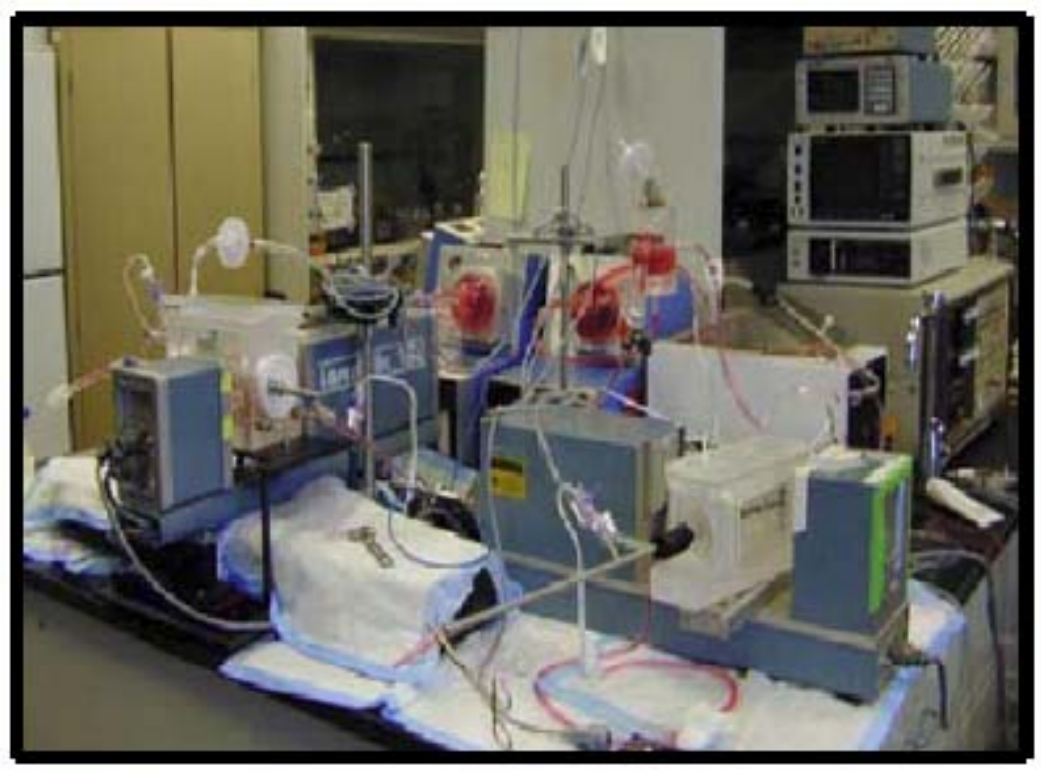

Figure 2.3 Earlier generation paired ex vivo vascular perfusion system. 


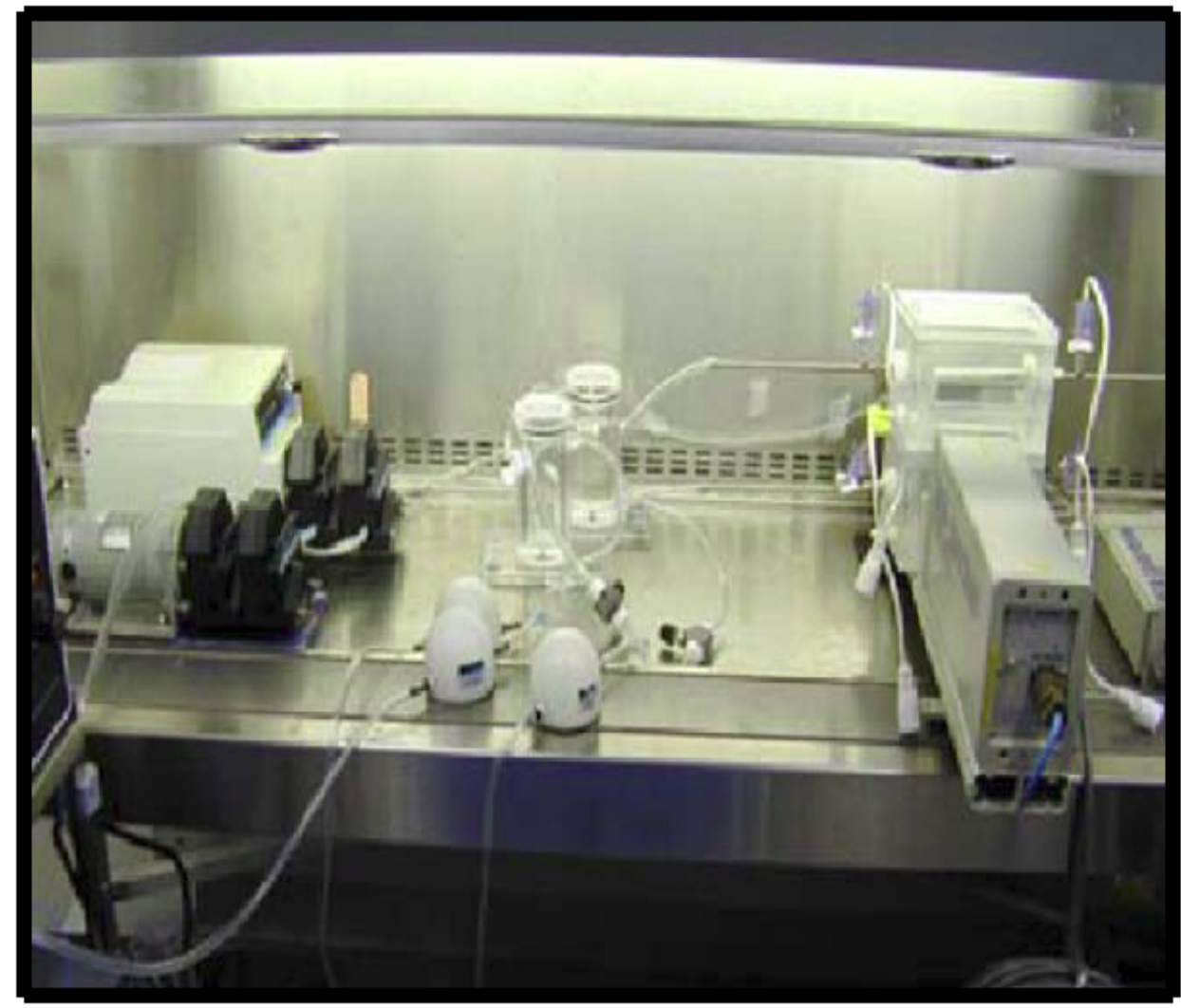

Figure 2.4 Modifications made to render our earlier generation ex vivo vascular perfusion system more compact so that it fits within a laminar flow hood. Note the stacked vessel housing chambers and single laser micrometer, reducing the size of the two independent flow loops. 


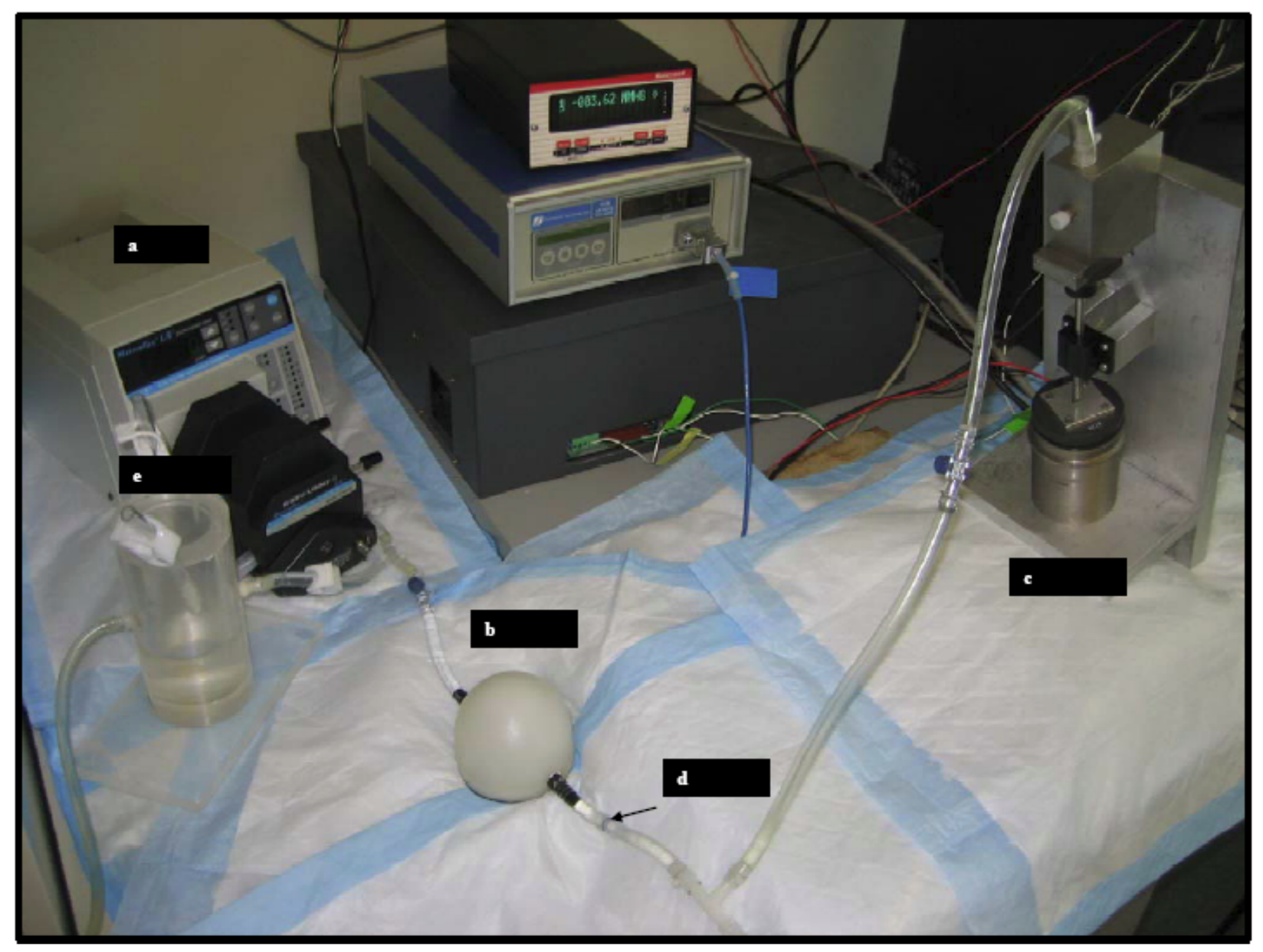

Figure 2.5 Photograph of the components within the closed loop ex vivo perfusion system. The bulk flow was generated using a Masterflex L/S computerized roller pump (a). A pulse-dampener (b) was added downstream to remove the very high frequency perturbations generated by the roller pump. Higher frequency components of the physiologic arterial pressure and flowrate waveforms were generated using a custom built piston/cylinder device (c). A one way valve (d), was placed between the pulse dampener and the piston to inhibit the flow or pressure from the piston to be dissipated by the pulse dampener. A fluid reservoir (e) completes the closed loop system. 


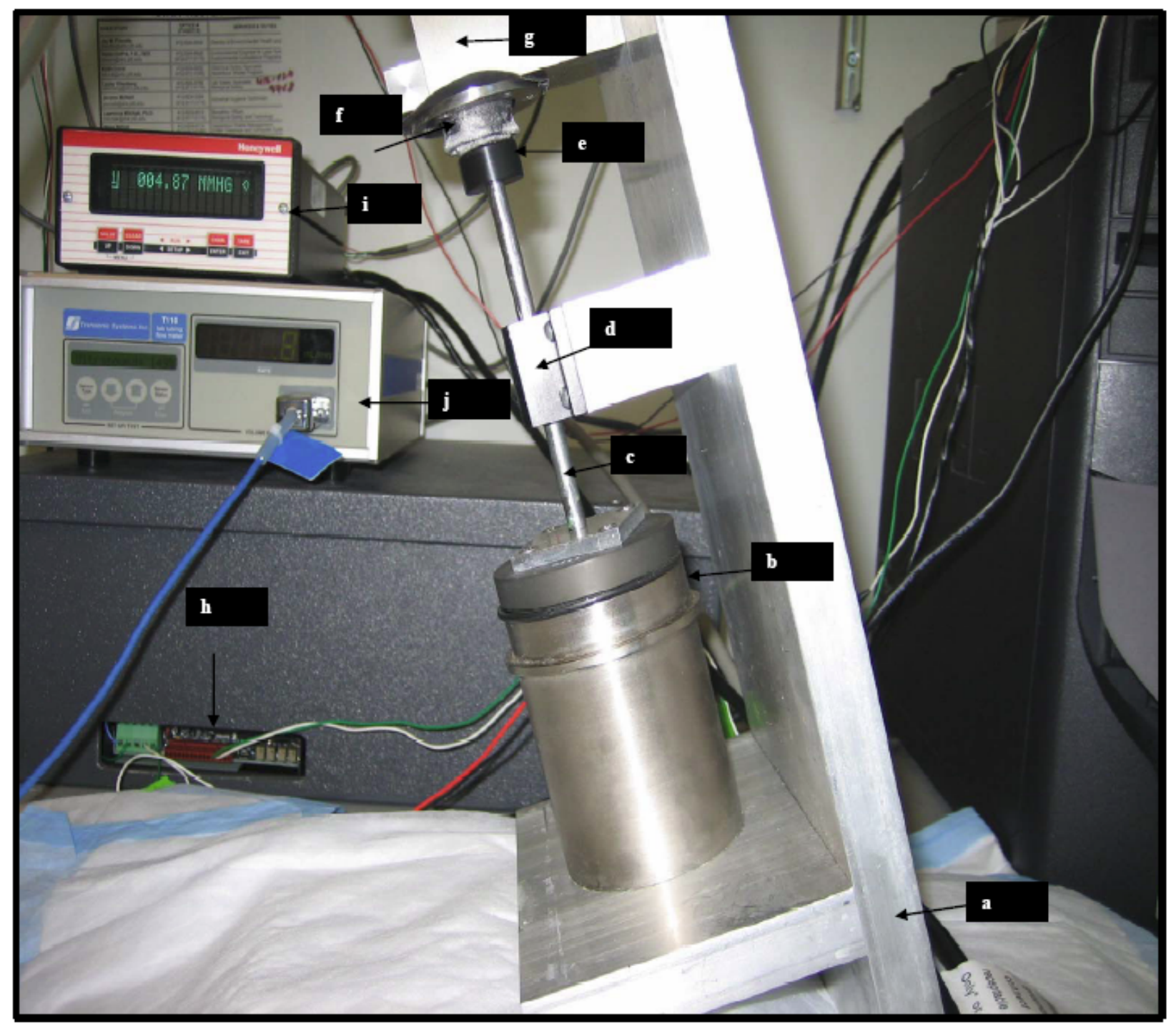

Figure 2.6 A close-up photograph of the piston/cylinder assembly. The components are held together with a custom built frame (a). The voice-coil (b) is coupled to a shaft (c) that translates linearly within the bearing (d). At the end of the shaft, a piston head (e) is connected to a water tight rolling diaphragm (f) that allows the piston head to have frictionless motion within the cylinder ( $\mathrm{g}$ ). The voice-coil is driven by analog signals sent to its own servoamplifier (h). Also seen are the pressure monitor (i) and the tubing flowmeter (j) used to process and transmit the pressure and flow transducer signals, respectively, to the data acquisition system. 


\subsubsection{Perfusate Reservoirs}

A standard media reservoir, used in our laboratory with previous generations of the EVPS $[3,39,248,249,272-274]$, was employed in each loop of the current paired EVPS design - the perfusate reservoir is shown schematically in

Figure 2.2(1), and pictorially in Figure 2.4 and Figure 2.5(e). Each reservoir consists of a plexiglass cylinder (height $=6 "$, outside diameter $=3.5 "$, and inside diameter $=2.5$ "), with detachable lids. Barbed, $1 / 4$ " normal pipe thread ports were drilled into this generic design. The reservoirs have ports at the following locations: (1) bottom center to allow for perfusate outflow; (2) halfway (approximately 3.5") up from the bottom on either side of the cylinder to allow for perfusate inflow and bypass circuit return; (3) approximately 4.5" up from the bottom and on either side of the cylinder - one for purging of physiologic gasses and one for media overflow return from the tissue housing chamber; and (4) in the center of the lid to allow for gas to escape to the atmosphere.

\subsubsection{Tissue Housing Chambers}

Two identical tissue housing chambers were designed for the paired EVPS. These are shown schematically in

Figure 2.2, pictorially in Figure 2.4, and in technical drawings in Figure A. 12 to Figure A. 26 in Appendix A. A plexiglass frame was used with side-mounted glass viewing panels. A detachable lid allowed easy access for implantation of blood vessels into the mounting fixture. The mounting fixture for the stainless steel cannulae facilitates positioning to accommodate blood vessels ranging from 1 to $20 \mathrm{~cm}$ in length. The glass viewing panels allow visualization of 
the specimen and scanning with a helium-neon laser micrometer, described below. The panels are offset at an angle of $5^{\circ}$ to prevent reflection of the incident laser beam. To secure a blood vessel to the mounting fixture, the lid is opened and the cannulated (using duck billed vessel cannulae) vessel segment is slid onto the proximal and distal stainless steel cannulae.

\subsubsection{EVPS Instrumentation}

\subsubsection{Maintaining Biophysical Homeostasis}

To eliminate the need for opening the perfusate reservoir and tissue housing chamber for sampling media or making temperature measurements, we incorporated the use of self-sealing sampling ports (Model IN4000, B. Braun Medical Inc., Bethlehem, PA) and inline Luerthermistors (Model CXTL, Terumo Co., Tokyo, Japan), respectively.

A handheld thermistor logger (Model 93210-50, Cole-Parmer Instrument Co., Bunker, CT) was used to read and record the temperature of the perfusate and bathing media. Temperature was maintained in each loop by using "heat-exchangers" formed by a $30 \mathrm{~cm}$ long stainless steel tube placed into a standard water bath (Model 51221052, Thermo Electron Co., Marietta, $\mathrm{OH}$ ) just downstream of the pumps. The water bath was set so that the media in contact with the tissue was at physiologic temperature $\left(37 \pm 1.5^{\circ} \mathrm{C}\right)$.

A 21 gauge needle was used to sample the media for subsequent blood gas analysis $(\mathrm{pH}$, $\mathrm{pO}_{2}$ and $\mathrm{pCO}_{2}$ ). Blood gasses were maintained at physiologic levels by mixing $5 \% \mathrm{CO}_{2}$ with room air. This gas mixture was then humidified using a clinically approved humidifier (Model 002620, Allegiance Healthcare Co., Salt Lake City, UT.). The humidified gas then passed through a sterile $0.2 \mu \mathrm{m}$ filter (Model 4251, PALL Co., East Hills, NY.) before being purged over the media. The flowrate of each gas was controlled with a ball-float type gas flow meter 
(Model S28A, Dwyer Instruments Inc., Michigan City, IN). Blood gas content $\left(\mathrm{pO}_{2}, \mathrm{pCO}_{2}\right.$, and pH) was measured using a blood gas analyzer (Model ABL5, Radiometer Copenhagen, West Sussex, UK).

Control of both the blood gas content and temperature of the perfusate and bathing media was essential for maintaining tissue viability. The recordings of $\mathrm{pH}, \mathrm{pO}_{2}, \mathrm{pCO}_{2}$ and temperature for several $(\mathrm{N}=18) 24$ hour ex vivo perfusion experiments are shown in

Figure 2.7. The panels on the left represent perfusate measurements, and the panels on the right represent adventitial bath media measurements. 


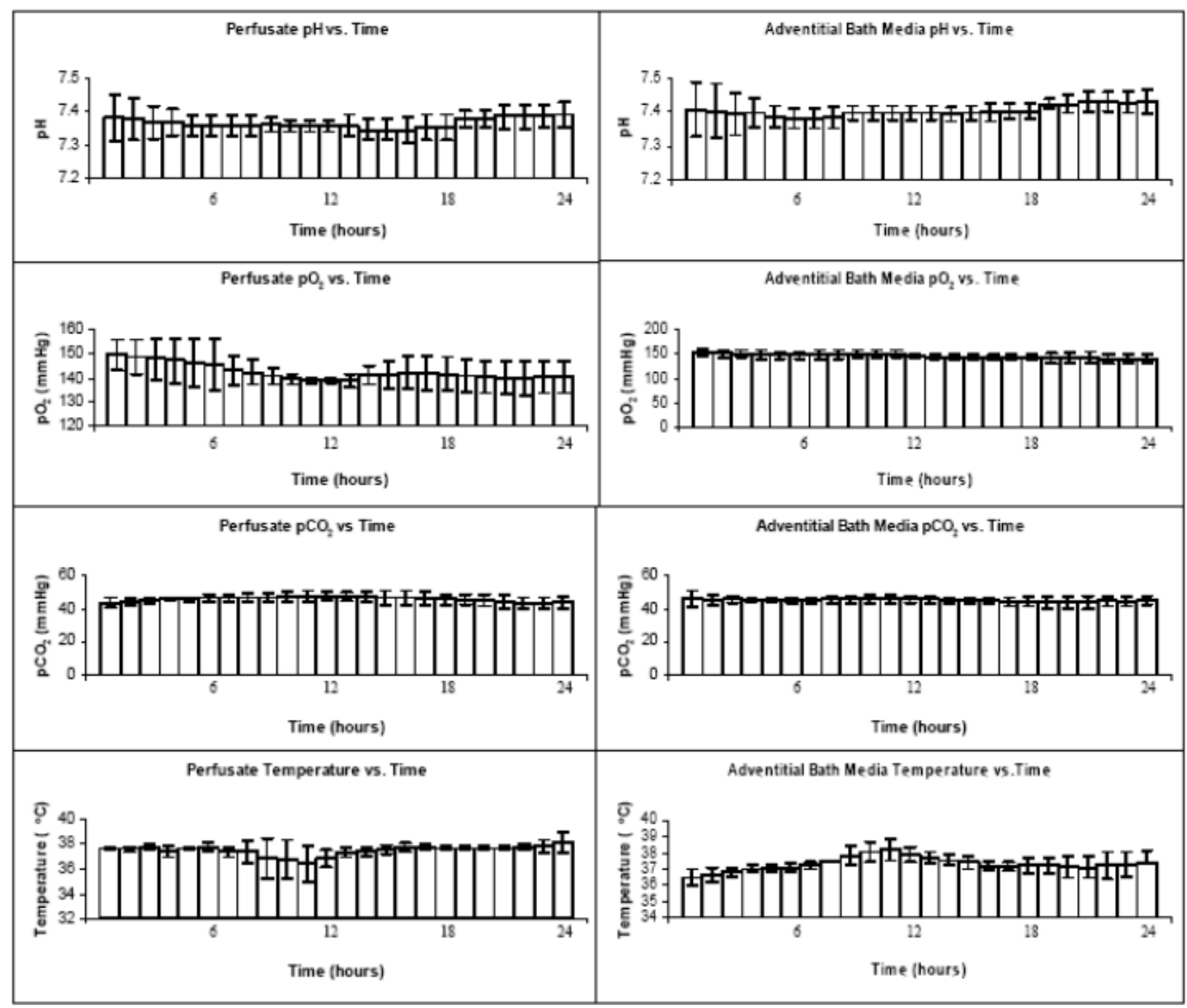

Figure 2.7 Blood gas and temperature control measurements made within our EVPS for several $(\mathrm{N}=18) 24$ hour $e x$ vivo perfusion experiments. The panels on the left are perfusate measurements and the panels on the right are bathing media measurements. Please note that measurements were recorded hourly. 


\subsubsection{Pressure Measurement}

We utilized two pressure transducers (Model TJE, Honeywell - Sensotec Co., Morristown, NJ), placed equidistant upstream and downstream of the vessel center in order to measure intraluminal pressure. The pressure in the center of the vessel was then calculated as the average between the proximal and distal pressure transducer measurements as has been done previously $[3,39,248,249,273,275]$. The pressure transducers sent signals to a pressure monitor (Model SC2000, Honeywell - Sensotec Co., Morristown, NJ) that supplied an analog signal to the data acquisition (DAQ) system.

\subsubsection{Flow Measurement}

Flow within the closed-loop was measured by an ultrasonic flow transducer (Model 4N32, Transonic Systems, Ithaca, NY) and flow meter (Model T110, Transonic Systems, Ithaca, NY). The flow meter generated an analog output signal which was sent to the DAQ system.

\subsubsection{Blood Vessel Outer Diameter Measurement}

Another significant component of the EVPS was the helium-neon laser micrometer (Model 162100, Beta Lasermike Co., Dayton, $\mathrm{OH}$ ), which was used to make non-contacting, continuous outer diameter measurements of implanted blood vessel segments while they were being perfused. The laser was powered and controlled by an "Intellipak" processor (Model 83744, Beta Lasermike Co., Dayton, $\mathrm{OH}$ ) which also provided an analog signal to the DAQ system.

\subsubsection{Analog Input and Output Capabilities}

The DAQ system consisted of a Pentium III personal computer that was equipped with a DAQ card (Model PCI-MIO-16XE-10, National Instruments Co., Austin, TX) that had both high 
frequency analog input and analog output capability. The DAQ card was connected to a shielded connector block (Model 776844-01, National Instruments Co., Austin, TX) via a 68 pin shielded cable (Model 184749-01, National Instruments Co., Austin, TX). All analog input and output signals were received and transmitted from the system via the shielded connector block.

\subsection{SUBSPACE SYSTEM IDENTIFICATION OF EVPS}

To achieve precise simulation of physiologic arterial hemodynamic waveforms from an EVPS, one must first select pumping components with a bandwidth capable of reproducing all the harmonics contained in these waveforms. Secondly, the most accurate mathematical representation of a system will facilitate the design of the most accurate controller for that system. EVPSs described to date have not achieved the ability to generate hemodynamic waveforms containing all the frequencies that are known to makeup physiologic hemodynamic waveforms. The selection and rigorous mathematical modeling of our pumping components has provided us with an EVPS that has a bandwidth capable of generating physiologic hemodynamic signals.

An application such as an EVPS is no different than most engineering applications in that one must "trade-off" model complexity versus accuracy. A complex model will lead to a complex design, while a simplistic model will deteriorate the overall performance and stability of the final implementation. The best model for simulation (for example, a set of partial differential equations which accurately model the system behavior) is not typically the best one for control of an EVPS since the complexity of a controller and the degree of difficulty associated with its implementation are directly proportional to the model complexity. Analytical models rarely 
match well with physical systems and usually require a considerable amount of "tweaking" to work properly when used with control systems. A more empirical approach is required to generate accurate simulation models for control system design [276]. All approaches to model building require that one have in mind a model order, which is usually increased for stability. A more robust alternative to classical techniques, such as frequency and transient response methods, is to use system identification to build linear simulation models of linear-time-invariant systems.

The concept of "system identification" was introduced in 1962 by Zadeh [277]. To paraphrase, "identification is the determination, on the basis of input and output, of a system within a specified class of systems, to which the system under test is equivalent”. In the early 1990s a new method for mathematically modeling dynamical systems, directly from input and output data, known as subspace state space system identification (SYSID), originated by combining concepts from the fields of system theory, geometry and numerical linear algebra [278].

The output of the Numerical algorithm for Subspace State Space System IDentification (N4SID) is a state space (SS) model of a dynamic system based on input and output data, requiring only specification of the system order $[279,280]$. An advantage of this technique is that the system order can be estimated by examining the singular values of a matrix that is calculated during the identification process as the product of the extended observability matrix and the state sequence [278-280].

The specific purpose of the work described in this section was to use N4SID to generate SS models of the flowrate and pressure generation capabilities within our EVPS for implementation into a feedback controller design. 


\subsubsection{SYSID Experiments}

An important aspect of the theory behind the SYSID algorithm is for the exciting signals to be spectrally dense [277-282]. Ideally, one would like to excite the system to be identified with a zero-mean white noise signal. In practicality, however, such signals may not be feasible to implement as was the case with our EVPS. For the SYSID experiments described in this study, physiologic arterial pressure and flowrate waveforms each containing ten harmonics were used to excite the system. These waveforms can be seen in Figure 2.8. Spectral analysis of the measured waveforms indicated power content up to 10 harmonics.

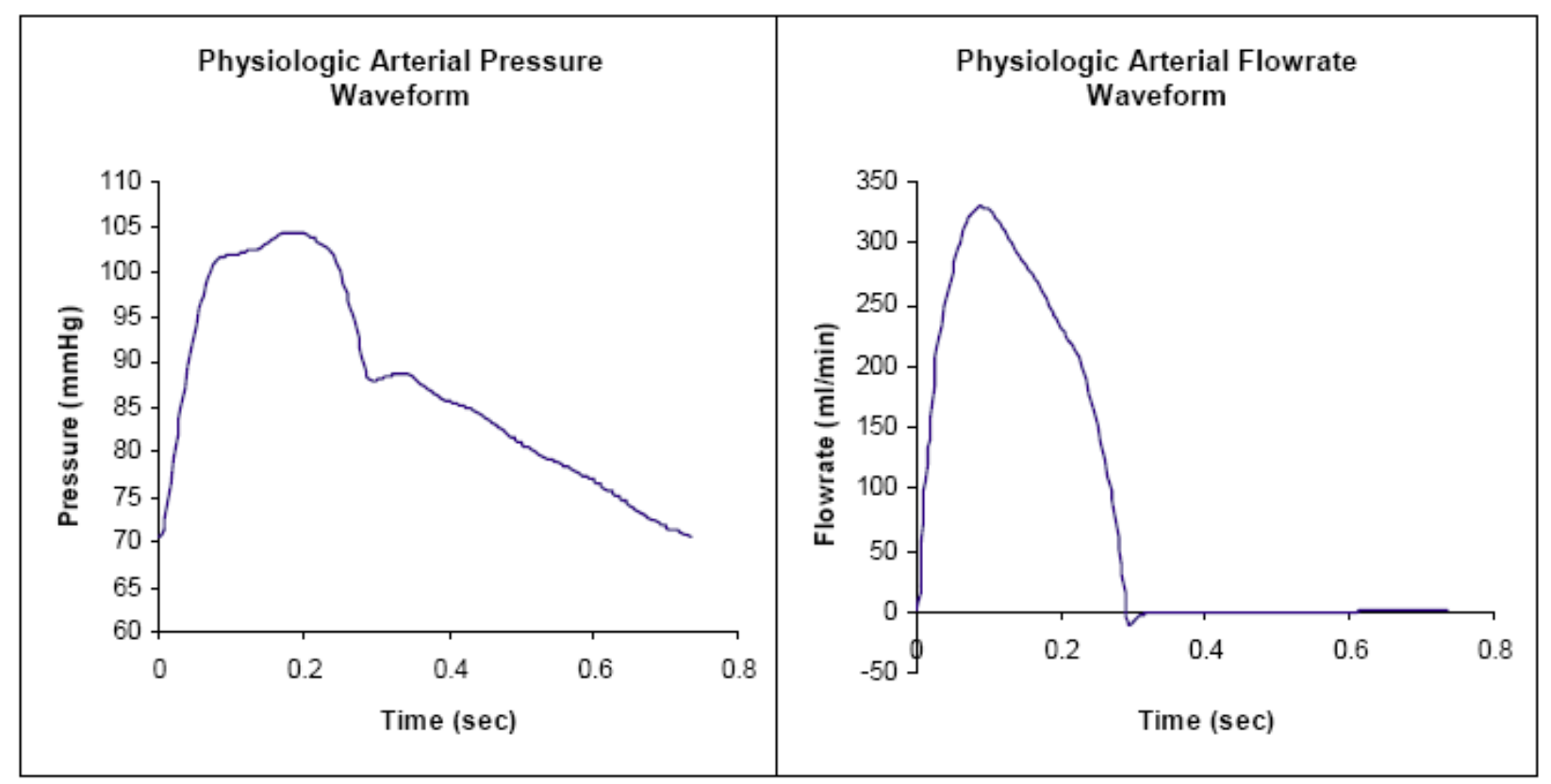

(A)

(B)

Figure 2.8 Physiologic arterial pressure (A) and flowrate (B) waveforms recorded from the aorta of a $45 \mathrm{Kg}$ pig using a $150 \mathrm{~Hz}$ sampling frequency. 
Separate sets of SYSID experiments were performed to identify the SS models representing the pressure and flowrate generating capabilities of the EVPS. For both sets of experiments the sampling frequency $\left(\mathrm{F}_{\mathrm{s}}\right)$ was set to $150 \mathrm{~Hz}$. The duration of each experiment was 60 seconds (60 waveforms), yielding a total of 9000 measured data points. Additionally, both sets of experiments were repeated five times $(\mathrm{N}=5)$ to demonstrate the reproducibility (via calculation of the standard deviation) of the model fit results. In order to better understand the interrelated dynamics of the pumping components within the EVPS, both the pressure and flowrate SYSID experiment sets were further subdivided into the SYSID of the roller pump alone and the SYSID of the series combination of the roller pump and piston. Two simple programs (simultaneous analog input and output) were written in $\mathrm{MATLAB}^{\circledR}$ to drive the roller pump and/or piston and to acquire and filter the measured pressure and flowrate signals. These MATLAB $^{\circledR}$ scripts can be found in Appendix B.

All SYSID algorithms consist of two steps [278-280,282,283]. The first step makes an orthogonal projection, onto a prescribed plane, of the subspaces generated via the data block Hankel matrices. This is followed by a singular value decomposition to find an estimate of the extended observability matrix and an estimate of the states of the unknown system [278-280], which completes the first step. The second step then retrieves the system matrices from either the extended observability matrix or the estimated states using a least-squares approach [278280]. The different SYSID algorithms have been automated and included as part of the SYSID toolbox in MATLAB ${ }^{\circledR}$. SS models were estimated directly from our input and output data.

To generate the discrete time SS model of the pressure generated by the roller pump, a step voltage input was used, and the resulting pressure in the system was measured. After being 
imported, the data were preprocessed by selecting the first one third of the data for estimation and the remaining two thirds for validation.

The N4SID algorithm was chosen because it provided the best results after comparing the model fits. Other algorithms that were tried include the Multivariable Output-Error State sPace (MOESP) [282] and Canonical Variate Analysis (CVA) [283] algorithms. All of these algorithms output SS models of the form:

$$
\begin{gathered}
x\left(t+T_{s}\right)=A \cdot x(t)+B \cdot u(t)+w(t) \\
y(t)=C \cdot x(t)+D \cdot u(t)+v(t)
\end{gathered}
$$

where $T_{s}=\frac{1}{F_{s}}, x$ is an $n$ (system order) by 1 vector representing the state, $y$ is the output vector of length $l, u$ is the input vector of length $m, A$ is the ( $n$ by $n)$ state matrix, $B$ is the ( $n$ by $m$ ) input matrix, $C$ is the ( $l$ by $n$ ) output matrix, $D$ is the feed-through (or feed-forward) matrix, $w$ is a vector representing the measurement noise, and $v$ is a vector representing the process noise.

Models of up to $10^{\text {th }}$ order were considered, and the focus of the identification was to minimize prediction errors. The resulting SS model was then converted into a discrete-time transfer function (TF) using a zero-order-hold transformation. The TFs were then factored into a "Zero-Pole-Gain" (ZPK) model. The model then had the form:

$$
H(Z)=k \frac{\left(Z-z_{1}\right)\left(Z-z_{2}\right) \ldots\left(Z-z_{m}\right)}{\left(Z-p_{1}\right)\left(Z-p_{2}\right) \ldots\left(Z-p_{n}\right)}
$$


where $H$ is the system output divided by the input (as with a TF), $z$ and $p$ are the vectors of realor complex-valued zeros and poles, and $k$ is the real- or complex-valued scalar gain. Note that a ZPK model is a factored TF.

The zeros, poles and gains from the five SYSID models were then averaged to yield an average ZPK model. This average ZPK model was then converted back into TF form:

$$
T F=\frac{\text { Output }}{\text { Input }}=\frac{a_{o}+a_{1} Z^{-1}+a_{2} Z^{-2}+\cdots+a_{n} Z^{-n}}{b_{o}+b_{1} Z^{-1}+b_{2} Z^{-2}+\cdots+b_{n} Z^{-n}}
$$

where $a$ and $b$ are the numerator and denominator polynomial coefficients, respectively, of the discrete-time TF. This is an averaged TF, representing the pressure generated by the roller pump divided by its input, and will henceforth be referred to as $\mathrm{H}_{\mathrm{RP}, \mathrm{p}}$.

The same procedure was performed for the series combination of the roller pump and piston pump except that a step voltage input was sent to the roller pump and a $1 \mathrm{~Hz}$ (60 beats per minute) physiologic arterial pressure waveform (seen in Figure 2.8(A)) input was sent to the piston pump. The resulting average TF will henceforth be referred to as $\mathrm{H}_{\mathrm{RPP}, \mathrm{p}}$. Please note that the input combination used to generate $\mathrm{H}_{\mathrm{RP}, \mathrm{p}}$, yielded the optimum $\mathrm{TF}$ representing the pressure generation capabilities of the roller pump and piston in series. The same is also true for the other identified TFs. That is, the different input signals that were ultimately used to drive the pump(s) yielded the optimum respective TFs.

To generate the SS model of the flowrate generated by the roller pump, a $1 \mathrm{~Hz}$ ( 60 beat per minute) physiologic arterial flowrate waveform (seen in Figure 2.8(B)) was input and the flowrate in the closed-loop system was measured. The resulting average TF will henceforth be referred to as $\mathrm{H}_{\mathrm{RP}, \mathrm{f} .}$ The process was repeated for the series combination of the roller pump and piston pump using a $1 \mathrm{~Hz}$ physiologic arterial flowrate waveform (seen in Figure 2.8(B)) input 
to both the roller pump and piston. The resulting average TF will henceforth be referred to as $\mathrm{H}_{\mathrm{RPP}, \mathrm{f} \cdot}$

The open loop step, impulse and frequency responses of $H_{R P, p}, H_{R P P, p}, H_{R P, f}$ and $H_{R P P, f}$ were generated for analysis.

\subsubsection{Statistical Analysis}

The SS model fit, computed as the percentage of the output variation that is reproduced by the model to a prescribed input, was calculated using the following equation:

where $y$ is the measured output, $\hat{y}$ is the simulated model output and $\bar{y}$ is the mean measured output. The || || symbol is the 2-norm operator that yields the Euclidean length of a vector which is defined as the square-root of the sum of the elements of a vector. The model fit results for each identified system are reported as the mean \pm the standard deviation of the model fit (of five SYSID experiments).

\subsubsection{Results}

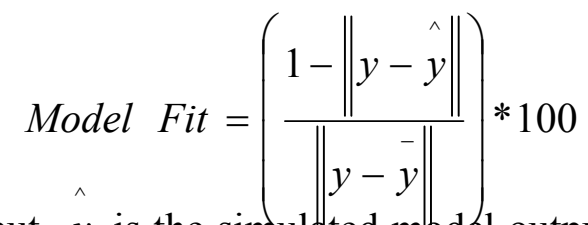

The mean model fits were $94.93 \pm 1.05 \%$ for $H_{R P, p}, 81.29 \pm 0.20 \%$ for $H_{R P P, p}, 94.45 \pm$ $0.73 \%$ for $\mathrm{H}_{\mathrm{RP}, \mathrm{f}}$ and $77.12 \pm 0.36 \%$ for $\mathrm{H}_{\mathrm{RPP}, \mathrm{f} .}$ A summary of these results can be seen in

Table 2.1. 
The step, impulse, and frequency responses for $H_{R P, p}, H_{R P P, p}, H_{R P, f}$, and $H_{R P P, f}$ can be seen in Figure 2.9 and Figure 2.10. From the step and impulse responses shown in Figure 2.9(A), it can be seen that $\mathrm{H}_{\mathrm{RP}, \mathrm{p}}$ has very slow, over-damped and open-loop-stable poles. The frequency response (Figure 2.9(B)) suggests that $\mathrm{H}_{\mathrm{RP}, \mathrm{p}}$ is low frequency and has a bandwidth of approximately $0.1 \mathrm{~Hz}$. Based on the step and impulse responses shown in Figure 2.9(C), $\mathrm{H}_{\mathrm{RPP}, \mathrm{p}}$ appears to have much faster, slightly under-damped, but open-loop-stable poles. The frequency response seen in Figure 2.9(D) suggests that $H_{R P P, p}$ has a band pass filter centered at approximately $37.5 \mathrm{~Hz}$. The combination of the roller pump and piston appeared to respond best from $10-75 \mathrm{~Hz}$, with respect to pressure generation, making the two components complimentary. The piston pump clearly provides the high-frequency content necessary to create the physiologic pressure waveform, while the roller pump is necessary for the mean pressure and flow in the system.

Similarly, from the step and impulse responses seen in Figure 2.10(A), it can be seen that $\mathrm{H}_{\mathrm{RP}, \mathrm{f}}$ has over-damped and open-loop-stable poles. The frequency response (Figure 2.10(B))

Table 2.1 Summary of SYSID model fit results. 


\begin{tabular}{|c|c|c|c|}
\hline $\begin{array}{l}\text { Transfer } \\
\text { Function }\end{array}$ & Model Fit (\%) & Pole Stability & Bandwidth \\
\hline $\mathrm{H}_{\mathrm{RP}, \mathrm{f}}$ & $94.45 \pm 0.73$ & $\begin{array}{l}\text { over-damped, open- } \\
\text { loop-stable }\end{array}$ & $\begin{array}{c}2 \mathrm{~Hz} \text {, but also } \\
\text { responds well b/w 18- } \\
30 \mathrm{~Hz}\end{array}$ \\
\hline $\mathrm{H}_{\mathrm{RPP}, \mathrm{f}}$ & $77.12 \pm 0.36$ & $\begin{array}{c}\text { slightly under-damped, } \\
\text { open-loop-stable }\end{array}$ & $\begin{array}{c}15 \mathrm{~Hz} \text { centered at } 22 \\
\mathrm{~Hz} \text { with a good } \\
\text { response in the } \\
\text { vicitnity of } 70 \mathrm{~Hz}\end{array}$ \\
\hline $\mathrm{H}_{\mathrm{RP}, \mathrm{p}}$ & $94.93 \pm 1.05$ & $\begin{array}{c}\text { very slow, over-damped, } \\
\text { open-loop-stable }\end{array}$ & $0.1 \mathrm{~Hz}$ \\
\hline $\mathrm{H}_{\mathrm{RPP}, \mathrm{p}}$ & $81.29 \pm 0.20$ & $\begin{array}{l}\text { much faster, slightly } \\
\text { under-damped, open- } \\
\text { loop-stable }\end{array}$ & $\begin{array}{c}\text { band pass filter } \\
\text { centered at } 37.5 \mathrm{~Hz}\end{array}$ \\
\hline
\end{tabular}

suggests that $\mathrm{H}_{\mathrm{RP}, \mathrm{f}}$ has a bandwidth of approximately $2 \mathrm{~Hz}$ but also responds well from 18-30 Hz. Based on the step and impulse responses shown in Figure 2.10(C), $H_{R P P, f}$ appears to have slightly under-damped, but open-loop-stable poles. The frequency response seen in Figure 2.10(D) suggests that $H_{R P P, f}$ has a bandwidth of approximately $15 \mathrm{~Hz}$ that is centered about 22 $\mathrm{Hz}$, with good response also observed at around $70 \mathrm{~Hz}$. Again, the responses of the roller pump and piston were complimentary, with respect to flowrate generation, and provided reasonable gain from $0-75 \mathrm{~Hz}$. With a heart rate of 1 to $1.5 \mathrm{~Hz}$ the first 10 harmonics would require a bandwidth of 10 to $15 \mathrm{~Hz}$, meaning that the system greatly exceeds the bandwidth considered necessary for good reproduction of the waveforms. 

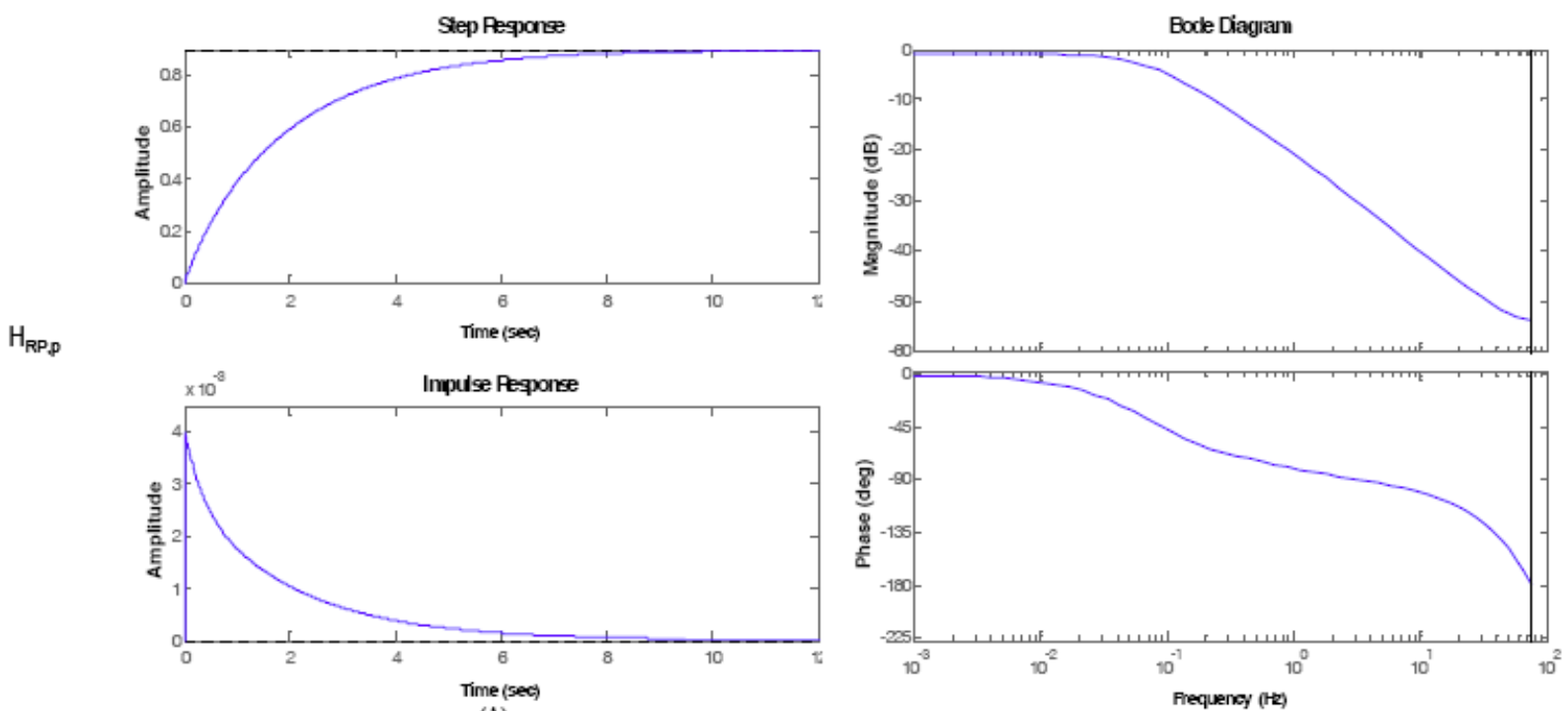

(A)

(B)
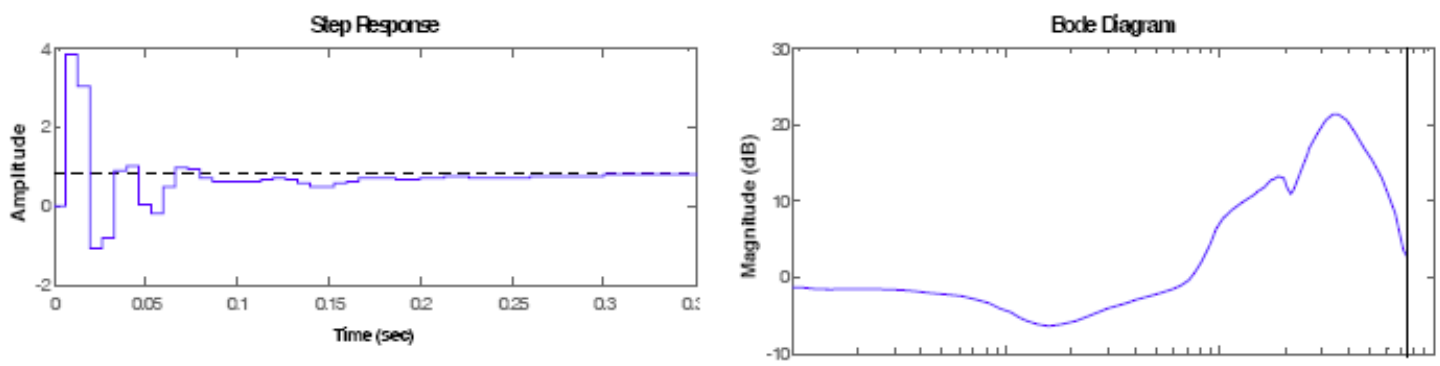

$\mathrm{H}_{\mathrm{RPp} p}$

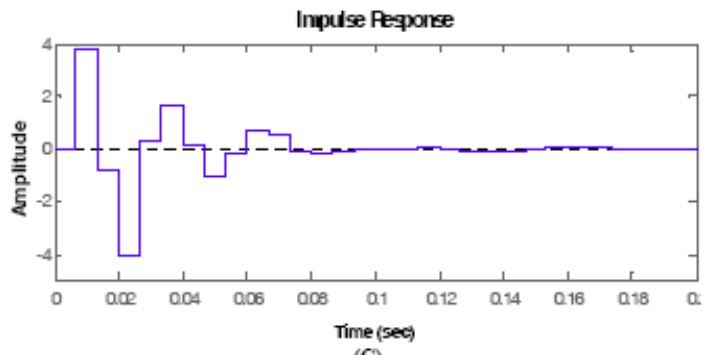

(C)

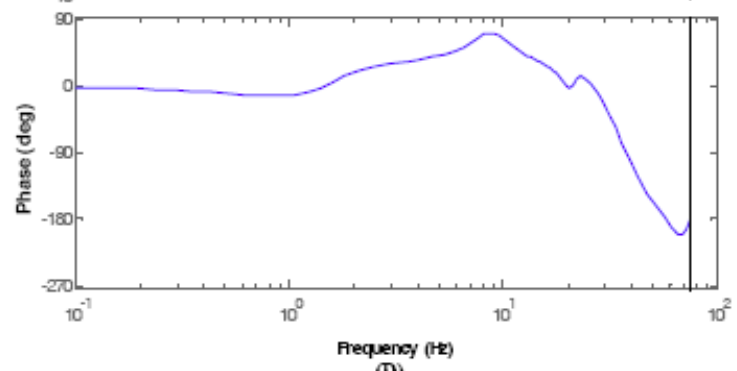

(D)

Figure 2.9 (A) The step and impulse responses suggest that $\mathrm{H}_{\mathrm{RP}, \mathrm{p}}$ has very slow, over-damped and open-loop-stable poles. (B) The frequency response suggests that $\mathrm{H}_{\mathrm{RP}, \mathrm{p}}$ is low frequency and has a bandwidth of approximately 0.1 Hz. (C) The step and impulse responses suggest that $\mathrm{H}_{\mathrm{RPP}, \mathrm{p}}$ appears to have much faster, slightly under-damped, but open-loop-stable poles. (D) The frequency response suggests that $\mathrm{H}_{\mathrm{RPP}, \mathrm{p}}$ has a band pass filter centered at approximately $37.5 \mathrm{~Hz}$. 

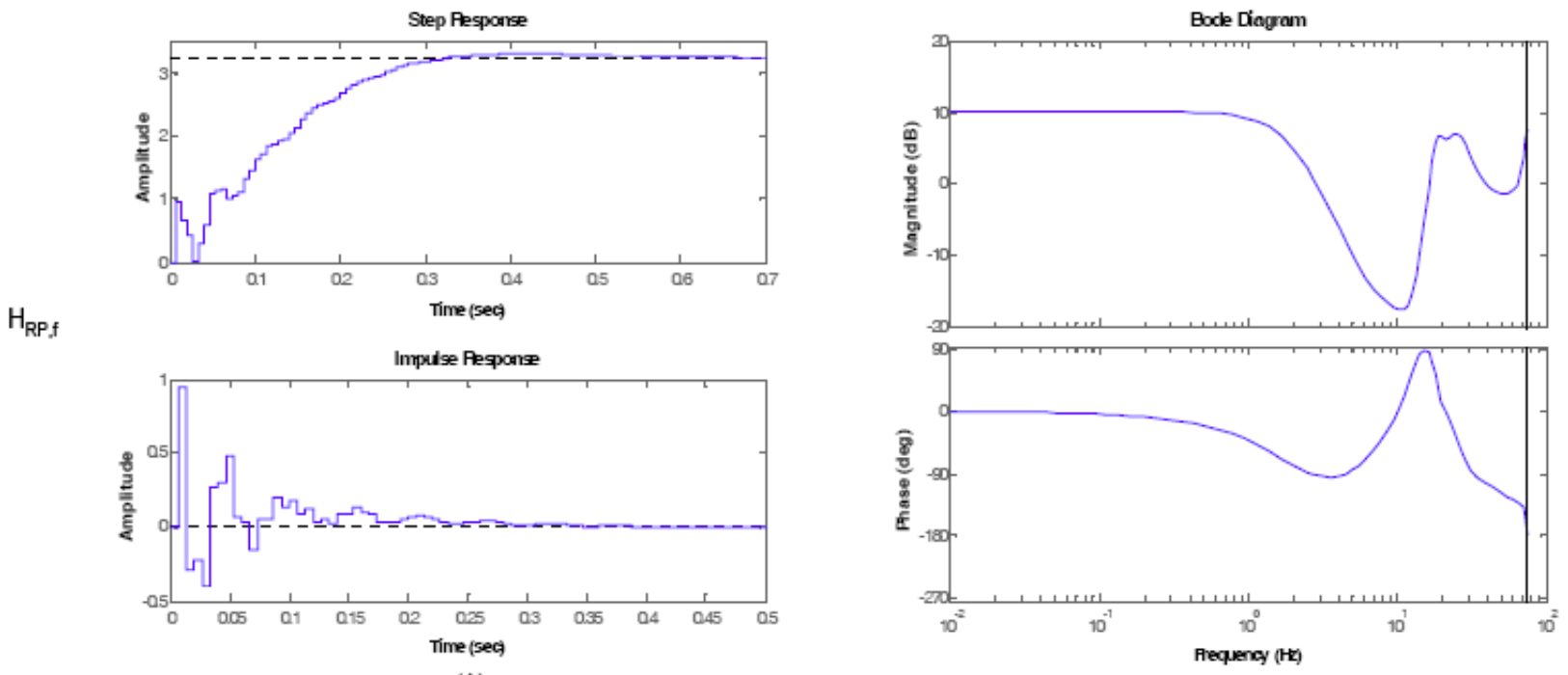

(A)

(B)
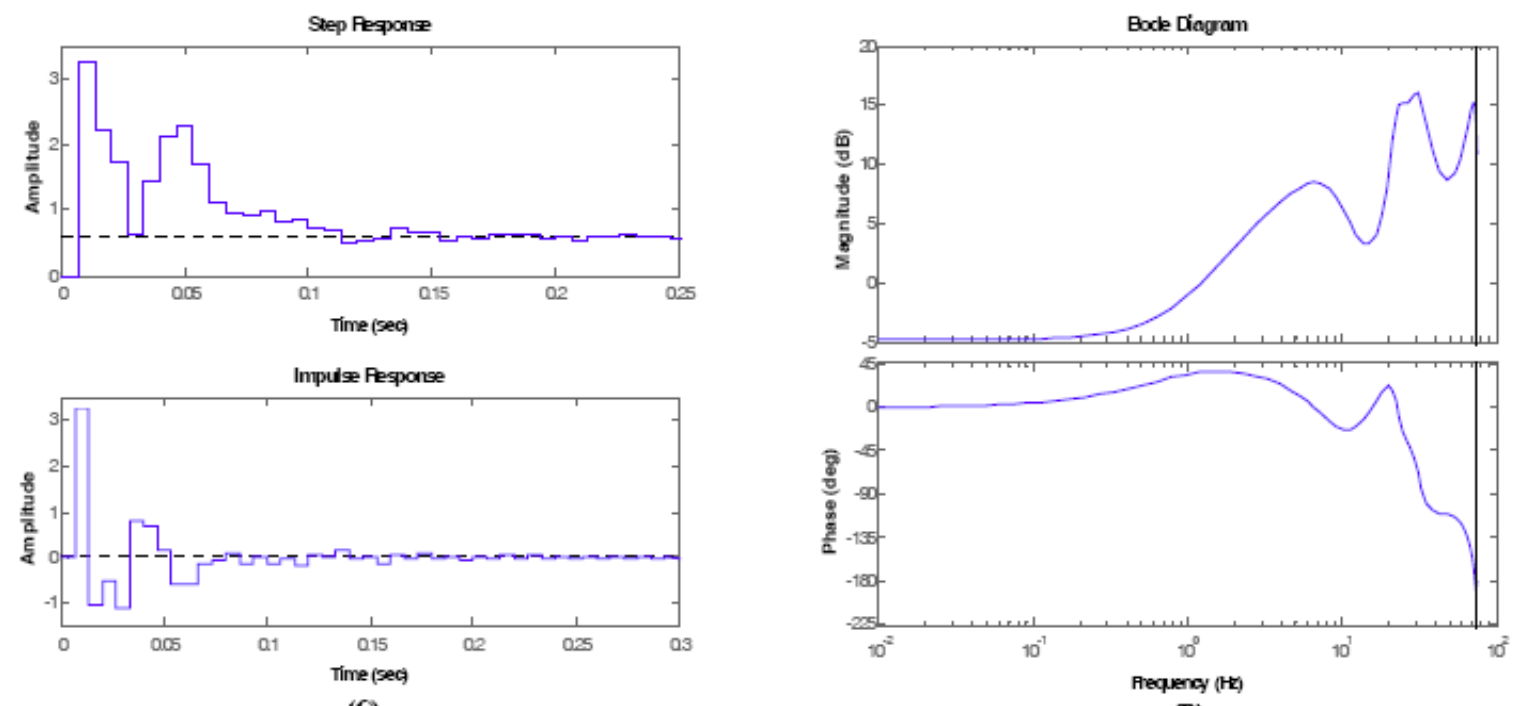

(C)

(D)

Figure 2.10 (A) The step and impulse responses show that $H_{R P, f}$ has over-damped and open-loop-stable poles. (B) The frequency response suggests that $\mathrm{H}_{\mathrm{RP}, \mathrm{f}}$ has a bandwidth of approximately $2 \mathrm{~Hz}$ but also responds well from 18 $30 \mathrm{~Hz}$. (C) The step and impulse responses show that $\mathrm{H}_{\mathrm{RPP}, \mathrm{f}}$ appears to have slightly under-damped, but open-loopstable poles. (D) The frequency response suggests that $\mathrm{H}_{\mathrm{RPP}, \mathrm{f}}$ has a bandwidth of approximately $15 \mathrm{~Hz}$ that is centered about $22 \mathrm{~Hz}$, with good response also observed in the vicinity of $70 \mathrm{~Hz}$. 


\subsubsection{Discussion}

The combined deterministic-stochastic SYSID approach used in this section, in the form of the N4SID, yielded accurate mathematical models of our EVPS. Such models are often used by engineers for industrial plants, especially for designing model-based controllers. We chose the N4SID approach since better accuracy was achieved than with any other available approach.

A precisely controlled EVPS with the capability of imposing and maintaining desired hemodynamic conditions can be an important tool for pathobiological studies using intact blood vessel segments. Although several EVPSs with pulsatile pressure and flow generating capabilities have previously been described, none to date have shown the ability to accurately mimic both physiologic arterial pressure and flowrate waveforms [256,262,265,284-286]. The system recently developed by Bergh et al. (2005) reported robust capabilities in that they were able to control pressure or flowrate, and they validated their control algorithm with disturbances [262]. However, the higher frequency harmonic content of neither the physiologic pressure nor the flowrate signals could be generated using the components of their system. Instead, their system was able to generate and control oscillatory, but not physiologically accurate, pressure or flowrate signals. That is, the harmonic content of physiologic pressure or flowrate signals can not be generated with their EVPS.

The implication of the results presented in this study, with respect to controller design, is that the system configuration we have chosen will allow us to generate both arterial pressure and flowrate waveforms containing all the necessary frequency components of the physiologic arterial pressure and flowrate signals. To our knowledge, SYSID has never been used to identify an EVPS or any other type of electromechanical biomedical device. However, earlier system identification methods have been employed over the years for biomedical applications in which 
this modeling framework has been useful in extracting information from cellular and physiologic measurements. Most recently, applications were in the areas of modeling molecular input and output processes within cells [287], predicting clinical parameters from physiologic measurements [288], and modeling physiologic systems [289].

The N4SID approach to SYSID allowed us to obtain the best possible mathematical representations of our EVPS. We feel it was necessary to perform this rigorous analysis of the EVPS dynamics in order to ensure that we had indeed designed and built a system capable of producing signals with a spectral density equal to or greater than the spectral density of physiologic hemodynamic signals. The ease and accuracy with which the N4SID algorithm was able to identify our EVPS lends to the robustness and applicability of this true "black box" approach for mathematically modeling dynamical systems. The models generated in this study can now be used for designing either a SS controller, by directly using the models output from the N4SID algorithm, or a proportional, integral and derivative controller using the TF model representations.

\subsubsection{Conclusions}

Use of the N4SID approach for mathematically modeling our EVPS was a better choice than any other available approach. This may perhaps be the case for other biomedical devices. The complexity of these devices, due to the complexity of the biological systems with which they interface, requires that an accurate mathematical representation be used for control system design. This could result in a more precisely controlled and hence more reliable device. 


\subsection{PROPORTIONAL INTEGRAL AND DERIVATIVE CONTROL OF CIRCUMFERENTIAL WALL STRESS AND LUMENAL SHEAR STRESS WITHIN VEINS IMPLANTED INTO THE EVPS}

\subsubsection{Rationale for Control System Architecture}

This section will describe the remaining modifications to the previous generation EVPS, and they include: 1) Implementation of safety precautions that allowed the EVPS to be left unattended for short periods of time; and 2) development of a PID algorithm to achieve feedback control over the CWS or luminal shear stress waveforms imposed on implanted vascular segments. Having precise control over the CWS and shear stress generation by our EVPS will help to facilitate the measurement of pathobiological related molecular responses to physiologic vascular biomechanical stimuli.

The PID control algorithm is used for the control of almost all feedback loops in the process industries, and is also the basis for many advanced control algorithms and strategies. In order for control loops to work properly, the PID loop must be properly tuned. For a complete description of the PID algorithm and the standard methods for tuning it, the book by Shaw (2005) is a comprehensive reference [290].

The PID algorithm continues to move the output in the direction that should move the process toward the set point until the process reaches the set point. The algorithm must have feedback (process measurement) in order to perform properly. To be able to tune a PID loop, each of the terms of the PID equation must be understood. The tuning is based on the dynamics of the process response and will be discussed below. 


\subsubsection{PID Control Algorithm}

The PID control algorithm is made of three basic responses, proportional (or gain), integral (or reset) and derivative. The most basic response is the proportional, or gain, response. In its pure form, the output of the controller is the error times the gain added to a constant known as "manual reset". The output is calculated as:

$$
\text { Output }=E \times G+K
$$

where:

Output $=$ the signal to be sent to the process

$E=$ error (difference between the measurement and the set point)

$G=$ gain, and

$K=$ manual reset, the value of the output when the measurement equals the set point.

The output is equal to the error times the gain plus the manual reset. A change in the process measurement, the set point, or the manual reset will cause a change in the output. If the process measurement, set point, and manual reset are held constant the output will be constant.

If we look only at the reset (or integral) contribution from a more mathematical point of view, the reset contribution is:

$$
\text { Output }=G \times K_{r} \times \int E d t
$$


where:

$$
\begin{aligned}
& G=\text { gain } \\
& K_{r}=\text { reset }, \text { and } \\
& E=\text { error } .
\end{aligned}
$$

At any time the rate of change of the output is the gain times the reset rate times the error. If the error is zero the output does not change; if the error is positive the output increases.

Derivative is the third and final element of PID control. Derivative responds to the rate of change of the process (or error). The derivative contribution can be expressed mathematically as:

$$
\text { Output }=G \times K_{d} \times d E / d t
$$

where:

$$
\begin{aligned}
& G=\text { gain } \\
& K_{d}=\text { is the derivative setting, and } \\
& E=\text { the error. }
\end{aligned}
$$

The amount of time that the derivative action advances the output is known as the "derivative time".

The PID control algorithm used in this study was adapted from the book by Shaw [290]. The PID equation is: 
where:

$$
\begin{aligned}
& K_{r}=\text { the reset rate } \\
& K_{d}=\text { the derivative setting } \\
& G=\text { gain, and } \\
& E=\text { error. }
\end{aligned}
$$

Equation 9 was implemented in the MATLAB ${ }^{\circledR}$ programming language and was the core design element of the PID control algorithms.

\subsubsection{Transfer Functions}

Rigorous mathematical modeling of our EVPS was performed and is described in detail in Section 2.4. In short, four TFs were estimated, two for a flowrate controller $\left(H_{R P, f} \& H_{R P P, f}\right)$ and two for a pressure controller $\left(\mathrm{H}_{\mathrm{RP}, \mathrm{p}} \& \mathrm{H}_{\mathrm{RPP}, \mathrm{p}}\right)$, with outputs of pressure (p) and flowrate (f). In each controller, one TF represents a roller-pump $\left(H_{R P, f} \& H_{R P, p}\right)$, and the second TF represents a roller-pump and piston in series $\left(\mathrm{H}_{\mathrm{RPP}, \mathrm{f}} \& \mathrm{H}_{\mathrm{RPP}, \mathrm{p}}\right)$. Using the outer diameter and intraluminal pressure transducer measurements in combination with $\mathrm{H}_{\mathrm{RP}, \mathrm{p}}$ and $\mathrm{H}_{\mathrm{RPP}, \mathrm{p}}$, a CWS controller was designed. Similarly, using the outer diameter and flowrate transducer measurements in combination with $\mathrm{H}_{\mathrm{RP}, \mathrm{f}}$ and $\mathrm{H}_{\mathrm{RPP}, \mathrm{f}}$, a shear stress controller was designed. 


\subsubsection{Mid-vein-wall CWS Calculation for Controller}

The specific intent of the EVPS described in this study was for the investigation of mechanopathobiological responses of vascular segments perfused ex vivo. In order to most accurately control the stresses imposed onto vascular segments, analytical models were derived to calculate the CWS and shear stress within the wall from intraluminal pressure, flowrate, and outer diameter measurements.

The classic Lamé solution (see for example Chandran, 1992 [291]) was employed for an open-ended, thick-walled cylinder under internal pressure $P_{i}$ and external pressure $P_{o}$. The circumferential stress, $\sigma_{\theta}$, at any radius, $r$, is:

$$
\sigma_{\theta}=\frac{R_{i}^{2} P_{i}-R_{o}^{2} P_{o}}{R_{o}^{2}-R_{i}^{2}}-\frac{\left(P_{i}-P_{o}\right)-R_{i}^{2} R_{o}^{2}}{\left(R_{o}^{2}-R_{i}^{2}\right) r^{2}}
$$

where $R_{i}$ and $R_{o}$ are the inner and outer radii, respectively.

We estimated $R_{i}$ from the outer diameter $\left(R_{o}\right)$ laser micrometer measurements. In order to do this, we assumed that blood vessels were incompressible materials and cylindrical in shape. For an incompressible material,

$$
V_{u}=V_{p}
$$

where $V_{u}$ and $V_{p}$ refer to the unpressurized and pressurized states, respectively. For a circular cylinder, equation 11 becomes: 


$$
\left[\pi\left(R_{o}^{2}-R_{i}^{2}\right) L\right]_{\iota}=\left[\pi\left(R_{o}^{2}-R_{i}^{2}\right) L\right]_{p}
$$

where $R_{o}$ and $R_{i}$ are the outer and inner radii, respectively, and $L$ is the cylinder length. So we obtained:

$$
R_{i, p}=\sqrt{\frac{R_{o, p}^{2} L_{p}-\left(R_{o, u}^{2}-R_{i, u}^{2}\right) L_{u}}{L_{p}}}
$$

By substituting equation 13 into equation 10, and assuming that $P_{o}=0$ (atmospheric pressure), we were able to calculate the CWS in the vascular wall. It should be noted that equation $\mathbf{1 0}$ was used to calculate the mid-vein-wall CWS. That is, the CWS was calculated at $r=\frac{R_{i}+R_{o}}{2}$.

\subsubsection{Lumenal Shear Stress Calculation for Controller}

To calculate the shear stress acting on the vessel lumen we used the Hagen-Poiseuille (see for example Nichols et al., 1990 [292]) equation:

$$
\tau=\frac{4 \mu Q}{\pi R_{i}^{3}}
$$


where $\mu$ is the viscosity of the flowing media (assumed to be equal to water), $Q$ is the measured flowrate and $R_{i}$ is the inner radius of the vessel (calculated using equation 13 as with the CWS calculation).

\subsubsection{CWS and Shear Stress Control Signal Calculations}

We determined the setpoint mid-wall CWS and wall shear stress waveforms in a segment of porcine carotid artery (PCA) exposed to a precisely controlled physiologic arterial pressure (Figure 2.11(A)) and flowrate (Figure 2.11(C)) waveforms, respectively, within our EVPS. Using equations 10 and 13, in combination with the intraluminal pressure and outer diameter measurements, the target arterial CWS waveform was calculated. Similarly, using equations $\mathbf{1 3}$ and $\mathbf{1 4}$ in combination with the flowrate and outer diameter measurements, the target lumenal shear stress waveform was calculated. The unpressurized dimensions were measured from rings which had been cut, at the time of harvest, proximally and distally to the PCA segment that was implanted into the EVPS. The rings were individually placed into a custom built holder [245] that allowed the unpressurized thickness, as well as the unpressurized inner and outer diameters to be measured via a helium-neon laser micrometer. A linear variation in dimensions was assumed along the longitudinal axis of the implanted PCA segment so that the dimensions could be estimated at any point along the length. The target waveforms that were calculated (from measured porcine pressure and flowrate waveforms) are shown in Figure 2.11. 


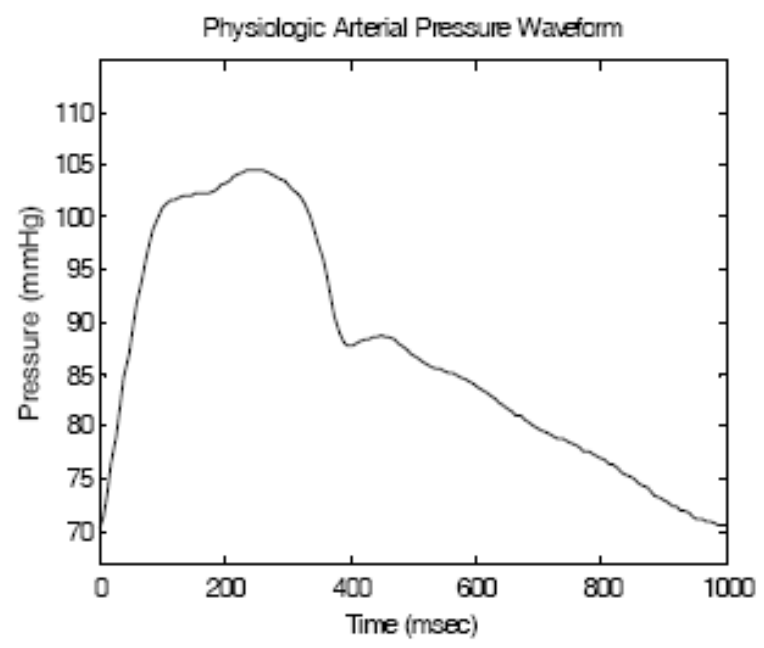

(A)

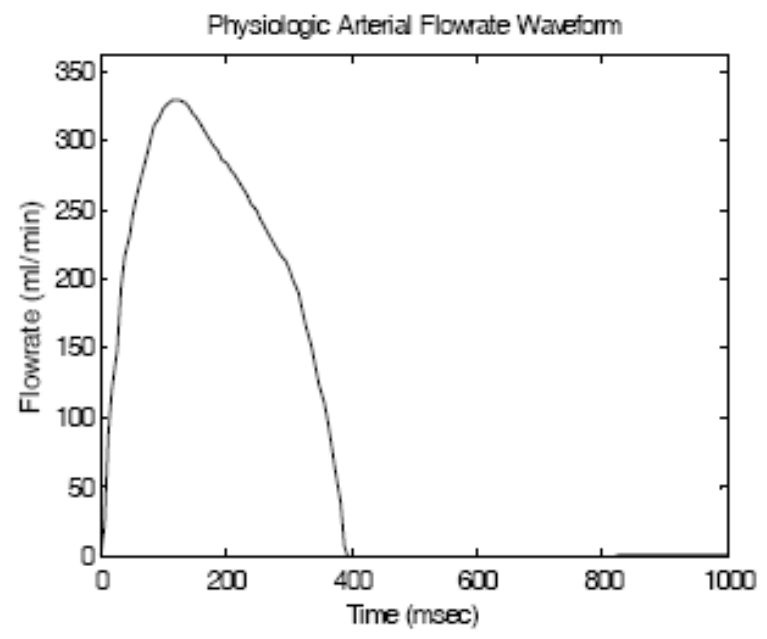

(C)

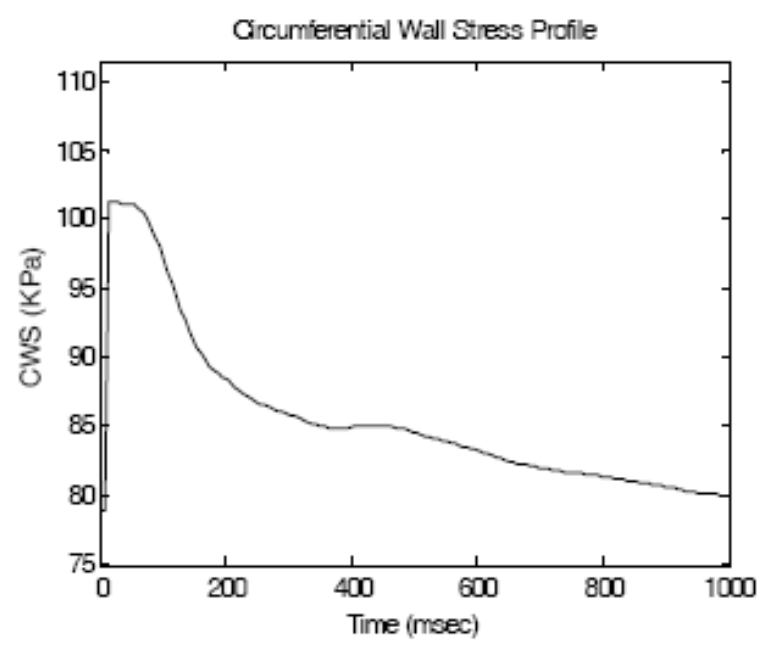

(B)

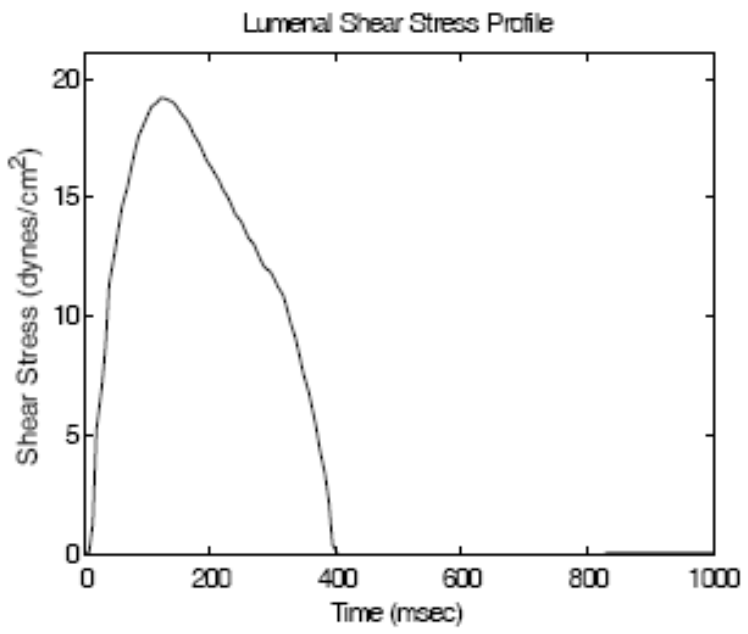

(D)

Figure 2.11 (A) Arterial pressure waveform that was used to calculate the circumferential wall stress profile (B). (C) Arterial flowrate waveform that was used to calculate the shear stress profile (D).

\subsubsection{PID Controller Performance Experiments}

Two separate PID control algorithms were developed using MATLAB ${ }^{\circledR}$, one for mid-wall CWS control and the second for lumenal shear stress control within vascular segments implanted into the EVPS. The MATLAB ${ }^{\circledR}$ scripts for both controllers can be seen in Appendix C. Using 
equation 15, the performance of each controller was assessed by calculating the root mean square of the error (RMSE) between the desired and measured process variables (CWS or shear stress) as:

$$
R M S E=\sqrt{\frac{\sum\left(y_{d}-y_{m}\right)^{2}}{n-n p}}
$$

where $y_{d}$ is the desired value of CWS or shear stress, $y_{m}$ is the calculated value of CWS or shear stress, $n$ is the number of data points, and $n p$ is the number of parameters. These experiments were run for 60 seconds each and repeated 10 times $(\mathrm{N}=10)$. An average RMSE was reported for each PID controller and standard deviations were calculated to demonstrate the reproducibility of the results.

\subsubsection{Implemented Safety Precautions}

As mentioned, the EVPS designed by Labadie et al. [3] could not be left unmanned for more than several minutes thus making perfusion experiments very labor intensive. If a leak developed from an implanted blood vessel, the perfusate would empty into the tissue housing chamber very quickly. In order to prevent this from happening, a safety feature was built into the EVPS. In the event of a leak, the system resistance, defined as pressure divided by the flowrate, decreased. The system was made to respond to this drop in resistance by stopping the piston pump and slowing the roller pump so that it supplied a minimal flowrate of $20 \mathrm{ml} / \mathrm{min}$ through the implanted blood vessel segment. An overflow port was put in place on the tissue housing chamber and was connected back to the perfusate reservoir (see dotted line in 
Figure 2.2) ensuring that the perfusate reservoir never ran dry. Media that leaked from the vessel into the tissue box flowed, via gravity, back to the reservoir. This did not eliminate the need for human interaction to correct a leak. However, it facilitated leaving the EVPS unmanned for short periods of time thus making the perfusion experiments less labor intensive and less costly in the event of a leak. We feel that this safety measure was of extreme importance since it also allowed us to respond to a leak with much less trauma to the implanted tissue. That is, no manual adjustments had to be made to the EVPS and the leak could be immediately addressed.

An experiment was performed to validate the implemented safety precautions. Briefly, the EVPS was set to operate under PID controlled arterial CWS conditions. To simulate a leak, a 21 gauge needle was used to create a hole through the wall of an implanted vein segment, which triggered the safety precautions to be initiated. The systemic pressure and flowrate were measured, and the total resistance of the system was calculated (as mean pressure divided by mean flowrate) during the entire simulation.

\subsubsection{Biological Validation of EVPS}

In order to futher validate our ex vivo vascular perfusion capabilities, we performed preliminary tissue viability analysis of vein segments perfused under VEN vs. ART conditions, as described in Section 3.1.3.1, and compared the results to baseline level of tissue viability. Scanning electron microscopy (SEM), hematoxylin and eosin (H\&E), Live/Dead ${ }^{\mathrm{TM}}$, and TUNEL analyses (as described in Section 3.2.6) were performed after 48 hours of ex vivo perfusion. 


\subsubsection{Results}

Figure 2.12 shows several representative waveforms from both the CWS and shear stress controller performance experiments. The "set" CWS and shear stress waveforms are those shown in Figure 2.11(B) and Figure 2.11(D), respectively, which were used as control signals for the EVPS performance experiments. The "measured" CWS and shear tress waveforms were recorded from the PID controlled EVPS in response to the input control signals. RMSE values for CWS control $(0.427 \pm 0.027 \mathrm{KPa})$ indicated that the system was able to generate a physiologic CWS waveform within $0.5 \%$ error of the peak desired CWS $(\sim 100 \mathrm{KPa})$ over each "cardiac" cycle. RMSE values for shear stress control $\left(0.05 \pm 0.0007 \mathrm{dynes} / \mathrm{cm}^{2}\right)$ indicated that the system was able to generate a physiologic shear stress waveform within $0.3 \%$ error of the peak desired shear stress $\left(\sim 18\right.$ dynes $\left./ \mathrm{cm}^{2}\right)$ over each "cardiac" cycle. 

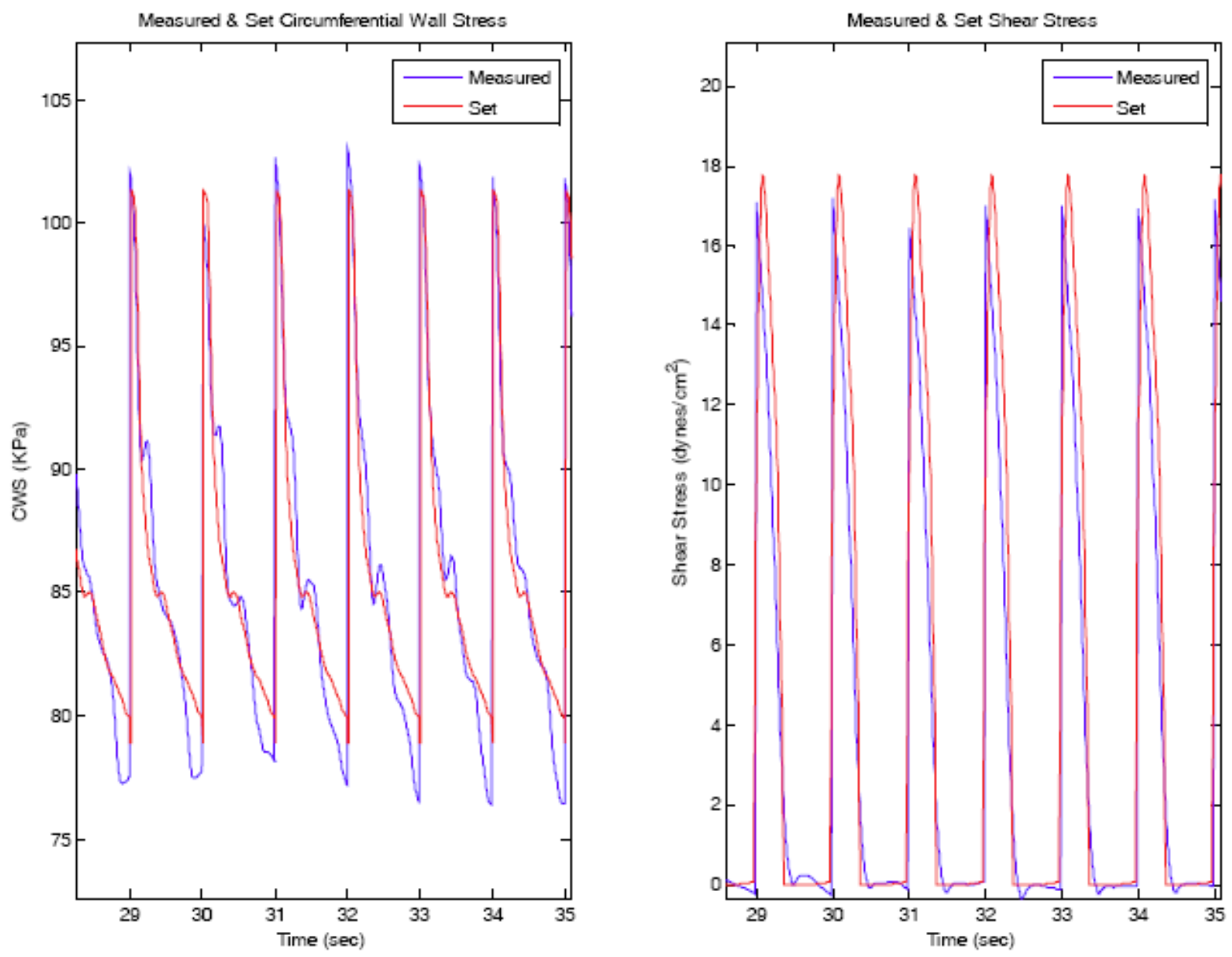

Figure 2.12 Several representative waveforms from the controller performance experiments. The left panel shows the measured and set CWS waveforms, and the right panel shows the measured and set shear stress waveforms.

The plots in Figure 2.13 show the pressure and flowrate measurements, as well as the calculated resistance, recorded during an experiment to validate the implemented safety precautions. It can be seen that, in the event of a leak, the piston stopped and the roller pump slowed to provide a minimal flowrate of approximately $20 \mathrm{ml} / \mathrm{min}$. 

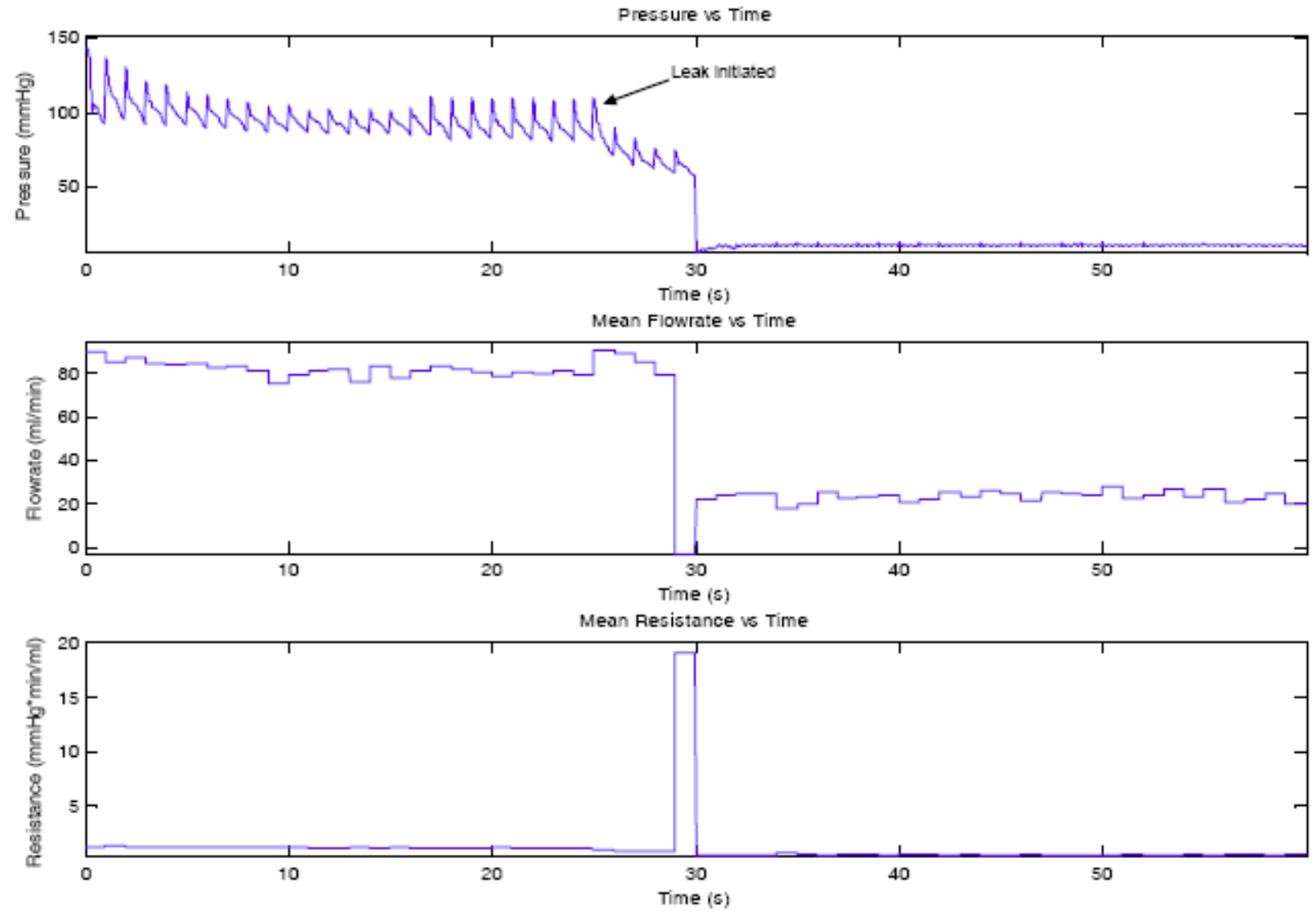

Figure 2.13 Leak simulation experiment results. As desired, in the event of a leak the piston stops and the roller pump slows to supply a minimal flowrate of approximately $20 \mathrm{ml} / \mathrm{min}$.

SEM and H\&E staining (Figure 2.14) indicated that the morphologic integrity of the tissue was intact after harvesting and after 48 hours of perfusion. Live/Dead ${ }^{\mathrm{TM}}$ and TUNEL (Figure 2.14) analyses showed no significant necrosis or apoptosis, respectively, in either the VEN or ART conditions when compared to baseline at 48 hours. This experiment laid the groundwork for the rest of the ex vivo vascular perfusion experiments described in this dissertation and demonstrated our ability to perform the ex vivo porcine internal jugular vein (PIJV) perfusions, with maintenance of sterile conditions and tissue viability. 


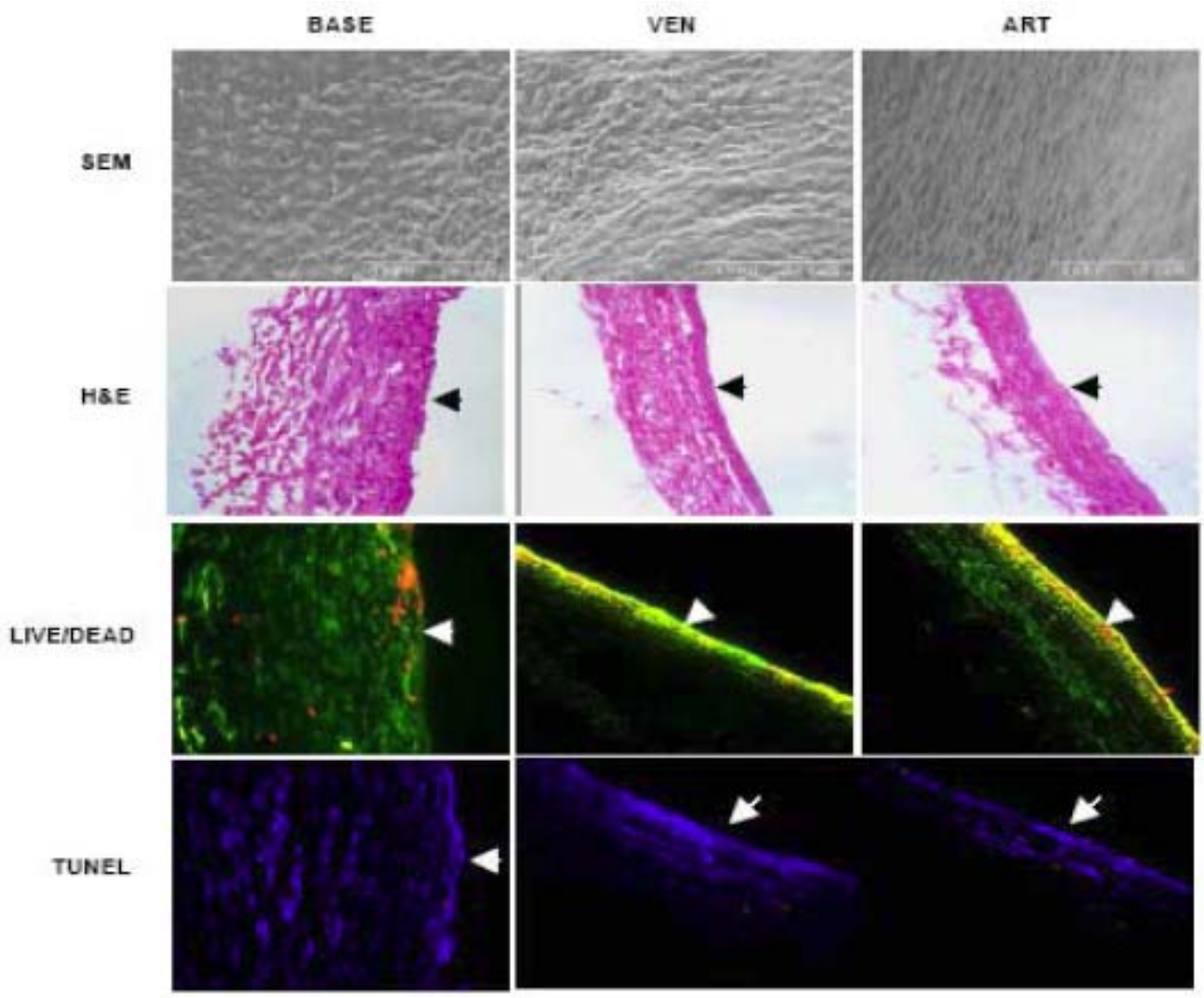

Figure 2.14 The top three panels show representative SEM images of the lumen of baseline control (BASE), "venous" 48 hour perfused control (VEN), and "arterial" 48 hour perfused (ART) PIJV segments. Note the cobblestone appearance of an intact endothelial cell layer. The second row of panels show representative microstructure and live nuclei via H\&E staining of each group (200x magnification). The third row of panels show representative live (green) and dead (red) cells within each tissue group (200x magnification). Note that there does not appear to be an increased level of necrosis in perfused tissue when compared to BASE control tissue. The bottom three panels show representative TUNEL assay images of tissue from the same 48 hour perfusion experiment (400x magnification under immersion oil). Note that there does not appear to be an increased level of apoptosis in perfused tissue when compared to BASE. In all panels the arrow designates the vessel lumen. 


\subsubsection{Discussion}

The purpose of this work was to improve upon the EVPS developed by Labadie et al. [3] by addressing some of its limitations. Maintenance of sterile culture conditions has been achieved for durations up to 14 days using a similar EVPS to the one described in this study [275]. These capabilities may allow the observation of vascular pathologies such as neointima formation in blood vessels perfused ex vivo. This has been reported to occur within 4 days in an in vivo model [171]. Our ability to successfully and reliably (see Figure 2.14) perfuse tissue for long-term experiments can be attributed to the improvements made to the previous generation EVPS [3]. Long-term (e.g., 4 weeks) ex vivo perfusion of intact vascular segments was reported recently in the literature [293]. Based on the previous work by Ligush et al., and based on preliminary experience with the new EVPS, we feel that our system will meet the challenge when used for ex vivo perfusions of extended duration, and will simply require regular media changes to maintain antibiotic and nutrient levels within the system.

Although several EVPSs with pulsatile pressure and flow generating capabilities have previously been described, none to date have shown the ability to accurately mimic both physiologic arterial pressure and flowrate waveforms [256,262,265,270,284-286]. A system described by Bergh et al. (2005) should be noted due to its robust capabilities [262]. They reported the ability to precisely control pressure, flowrate, $\mathrm{pH}, \mathrm{pO}_{2}$ and temperature within their EVPS, and validated their control algorithm with prescribed perturbations to systemic conditions. However, the higher frequency harmonic content of neither the physiologic pressure nor the flowrate signals could be generated using the components of their system. Instead, their system was able to generate and control oscillatory, but not physiologically accurate, pressure or flowrate signals. That is, the harmonic content of physiologic pressure or flowrate signals can 
not be generated with their EVPS. Our EVPS improves upon other EVPS designs in that we are able to generate CWS and shear stress (possibly more pertinent than pressure and flowrate) signals that closely mimic physiologic signals. This was made possible with the customdesigned high-fidelity piston-cylinder device described in Section 2.3.2. Secondly, we believe that feedback control over blood gases and temperature would have been an engineering improvement for the current generation of our EVPS, however, the stability with which we maintained these parameters was sufficient and did not warrant it. The methods used to maintain physiologic blood gases and temperature are similar to those used by Labadie et al. [3]. However, placement of media sampling ports and inline thermistors was helpful in maintaining sterility within the EVPS. Humidification of the gas prior to purging over the media helped to prevent evaporation making all system parameters (including $\mathrm{CWS}$, shear stress, $\mathrm{pH}, \mathrm{pO}_{2}, \mathrm{pCO}_{2}$ and temperature) much more stable.

A limitation of our current EVPS is that when a leak occurred there was mixing of perfusate and bath media. It should be noted that two different media types were used, M199 was used as perfusate and DMEM was used as bath media. Even with successful implementation of the safety precautions described in this report, when major leaks occurred, the media had to be drained and the system refilled once the leak was fixed. Another limitation comes as a result of the notion that simultaneous control of the CWS, shear stress, and the phase angle between them would be ideal. However, it is expected that this type of control system requires a multivariable SS controller. That is, the PID control architecture used in this study is insufficient for controlling multi-input-multi-output systems. Instead PID control is limited to single-input-single output control systems. Efforts to achieve this capability were beyond the scope of the current work. 
In order to most accurately assess molecular changes occurring within the vasculature in response to biomechanical signals, a precisely controlled EVPS is the optimum model to use. This model eliminates the confounding conditions associated with both cell culture and in vivo models by providing an isolated system in which desired stimuli can be imposed on intact vascular segments. The observed molecular changes can then be directly correlated to the imposed stimulus.

\subsubsection{Conclusions}

The PID controllers to achieve CWS and shear stress control make the EVPS described in this chapter unique. That is, stress control within the EVPS may be more pertinent to understanding the molecular responses of vascular tissue to the biomechanical environment than is control of simply pressure and flowrate. The analytical models that were derived make the EVPS control systems even more robust by incorporating tissue-specific geometry. Of course, any stress model could be substituted depending on the geometry of the implanted tissue. Based on the EVPS controller performance results we believe that the choice of using a PID algorithm to achieve these capabilities was justified. 


\subsection{EX VIVO PERFUSION EXPERIMENTS}

Specific Aims 2 and 3 were accomplished by performing several sets of ex vivo vascular perfusion experiments. Initially, one set of experiments (see Section 3.1.3.1) was performed to establish the acute hyperplastic response of PIJVs abruptly exposed to arterial biomechanical conditions, and to compare this response to PIJVs exposed to native venous conditions. We then attempted to attenuate this acute hyperplastic response by gradually exposing PIJVs to desired CWS profiles via manual adjustment of EVPS pressure (see Section 3.1.3.2). These experiments were directly related to Specific Aim 2. In Specific Aim 3 we wanted to tune the degradation rate of an adventitial biodegradable polymer wrap so as to achieve the same CWS profiles as in Specific Aim 2, and then to use this wrap to attenuate the acute hyperplastic response in PIJVs compared to unwrapped controls (see Section 3.1.3.3).

Each of these experiments was "paired" to account for animal-to-animal variability, and generally, proceeded as follows. Bilateral PIJVs were surgically harvested from juvenile pigs (see Section 3.1.1) and tied into separate, independent EVPSs (see Section 2.1). Vascular perfusion experiments were carried out for 24 or 72 hours since the majority of the endpoints under investigation have been successfully detected within a few hours of these time points (see references in Table 1.2). At the conclusion of each experiment, the tissue was processed (see Section 3.1.6) for biological assays to asses the endpoints outlined in Table 1.2. 
This chapter will describe in detail the methods used and the results obtained from these ex vivo experiments. It will conclude with a discussion of the implication of the results.

\subsection{METHODS}

\subsubsection{Tissue Harvest and Transport}

All animal procedures were performed under a protocol approved by the Institutional Animal Care and Use Committee of the University of Pittsburgh. The porcine internal jugular vein (PIJV) was chosen as a model because of its similarity in inner diameter and wall thickness to the human greater saphenous vein, and because this tissue has previously been used to investigate the pathologic response of veins exposed to arterial hemodynamic conditions $[43,49,51,81,163,221,248,273,294-296]$. The surgical harvest procedure was performed in the

manner of a saphenectomy for bypass. Briefly, the anesthetized animal was placed in supine position, cervical incisions were made bilaterally, and dissection was done in layers to the vascular fascia of the neck. Each PIJV was identified and dissected proximal to the jugular confluence and distal to the jugular foramen. All tributaries were identified and carefully ligated to avoid leakage. After the desired length $(6-8 \mathrm{~cm})$ was exposed, the segment was cannulated on each end with duck billed vessel cannulae. Just prior to explant, a custom-designed vascular clamp [275] (see technical drawings in Figure A. 27 to Figure A. 32 in Appendix A) was attached onto the ends of the cannulae to maintain the in vivo length of the vessel following removal. The vessel was then cut on either side between the clamped cannulae and the ligations. Immediately after removal, the vessels were placed in a sterile transport box (containing lactated 
ringers solution supplemented with heparin (500 units/liter), papaverine (60 mg/liter), and Cefoxitin (1.0 g/liter). Technical drawings of the transport box are given in Figure A. 33 to Figure A. 51 Appendix A. Figure 3.1 shows a PIJV segment within the clamp which is immersed in media within the transport box. The time between tissue harvest and mounting into the perfusion system described below was always less than one hour. The animal was euthanized upon completion of surgery according to university guidelines.

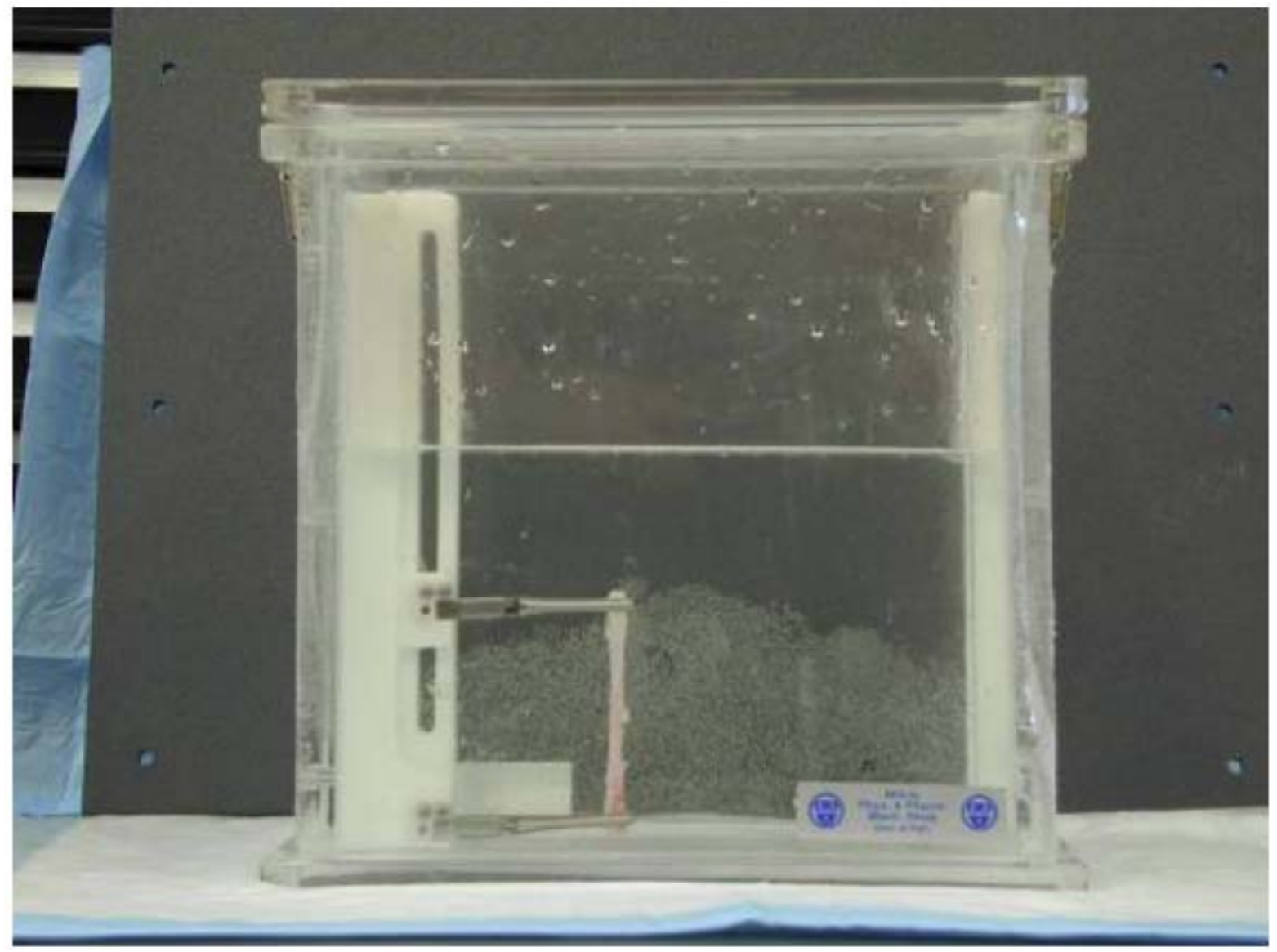

Figure 3.1 Harvested PIJV segment within custom designed in vivo-length clamp and immersed in media within the transport box. 


\subsubsection{Perivascular Placement of Electrospun Biodegradable Polymer Wrap}

The biodegradable polymer composite used to form the adventitial wrap was based on the poly(ester urethane)urea (PEUU) material developed by Guan et al. [297] and further characterized in electrospun format by Stankus et al. [57,242]. This polymer undergoes hydrolytic degradation in vitro into non-cytotoxic degradation products and has been shown to degrade to near completion in vivo at approximately 3 months [298,299]. To control the degradation rate of the wrap, a composite of PEUU, collagen, and elastin proteins was utilized, with protein addition used to hasten mass loss.

PEUU was synthesized from poly( $\varepsilon$-caprolactone $)$ diol and 1,4-diisocyanatobutane with putrescine chain extension. PEUU, collagen, and elastin were combined in solution in 1,1,1,3,3,3-hexafluoro-2-propanol (HFIP), and then electrospun onto a PIJV segment using a procedure explained in detail elsewhere [57]. Briefly, electrospinning conditions included a mixture solution volumetric flowrate of $0.28 \mu \mathrm{L} / \mathrm{s}$, a distance between nozzle and target of 17 $\mathrm{cm}$, and electrical charges of $+12 \mathrm{kV}$ to the nozzle and $-3 \mathrm{kV}$ to the target. The target used for fabrication of spun AVGs for implantation was a Type 316 stainless steel mandrel of $3 \mathrm{~mm}$ diameter that was carefully inserted into the AVG lumen to avoid endothelial injury. The mandrel and coaxial vein were rotated together at $250 \mathrm{rpm}$, and translated axially on a linear stage at a speed of approximately $8 \mathrm{~cm} / \mathrm{sec}$ over $10 \mathrm{~cm}$ to produce a more uniform coating thickness.

There were three parameters used to tune the mechanical properties and degradation rate of the polymer: 1) the final polymer concentration in a mixture solution; 2) the PEUU:collagen:elastin ratio in the mixture solution; and 3) the wrap thickness, which was proportional to electrospinning time. In our first attempt (combination D, Table 3.1), we used a 
50:50 PEUU:collagen ratio with a final concentration of $12 \%$ that was electrospun onto a PIJV segment for 20 minutes. This combination was chosen based on the mechanical properties, being somewhat similar to arterial mechanical properties, reported by Stankis et al. for this polymer [57]. This provided a starting point for us to tune the polymer wrap in order to achieve mechanical properties that were similar to arterial tissue. In order to show that we could tune the polymer wrap to biodegrade in a short a time period, we decided to use 24 hours as the desired biodegradation timeframe. Several combinations were attempted in order to achieve this biodegradation rate and a summary of all tested parameter combinations is shown in Table 3.1. Combination "C" was our second attempt, where we shortened the electrospinning time and reduced the final polymer concentration in an effort to increase the biodegradation rate. Combination "B" was the third attempt, where we increased the proportion of collagen in order to hasten mass loss in an effort to further increase the rate of biodegradation. Combination "A" was the final choice where we incorporated elastin into the wrap, as well as slightly increased the electrospinning time. This combination satisfied our design requirements and was chosen for both the ex vivo and in vivo experiments described in the rest of this dissertation.

Table 3.1 Summary of polymer tuning parameter combinations.

\begin{tabular}{|c|c|c|c|}
\hline Combination & $\begin{array}{c}\text { PEUU:collagen:elastin } \\
(\mathbf{\%})\end{array}$ & $\begin{array}{c}\text { Electrospinning Time } \\
\text { (min) }\end{array}$ & $\begin{array}{c}\text { Final Concentration } \\
\text { (\%) }\end{array}$ \\
\hline A & $14.3: 42.3: 42.3$ & 20 & 6 \\
\hline B & $25: 75: 0$ & 15 & 6 \\
\hline C & $50: 50: 0$ & 15 & 6 \\
\hline D & $50: 50: 0$ & 20 & 12 \\
\hline
\end{tabular}


Following electrospinning, the PIJV segments were placed back into the vascular clamps, again maintaining the in vivo length of the tissue [275]. The clamp was then placed back into the transport box (Figure 3.1) so the spun PIJV could be taken to he laboratory and implanted into the EVPS as described in Section 3.1.3.3.

\subsubsection{Ex vivo Perfusion Conditions}

Vein segments with and without the wrap were mounted in our well established, validated earlier version EVPS (not the version described in Chapter 2.0) [3,39,245,246,248,249,273,275]. Briefly, the closed loop perfusion design allows the circulation of sterile perfusate (tissue culture Media 199 supplemented with $1 \%$ fetal bovine serum and $1.0 \mathrm{~g} /$ liter cefoxitin) through the vascular segment as well as circulation of an adventitial bath (DMEM with $1 \%$ fetal bovine serum and $1.0 \mathrm{~g}$ /liter cefoxitin) within a sealed chamber. Both the perfusate and bathing media were maintained at $37^{\circ} \mathrm{C}$ and physiologic levels of dissolved gasses. The first set of experiments utilized one of two simulated hemodynamic conditions [248,273] - either native venous (VEN) or arterial (ART) conditions. To simulate VEN hemodynamics the perfusion loop was set to provide nonpulsatile flow of $20 \mathrm{ml} / \mathrm{min}$ and pressure of $20 \mathrm{mmHg}$. To simulate ART hemodynamics, the system was set to provide a pulsatile pressure waveform of $120 / 80 \mathrm{mmHg}$ with a mean perfusate flow of $100 \mathrm{ml} / \mathrm{min}$. The second set of experiments involved the manual ramping of hemodynamic conditions from VEN to ART over 24 or 72 hours. These mechanical conditioning regimens were termed cART hemodynamic conditions, and PIJVs perfused under cART conditions were compared to ART controls. Finally, experiments were performed to examine unwrapped PIJVs under ART conditions, which were compared to wrapped PIJVs under ART conditions (wART). Each of the VEN vs. ART and ART vs. wART perfusion 
experiments lasted for 24 hours. Using our DAQ system, the intraluminal pressure, flowrate, and outer diameter were recorded hourly over a 5 second duration with a sampling frequency of 150 $\mathrm{Hz}$ as described in Section 2.3.3. $\mathrm{pO}_{2}, \mathrm{pCO}_{2}, \mathrm{pH}$, perfusate temperature, and bathing media temperature were measured within each loop of the paired EVPS every hour. After each perfusion experiment, the unpressurized vessel dimensions were measured from rings cut from the proximal and distal ends of the PIJV sengments using a custom-designed "chuck" as described in Brant et al. [240]. The values obtained from each ring were then averaged, and these measurements were then used with the pressurized outer diameter and intraluminal pressure measurements to calculate the CWS as described in Section 2.5.4. Vein segments were then analyzed either histologically or via immunohistochemistry as described in Section 3.1.7. Table 3.2 lists all of the ex vivo experiments performed in this study and the respective endpoints analyzed for each experiment.

\subsubsection{VEN vs. ART Experiments}

Figure 3.2 is a schematic depicting the first set of ex vivo experiments that were performed related to Specific Aim 2. In these experiments we evaluated the acute hyperplastic response of PIJVs abruptly exposed to ART conditions vs. PIJVs exposed to VEN conditions for 24 hours. The endpoints and timepoints that were chosen for these experiments are based on the information outlined in Table 1.1 and Table 1.2. All pairs of PIJVs used in these experiments were harvested and transported to the laboratory as described in Section 3.1.1. For the duration of these experiments hourly recordings were made of many EVPS experimental parameters. These recordings were made manually and are included in Table D. 1 to Table D. 6 in Appendix D. 
Table 3.2 Summary of all ex vivo vascular perfusion experiments that were performed and the respective endpoints that were analyzed. Note that VMC: vasomotor challenge; PCNA: proliferating cell nuclear antigen; TUNEL: terminal deoxynucleotidyl transferase-mediated dUTP-biotin in situ nick end labeling; GOLGI: Golgi Complex; H\&E: hematoxylin and eosin; MTC: Masson's trichrome; MPC: Movat's pentachrome; PSR: picrosirius red; SEM: scanning electron microscopy; and TEM: transmission electron microscopy. An " $\mathrm{X}$ " indicates that the analysis was performed.

\begin{tabular}{|c|c|c|c|c|c|c|c|c|c|c|}
\hline \multicolumn{2}{|c|}{ Experiment/Duration } & \multicolumn{3}{|c|}{ Immunohistochemistry } & \multicolumn{4}{|c|}{ Histology } & \multicolumn{2}{|c|}{ EM } \\
\hline Date & Condition & PCNA & TUNEL & GOLGI & T\&E & MTIC & TMPC & PSR & SEM & TEM \\
\hline $\begin{array}{c}4 / 23 / 2003 \\
24 \text { hours }\end{array}$ & $\begin{array}{l}\text { VEN } \\
\text { ART }\end{array}$ & $\begin{array}{l}\mathrm{X} \\
\mathrm{X}\end{array}$ & $\begin{array}{l}\mathrm{X} \\
\mathrm{X}\end{array}$ & $\begin{array}{l}\mathrm{X} \\
\mathrm{X}\end{array}$ & & & & & & \\
\hline $\begin{array}{l}5 / 7 / 2003 \\
24 \text { hours }\end{array}$ & $\begin{array}{l}\text { VEN } \\
\text { ART }\end{array}$ & $\begin{array}{l}\bar{X} \\
x\end{array}$ & $\begin{array}{l}\bar{X} \\
X\end{array}$ & $\begin{array}{l}\bar{X} \\
x\end{array}$ & $\begin{array}{l}X \\
x\end{array}$ & & & & & $\begin{array}{l}x \\
x\end{array}$ \\
\hline $\begin{array}{c}10 / 9 / 2004 \\
24 \text { hours }\end{array}$ & $\begin{array}{l}\text { VEN } \\
\text { ART }\end{array}$ & $\begin{array}{l}x \\
X\end{array}$ & $\begin{array}{l}X \\
X\end{array}$ & $\begin{array}{l}x \\
x\end{array}$ & & & & & & $\begin{array}{l}x \\
x\end{array}$ \\
\hline $\begin{array}{c}10 / 16 / 2004 \\
24 \text { hours }\end{array}$ & $\begin{array}{l}\text { VEN } \\
\text { ART }\end{array}$ & $\begin{array}{l}\mathrm{X} \\
\mathrm{X}\end{array}$ & $\begin{array}{l}X \\
X\end{array}$ & $\begin{array}{l}\mathrm{X} \\
\mathrm{x}\end{array}$ & & & & & & $\begin{array}{l}x \\
x\end{array}$ \\
\hline $\begin{array}{c}5 / 24 / 2006 \\
24 \text { hours }\end{array}$ & $\begin{array}{l}\text { VEN } \\
\text { ART }\end{array}$ & $\begin{array}{l}x \\
X\end{array}$ & $\begin{array}{l}X \\
X\end{array}$ & $\begin{array}{l}\bar{X} \\
x\end{array}$ & & & & & & \\
\hline $\begin{array}{c}9 / 14 / 2006 \\
24 \text { hours }\end{array}$ & $\begin{array}{l}\text { VEN } \\
\text { ART }\end{array}$ & $\begin{array}{l}X \\
x\end{array}$ & $\begin{array}{l}X \\
X\end{array}$ & $\begin{array}{l}X \\
x\end{array}$ & & & $\begin{array}{l}X \\
x\end{array}$ & $\begin{array}{l}X \\
x\end{array}$ & & \\
\hline $\begin{array}{c}10 / 22 / 2004 \\
24 \text { hours }\end{array}$ & $\begin{array}{l}\text { ART } \\
\text { cART }\end{array}$ & $\begin{array}{l}\bar{X} \\
x\end{array}$ & $\begin{array}{l}\bar{X} \\
x\end{array}$ & $\begin{array}{l}\bar{x} \\
\mathrm{x}\end{array}$ & & & & & & $\begin{array}{l}x \\
x\end{array}$ \\
\hline $\begin{array}{l}11 / 1 / 2004 \\
24 \text { hours }\end{array}$ & $\begin{array}{l}\text { ART } \\
\text { CART }\end{array}$ & $\begin{array}{l}x \\
X\end{array}$ & $\begin{array}{l}\bar{X} \\
X\end{array}$ & $\begin{array}{l}x \\
X\end{array}$ & & & & & & \\
\hline $\begin{array}{c}11 / 11 / 2004 \\
24 \text { hours }\end{array}$ & $\begin{array}{l}\text { ART } \\
\text { cART }\end{array}$ & $\begin{array}{l}X \\
X\end{array}$ & $\begin{array}{l}X \\
X\end{array}$ & $\begin{array}{l}\mathrm{X} \\
\mathrm{X}\end{array}$ & & & & & & $\begin{array}{l}X \\
x\end{array}$ \\
\hline $\begin{array}{c}9 / 21 / 2006 \\
24 \text { hours }\end{array}$ & $\begin{array}{l}\text { ART } \\
\text { cART }\end{array}$ & $\begin{array}{l}\bar{x} \\
\mathrm{x}\end{array}$ & $\begin{array}{l}x \\
x\end{array}$ & $\begin{array}{l}\bar{X} \\
\mathrm{x}\end{array}$ & & & & & & \\
\hline $\begin{array}{c}10 / 16 / 2006 \\
24 \text { hours }\end{array}$ & $\begin{array}{l}\text { ART } \\
\text { cART }\end{array}$ & $\begin{array}{l}\bar{X} \\
x\end{array}$ & $\begin{array}{l}\bar{X} \\
x\end{array}$ & $\begin{array}{l}\bar{X} \\
x\end{array}$ & & & $\begin{array}{l}\bar{x} \\
x\end{array}$ & & & \\
\hline $\begin{array}{c}10 / 23 / 2006 \\
24 \text { hours }\end{array}$ & $\begin{array}{c}\text { ART } \\
\text { WART }\end{array}$ & $\begin{array}{l}\bar{X} \\
\mathrm{X}\end{array}$ & $\begin{array}{l}x \\
X\end{array}$ & $\begin{array}{l}\bar{X} \\
\mathrm{X}\end{array}$ & & & $\begin{array}{l}x \\
x\end{array}$ & $\begin{array}{l}x \\
x\end{array}$ & & \\
\hline $\begin{array}{c}10 / 30 / 2006 \\
24 \text { hours }\end{array}$ & $\begin{array}{c}\text { ART } \\
\text { WART }\end{array}$ & $\begin{array}{l}\bar{X} \\
x\end{array}$ & $\begin{array}{l}\bar{X} \\
X\end{array}$ & $\begin{array}{l}\bar{x} \\
\mathrm{x}\end{array}$ & & & & & & \\
\hline $\begin{array}{l}11 / 6 / 2006 \\
24 \text { hours }\end{array}$ & $\begin{array}{c}\text { ART } \\
\text { WART }\end{array}$ & $\begin{array}{l}\bar{X} \\
x\end{array}$ & $\begin{array}{l}x \\
x\end{array}$ & $\begin{array}{l}\bar{X} \\
x\end{array}$ & & & & & & \\
\hline $\begin{array}{c}11 / 13 / 2006 \\
24 \text { hours }\end{array}$ & $\begin{array}{c}\text { ART } \\
\text { WART }\end{array}$ & $\begin{array}{l}\bar{X} \\
\mathrm{x}\end{array}$ & $\begin{array}{l}\bar{X} \\
x\end{array}$ & $\begin{array}{l}\bar{X} \\
\mathrm{X}\end{array}$ & & & & & & \\
\hline $\begin{array}{c}11 / 20 / 2006 \\
24 \text { hours }\end{array}$ & $\begin{array}{c}\text { ART } \\
\text { WART }\end{array}$ & $\begin{array}{l}\bar{X} \\
x\end{array}$ & $\begin{array}{l}\bar{X} \\
x\end{array}$ & $\begin{array}{l}\bar{x} \\
\mathrm{x}\end{array}$ & $\begin{array}{l}\bar{X} \\
x\end{array}$ & $\begin{array}{l}x \\
x\end{array}$ & $\begin{array}{l}x \\
x\end{array}$ & & $\begin{array}{l}\bar{x} \\
\mathrm{x}\end{array}$ & \\
\hline $\begin{array}{c}11 / 27 / 2006 \\
24 \text { hours }\end{array}$ & $\begin{array}{c}\text { ART } \\
\text { WART }\end{array}$ & $\begin{array}{l}\bar{X} \\
x\end{array}$ & $\begin{array}{l}\bar{X} \\
X\end{array}$ & $\begin{array}{l}x \\
x\end{array}$ & & & & & & \\
\hline $\begin{array}{c}2 / 16 / 2007 \\
72 \text { hours }\end{array}$ & $\begin{array}{l}\text { ART } \\
\text { cART }\end{array}$ & $\begin{array}{l}\bar{x} \\
x\end{array}$ & $\begin{array}{l}\bar{X} \\
x\end{array}$ & $\begin{array}{l}\bar{x} \\
x\end{array}$ & & & & & & \\
\hline $\begin{array}{l}3 / 7 / 2007 \\
72 \text { hours }\end{array}$ & $\begin{array}{l}\text { ART } \\
\text { cART }\end{array}$ & $\begin{array}{l}\bar{X} \\
X\end{array}$ & $\begin{array}{l}\bar{X} \\
X\end{array}$ & $\begin{array}{l}\bar{X} \\
X\end{array}$ & & & & & & \\
\hline $\begin{array}{c}3 / 15 / 2007 \\
72 \text { hours }\end{array}$ & $\begin{array}{l}\text { ART } \\
\text { cART }\end{array}$ & $\begin{array}{l}X \\
X\end{array}$ & $\begin{array}{l}X \\
X\end{array}$ & $\begin{array}{l}X \\
X\end{array}$ & & & & & & \\
\hline $\begin{array}{l}4 / 2 / 2007 \\
72 \text { hours }\end{array}$ & $\begin{array}{l}\text { ART } \\
\text { CART }\end{array}$ & $\begin{array}{l}\bar{X} \\
x\end{array}$ & $\begin{array}{l}\bar{X} \\
x\end{array}$ & $\begin{array}{l}\bar{x} \\
x\end{array}$ & & & & & & \\
\hline $\begin{array}{c}4 / 26 / 2007 \\
72 \text { hours }\end{array}$ & $\begin{array}{l}\text { ART } \\
\text { CART }\end{array}$ & $\begin{array}{l}\bar{x} \\
x\end{array}$ & $\begin{array}{l}\bar{X} \\
x\end{array}$ & $\begin{array}{l}\bar{X} \\
x\end{array}$ & & & & & & \\
\hline $\begin{array}{c}3 / 22 / 2007 \\
\text { acute (VMC) }\end{array}$ & $\begin{array}{c}\text { unwrapped } \\
\text { wrapped }\end{array}$ & & & & & & & & & \\
\hline $\begin{array}{c}5 / 17 / 2007 \\
\text { acute (VMC) }\end{array}$ & $\begin{array}{c}\text { unwrapped } \\
\text { wrapped }\end{array}$ & & & & & & & & & \\
\hline $\begin{array}{c}5 / 23 / 2007 \\
\text { acute(VMC) }\end{array}$ & $\begin{array}{c}\text { unwrapped } \\
\text { wrapped }\end{array}$ & & & & & & & & & \\
\hline $\begin{array}{c}6 / 7 / 2007 \\
\text { acute(VMC) }\end{array}$ & $\begin{array}{c}\text { unwrapped } \\
\text { wrapped }\end{array}$ & & & & & & & & & \\
\hline
\end{tabular}




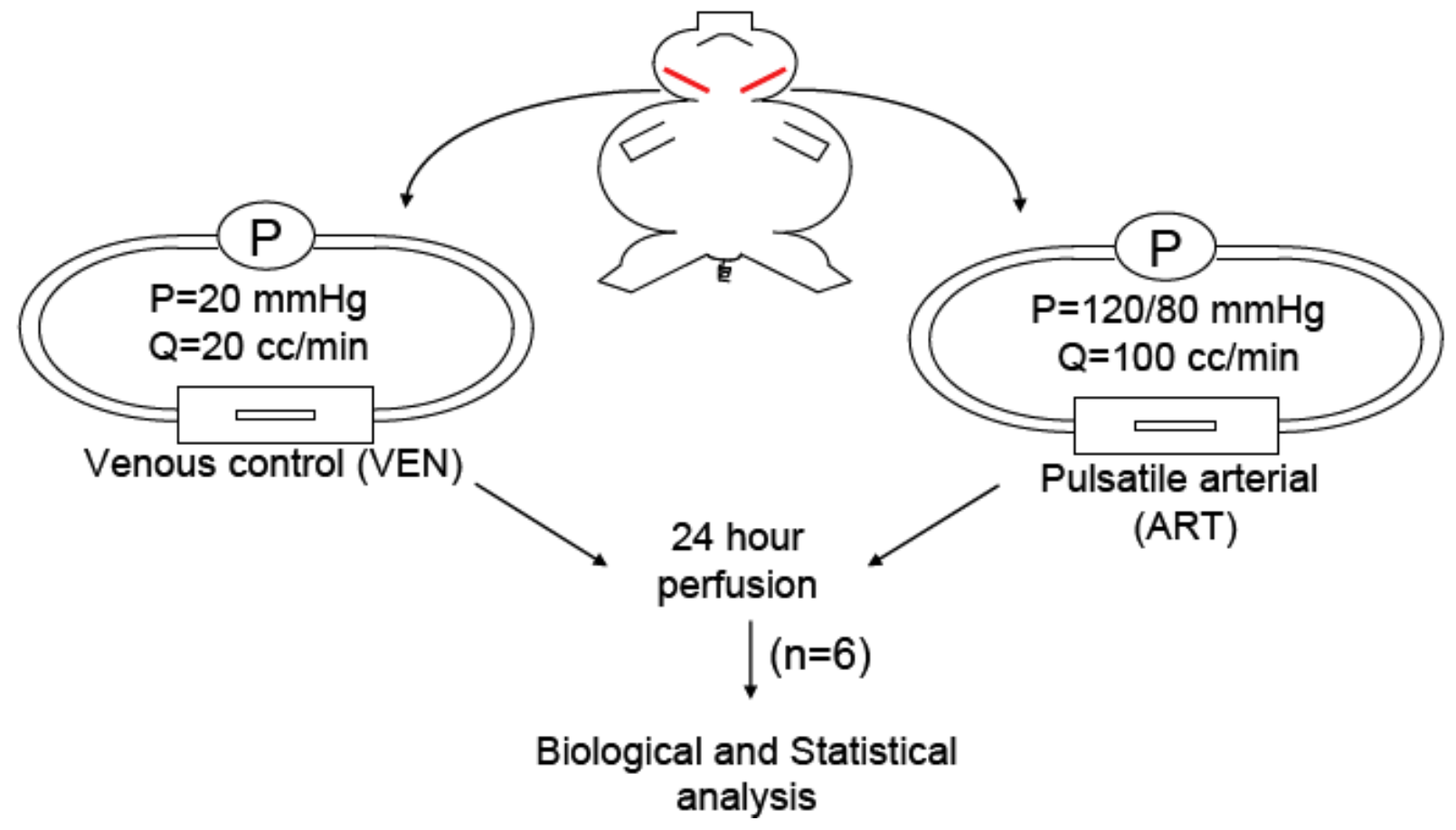

Figure 3.2 Schematic depicting the paired VEN vs. ART ex vivo perfusion experiments.

\subsubsection{ART vs. cART Experiments}

Figure 3.3 is a schematic depicting the second set of ex vivo experiments that were performed related to Specific Aim 2. In these experiments we evaluated the acute hyperplastic response of PIJVs in response to a mechanical arterial conditioning paradigm (cART conditions) vs. PIJVs abruptly exposed to ART conditions for 24 and 72 hours. The endpoints and timepoints that were chosen for these experiments are based on the information outlined in Table 1.1 and Table 1.2 . 
All pairs of PIJVs used in these experiments were harvested and transported to the laboratory as described in Section 3.1.1. In the first set of paired experiments one PIJV segment was abruptly exposed to ART conditions (control) and the other to cART (test) conditions for 24 hours. For the cART conditions, the test PIJV was implanted into the EVPS, exposed initially to VEN conditions, and then the pressure and flow were manually, and linearly, increased over 24 hours until ART conditions were attained. Specifically, the mean pressure and flowrate were incrementally increased by $10 \mathrm{mmHg}$ (from 20 to $100 \mathrm{mmHg}$ ) and $10 \mathrm{ml} / \mathrm{min}$ (from 20 to 100 $\mathrm{ml} / \mathrm{min}$ ), respectively, every 3 hours. As the mean pressure and flowrate were increased, pulsatility was also increased. The pressure "pulse" was incrementally increased from 0 to 40 $\mathrm{mmHg}$, and the flowrate "pulse" was incrementally increased from approximately 0 to $80 \mathrm{ml} / \mathrm{min}$ over 24 hours by manual adjustment of the centrifugal pump.

In the second set of paired ART vs. cART experiments the test PIJV was implanted into VEN conditions and then the pressure and flow were manually, and linearly, increased over 72 hours upto ART conditions. The mean pressure and flowrate were incrementally increased by 10 $\mathrm{mmHg}$ (from 20 to $100 \mathrm{mmHg}$ ) and $10 \mathrm{ml} / \mathrm{min}$ (from 20 to $100 \mathrm{ml} / \mathrm{min}$ ), respectively, every 9 hours. As the mean pressure and flowrate were increased, pulsatility was also increased. The pressure "pulse" was incrementally increased from 0 to $40 \mathrm{mmHg}$, and the flowrate "pulse" was incrementally increased from approximately 0 to $80 \mathrm{ml} / \mathrm{min}$ over 72 hours. 


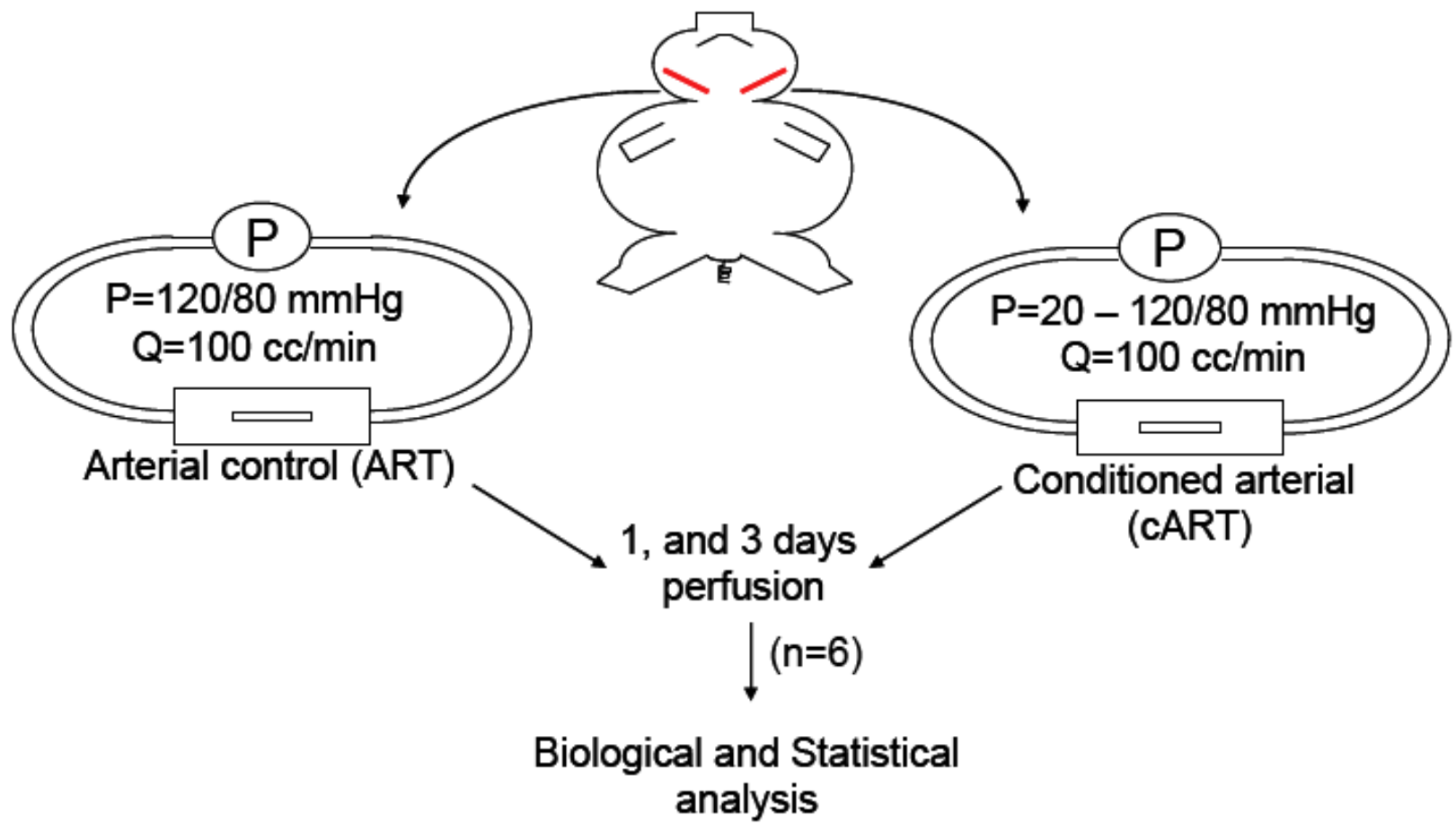

Figure 3.3 Schematic depicting the paired ART vs. cART ex vivo perfusion experiments.

\subsubsection{ART vs. wART Experiments}

Figure 3.4 is a schematic depicting the third set of ex vivo experiments that were performed. These experiments were related to Specific Aim 3. We evaluated the beneficial effects of a tuned biodegradable polymer wrap on the hyperplastic response of PIJVs exposed to ART conditions (wART conditions) vs. unwrapped PIJVs exposed to ART conditions for 24 hours. The endpoints and timepoints that were chosen for these experiments are based on the information outlined in Table 1.1 and Table 1.2.

All pairs of PIJVs used in these experiments were harvested and transported to the laboratory as described in Section 3.1.1. One of the PIJVs was then electrospun, using polymer wrap "combination A" from Table 3.1, (as described in Section 3.1.2) and then implanted into 
the EVPS and exposed to ART conditions for 24 hours. We chose to use "combination A", over the other tested combinations, for the vein wrap based on the CWS profile that was achieved with this polymer. The other PIJV was treated exactly the same as the wrapped PIJV except that no wrap was applied. This included placement of the mandrel through the cannulated vein, and exposure to the electrical field of the electrospinning device. The PIJVs were then implanted into the EVPS and exposed to ART conditions for 24 hours.

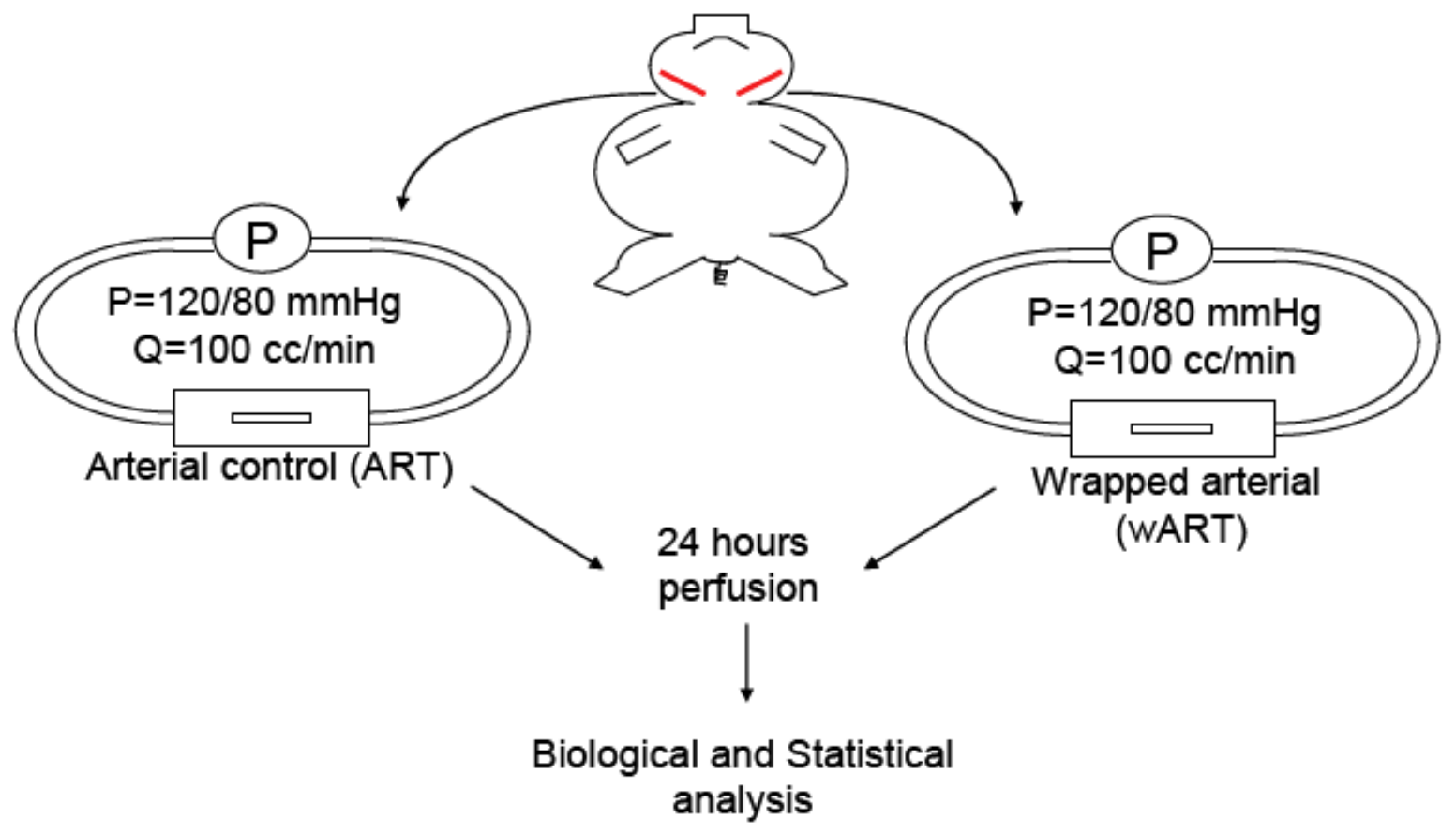

Figure 3.4 Schematic depicting the paired ART vs. wART ex vivo perfusion experiments. 


\subsection{CWS Calculation in a Compound Cylinder}

Since it is believed that an abrupt exposure of AVGs to arterial levels of CWS may contribute to their failure modalities [27-37], we believe that one potential application of the electrospun biodegradable polymer wrap would be to gradually expose AVGs to arterial levels of CWS. Previous attempts to limit CWS using an external sheath have not been fully successful because they were either biodurable and/or loose fitting $[31,40-52,54,300]$. To demonstrate how the wrap may modulate CWS, and how the wrap may be tuned to achieve desired results, we examined the CWS-over-time profile for each of the wrap combinations given in Table 3.1 and compared these to unwrapped vein segments exposed to venous or arterial conditions. This was achieved using the data collected from ex vivo perfusion experiments and a mathematical model for CWS.

For biomechanical modeling purposes, consider the schematic in Figure 3.5 showing an idealized cross section of the vein/wrap complex. The outer layer of the bi-layer compound tube is taken as the electrospun polymer wrap and the concentric inner layer is the vein segment. 


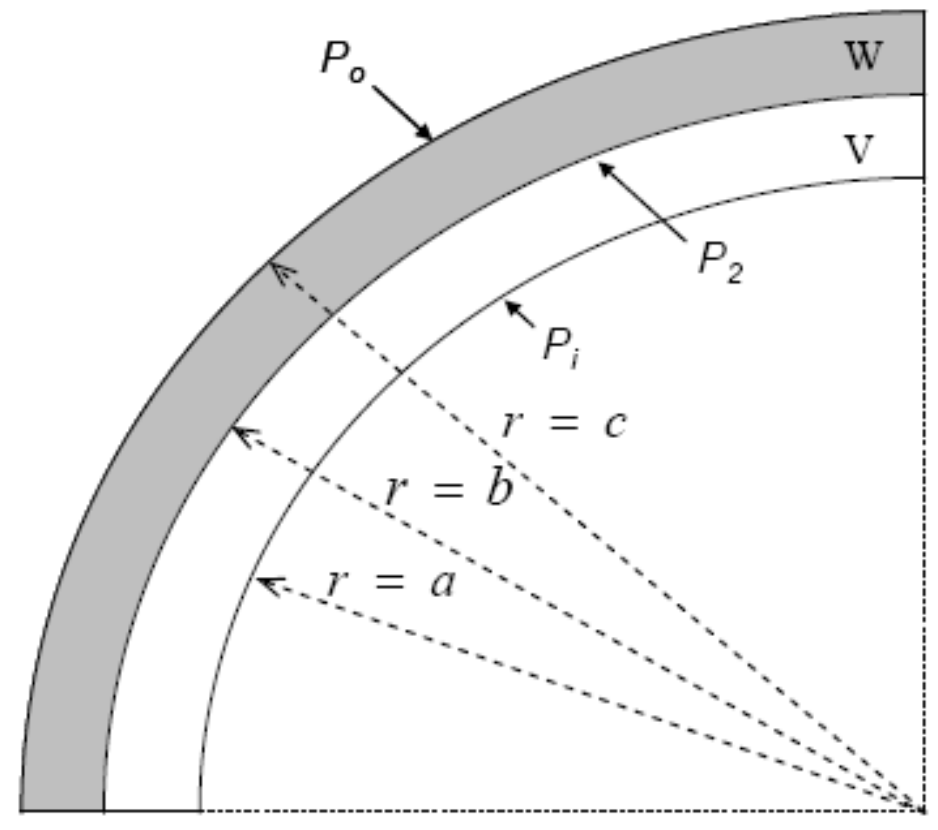

Figure 3.5 Schematic showing a cross-sectional view of the vein /wrap complex. Note W: electrospun polymer wrap; V: vein segment; $P_{i}$ : intraluminal pressure; $P_{2}$ : interfacial pressure; $P_{o}$ : atmospheric pressure.

The following assumptions were then made [301]:

i) There is no slipping or detachment between layers

ii) Compatibility of deformation across the interface is maintained

iii) There is only a small deformation under mean arterial pressure

iv) The system is under a state of plane stress

v) Both layers are incompressible, isotropic, homogeneous and linearly elastic materials

vi) Each separate layer may be generalized as a single, thick-walled cylinder subjected to internal and external pressure 
The mathematical model developed by Vorp et al. [301] was adapted for the model represented by Figure 3.5. In short, we used the classic Lamé solution for radial and circumferential wall stresses ( $\sigma_{r}$ and $\sigma_{\theta}$, respectively), and radial deformation $\left(u_{r}\right)$ at any radius, $r$, in an open-ended, thick-walled cylinder under the action of internal and external pressures [301]. For the inner (vein) layer shown in Figure 3.5, we obtain [301]:

$$
\begin{aligned}
& \sigma_{r, V}=\frac{a^{2} P_{i}-b^{2} P_{2}}{b^{2}-a^{2}}-\frac{\left(P_{i}-P_{2}\right) a^{2} b^{2}}{\left(b^{2}-a^{2}\right) r^{2}} \\
& \sigma_{\theta, V}=\frac{a^{2} P_{i}-b^{2} P_{2}}{b^{2}-a^{2}}+\frac{\left(P_{i}-P_{2}\right) a^{2} b^{2}}{\left(b^{2}-a^{2}\right) r^{2}} \\
& u_{r, V}=\frac{1-v_{V}}{E_{V}} \frac{\left(a^{2} P_{i}-b^{2} P_{2}\right) r}{b^{2}-a^{2}}+\frac{1+v_{V}}{E_{V}} \frac{\left(P_{i}-P_{2}\right) a^{2} b^{2}}{\left(b^{2}-a^{2}\right) r}
\end{aligned}
$$

18

where the " $V$ " subscript refers to quantities with respect to the vein, and $a$ and $b$ are the inner and outer radii, respectively, of the vein layer. $P_{i}$ is the internal pressure, and $P_{2}$ is the interfacial pressure acting between the two layers of the concentric cylinder resulting from their difference in mechanical properties. $\quad v$ is the Poisson's ratio and $E$ is the Young's modulus of elasticity. For the outer (wrap) layer shown in Figure 3.5, we have: 


$$
\begin{aligned}
& \sigma_{r, W}=\frac{b^{2} P_{2}-c^{2} P_{o}}{c^{2}-b^{2}}-\frac{\left(P_{2}-P_{o}\right) b^{2} c^{2}}{\left(c^{2}-b^{2}\right) r^{2}} \\
& \sigma_{\theta, W}=\frac{b^{2} P_{2}-c^{2} P_{o}}{c^{2}-b^{2}}+\frac{\left(P_{2}-P_{o}\right) b^{2} c^{2}}{\left(c^{2}-b^{2}\right) r^{2}} \\
& u_{r, W}=\frac{1-v_{W}}{E_{W}} \frac{\left(b^{2} P_{2}-c^{2} P_{o}\right) r}{c^{2}-b^{2}}+\frac{1+v_{W}}{E_{W}} \frac{\left(P_{2}-P_{o}\right) b^{2} c^{2}}{\left(c^{2}-b^{2}\right) r}
\end{aligned}
$$

where the " $W$ " subscript refers the quantities to the region occupied by the wrap, and $b$ and $c$ are the inner and outer radii, respectively, of the wrap layer. $P_{o}$ is the external pressure. With compatibility of deformations across the interface between the layers, it must be that:

$$
u_{r, V}=u_{r, W} \quad \text { at } r=b
$$

Substituting (16) and (18) into (19), letting $v_{W}=v_{V}=v=0.5$ (both materials assumed to be incompressible), setting $P_{o}=0$ (i.e., atmospheric pressure), and solving for $P_{2}$ we obtain:

$$
P_{2}=\frac{a^{2} P_{i}(1-v) E_{W} b\left(c^{2}-b^{2}\right)+(1+v) E_{W}\left(c^{2}-b^{2}\right) b a^{2} P_{i}}{b^{2}(1-v) E_{W} b\left(c^{2}-b^{2}\right)+(1-v) a^{3} E_{V}\left(b^{2}-a^{2}\right)+(1+v) E_{W}\left(c^{2}-b^{2}\right) b a^{2}+c^{2} E_{V} b\left(b^{2}-a^{2}\right)}
$$

Recall that $P_{i}$ and outer diameter (i.e., $c$ ) were measured in our ex vivo perfusion experiments. Therefore we had to estimate the inner $(r=a)$ and interfacial $(r=b)$ radii for 
each set of measured $P_{i}$ and $c$. Since we utilized the assumption that both the vein and wrap are incompressible materials, which requires the volume of each cylinder to be constant at any state of deformation, it must be that:

$$
\left[\pi\left(r_{o}^{2}-r_{i}^{2}\right) L\right]_{u}=\left[\pi\left(r_{o}^{2}-r_{i}^{2}\right) L\right]_{p}
$$

where $r_{o}$ and $r_{i}$ are the outer and inner radii, respectively, and $L$ is the length of each cylinder, and the subscripts $u$ and $p$ refer to the unpressurized and pressurized states, respectively. Applying equation 24 to the geometry of the "wrap" cylinder in Figure 3.5, yields:

$$
b_{p}=\sqrt{\frac{c_{p}^{2} L_{p}-\left(c_{u}^{2}-b_{u}^{2}\right) L_{u}}{L_{p}}}
$$

Therefore for any measured $c_{p}$ and $L_{p}$, a value of $b_{p}$ can be calculated. Similarly, considering only the "vein" cylinder in Figure 3.5 and utilizing equation 25 for $b_{p}$, we find:

$$
a_{p}=\sqrt{\frac{\left(\frac{c_{p}^{2} L_{p}\left(c_{u}^{2}-b_{u}^{2}\right) L_{u}}{L_{p}}\right) L_{p}-\left(b_{u}^{2}-a_{u}^{2}\right) L_{u}}{L_{p}}}
$$


Substituting equations 23, 25 and 26 into equation 20, and evaluating at the mean

arterial pressure and at the mid-wall radius of the vein (i.e., $r=\frac{a_{p}+b_{p}}{2}$ ), we can calculate the mid-wall CWS in the polymer wrapped vein. We assumed that $E_{W}=7.5 \mathrm{MPa}$ [57], and $E_{V}=600$ $\mathrm{KPa}$ [228] in our calculations. The modulus used for the wrap represents a value that was measured using a similar mixture of PEUU and collagen as was used in combination A in Table 3.1. The modulus used for the vein represents a value that was measured in a dog jugular vein [228].

\subsubsection{Vasomotor Challenge Experiments}

We performed several experiments that were dedicated to ensure that the functional viability of the tissue was maintained in PIJVs perfused within our EVPS, and to assess the effects of the electrospinning process on tissue functionality. Tissue functionality was assessed using an ex vivo vasomotor challenge as previously described [3,275]. In short, vessel segments were cannulated, placed under a constant intraluminal pressure of $20 \mathrm{mmHg}$, and exposed to incremental doses of epinephrine (EPI). Throughout the experiment, outer vessel diameter $(D)$ was continuously measured with a laser micrometer [3,245,275]. The baseline diameter ( $\left.D_{\text {baseline }}\right)$ was measured before injection of the first dose of EPI. EPI was subsequently injected to final concentrations of $2 \times 10^{-5}, 2 \times 10^{-4}$, and $2 \times 10^{-3} \mathrm{mg} / \mathrm{ml}$ at $1,4.5$, and 10 minutes, respectively. Following observation of maximal vasoconstriction with each dose, each subsequent dose was administered. After administration of the maximal dose of EPI, and observation of maximal level of constriction ( $D_{\text {constricted }}$ ), a $2 \mathrm{ml}$ bolus of $25 \mathrm{mg} / \mathrm{ml}$ sodium nitroprusside (SNP) was injected to give a final concentration of $0.125 \mathrm{mg} / \mathrm{ml}$. When full 
dilation was observed, $D_{\text {dilated }}$ was recorded. The level of constriction in response to EPI was calculated as:

$$
\% \text { Constriction }=\frac{D_{\text {baseline }}-D_{\text {constricted }}}{D_{\text {constricted }}} * 100
$$

Similarly, the level of dilation in response to SNP was calculated as:

$$
\% \text { Dilation }=\frac{D_{\text {dilated }}-D_{\text {constricted }}}{D_{\text {constricted }}} * 100
$$

\subsubsection{Compliance and $\beta$-stiffness Measurements}

Hourly measurements of outer diameter $(O D)$ and intraluminal pulsatile pressure $(P)$ were made during the ART vs. wART 24-hour perfusion experiments $(\mathrm{N}=6)$ described in Section 3.1.3.3. These measurements were used to calculate the compliance $(C)$ and $\beta$-stiffness $(\beta)$ of both spun and sham control PIJVs. Using a sampling frequency of $150 \mathrm{~Hz}$, the hourly measurements were made for 5 seconds so that approximately 5 complete "cardiac cycles" of data were collected. The acquired signals were then filtered and plotted. Using the maximum $\left(O D_{s}\right.$ and $\left.P_{s}\right)$ and minimum $\left(O D_{d}\right.$ and $\left.P_{d}\right)$ values for each cycle. The 5 values were averaged and single values of $C$ and $\beta$ were calculated every hour. The compliance was calculated as:

$$
C=\frac{\left(O D_{s}-O D_{d} / O D_{d}\right)}{P_{s}-P_{d}}
$$


Similarly, the $\beta$-stiffness was calculated as [302]:

$$
\beta=\frac{\ln \left(P_{s}-P_{d}\right)}{\left(O D_{s}-O D_{d} / O D_{d}\right)}
$$

\subsubsection{Post-perfusion Tissue Processing}

In Specific Aims 2 and 3, the hyperplastic response of the PIJVs was quantified by measuring the various carefully-chosen endpoints summarized in Section 1.2. These endpoints were grouped into three categories based on the required tissue processing: i) histology (including micro/ultrastructure); ii) RNA analysis; and iii) protein analysis. All PIJV segments from the $e x$ vivo experiments were segmented and processed according to Figure 3.6. After segmentation the samples were immediately placed into separate containers and snap frozen in liquid nitrogen. The frozen samples were then stored in a $-80{ }^{\circ} \mathrm{C}$ freezer.

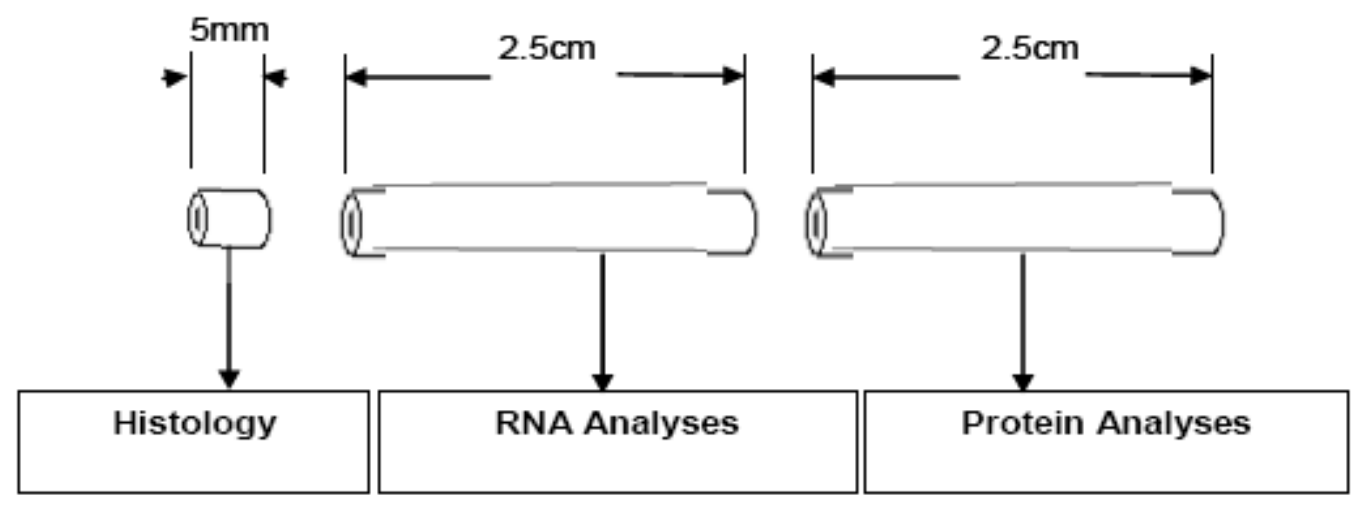

Figure 3.6 Schematic of post perfusion PIJV segment processing for endpoint analysis. Lengths given represent unloaded vessel resting lengths. 


\subsubsection{Biological Analyses}

The biological endpoints related to Specific Aims 2 and 3 were characterized as either histological or molecular-based with respect to the type of assay required. The histological endpoints included evaluation of microstructure, apoptosis, proliferation, and a SMC phenotype marker. The endpoints related to de-adhesion/migration of SMCs were attempted but could not be reliably evaluated due to difficulty in troubleshooting both the histological and molecularbased assays for these endpoints. The protein and gene expression endpoints required isolation of protein and RNA and are thus classified as molecular. As stated in Section 1.6, some of the histological and molecular endpoints considered were experimentally difficult to obtain. Further description of the methods, experimental difficulties, preliminary results, and recommendations for future investigation with regards to the molecular endpoints can be found in Appendix H.

The samples dedicated for histological analysis (Figure 3.6) were taken from the $-80^{\circ} \mathrm{C}$ freezer and immediately embedded in Tissue Freezing Medium ${ }^{\mathrm{TM}}$ (Triangle Biomedical Sciences, Durham, NC) and frozen at $-65^{\circ} \mathrm{C}$. Five-micron cross-sections were cut using a cryotome and placed on positively charged, glass microscope slides. Slides were stored at $-80^{\circ} \mathrm{C}$ until they could be processed for histological or immunohistochemical assays. The detailed protocols for each endpoint are provided in Appendix E.

\subsubsection{Scanning Electron Microscopy}

Some representative PIJVs (see Table 3.2 for details) were examined under SEM. In short, tissue segments designated for SEM were fixed in ultrapure $2.5 \%$ gluteraldehyde, dehydrated through a graded series of ethanol solutions (30-100\%), critical point dried (Emscope, CPD 750, 
Ashford, Kent, UK), then overcoated with vaporized carbon (Cressington Freeze Fracture Device, Cressington, Cranberry, PA, USA). A detailed protocol for sample preparation is provided on in Appendix E (Protocol E.1). The tissue was visualized using a JEOL JEM-6335F field emission gun SEM (JEOL, Peabody, MA, USA).

\subsubsection{Histology}

The PIJV segment dedicated for histology (Figure 3.6) was taken from the $-80^{\circ} \mathrm{C}$ freezer and then fixed in $4 \%$ paraformaldehyde for 4 hours at $4{ }^{\circ} \mathrm{C}$ followed by $30 \%$ sucrose at $4^{\circ} \mathrm{C}$ overnight. $5 \mathrm{~mm}$ tissue rings were cut, washed with PBS, embedded in Tissue Freezing Medium $^{\mathrm{TM}}$ (Triangle Biomedical Sciences, Durham, NC), and cut into $5 \mu \mathrm{m}$ sections. The tissue sections were either stained with H\&E, Masson's trichrome (MTC), picrosirius red (PSR), or Movat's pentachrome stains (MPC). The detailed protocols for H\&E, MTC, PSR, and MPC staining are provided in Appendix E (Protocols E.2 to E.5). Stained tissue sections were then visualized using an Olympus Provis light microscope (Olympus, Center Valley, PA, USA) and compared qualitatively.

\subsubsection{Necrosis}

To assess the effects of the electrospinning process on tissue viability we examined spun and sham PIJV segments, as well as untreated freshly excised ("control") tissue. Tissue necrosis was examined using Live/Dead ${ }^{\mathrm{TM}}$ staining (Molecular Probes, Carlsbad, CA, USA) of cryosections, according to manufacturer's instructions. Each segment (control, sham control and spun) intended for Live/Dead ${ }^{\mathrm{TM}}$ staining was cut in half and placed in static culture within a Petri-dish under standard incubator conditions. One-half of each segment was assessed after 18 hours of culture, the other after 92 hours. $5 \mathrm{~mm}$ rings were cut from each sample and embedded in 
cryomatrix (TBS, Durham, NC) then frozen. Five $8 \mu \mathrm{m}$ sections were cut from each ring and imaged under 20x magnification using an epifluorescent microscope (Nikon, Model E800, Melville, NY, USA). Two images were taken per section so that a total of 10 fields of view were quantified per PIJV segment. Scion Image (Version Beta 4.02, NIH, Bethesda, MD) was used to count the total number of cells in a field of view. To determine the percentage of live cells in a field of view, dead cells were counted manually, divided by the total number of cells, and multiplied by $100 \%$. The percentage of dead cells was subtracted from $100 \%$ to calculate the percentage of live cells.

\subsubsection{Apoptosis}

Apoptosis was assessed using the In Situ Cell Death Kit, fluorescein (TUNEL) (Roche Applied Science, Indianapolis, IN). This assay uses the TUNEL technology which identifies the genomic DNA cleavage component of apoptosis. Briefly, cross-sections were dried at $37^{\circ} \mathrm{C}$ for 20 minutes, fixed in $4 \%$ paraformaldehyde for 20 minutes, and rehydrated in phosphate buffered saline (PBS) for 30 minutes. Samples were then incubated at room temperature for 10 minutes each in $10 \mu \mathrm{g} / \mathrm{ml}$ Proteinase K followed by a freshly prepared solution of $0.1 \%$ Triton X-100 and $0.1 \%$ sodium citrate for permeabilization of membranes. DNA strand breaks were identified by incubation at $37^{\circ} \mathrm{C}$ for one hour with Terminal deoxynucleotidyl transferase and fluorescein labeled dUTP (both provided in the kit from Roche). Nuclei were counterstained with Hoechst 33258. A small set of samples was treated with $100 \mathrm{U} / \mathrm{ml}$ of DNase I to serve as positive controls each time the assay was performed to ensure efficacy. All sample preparation parameters including incubation times, temperatures, and reagent concentrations were optimized using DNase I treated positive controls. Negative controls were incubated with labeled dUTP without 
the transferase enzyme. The detailed protocol for TUNEL staining is provided in Appendix E (Protocol E.6).

Quantification of the percent of TUNEL positive cells was performed using a manual counting procedure. Numbers of positive cells from each of 5 FOVs (field of views) from a given $5 \mu \mathrm{m}$ cross-section were averaged to define the mean percent TUNEL positive cells for a PIJV segment (see Figure 3.6).

\subsubsection{Proliferation}

Proliferation was assessed by the expression of proliferating cell nuclear antigen (PCNA) determined by immunohistochemistry. Five-micron cross-sections were dried, fixed, and permeabilized as described for the TUNEL assay in Section 3.1.7.4. Nonspecific binding of antibodies was blocked by incubating the samples for 30 minutes with $1 \%$ horse serum in PBS. Following this, the samples were incubated with a primary mouse monoclonal antibody against human PCNA (Dako Cytomation, Clone PC10, Denmark) overnight at $4{ }^{\circ} \mathrm{C}$ in a moist chamber to prevent sample drying. Unbound primary antibody was removed by subsequent washes in PBS. The detailed protocol for primary PCNA antibody incubation is provided in Appendix E (Protocol E.7). Next, cross-sections were incubated with a universal (anti-mouse and antirabbit) biotinylated secondary antibody which was part of the Vectastain Elite ${ }^{\mathrm{TM}}$ horse-radish peroxidase and avidin-biotin-complex (HRP/ABC) detection system (Vector Labs, Cat.\# PK6200, Burlingame, CA) for 60 minutes at $37{ }^{\circ} \mathrm{C}$ in a moist chamber and then rinsed 3 times with PBS. Incubation with the Vectastain ${ }^{\mathrm{TM}}$ reagent was then performed for 30 minutes at room temperature. To detect positively stained cells, a diaminobenzidine (DAB) substrate (Vector Labs, Cat.\# SK-4100, Burlingame, CA) was used. The enzymatic reaction caused PCNA positive cells to stain brown which was visualized via microscope (100x magnification) until the 
desired level of staining was achieved. The reaction was then stopped by placing the slides into deionized water. The detailed protocol for the HRP/ABC detection system is given in Appendix E (Protocol E.8). For nuclear visualization, cells were counter-stained with Hematoxylin (Vector Labs, Cat.\# H-3401, Burlingame, CA) according to manufacturer's instructions. Quantification of the percent PCNA positive cells was performed using the same methodology as for TUNEL as described in Section 3.1.7.4.

\subsubsection{SMC Phenotype}

To detect a synthetic SMC phenotype, we used a mouse monoclonal antibody raised against human Golgi complex (Abcam, Cat.\# ab14487, Cambridge, MA). The detailed protocol for Golgi complex primary antibody incubation is given in Appendix E (Protocol E.9). Essentially the exact same procedure as described in Section 3.1.7.5 (PCNA) was used to detect the expression of, and subsequently to quantify the mean percentage of Golgi complex positive cells per segment of PIJV.

Initially we attempted to visualize changes in Golgi complex and rough endoplasmic reticulum expression, as markers of synthetic SMCs, via transmission electron microscopy (TEM) as described in the following sub-section. However it was determined that immunohistochemistry would provide more easily quantifiable results.

\subsection{Transmission Electron Microscopy}

Tissue from several experiments (see Table 3.2) was devoted for TEM analysis. Briefly, tissue rings were fixed in $2.5 \%$ gluteraldehyde, dehydrated through a graded series of ethanol solutions (30-100\%), then cured and embedded in epon. A detailed protocol for TEM sample preparation 
is provided in Appendix E (Protocol E.10). The tissue was visualized using a JEOL 1011CX TEM (JEOL, Peabody, MA, USA).

\subsubsection{Statistics}

For the vasomotor challenge data, and the immunohistochemistry image quantification data a paired student's t-test for means was performed, and $\mathrm{P}<0.05$ was considered statistically significant. Unless otherwise stated all data is presented as mean \pm standard error of the mean.

\subsection{RESULTS}

\subsubsection{Ex vivo Biomechanical Conditioning Perfusion Experiments}

Figure 3.7 shows representative mean intraluminal pressure vs. time, outer diameter vs. time, and mean CWS vs. time profiles from a representative 24 hour ART vs. cART experiment, and Figure 3.8 shows the same from a representative 72 hour ART vs. cART experiment. Similar data for all other experiments are shown in Figure D. 1 to Figure D. 9 in Appendix D. 

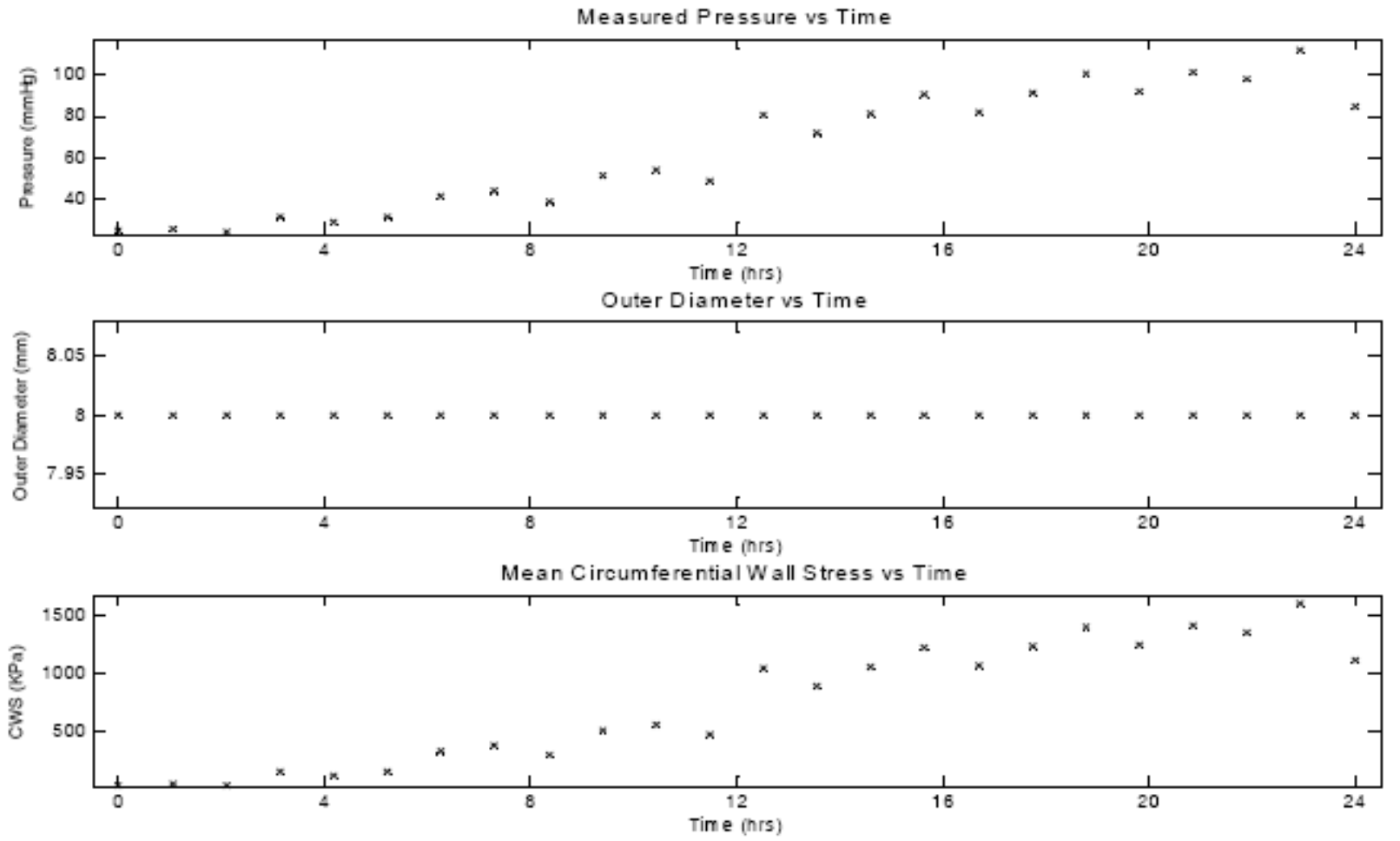

Figure 3.7 Representative mean pressure, outer diameter, and CWS vs. time profiles from a 24 hour ART vs. cART experiment. 

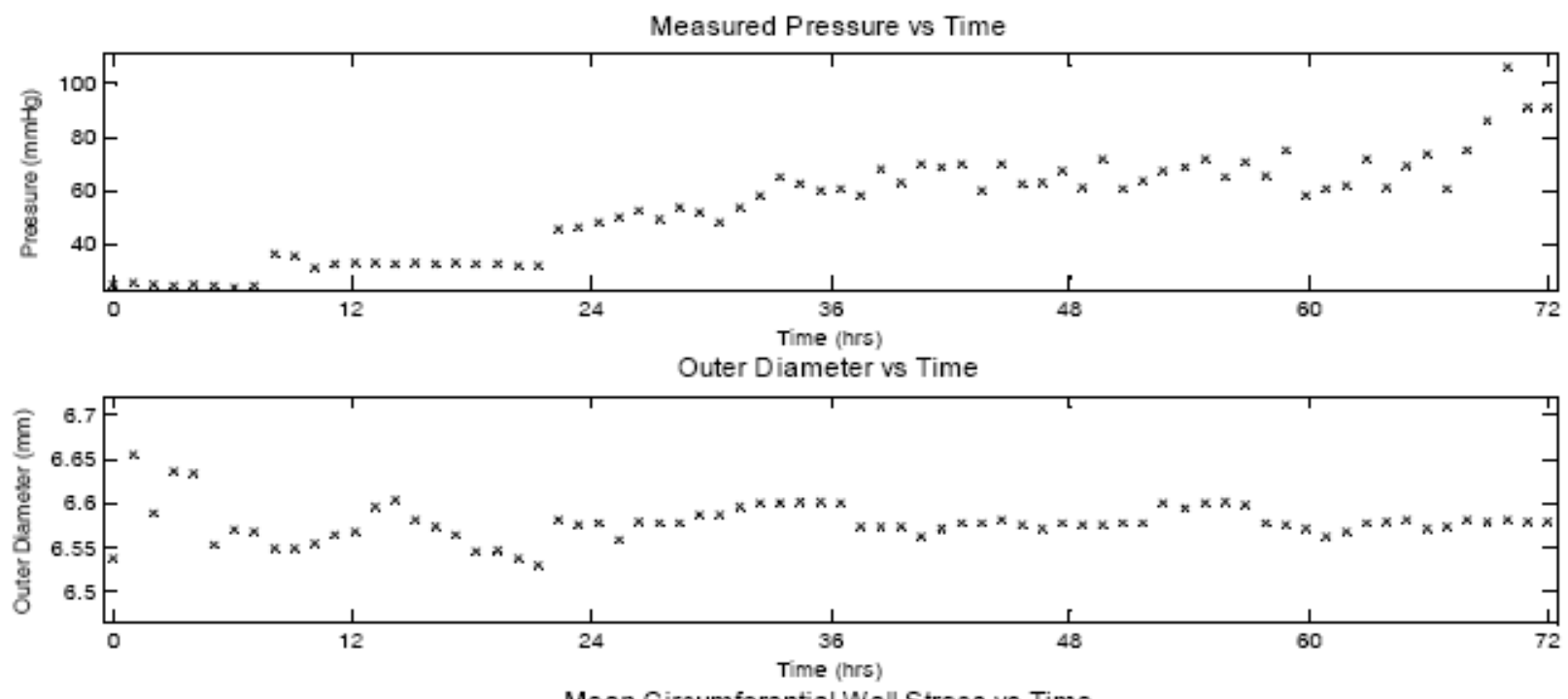

Mean Circumferential Wall Stress vs Time

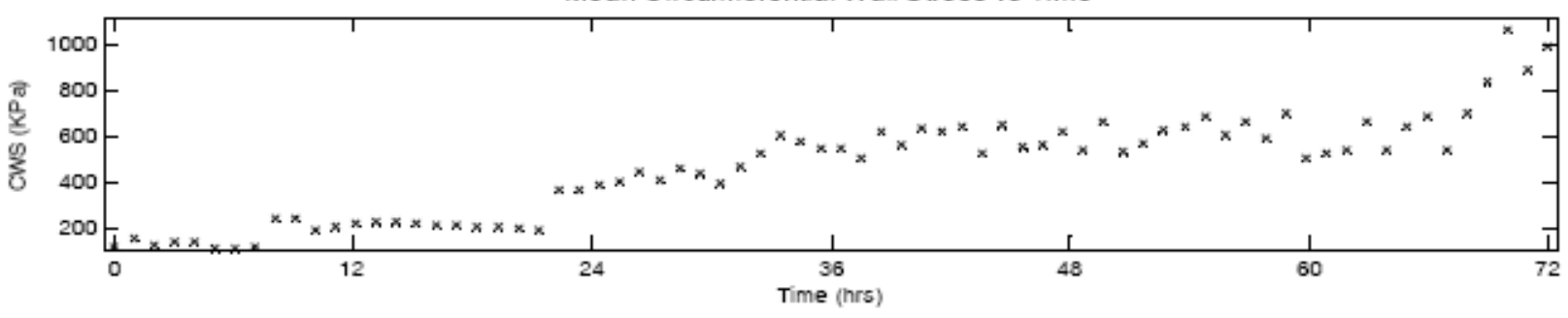

Figure 3.8 Representative mean pressure, outer diameter, and CWS vs. time profiles from a 72 hour ART vs. cART experiment.

\subsubsection{ART vs. wART Diameter Profiles}

The structural support offered to a vein by the wrap is evident when we examine the outer diameter profiles in Figure 3.9. It was shown that a vein with a wrap does not expand to the same degree under ART conditions as a vein without a wrap. 


\subsubsection{CWS Profiles}

The CWS-over time profile for the polymer solution combinations of Table $\mathbf{3 . 1}$ were quite variable (Figure 3.10). In one case (combination B), the wrap degraded too quickly and resulted in a rapid increase in CWS under ART conditions. Other combinations (C and D) did not degrade quickly enough and resulted in no appreciable increase in CWS over a 24-hour period. Combination A degraded at a rate which resulted in a nearly linear variation over the 24-hour period between VEN and ART levels of CWS. This combination was repeated $(\mathrm{N}=7)$ and the effect was found to be repeatable. 


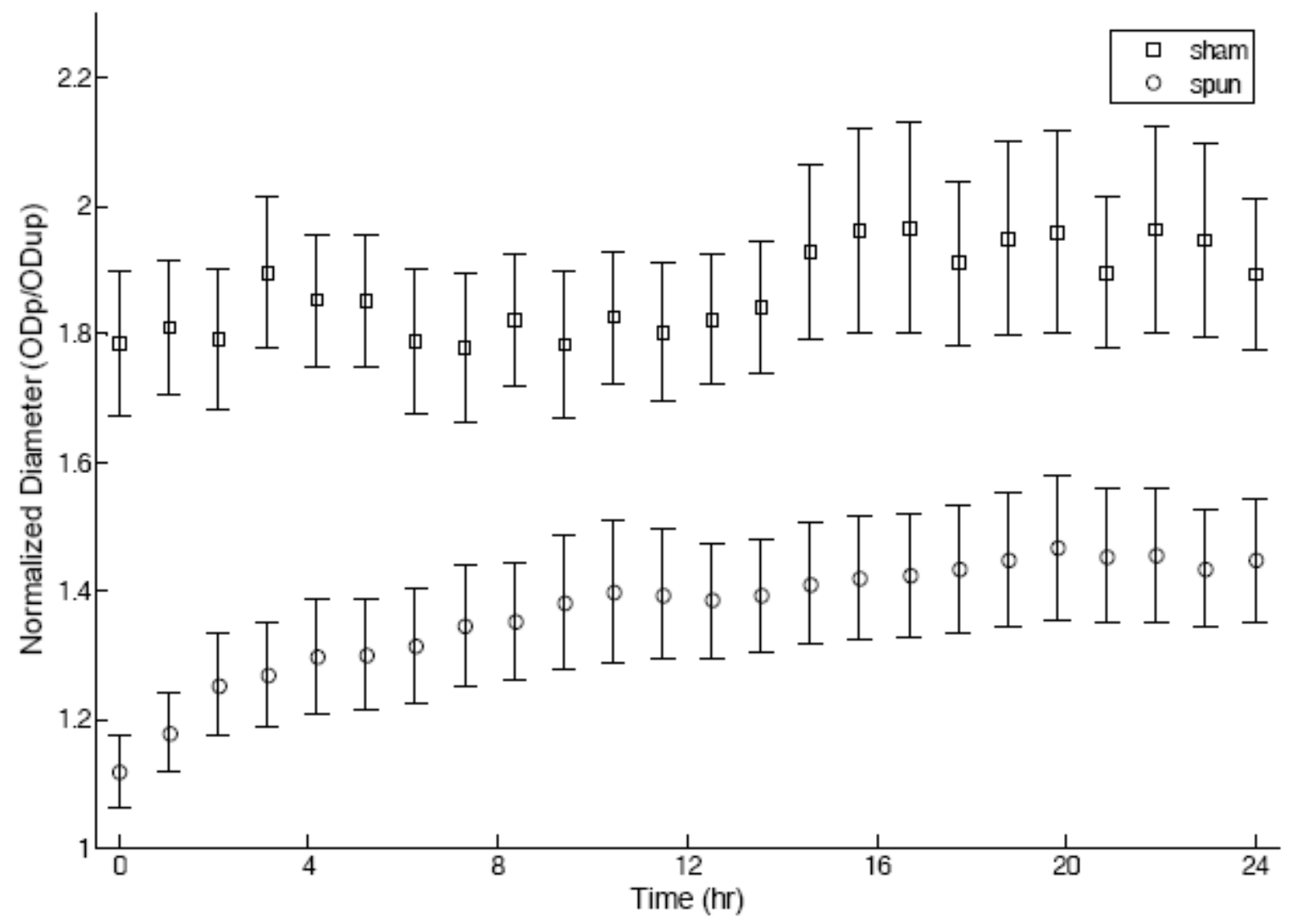

Figure 3.9 Normalized outer diameter response profiles of PIJVs for both sham and spun PIJVs. Both spun (wART) and sham control PIJVs were perfused under ART conditions of $120 / 80 \mathrm{mmHg}$ pressure and $100 \mathrm{ml} / \mathrm{min}$ mean flowrate. Note that the normalized diameter of the spun veins $(\mathrm{N}=7)$ is dramatically reduced when compared to sham controls $(\mathrm{N}=5)$. Pressurized outer diameter (ODp) was normalized to unpressurized outer diameter (ODup) and data is shown as mean \pm standard error of the mean.

\subsubsection{Vasomotor Challenge Results}

The results of a typical vasomotor challenge experiment are shown in Figure 3.11. The sham PIJV segment responded in a predictable dose-dependent manner to stimulation with EPI, while the spun PIJV exhibited a single contraction commencing with the lowest dose of EPI. 
Vasodilation in response to SNP was similar for both the control and spun PIJVs, each resulting in a larger outer diameter than that at baseline, suggesting a certain level of basal tone in both the sham and spun PIJVs. All of the vasomotor challenge experiment results are provided in Figure D. 10 to Figure D. 13 in Appendix D. Overall, there was no significant difference in the level of contraction (Figure 3.12(A)) or dilation (Figure 3.12(B)) between sham and spun PIJV segments. The individual percent constriction and dilation values for each experiment are provided in Table D. 7 and Table D. 9, respectively, in Appendix D. The Microsoft Excel student's t-test output tables for the percent constriction and percent dilation data are provided in Table D. 8 and Table D. 10, respectively, in Appendix D. 


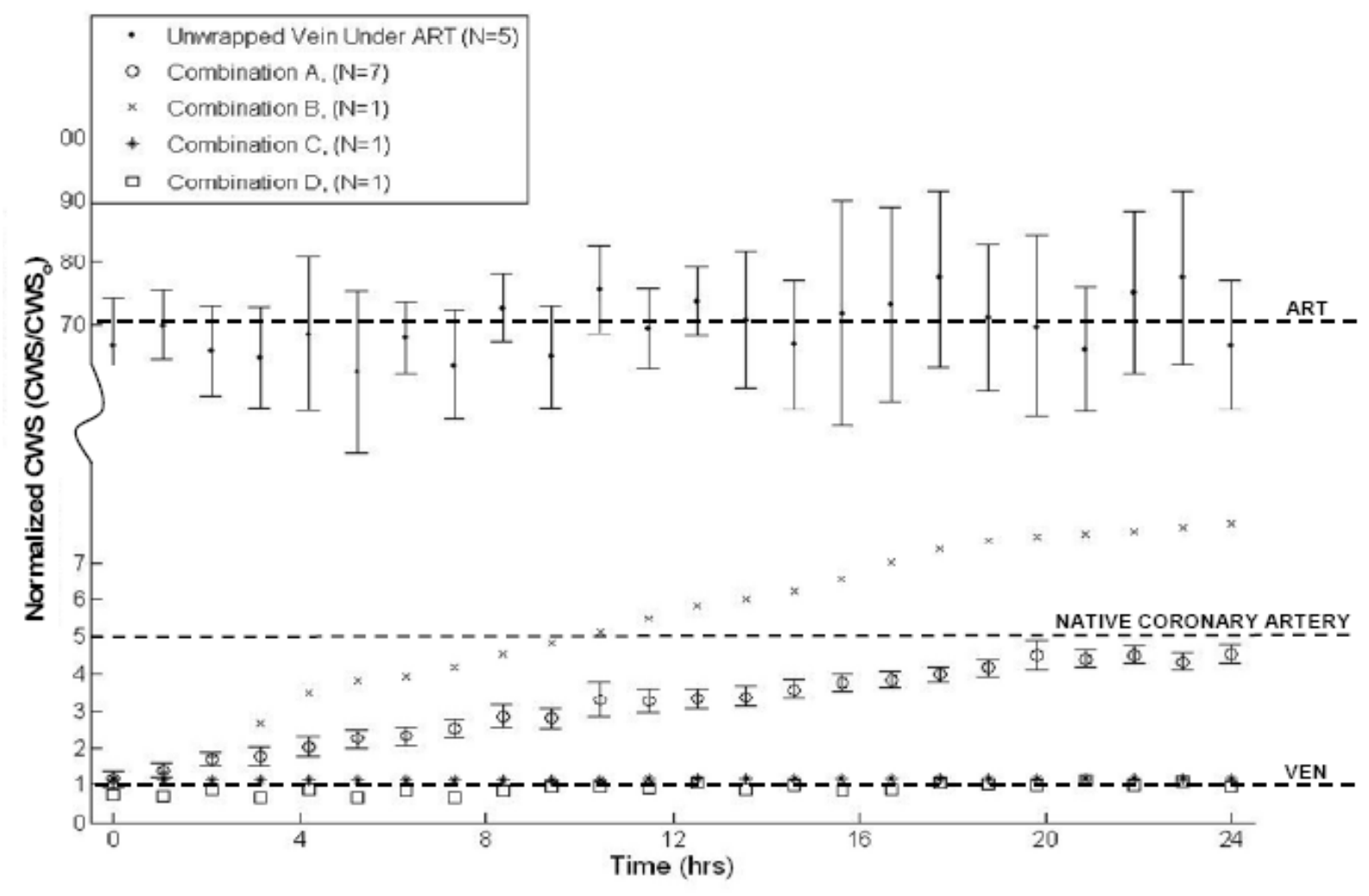

Figure 3.10 CWS vs. time results from 24 hour ex vivo perfusions of electrospun polymer wrapped PIJV segments for each combination in Table 3.1. The lower dashed horizontal line indicates the mean CWS level measured in an unwrapped vein under venous conditions (CWSo $\sim 25 \mathrm{KPa}$ ), and the middle dashed horizontal line indicates the mean CWS in a coronary artery $(\sim 120 \mathrm{KPa})[4]$. The upper dashed line represents the mean CWS measured in an unwrapped vein (sham control) under ART conditions. In the legend, ET stands for electrospinning time. All CWS values were normalized to $\mathrm{CWS}_{\mathrm{o}}$. The data are presented as mean \pm standard error of the mean. 


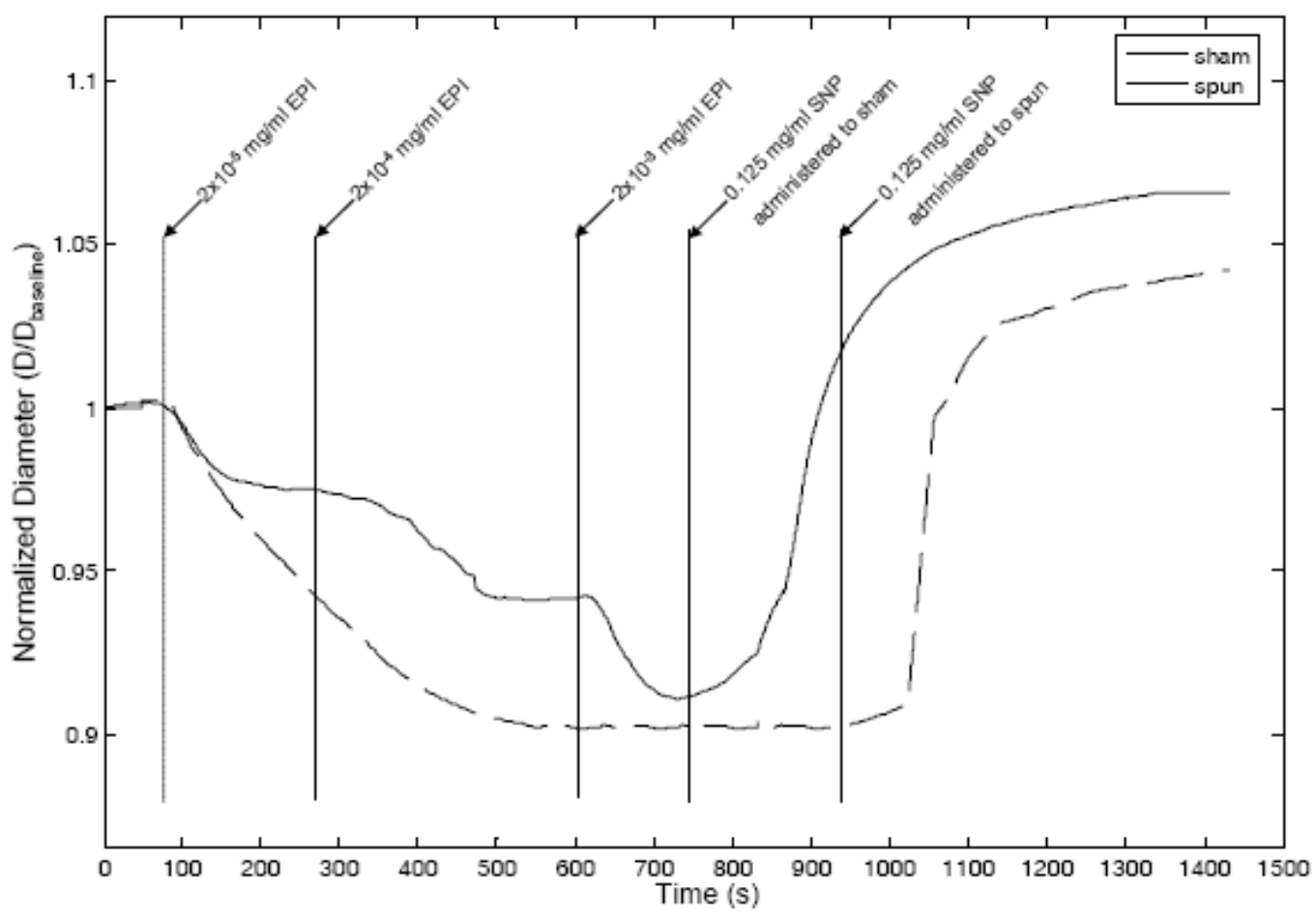

Figure 3.11 Representative vasomotor challenge results obtained using epinephrine (EPI) and sodium nitroprusside (SNP) to stimulate both a spun and a sham control PIJV segment. Please note that SNP was administered immediately upon observing a natural relaxation of the tissue post-stimulation with EPI. That is, SNP was administered at different times for the sham and spun PIJVs, depending on when the natural relaxation of the tissue (post stimulation with EPI) was observed. Outer diameter measurements of each PIJV segment over the duration of the experiments were normalized to the baseline outer diameter which was measured prior to administration of the first dose of EPI. 


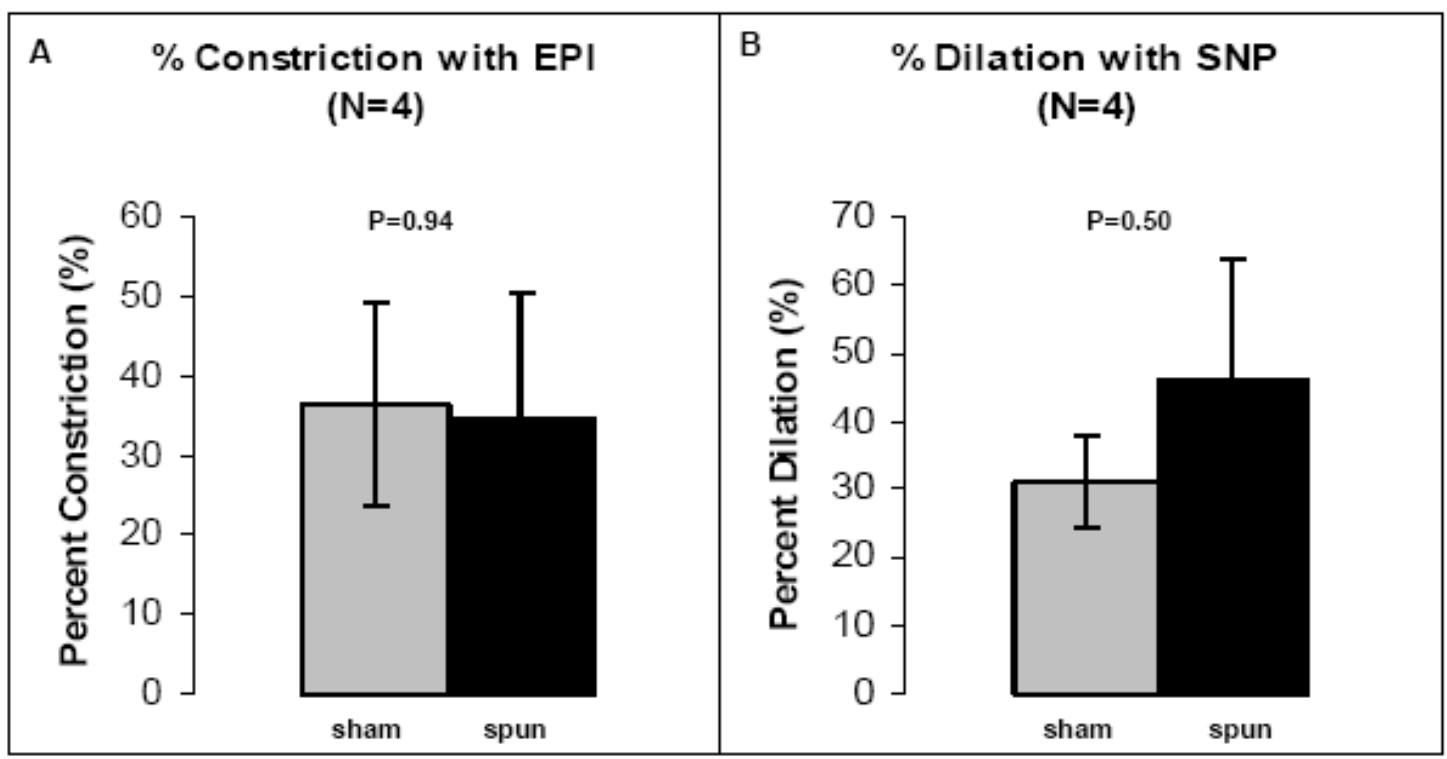

Figure 3.12 Results from vasomotor challenge experiments $(\mathrm{N}=4)$. There appears to be no significant difference in the level of contraction or dilation between the sham control and spun PIJVs. The data are presented as mean \pm standard error of the mean.

\subsubsection{Compliance and $\beta$-stiffness}

In Figure 3.13(A) and (C), we see that PIJVs are very stiff (and hence much less compliant) when exposed to arterial levels of pressure. Under the same hemodynamic conditions, the tuned polymer wrap that was spun onto the adventitial surface of the PIJVs offered structural support which is evident by the decreased stiffness (Figure 3.13(B)) and increased compliance (Figure 3.13(D)). Please note that due to technical issues, the pressure and diameter measurements for one of the sham controls were not possible and thus there was one less data set $(\mathrm{N}=5)$ than in the spun group $(\mathrm{N}=6)$. 


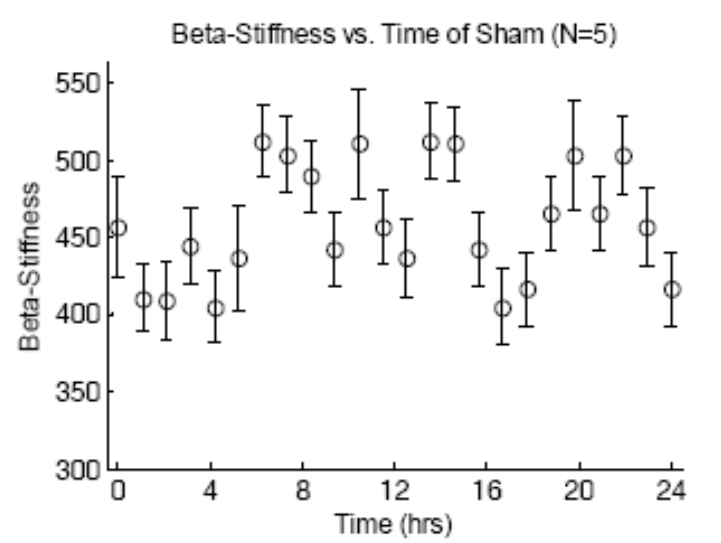

(A)

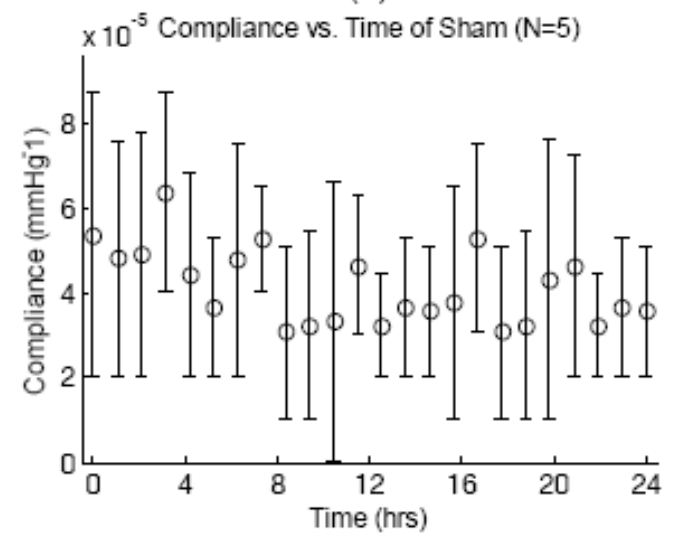

(C)

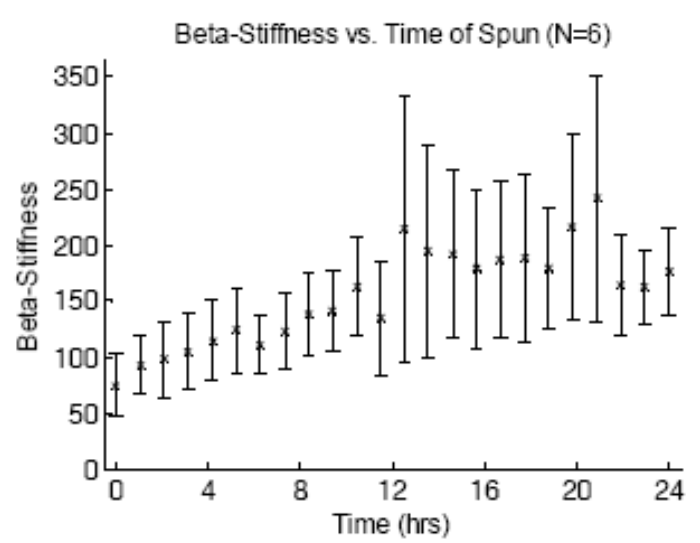

(B)

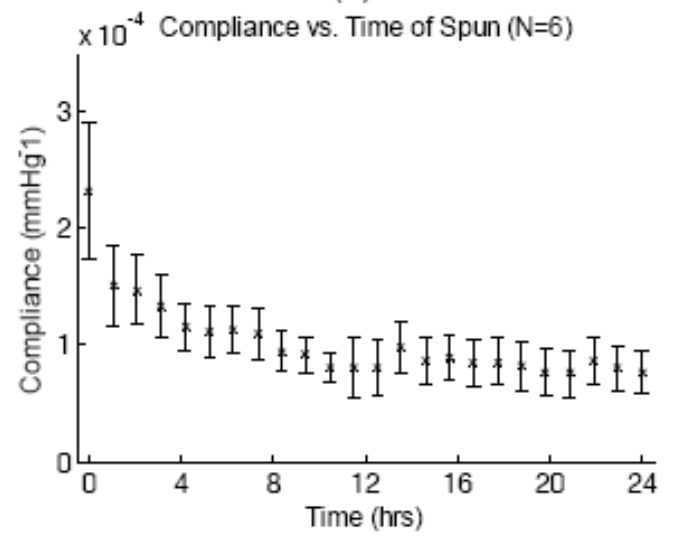

(D)

Figure 3.13 Results from the compliance and $\beta$-stiffness calculations for both sham (A \& C) and spun (B \& D) PIJVs over 24 hours. The data are presented as mean \pm standard error of the mean.

\subsubsection{Biological Analyses}

\subsubsection{Scanning Electron Microscopy}

SEM analysis was performed on tissue samples from a representative ex vivo ART vs. wART experiment (see Table 3.2). The electrospun adventitial wrap exhibited high porosity and tight adherence to the adventitial surface of the veins (Figure 3.14(A)-(C)), which suggests that the wrap would provide structural support to an AVG without inhibiting adventitial nutrient and gas 
diffusion into the tissue. Another important observation was that the electrospinning process did not appear to damage the endothelial layer, which remained continuous (Figure 3.14(D)).

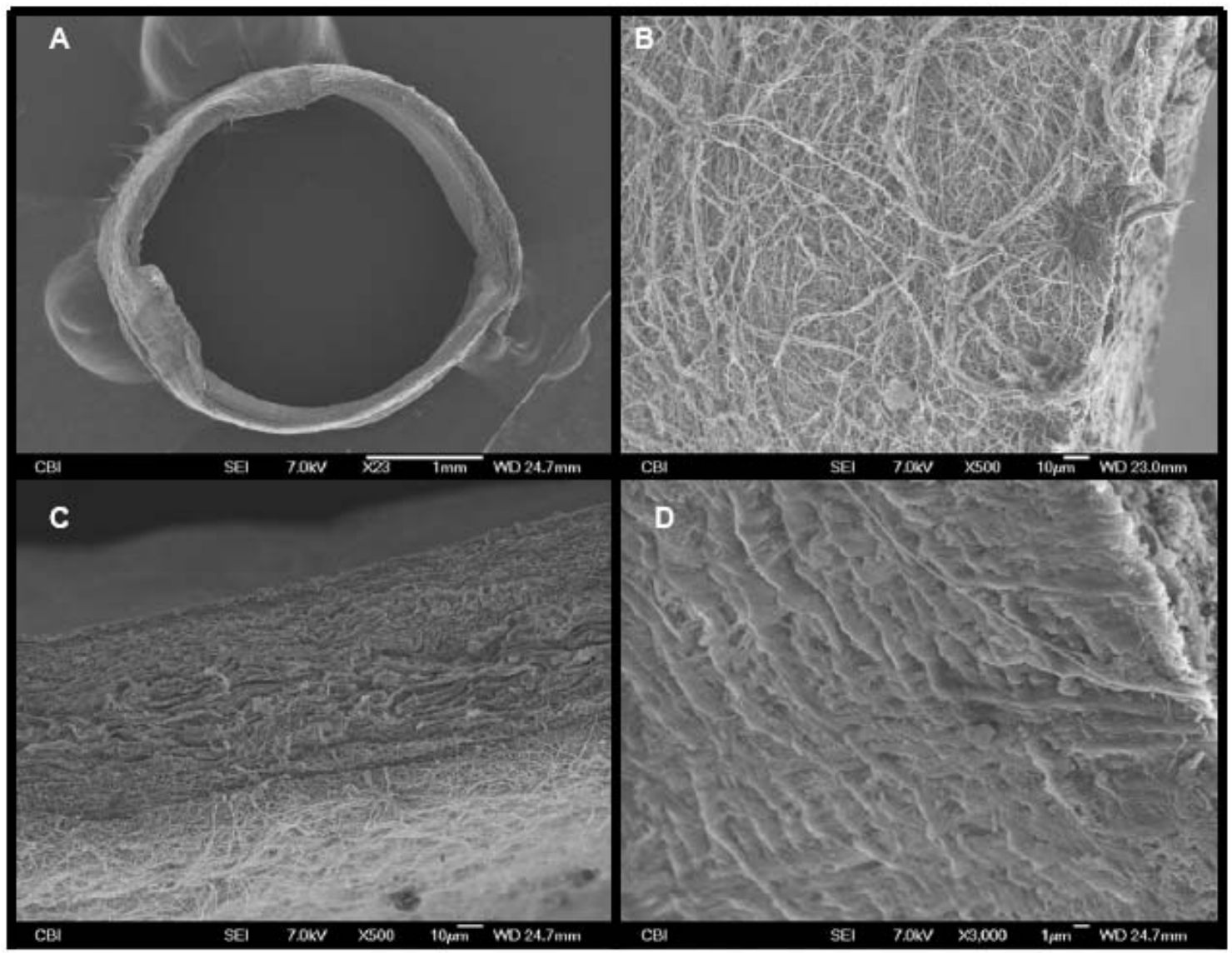

Figure 3.14 (A) shows a low magnification SEM image of the PIJV segment with the electrospun polymer deposited onto its adventitial surface. (B) is an SEM image (taken at 500x magnification) of the adventitial surface of the PIJV after the polymer wrap was applied. Note the high porosity of the polymer wrap. (C) is an SEM image (taken at 500x magnification) showing the attachment of the polymer wrap to the vein. (D) is an SEM image (taken at 500x magnification of the luminal surface of the vein and shows a continuous endothelium layer which appears to have remained intact. 


\subsubsection{Histology}

Histological analysis was performed on tissue samples from representative ex vivo experiments (see Table 3.2). H\&E and MTC images were consistent with the SEM images in that they also showed the polymer wrap to be well attached to the adventitial surface of the vein and that it can be electrospun with an approximately uniform thickness (Figure 3.15(A) and (C)). Further, the

polymer degraded nearly completely following the 24 hour perfusion period (Figure 3.15(B) and (D)). 


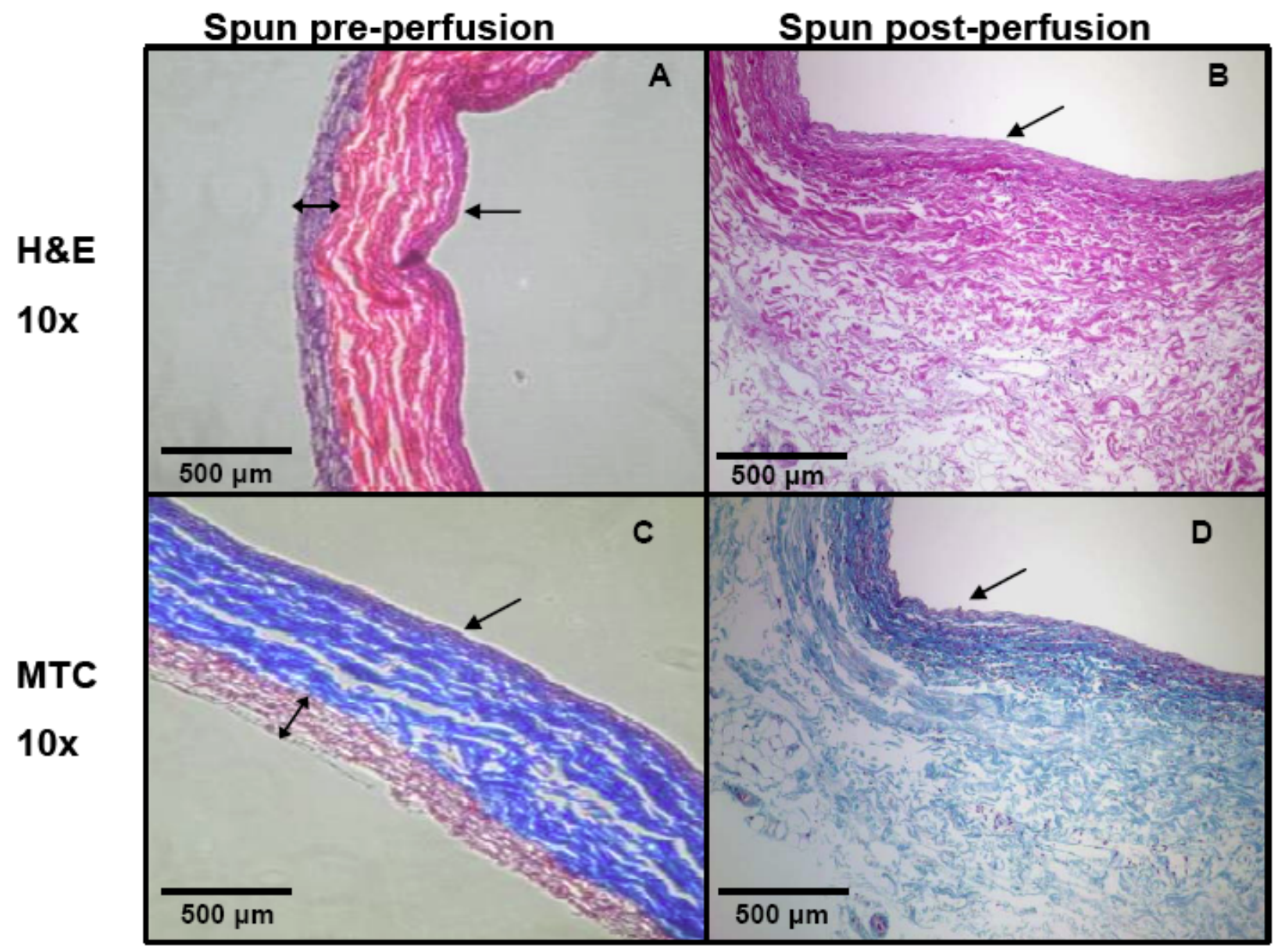

Figure 3.15 Representative hematoxylin and eosin (A,B) and Masson's trichrome images (C,D) for both before perfusion and after wrapping procedure $(\mathrm{A}, \mathrm{C})$ and after 24 hours of ex vivo perfusion $(\mathrm{B}, \mathrm{D})$. Note the uniform thickness of the polymer wrap prior to perfusion, and the absence of the polymer wrap in the post-perfusion images. The single-headed arrow indicates the vessel lumen. The double-headed arrow in (A) and (C) indicates the thickness of the polymer wrap, which was not detectable in (B) or (D).

Figure 3.16 shows birefringence images of vein sections stained with PSR. In each image, the color range from red to green indicates a range of collagen fiber organization with red being most organized and green being less organized. The granulated appearance of the staining indicates the natural crimped collagen fiber state, whereas stretched fibers appear striated rather than granulated. These results suggest that the polymer wrap reduces the level of collagen fiber 
stretching (including greater organization and reduced crimping) when compared to a control PIJV segment perfused ex vivo under ART conditions for 24 hours.

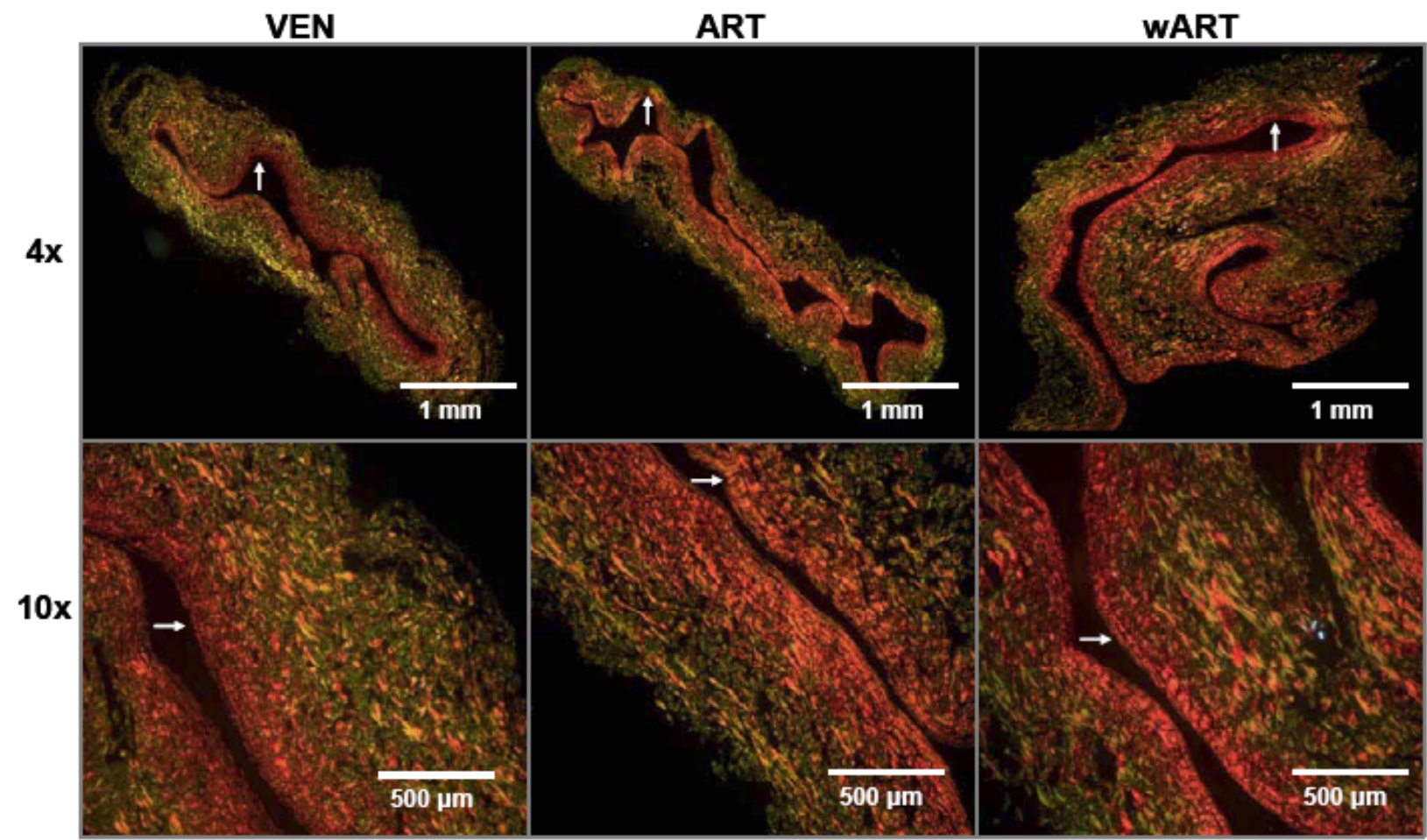

Figure 3.16 Representative birefringence images of vein sections stained with picrosirius red. The experimental conditions are defined as: Venous (VEN) conditions of $20 \mathrm{mmHg}$ pressure and $20 \mathrm{ml} / \mathrm{min}$ flowrate; pulsatile arterial (ART) conditions of $120 / 80 \mathrm{mmHg}$ pressure and $100 \mathrm{ml} / \mathrm{min}$ mean flowrate; and wrapped arterial (wART) conditions where the wrapped vein segments were perfused under ART conditions for 24 hours ex vivo. The arrow indicates the vessel lumen.

Images of MPC stained tissue sections are shown in Figure 3.17. The internal elastic lamina appears disrupted in the PIJVs perfused under ART conditions when compared to both VEN and wART conditions. As with the PSR staining, this data suggests that the polymer wrap 
was successful in reducing the level of stretch within the vein wall when exposed to ART conditions.

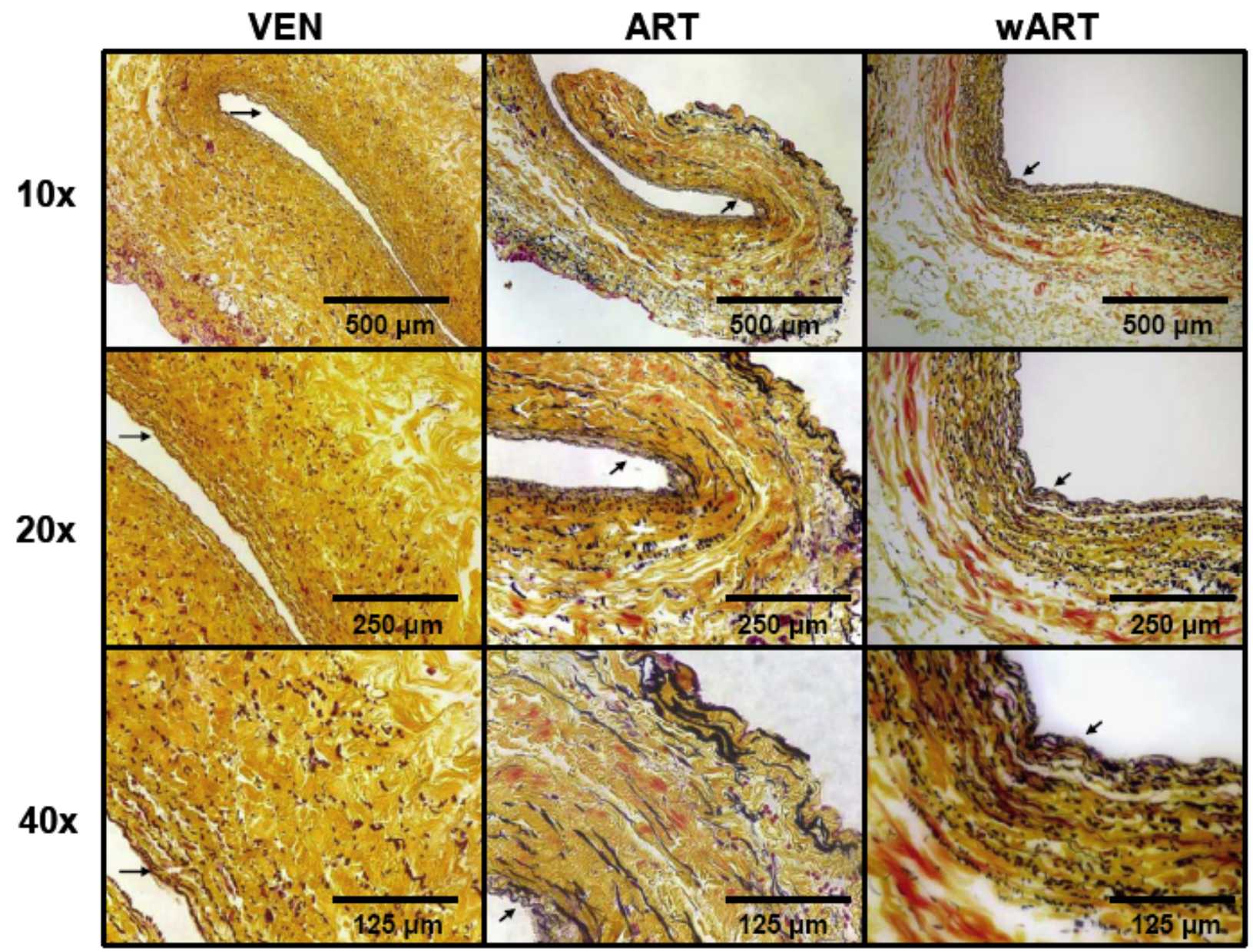

Figure 3.17 Representative Movat's pentachrome staining of vein tissue sections. In each image collagen stains yellow, elastin and nuclei stain black, and muscle stains red. The red staining in the adventitial side of the wART sections is unspecific staining of culture media proteins that become entrapped within the polymer during ex vivo perfusion experiments. The arrow indicates the vessel lumen. 


\subsubsection{Necrosis}

Figure 3.18 (left panel) shows a representative image of a Live/Dead ${ }^{\mathrm{TM}}$ stained tissue section. There was no significant difference in tissue necrosis between each experimental group for each timepoint (Figure 3.18 right panel). Representative images from each condition (control, sham and spun) from the 92 hour timepoint are provided in Figure F. 1 in Appendix F.
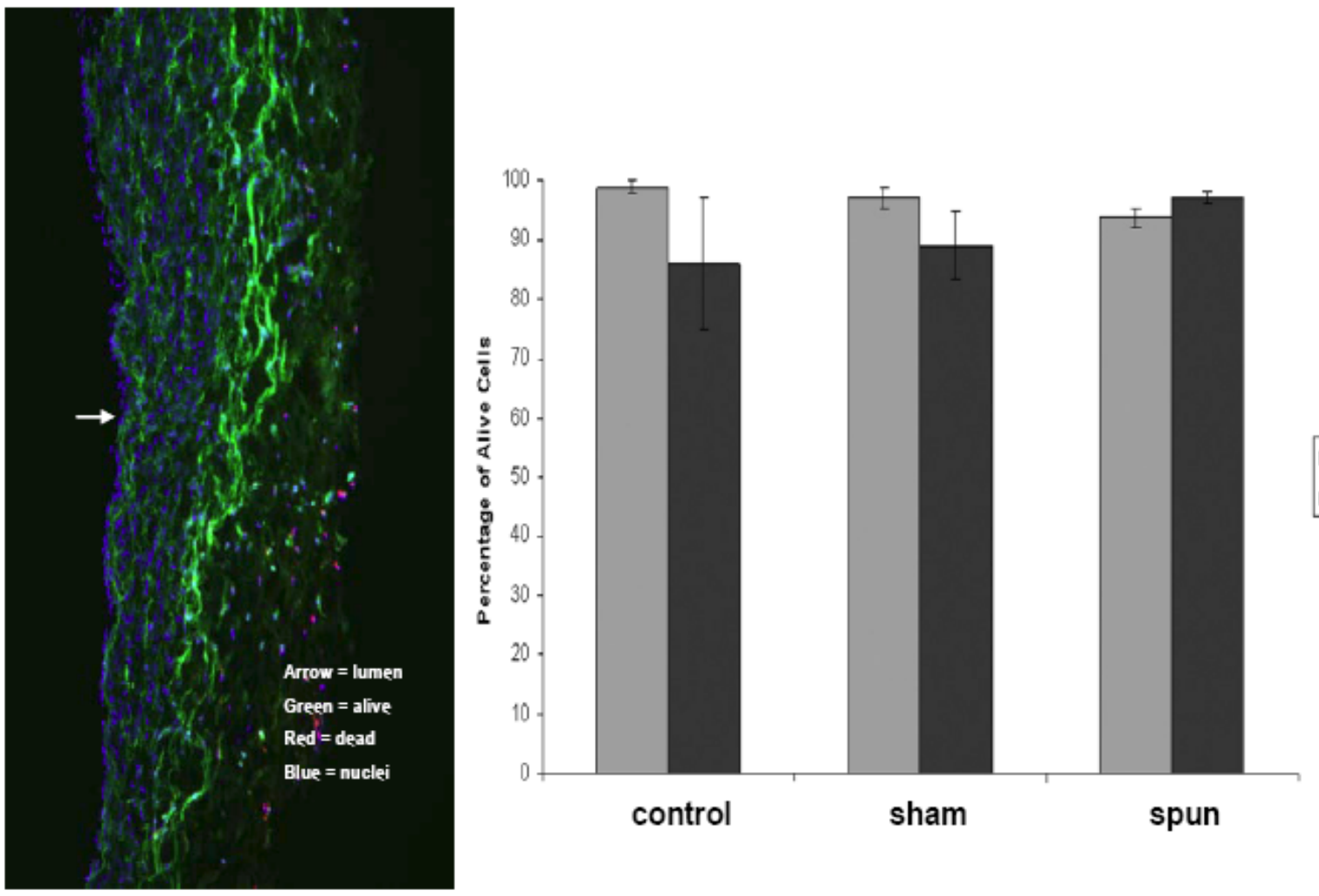

Figure 3.18 Left: Representative image of Live/Dead ${ }^{\mathrm{TM}}$ stained tissue section from a spun PIJV. Right: Quantified Live/Dead ${ }^{\mathrm{TM}}$ results to assess the level of necrosis in PIJVs after electrospinning, and after 18 and 92 hours of postelectrospinning static culture. The data shown was for a single experiment, and the error bars result from the 10 fields of view that were analyzed per PIJV segment. The data are presented as mean \pm standard error of the mean. 


\subsubsection{Apoptosis}

Figure 3.19 shows representative paired fluorescent immunohistochemistry images of TUNEL staining from all four ex vivo vascular perfusion experiments described in Section 3.1.3. Figure 3.20 shows the quantified TUNEL analysis results from these experiments. It can be seen that there is a statistically significant increase in apoptotic cells within PIJVs abruptly exposed to ART conditions vs. VEN controls. However, the mechanical conditioning paradigm imposed via cART conditions (for both 24 and 72 hours) and via the biodegradable electrospun polymer wrap (wART conditions) statistically significantly reduced the number of apoptotic cells within PIJVs vs. ART control conditions. Representative TUNEL staining images from all ex vivo perfusion experiments are provided in Figure F. 2 to Figure F. 23 in Appendix F. The mean percent values of TUNEL positive cells, from all experiments, are provided in Table F. 1 in Appendix F. The Microsoft Excel student's t-test output tables for the mean percent TUNEL positive cells, for each comparison shown in Figure 3.20, are provided in Table F. 2 to Table F. 5 in Appendix F. 


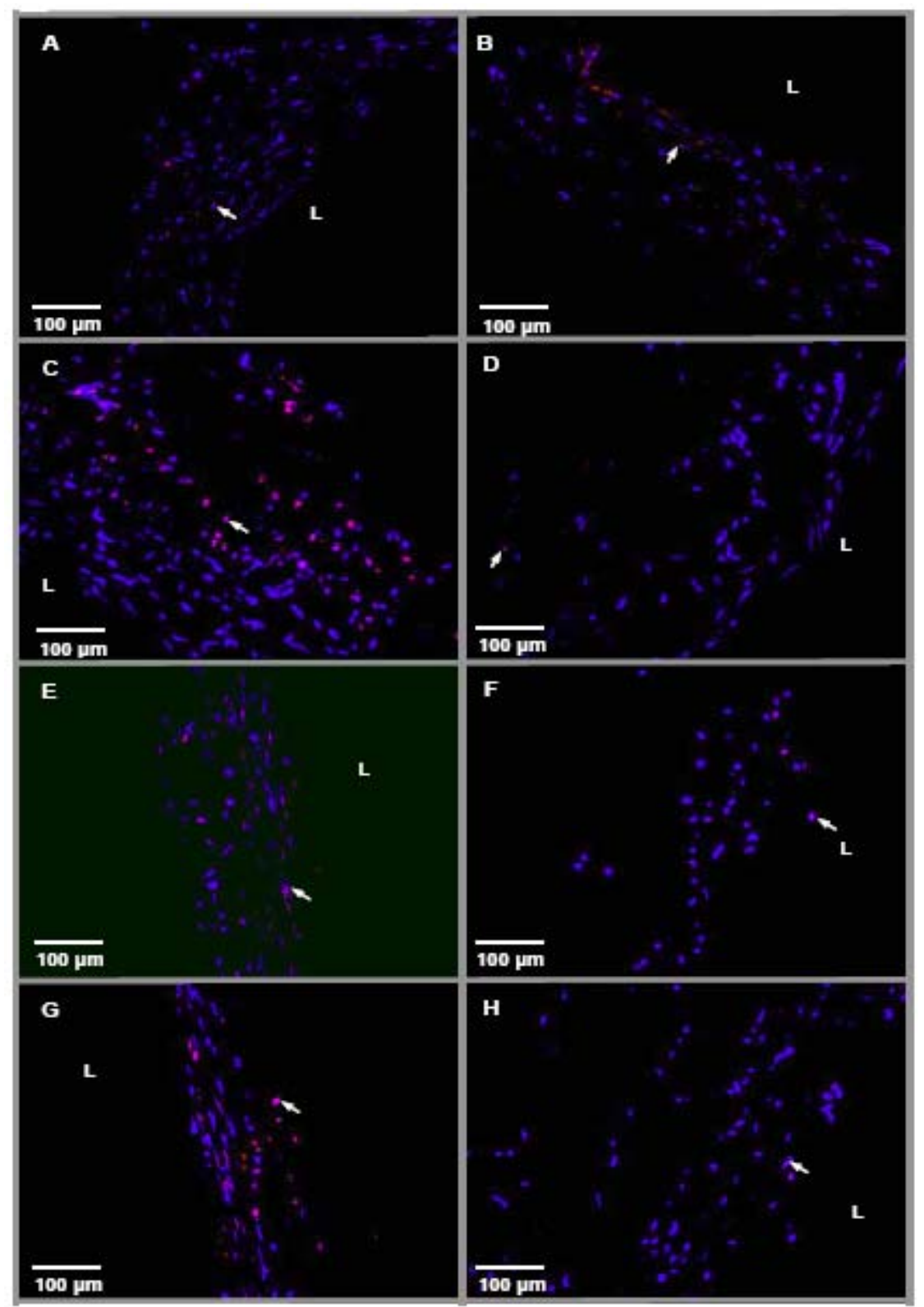

Figure 3.19 Representative immunohistochemistry images from the fluorescent based TUNEL analysis. The top two panels are from a 24-hour VEN (A) vs. ART (B) experiment. The next two panels are from a 24-hour ART (C) vs. cART (D) experiment. The third row of panels are from a 72-hour ART (E) vs. cART (F) experiment. The bottom two panels are from a 24-hour ART $(\mathrm{G})$ vs. wART $(\mathrm{H})$ experiment. The arrows indicate apoptotic cells. L indicates the PIJV lumen. 


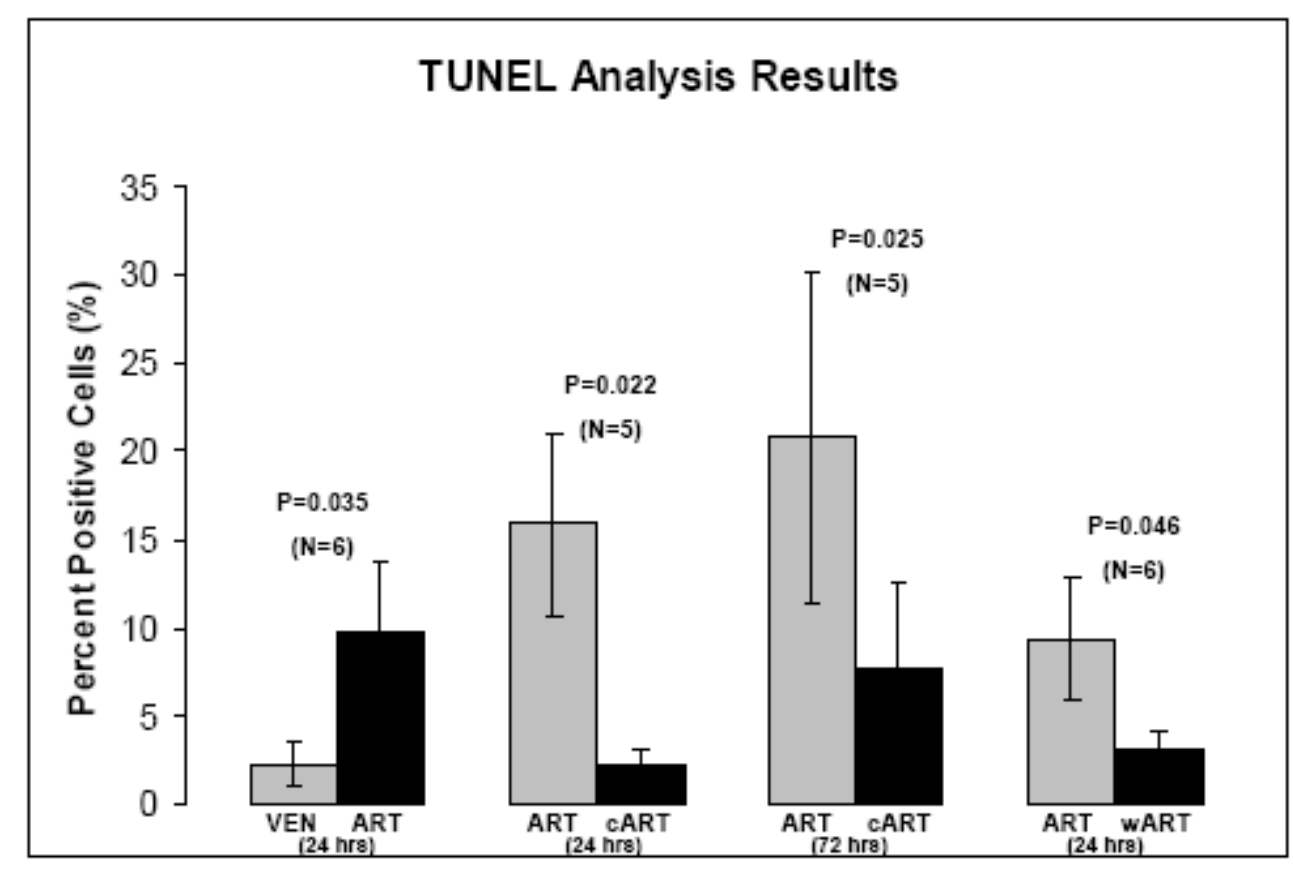

Figure 3.20 Quantified immunohistochemistry results from fluorescent based TUNEL analysis to assess the percentage of apoptotic cells within PIJVs from all the ex vivo vascular perfusion experiments. The data are presented as mean \pm standard error of the mean.

\subsubsection{Proliferation}

Figure 3.21 shows representative paired HRP/ABC based immunohistochemistry images of PCNA staining from all four ex vivo vascular perfusion experiments described in Section 3.1.3. Figure 3.22 shows the quantified PCNA analysis results from these experiments. It can be seen that there is a statistically significant decrease in proliferating cells within PIJVs abruptly exposed to ART conditions vs. VEN controls. However, the mechanical conditioning paradigm imposed via cART conditions (24 hours) and via the biodegradable electrospun polymer wrap (wART conditions) statistically significantly inhibited the decrease in the number of proliferating cells within PIJVs vs. ART control conditions. The number of proliferating cells within PIJVs 
exposed to cART conditions for 72 hours was not statistically significantly different than ART controls. Representative PCNA staining images from all ex vivo perfusion experiments are provided in Figure F. 24 to Figure F. 45 in Appendix F. The mean percent values of PCNA positive cells, from all experiments, are provided in Table F. 6 in Appendix F. The Microsoft Excel student's t-test output tables for the mean percent PCNA positive cells, for each comparison shown in Figure 3.22, are provided in Table F. 7 to Table F. 10 in Appendix F. 


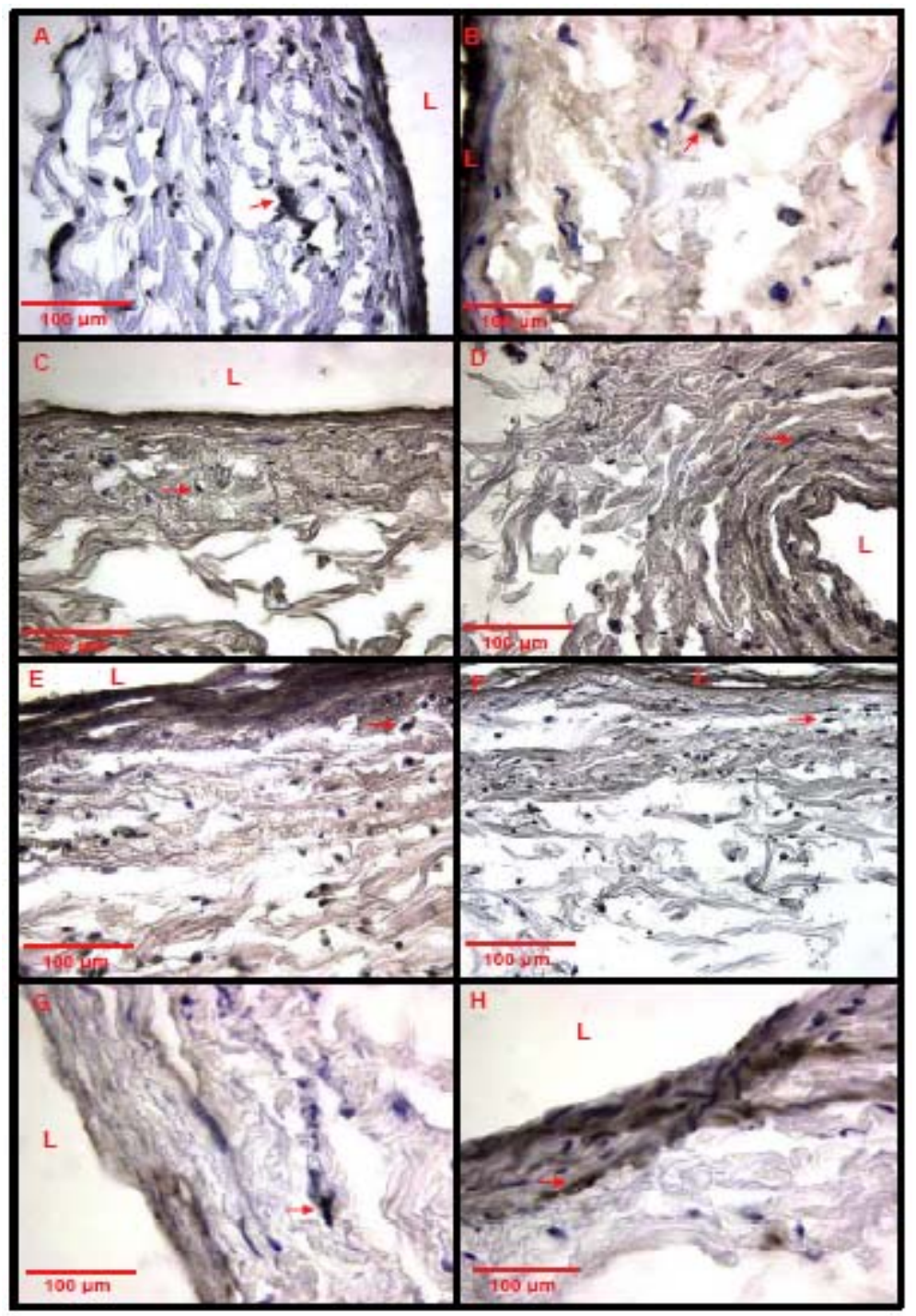

Figure 3.21 Representative immunohistochemistry images from the HRP/ABC based PCNA analysis. The top two panels are from a 24-hour VEN (A) vs. ART (B) experiment. The next two panels are from a 24-hour ART (C) vs. cART (D) experiment. The third row of panels are from a 72-hour ART (E) vs. cART (F) experiment. The bottom two panels are from a 24-hour ART $(\mathrm{G})$ vs. wART $(\mathrm{H})$ experiment. The arrows indicate proliferating cells. L indicates the PIJV lumen. 


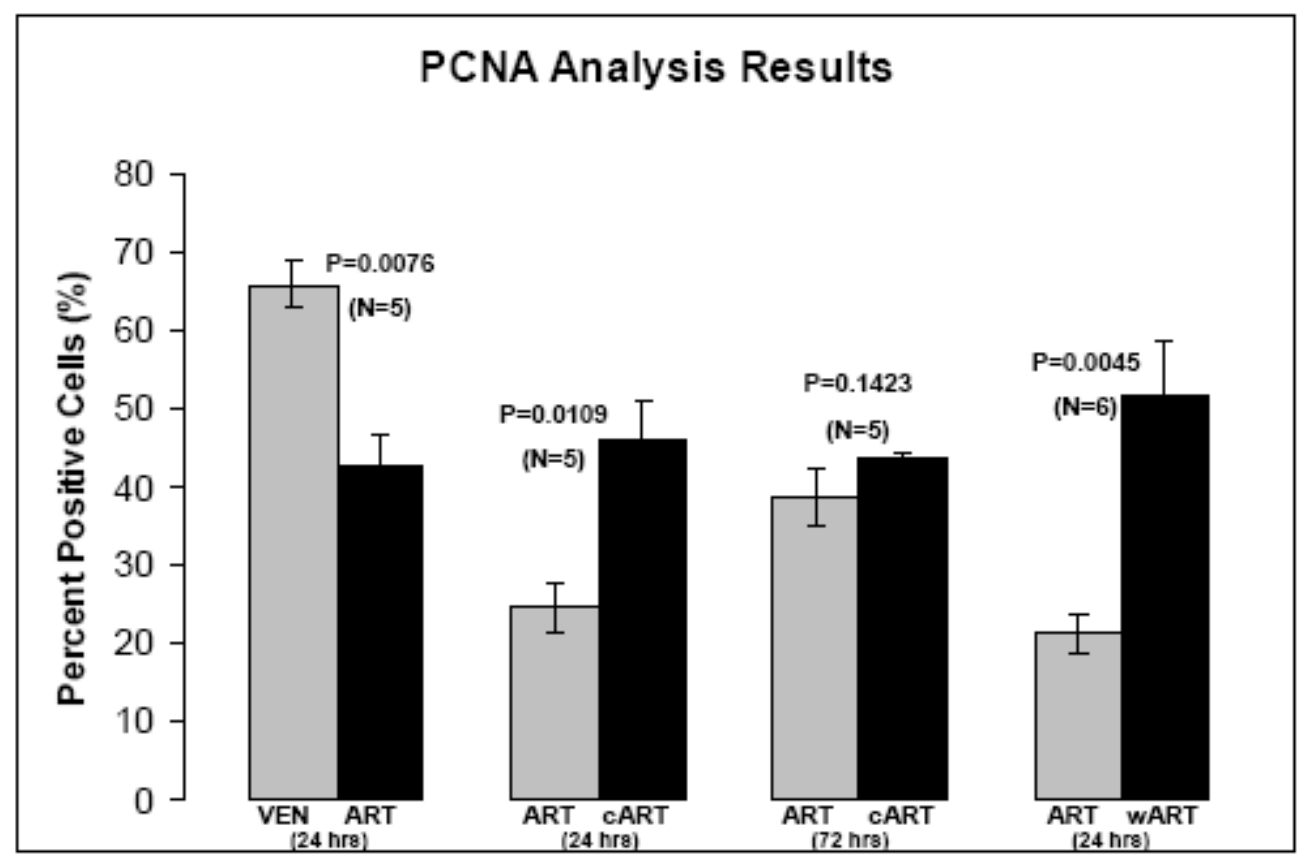

Figure 3.22 Quantified immunohistochemistry results from HRP/ABC based PCNA expression analysis to assess the percentage of proliferating cells within PIJVs from all the ex vivo vascular perfusion experiments. The data are presented as mean \pm standard error of the mean.

\subsubsection{SMC Phenotype}

Figure 3.23 shows representative paired HRP/ABC based immunohistochemistry images of Golgi complex staining from all four ex vivo vascular perfusion experiments described in Section 3.1.3. Figure 3.24 shows the quantified Golgi complex analysis results from these experiments. It can be seen that there is a statistically significant increase in the number of cells staining positive for Golgi complex within PIJVs abruptly exposed to ART conditions vs. VEN controls. The mechanical conditioning paradigm imposed via cART conditions (for both 24 and 72 hours) and via the biodegradable electrospun polymer wrap (wART conditions) suggests only a trend towards statistically significantly inhibiting the increase in the number of cells positively stained for Golgi complex within PIJVs vs. ART control conditions. Representative Golgi 
complex staining images from all ex vivo perfusion experiments are provided in Figure F. 46 to Figure F. 67 in Appendix F. The mean percent values of Golgi complex positive cells, from all experiments, are provided in Table F. 11 in Appendix F. The Microsoft Excel student's t-test output tables for the mean percent PCNA positive cells, for each comparison shown in Figure 3.24, are provided in Table F. 12 to Table F. 15 in Appendix F. 


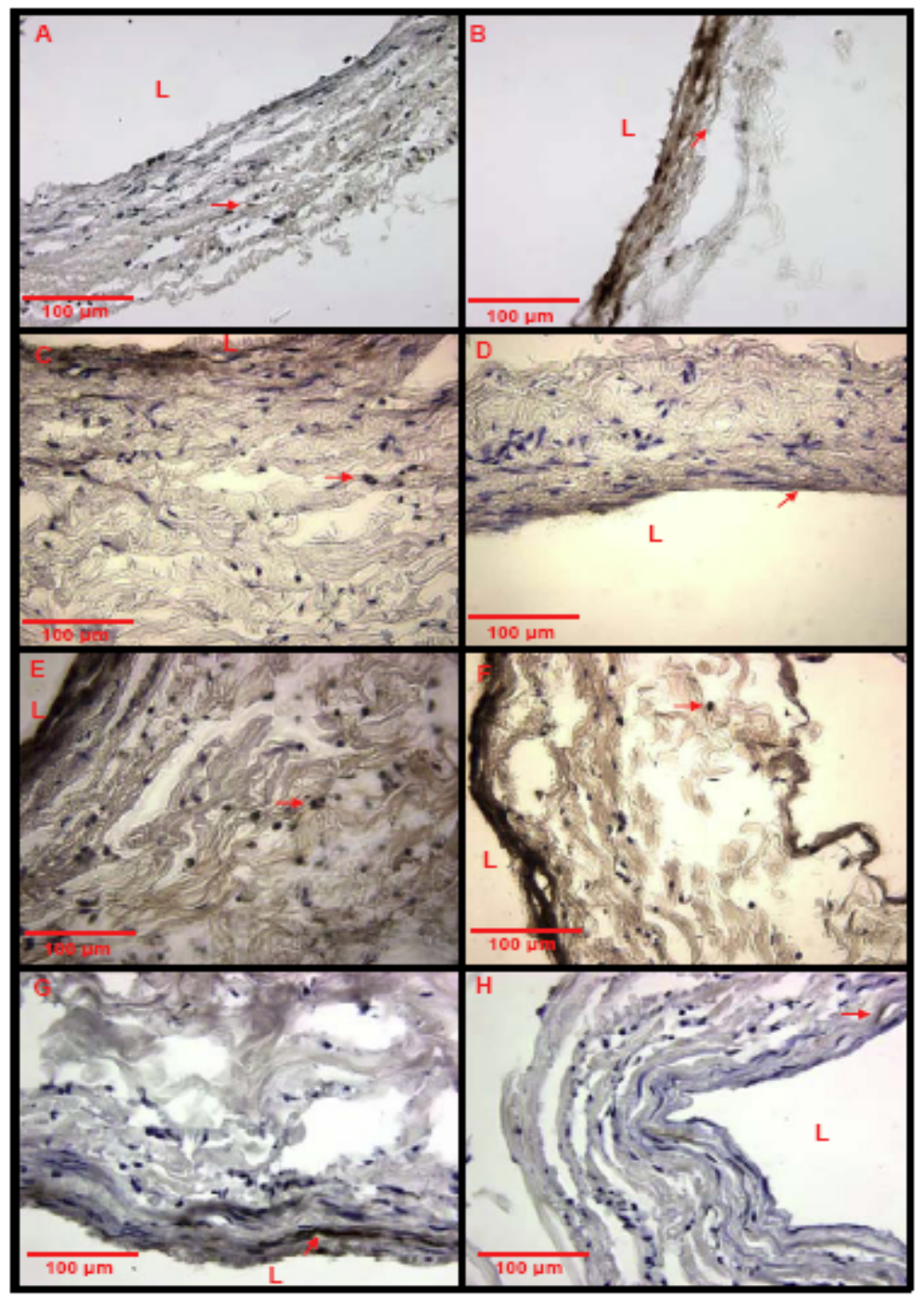

Figure 3.23 Representative immunohistochemistry images from the HRP/ABC based Golgi complex analysis. The top two panels are from a 24-hour VEN (A) vs. ART (B) experiment. The next two panels are from a 24-hour ART (C) vs. cART (D) experiment. The third row of panels are from a 72-hour ART (E) vs. cART (F) experiment. The bottom two panels are from a 24-hour ART $(\mathrm{G})$ vs. wART $(\mathrm{H})$ experiment. The arrows indicate positively stained cells. L indicates the PIJV lumen. 


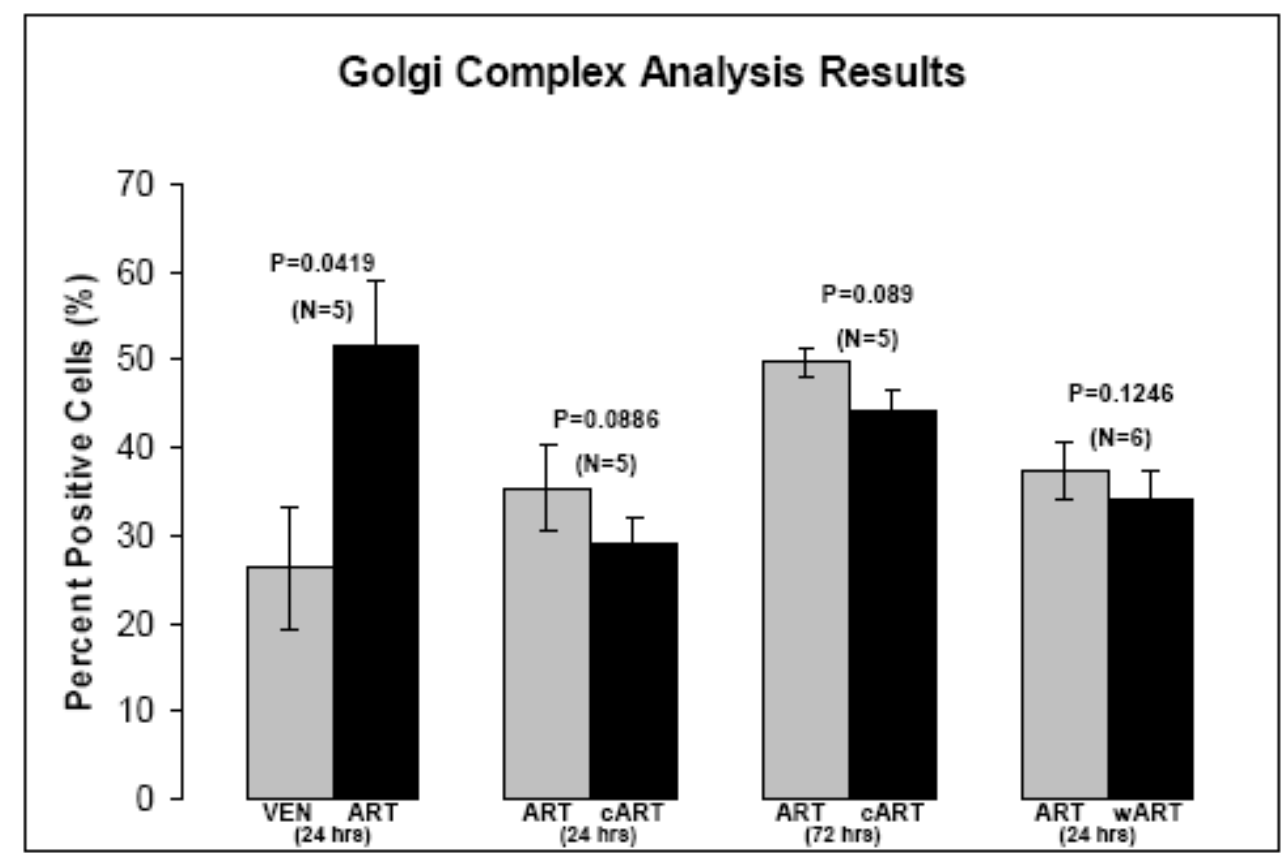

Figure 3.24 Quantified immunohistochemistry results from HRP/ABC based Golgi complex expression analysis to assess the percentage cells staining positive for Golgi complex within PIJVs from all the ex vivo vascular perfusion experiments. The data are presented as mean \pm standard error of the mean.

\subsection{Transmission Electron Microscopy}

The level of Golgi complex and rough endoplasmic reticulum expression could not be quantified from the TEM images. However, the representative TEM images seen in Figure 3.25 show that the SMCs within the PIJVs perfused under VEN and cART conditions (Figure 3.25(A) and Figure 3.25(C), respectively) have a very randomly oriented actin cytoskeleton in contrast to ART conditions (Figure 3.25(B)) which shows very aligned and more dense actin fibers. It should be noted that the alignment is parallel to the direction of the CWS. All other TEM images are provided in Figure H. 11 to Figure H. 13 in Appendix H. 

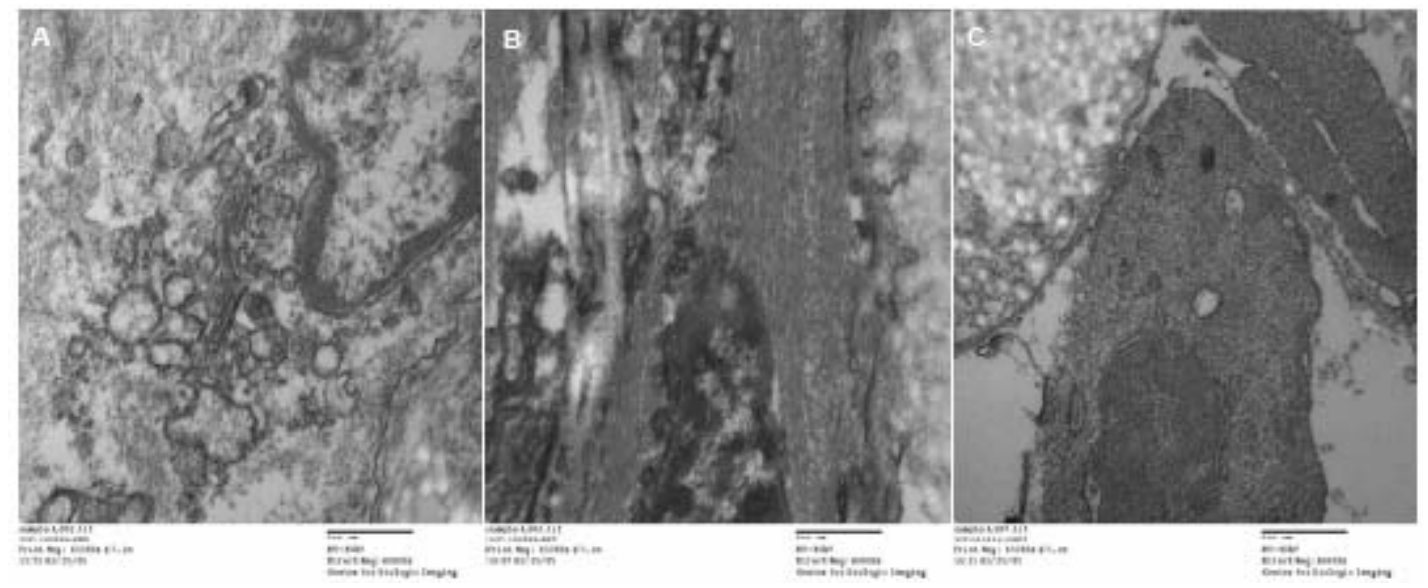

Figure 3.25 Transmission electron microscopy (TEM) images of sections prepared from vein segments perfused, ex vivo, for 24 hours under (A) venous (VEN) conditions of $20 \mathrm{mmHg}$ pressure and $20 \mathrm{ml} / \mathrm{min}$ flowrate, (B) pulsatile arterial (ART) conditions of $120 / 80 \mathrm{mmHg}$ pressure and $100 \mathrm{ml} / \mathrm{min}$ mean flowrate, and (C) conditioned arterial (cART) conditions where the pressure and flowrate were ramped in equal increments, gradually over 24 hours, from VEN to ART conditions. Note the random orientation of the actin cytoskeleton in (A) and (C) and the aligned orientation of the fibers in (B). Images were taken at a magnification of $60,000 \mathrm{x}$.

\subsection{DISCUSSION}

The work presented in this chapter shows that a biodegradable electrospun polymer wrap can be uniformly (Figure 3.15) and safely (Figure 3.11, Figure 3.12, and Figure 3.18) electrospun onto vein segments, and that the wrap can be tuned to completely degrade (Figure 3.15) such that CWS is applied to an AVG at a desired rate (Figure 3.10). Having control over the biodegradation rate of an adventitially placed electrospun polymer wrap could lend itself to three potentially beneficial support modalities for attenuating IH in AVGs. As shown here, biomechanical support can be delivered at a desired rate. Consequently, delivery of both biochemical (drugs), and biological (cellular) support might theoretically be achieved using the 
same approach $[242,303]$. The potentially beneficial effects of the polymer wrap on AVG microstructure were observed from the PSR and MPC staining (Figure 3.12 and Figure 3.13, respectively). The polymer wrap seems to provide structural support to AVGs resulting in a more naturally crimped configuration of the collagen fibers (Figure 3.16), as well as less damage to the internal elastic lamina (Figure 3.17). Maintaining integrity of the structural proteins that comprise the AVG wall may help to minimize the detrimental mechanical triggers received by the vascular ECs and SMCs and hence could help to attenuate IH in AVGs. We also assessed the level of necrosis via Live/Dead ${ }^{\mathrm{TM}}$ staining in the electrospun PIJVs and showed no appreciable increase in necrosis due to electrospinning over sham and static controls (Figure 3.18). This data in addition to the vasomotor challenge data (Figure 3.11 and Figure 3.12) is more evidence to show that tissue viability is not affected by electropsinning.

The immunohistochemistry results suggest that gradual vs. abrupt exposure of AVGs to arterial levels of CWS may be beneficial. The balance between apoptosis and proliferation, as seen in Figure 3.20 and Figure 3.22 respectively, was shown to be disrupted due to abrupt exposure of PIJVs to ART conditions over VEN controls. The observed increase in apoptosis and reduction in proliferation in PIJVs perfused under ART conditions suggests that there is an immediate shift in cellular function due to the altered biomechanical environment of the vein. This shift in cellular function within veins was shown to be inhibited by more gradual imposition of arterial levels of CWS via cART and wART ex vivo perfusion conditions. In addition, the level of Golgi complex expression in PIJVs exposed to ART conditions was increased over VEN controls (Figure 3.24), suggesting a modulation in SMC phenotype to a more synthetic state. This observed shift in cellular function was not significantly inhibited by gradual exposure to ART levels of CWS via cART or wART conditions. However, a trend towards inhibition of this 
shift was shown (see Figure 3.24). Additional experiments are required to determine if this trend becomes statistically significant.

The observed alteration in SMC phenotype that resulted from exposing PIJVs to ART conditions agrees with previously reported data [71,131,163]. The concept of more gradual imposition of arterial levels of CWS to AVGs has not previously been reported but could result in a means to slow down or inhibit SMC phenotypic modulation which could consequently attenuate the hyperplastic response. The reduction in apoptosis in PIJVs exposed to ART vs. VEN conditions also agrees with published results [108,124,203-206]. However, the reduction in proliferation in ART perfused PIJVs vs. VEN, cART, and wART groups was inconsistent with some published data [209,212,213]. Liu et al. suggested however that mechanical stretch due to arterial hemodynamics induces cell death, which possibly mediates subsequent cell proliferation [108]. The short-term timepoints studied in this dissertation may not have been long enough to see a rise in proliferation after the initial increase in apoptosis in the ART perfused PIJVs.

Several limitations of the work presented in this chapter should be noted. Although the Live/Dead ${ }^{\mathrm{TM}}$ assay is widely used to evaluate necrosis in living cells and tissues, it arguably was not ideally suited for our application. This was due to the limited distance the reagents were able to diffuse through the thickness of vascular tissue. It was observed that the staining occurred predominantly in the intimal and adventitial layers of the vein wall, while the media was much less intensely stained. It is true that the adverse effect of the electrospinning process would be in the area of contact between the polymer wrap and the vein wall (i.e., the adventitia), as well as in the area of contact between the mandrel and the vein wall (i.e., the lumen). The Live/Dead ${ }^{\mathrm{TM}}$ assay appeared to work well in both of these areas and showed no appreciable increase in the 
level of necrosis when compared to control tissue. Additionally, the vasomotor challenge data indicated that the spun PIJV was able to contract with the same intensity as the sham control which demonstrated the viability of the SMCs comprising the medial layer of the tissue. Finally, we would have ideally compared the vasomotor responses of the sham and spun PIJVs to a baseline control response - that is, with a freshly excised PIJV segment. However, obtaining a third segment of PIJV for immediate testing was not feasible since we could only harvest two PIJV segments per animal. We feel that the choice of a sham control over a baseline control was acceptable in that we wanted to assess the differences associated only with electrospinning.

\subsection{CONCLUSION}

We showed here that a tunable polymer wrap can be applied to vein segments without compromising viability or function, and demonstrated one potential application; i.e., gradually imposing the mid-wall CWS in wrapped veins exposed to arterial levels of pressure. The gradual imposition of arterial levels of CWS, rather than abrupt exposure, may be an important new means to reduce the hyperplastic response of AVGs, promoting instead safe arterialization.

Incorporation of either pharmaceuticals or biologicals into an adventitial polymer wrap represents a possible future application, and may further enhance the patency of AVGs. To our knowledge, controlled delivery of cellular support via a biodegradable AVG wrap/sheath has not been previously reported and hence this possible future application of the adventitial wrap would be novel. The polymer that was used in this study has been characterized [57,297,304], and successfully micro-integrated with viable SMCs [242], and would lend itself to this possible future application. 


\subsection{IN VIVO ARTERIAL VEIN GRAFTING}

Figure 4.1 is a schematic depicting the set of in vivo experiments that were performed related to Specific Aim 4. In order to evaluate the mitigating effect of the electrospun PEUU adventitial wrap on the acute and chronic hyperplasic response of vein segments implanted as carotid interposition grafts in a preclinical model, "proof of concept" carotid interposition vein graft experiments were performed in a pig model. For this, we utilized a unilateral autologous carotid interposition internal jugular vein graft. This model was chosen because PIJVs are similar in both size and anatomy to the human saphenous vein, and the physiology and pathobiology of the pig's cardiovascular system is known to closely approximate that of humans [305]. This model of AVG IH has previously been used extensively by others [27,41,43,49,51,294-296,306].

\subsection{METHODS}

Pigs were divided into two groups: a "spun" AVG group and a "sham control" AVG group. Each animal served as its own vein graft donor. In brief, PIJVs were harvested according to Section 3.1.1 and were either spun with the a same wrap composition and thickness as in Specific Aim 3 using the electrospinning process described in Section 3.1.2, or designated as sham controls. The AVGs were then implanted as carotid interposition grafts (as described in Section 4.1.1) for 30 days (or upon observing irreversible complications), an implant duration 
sufficient to allow IH to be grossly apparent in the sham control group $[27,43,48,49,51,241,294-$ $296]$ to which the spun group was compared. In addition to evaluating patency via angiography, the explanted AVGs were processed for histological evaluation of IH, and for SEM as described in Section 3.2.6.

Table 4.1 summarizes the in vivo experiments performed in this study and the respective endpoints analyzed for each experiment. Please note that the quantified endpoints of the in vivo studies were strictly histological in nature.

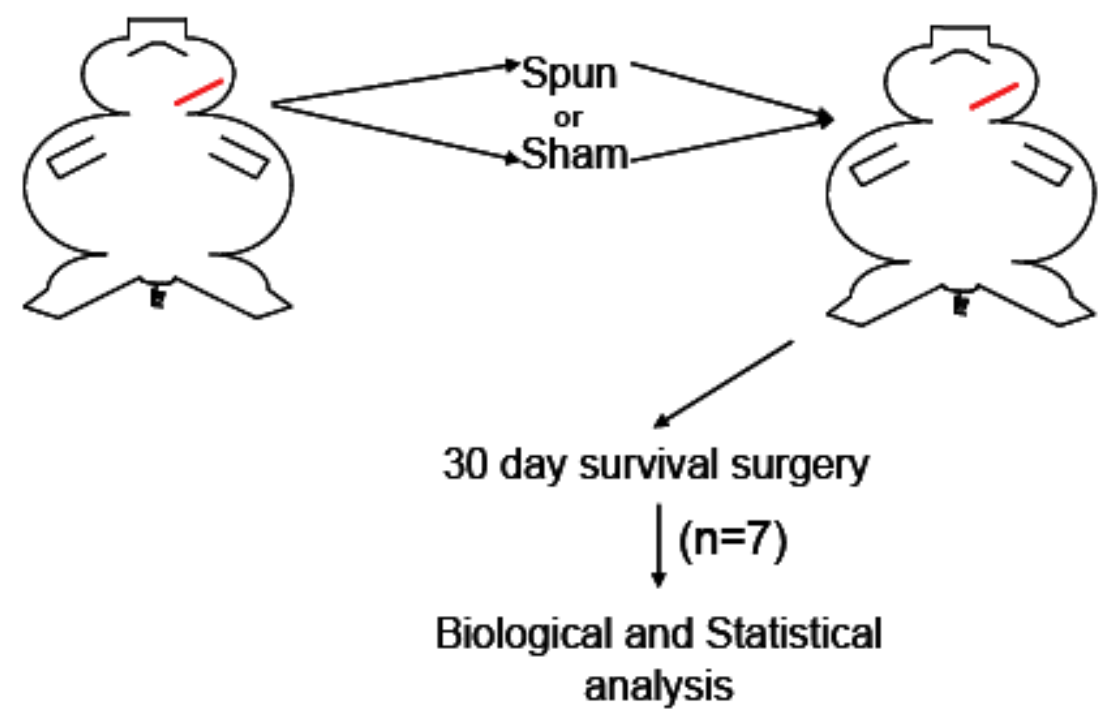

Figure 4.1 Schematic depicting the un-paired spun vs. sham AVG in vivo experiments. 
Table 4.1 Summary of all in vivo AVG experiments that were performed and the respective endpoints that were analyzed. Note that H\&E: hematoxylin and eosin; MPC: Movat's pentachrome; and SEM: scanning electron microscopy. An " $\mathrm{X}$ " indicates that the analysis was performed.

\begin{tabular}{|c|c|c|c|c|}
\hline \multicolumn{2}{|c|}{ Experiment/Duration } & \multicolumn{2}{|c|}{ Histology } & EM \\
\hline Date & condition & H\&E & TMPC & SEM \\
\hline $\begin{array}{c}12 / 4 / 2006 \\
30 \text { day survival }\end{array}$ & $\begin{array}{l}\text { sham } \\
\text { spun }\end{array}$ & & $\begin{array}{l}\bar{X} \\
x\end{array}$ & \\
\hline $\begin{array}{c}12 / 5 / 2006 \\
30 \text { day survival }\end{array}$ & $\begin{array}{l}\text { Sham } \\
\text { spun }\end{array}$ & & $\begin{array}{l}x \\
x \\
\end{array}$ & \\
\hline $\begin{array}{c}12 / 6 / 2006 \\
30 \text { day survival }\end{array}$ & $\begin{array}{l}\text { sham } \\
\text { spun }\end{array}$ & & $\begin{array}{l}\bar{X} \\
x \\
\end{array}$ & \\
\hline $\begin{array}{c}6 / 11 / 2007 \\
30 \text { day survival }\end{array}$ & $\begin{array}{l}\text { Sham } \\
\text { spun }\end{array}$ & & $\begin{array}{l}x \\
x \\
\end{array}$ & $\begin{array}{l}X \\
X\end{array}$ \\
\hline $\begin{array}{c}6 / 26 / 2007 \\
30 \text { day survival }\end{array}$ & $\begin{array}{l}\text { sham } \\
\text { spun }\end{array}$ & $\begin{array}{l}X \\
X\end{array}$ & $\begin{array}{l}X \\
x\end{array}$ & $\begin{array}{l}X \\
X\end{array}$ \\
\hline $6 / 27 / 2007$ & sham & & $\bar{x}$ & \\
\hline 30 day survival & spun & $\begin{array}{r}\text { died } \\
\text { res }\end{array}$ & $\begin{array}{l}\text { erati } \\
\text { comp }\end{array}$ & \\
\hline $6 / 28 / 20007$ & sham & & $x$ & $\mathrm{X}$ \\
\hline 30 day survival & spun & sacrifice & $\begin{array}{l}05 / 07 \\
\text { sero }\end{array}$ & severe \\
\hline
\end{tabular}

\subsubsection{Unilateral Porcine Carotid Interposition Grafting}

All pig surgeries were performed either in the Preclinical Testing facilities of the McGowan Institute for Regenerative Medicine, which has a suite of operating rooms (small and large) and extensive core support facilities, or within the animal facility on the $9^{\text {th }}$ floor of the Biomedical Science Tower. Figure 4.2 shows some pictures taken within the facilities. Animals were brought into the facility 7-10 days prior to the day of the experiment, and kept NPO 12 hours prior to surgery. Prior to surgery, animals were anesthetized with Acepromazine $(0.15 \mathrm{mg} / \mathrm{kg}$ IM) and Ketamine (15.0 mg/kg, IM combination), intubated and maintained at a surgical plane of anesthesia with Isoflurane (1-3\% in oxygen). Once each animal was clipped and prepped for 
the procedures it was moved into the surgical suite, placed on positive pressure ventilation and instrumented with monitoring equipment (ECG). Pulse oximetry and blood pressure were monitored throughout the surgical procedure. After the induction of anesthesia, aseptic surgery was performed.

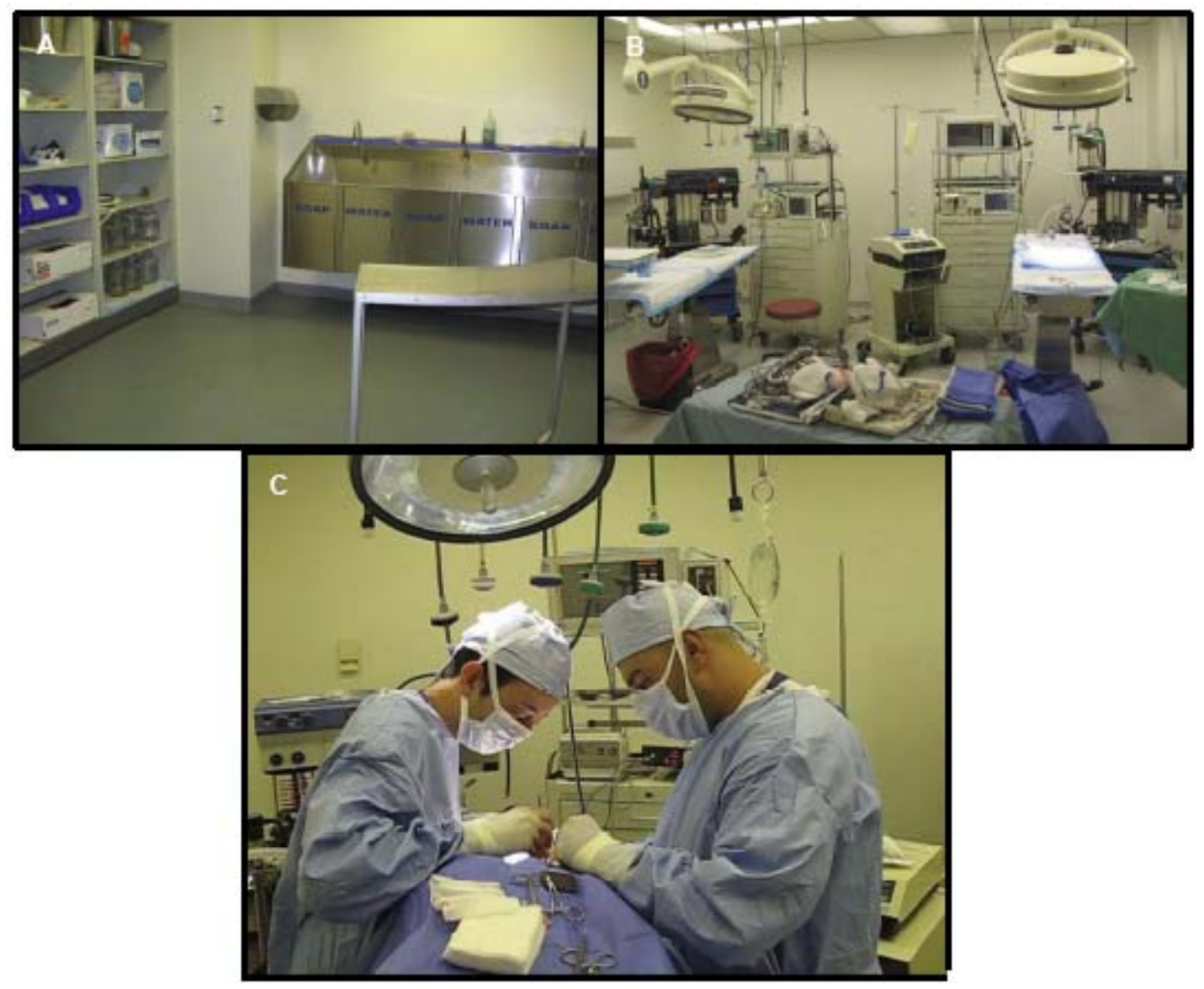

Figure 4.2 Photographs taken within the animal facility. A) Hand-washing "scrub" area. B) Operating room equipped for two surgeries. C) Alejandro Nieponice, MD (left) and I performing one of the AVG implants. 
The surgical procedure was as follows. Animals were placed in the supine position and a unilateral cervical incision was made to expose the PIJV and common carotid artery. A fresh unilateral PIJV harvest was performed on the pig according to Section 3.1.1. A picture of a cannulated PIJV within the custom "in vivo length" clamp is shown in Figure 4.3. The harvested PIJV was then either spun (as described in Section 3.1.2 and in Stankus et al. [57]) or designated as the sham control. A picture showing a cannulated PIJV segment within the electrospinning device, and within the clamp immediately post-electrospinning are shown in Figure 4.4(A) and (B), respectively. The animal was then heparinized (300 UI/Kg), and the carotid artery clamped proximally and distally using atraumatic vascular clamps. The segment between clamps was excised $(\sim 6 \mathrm{~cm})$. The vein segments were then implanted as unilateral carotid interposition grafts (end to end) using interrupted 7-0 prolene sutures. A picture of the spun AVG just prior to implantation is shown in Figure 4.5.

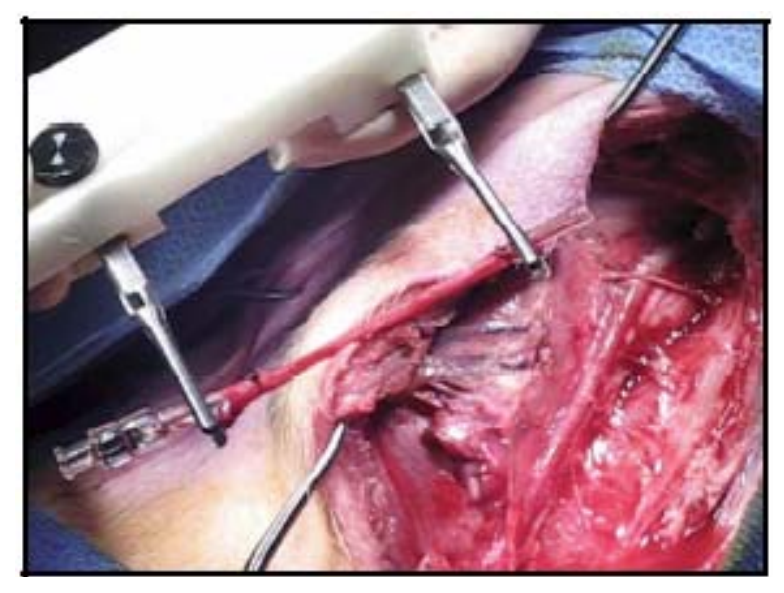

Figure 4.3 Photograph showing a cannulated PIJV segment within the custom clamp immediately upon harvest. 


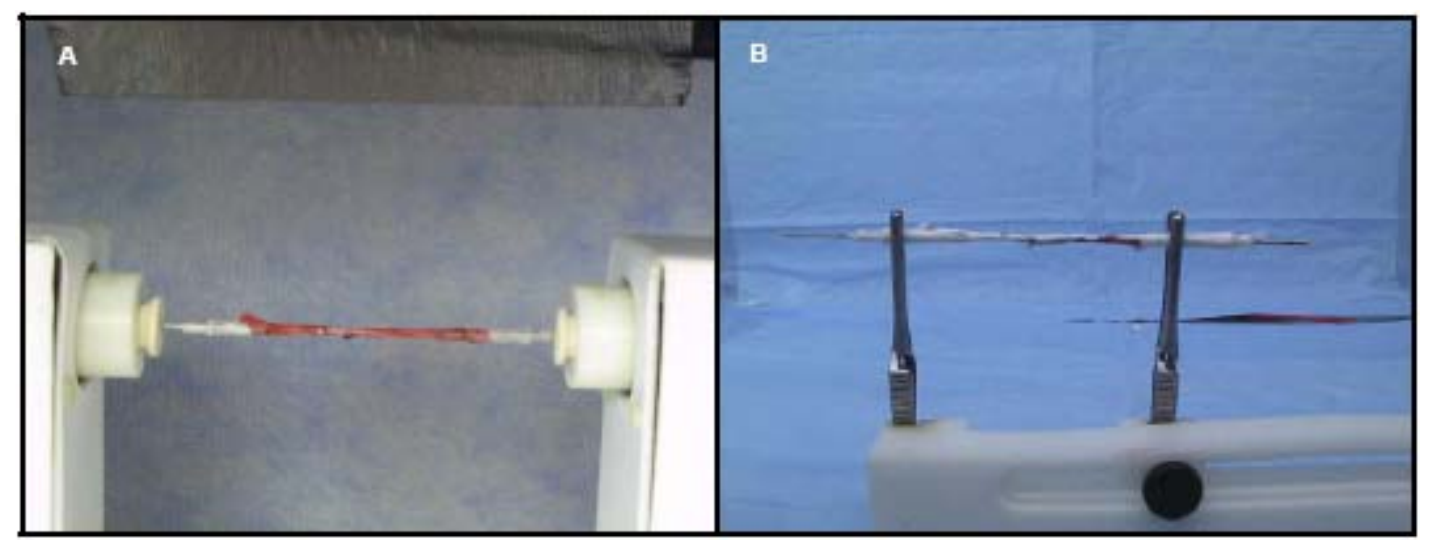

Figure 4.4 Photograph showing a cannulated PIJV segment within (A) the electrospinning device and (B) within the custom clamp immediately post-electrospinning.

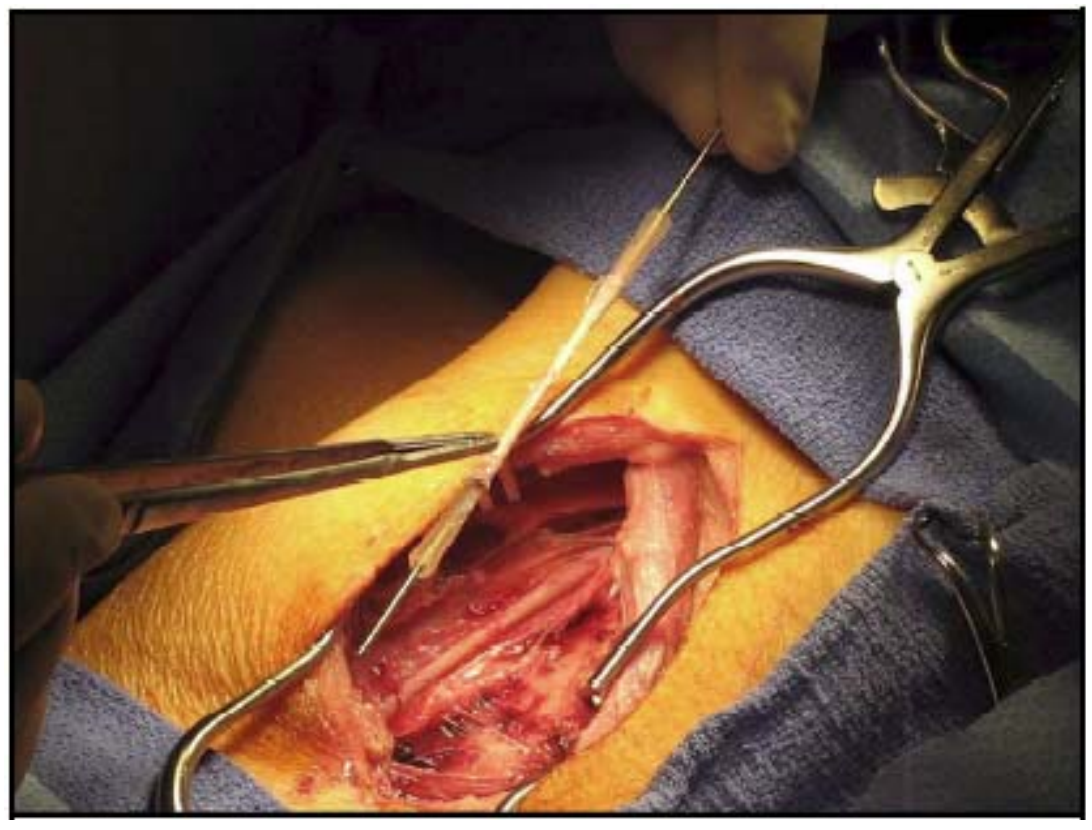

Figure 4.5 Photograph showing a spun cannulated PIJV segment just prior to being implanted as an AVG. 
Post-operatively, animals were recovered and housed in the MIRM intensive care unit. Following the surgical procedure and cessation of inhalation anesthesia, the animals were extubated when it exhibited a swallowing reflex and the protective cough reflexes were functional. The animals were continually monitored for 24 hours, and the following parameters were recorded every hour: pulse rate, strength of pulse, capillary refill time, respiratory rate, urinary output, and defecation. Body temperature was determined and recorded every 2 hours. The animal was kept warm and dry to prevent hypothermia. Buprenorphine hydrochloride $(0.005-0.01 \mathrm{mg} / \mathrm{kg}, \mathrm{IM}, \mathrm{q} 12 \mathrm{~h})$ was administered at regular intervals for 4 days for pain and continued to be administered for pain management if signs of pain were exhibited. Acute pain in animals is expressed by guarding, vocalization, mutilation, restlessness, recumbency for an unusual length of time, depression (reluctance to move or difficulty in rising), or abnormal appearance (head down, tucked abdomen, hunched). Animals exhibiting any of these behaviors were treated with pain medication. Skin staples/sutures were removed 10 days post-op. All animals were monitored daily by a trained staff of veterinarians, registered veterinary technicians, and animal care personnel.

An anti-coagulation regimen was used to battle acute AVG failure via thrombosis. Oral doses of aspirin ( $325 \mathrm{mg} /$ day) and Plavix ( $75 \mathrm{mg} /$ day) were both started 3 days pre-operatively. The aspirin was administered daily for the entire 30 day post-operative period, and Plavix was administered daily for only 14 days post-operatively.

After a 30-day survival time (or upon observing irreversible complications), the animals were euthanized. The pigs were deeply anesthetized with Acepromazine $(0.15 \mathrm{mg} / \mathrm{kg} \mathrm{IM})$ and Ketamine $(30.0 \mathrm{mg} / \mathrm{kg}$, IM combination), then euthanized by injection of an overdose of intravenous potassium chloride to induce cardiac arrest. Vital signs were monitored to effect. 


\subsubsection{Fluoroscopic Angiography}

After euthanasia and just prior to graft explant, fluoroscopic angiography was performed to assess graft patency. The carotid artery was clamped approximately $3 \mathrm{~cm}$ upstream of the proximal graft anastomosis, and contrast medium was infused into the carotid artery between the clamp and proximal anastomosis. Angiograms were recorded (Model OEC 9800 Plus, General Electric Inc.) to verify flow through the entire graft segment. If flow could not be established through a graft (i.e., due to occlusion), angiography was not performed.

\subsubsection{Post-explant Tissue Processing}

The grafts (including $1 \mathrm{~cm}$ of carotid artery proximal and distal to graft) were extracted and cut in half so that each half included one anasotomosis and a segment from the mid-graft region. One half of the tissue was immediately fixed in $4 \%$ paraformaldehyde and analyzed histologically as described below. The other half of the tissue was fixed in ultrapure $2.5 \%$ gluteraldehyde for SEM analysis as described in Section 3.1.7.1.

\subsubsection{Histological Measurements of IH}

Morphometric analysis was performed on sections from the central region of all of the explanted AVGs, except from the two animals that expired prematurely. Five $5 \mu \mathrm{m}$ tissue sections from each AVG segment were stained with MPC as described in protocol E.4 in Appendix E. The intimal thickness was defined as the thickness measured from the internal elastic lamina to the vessel lumen, and the medial thickness was defined as the thickness measured from the external 
elastic lamina to the internal elastic lamina. Intimal $\left(T_{I}\right)$ and medial $\left(T_{M}\right)$ thicknesses were measured from the MPC images, and the intimal-to-medial $\left(\mathrm{T}_{I} / \mathrm{T}_{\mathrm{M}}\right)$ thickness ratios were calculated from these measurements. Measurements were made from 4 fields of view per section yielding a total of 20 measurements that were then averaged to yield a single value for each AVG segment.

\subsubsection{Scanning Electron Microscopy}

The same procedure as described in Section 3.1.7.1 was used to process and image the explanted AVGs from the in vivo experiments. The SEM sample was filleted longitudinally so that the luminal surface was visible and both the anastomotic interface and mid-graft region could be imaged. A low magnification SEM image was taken for some (see Table 4.1) of the AVGs post explant.

\subsubsection{Statistics}

An unpaired student's t-test was performed on the intimal-to-medial thickness ratio data, and $\mathrm{P}<0.05$ was considered statistically significant. Unless otherwise indicated, data are presented as mean \pm standard error of the mean. 


\subsection{RESULTS}

The adventitial polymer wrap had an immediately apparent effect of maintaining the AVG at a diameter consistent with that for the native vein (see Figure 4.6) under arterial pressure. Images from the implanted AVGs, immediately upon re-establishing flow, for the last 4 experiments are provided in Figure G. 1 to Figure G. 4 in Appendix G. In addition, the wrapped AVGs exhibited pulsatile radial excursions (i.e., compliance) similar to the native carotid artery, whereas the distended un-wrapped AVG appeared to be a rigid tube with no detectable pulsations. That is, upon establishing flow through the control grafts, it was observed that unlike the native carotid arteries and spun veins, the sham control veins did not change in diameter in response to the pulsatile pressure. 


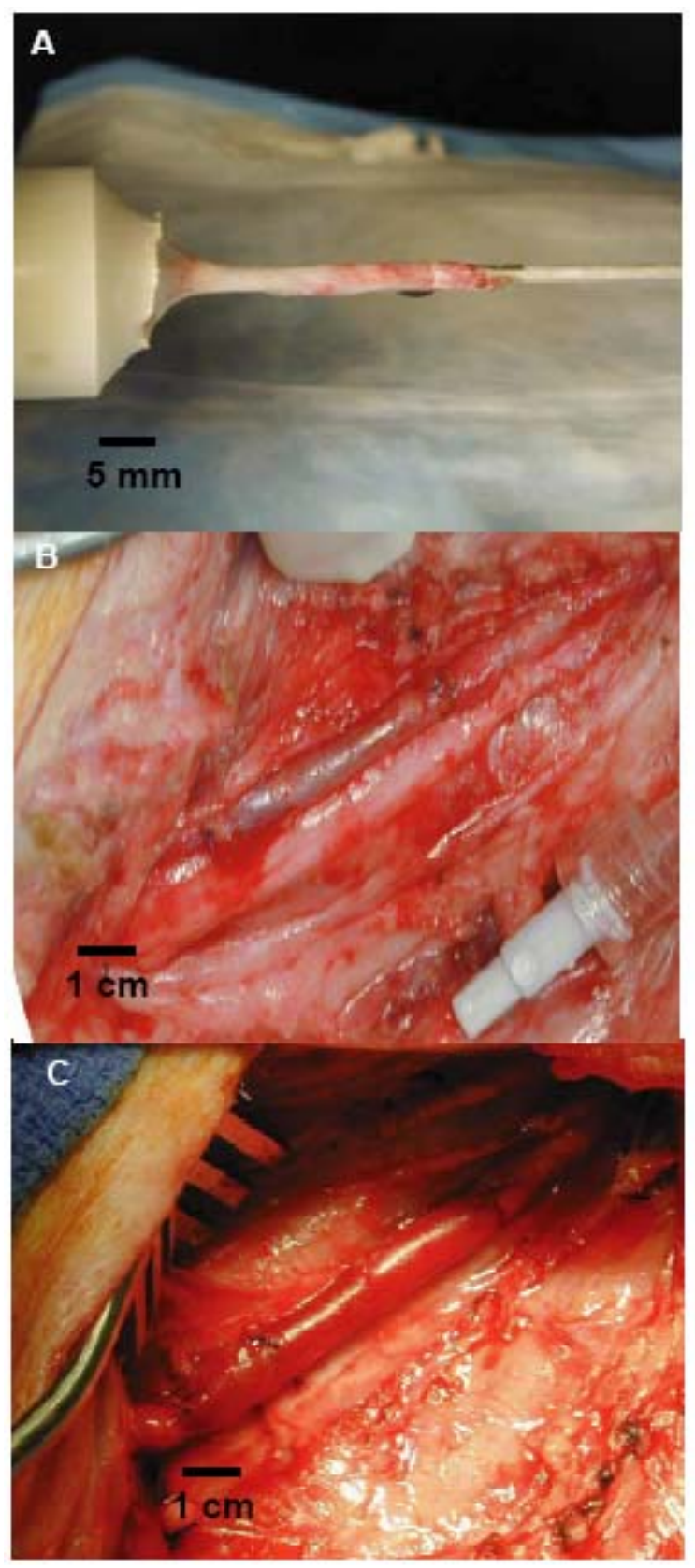

Figure 4.6 A) Wrapped PIJV segment during the electrospinning process. B) Wrapped PIJV implanted as a carotid interposition graft. C) Unwrapped PIJV graft. Note that the wrapped PIJV (B) does not expand under arterial pressure as does the unwrapped vein $(\mathrm{C})$. 
Out of the 7 in vivo experiments that were performed, only 1 experiment (spun: $\mathrm{N}=1$; sham: $\mathrm{N}=1$ ) was completely successful. That is, the AVGs from both the spun and sham pigs were $100 \%$ patent after 30 days. Angiography images of these AVGs can be seen in Figure 4.7. The rest of the experiments were deemed unsuccessful due to one of 3 reasons: 1) total occlusion of either the spun $(\mathrm{N}=1)$ or sham $(\mathrm{N}=1)$ AVGs due to IH or thrombosis; 2) post-operative (respiratory related) complications leading to the death of an animal in the spun ( $\mathrm{N}=1)$ group; and 3) infection resulting in the need to euthanize an animal in the spun ( $\mathrm{N}=1)$ group after 1 week post-op. Two of the AVGs (1 sham and 1 spun) were $100 \%$ patent, and 8 of the AVGs (5 sham and 3 spun) were only partially occluded. We performed morphometric measurements to assess IH development for comparison between the two groups (sham, $\mathrm{N}=6$; spun, $\mathrm{N}=4$ ). The AVGs from the two animals in the spun group that died prematurely were not included in the morphometric analysis. Representative images of MPC staining that were used in the morphometric analysis are shown in Figure 4.8. The other MPC images (see Table 4.1) are provided in Figure G. 5 to Figure G. 10 in Appendix G. The quantified results can be seen in Figure 4.9. Though not quite statistically significant, there was a trend $(\mathrm{P}=0.086)$ of the intimalto-medial thickness ratios for the spun group to be less than that for the sham controls. The Microsoft Excel student's t-test output table for the comparison shown in Figure 4.9, is provided in Table G. 1 in Appendix G. 

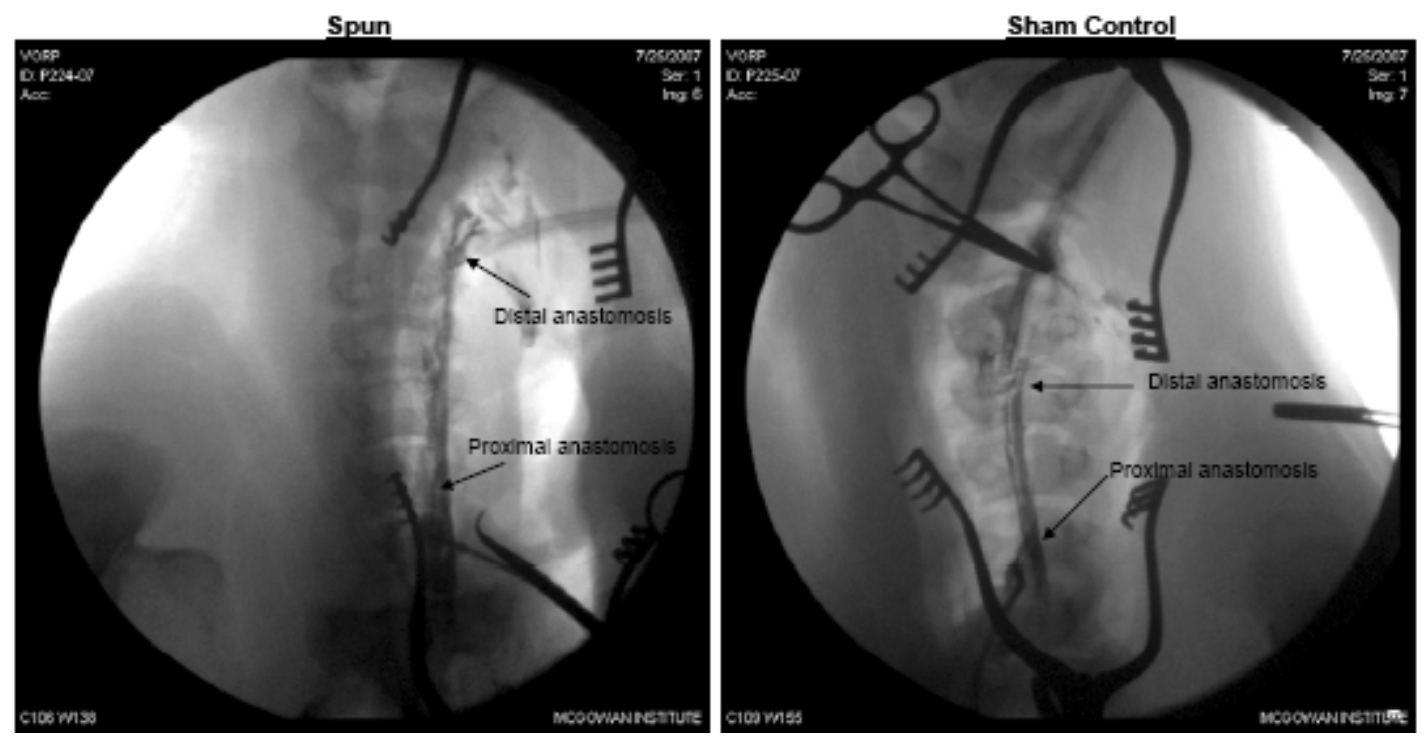

Figure 4.7 Fluoroscopic angiography images from both spun and sham AVGs.

After the first three in vivo experiments were performed, it was decided to include SEM analysis in the following four experiments. SEM images were taken of the AVGs from one completely successful experiment (Figure 4.10(A) and (B)) as well as from another experiment where the AVGs were stenotic, but not completely occluded (Figure 4.10(C) and (D)). The anastomotic interface between the vein graft and artery, evidenced by the suture line, can be seen in each image. Also, a portion of the mid-AVG region can be seen in each SEM image. The other SEM images (see Table 4.1) are provided in Figure G. 11 to Figure G. 15 in Appendix G. Please note that one sham AVG was not imaged via SEM because it was too difficult to visualize a continuous lumen. The AVGs from the two animals in the spun group that died prematurely were also not imaged via SEM. 


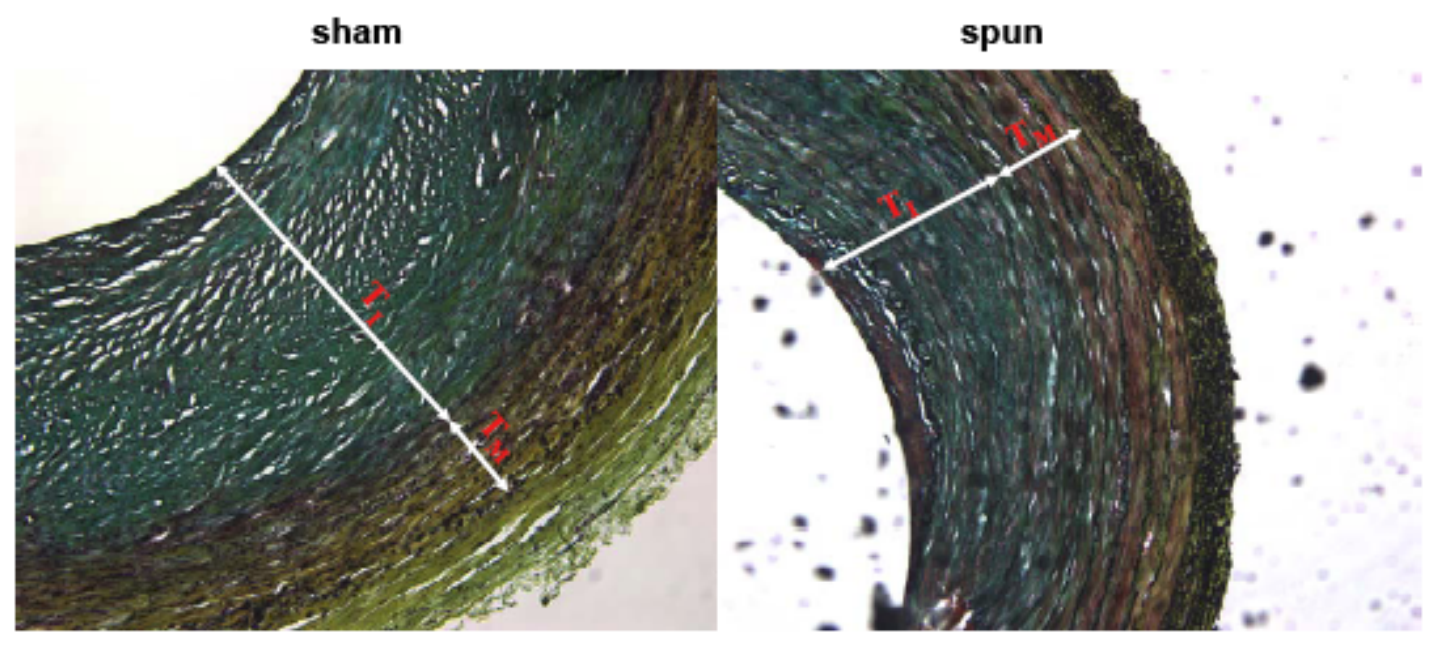

Figure 4.8 Representative MPC staining images that were used for morphometric measurements of IH. Note: $T_{I}=$ intimal thickness; $\mathrm{T}_{\mathrm{M}}=$ medial thickness.

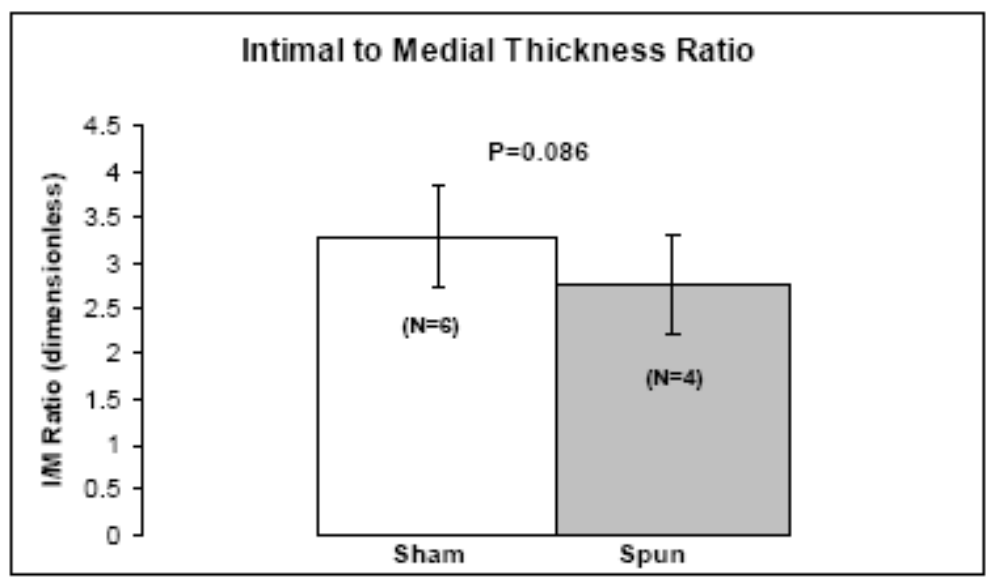

Figure 4.9 Summary of quantified results from morphometric measurements of $\mathrm{IH} . \quad \mathrm{P}<0.05$ was considered statistically significant. Note only a trend towards statistical significance was observed. 


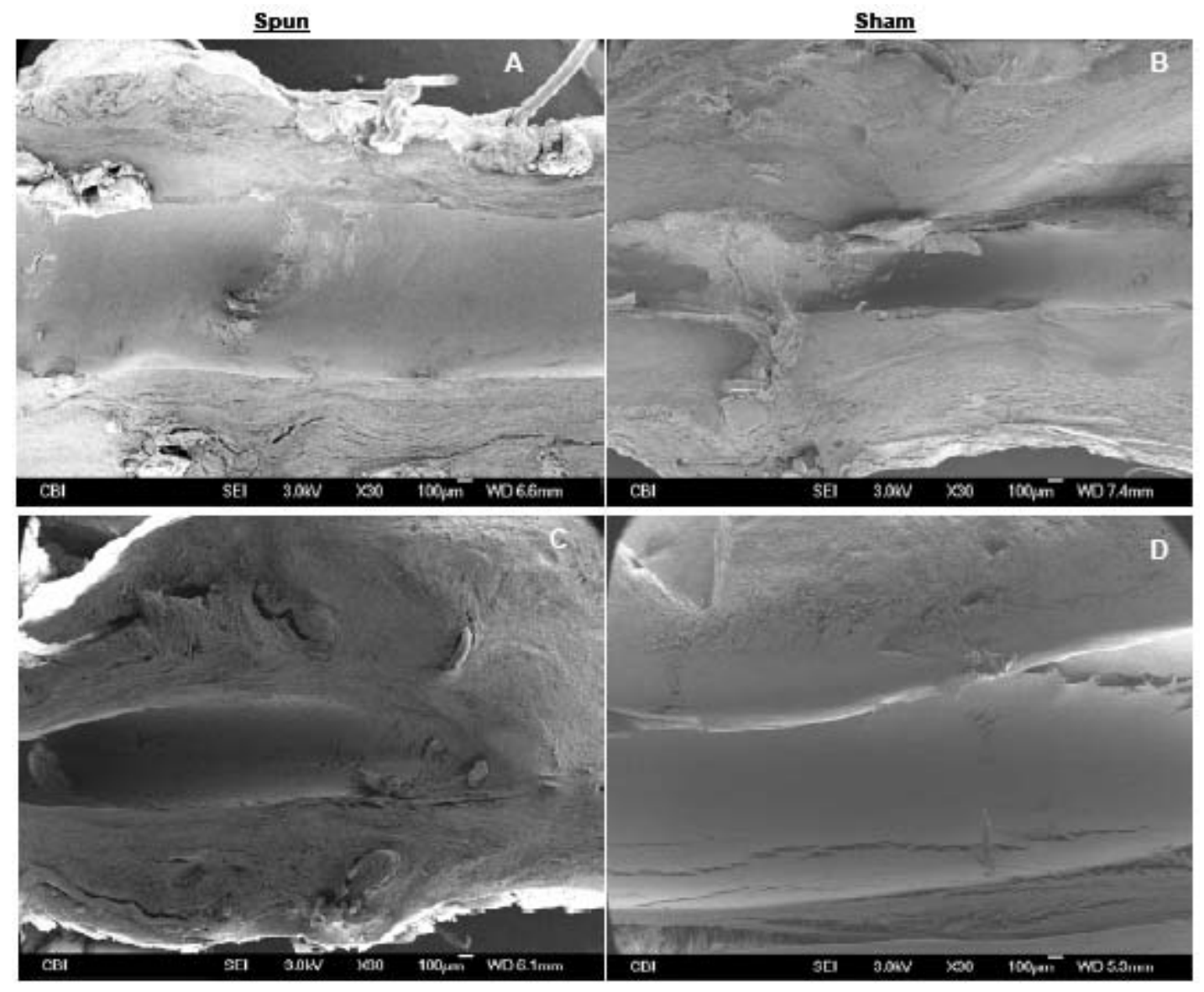

Figure 4.10 Low magnification (30x) SEM images from two in vivo experiments where the AVGs were not occluded. A and B were from an experiment where the grafts were fully patent. C and D are from an experiment where the grafts were only partially occluded. These images show the anastomotic interface between the vein graft and the carotid artery.

\subsection{DISCUSSION}

A trend towards a significant reduction in the intimal-to-medial thickness ratios in the spun vs. sham groups was observed (Figure 4.9). It is likely that this difference would become statistically significant if the number of experiments was increased. These results, as well as the 
qualitative SEM results (Figure 4.10), suggest that the electrospun biodegradable polymer wrap was beneficial in reducing IH in AVGs over sham controls. However, further investigation is necessary to determine if these effects are in fact persistently beneficial. In addition to the inherent variability associated with mechanopathobiological data, there was also variability introduced into our results by having 3 different surgeons, of varying experience, perform the surgeries. It is also true that there is a "learning curve" associated with creating anastomoses using a two layered AVG (spun group) instead of the normal one layered AVG (sham group). As with any new surgical procedure, as the comfort level of the surgeon performing the surgery increases, the success rate of the surgery will consequently increase.

Section 3.3 provides a summary of the previous studies that have showed similar results to ours by using an external sheath to reduce AVG IH $[42-44,46,47,49-52,55,240,241]$. These studies focused on the delivery of mechanical (as described in this dissertation) and biochemical support to AVGs in various animal models. The previous in vivo animal studies performed by Huynh et al. [31,42], Jeremy et al. [43], Vijayan et al. [51,52], and Liao et al. [55] showed a significant reduction in IH development within AVGs that were supported by biodegradable wraps over unwrapped controls. The in vivo results presented in this dissertation are supportive of these previous studies, however, our results did not reach statistical significance but showed a definite trend (see Figure 4.9) towards reducing AVG IH. Additional experiments would likely make our results more pronounced and could lead to a statistically significant reduction in AVG IH over sham controls.

Clinical translation of these previous approaches was not achieved due to two main limitations. Specifically, they all used either loose-fitting/biodegradable or loosefitting/biodurable sheaths. In this work, we desired to address these limitations by developing a 
means to safely "wrap" an AVG with a tight-fitting and biodegradable polymer. The AVG wrap used in this study to provide transient structural support is superior to any previous design because it has the following distinguishing characteristics: 1) it is custom-fit to each AVG;2) it has tunable biodegradation kinetics; 3) it is a peri-surgical tool; 4) it can be used to deliver structural, pharmacological, and biological support either individually or in any desired combination; and 5) it is rapidly manufactured.

As in this study, AVGs have previously been shown to develop IH within 4 weeks in a pig model $[43,48,49,51,241,294,296]$. Although we observed IH development in AVGs at 4 weeks, it is yet unclear if the beneficial effects of our polymer wrap would also be apparent at longer timepoints. Longer term experiments (upto 6 months) are required to more fully assess whether or not the spun AVGs would perform better than sham controls over a longer time period. The study by Vijayan et al., included both 1 and 6 month timepoints, and reported that the beneficial effect of external structural support to AVGs was preserved at 6 months [51]. Any reduction in the rate of development of $\mathrm{IH}$, which is essentially the current focus of this field of research, could have a considerable impact on the mortality and/or re-operation rates in patients undergoing arterial bypass grafting.

Of course there are limitations to the work presented here. The fact that the sham controls were not paired to the spun AVGs (i.e., from the same pig) provides us with less statistical power in the study. However, the unpaired experimental design that we used was deemed necessary in order to avoid post-operative complications in the animals. We felt it was safer to perform unilateral surgeries instead of bilateral so that the venous blood return from the brain would not be excessively altered. Another limitation stems from the varying experience of the surgeons who performed the procedures. It is likely that the results would be more 
statistically significant if the patency rate of the AVGs was increased. That is, if the procedures were all performed by the most experienced surgeon, the electrospun biodegradable polymer wrap may have significantly reduced IH in the AVGs over sham controls. A third limitation is that the 30-day implant duration may not have been long enough. Longer term experiments, perhaps as long as 6 months, are required to determine if the efficacy of our approach in reducing AVG IH is sustained over time.

The delivery of the aforementioned "3 modes of support" (see Section 1.4) to AVGs via a peri-adventitially placed biodegradable polymer wrap may lead to improved AVG patency which could consequently reduce both mortality and the rate of re-operation associated with arterial bypass grafting. The technique described in this study lends itself to delivery of all 3 modes of support to AVGs. Future work for this ongoing project should focus on combining the delivery of the other two modes of support (pharmacological and biological, described in Section 1.4) to the delivery of structural support. We feel this will enhance the beneficial effects with respect to IH development in AVGs.

\subsection{CONCLUSIONS}

The in vivo experiments reported in this dissertation were "proof-of-concept" in nature. That is, we desired to assess the feasibility of safely applying an adventitial biodegradable polymer wrap onto a vein segment via electrospinning and then implanting the spun vein as an AVG in a preclinical model. This goal was achieved and we feel that the reported results warrant further investigation of this approach as a treatment alternative to improve AVG patency. Based on what has previously been reported in the literature, we feel that our preliminary findings fit well 
within the context of this field of research. Our novel approach could provide a unique and effective means to safely and functionally arterialize AVGs in situ. 


\subsection{STUDY SUMMARY}

To summarize the work presented in this dissertation, a description of the results related to each Specific Aim is provided. Also, some recommendations for future directions are made.

\subsection{SUMMARY OF PERTINENT FINDINGS}

\subsubsection{Specific Aim 1}

The paired ex vivo vascular perfusion system described here has improved on any previously existing apparatus in that we were able to precisely control imposed physiologic arterial CWS and shear stress waveforms. The use of the N4SID algorithm yielded the most rigorous mathematical models of the system which were subsequently used to develop the PID controllers. This novel approach allowed us to reproduce the arterial biomechanical environment with great accuracy.

\subsubsection{Specific Aim 2}

We have shown that SMC apoptosis, proliferation, and Golgi complex expression was altered in PIJVs exposed to ART vs. VEN conditions, and that mechanical conditioning (cART conditions) 
modulated this altered protein expression. Specifically, apoptosis was increased in PIJVs exposed to ART conditions for 24 hours vs. VEN controls. This abrupt increase was attenuated in PIJVs perfused under cART conditions (both for 24 and 72 hours) vs. ART controls. Proliferation was observed to be reduced in PIJVs perfused for 24 hours under ART conditions vs. VEN controls, and this reduction was subsequently shown to be attenuated in PIJVs perfused under cART conditions (24 hours only) vs. ART controls. Finally, the expression of Golgi complex was increased in PIJVs perfused under ART conditions for 24 hours vs. VEN controls. However, only a trend towards inhibition of this increase was observed for both 24 and 72 hour cART perfused PIJVs vs. ART controls. These results are all in support of our hypothesis that a biodegradable polymer wrap can be placed peri-adventitially on AVGs and tuned to provide, in situ, the CWS profile necessary to achieve a reduced hyperplastic response, and thus warrant further investigation.

\subsubsection{Specific Aim 3}

By varying the composition, electrospinning time, and final polymer solution concentration we were able to tune the degradation rate of the wrap so as to gradually expose PIJVs to arterial levels of CWS at a desired rate (wART conditions). Several combinations were attempted until we achieved a wrap that resulted in an approximately linear increase in CWS, from venous to arterial levels, within PIJVs implanted into our ex vivo vascular perfusion system over a 24-hour period. As desired, the effect of the wrap on the acute hyperplastic response was similar to the results observed using cART conditions in Specific Aim 2. WART conditions significantly reduced the number of apoptotic cells, and inhibited the decrease in the number of proliferating cells within PIJVs vs. ART control conditions. However, only a trend towards significance was 
observed for wART conditions inhibiting the increase in the number of cells positively stained for Golgi complex. vs. ART control conditions. These results are in support of our hypothesis.

\subsubsection{Specific Aim 4}

The biodegradable electrospun polymer wrap developed in Specific Aim 3 was used to create the spun AVGs for the in vivo preclinical experiments. A trend towards a statistically significant reduction in AVG IH was observed in the spun vs. sham control group. Although the efficacy of the wrap in reducing AVG IH appeared to be favorable, additional experiments of longer timepoints must be performed to determine if the results are persistently beneficial.

\subsection{SUMMARY OF ACCOMPLISHMENTS}

The work presented in this dissertation has produced the following patent applications and manuscripts:

1. Provisional Patent Application:

- El-Kurdi MS, Hong Y, Stankus JJ, Soletti L, Wagner WR, Vorp DA, Filed January 30, 2007, "Bioerodible Wraps and Uses Therefor". Serial Number: 60/898,356.

2. Utility Patent Application:

- El-Kurdi MS, Hong Y, Stankus JJ, Soletti L, Wagner WR, Vorp DA, Filed January 30, 2008, "Bioerodible Wraps and Uses Therefor".

Serial Number: 12/022430. 
3. PCT Patent Application:

- El-Kurdi MS, Hong Y, Stankus JJ, Soletti L, Wagner WR, Vorp DA, Filed January 30, 2008, "Bioerodible Wraps and Uses Therefor".

Serial Number: PCT/US08/52408.

4. El-Kurdi MS, Vipperman JS, Vorp DA, "Proportional Integral and Derivative Control of Arterial Circumferential Wall Stress and Shear Stress Waveform Generation within an Ex vivo Vascular Perfusion System”, J Biomech Eng, In press.

5. El-Kurdi MS, Vipperman JS, Vorp DA, "Design and Subspace System Identification of an Ex vivo Vascular Perfusion System”, J Biomech Eng, Accepted 2008.

6. El-Kurdi MS, Hong Y, Stankus JJ, Soletti L, Wagner WR, Vorp DA, “Transient Elastic Support for Vein Grafts Using a Constricting Microfibrillar Polymer Wrap", Biomaterials. 2008 Aug;29(22):3213-3220.

\subsection{FUTURE DIRECTIONS}

The purpose of this work was to further our understanding of the influence of arterial biomechanical signals on the progression of AVG IH. The specific biomechanical signal that we chose to focus our attention on controlling was mid-vein CWS. There is significant evidence that the severe and abrupt increase in CWS that occurs when flow is re-established in AVGs may lead to the eventual failure of a large percentage due to IH. To counteract the likely development of AVG IH, we desired to develop a peri-surgical tool that would facilitate the in situ bioengineering of AVGs. That is, we wanted to develop a safe means that allows AVGs time to adapt to the arterial biomechanical environment. The custom-fit biodegradable polymer wrap 
that we used to gradually impose arterial levels of CWS showed promising results in reducing the level of IH development in AVGs vs. sham controls in a pre-clinical model. The delivery of mechanical support to AVGs in an effort to battle IH is but one approach under investigation by us and others. The use of electropsinning offers a wide range of possibilities for the delivery of biomechanical, biochemical, and biological support, individually or in any combination, to AVGs. The controlled delivery of both drugs and stem cells to AVGs via an adventitially placed electrospun biodegradable polymer wrap is currently under investigation by our laboratory.

The advancements made to our ex vivo vascular perfusion capabilities was the second major area of focus in this dissertation. The single-input-single-output PID controllers that were developed to individually modulate the imposed CWS and shear stress waveforms within the system were the best that could be achieved with the available hardware. The development of a multi-input-multi-output state space controller to simultaneously control CWS and shear stress would be the natural next step in the advancement of our system. This capability requires more complex hardware and is currently under investigation. 
APPENDIX A

CUSTOM BUILT EVPS COMPONENT DRAWINGS

Several of the EVPS components were custom designed and built. The machine drawings for all these components are included in this Appendix. 


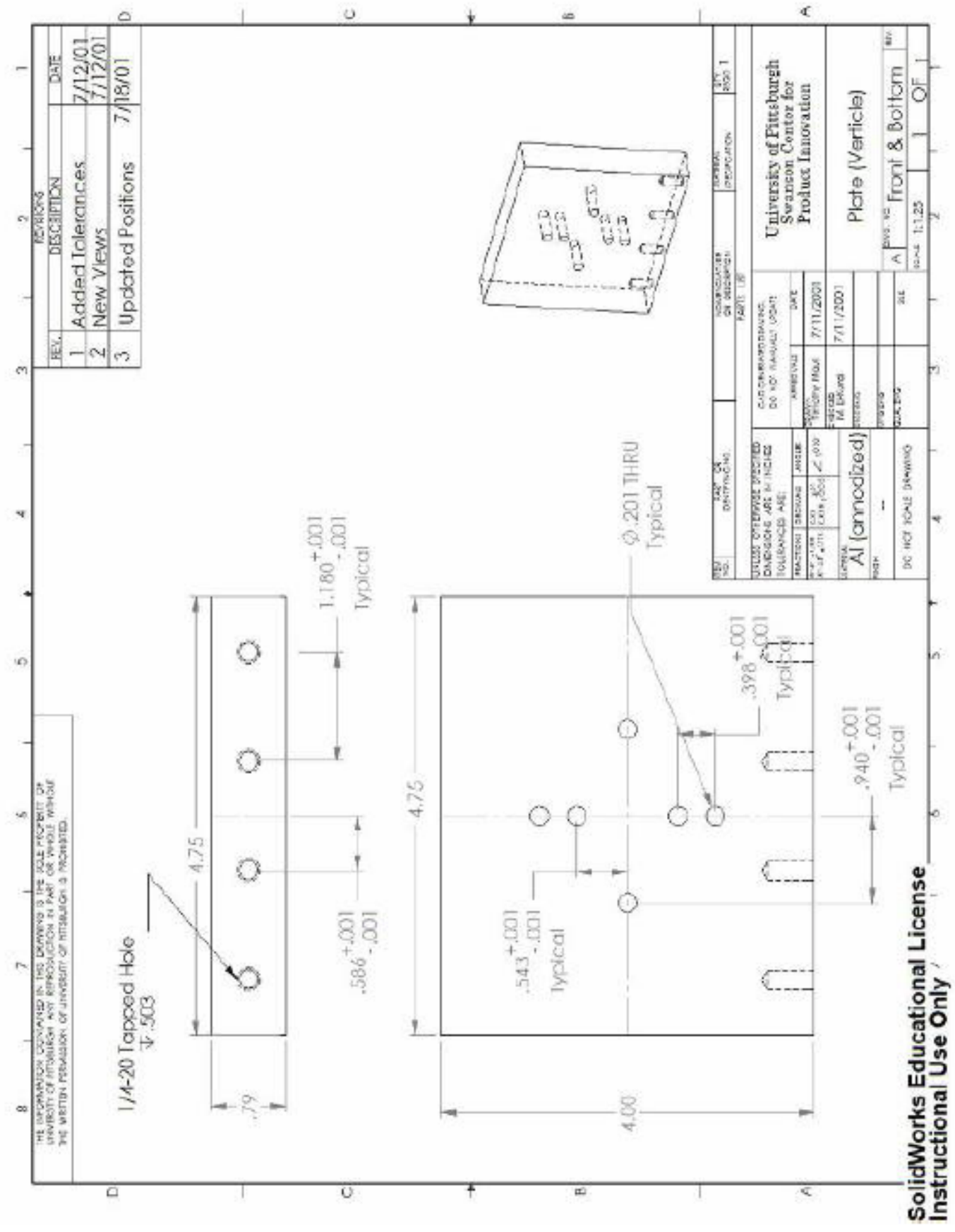

Figure A. 1 Solidworks technical drawing of vertical plate for piston/cylinder device frame. 


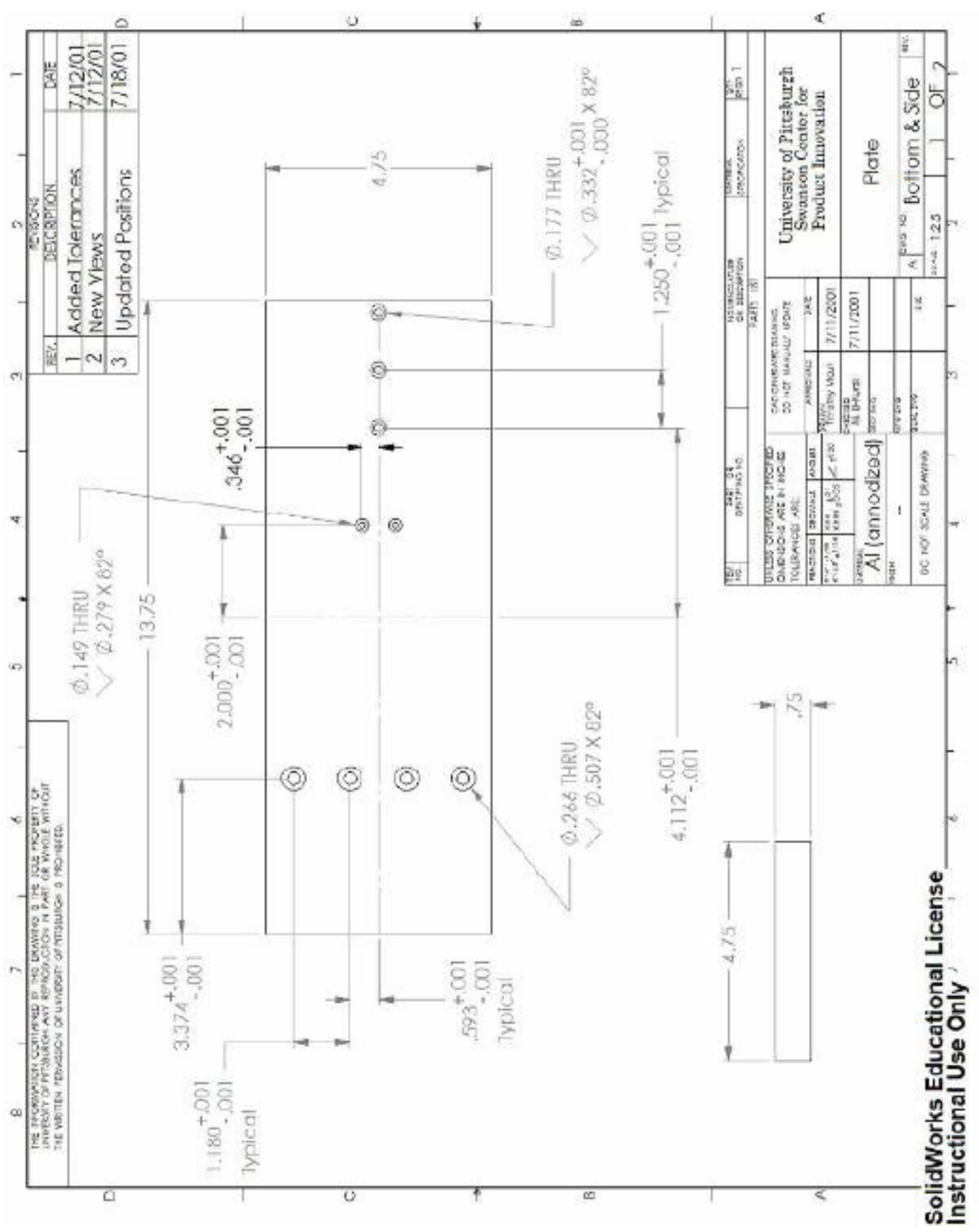

Figure A. 2 Solidworks technical drawing of horizontal plate for piston/cylinder device frame. 


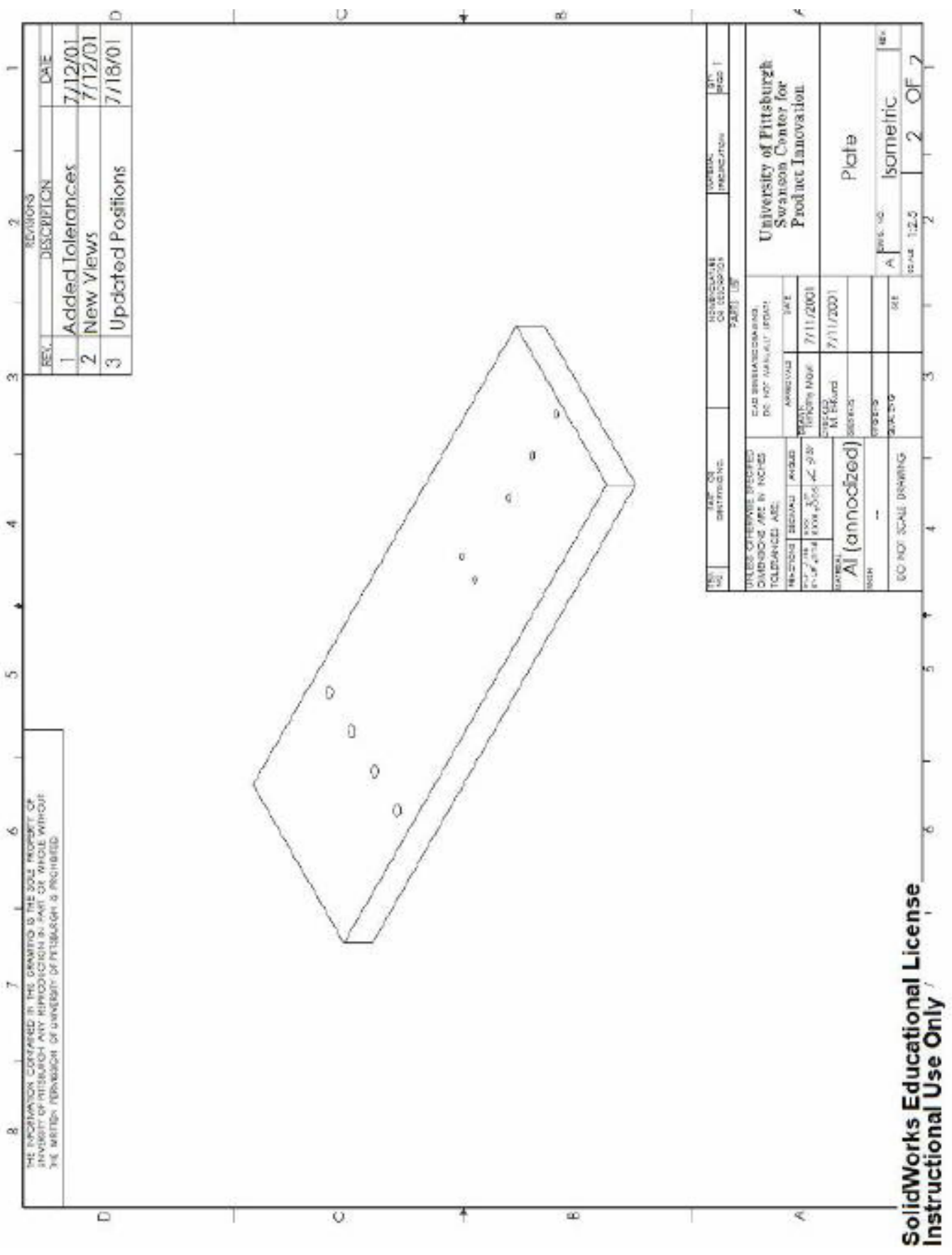

Figure A. 3 Solidworks technical drawing of isometric view of horizontal plate for piston/cylinder device frame. 


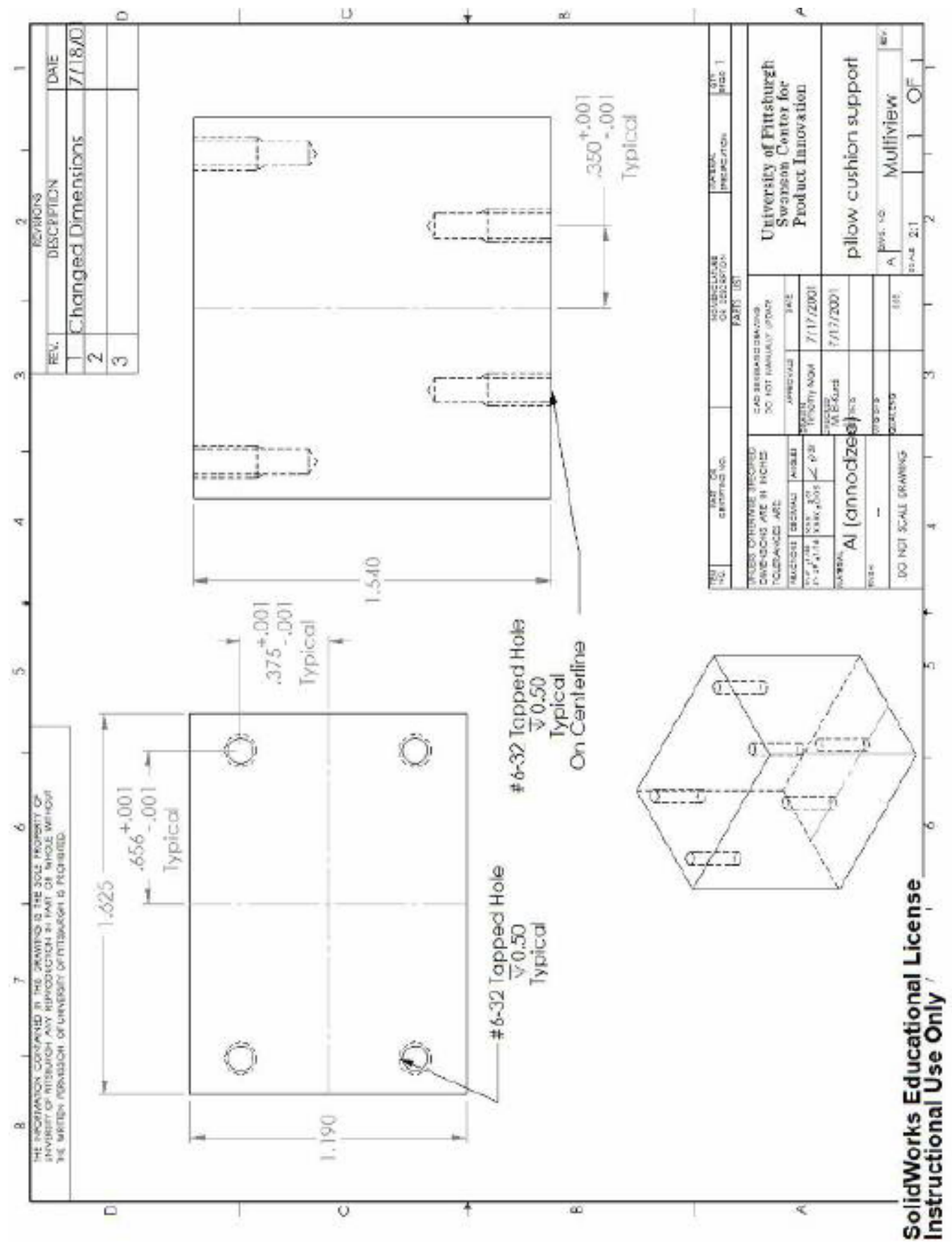

Figure A. 4 Solidworks technical drawing of pillow cushion support for piston/cylinder device frame. 


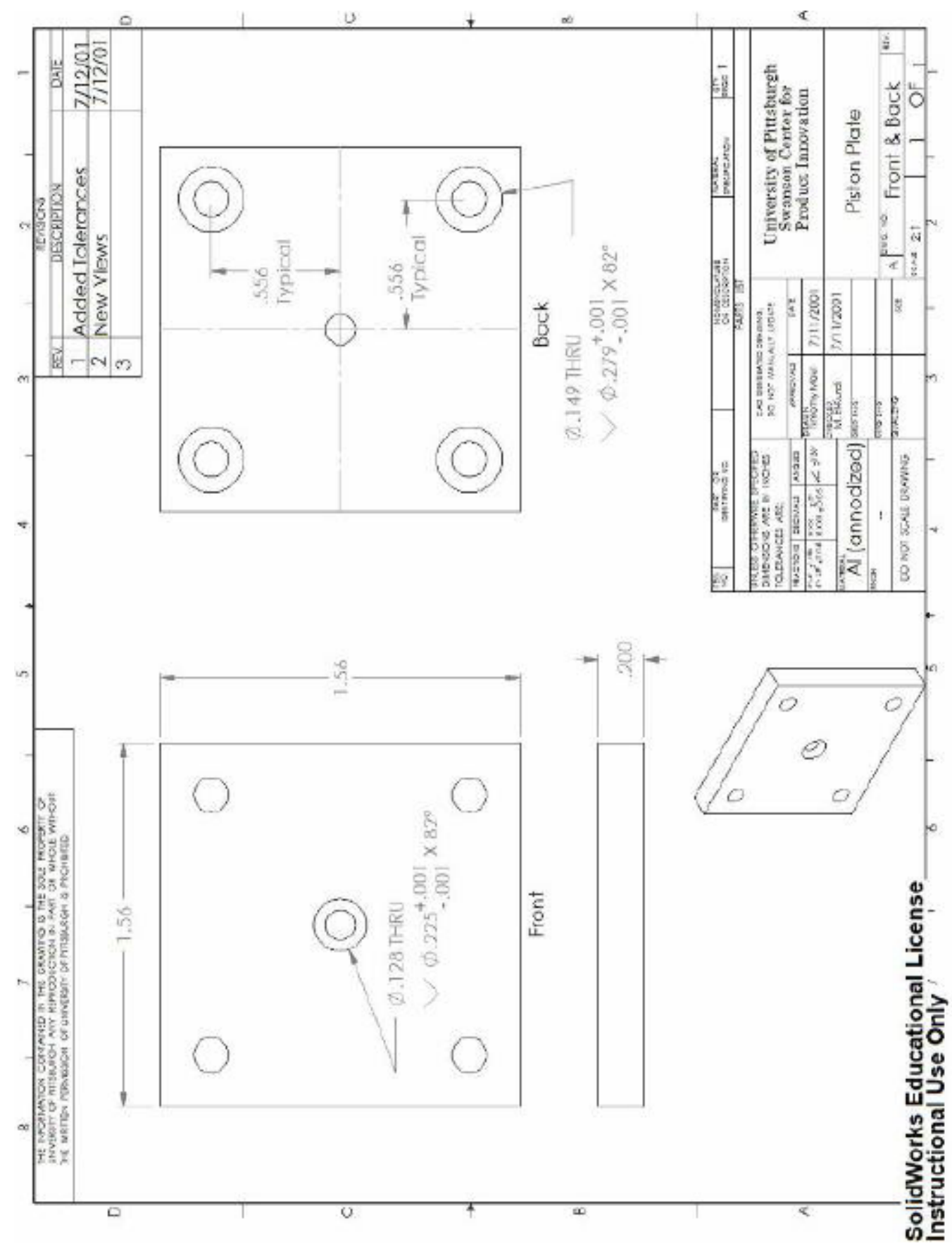

Figure A. 5 Solidworks technical drawing of piston plate for piston/cylinder device frame. 


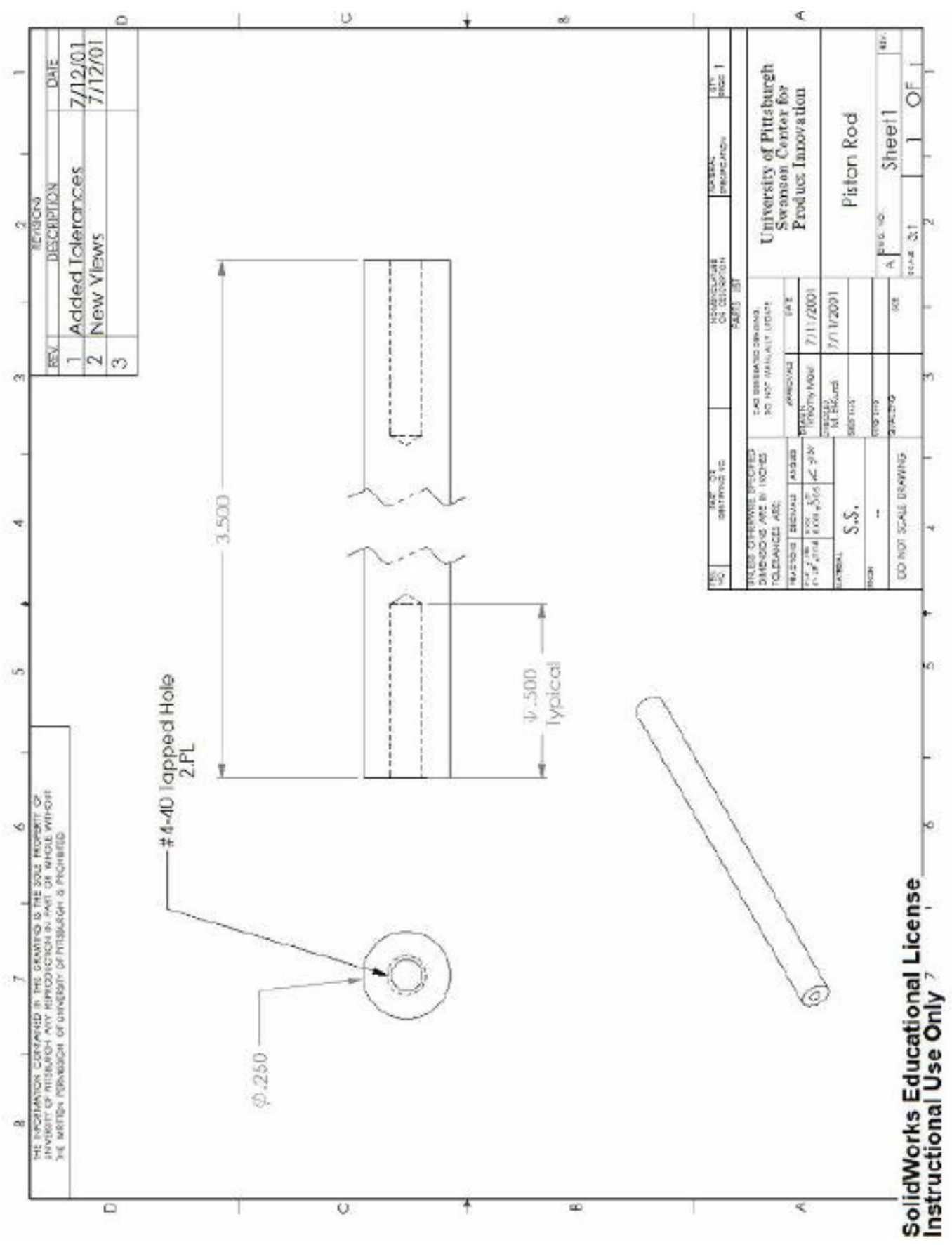

Figure A. 6 Solidworks technical drawing of piston rod for piston/cylinder device. 


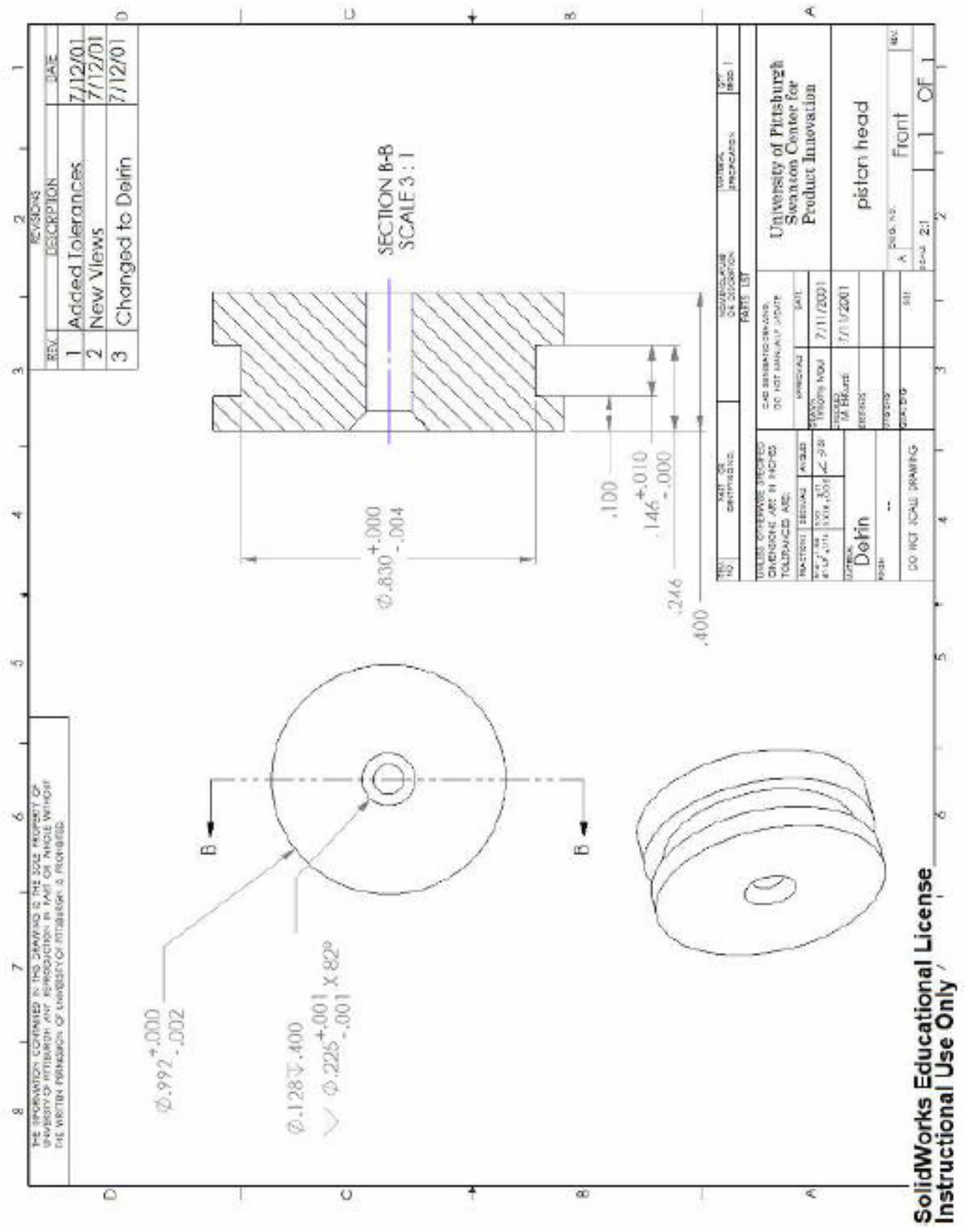

Figure A. 7 Solidworks technical drawing of piston head for piston/cylinder device. 


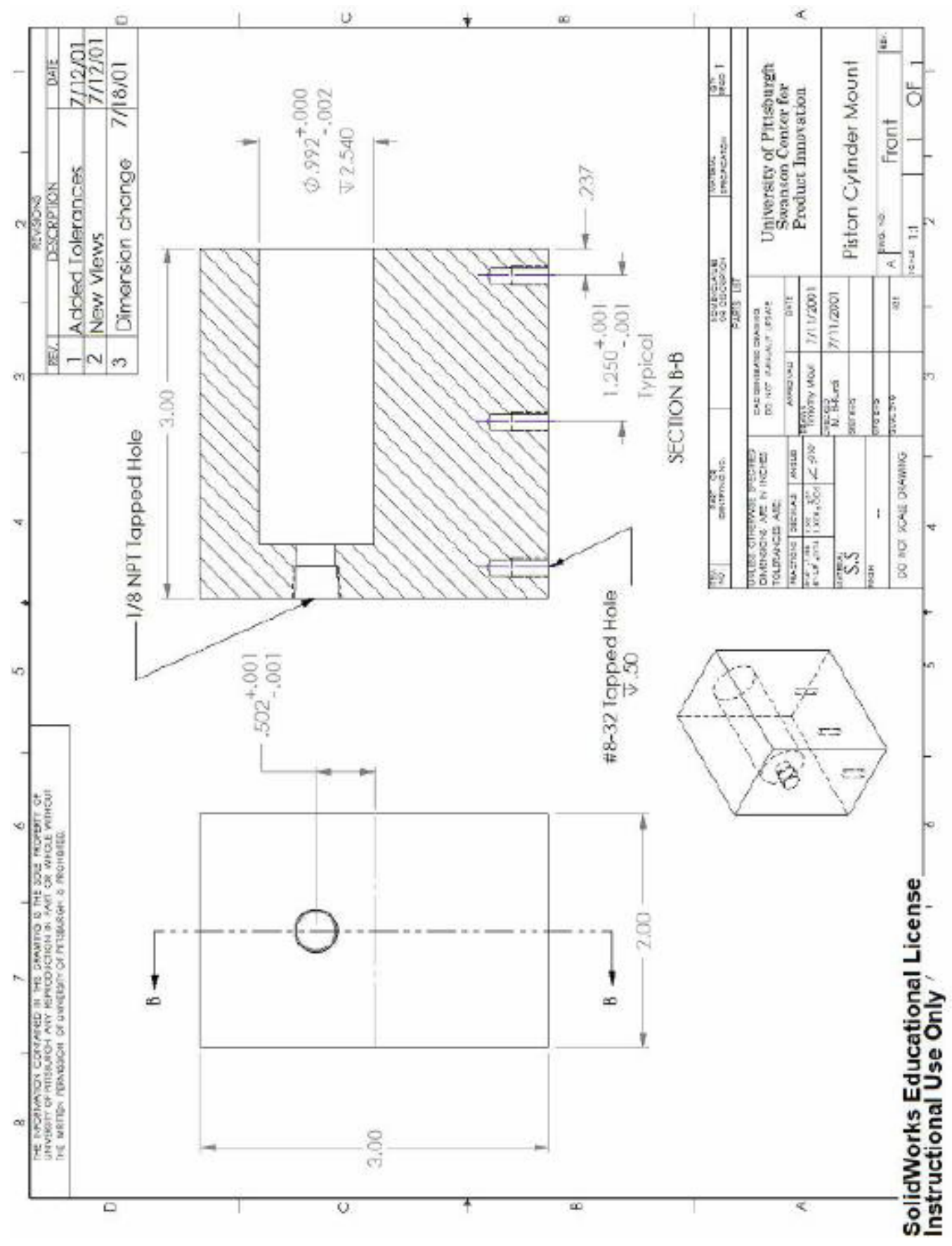

Figure A. 8 Solidworks technical drawing of the piston cylinder mount for piston/cylinder device. 


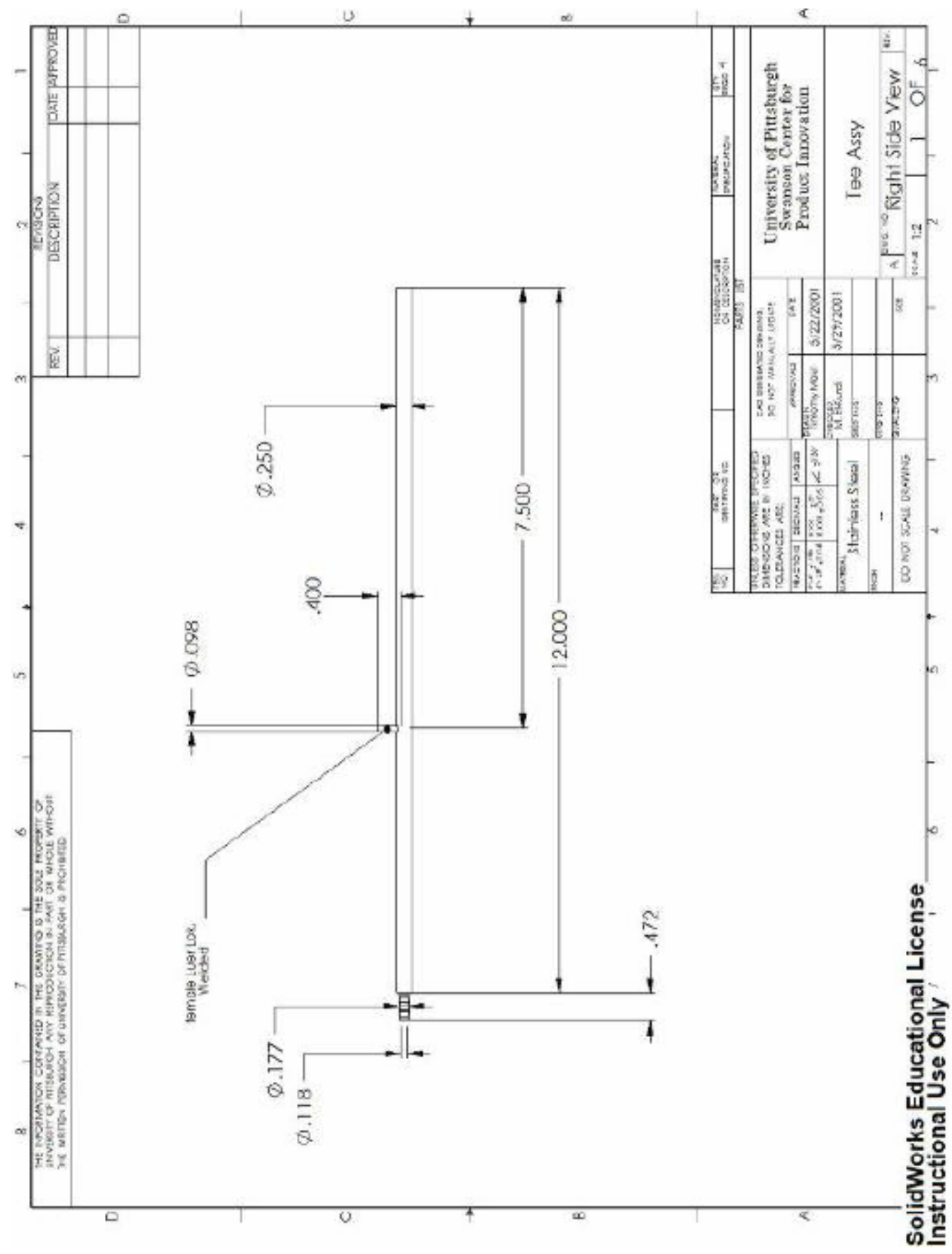

Figure A. 9 Solidworks technical drawing of right side view of vessel tee assembly. 


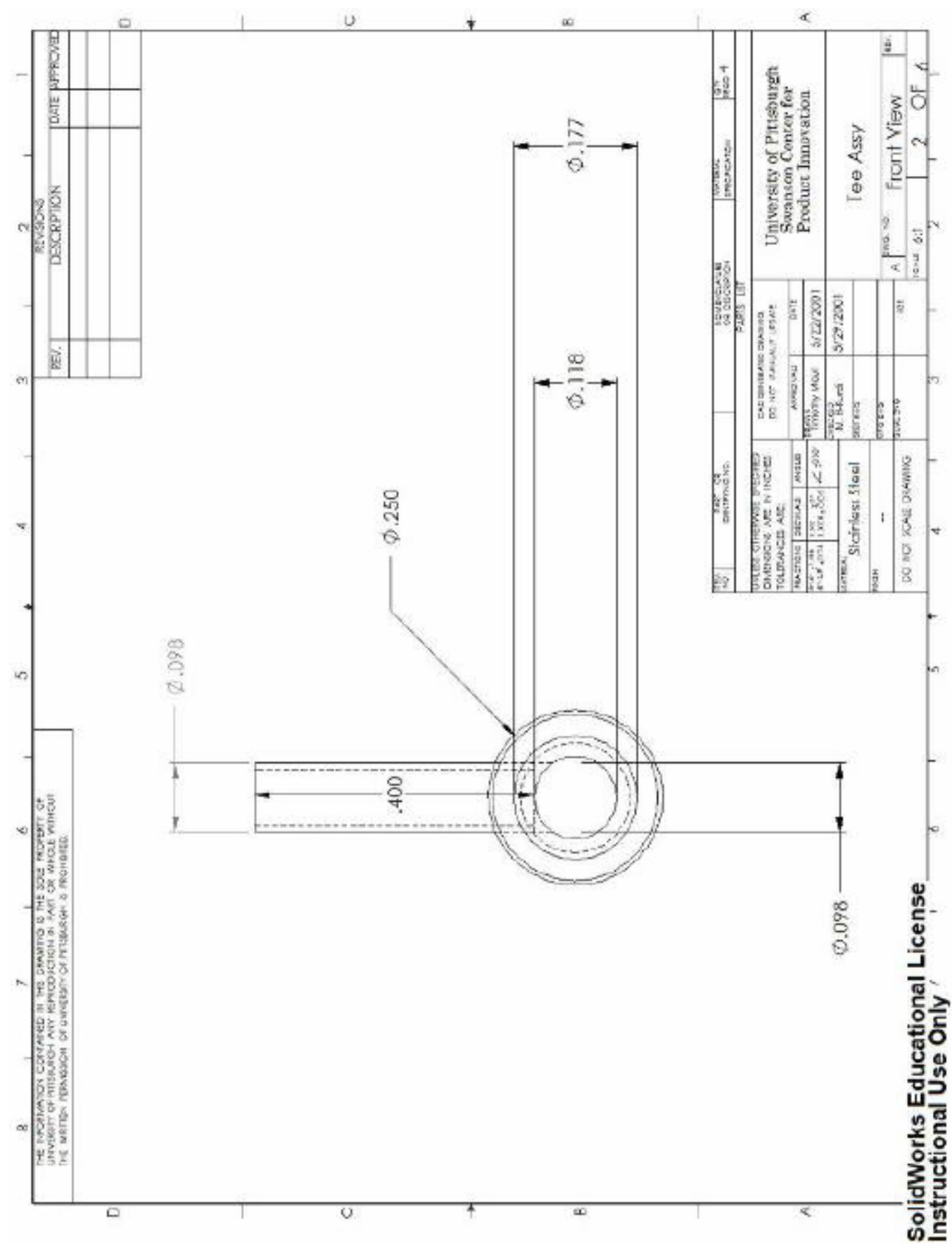

Figure A. 10 Solidworks technical drawing of top view of vessel tee assembly. 


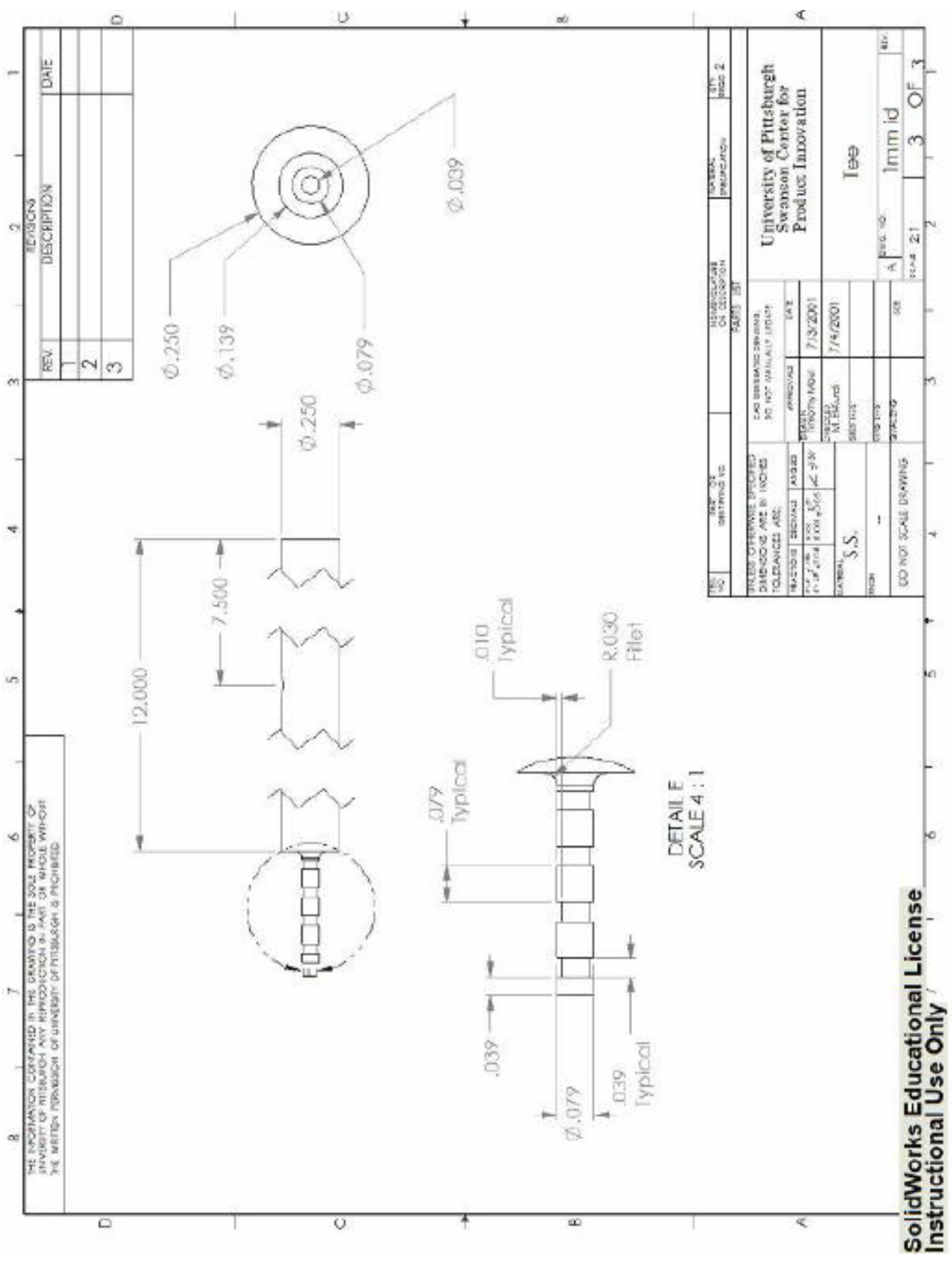

Figure A. 11 Solidworks technical drawing of vessel tee assembly. 


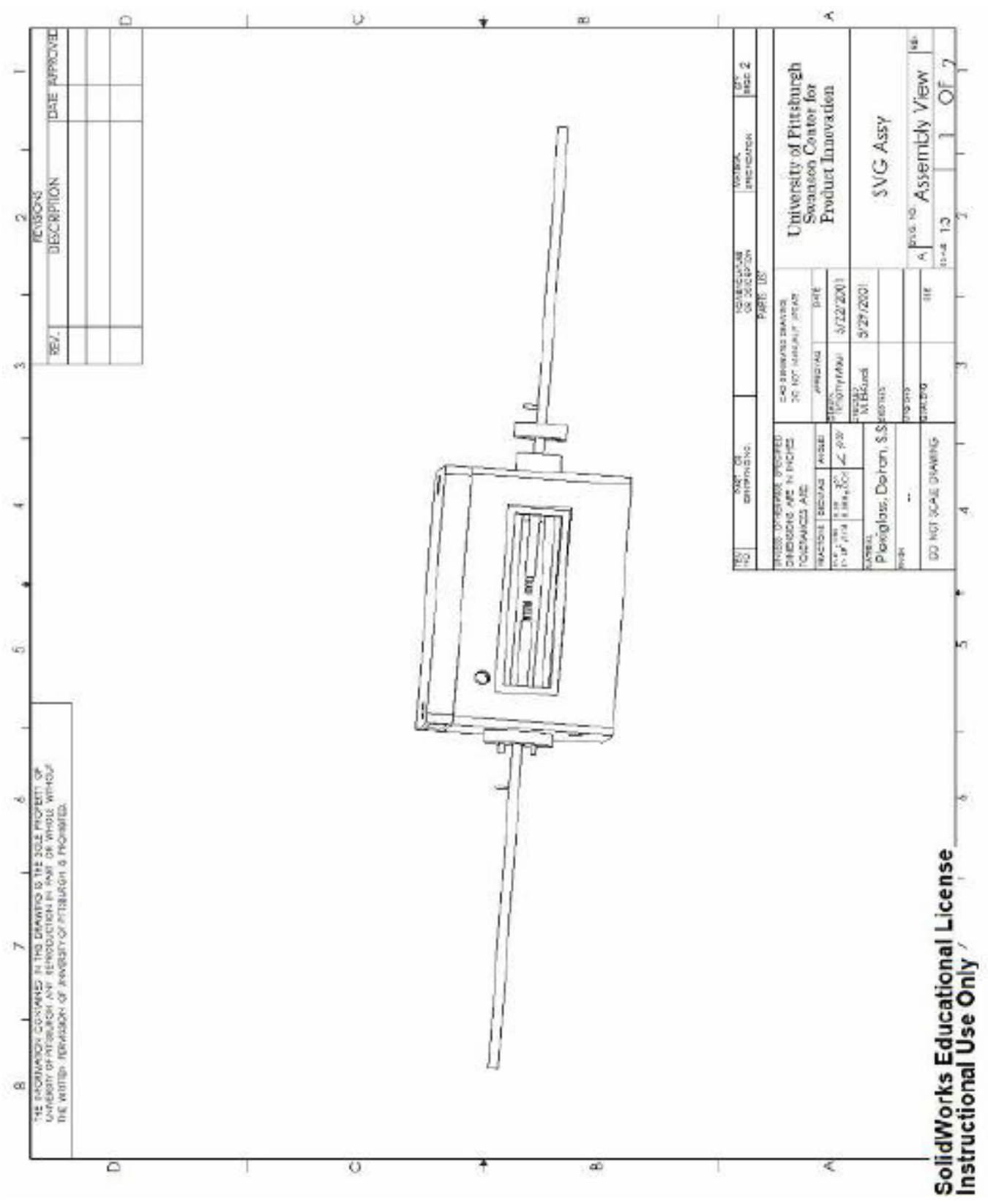

Figure A. 12 Solidworks technical drawing of isometric view of tissue housing chamber assembly. 


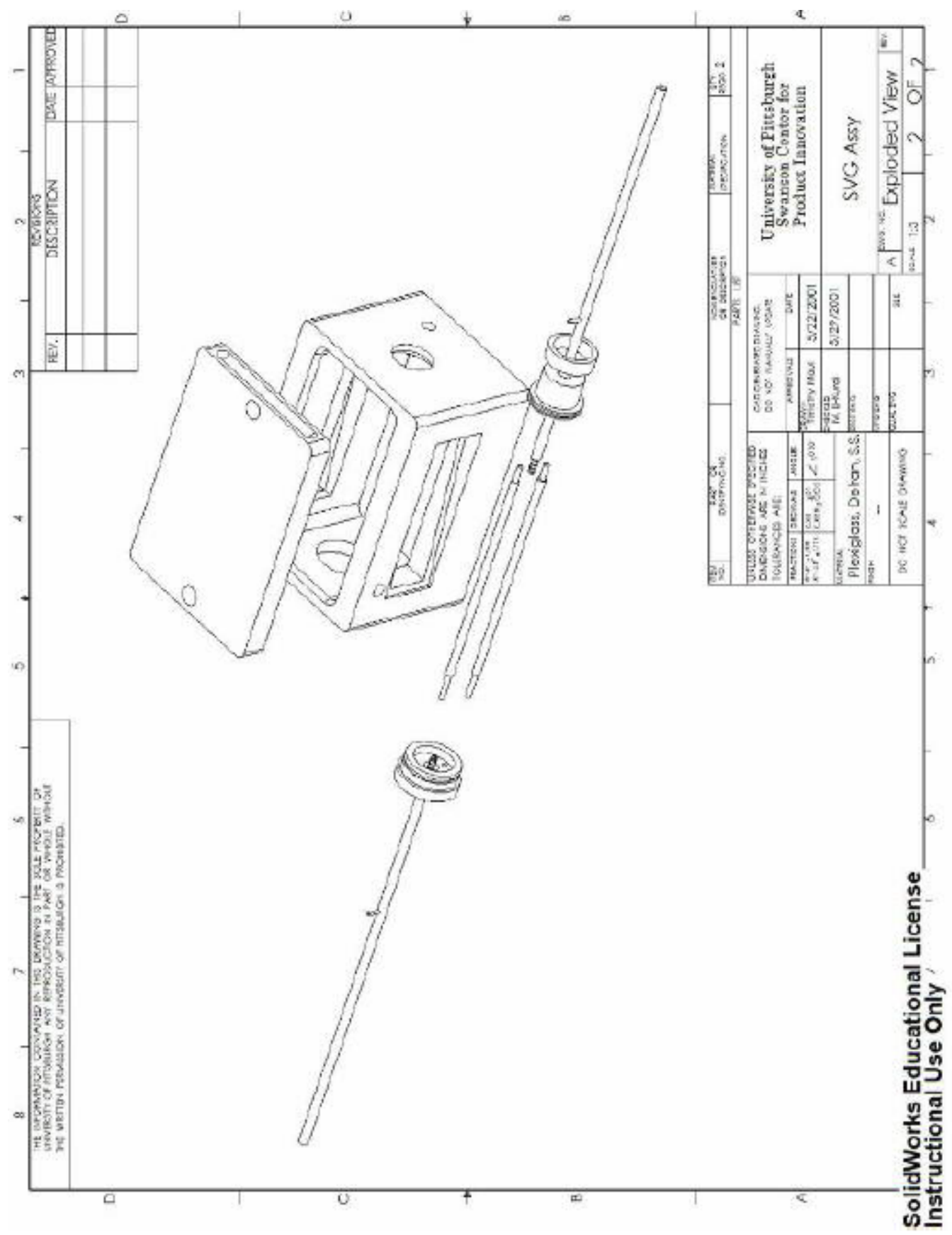

Figure A. 13 Solidworks technical drawing of exploded isometric view of tissue housing chamber assembly. 


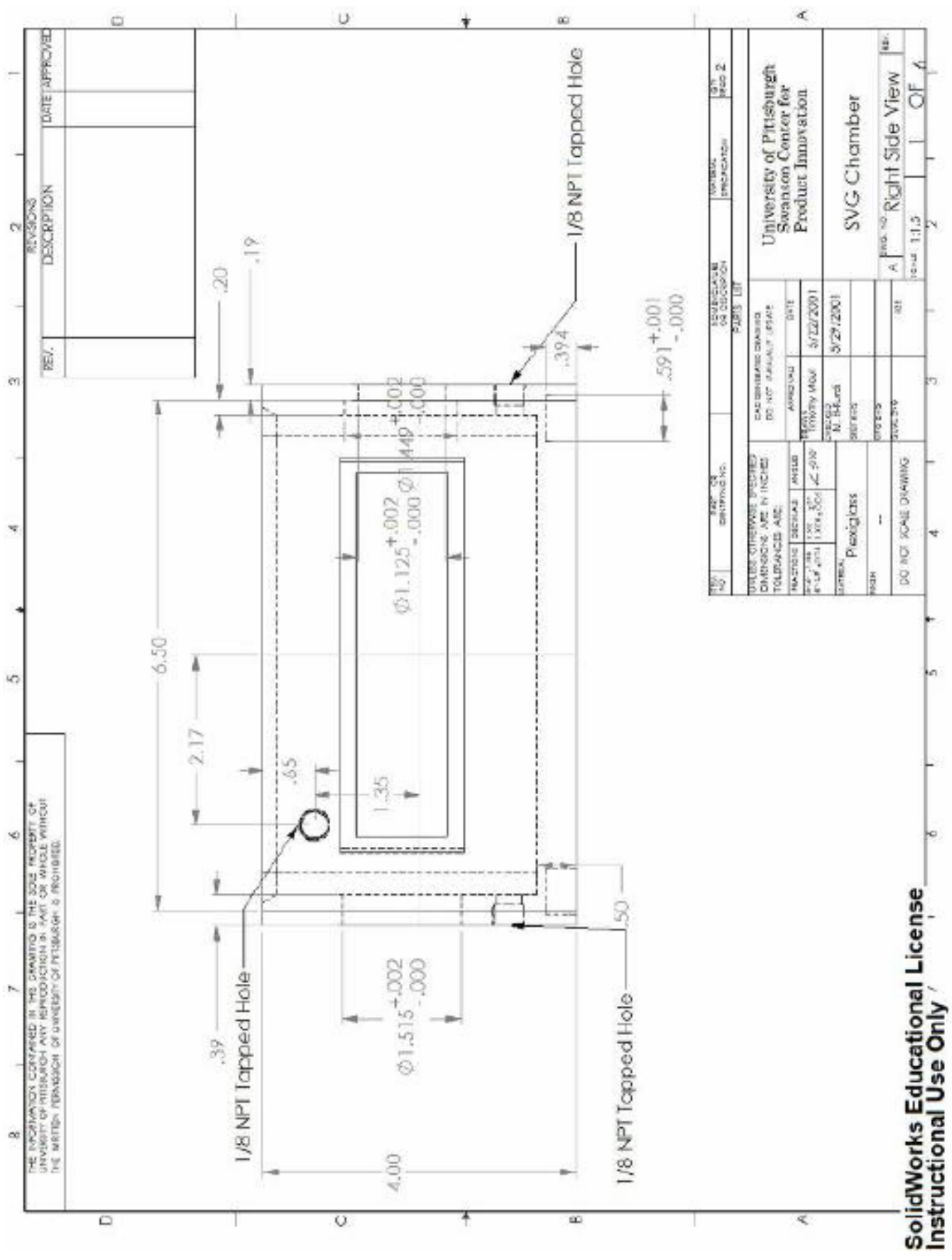

Figure A. 14 Solidworks technical drawing of right side view of tissue housing chamber assembly. 


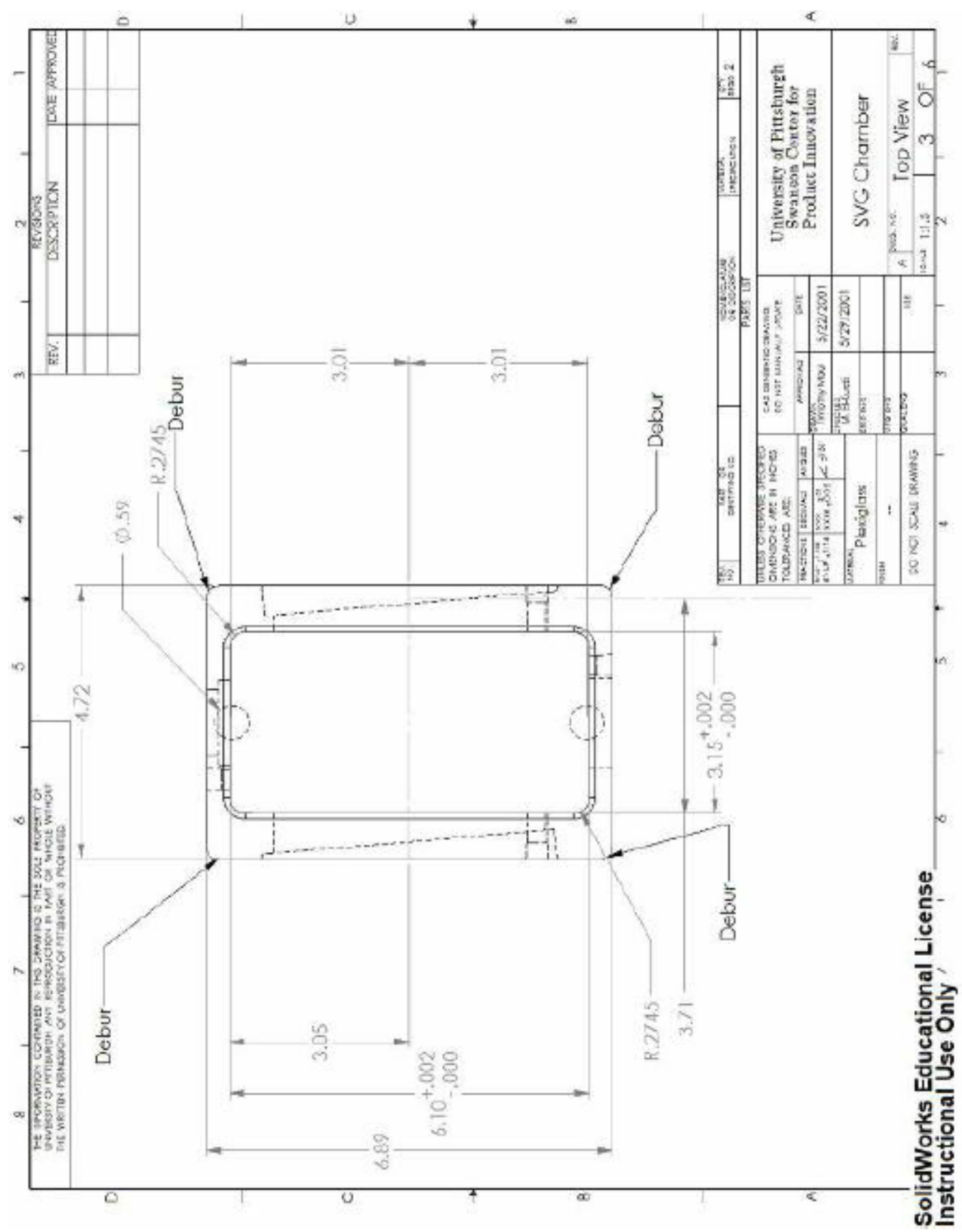

Figure A. 15 Solidworks technical drawing of top view of tissue housing chamber assembly. 


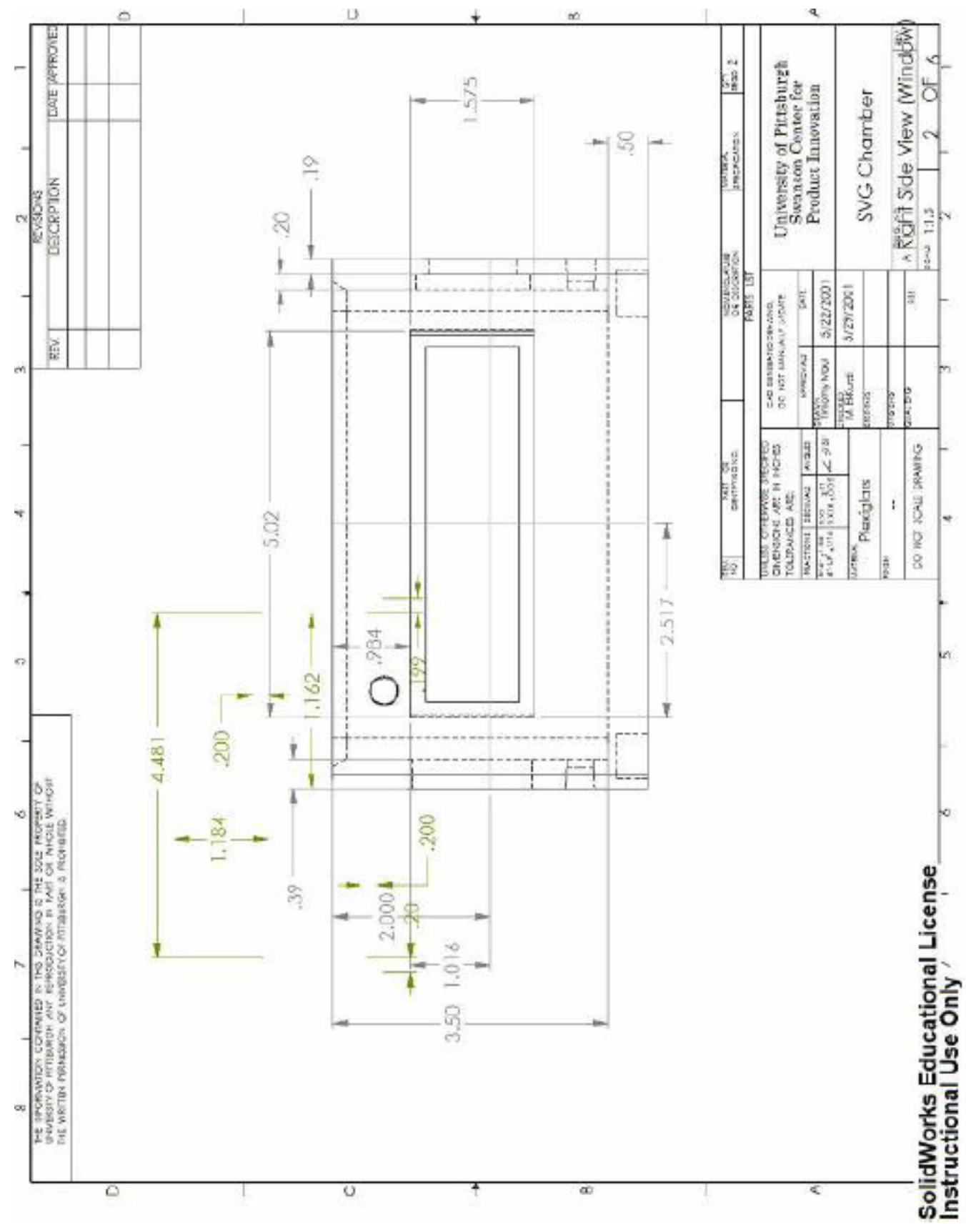

Figure A. 16 Solidworks technical drawing of right side view (window) of tissue housing chamber assembly. 


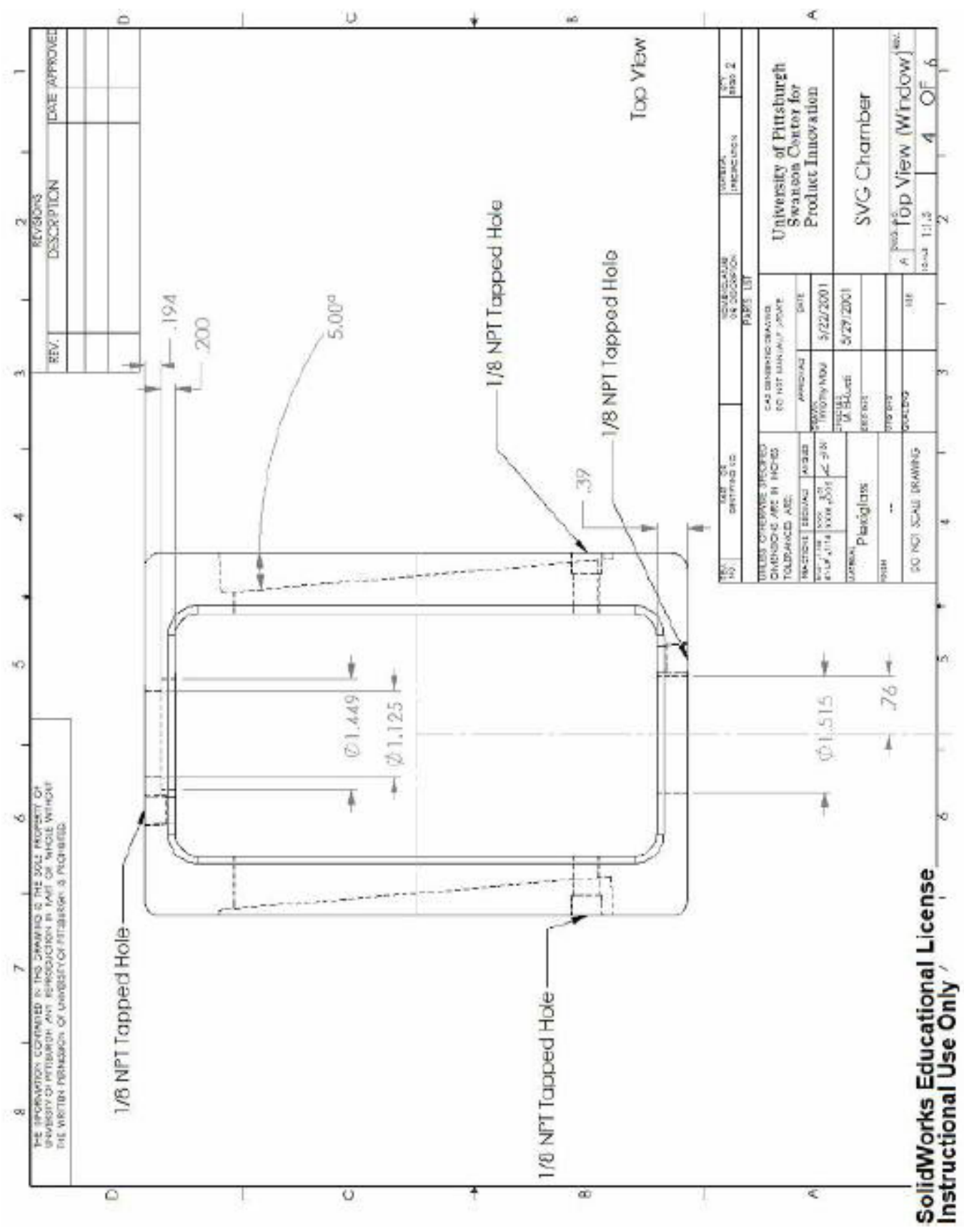

Figure A. 17 Solidworks technical drawing of top view (window) of tissue housing chamber assembly. 


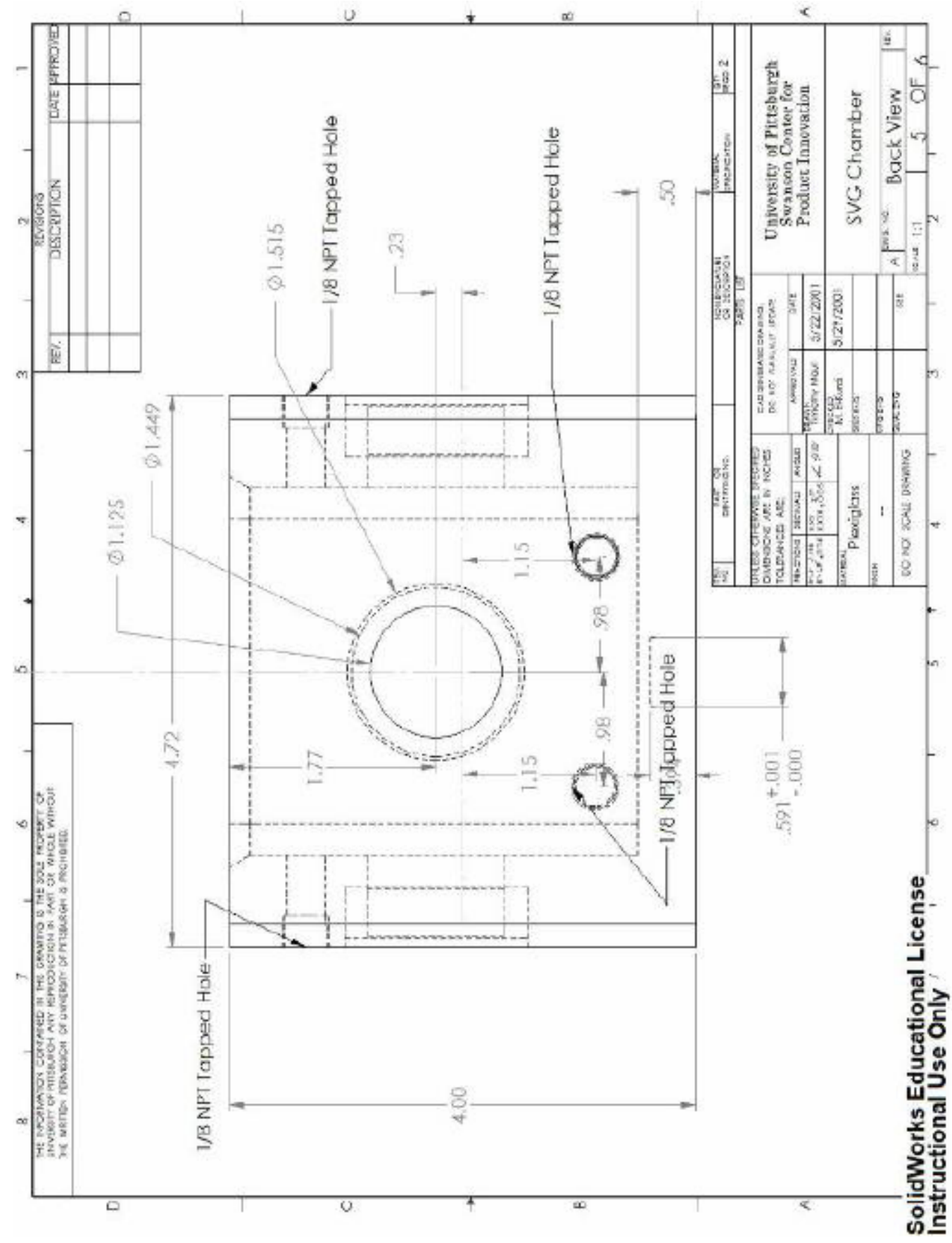

Figure A. 18 Solidworks technical drawing of back view of tissue housing chamber assembly. 


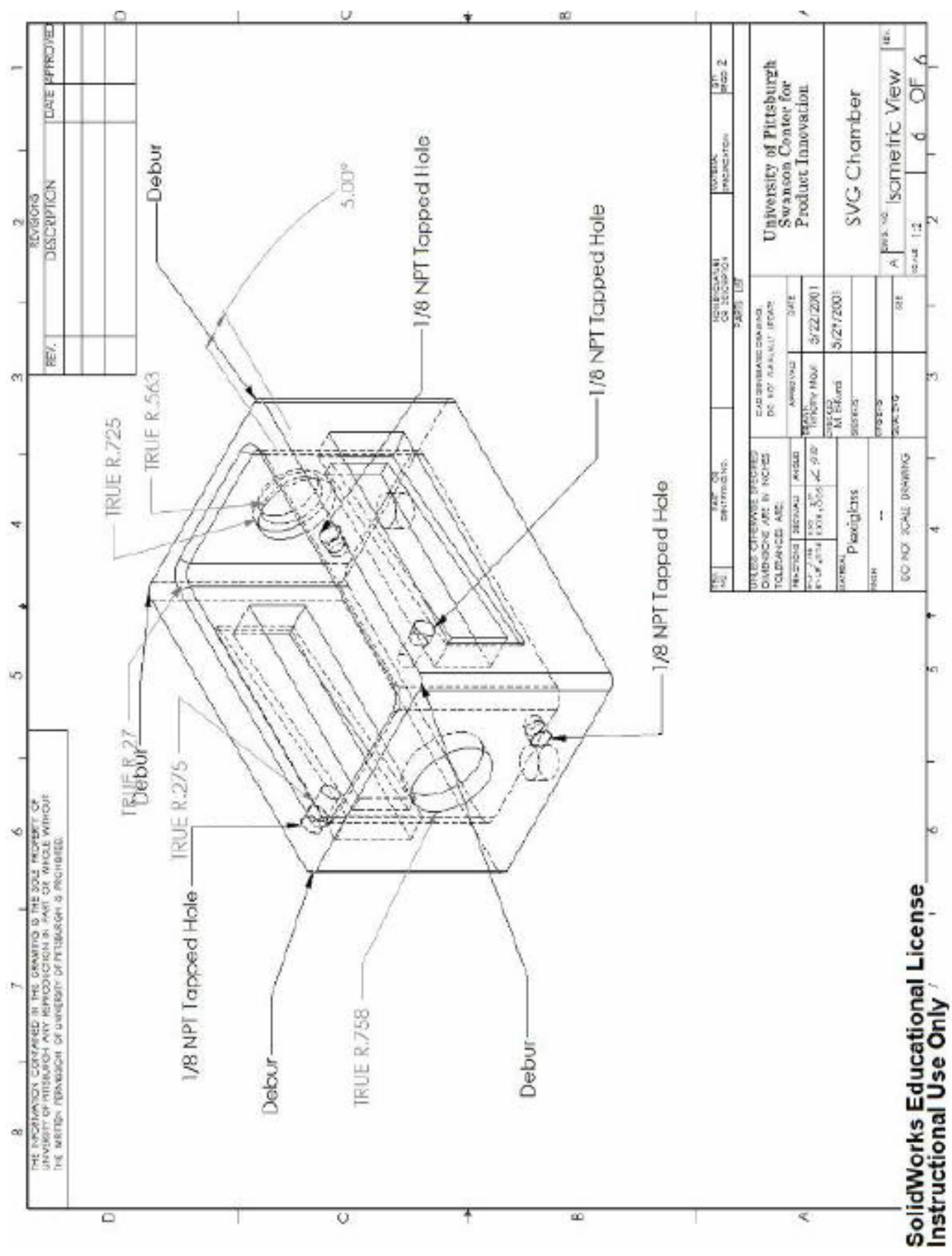

Figure A. 19 Solidworks technical drawing of isometric view of tissue housing chamber assembly. 


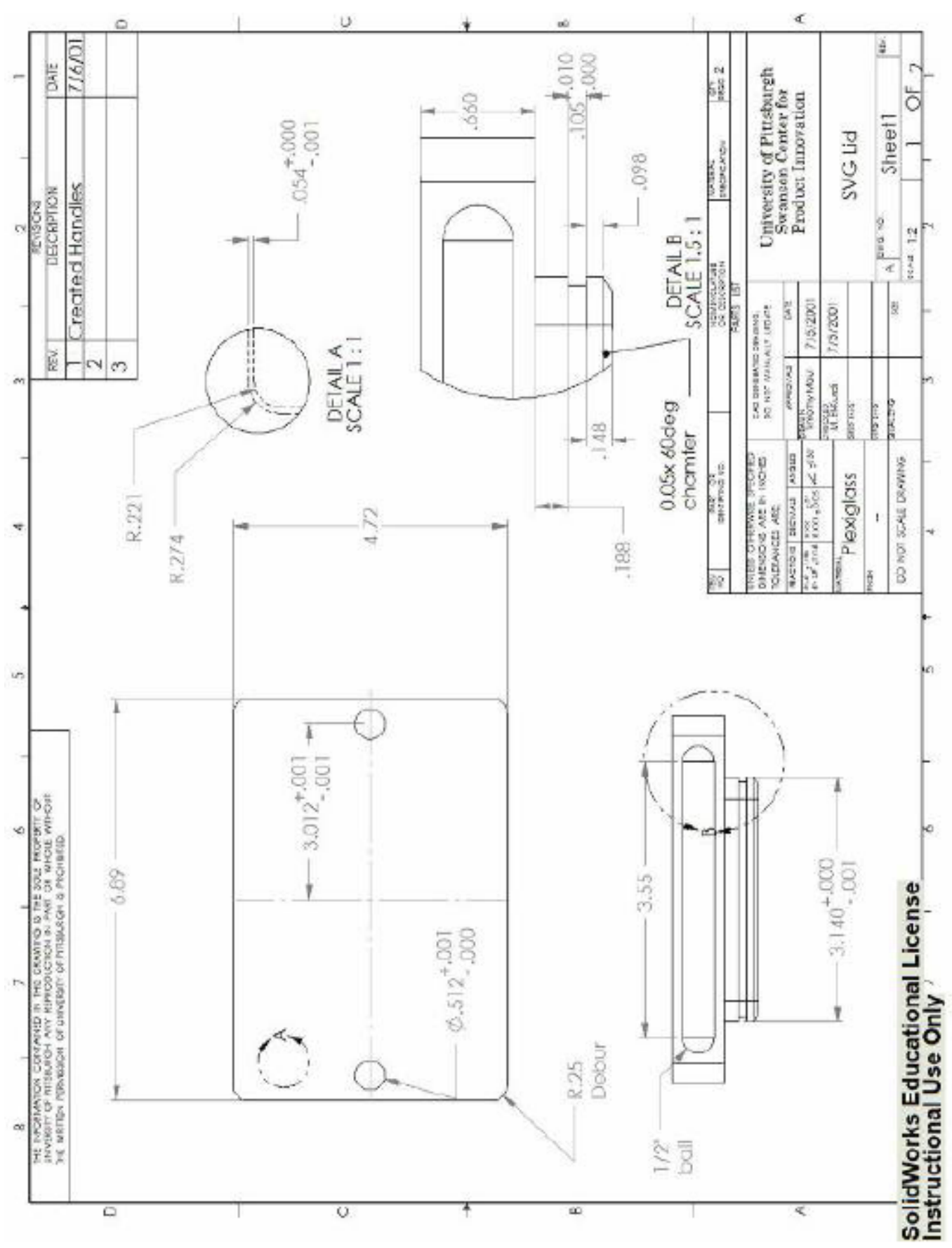

Figure A. 20 Solidworks technical drawing of lid for tissue housing chamber assembly. 


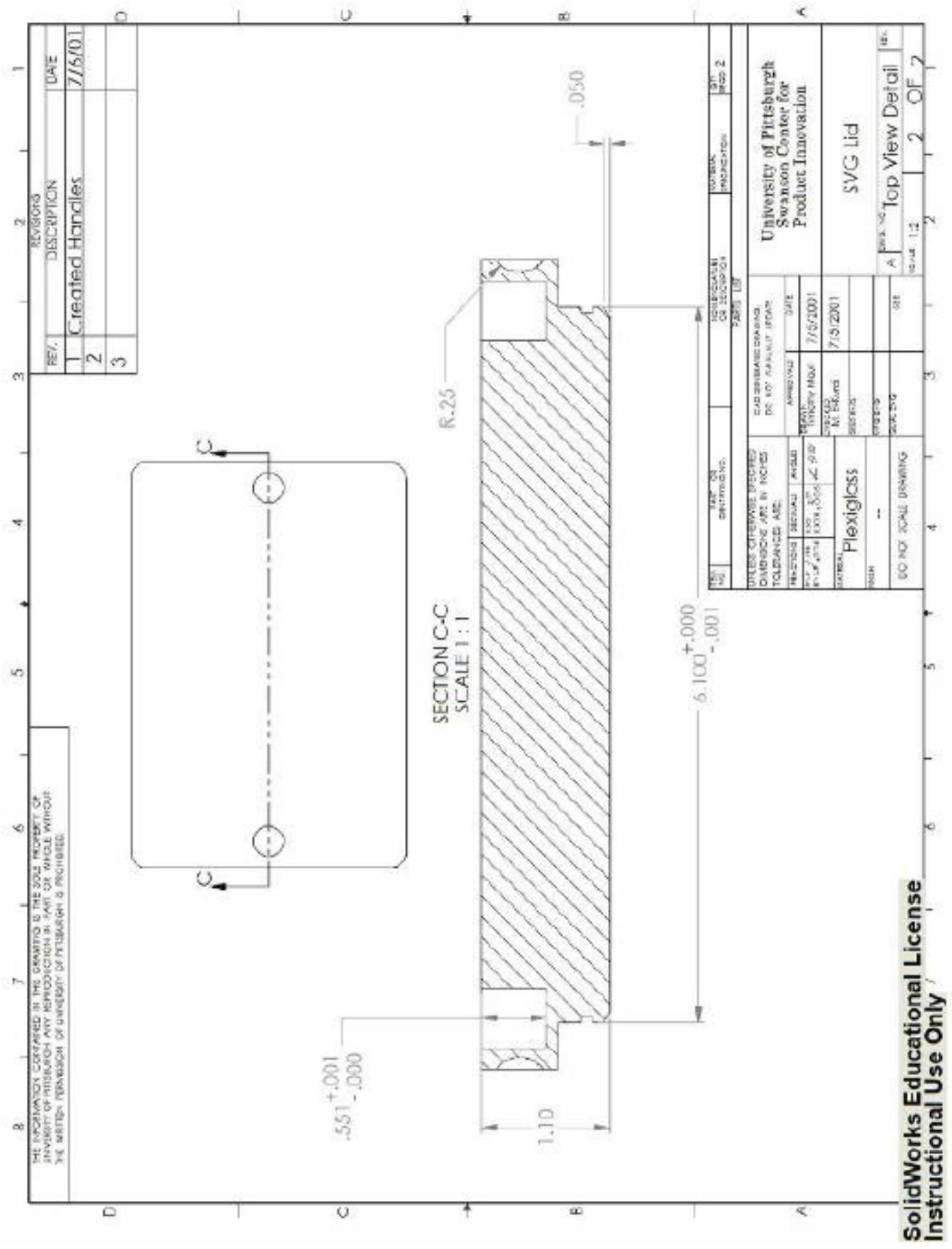

Figure A. 21 Solidworks technical drawing of lid for tissue housing chamber assembly. 


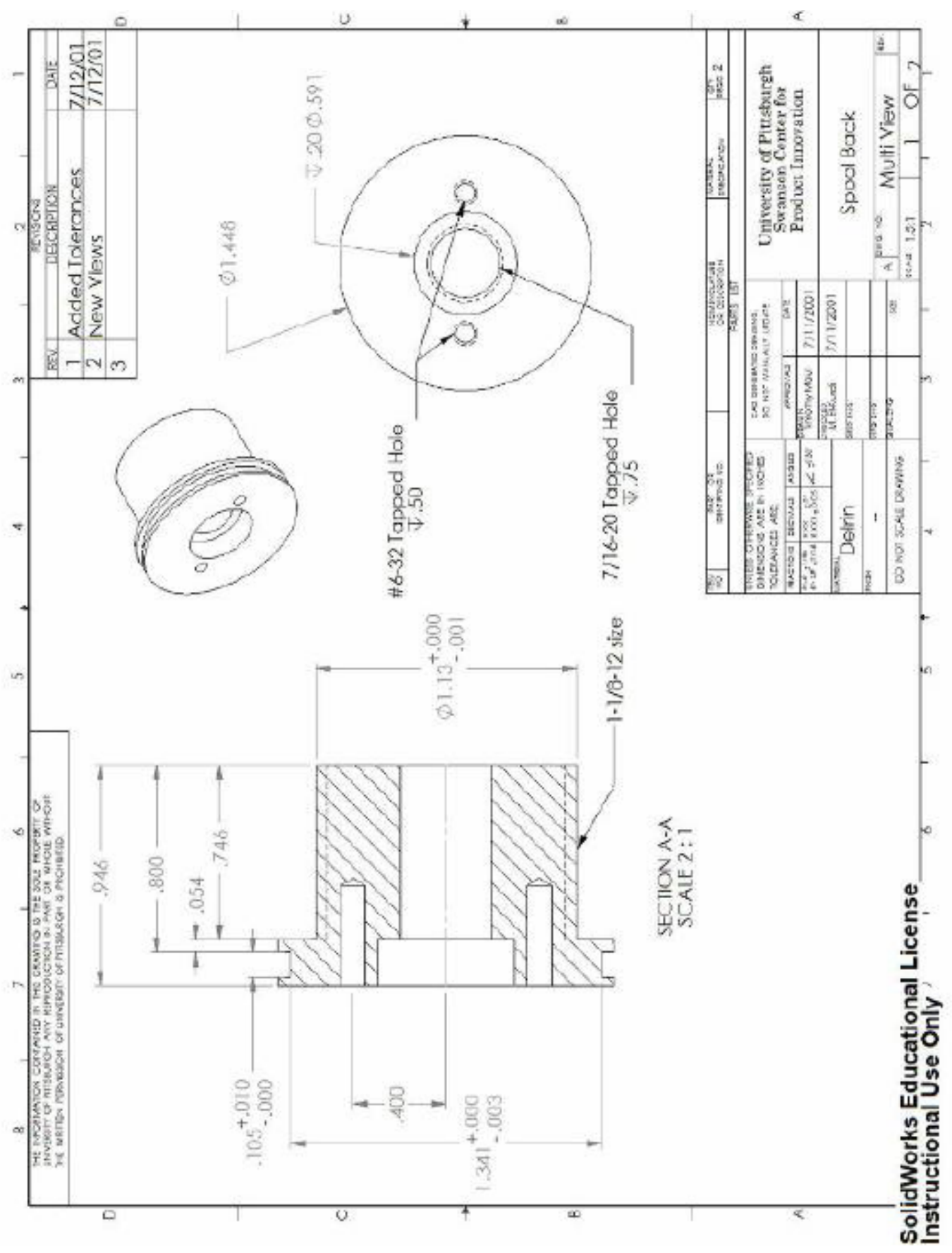

Figure A. 22 Solidworks technical drawing of spool-block for tissue housing chamber assembly. 


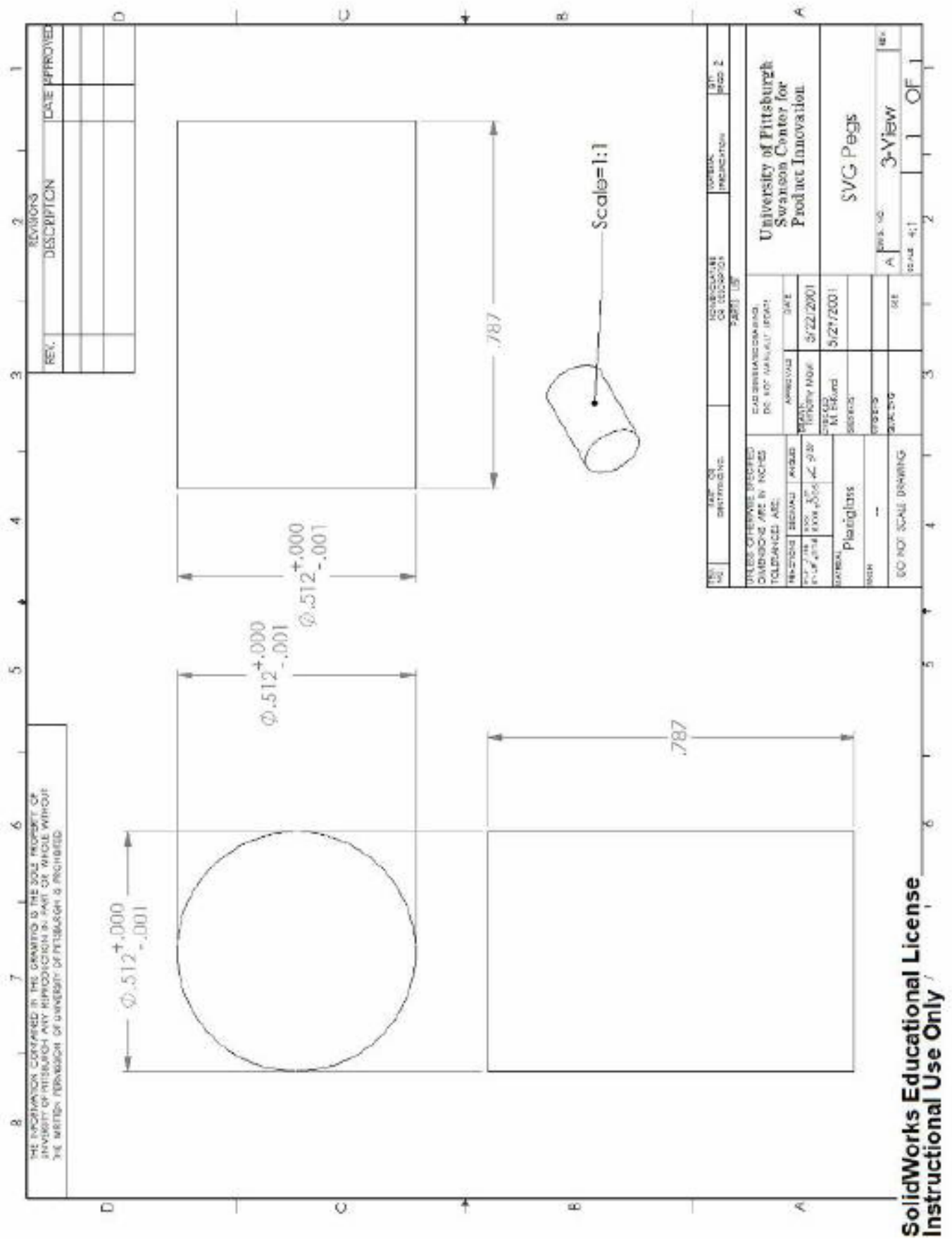

Figure A. 23 Solidworks technical drawing of peg for tissue housing chamber assembly. 


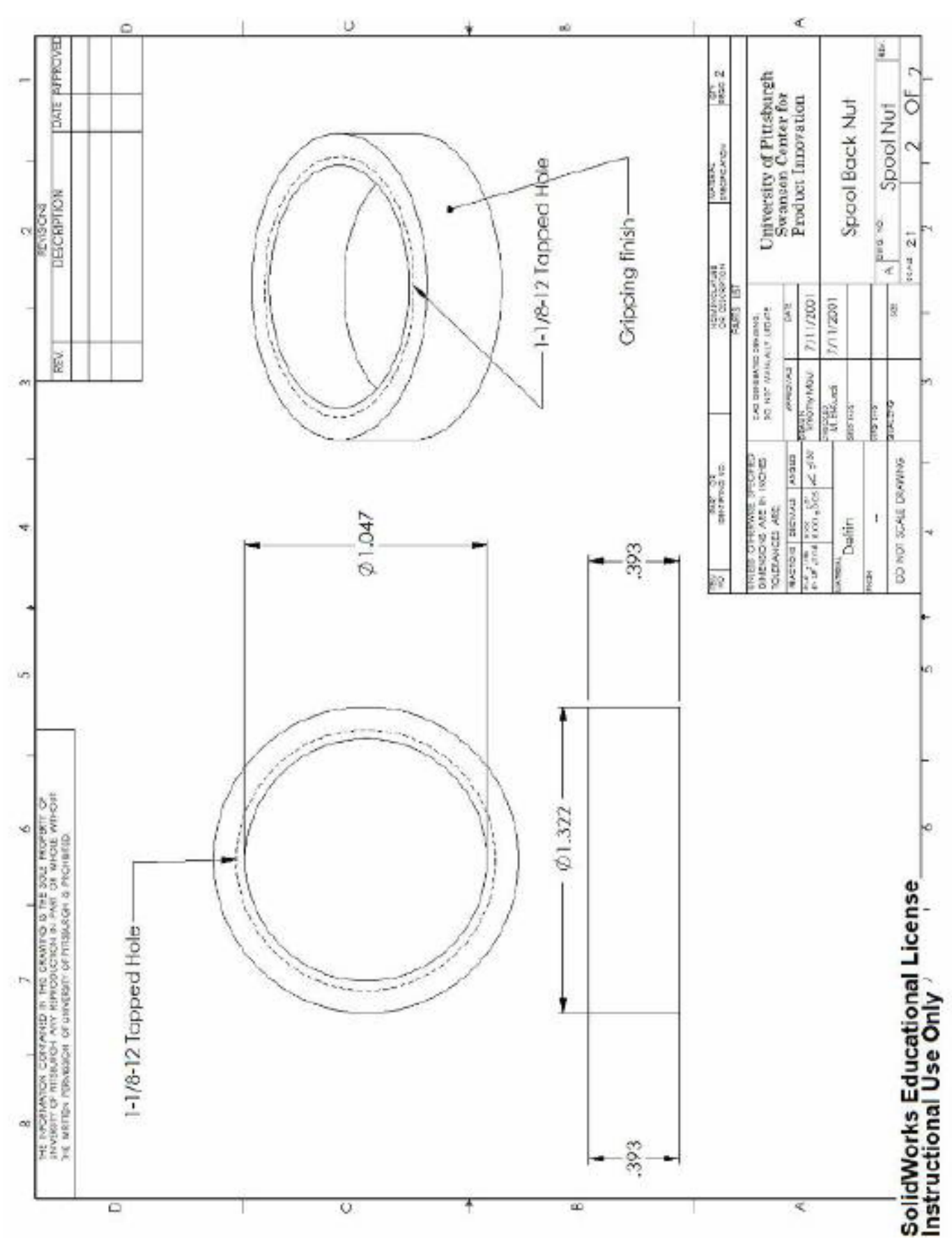

Figure A. 24 Solidworks technical drawing of spool-block nut for tissue housing chamber assembly. 


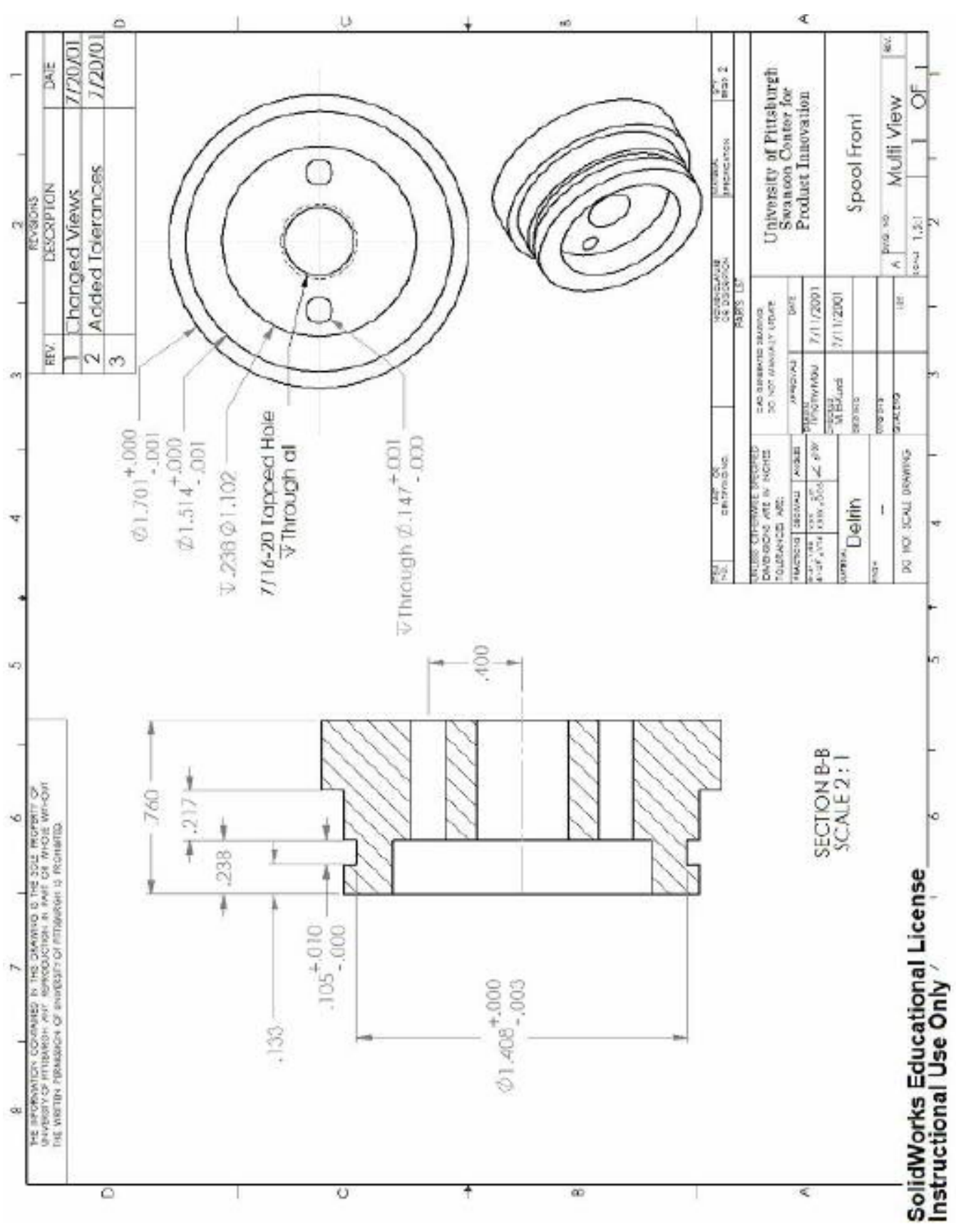

Figure A. 25 Solidworks technical drawing of front spool for tissue housing chamber assembly. 


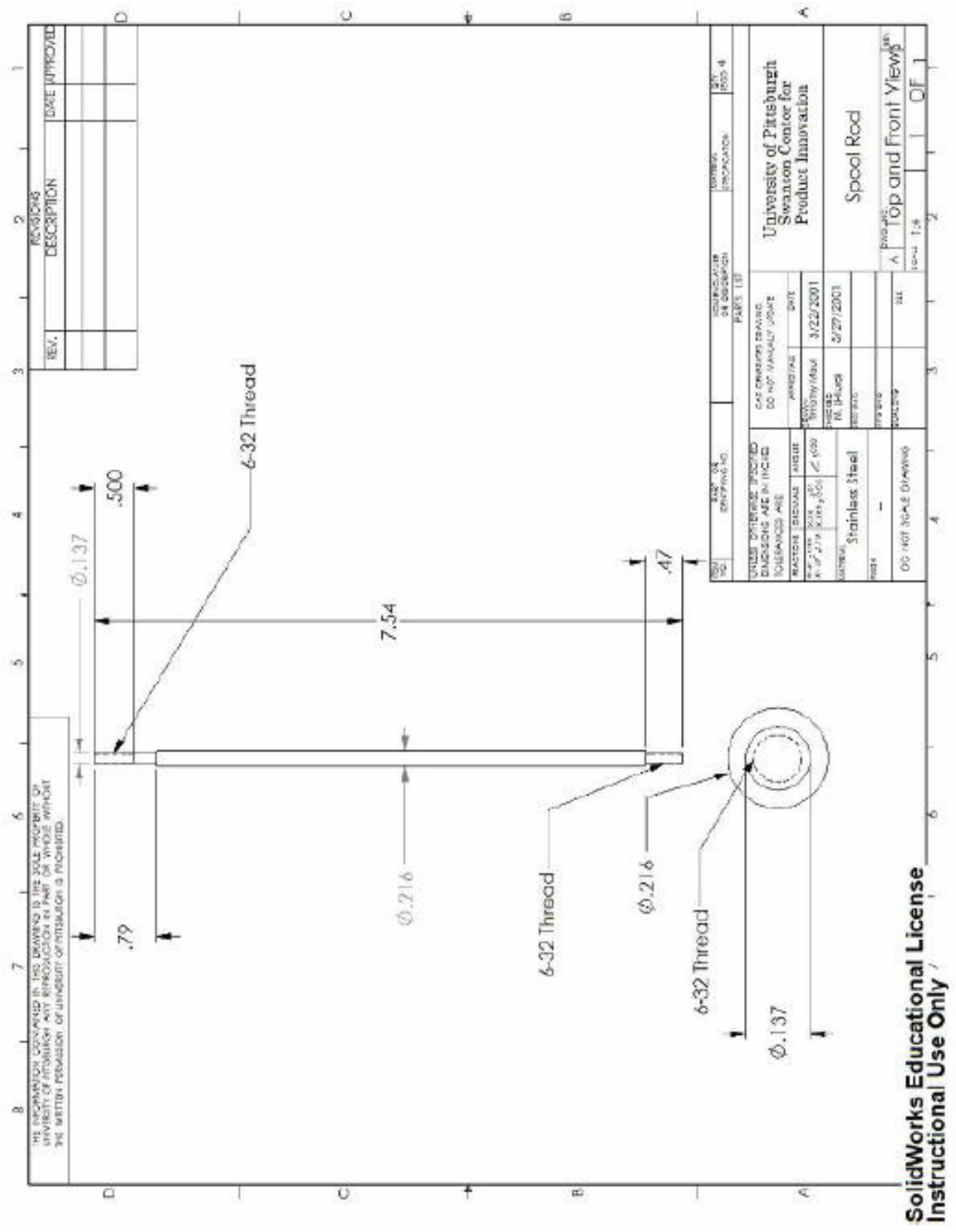

Figure A. 26 Solidworks technical drawing of spool-rod for tissue housing chamber assembly. 


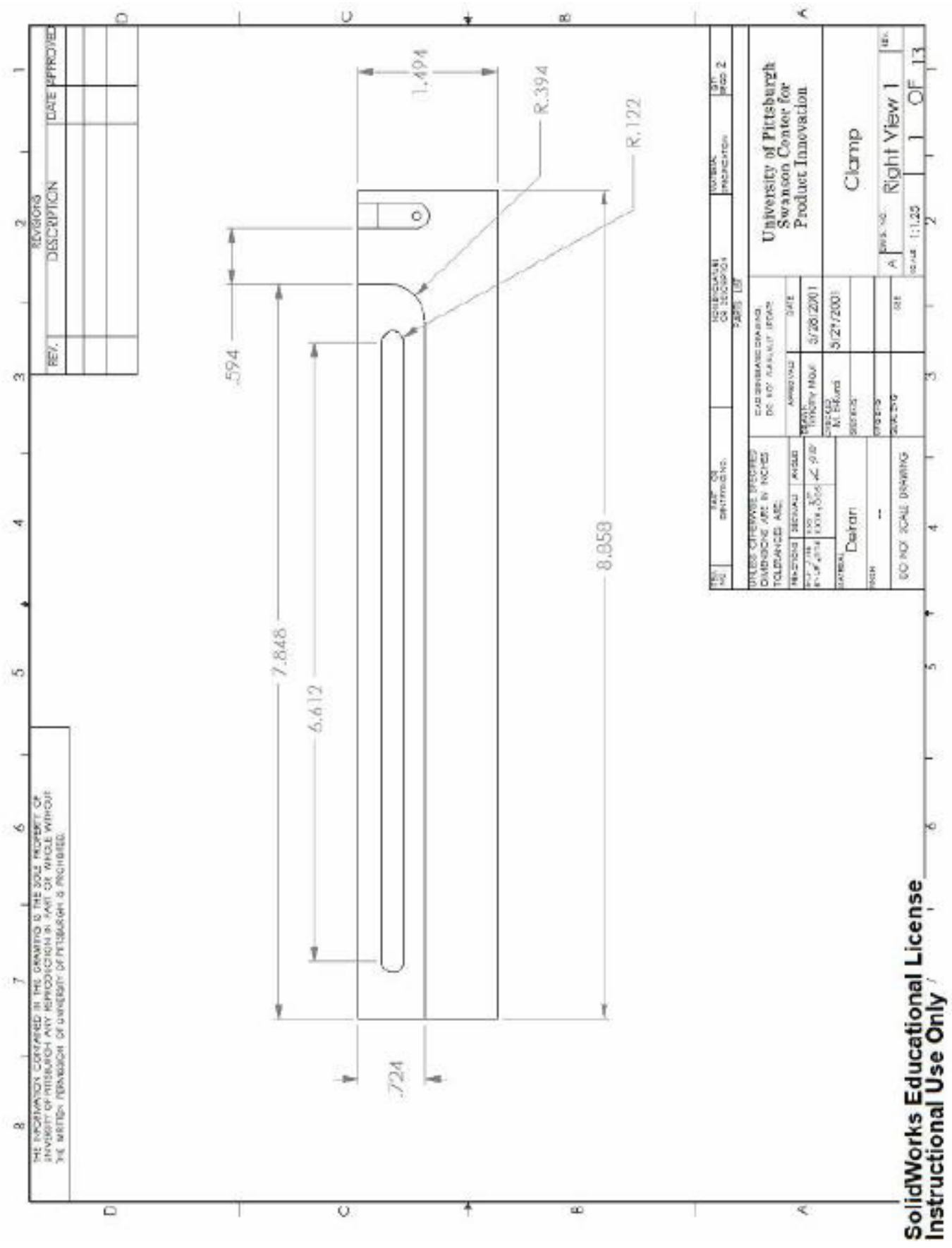

Figure A. 27 Solidworks technical drawing of right side view of in vivo length blood vessel clamp assembly. 


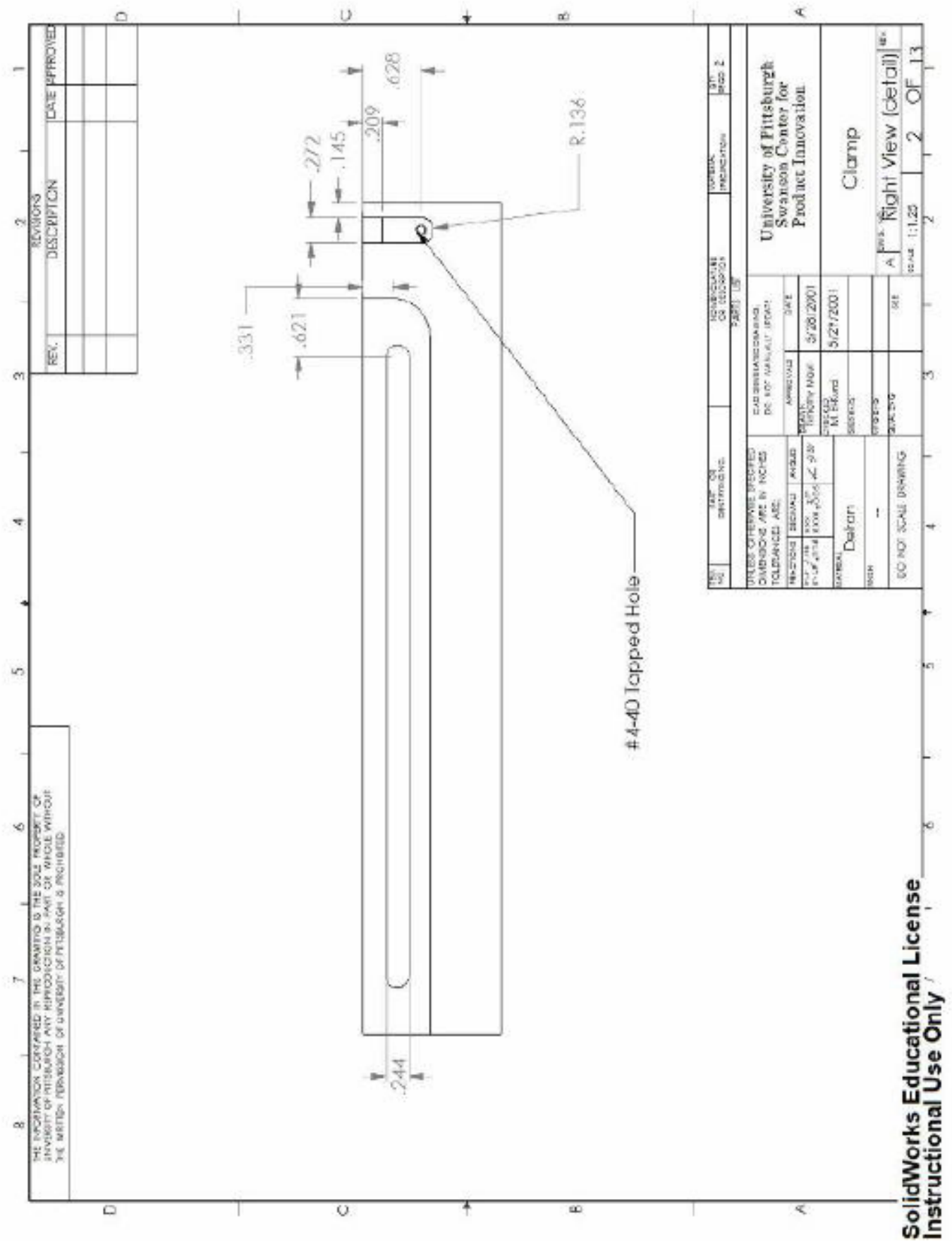

Figure A. 28 Solidworks technical drawing of right side view (detail) of in vivo length blood vessel clamp assembly. 


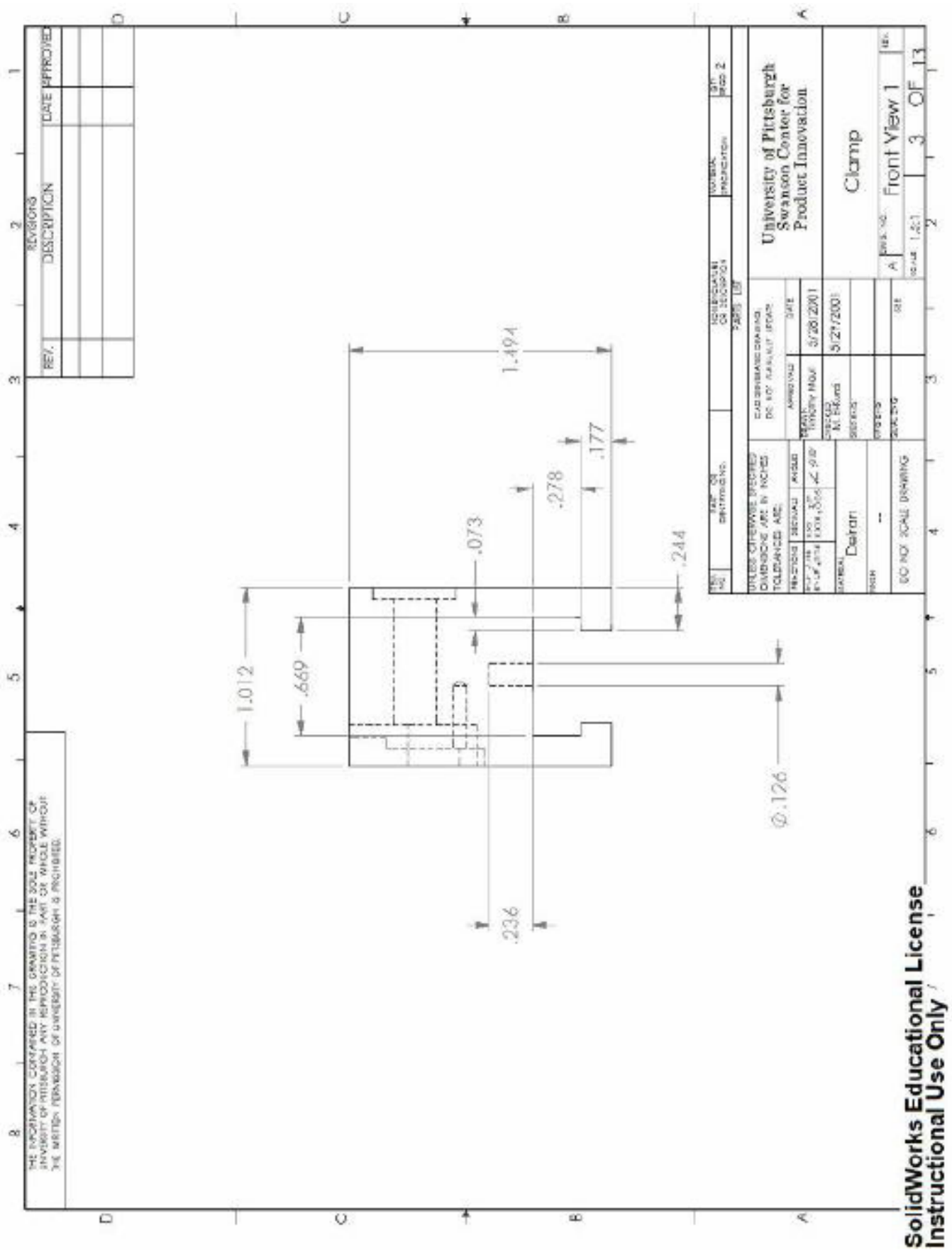

Figure A. 29 Solidworks technical drawing of front view of in vivo length blood vessel clamp assembly. 


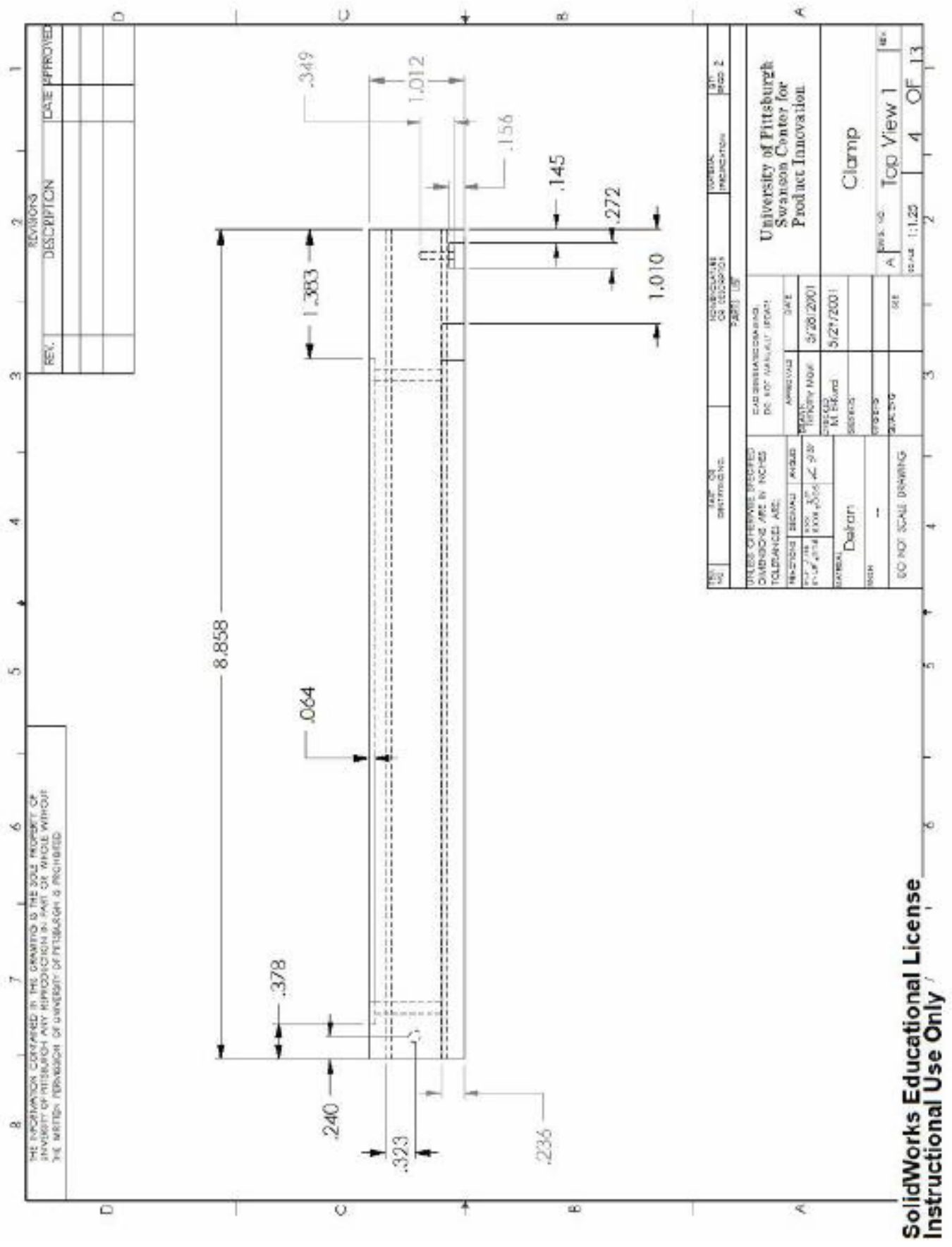

Figure A. 30 Solidworks technical drawing of top view of in vivo length blood vessel clamp assembly. 


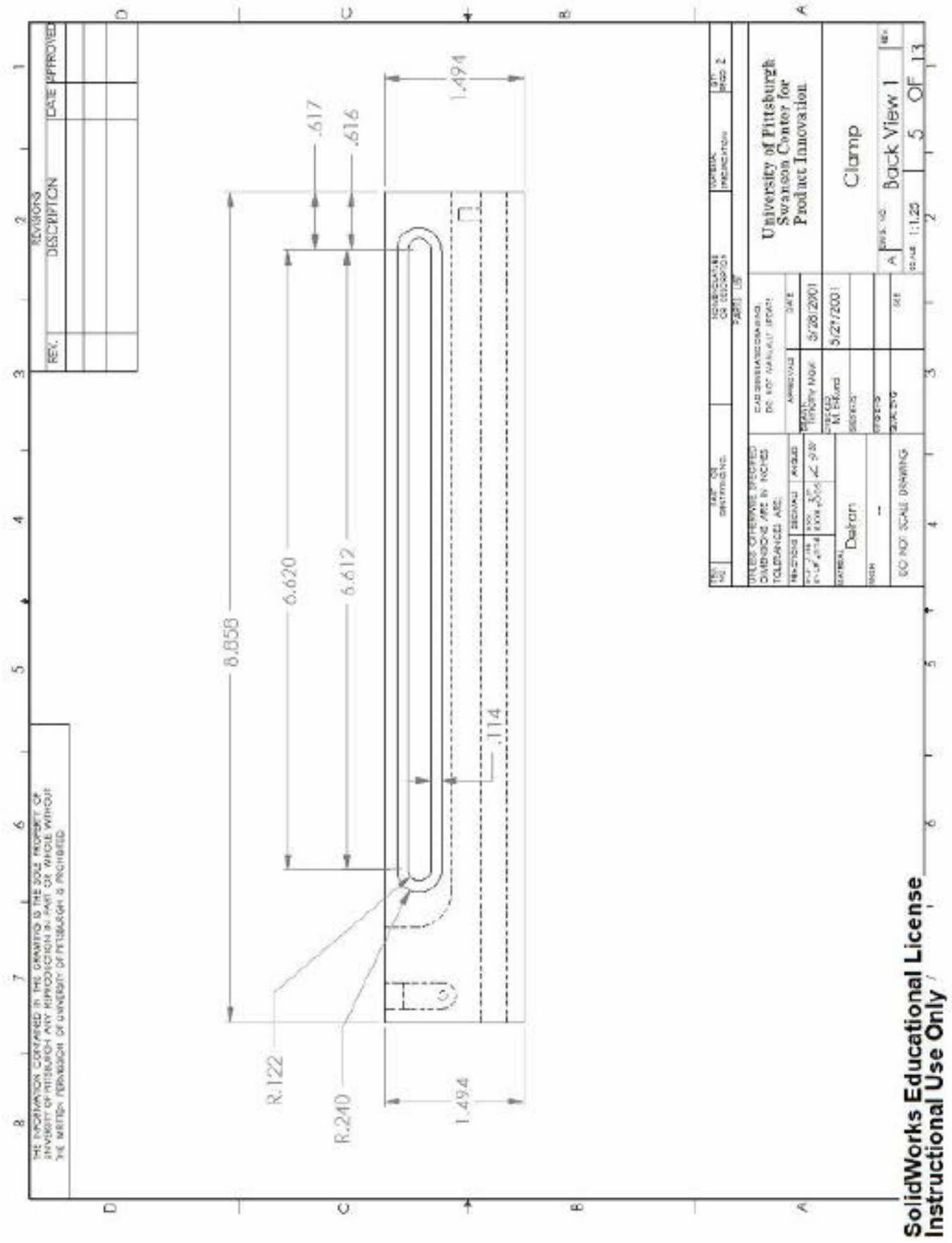

Figure A. 31 Solidworks technical drawing of back view of in vivo length blood vessel clamp assembly. 


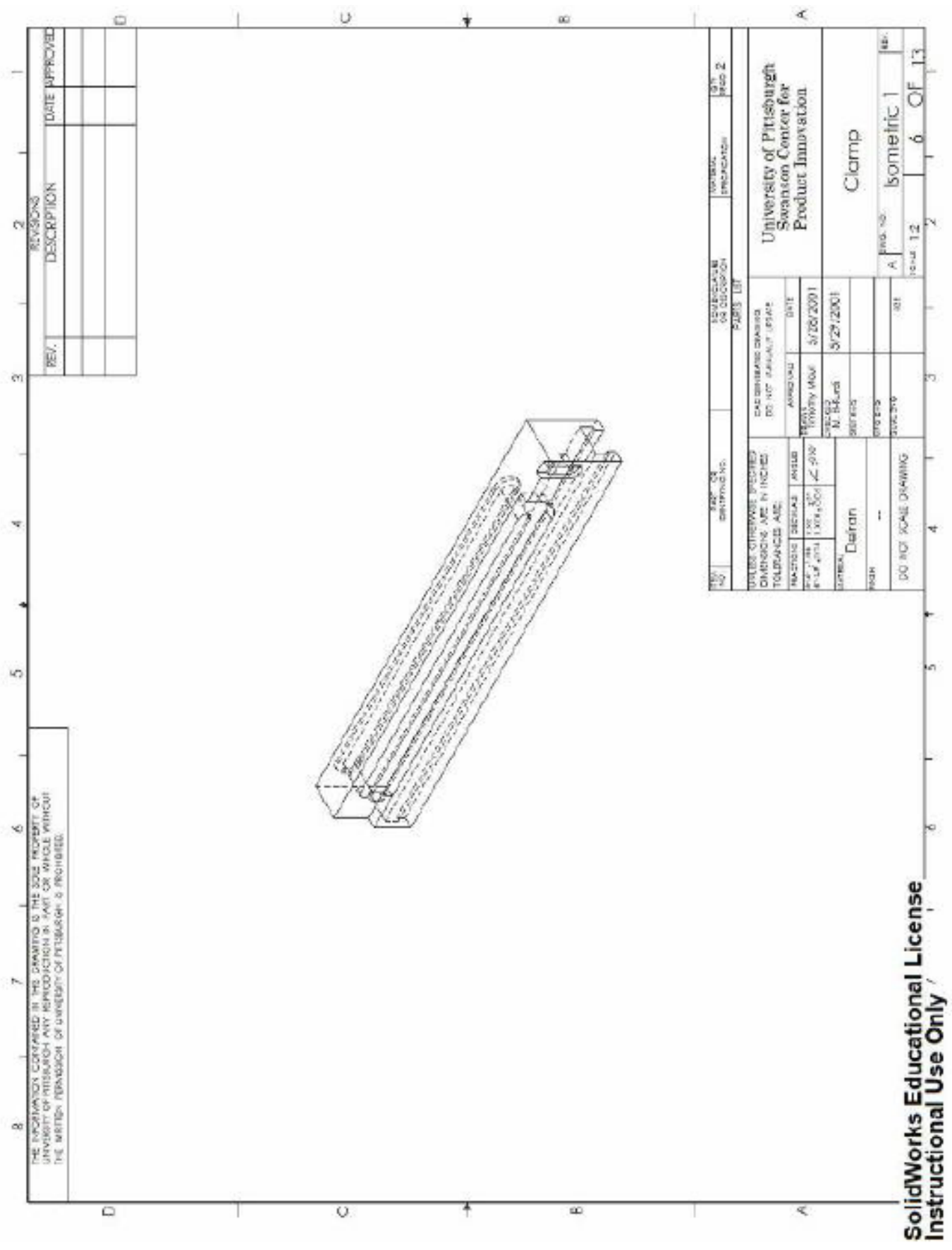

Figure A. 32 Solidworks technical drawing of isometric view of in vivo length blood vessel clamp assembly. 


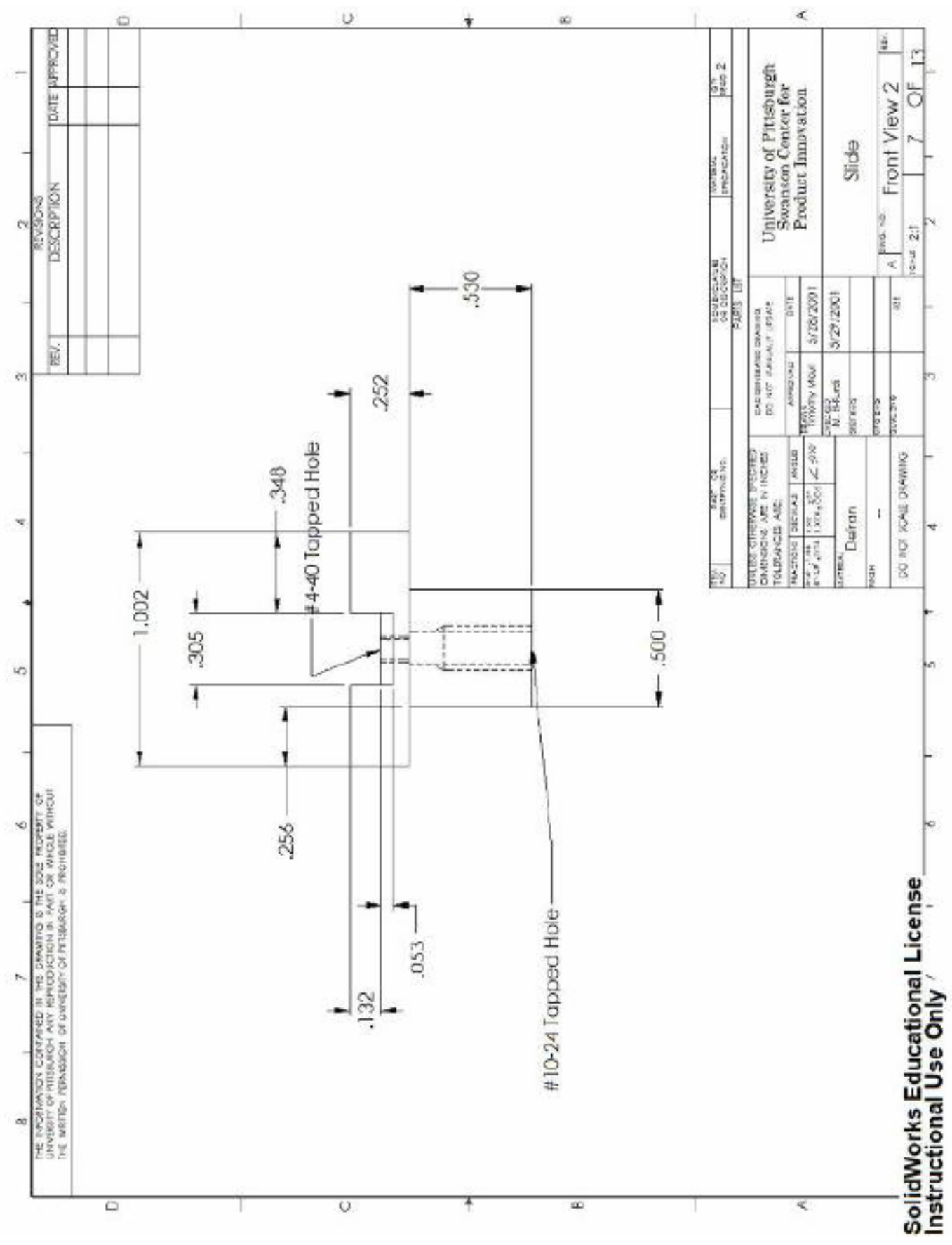

Figure A. 33 Solidworks technical drawing of front view of slider for in vivo length blood vessel clamp assembly. 


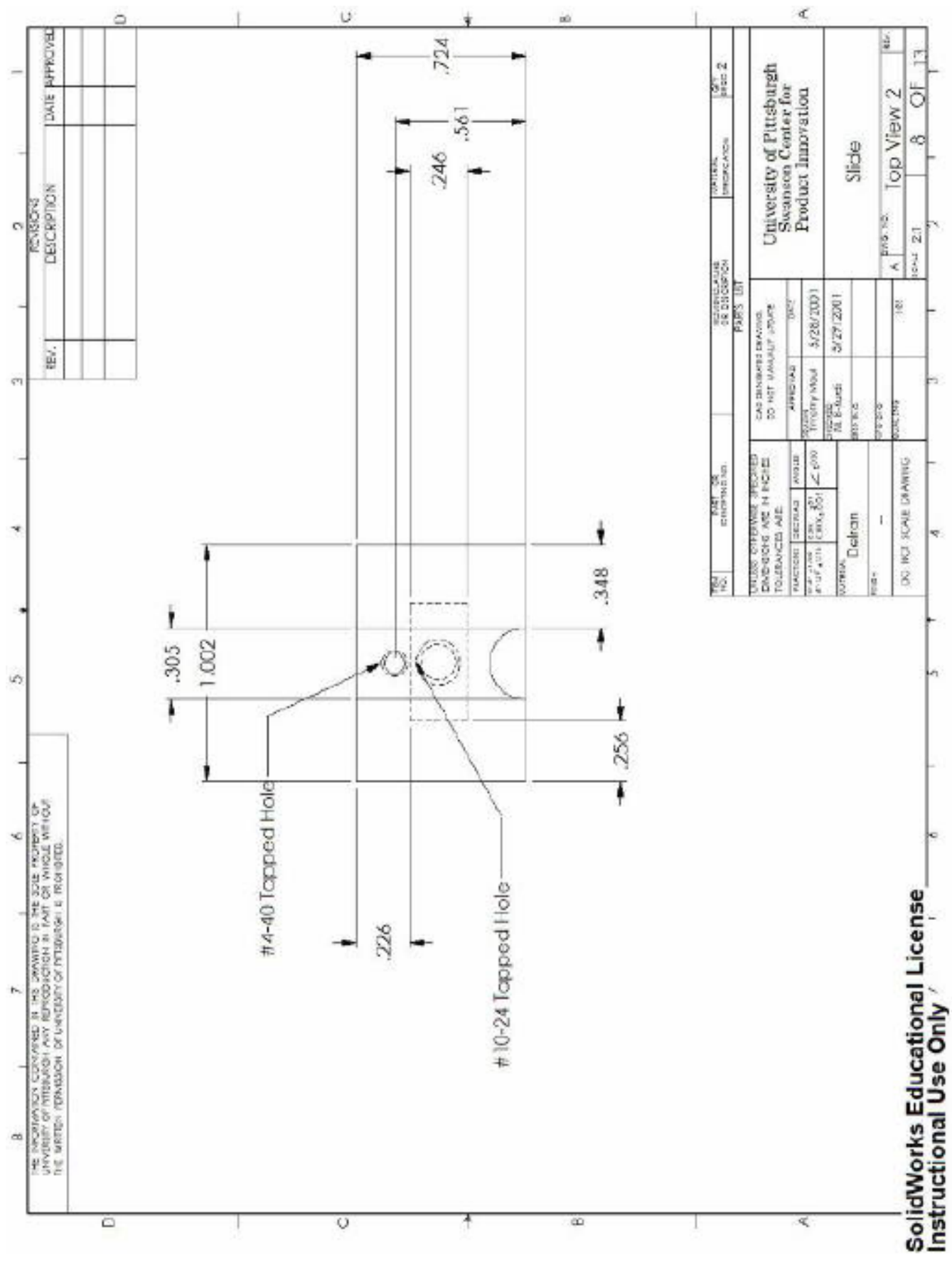

Figure A. 34 Solidworks technical drawing of top view of slider for in vivo length blood vessel clamp assembly. 


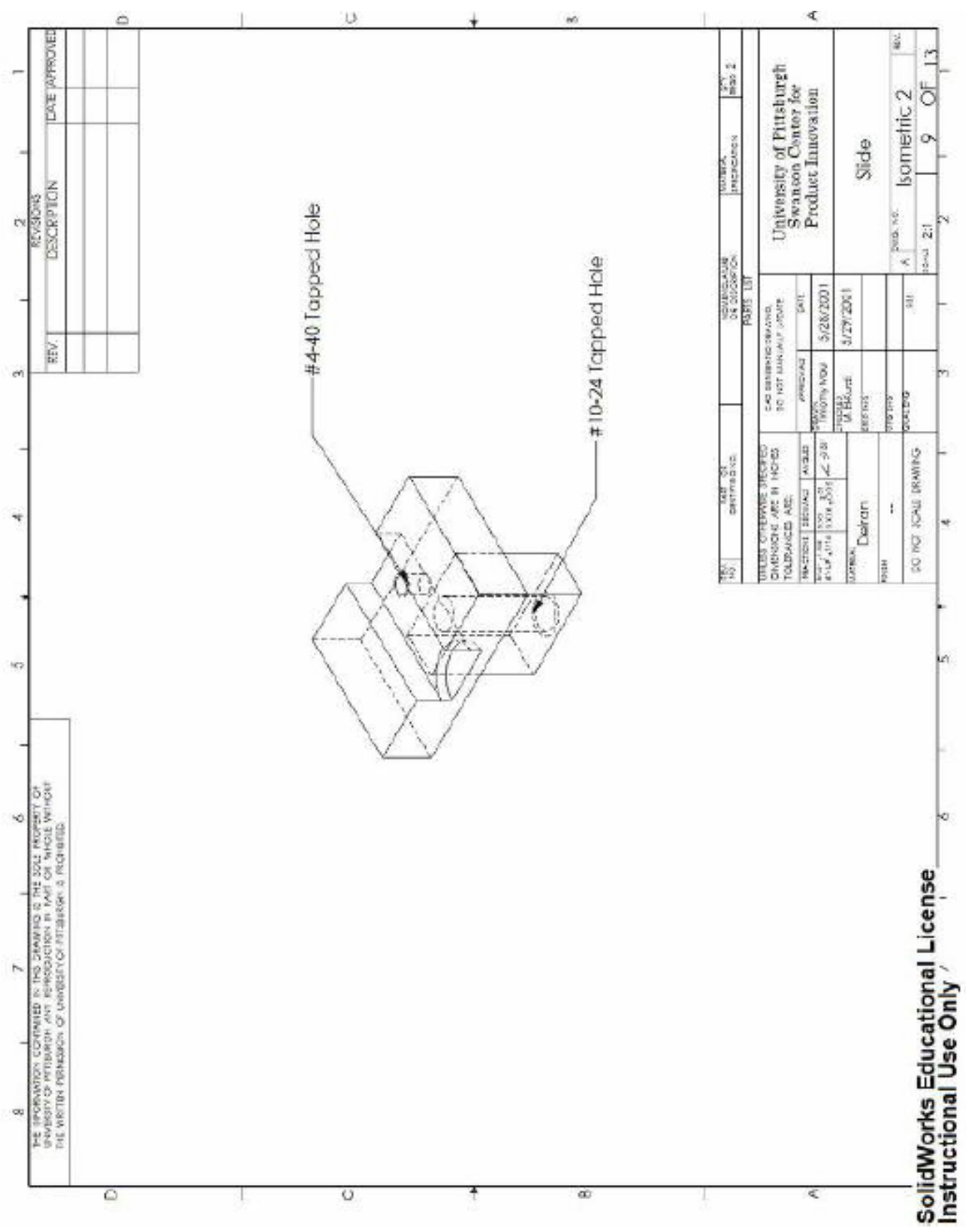

Figure A. 35 Solidworks technical drawing of isometric view of slider for in vivo length blood vessel clamp assembly. 


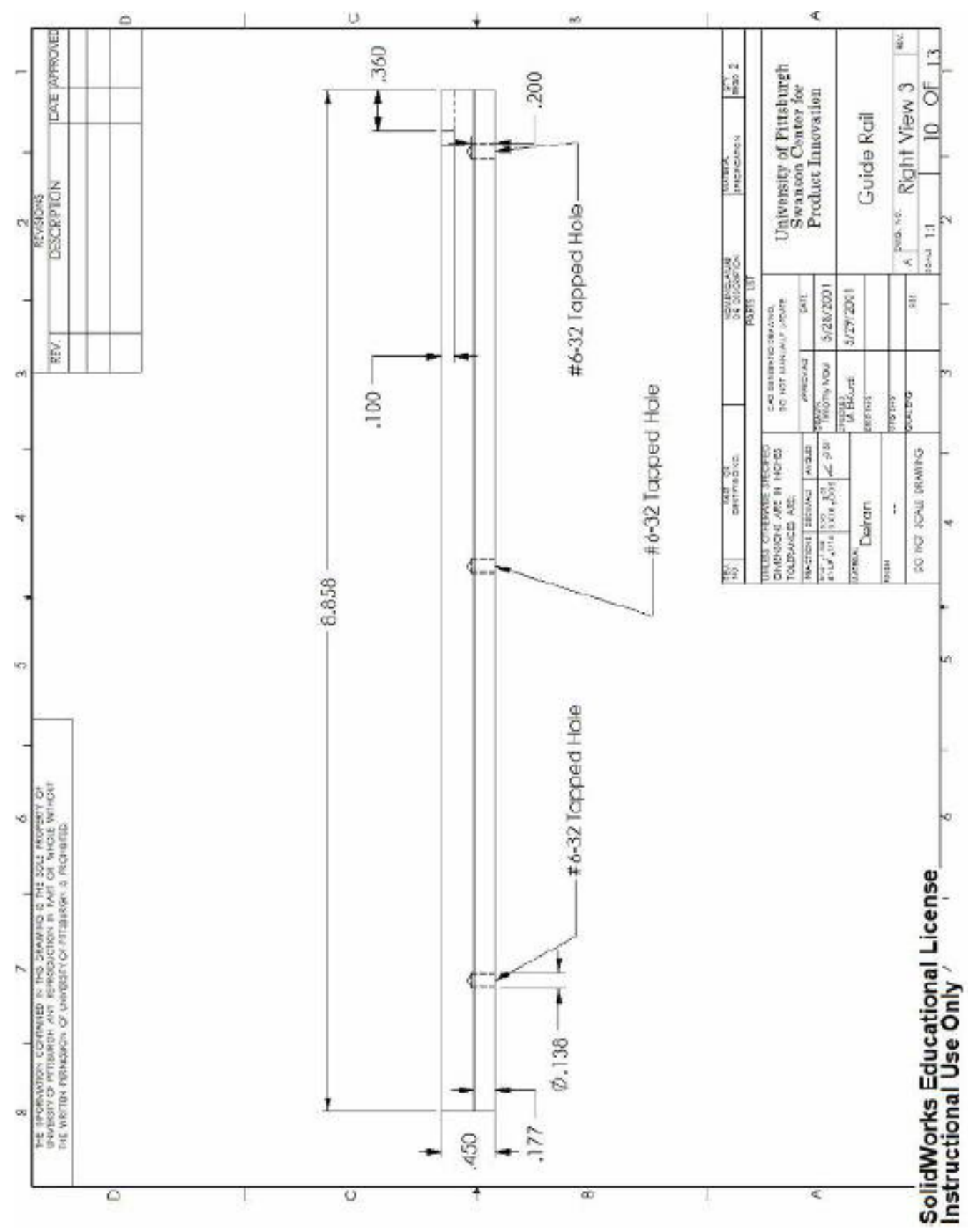

Figure A. 36 Solidworks technical drawing of right side view of guide-rail for transport box assembly. 


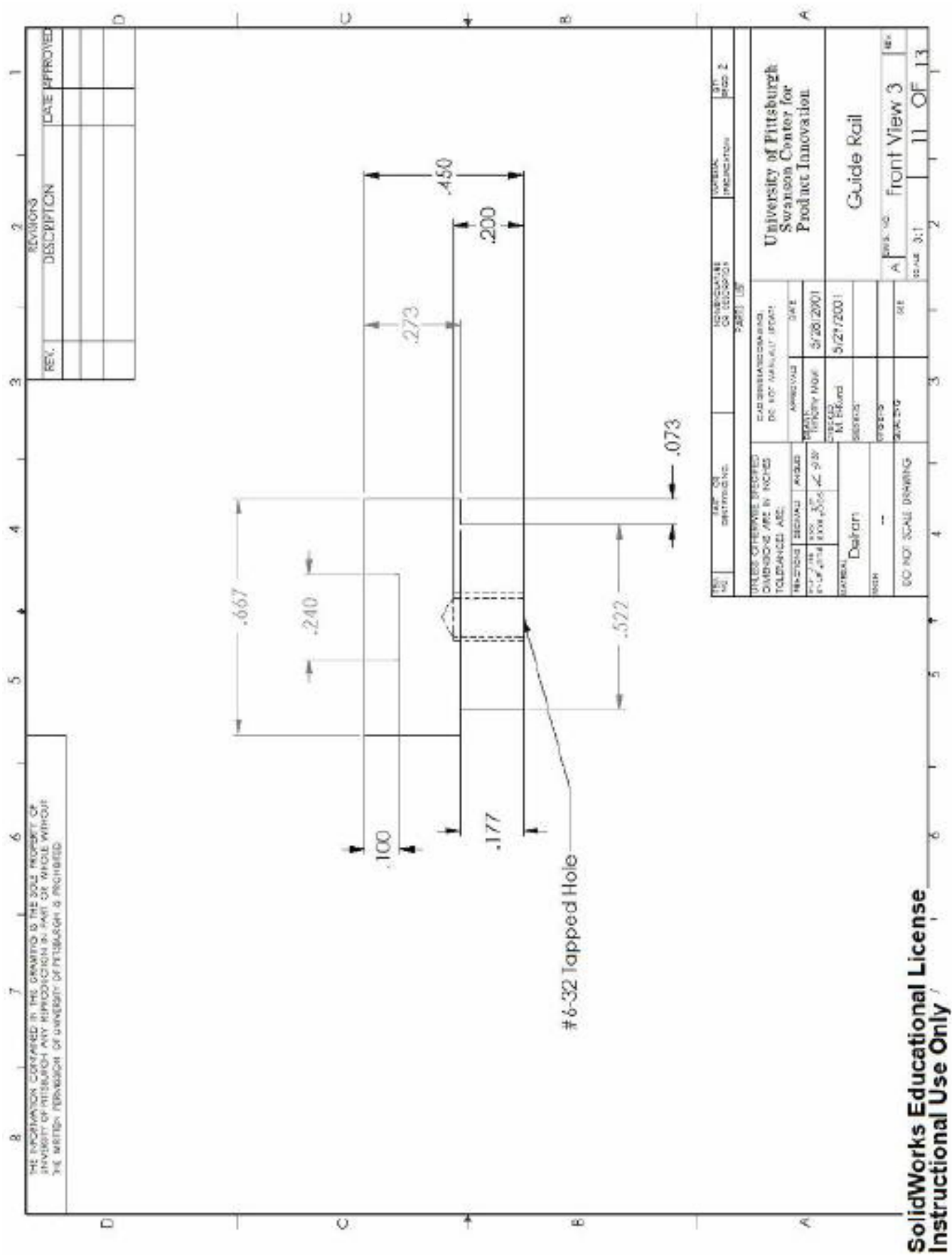

Figure A. 37 Solidworks technical drawing of front view of guide-rail for transport box assembly. 


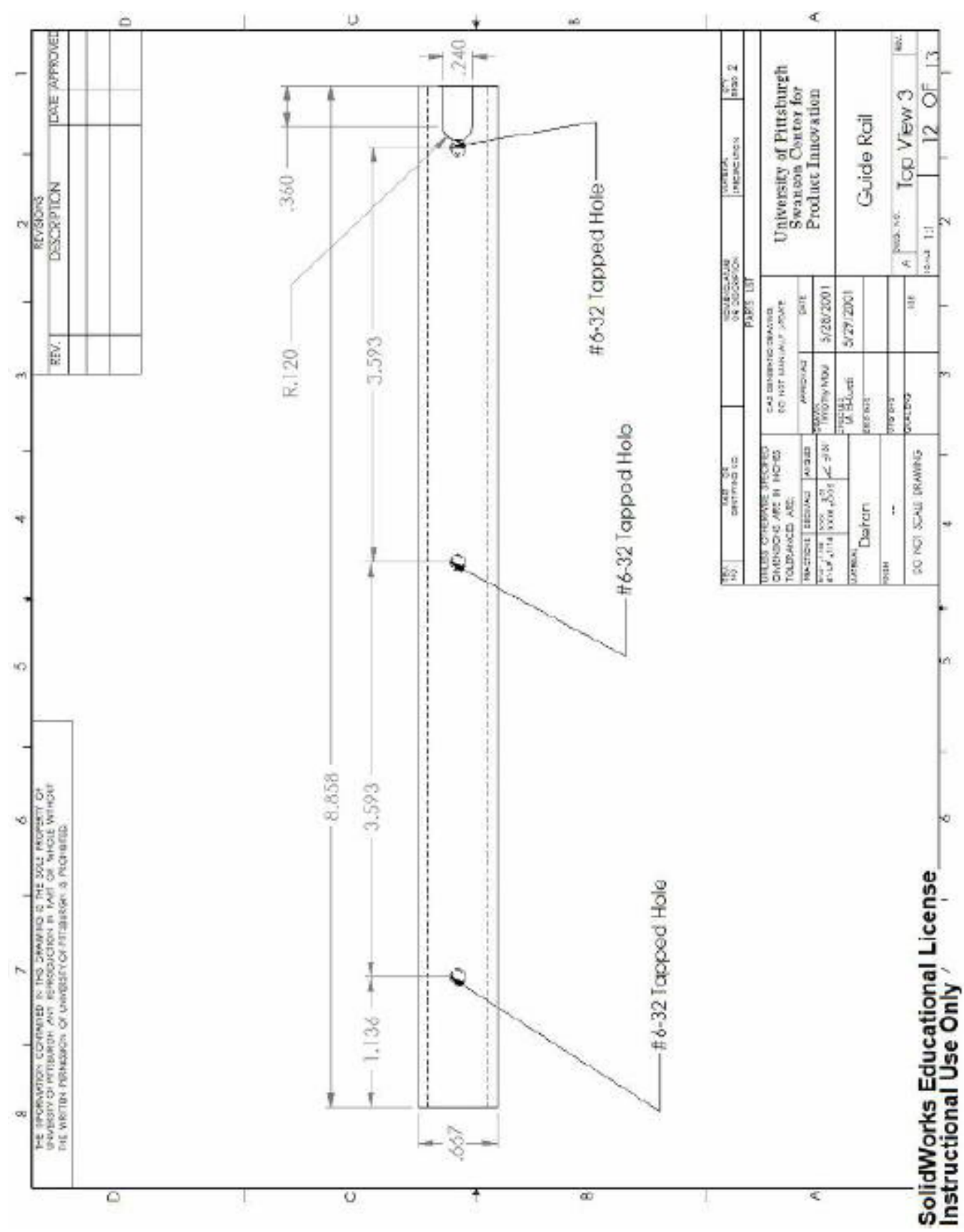

Figure A. 38 Solidworks technical drawing of top view of guide-rail for transport box assembly. 


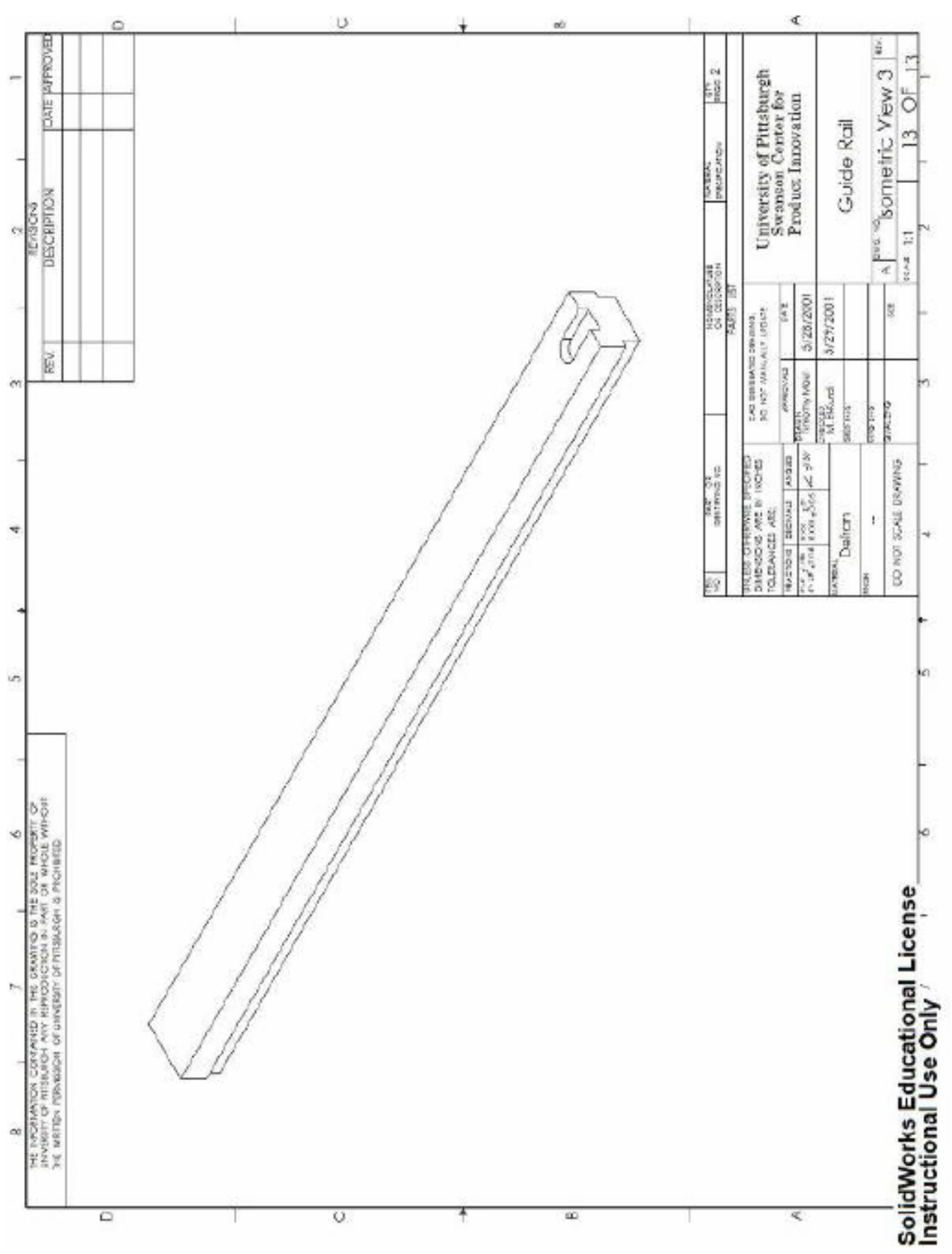

Figure A. 39 Solidworks technical drawing of isometric view of guide-rail for transport box assembly. 


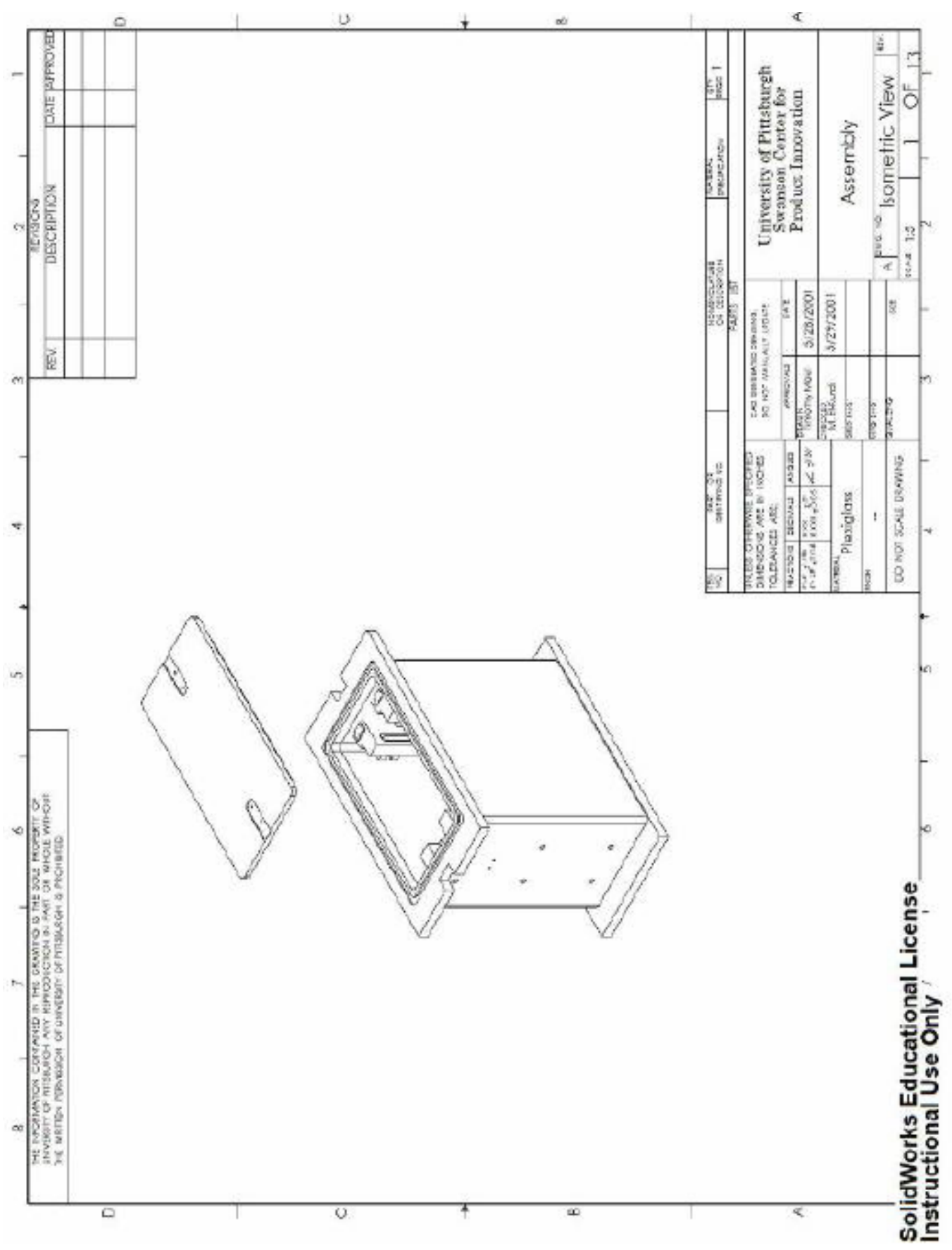

Figure A. 40 Solidworks technical drawing of isometric view of transport box assembly. 


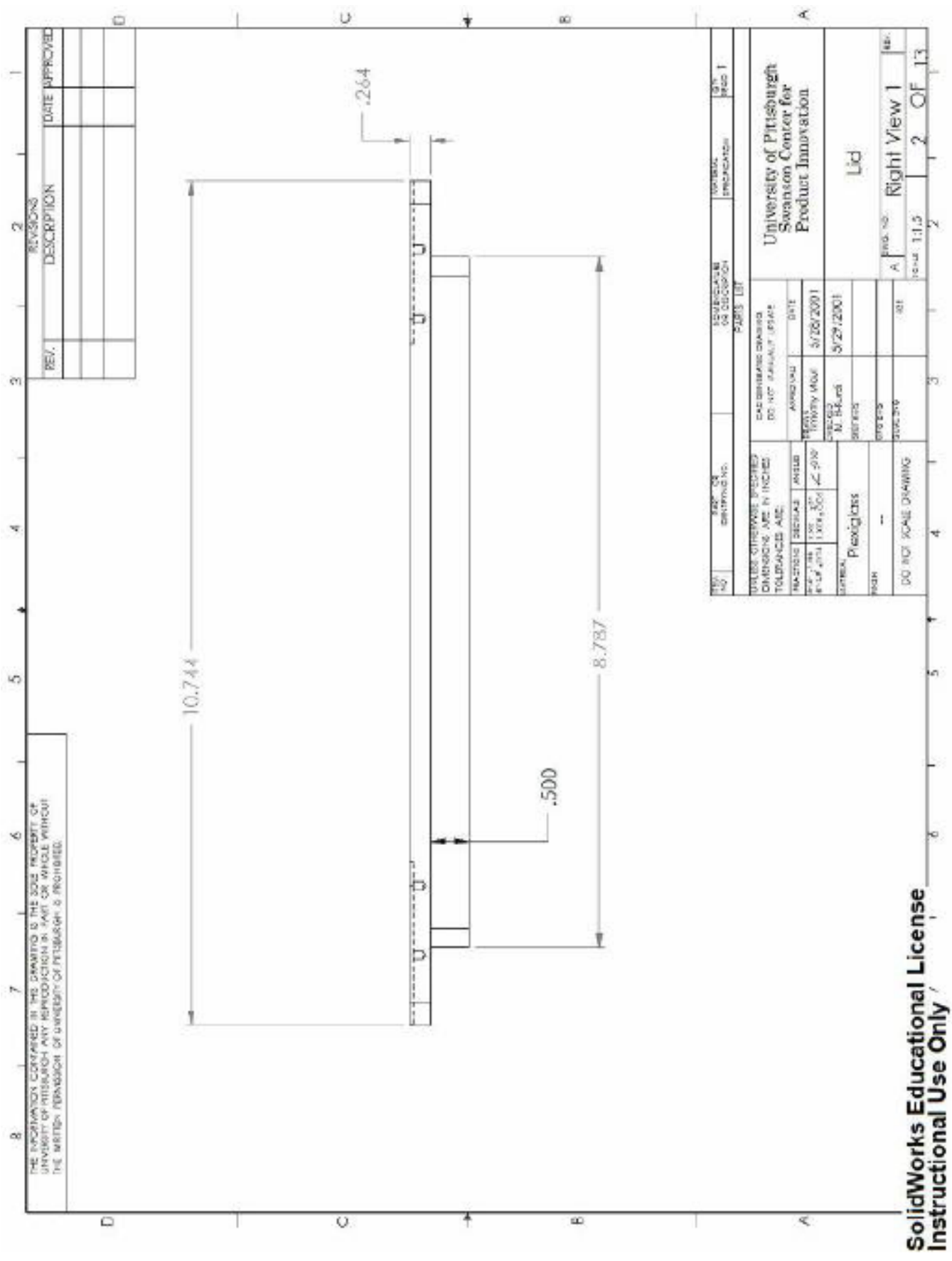

Figure A. 41 Solidworks technical drawing of right side view of lid for transport box assembly. 


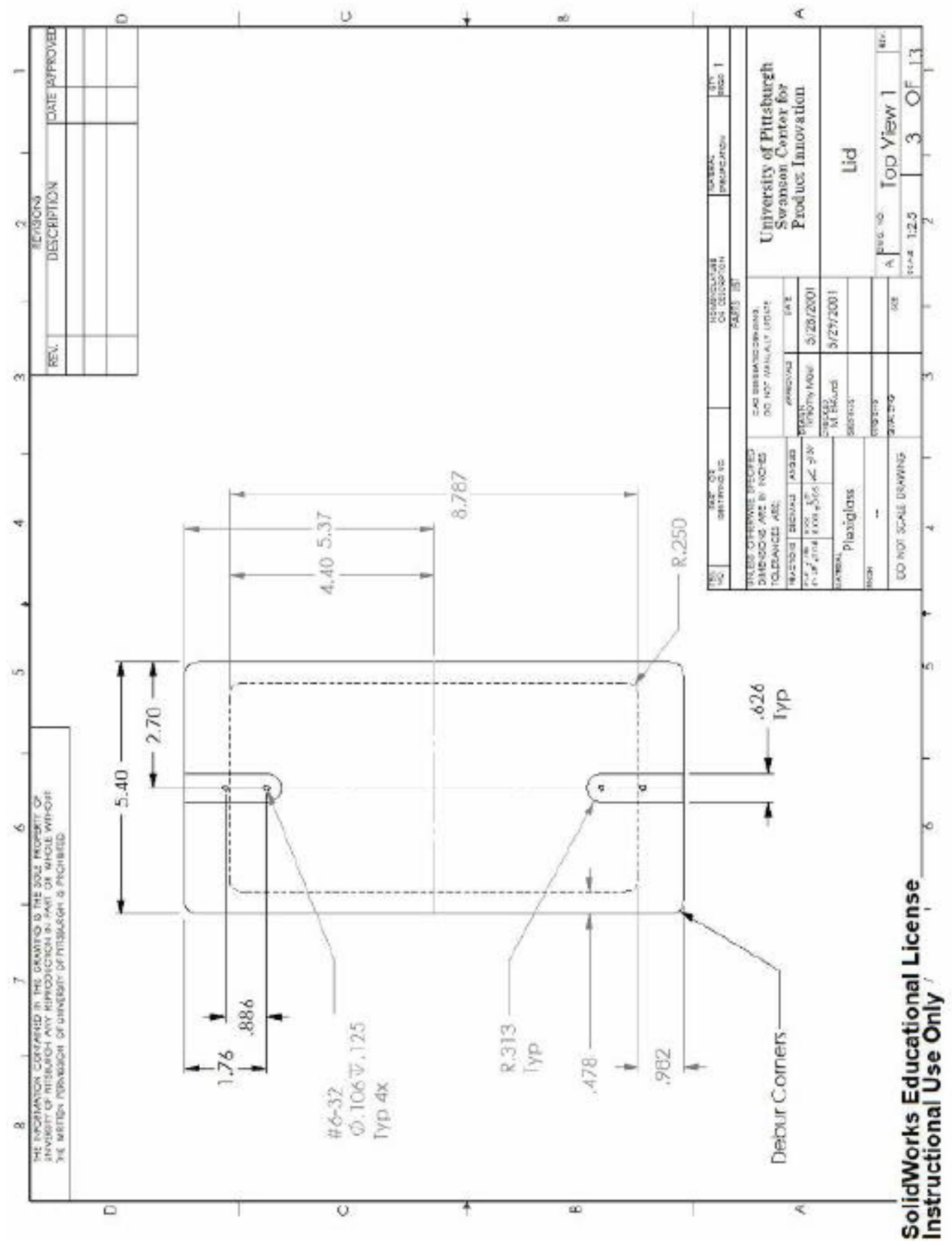

Figure A. 42 Solidworks technical drawing of top view of lid for transport box assembly. 


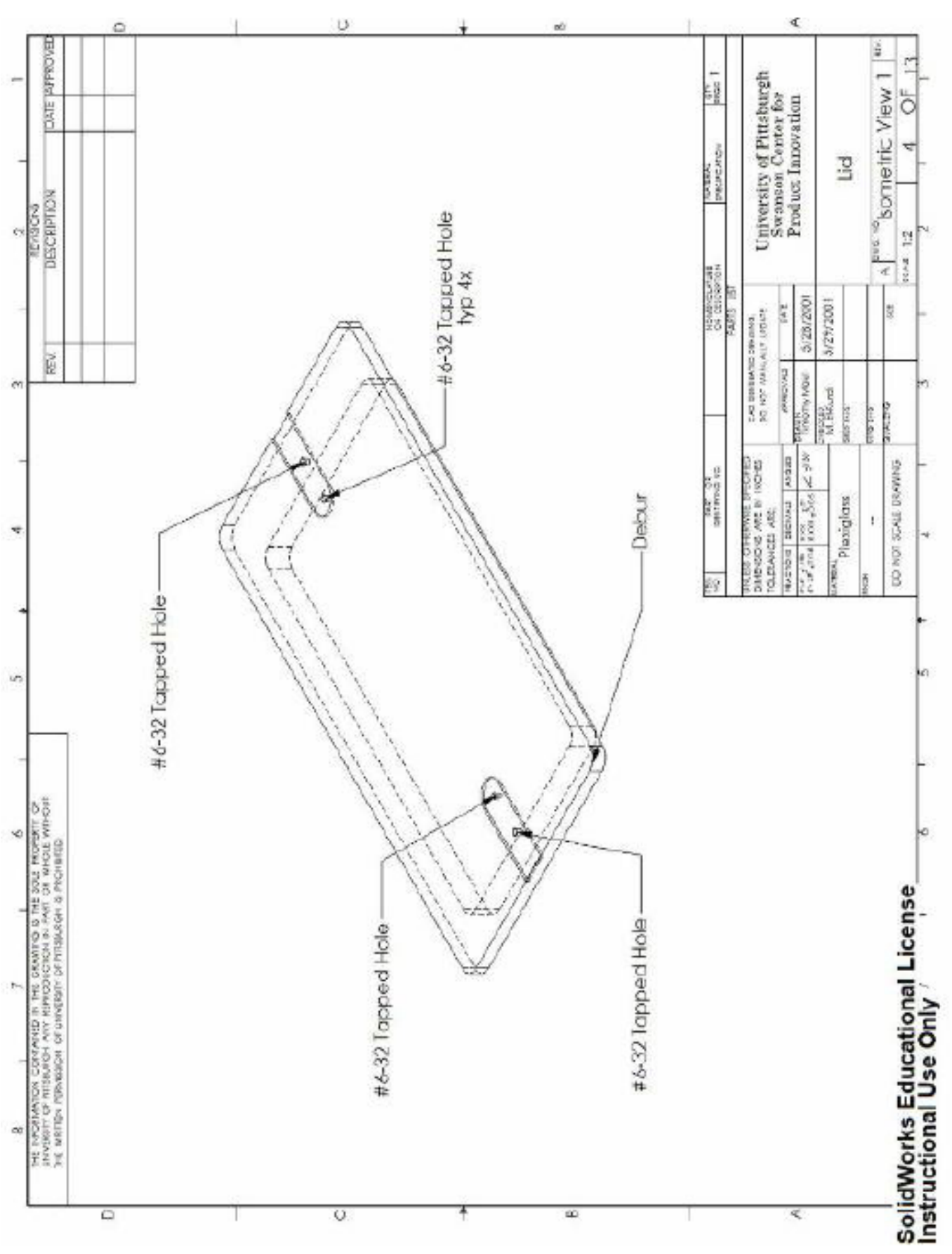

Figure A. 43 Solidworks technical drawing of isometric view of lid for transport box assembly. 


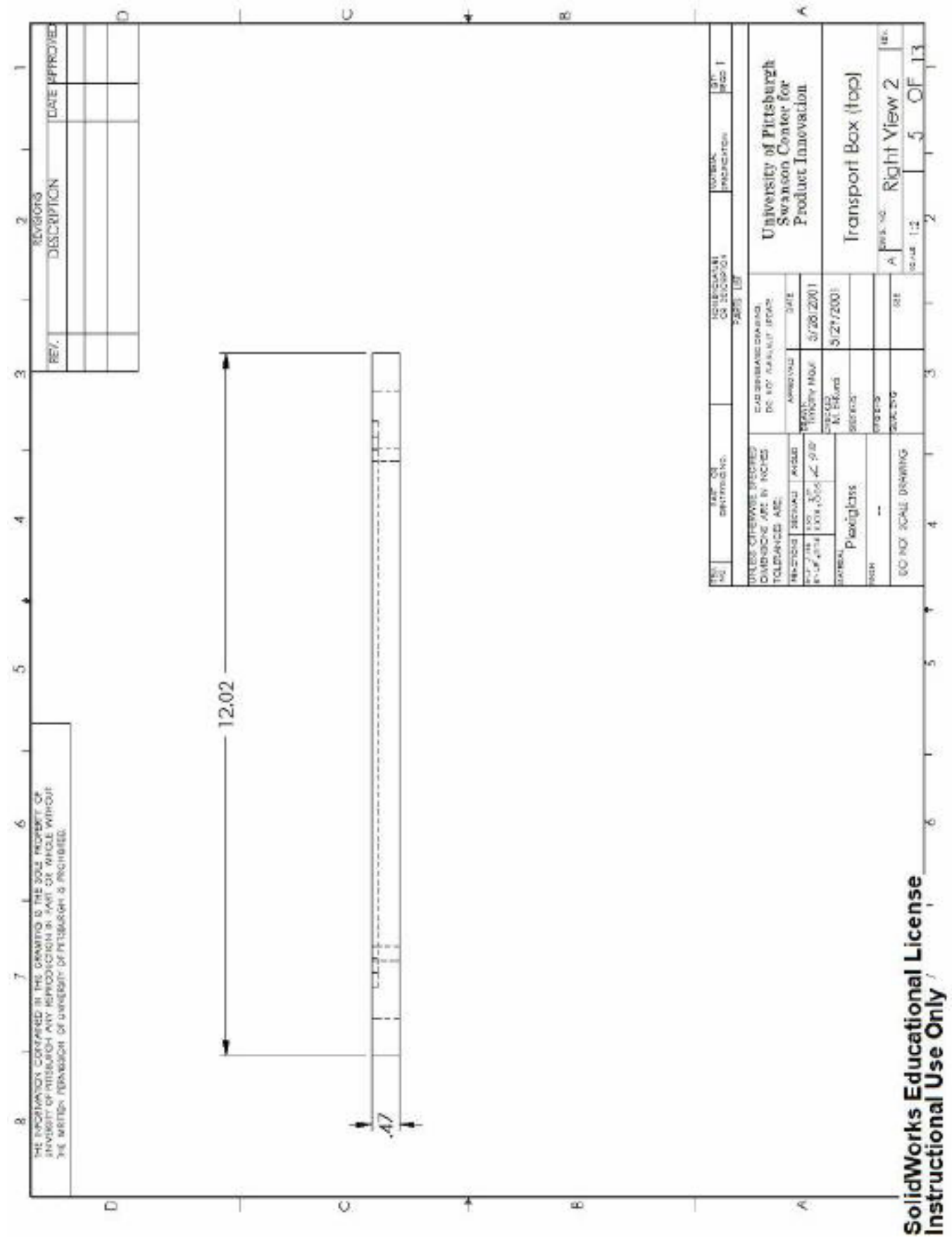

Figure A. 44 Solidworks technical drawing of right side view of top for transport box assembly. 


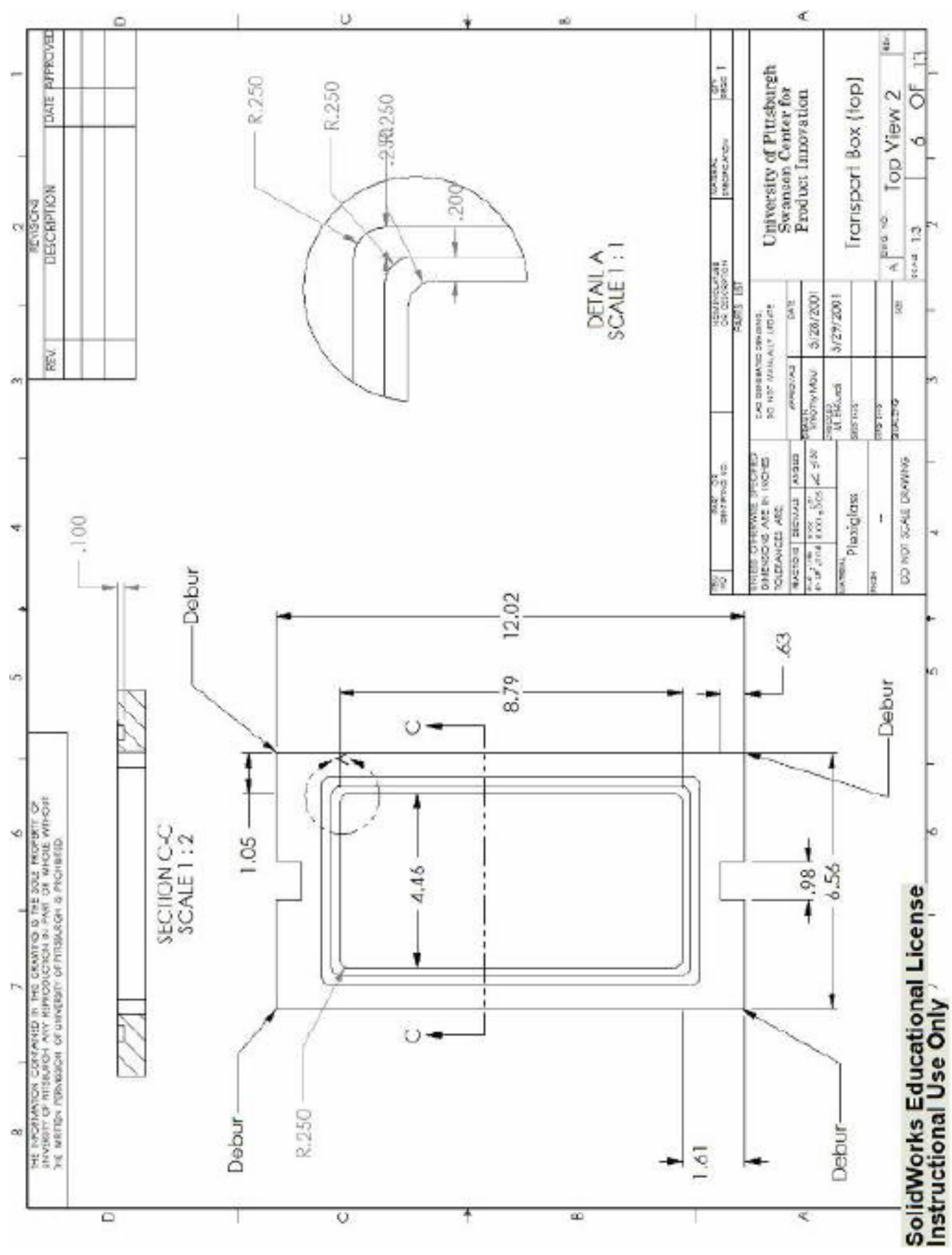

Figure A. 45 Solidworks technical drawing of top view of top for transport box assembly. 


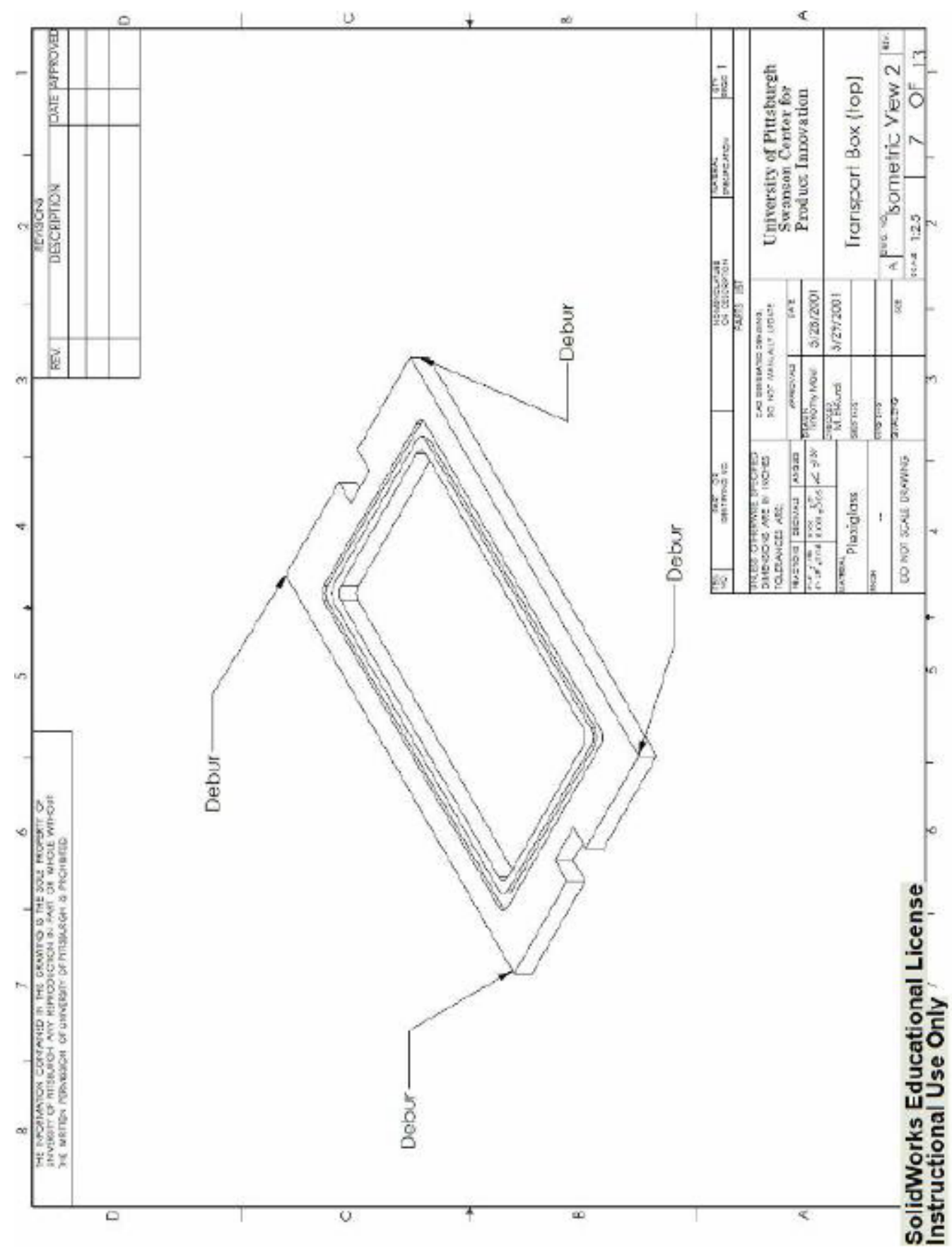

Figure A. 46 Solidworks technical drawing of isometric view of top for transport box assembly. 


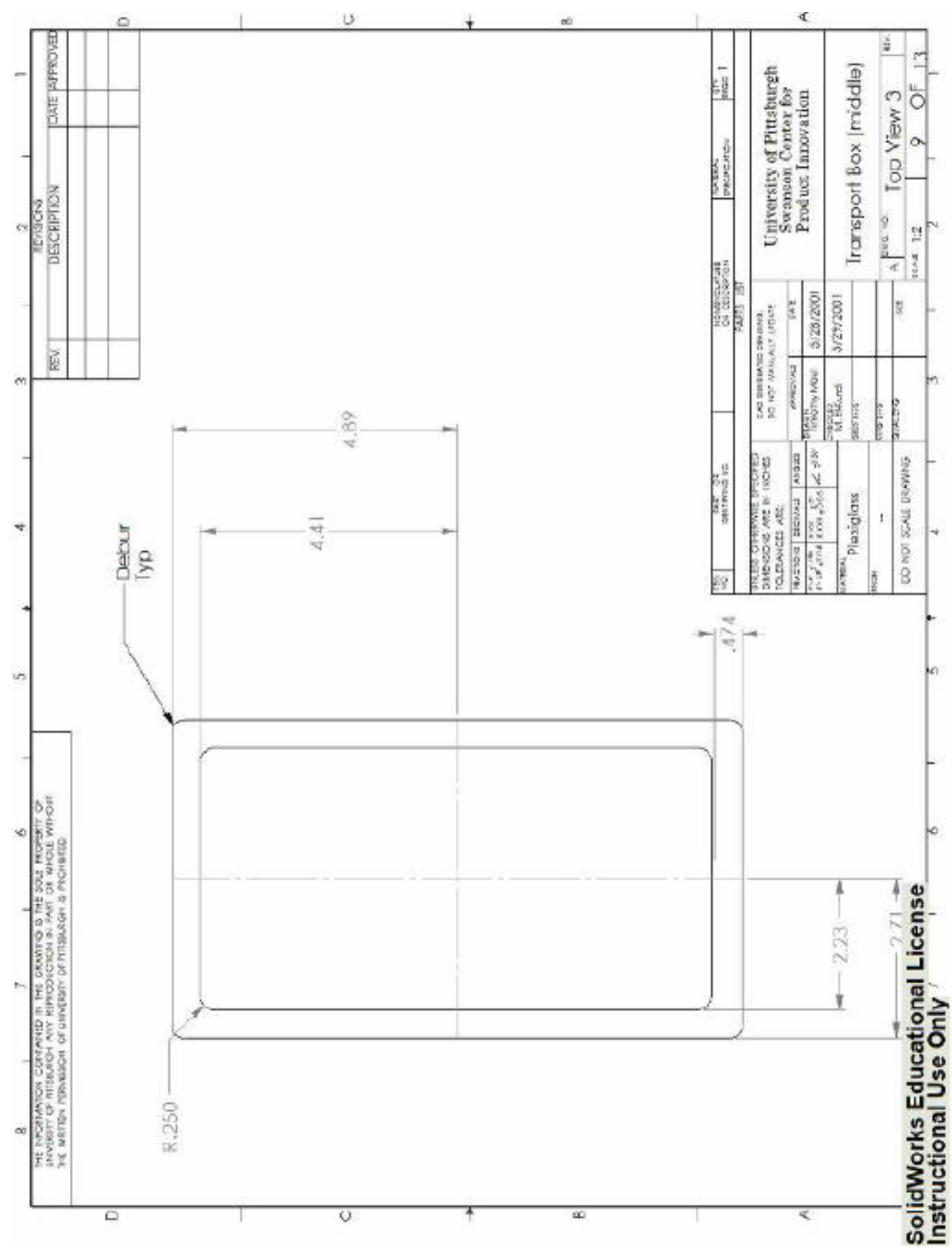

Figure A. 47 Solidworks technical drawing of top view of middle for transport box assembly. 


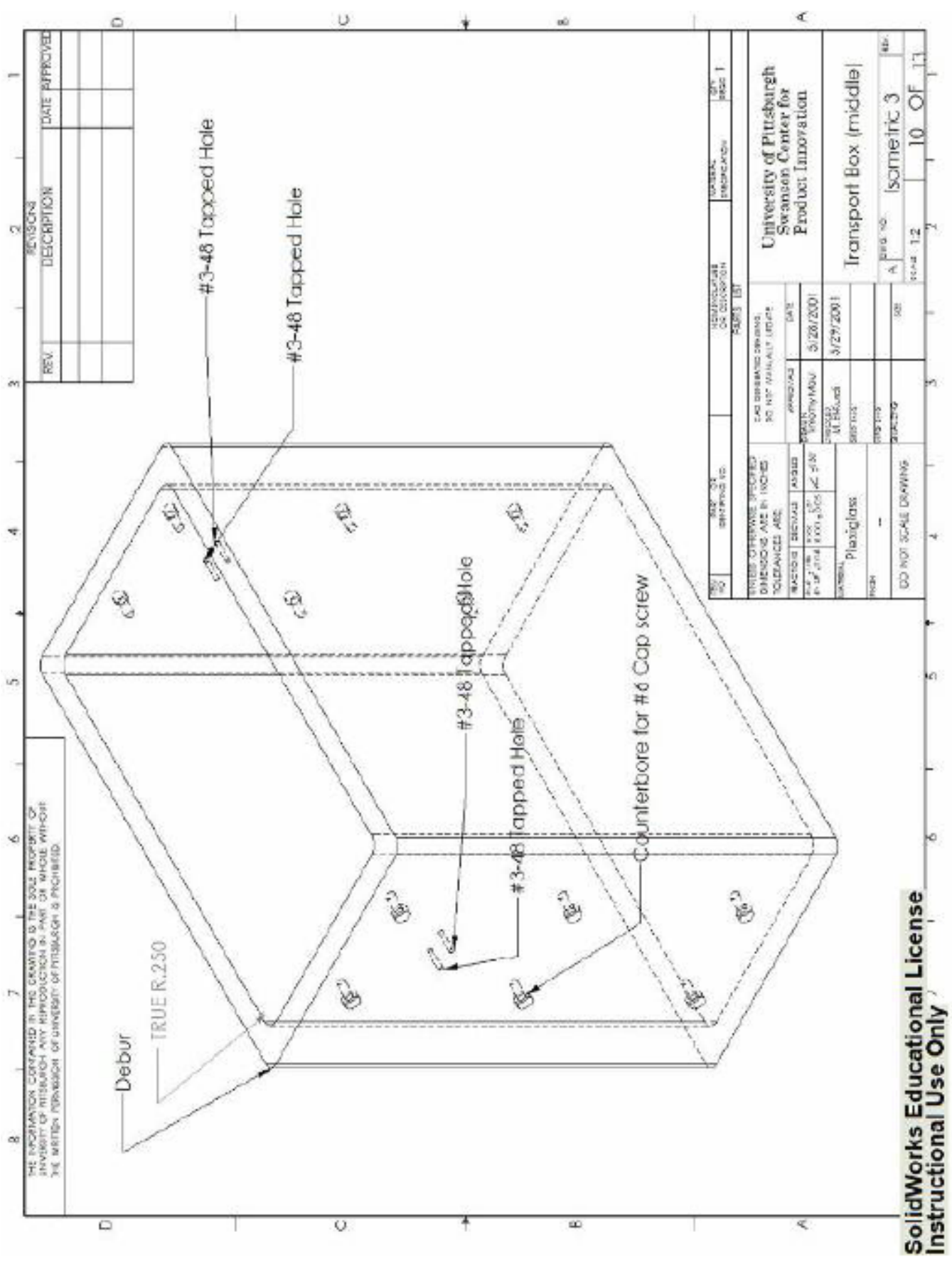

Figure A. 48 Solidworks technical drawing of isometric view of middle for transport box assembly. 


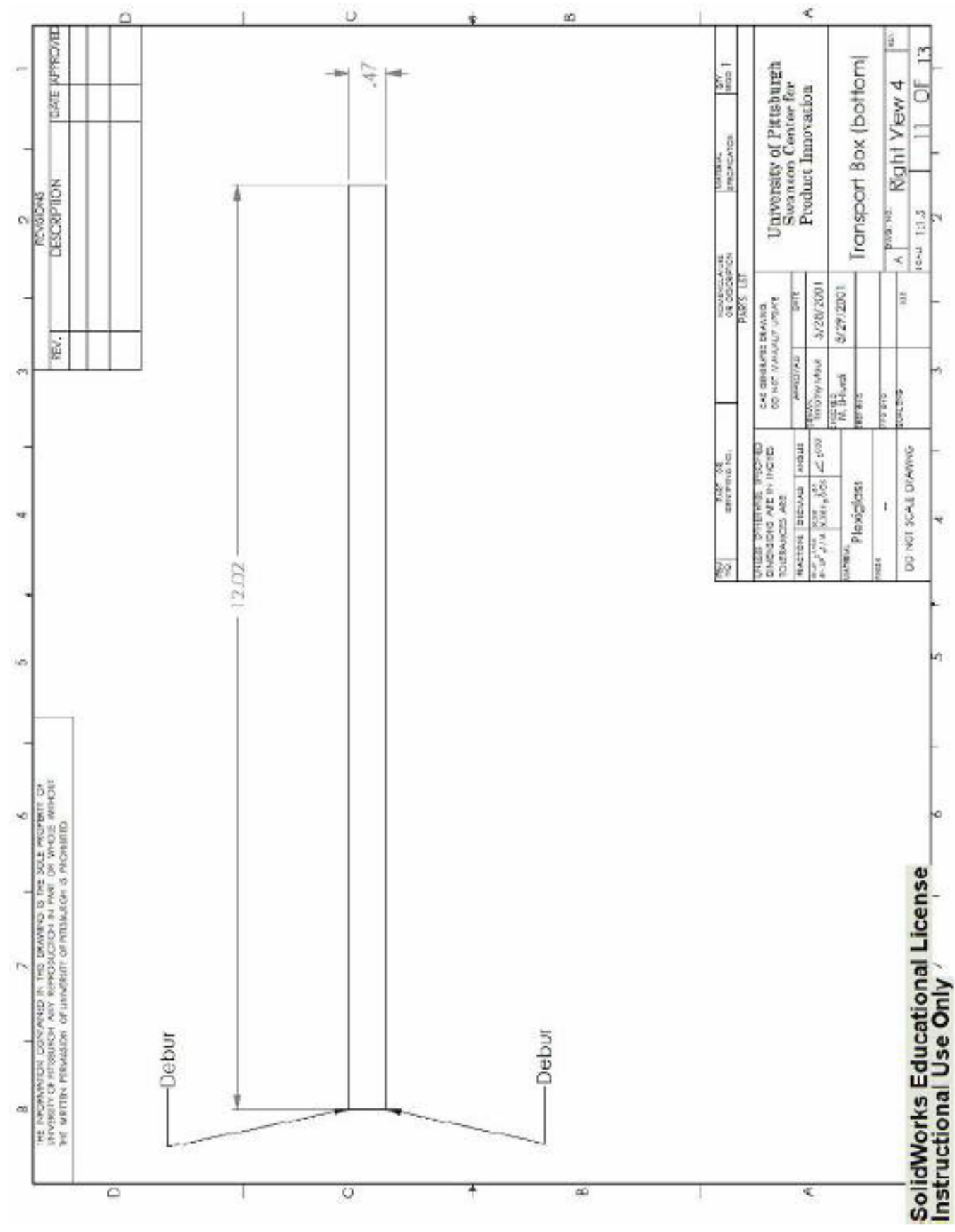

Figure A. 49 Solidworks technical drawing of right side view of bottom for transport box assembly. 


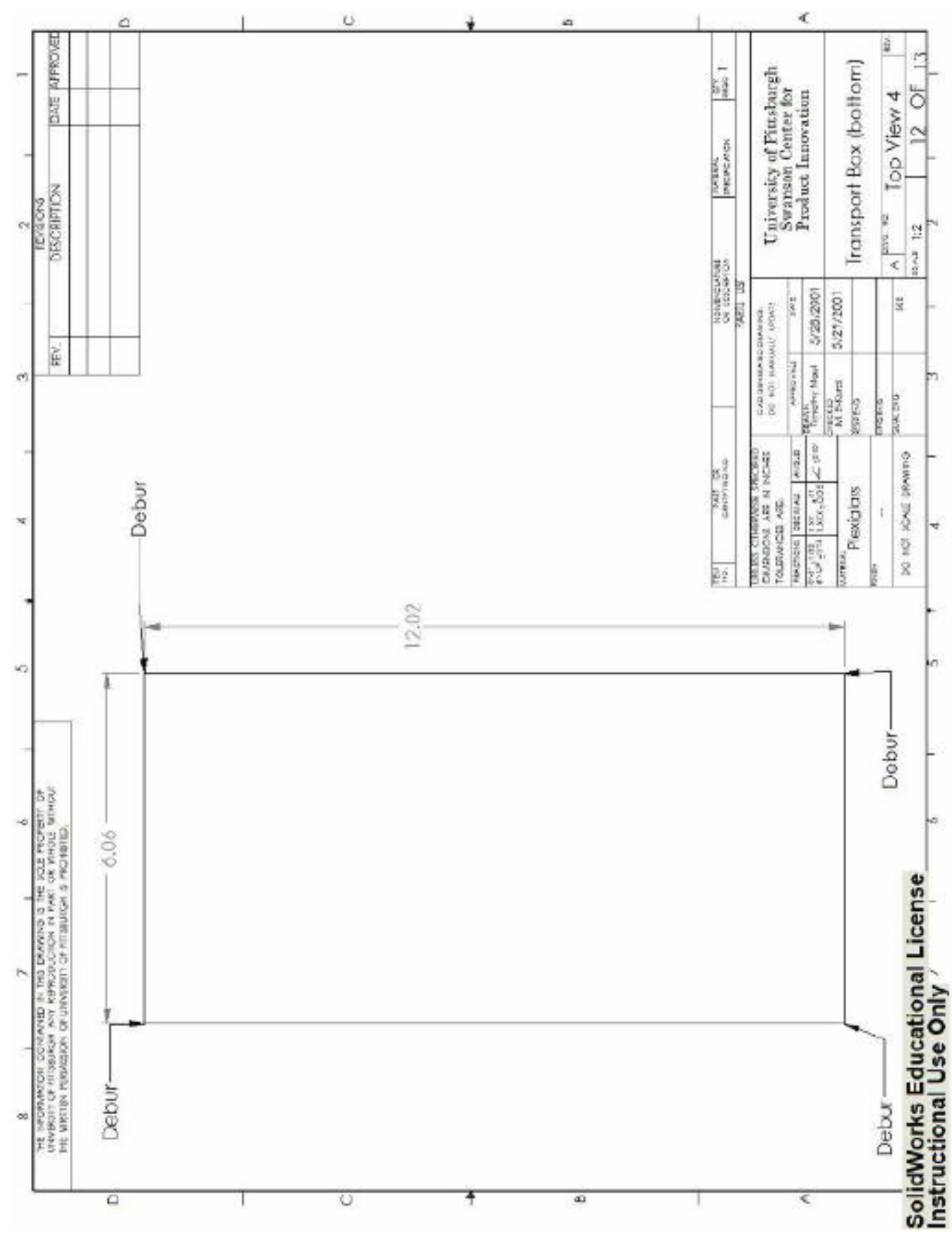

Figure A. 50 Solidworks technical drawing of top view of bottom for transport box assembly. 


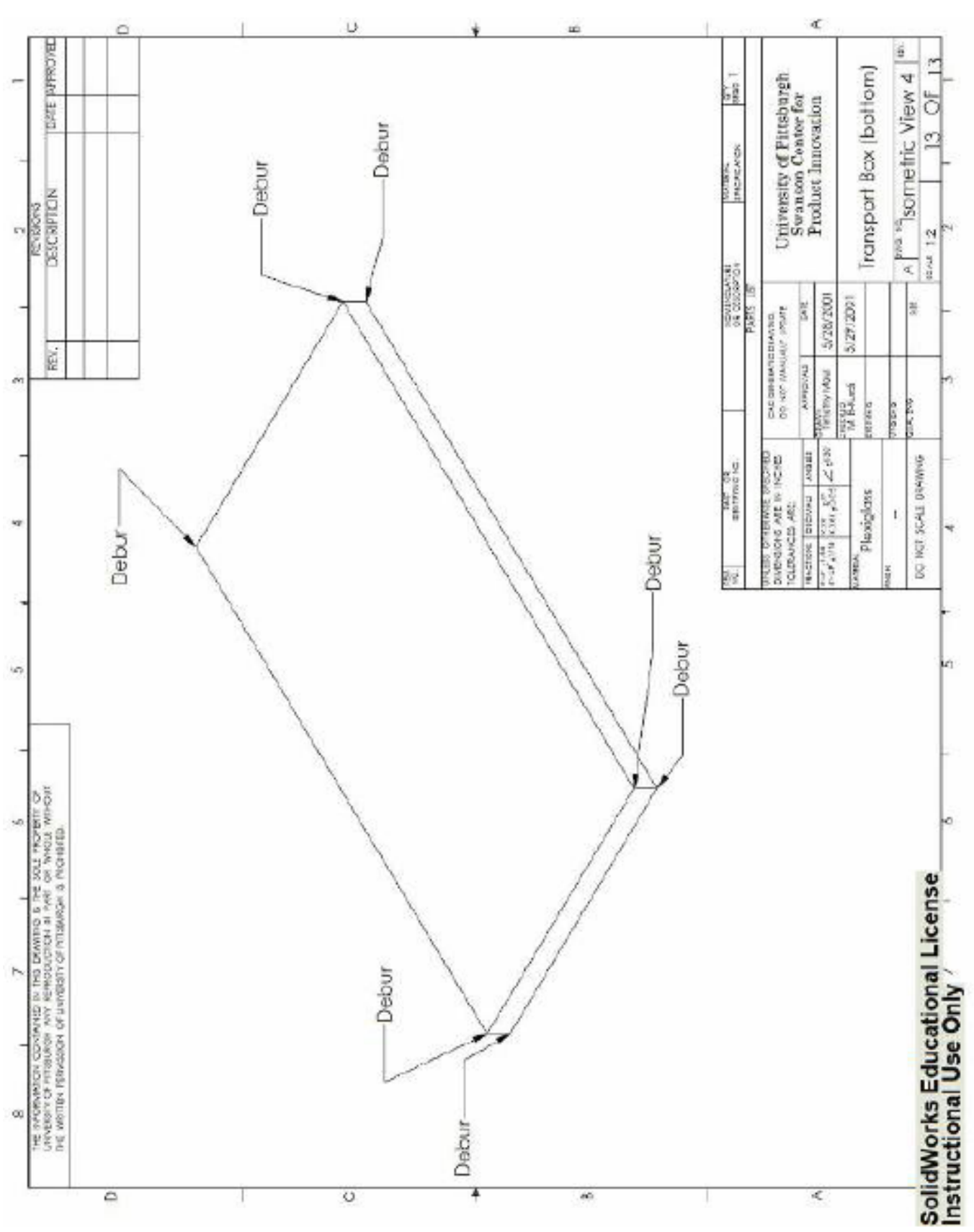

Figure A. 51 Solidworks technical drawing of isometric view of bottom for transport box assembly. 


\section{APPENDIX B}

\section{SYSID MATLAB ${ }^{\circledR}$ CODE}

The Matlab ${ }^{\circledR}$ programs that were used to generate the input/output data used in the SYSID algorithm are presented in this Appendix. Also, the program that was used to convert the SYSID generated state-space models into transfer functions is provided.

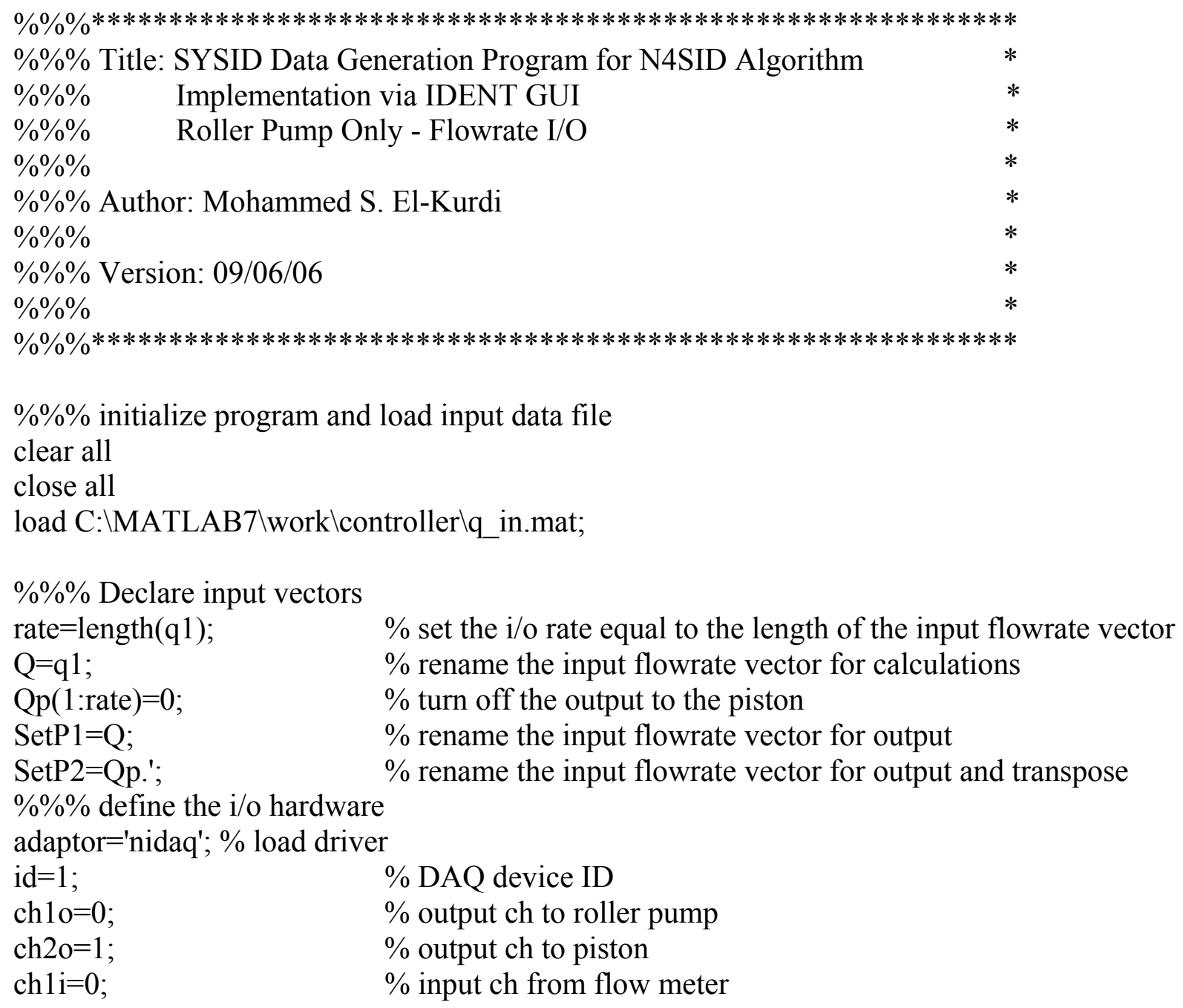




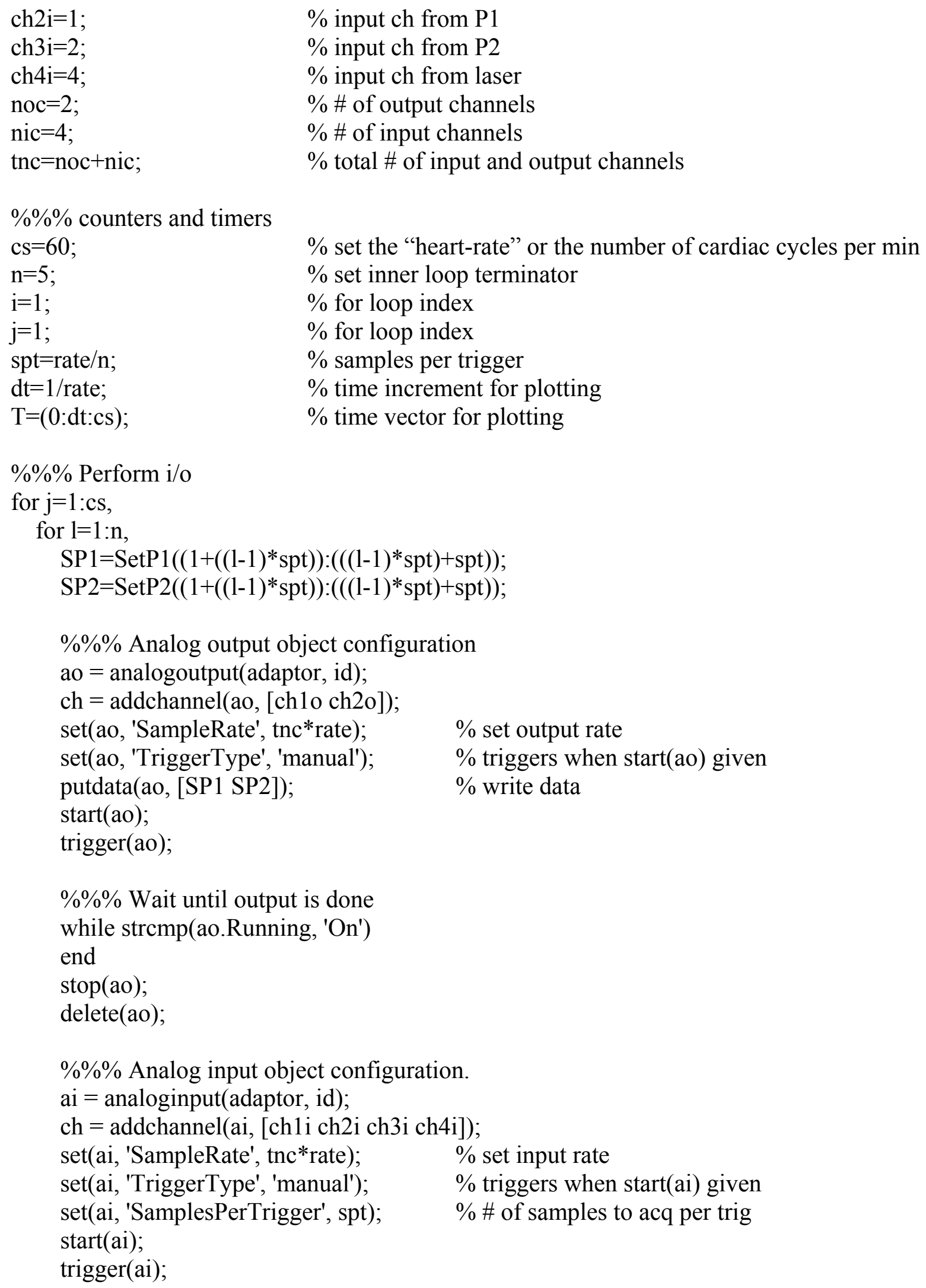


$\% \% \%$ Append acquired data

data $=$ getdata(ai);

Flow $\left(\left(1+\left((1-1)^{*}\right.\right.\right.$ spt $\left.)\right):\left(\left((1-1)^{*}\right.\right.$ spt $)+$ spt $\left.)\right)=$ data(:,1).';

pressure $\left.1\left((1+((1-1) * \mathrm{spt})):\left(\left((1-1)^{*} \mathrm{spt}\right)+\mathrm{spt}\right)\right)=\mathrm{data}(:, 2) \cdot\right)^{\prime} ;$

pressure $2\left(\left(1+\left((1-1)^{*} \mathrm{spt}\right)\right):\left(\left((1-1)^{*} \mathrm{spt}\right)+\mathrm{spt}\right)\right)=\mathrm{data}(:, 3) \cdot ;$

$\operatorname{OutP}((1+((1-1) * \mathrm{spt})):(((1-1) * \mathrm{spt})+\mathrm{spt}))=(\operatorname{pressure} 1((1+((1-1) * \mathrm{spt})):(((1-$

$\left.\left.\left.1)^{*} \mathrm{spt}\right)+\mathrm{spt}\right)\right)+$ pressure2((1+((1-1)*spt)):(((1-1)*spt)+spt)))./2;

OuterDiameter((1+((1-1)*spt)):(((1-1)*spt)+spt))=data(:,4).';

$\% \%$ Wait until intput is done

while strcmp(ai.Running, 'On')

end

stop(ai);

delete(ai);

$1=1+1$;

end

$\% \% \%$ Filter output using a first order Savitzky-Golay filter

$\mathrm{n} 1=1$

$\%$ order of filters for all raw data

$\mathrm{m} 1=71$

$\%$ frame size of filter for raw pressure data

$\mathrm{m} 2=31$

$\%$ frame size of filter for raw flowrate data

$\mathrm{m} 3=13$;

$\%$ frame size of filter for raw outer diameter data

OutPf=sgolayfilt(OutP,n1,m1);

$\%$ filter pressure data

Flowf=sgolayfilt(Flow,n1,m2);

$\%$ filter flowrate data

ODf=sgolayfilt(OuterDiameter,n1,m3); \% filter outer diameter data

$\% \% \%$ Filter output again using a first order Savitzky-Golay filter

OutPf=sgolayfilt(OutPf,n1,m1); $\quad \%$ filter pressure data

Flowf=sgolayfilt(Flowf,n1,m2); $\quad \%$ filter flowrate data

$\mathrm{ODf}=$ sgolayfilt(ODf,n1,m3); $\quad \%$ filter outer diameter data

$\% \% \%$ Transpose acquired data to column vectors

OutPf=OutPf.';

Flowf=Flowf.';

ODf=ODf.';

$\% \% \%$ Append $\mathrm{i} / \mathrm{o}$ vectors for plotting

SetPMasterPlot $((1+((\mathrm{j}-1) *$ rate $)):((\mathrm{j}-1) *$ rate $)+$ rate $))=\mathrm{q} 1(:) ;$

MFlow $((1+((\mathrm{j}-1) *$ rate $)):(((\mathrm{j}-1) *$ rate $)+$ rate $))=$ Flowf $(:) . * 400$;

$\operatorname{MP}((1+((\mathrm{j}-1) *$ rate $)):((\mathrm{j}-1) *$ rate $)+$ rate $))=\operatorname{OutPf}(:) . * 56.76$;

$\operatorname{MOD}((1+((j-1) *$ rate $)):(((j-1) *$ rate $)+$ rate $))=\operatorname{ODf}(:) ;$

$\mathrm{j}=\mathrm{j}+1$;

looper $=\mathrm{j}$

end

$\% \% \%$ eliminate skewness of data 
$\mathrm{i}=1$;

$\mathrm{z}=3$;

for $\mathrm{i}=1$ :length(SetPMasterPlot)-z,

$\%$ shifts input $\mathrm{z}$ pts to the right

SetPMasterPlot $(\mathrm{i})=\operatorname{SetPMasterPlot}(\mathrm{i}+\mathrm{z})$;

end

$\% \% \%$ Create IDDATA object for importation into IDENT GUI

y=MFlow.';

$\mathrm{u}=$ SetPMasterPlot.';

dat=iddata $(y, u, d t)$

$\% \%$ stop pump and piston

waveform $1 \mathrm{a}=0$;

ao = analogoutput(adaptor, id);

$\mathrm{ch}=\operatorname{addchannel}(\mathrm{ao},[\mathrm{ch} 1 \mathrm{o} \operatorname{ch} 2 \mathrm{o}])$;

set(ao, 'SampleRate', rate);

set(ao, 'TriggerType', 'immediate');

putdata(ao, [waveformla waveform1a]);

start(ao);

stop(ao);

delete(ao);

$\% \%$ Plot i/o data

$\mathrm{T}=\mathrm{T}(1:$ length(MFlow $)$;

figure;

plot(T,MFlow,'b-');

hold on

plot(T,SetPMasterPlot,'r-');

axis([0 T(length(MFlow)) $01.05 *(\max ($ SetPMasterPlot $))])$;

title('Measured vs Set Flowrate')

xlabel('Time (s)');

ylabel('Flowrate (cc/min)');

legend('Measured', 'SetPoint');

hold off 


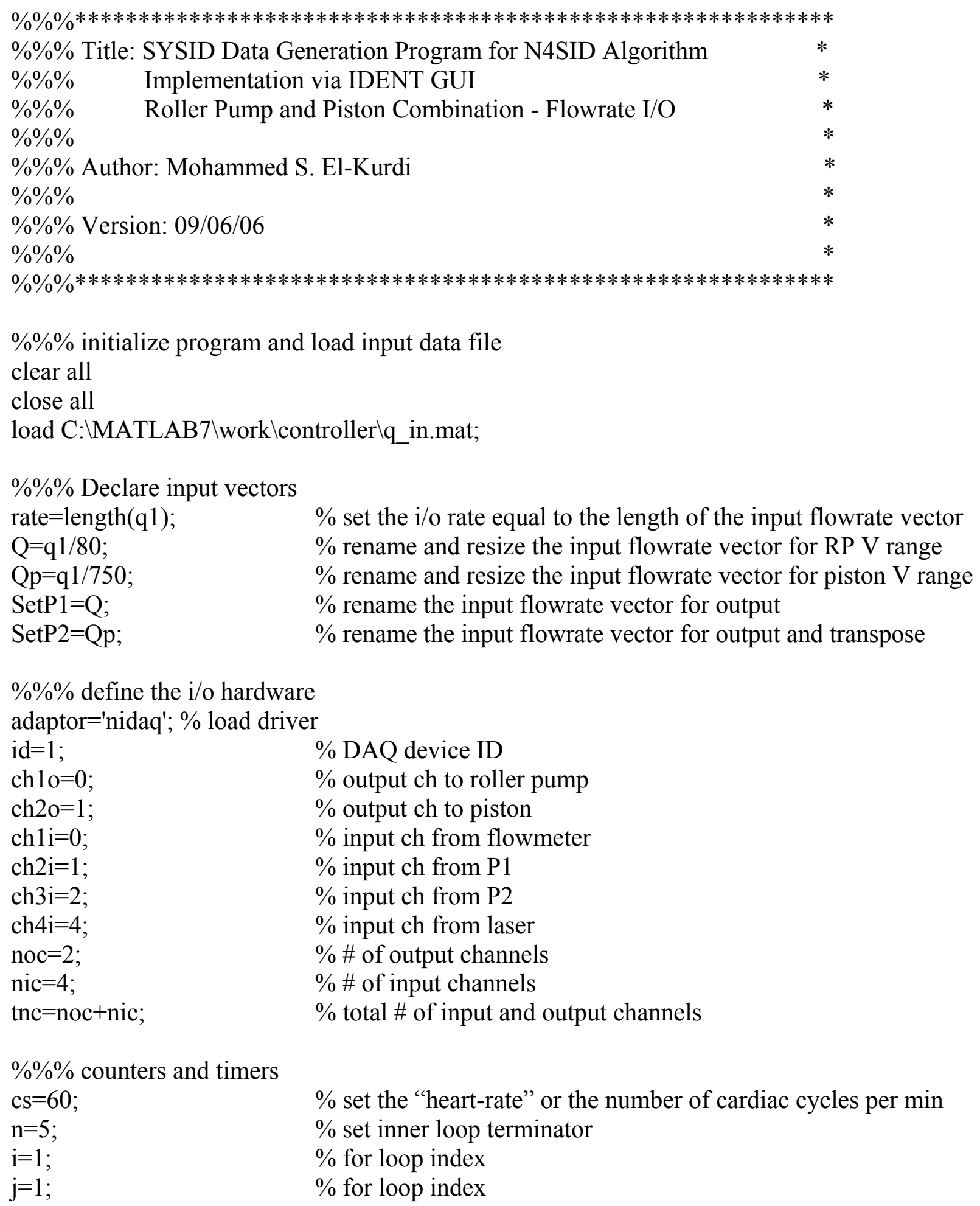









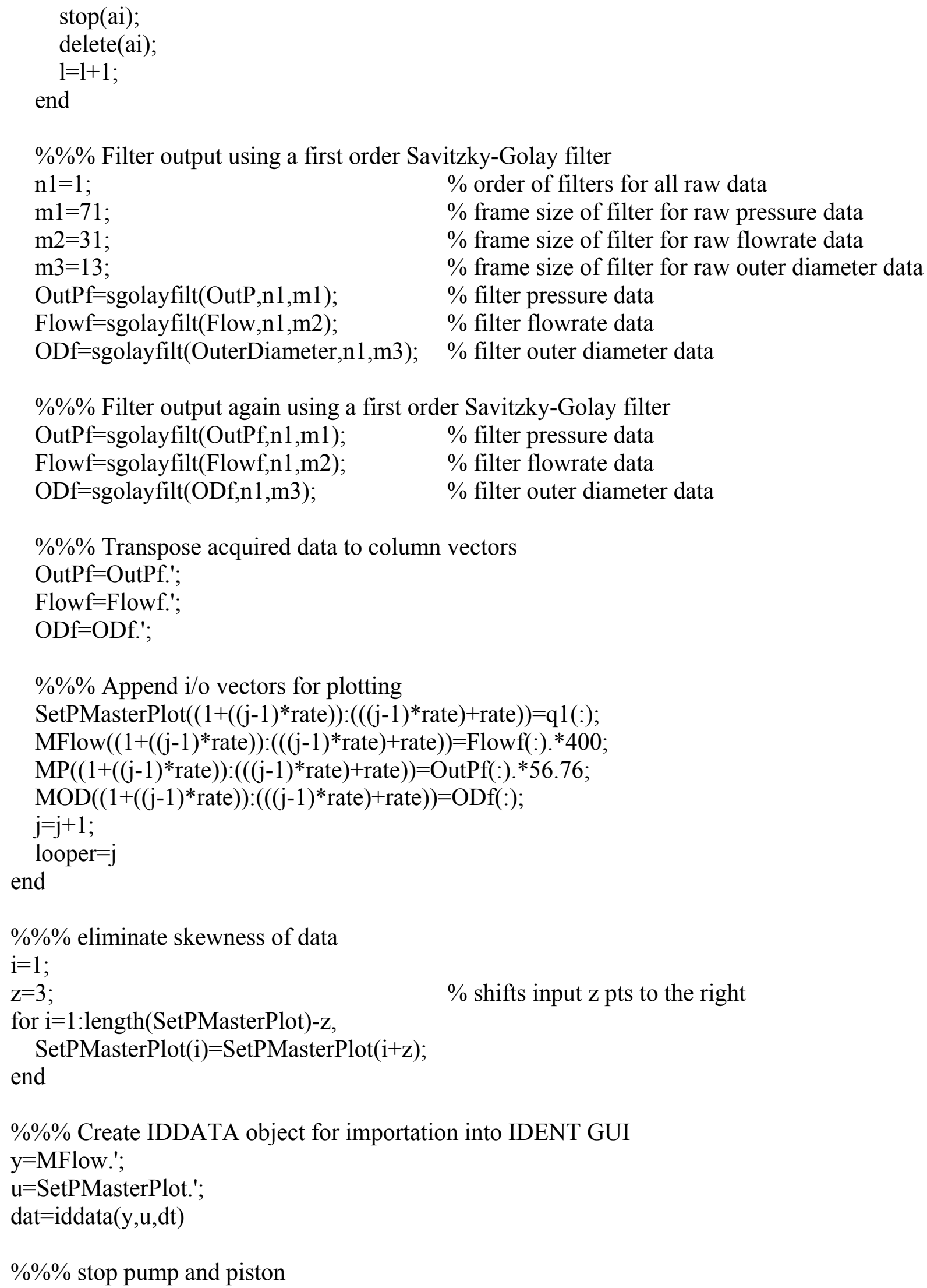




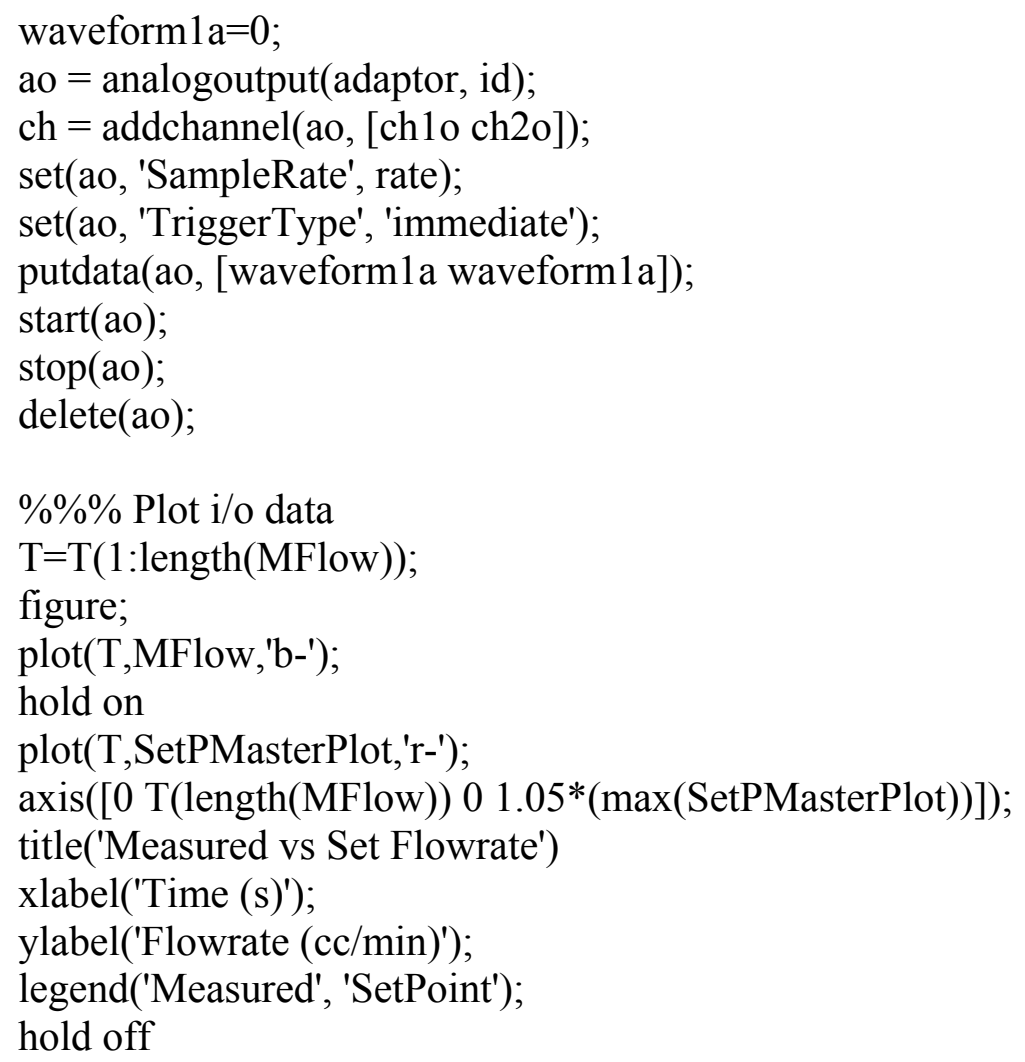




\begin{tabular}{|c|c|c|}
\hline$\% \% \%$ & SYSID Data Generation Program for N4SID Algorithm & $*$ \\
\hline$\% \% \%$ & Implementation via IDENT GUI & $*$ \\
\hline$\% \% \%$ & Roller Pump Only - Pressure I/O & * \\
\hline$\% \% \%$ & & * \\
\hline$\% \% \%$ & or: Mohammed S. El-Kurdi & $*$ \\
\hline$\% \% \%$ & & $*$ \\
\hline$\% \% \%$ & on: $09 / 06 / 06$ & $*$ \\
\hline$\% \% \%$ & & $*$ \\
\hline
\end{tabular}

$\% \% \%$ initialize program and load input data file

clear all

close all

load C: MMATLAB7\work\controller $\backslash p \_$in.mat;

$\% \% \%$ Declare input vectors

rate $=$ length $(\mathrm{p} 1) ; \quad \%$ set the $\mathrm{i} / \mathrm{o}$ rate equal to the length of the input pressure vector

$\mathrm{P}(1:$ rate $)=1 ; \quad \%$ step input voltage to $\mathrm{RP}$

$\operatorname{Pp}(1:$ rate $)=0 ; \quad \%$ turn off pitson

SetP1=P.'; $\quad \%$ rename and transpose RP output voltage

SetP2=Pp.'; $\quad \%$ rename and transpose piston voltage

$\% \% \%$ define the $\mathrm{i} / \mathrm{o}$ hardware

adaptor='nidaq'; $\quad \%$ load driver

$\mathrm{id}=1 ; \quad \%$ DAQ device ID

$\operatorname{ch} 1 \mathrm{o}=0 ; \quad \%$ output ch to roller pump

$\operatorname{ch} 2 \mathrm{o}=1 ; \quad \%$ output ch to piston

ch $1 \mathrm{i}=0 ; \quad \%$ input ch from flowmeter

$\operatorname{ch} 2 \mathrm{i}=1 ; \quad \%$ input ch from $\mathrm{P} 1$

$\operatorname{ch} 3 \mathrm{i}=2 ; \quad \%$ input ch from $\mathrm{P} 2$

ch $4 \mathrm{i}=4 ; \quad \%$ input ch from laser

noc $=2 ; \quad \%$ \# of output channels

nic $=4 ; \quad \%$ \# of input channels

tnc $=$ noc + nic; $\quad \%$ total $\#$ of input and output channels

$\% \% \%$ counters and timers

$\mathrm{cs}=60 ; \quad \%$ set the "heart-rate" or the number of cardiac cycles per min

$\mathrm{n}=5$; $\quad \%$ set inner loop terminator

$\mathrm{i}=1 ; \quad \%$ for loop index

$\mathrm{j}=1 ; \quad \%$ for loop index 


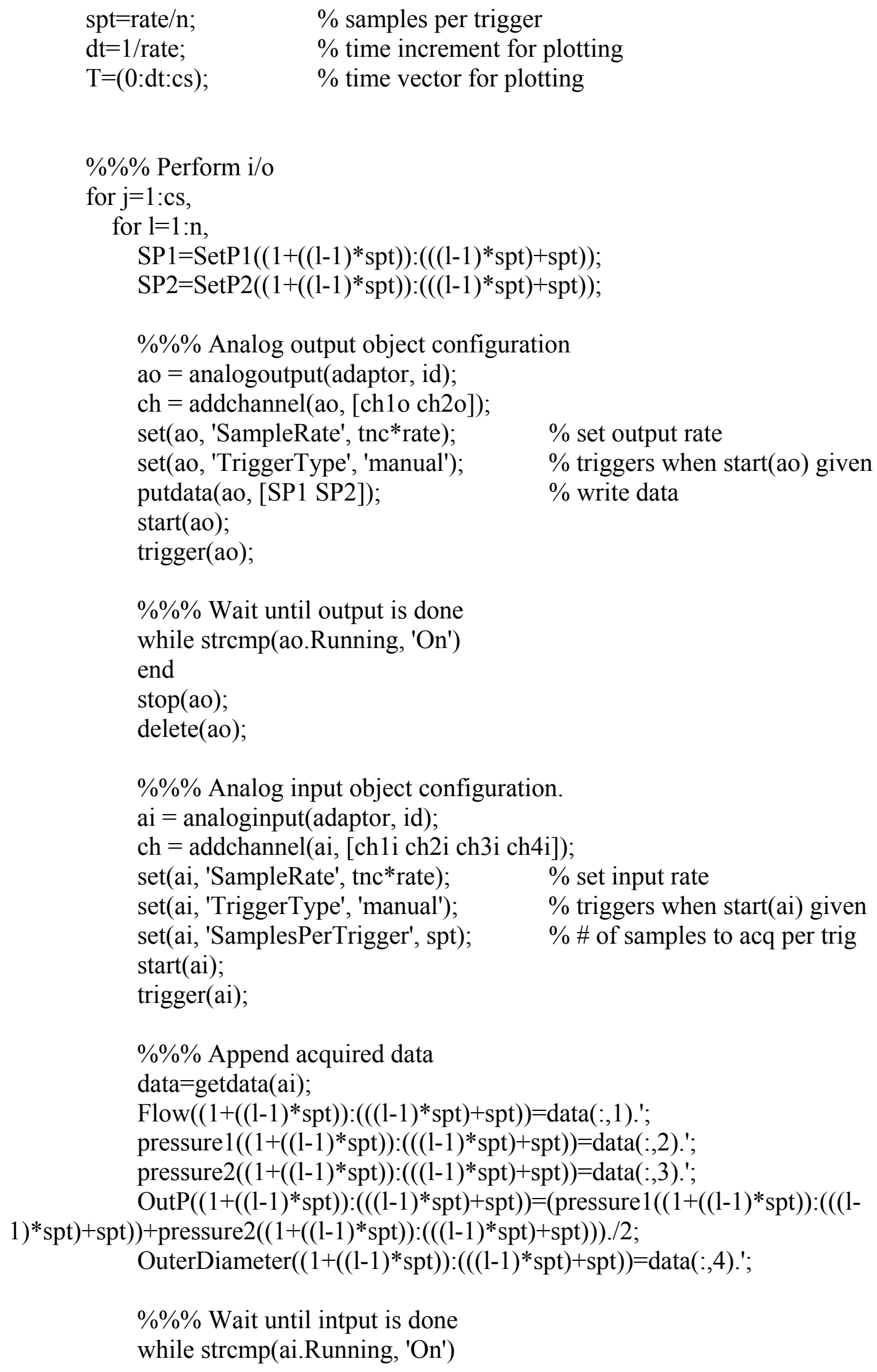




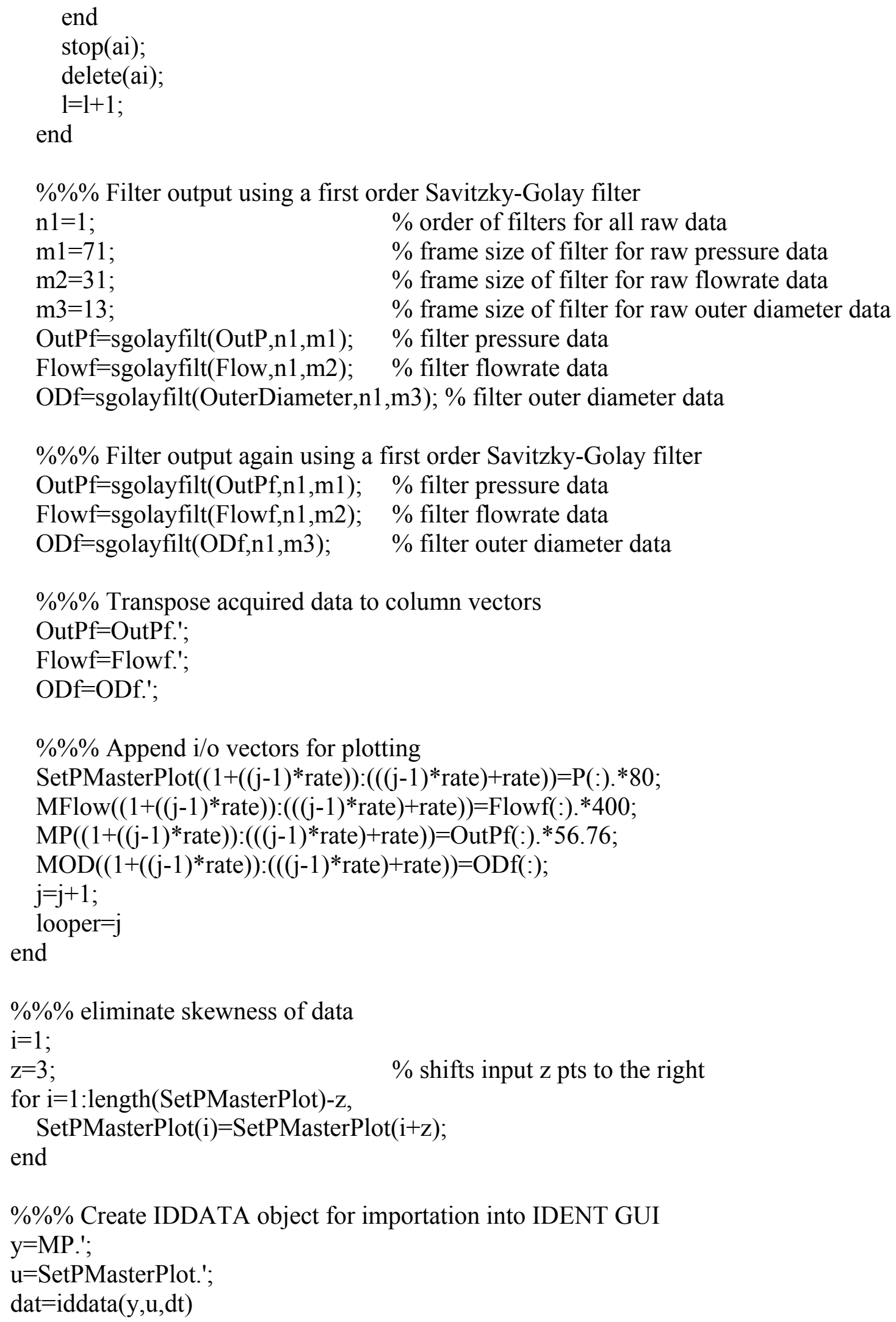


$\% \% \%$ stop pump and piston

waveform $1 \mathrm{a}=0$;

ao = analogoutput(adaptor, id);

$\mathrm{ch}=\operatorname{addchannel}(\mathrm{ao}$, [ch1o ch2o]);

set(ao, 'SampleRate', rate);

set(ao, 'TriggerType', 'immediate');

putdata(ao, [waveform1a waveformla]);

start(ao);

stop(ao);

delete(ao);

$\% \% \%$ Plot i/o data

$\mathrm{T}=\mathrm{T}(1:$ length(MP));

figure;

$\operatorname{plot}\left(\mathrm{T}, \mathrm{MP}, \mathrm{b}^{-}\right)$;

hold on

plot(T,SetPMasterPlot,'r-');

axis([0 T(length(MP)) 0 1.05*(max(SetPMasterPlot)) $])$;

title('Measured vs Set Pressure')

xlabel('Time (s)');

ylabel('Pressure $(\mathrm{mmHg})$ ');

legend('Measured', 'SetPoint');

hold off 
$\begin{array}{lr}\% \% \% * * * * * * * * * * * * * * * * * * * * * * * * * * * * * * * * * * * * * * * * * * * * * * * * * * * * * * * * * * * * \\ \% \% \% \text { Title: SYSID Data Generation Program for N4SID Algorithm } & * \\ \% \% \% & \text { Implementation via IDENT GUI } \\ \% \% \% & * \\ \% \% \% & \text { Roller Pump Plus Piston Combination - Pressure I/O } \\ \% \% \% & * \\ \% \% \% \text { Author: Mohammed S. El-Kurdi } & * \\ \% \% \% & * \\ \% \% \% \text { Version: } 09 / 06 / 06 & * \\ \% \% \% & \\ \% \% \% * * * * * * * * * * * * * * * * * * * * * * * * * * * * * * * * * * * * * * * * * * * * * * * * * * * * * * * * * * * *\end{array}$

$\% \% \%$ initialize program and load input data file

clear all

close all

load C: MATLAB7\work\controller $\backslash p \_$in.mat;

$\% \% \%$ Declare input vectors

rate $=$ length $(\mathrm{p} 1) ; \quad \%$ set the $\mathrm{i} / \mathrm{o}$ rate equal to the length of the input pressure vector

$\mathrm{P}(1:$ rate $)=1 ; \quad \%$ step input voltage to $\mathrm{RP}$

$\mathrm{Pp}=\mathrm{p} 1 . / 357.5 ; \quad \%$ scale input to piston to proper voltage range

SetP1=P.'; $\quad \%$ rename and transpose RP output voltage

$\mathrm{SetP} 2=\mathrm{Pp} ; \quad \%$ rename and transpose piston voltage

$\% \% \%$ define the $\mathrm{i} / \mathrm{o}$ hardware

adaptor='nidaq'; $\quad \%$ load driver

$\mathrm{id}=1 ;$

$\operatorname{ch} 1 \mathrm{o}=0 ;$

$\operatorname{ch} 2 \mathrm{o}=1 ; \quad \%$ output ch to piston

ch $1 \mathrm{i}=0 ; \quad \%$ input ch from flowmeter

$\operatorname{ch} 2 \mathrm{i}=1 ; \quad \%$ input ch from $\mathrm{P} 1$

$\operatorname{ch} 3 \mathrm{i}=2 ; \quad \%$ input ch from $\mathrm{P} 2$

$\operatorname{ch} 4 \mathrm{i}=4 ; \quad \%$ input ch from laser

noc $=2 ; \quad \%$ \# of output channels

nic $=4 ; \quad \%$ \# of input channels

tnc $=$ noc + nic; $\quad \%$ total $\#$ of input and output channels

$\% \% \%$ counters and timers

$\mathrm{cs}=60 ; \quad \%$ set the "heart-rate" or the number of cardiac cycles per min

$\mathrm{n}=5$; $\quad \%$ set inner loop terminator

$\mathrm{i}=1 ; \quad \%$ for loop index

$\mathrm{j}=1 ; \quad \%$ for loop index

$\mathrm{spt}=$ rate $/ \mathrm{n} ; \quad \%$ samples per trigger 
$\begin{array}{ll}\mathrm{dt}=1 / \text { rate; } & \% \text { time increment for plotting } \\ \mathrm{T}=(0: \mathrm{dt}: \mathrm{cs}) ; & \% \text { time vector for plotting }\end{array}$

$\% \% \%$ Perform i/o

for $\mathrm{j}=1: \mathrm{cs}$,

for $\mathrm{l}=1: \mathrm{n}$,

$\mathrm{SP} 1=\operatorname{SetP} 1\left(\left(1+\left((1-1)^{*} \mathrm{spt}\right)\right):\left(\left((1-1)^{*} \mathrm{spt}\right)+\mathrm{spt}\right)\right)$;

$\mathrm{SP} 2=\operatorname{SetP} 2\left(\left(1+\left((1-1)^{*} \mathrm{spt}\right)\right):\left(\left((1-1)^{*} \mathrm{spt}\right)+\mathrm{spt}\right)\right)$;

$\% \% \%$ Analog output object configuration

ao $=$ analogoutput(adaptor, id);

$\mathrm{ch}=$ addchannel(ao, [ch1o ch2o]);

set(ao, 'SampleRate', tnc*rate); $\quad \%$ set output rate

set(ao, 'TriggerType', 'manual'); $\quad \%$ triggers when start(ao) given

putdata(ao, [SP1 SP2]);

$\%$ write data

start(ao);

trigger(ao);

$\% \% \%$ Wait until output is done

while strcmp(ao.Running, 'On')

end

stop(ao);

delete(ao);

$\% \% \%$ Analog input object configuration.

ai $=$ analoginput(adaptor, id);

ch = addchannel(ai, [ch1i ch2i ch3i ch4i]);

set(ai, 'SampleRate', tnc*rate); $\quad \%$ set input rate

set(ai, 'TriggerType', 'manual'); $\quad \%$ triggers when start(ai) given

set(ai, 'SamplesPerTrigger', spt); $\quad \%$ \# of samples to acq per trig

start(ai);

trigger(ai);

$\% \% \%$ Append acquired data

data $=$ getdata(ai);

Flow $\left(\left(1+\left((1-1)^{*} \mathrm{spt}\right)\right):\left(\left((1-1)^{*} \mathrm{spt}\right)+\mathrm{spt}\right)\right)=\operatorname{data}(:, 1)$. ';

pressure $\left.1\left(\left(1+\left((1-1)^{*} \mathrm{spt}\right)\right):\left(\left((1-1)^{*} \mathrm{spt}\right)+\mathrm{spt}\right)\right)=\operatorname{data}(:, 2)\right)^{\prime}$;

pressure $2\left(\left(1+\left((1-1)^{*} \mathrm{spt}\right)\right):\left(\left((1-1)^{*} \mathrm{spt}\right)+\mathrm{spt}\right)\right)=\operatorname{data}(:, 3) .{ }^{\prime}$;

OutP $((1+((1-1) * \operatorname{spt})):(((1-1) *$ spt $)+$ spt $))=($ pressure $1((1+((1-1) * \operatorname{spt})):(((1-$

$\left.\left.\left.\left.1)^{*} \mathrm{spt}\right)+\mathrm{spt}\right)\right)+\operatorname{pressure} 2\left(\left(1+\left((1-1)^{*} \mathrm{spt}\right)\right):\left(\left((1-1)^{*} \mathrm{spt}\right)+\mathrm{spt}\right)\right)\right) \cdot / 2$

OuterDiameter $\left(\left(1+\left((1-1)^{*} \mathrm{spt}\right)\right):\left(\left((1-1)^{*} \mathrm{spt}\right)+\mathrm{spt}\right)\right)=\mathrm{data}(:, 4) . '$;

$\% \% \%$ Wait until intput is done while strcmp(ai.Running, 'On')

end 


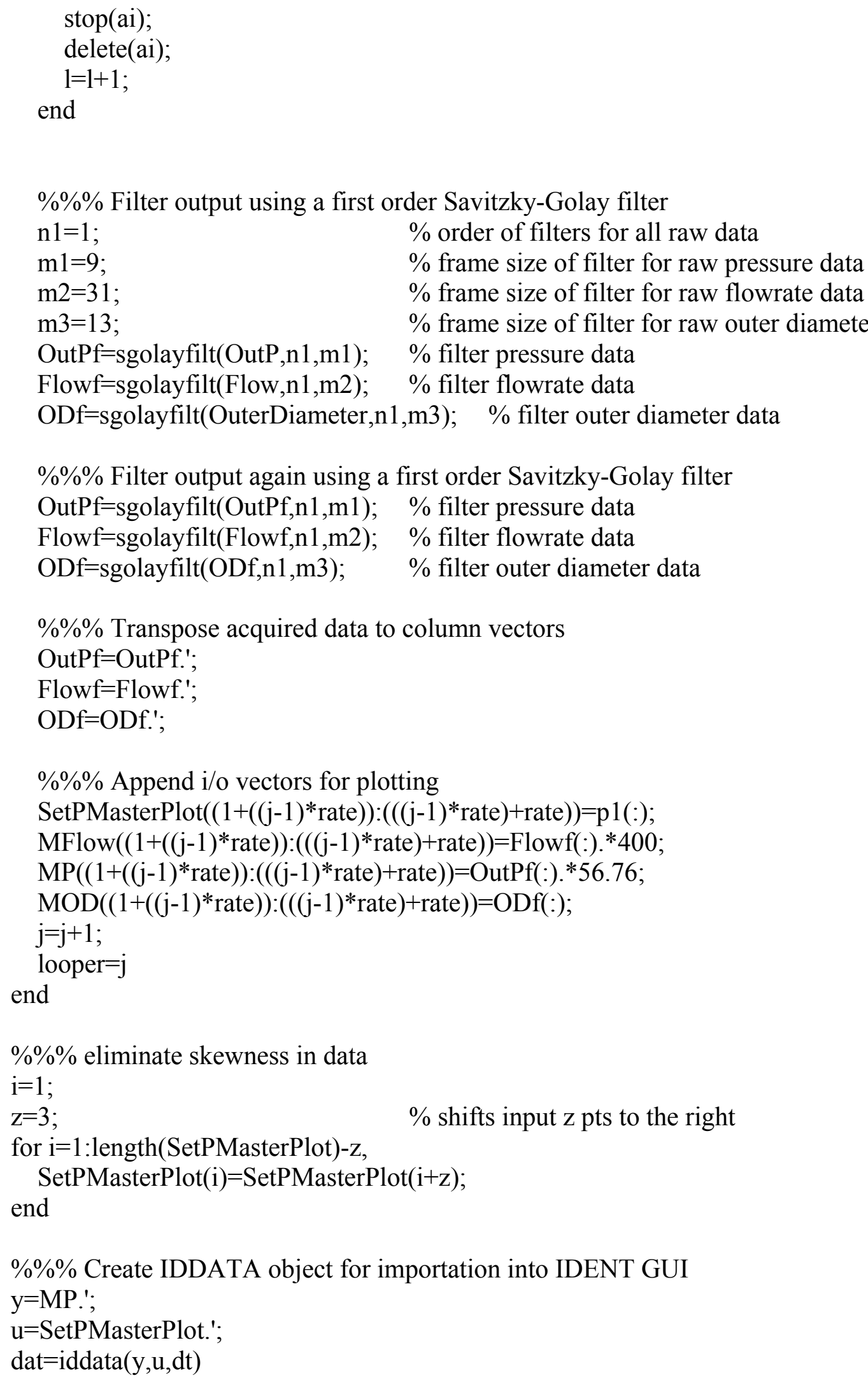


$\% \%$ stop pump and piston

waveform $1 \mathrm{a}=0$;

ao = analogoutput(adaptor, id);

$\mathrm{ch}=\operatorname{addchannel}(\mathrm{ao},[\mathrm{ch} 1 \mathrm{o} \operatorname{ch} 2 \mathrm{o}])$;

set(ao, 'SampleRate', rate);

set(ao, 'TriggerType', 'immediate');

putdata(ao, [waveform1a waveformla]);

start(ao);

stop(ao);

delete(ao);

$\% \% \%$ Plot i/o data

$\mathrm{T}=\mathrm{T}(1:$ length(MP));

figure;

$\operatorname{plot}\left(\mathrm{T}, \mathrm{MP}, \mathrm{b}^{-}\right)$;

hold on

plot(T,SetPMasterPlot,'r-');

axis([0 T(length(MP)) $\left.\left.01.05^{*}(\max (\mathrm{MP}))\right]\right)$;

title('Measured vs Set Pressure')

xlabel('Time (s)');

ylabel('Pressure $(\mathrm{mmHg})$ ');

legend('Measured', 'SetPoint');

hold off 


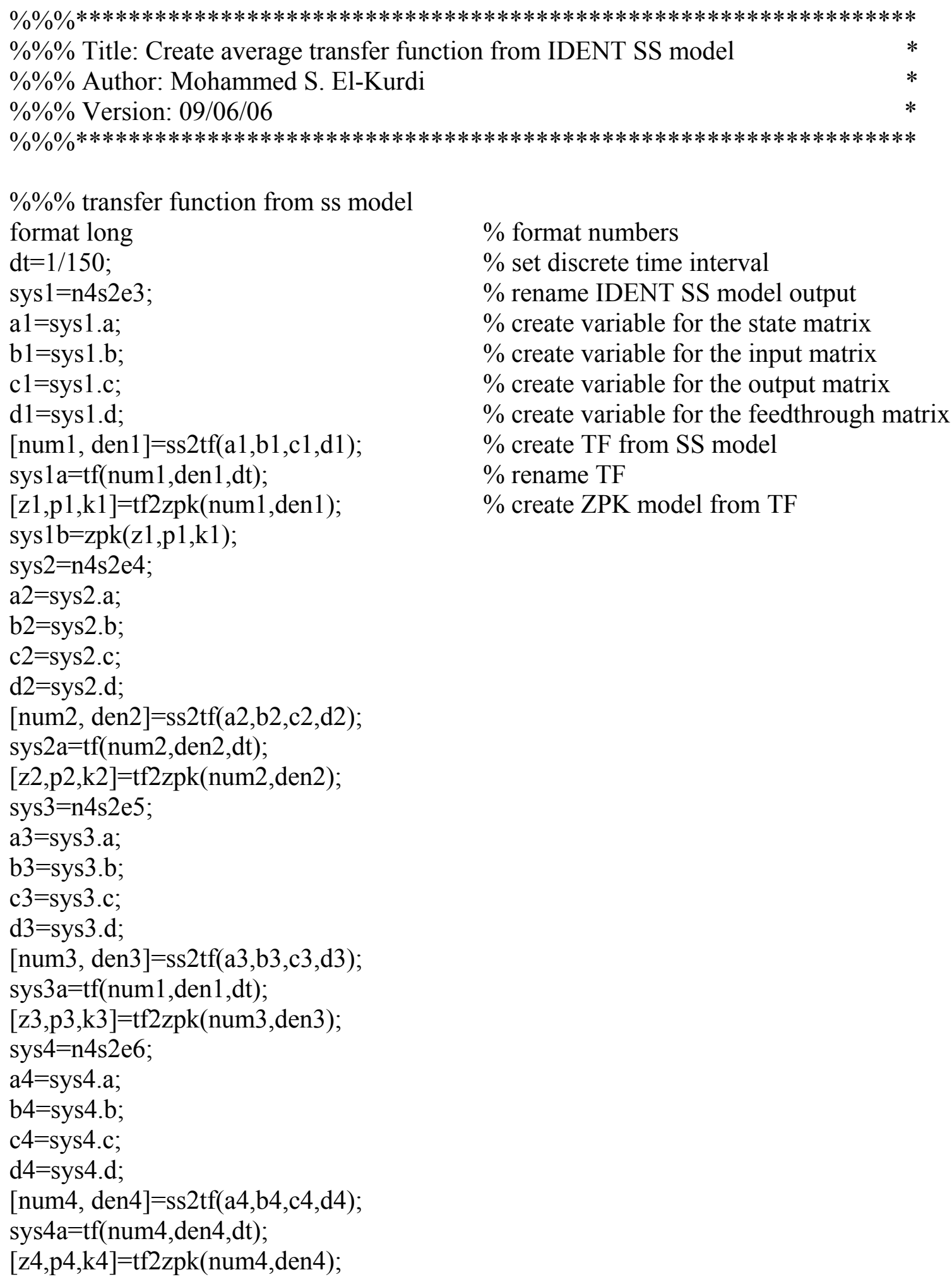

$\%$ format numbers

$\%$ set discrete time interval

$\%$ rename IDENT SS model output

$\%$ create variable for the state matrix

$\%$ create variable for the input matrix

$\%$ create variable for the output matrix

$\%$ create variable for the feedthrough matrix

$\%$ create TF from SS model

$\%$ rename $\mathrm{TF}$

$\%$ create $\mathrm{ZPK}$ model from $\mathrm{TF}$ 


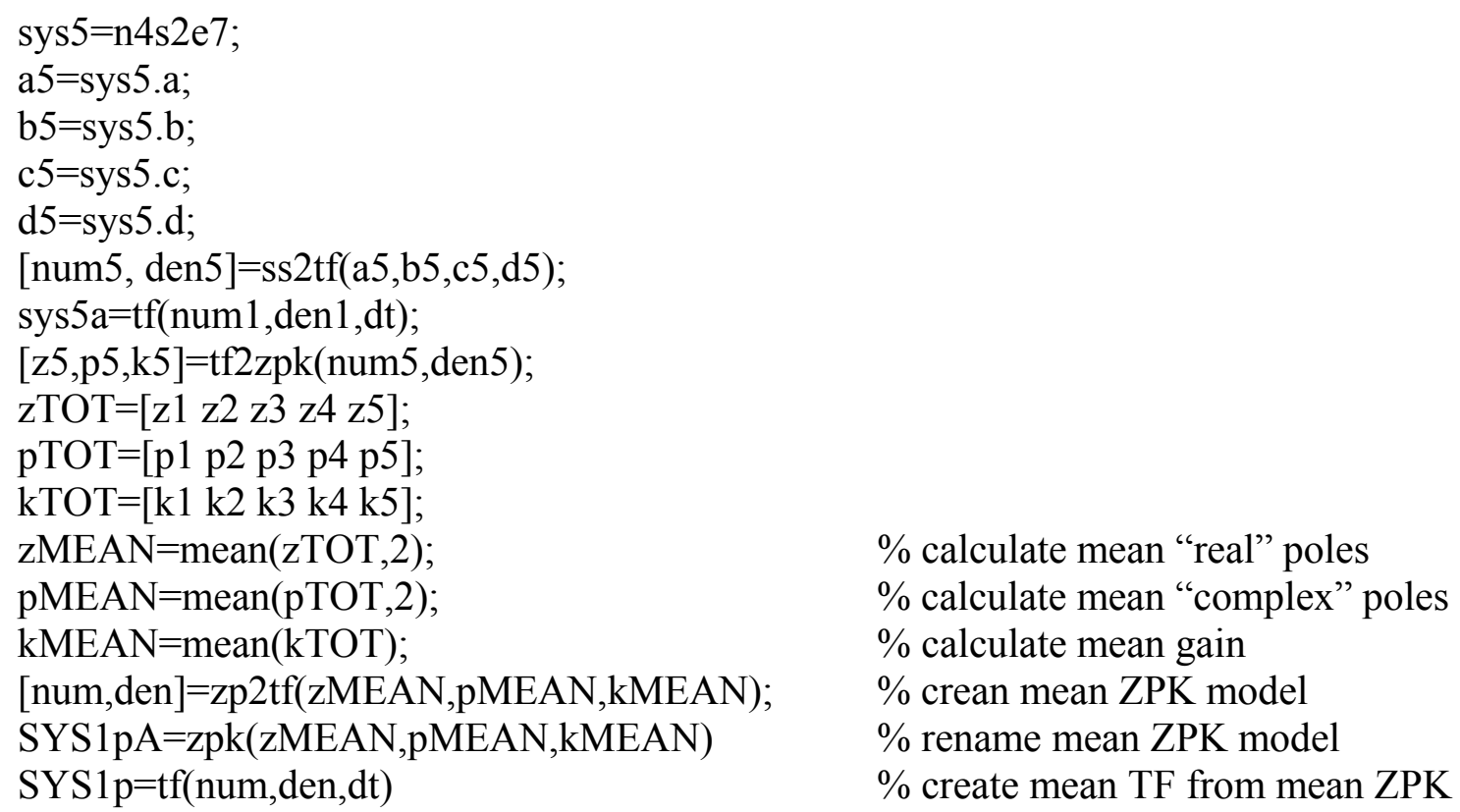

SYS1pA=zpk(zMEAN,pMEAN,kMEAN) $\quad \%$ rename mean ZPK model

$\%$ calculate mean "real" poles

$\%$ calculate mean "complex" poles

$\%$ calculate mean gain

$\%$ create mean $\mathrm{TF}$ from mean $\mathrm{ZPK}$ 


\section{APPENDIX C}

\section{PID CONTROLLER MATLAB ${ }^{\circledR}$ CODE}

The Matlab ${ }^{\circledR}$ programs that were used to separately control the CWS and shear stress waveforms within our EVPS are presented in this Appendix.

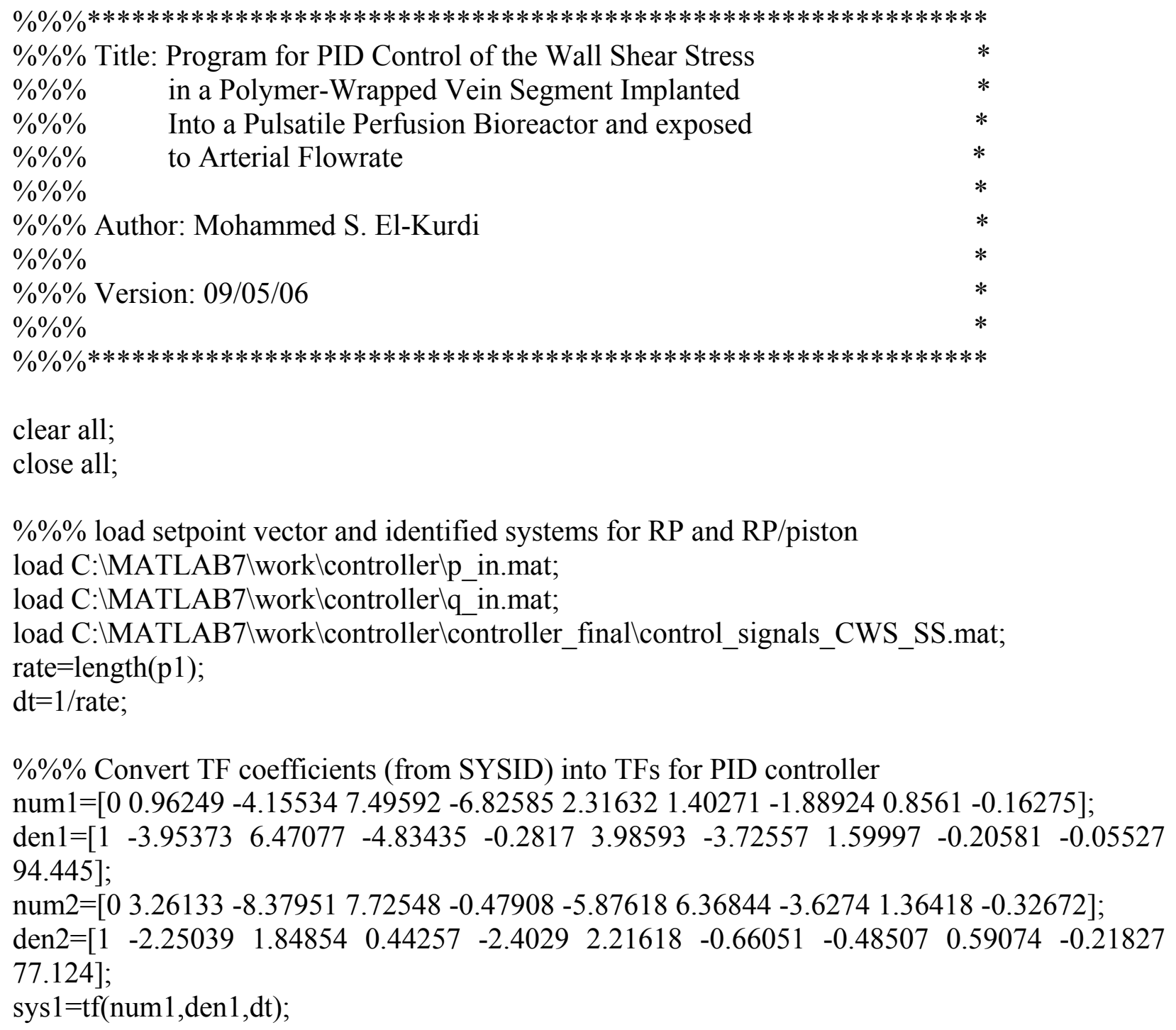


sys2=tf(num2,den2,dt);

$\% \% \%$ declare and initialize setpoints for OL priming cycles

SetP1 $(1$ :rate $)=0.25$;

SetP1=SetP1.';

SetP2a $(1:$ rate $)=0$;

SetP2a=SetP2a.';

$\%$ transpose to column vector

$\% \% \%$ Define the i/o hardware

adaptor='nidaq';

id $=1$;

$\operatorname{ch} 1 \mathrm{o}=0$;

$\operatorname{ch} 2 \mathrm{o}=1$;

ch1 $1=0$;

$\operatorname{ch} 2 \mathrm{i}=1$;

$\operatorname{ch} 3 \mathrm{i}=2$;

$\operatorname{ch} 4 \mathrm{i}=4$;

noc $=2$;

$\mathrm{nic}=4$;

tnc $=$ noc + nic;

$\%$ load driver

$\%$ DAQ device ID

$\%$ output ch to roller pump

$\%$ output ch to piston

$\%$ input ch from flowmeter

$\%$ input ch from $\mathrm{P} 1$

$\%$ input ch from $\mathrm{P} 2$

$\%$ input ch from laser

$\%$ \# of output channels

$\%$ \# of input channels

$\%$ total \# of input and output channels

$\% \% \%$ Counters and timers

$\mathrm{cs}=10$

$\mathrm{n}=10$;

$\mathrm{i}=1$;

$\mathrm{j}=1$;

$\mathrm{k}=1$;

$1=1$;

$\mathrm{e}=1$;

$\mathrm{p}=1$;

$\mathrm{m}=10$;

$\mathrm{o}=10$;

$\mathrm{q}=149$;

$\mathrm{N}=10$;

$\mathrm{spt}=\mathrm{rate} / \mathrm{m}$;

spt $2=$ rate/o;

$\mathrm{dt}=1 /$ rate;

$\mathrm{T}=(0: \mathrm{dt}: \mathrm{n})$;

Time $=(0: \mathrm{dt}: \mathrm{N})$;

$\% \#$ of open loop priming cycles to execute

$\% \#$ of controlled cycles to execute (at $1 \mathrm{~Hz}, \mathrm{n}=\mathrm{sec}$ )

$\%$ for loop index

$\%$ for loop index

$\%$ for loop index

$\%$ for loop index

$\%$ for loop index

$\%$ for loop index

$\%$ loop terminator for OL cycles

$\%$ loop terminator for CL cycles

$\%$ loop increment for CL cycles

$\%$ time in sec for simulation

$\%$ samples per trigger for open loop cycles

$\%$ sampls per trigger for closed loop cycles

$\%$ time increment for simulation and plotting

$\%$ time vector for plotting

$\%$ time vector for simulation

$\% \% \%$ Open loop priming cycles

for $\mathrm{j}=1$ :cs, for $\mathrm{i}=1: \mathrm{m}$,

SetPone $=\operatorname{SetP} 1\left(\left(1+\left((\mathrm{i}-1)^{*} \mathrm{spt}\right)\right):\left(\left((\mathrm{i}-1)^{*} \mathrm{spt}\right)+\mathrm{spt}\right)\right)$;

SetPtwo $=\operatorname{SetP} 2 \mathrm{a}\left(\left(1+\left((\mathrm{i}-1)^{*} \mathrm{spt}\right)\right):(((\mathrm{i}-1) * \mathrm{spt})+\mathrm{spt})\right)$; 


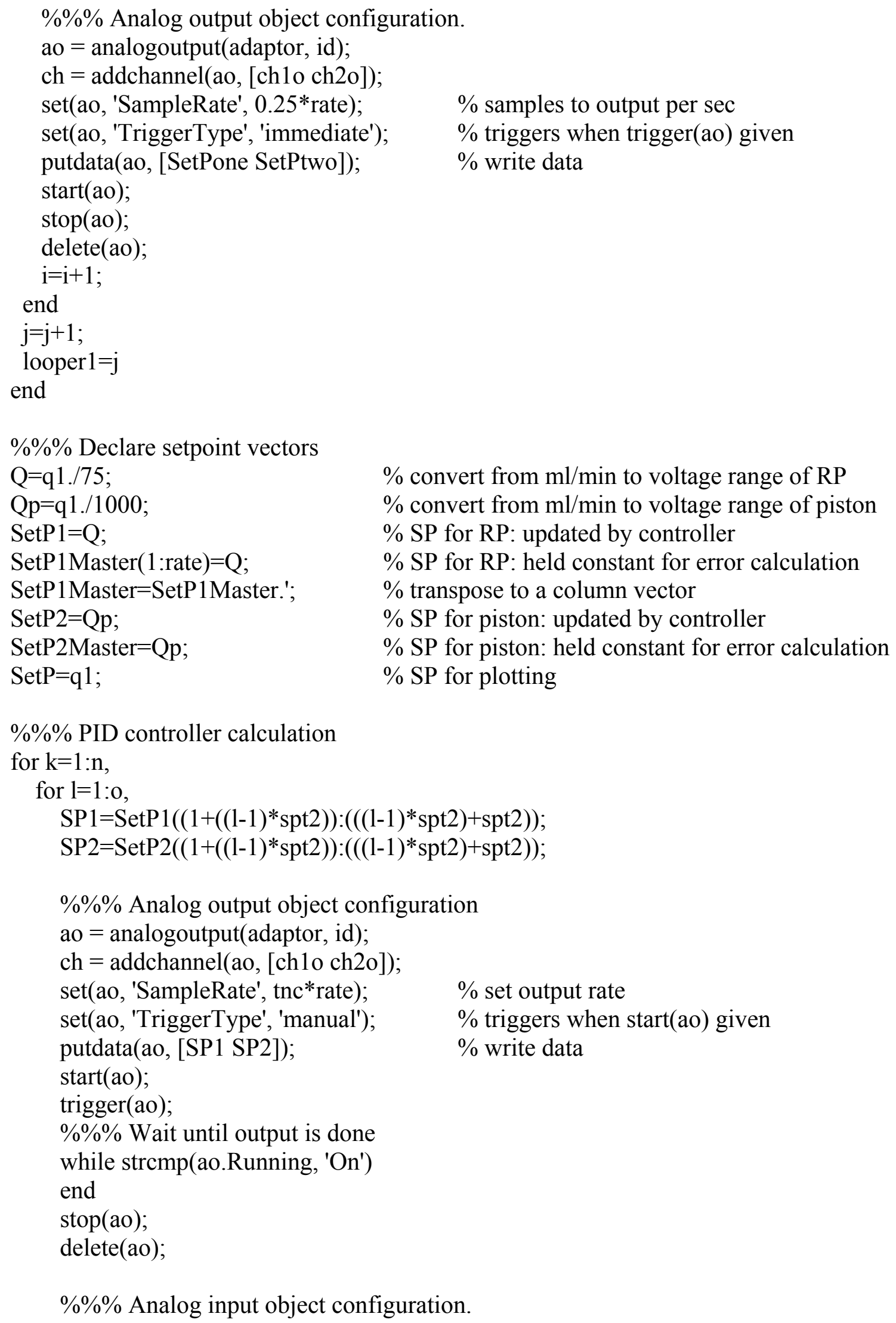




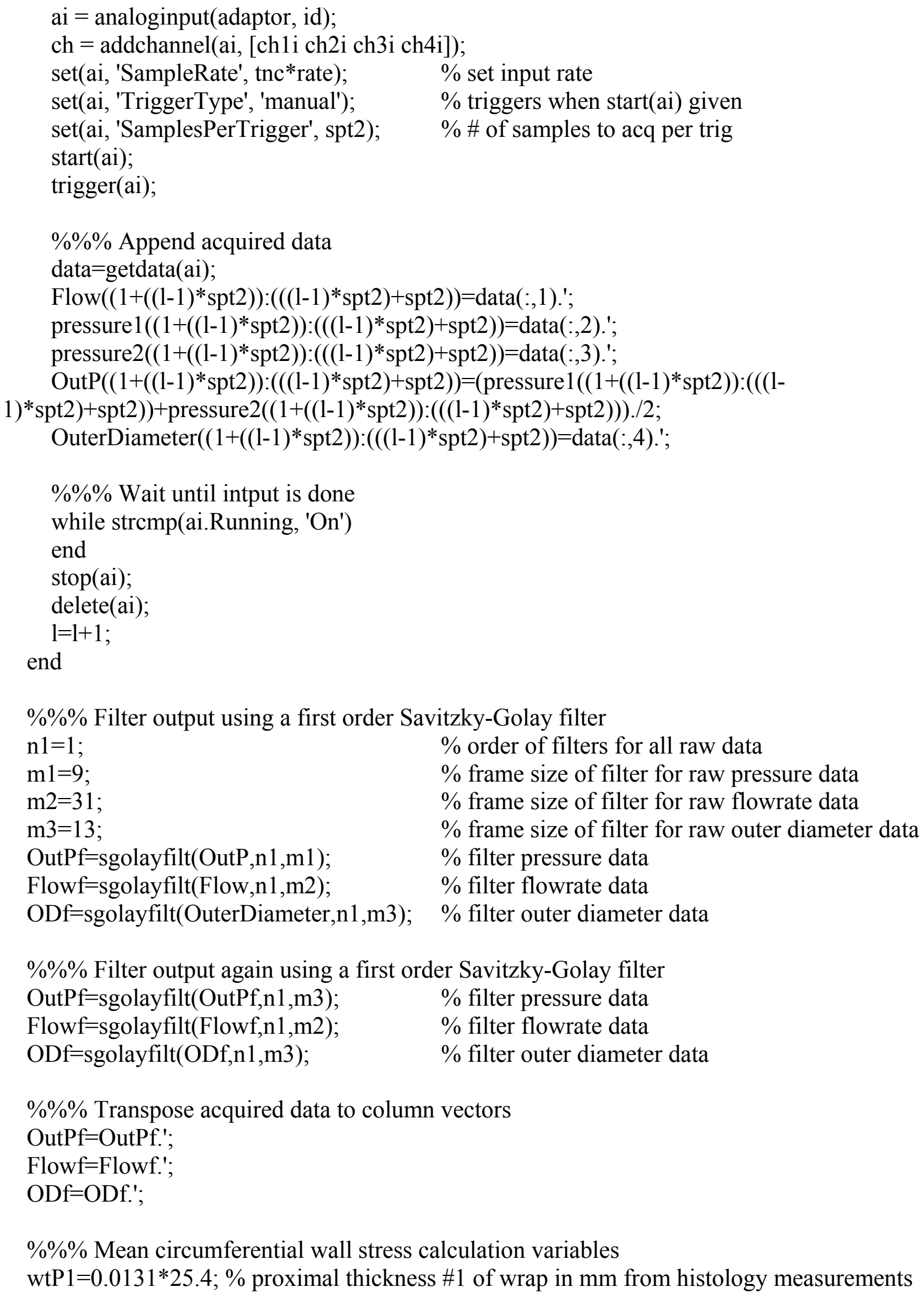


widP $=0.1086 * 25.4 ; \%$ proximal unpressurized inner diameter of wrap in $\mathrm{mm}$ from histology measurements

${ }_{\mathrm{wtP}}=0.0133 * 25.4 ; \%$ proximal thickness $\# 2$ of wrap in $\mathrm{mm}$ from histology measurements $\mathrm{wtD} 1=0.0131 * 25.4 ; \%$ distal thickness \#1 in of wrap mm from histology measurements widD $=0.1086 * 25.4 ; \%$ distal unpressurized inner diameter of wrap in $\mathrm{mm}$ from histology measurements

$\mathrm{wtD} 2=0.0133 * 25.4 ; \%$ distal thickness $\# 2$ of wrap in $\mathrm{mm}$ from histology measurements $\mathrm{wtP}=(\mathrm{wtP} 1+\mathrm{wtP} 2) / 2 ; \%$ proximnal thickness of wrap in $\mathrm{mm}$

$\mathrm{wtD}=(\mathrm{wtD} 1+\mathrm{wtD} 2) / 2 ; \%$ distal thickness of wrap in $\mathrm{mm}$

$\mathrm{wtu}=(\mathrm{wtP}+\mathrm{wtD}) / 2 ; \%$ unpressurized thickness of wrap in $\mathrm{mm}$

$\mathrm{wIDu}=(\mathrm{widP}+\mathrm{widD}) / 2 ; \%$ unpressurized inner diameter of wrap in $\mathrm{mm}$

$\mathrm{bu}=\mathrm{wIDu} / 2 ; \%$ unpressurized inner radius of wrap in $\mathrm{mm}$

$\mathrm{cu}=\mathrm{bu}+\mathrm{wtu}$;

$\mathrm{vtP} 1=0.0131 * 25.4 ; \%$ proximal thickness $\# 1$ of vein in $\mathrm{mm}$ from histology measurements

vidP $=0.1086 * 25.4 ; \%$ proximal unpressurized inner diameter of vein in $\mathrm{mm}$ from histology measurements

$\mathrm{vtP} 2=0.0133 * 25.4 ; \%$ proximal thickness $\# 2$ of vein in $\mathrm{mm}$ from histology measurements $\mathrm{vtD} 1=0.0131 * 25.4 ; \%$ distal thickness $\# 1$ in of vein $\mathrm{mm}$ from histology measurements

$\operatorname{vidD}=0.1086 * 25.4 ; \%$ distal unpressurized inner diameter of vein in $\mathrm{mm}$ from histology measurements

$\mathrm{vtD} 2=0.0133 * 25.4 ; \%$ distal thickness $\# 2$ of vein in $\mathrm{mm}$ from histology measurements $\mathrm{vtP}=(\mathrm{vtP} 1+\mathrm{vtP} 2) / 2 ; \%$ proximnal thickness of vein in $\mathrm{mm}$

$\mathrm{vtD}=(\mathrm{vtD} 1+\mathrm{vtD} 2) / 2 ; \%$ distal thickness of vein in $\mathrm{mm}$

$\mathrm{vtu}=(\mathrm{vtP}+\mathrm{vtD}) / 2 ; \%$ unpressurized thickness of vein in $\mathrm{mm}$

$\mathrm{vIDu}=(\mathrm{vidP}+\mathrm{vidD}) / 2 ; \%$ unpressurized inner diameter of vein in $\mathrm{mm}$

$\mathrm{au}=\mathrm{vIDu} / 2 ; \%$ unpressurized inner radius of vein in $\mathrm{mm}$

$\mathrm{Lu}=50 ; \%$ unstretched length of vessel segment in $\mathrm{mm}$

$\mathrm{Lp}=1.3 * \mathrm{Lu} ; \%$ in-vivo length of vessel segment in $\mathrm{mm}$

$\mathrm{ODp}=\mathrm{ODf} ; \%$ pressurized outer diameter in $\mathrm{mm}$

$\mathrm{cp}=\mathrm{ODp} . / 2 ; \%$ pressurized outer radius in $\mathrm{mm}$

$\% \% \%$ calculate pressurized inner radius assuming incompressibility

$\operatorname{IRp}=\operatorname{zeros}(150,1)$

TEMPVAR1=zeros $(150,1)$;

TEMPVAR2 $=$ zeros $(150,1)$;

TEMPVAR1 $=\left(\left((\mathrm{cp} . \wedge 2) \cdot{ }^{*} \mathrm{Lp} .{ }^{*}\left(\mathrm{cu}^{\wedge} 2-\mathrm{bu}{ }^{\wedge} 2\right) * \mathrm{Lu}\right) . / \mathrm{Lp}\right.$;

$\mathrm{v}=1$;

for $\mathrm{v}=1$ :rate, $\mathrm{bp}(\mathrm{v})=\operatorname{sqrt}(\operatorname{TEMPVAR} 1(\mathrm{v}))$;

end

$\mathrm{bp}=\mathrm{bp} .{ }^{\prime} \%$ pressurized interfacial radius in $\mathrm{mm}$

TEMPVAR2 $=\left(\left(\left(c p .{ }^{\wedge} 2\right) \cdot{ }^{*} \mathrm{Lp} .{ }^{*}\left(\mathrm{cu}^{\wedge} 2-\mathrm{bu}^{\wedge} 2\right) * \mathrm{Lu} / \mathrm{Lp}\right)-\left(\mathrm{bu}^{\wedge} 2-\mathrm{au}{ }^{\wedge} 2\right) * \mathrm{Lu}\right) . / \mathrm{Lp}$;

$\mathrm{v}=1$;

for $\mathrm{v}=1$ :rate, $\operatorname{ap}(\mathrm{v})=\operatorname{sqrt}(\operatorname{TEMPVAR} 2(\mathrm{v}))$;

end 
ap=ap.' \% pressurized inner radius in $\mathrm{mm}$

$\% \% \%$ Interfacial pressure calculation

$\mathrm{P}=1.333224 . *$ (OutPf.*46.76)./1e3; \% Convert intraluminal pressure from $\mathrm{mmHg}$ to $\mathrm{KPa}$

$\mathrm{Nu}=0.5 ; \%$ Poisson's ratio of wrap and vein

$\mathrm{Ew}=7500 ; \%$ Young's modulus of wrap in $\mathrm{KPa}$

$\mathrm{Ev}=600 ; \%$ Young's modulus of vein in $\mathrm{KPa}$

$\mathrm{P}$ int num $=\left(\right.$ ap. ${ }^{\wedge} 2 . * P . *(1-\mathrm{Nu}) . *$ Ew. $*$ bp.*(cp.^2-bp. $\left.{ }^{\wedge} 2\right)+(1+\mathrm{Nu}){ }^{*} \mathrm{Ew} . *\left(\mathrm{cp} .{ }^{\wedge} 2-\right.$

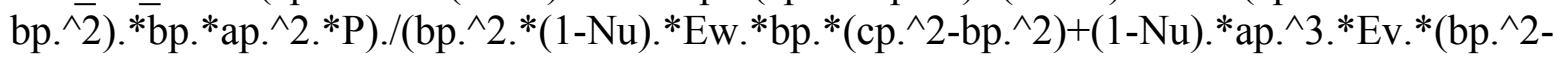

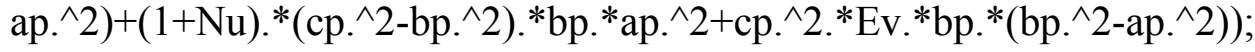

$\% \% \%$ Mid-vein-wall circumferential wall stress calculation

rho $=($ bp-ap). $/ 2 ; \%$ prescribes midwall cws calc

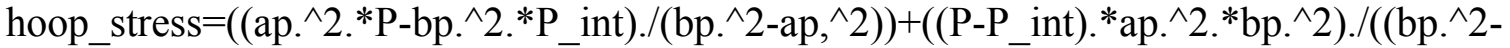

ap.^2)./rho);

$\% \% \%$ Lumenal shear stress calculation

$\mathrm{Mu}=1.02 ; \%$ viscosity of perfusate in dynes* ${ }^{*} / \mathrm{cm}^{\wedge} 2$

shear_stress $=(4 *$ Mu.*Flowf)./(pi.*ap.^3);

$\% \% \%$ Prescribe size of and calculate error vectors

Errl=zeros(rate, 1$)$;

Err2=Err1;

$\operatorname{Err} 1(1:$ rate $)=$ mean (shear_stress)-mean $(\mathrm{SSc}) ; \%$ err1 calc for direct action

lag $=1$;

for $\mathrm{e}=1$ :rate-lag,

Err2(e)=shear_stress(e)-SSc(e+lag); \% err2 calc for direct action end

$\% \% \%$ Append i/o vectors for plotting

SetPMasterPlot $((1+((\mathrm{k}-1) *$ rate $)):(((\mathrm{k}-1) *$ rate $)+$ rate $))=\mathrm{p} 1(:)$;

SetSSMasterPlot $((1+((\mathrm{k}-1) *$ rate $)):(((\mathrm{k}-1) *$ rate $)+$ rate $))=\operatorname{SSc}(:)$;

MFlow $((1+((\mathrm{k}-1) *$ rate $)):(((\mathrm{k}-1) *$ rate $)+$ rate $))=$ Flowf $(:) * 330$;

$\operatorname{MP}((1+((\mathrm{k}-1) *$ rate $)):(((\mathrm{k}-1) *$ rate $)+$ rate $))=\operatorname{OutPf}(:) * 46.76$;

$\operatorname{MMP}((1+((\mathrm{k}-1) *$ rate $)):(((\mathrm{k}-1) *$ rate $)+$ rate $))=\operatorname{mean}(\operatorname{MP}((1+((\mathrm{k}-1) *$ rate $)):(((\mathrm{k}-1) *$ rate $)+$ rate $)))$;

MMFlow $((1+((\mathrm{k}-1) *$ rate $)):(((\mathrm{k}-1) *$ rate $)+$ rate $))=\operatorname{mean}(\operatorname{MFlow}((1+((\mathrm{k}-1) *$ rate $)):(((\mathrm{k}-$

$1)^{*}$ rate $)+$ rate $\left.)\right)$;

$\operatorname{MMR}((1+((\mathrm{k}-1) *$ rate $)):(((\mathrm{k}-1) *$ rate $)+$ rate $))=\operatorname{MMP}((1+((\mathrm{k}-1) *$ rate $)):(((\mathrm{k}-$

$1)^{*}$ rate $)+$ rate $\left.)\right)$ /MMFlow $((1+((\mathrm{k}-1) *$ rate $)):(((\mathrm{k}-1) *$ rate $)+$ rate $))$;

$\operatorname{MOD}((1+((\mathrm{k}-1) *$ rate $)):(((\mathrm{k}-1) *$ rate $)+$ rate $))=\operatorname{ODf}(:) ;$

$\operatorname{MCWS}((1+((\mathrm{k}-1) *$ rate $)):(((\mathrm{k}-1) *$ rate $)+$ rate $))=$ hoop_stress $(:)$;

$\operatorname{MSS}((1+((\mathrm{k}-1) *$ rate $)):(((\mathrm{k}-1) *$ rate $)+$ rate $))=$ shear_stress $(:)$;

Error1 $((1+((\mathrm{k}-1) *$ rate $)):((\mathrm{k}-1) *$ rate $)+$ rate $))=\operatorname{Err1}(:)$;

Error2 $((1+((\mathrm{k}-1) *$ rate $)):(((\mathrm{k}-1) *$ rate $)+$ rate $))=\operatorname{Err} 2(:)$; 
$\% \% \%$ Initialize variables for PID algorithm

ErrLast $1=$ Err1;

ErrLast2=Err2;

ErrLastLast $1=$ ErrLast 1 ;

ErrLastLast $2=$ ErrLast 2 ;

Input $1 \mathrm{a}=75 .{ }^{*} \mathrm{SetP} 1 ; \%$ input signal for simulation $\# 1$

Input $2 \mathrm{a}=$ Input $1 \mathrm{a}+1000 .{ }^{*} \mathrm{SetP} 2 ; \%$ input signal for simulation \#2

$\% \% \%$ PID control variables for simulation \#1

$\mathrm{kd} 1=0$;

$\%$ derivative gain

$\mathrm{kp} 1=0$;

$\%$ proportional gain

ki1 $=0$;

$\%$ integral gain

numPID1=[kd1 kp1 ki1];

denPID1=[1 0$]$;

numc $1=\operatorname{conv}($ num1, denPID1);

denc1=polyadd(conv(denPID1,den1), conv(numPID1,num1));

sys1 $\mathrm{a}=\mathrm{tf}($ numc $1, \mathrm{denc} 1, \mathrm{dt})$;

$\% \% \%$ PID control variables for simulation \#2

$\mathrm{kd} 2=0$;

$\mathrm{kp} 2=0$;

$\%$ derivative gain

ki2 $=0$;

$\%$ proportional gain

numPID2 $=[\mathrm{kd} 2 \mathrm{kp} 2 \mathrm{ki} 2]$;

$\%$ integral gain

denPID2=[1 0$]$;

numc $2=\operatorname{conv}($ num 2 ,denPID2);

denc2 $=$ polyadd( $\operatorname{conv}(\operatorname{denPID} 2, \operatorname{den} 2), \operatorname{conv}($ numPID2,num2 $))$;

sys2 $\mathrm{a}=\mathrm{tf}($ numc2, denc2, dt);

$\% \% \%$ Declare variables for PID control algorithm

$\mathrm{Kp}=1$

$\mathrm{Ki}=20$;

$\%$ gain

$\mathrm{Kd}=1$;

$\%$ reset rate in repeats per minutes

$\%$ derivative time in minites

$\% \% \%$ Set controller tolerances

iteration $=0$;

iteration_max $=20$;

Err_tol=zeros(rate, 1$)$;

Err_tol(1:rate $)=0.05 ; \quad \%$ tolerance in dynes $/ \mathrm{cm}^{\wedge} 2$

for $\mathrm{p}=1$ :rate- $\mathrm{q}$,

if $((0-\operatorname{Err} 2(p: p+q))>\operatorname{Err}$ tol $(p: p+q)) \mid\left((0-\operatorname{Err} 1(p: p+q))>\operatorname{Err} \_t o l(p: p+q)\right)$,

Message $1=$ 'Error is -'

While

$\left.\operatorname{Errl}(\mathrm{p}: \mathrm{p}+\mathrm{q}))>\operatorname{Err} \_t o l(p: p+q)\right) \&($ iteration $<=$ iteration_max $)$,

$\left((0-\operatorname{Err} 2(p: p+q))>\operatorname{Err} \_t o l(p: p+q)\right) \&((0-$

$\% \% \%$ simulate the system response to previous input

Input $1 \mathrm{i}=$ interp(Input1 $\mathrm{a}(\mathrm{p}: \mathrm{p}+\mathrm{q}), \mathrm{N}) ; \%$ resize input for siumulation 
Input2_i=interp(Input2a(p:p+q),N); \% resize input for siumulation $\operatorname{IRp}=\operatorname{interp}(\operatorname{IRp}(\mathrm{p}: \mathrm{p}+\mathrm{q}), \mathrm{N}) ; \%$ resize input for siumulation $\mathrm{ORp}=\operatorname{interp}(\mathrm{ORp}(\mathrm{p}: \mathrm{p}+\mathrm{q}), \mathrm{N}) ; \%$ resize input for siumulation OutPS1a=1sim(sys1a,Input1_i,Time(1:length(Input1_i))); OutPS2a $=1$ sim(sys2a,Input2_i,Time(1:length(Input2_i )));

$\% \% \%$ calculate the SS from the simulated pressure output

OutPS1b=(4*Mu.*OutPS1a)./(pi.*IRp.^3);

OutPS2b=(4*Mu.*OutPS2a)./(pi.*IRp.^3);

OutPS1 $(\mathrm{p}: \mathrm{p}+\mathrm{q})=$ resample(OutPS1b,1,N); \% resisize input to system OutPS2 $(p: p+q)=$ resample(OutPS2b,1,N); \% resisize input to system IRp=resample(IRp, $1, \mathrm{~N}) ; \%$ resize input for siumulation $\mathrm{ORp}=$ resample $(\mathrm{ORp}, 1, \mathrm{~N}) ; \%$ resize input for siumulation

$\% \% \%$ calculate the change in output using the derivative of the $\% \% \%$ PID algorithm then add to the previous output

$\operatorname{Err} 1(p: p+q)=\operatorname{mean}(\operatorname{OutPS} 1(p: p+q))-\operatorname{mean}(\operatorname{SSc}(p: p+q))$;

$\operatorname{Err} 2(p: p+q)=\operatorname{OutPS} 2(p: p+q)-S S c(p: p+q)$; $\operatorname{dOutP} 1(p: p+q)=K p \cdot *(\operatorname{Err} 1(p: p+q)-$

$\operatorname{ErrLast} 1(\mathrm{p}: \mathrm{p}+\mathrm{q})+\mathrm{Ki} .{ }^{*} \operatorname{Err} 1(\mathrm{p}: \mathrm{p}+\mathrm{q}) . / \mathrm{rate}+\mathrm{Kd} .{ }^{*}(\operatorname{Err1}(\mathrm{p}: \mathrm{p}+\mathrm{q})-$

2. $\left.\left.{ }^{*} \operatorname{ErrLast} 1(\mathrm{p}: \mathrm{p}+\mathrm{q})+\operatorname{ErrLastLast} 1(\mathrm{p}: \mathrm{p}+\mathrm{q})\right)\right)$;

dOutP2(p:p+q) $=K p \cdot *(E r r 2(p: p+q)-$

$\operatorname{ErrLast} 2(p: p+q)+K i .{ }^{*} \operatorname{Err} 2(p: p+q) . / r a t e+K d . *(\operatorname{Err} 2(p: p+q)-$

2. $\left.\left.{ }^{*} \operatorname{ErrLast} 2(\mathrm{p}: \mathrm{p}+\mathrm{q})+\operatorname{ErrLastLast} 2(\mathrm{p}: \mathrm{p}+\mathrm{q})\right)\right)$;

$\% \% \%$ Update input - recaclculate flowrate from simulated

$\% \% \%$ stress calcs

$\operatorname{Input} 1(\mathrm{p}: \mathrm{p}+\mathrm{q})=\operatorname{OutPS} 1(\mathrm{p}: \mathrm{p}+\mathrm{q})+\mathrm{dOutP} 1(\mathrm{p}: \mathrm{p}+\mathrm{q})$;

Input1 $a(p: p+q)=\left(\operatorname{Input1}(p: p+q) .{ }^{*} \operatorname{IRp} \wedge^{\wedge} 3 . * p i\right) . /(4 * \mathrm{Mu})$;

Input2(p:p+q)=OutPS2 $(p: p+q)+\operatorname{dOutP} 2(p: p+q)$;

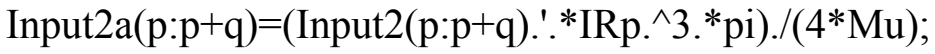

$\% \% \%$ Update variables for PID algorithm

ErrLastLast $1(p: p+q)=\operatorname{ErrLast} 1(p: p+q)$;

ErrLastLast $2(p: p+q)=\operatorname{ErrLast} 2(p: p+q)$;

$\operatorname{ErrLast} 1(p: p+q)=\operatorname{Err} 1(p: p+q)$;

$\operatorname{ErrLast} 2(p: p+q)=\operatorname{Err} 2(p: p+q)$;

iteration $=$ iteration +1 ;

end

$\% \% \%$ Update setpoints

$\operatorname{SetP} 1(p: p+q)=\operatorname{Input} 1 \mathrm{a}(\mathrm{p}: \mathrm{p}+\mathrm{q}) \cdot / 75$

$\operatorname{SetP} 2(p: p+q)=(\operatorname{Input} 2 a(p: p+q) \cdot / 75)-\operatorname{SetP} 1(p: p+q)$;

elseif $\left(\operatorname{Err} 2(p: p+q)>\operatorname{Err} \_t o l(p: p+q)\right) \mid\left(\operatorname{Err1}(p: p+q)>\operatorname{Err} \_t o l(p: p+q)\right)$, Message $1=$ 'Error is $+^{\prime}$ 
while

$\left(\operatorname{Err} 2(p: p+q)>\operatorname{Err} \_t o l(p: p+q)\right) \&\left(\operatorname{Err} 1(p: p+q)>\operatorname{Err} \_t o l(p: p+q)\right) \&($ iteration $<=$ iteration_max $)$, $\% \% \%$ simulate the system response to previous input

Input1_i $=$ interp(Inputla $(p: p+q), N) ; \%$ resize input for siumulation Input2_ $\mathrm{i}=$ interp(Input2a(p:p+q),N); \% resize input for siumulation $\operatorname{IRp}=\operatorname{interp}(\operatorname{IRp}(\mathrm{p}: \mathrm{p}+\mathrm{q}), \mathrm{N}) ; \%$ resize input for siumulation $\mathrm{ORp}=\operatorname{interp}(\mathrm{ORp}(\mathrm{p}: \mathrm{p}+\mathrm{q}), \mathrm{N}) ; \%$ resize input for siumulation OutPS1a=1sim(sys1a,Input1_i,Time(1:length(Input1_i))); OutPS2a $=1 \operatorname{sim}($ sys2a,Input2_i,Time(1:length(Input2_i $)$ ));

$\% \% \%$ calculate the SS from the simulated pressure output OutPS1b=(4*Mu.*OutPS1a)./(pi.*IRp.^3); OutPS2b=(4*Mu.*OutPS2a)./(pi.*IRp.^3);

$\operatorname{OutPS1}(\mathrm{p}: \mathrm{p}+\mathrm{q})=$ resample(OutPS1b, $1, \mathrm{~N}) ; \%$ resisize input to system OutPS2 $(p: p+q)=$ resample $($ OutPS2b,1,N); \% resisize input to system $\mathrm{IRp}=$ resample(IRp, $1, \mathrm{~N}) ; \%$ resize input for siumulation $\mathrm{ORp}=$ resample $(\mathrm{ORp}, 1, \mathrm{~N}) ; \%$ resize input for siumulation

$\% \% \%$ calculate the change in output using the derivative of the $\% \% \%$ PID algorithm then add to the previous output $\operatorname{Err} 1(p: p+q)=\operatorname{mean}(\operatorname{OutPS} 1(\mathrm{p}: \mathrm{p}+\mathrm{q}))-\operatorname{mean}(\operatorname{SSc}(\mathrm{p}: \mathrm{p}+\mathrm{q}))$; $\operatorname{Err} 2(p: p+q)=\operatorname{OutPS} 2(p: p+q)-S S c(p: p+q)$; $\operatorname{dOutP} 1(\mathrm{p}: \mathrm{p}+\mathrm{q})=\mathrm{Kp} . *(\operatorname{Err} 1(\mathrm{p}: \mathrm{p}+\mathrm{q})-$

$\operatorname{ErrLast} 1(\mathrm{p}: \mathrm{p}+\mathrm{q})+\mathrm{Ki} .{ }^{*} \operatorname{Err} 1(\mathrm{p}: \mathrm{p}+\mathrm{q}) . / \mathrm{rate}+\mathrm{Kd} .{ }^{*}(\operatorname{Err} 1(\mathrm{p}: \mathrm{p}+\mathrm{q})-$

2. $\left.\left.{ }^{*} \operatorname{ErrLast} 1(\mathrm{p}: \mathrm{p}+\mathrm{q})+\operatorname{ErrLastLast} 1(\mathrm{p}: \mathrm{p}+\mathrm{q})\right)\right)$; $\operatorname{dOutP} 2(p: p+q)=K p \cdot *(\operatorname{Err} 2(p: p+q)-$

ErrLast2(p:p+q)+Ki. ${ }^{*} \operatorname{Err} 2(p: p+q) . / r a t e+K d . *(\operatorname{Err} 2(p: p+q)-$

2.*ErrLast2(p:p+q)+ErrLastLast2(p:p+q)));

$\% \% \%$ Update input - recaclculate flowrate from simulated $\% \% \%$ stress calcs

$\operatorname{Input} 1(\mathrm{p}: \mathrm{p}+\mathrm{q})=\operatorname{OutPS} 1(\mathrm{p}: \mathrm{p}+\mathrm{q})+\operatorname{dOutP} 1(\mathrm{p}: \mathrm{p}+\mathrm{q})$;

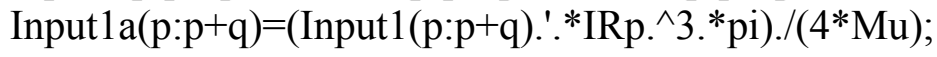

Input $2(p: p+q)=\operatorname{OutPS} 2(p: p+q)+\operatorname{dOutP} 2(p: p+q)$;

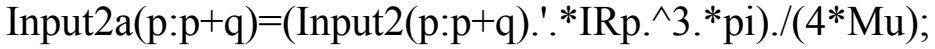

$\% \% \%$ Update variables for PID algorithm

$\operatorname{ErrLastLast} 1(p: p+q)=\operatorname{ErrLast} 1(p: p+q)$;

ErrLastLast $2(p: p+q)=\operatorname{ErrLast} 2(p: p+q)$;

$\operatorname{ErrLast} 1(p: p+q)=\operatorname{Err1}(p: p+q)$;

ErrLast2 $(p: p+q)=\operatorname{Err} 2(p: p+q)$;

iteration $=$ iteration +1 ;

end

$\% \% \%$ Update setpoints

$\operatorname{SetP} 1(p: p+q)=\operatorname{Input} 1 a(p: p+q) \cdot / 75$

$\operatorname{SetP} 2(p: p+q)=(\operatorname{Input} 2 a(p: p+q) \cdot / 75)-\operatorname{SetP} 1(p: p+q)$; 


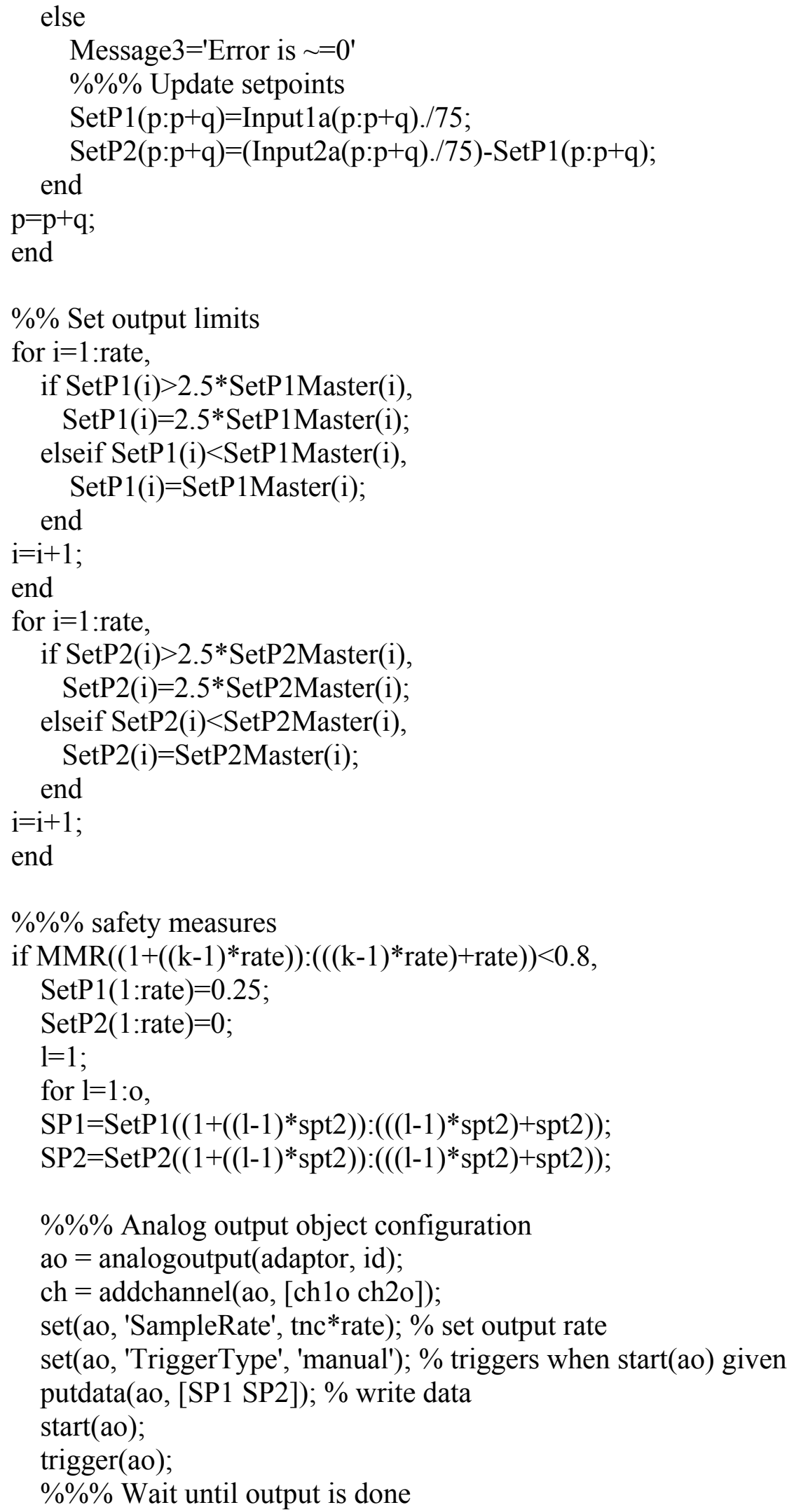


while strcmp(ao.Running, 'On')

end

$\operatorname{stop}($ ao);

delete(ao);

$\% \% \%$ Analog input object configuration.

ai $=$ analoginput(adaptor, id);

ch = addchannel(ai, [ch1i ch2i ch3i ch4i]);

set(ai, 'SampleRate', tnc*rate); \% set input rate

set(ai, 'TriggerType', 'manual'); \% triggers when start(ai) given

set(ai, 'SamplesPerTrigger', spt2); \% \# of samples to acq per trig

start(ai);

trigger(ai);

$\% \% \%$ Append acquired data

data $=$ getdata(ai);

FlowS((1+((1-1)*spt2)):(((1-1)*spt2)+spt2))=data(:,1).';

pressure1S $((1+((1-1) * \operatorname{spt} 2)):(((1-1) * \operatorname{spt} 2)+\operatorname{spt} 2))=\operatorname{data}(:, 2) . ' ;$

pressure $2 \mathrm{~S}\left(\left(1+\left((1-1)^{*} \mathrm{spt} 2\right)\right):\left(\left((1-1)^{*} \mathrm{spt} 2\right)+\mathrm{spt} 2\right)\right)=\operatorname{data}(:, 3) . ;$

OutPS $\left(\left(1+\left((1-1)^{*} \operatorname{spt} 2\right)\right):\left(\left((1-1)^{*} \operatorname{spt} 2\right)+\operatorname{spt} 2\right)\right)=(\operatorname{pressure} 1((1+((1-1) * \operatorname{spt} 2)):(((1-$

$\left.\left.\left.1)^{*} \operatorname{spt} 2\right)+\operatorname{spt} 2\right)\right)+$ pressure $\left.2\left(\left(1+\left((1-1)^{*} \operatorname{spt} 2\right)\right):\left(\left((1-1)^{*} \operatorname{spt} 2\right)+\operatorname{spt} 2\right)\right)\right) \cdot / 2$;

OuterDiameterS((1+((1-1)*spt2)):(((1-1)*spt2)+spt2))=data(:,4).';

$\% \% \%$ Wait until intput is done

while strcmp(ai.Running, 'On')

end

stop(ai);

delete(ai);

$1=1+1$;

end

$\% \% \%$ Filter output using a first order Savitzky-Golay filter

$\mathrm{n} 1=1$;

$\%$ order of filters for all raw data

$\mathrm{ml}=9$;

$\%$ frame size of filter for raw pressure data

$\mathrm{m} 2=31$;

$\%$ frame size of filter for raw flowrate data

$\mathrm{m} 3=13$;

$\%$ frame size of filter for raw outer diameter data

OutPSf=sgolayfilt(OutPS,n1,m1);

$\%$ filter pressure data

FlowSf=sgolayfilt(FlowS,n1,m2);

$\%$ filter flowrate data

ODSf=sgolayfilt(OuterDiameterS,n1,m3); \% filter outer diameter data

$\% \% \%$ Filter output again using a first order Savitzky-Golay filter

OutPSf=sgolayfilt(OutPSf,n1,m3); $\quad \%$ filter pressure data

FlowSf=sgolayfilt(FlowSf,n1,m2); $\quad \%$ filter flowrate data

ODSf=sgolayfilt(ODSf,n1,m3); $\quad \%$ filter outer diameter data 


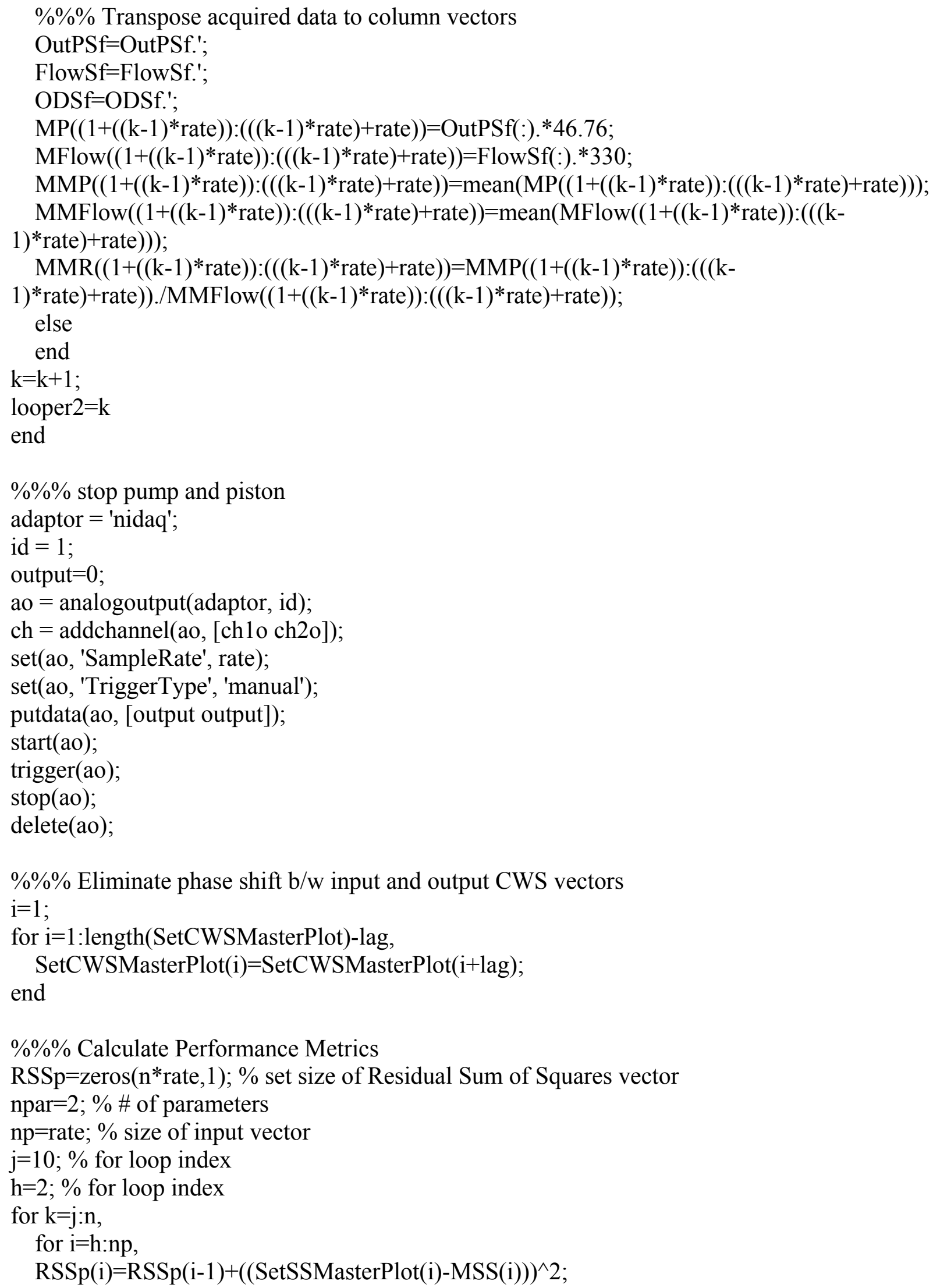




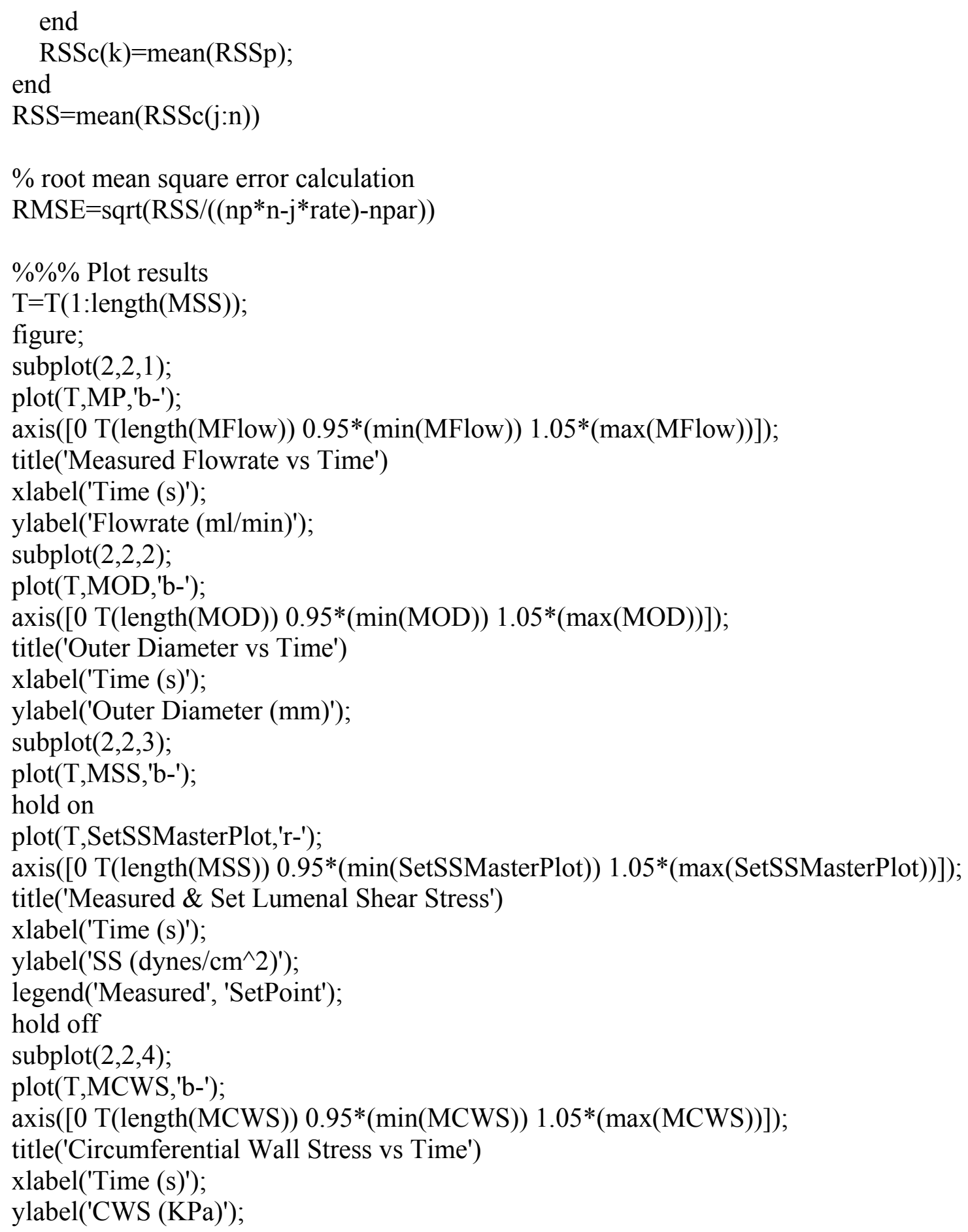


$0 \% 0 \% * * * * * * * * * * * * * * * * * * * * * * * * * * * * * * * * * * * * * * * * * * * * * * * * * * * * * * * * * * * *$

$\% \% \%$ Title: Program for PID Control of the Mid-Vein-Wall CWS

$\% \% \% \quad$ in a Polymer-Wrapped Vein Segment Implanted *

$\% \% \% \quad$ Into a Pulsatile Perfusion Bioreactor and exposed *

$\% \% \%$ to Arterial Pressure *

$\% \% \%$ *

$\% \% \%$ Author: Mohammed S. El-Kurdi $\quad$ *

$\% \% \%$ *

$\% \% \%$ Version: 09/01/06 * *

$\% \% \% \quad *$

$0 \% 0 \% * * * * * * * * * * * * * * * * * * * * * * * * * * * * * * * * * * * * * * * * * * * * * * * * * * * * * * * * * * * *$

clear all;

close all;

$\% \% \%$ load setpoint vector and identified systems for RP and RP/piston

load C:IMATLAB7 \worklcontrollerlp_in.mat;

load C:IMATLAB7 \worklcontrollerlq_in.mat;

load C:MMATLAB7\work|controller|controller_final lcontrol_signals_CWS_SS.mat;

rate $=$ length $(\mathrm{p} 1)$;

$\mathrm{dt}=1 /$ rate;

$\% \% \%$ Convert TF coefficients (from SYSID) into TFs for PID controller

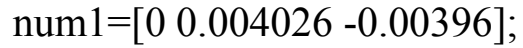

$\operatorname{den} 1=\left[\begin{array}{lll}1 & -1.975 & 0.9748\end{array}\right]$;

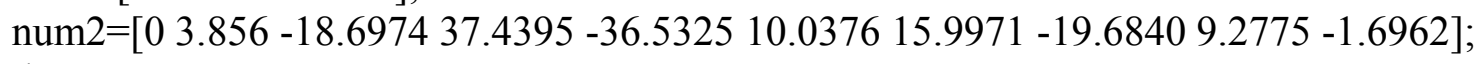

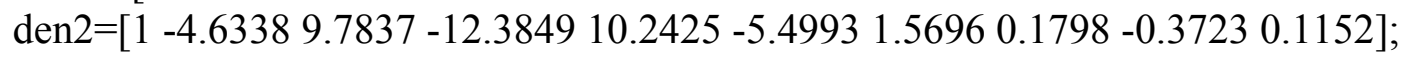

sys $1=\operatorname{tf}($ num $1, \mathrm{den} 1, \mathrm{dt})$

sys $2=\mathrm{tf}($ num $2, \mathrm{den} 2, \mathrm{dt})$

$\% \% \%$ declare and initialize setpoints for OL priming cycles

SetP1(1:rate $)=1$;

SetP1=SetP1.';

SetP2a $(1:$ rate $)=0$;

SetP2a=SetP2a.';

$\% \% \%$ Define the $\mathrm{i} / \mathrm{o}$ hardware

adaptor='nidaq'; $\quad \%$ load driver

id $=1$;

$\%$ DAQ device ID 


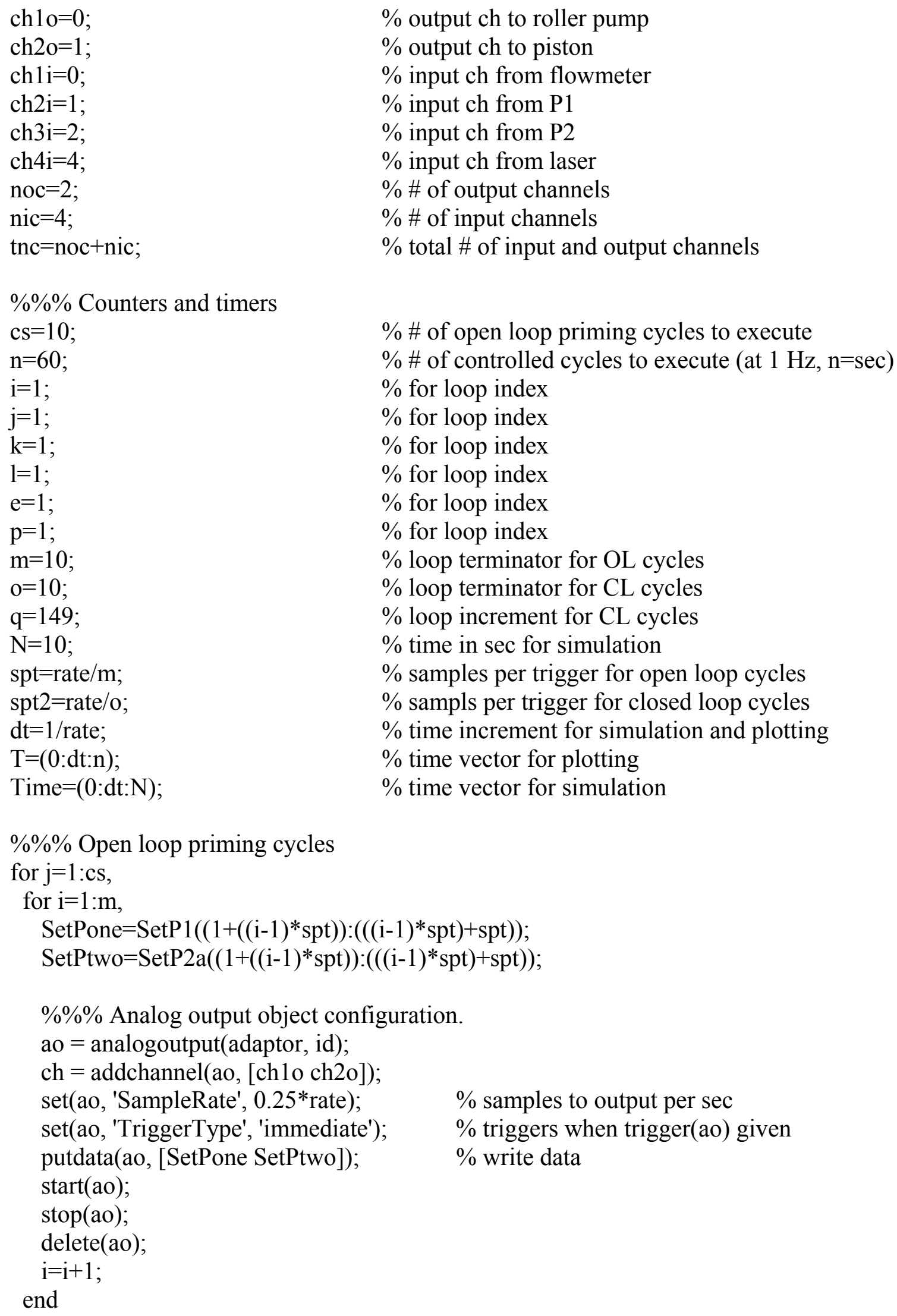




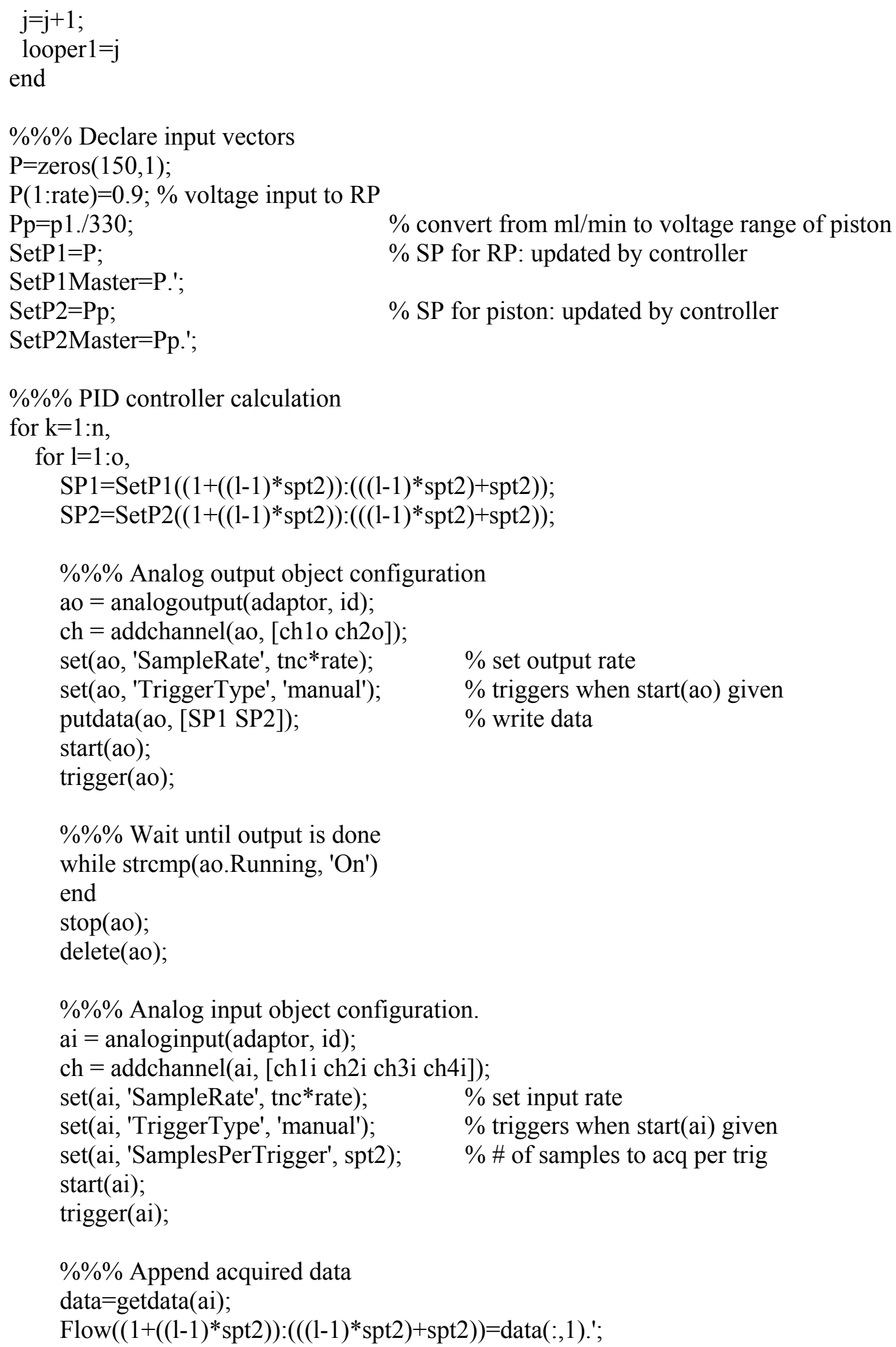




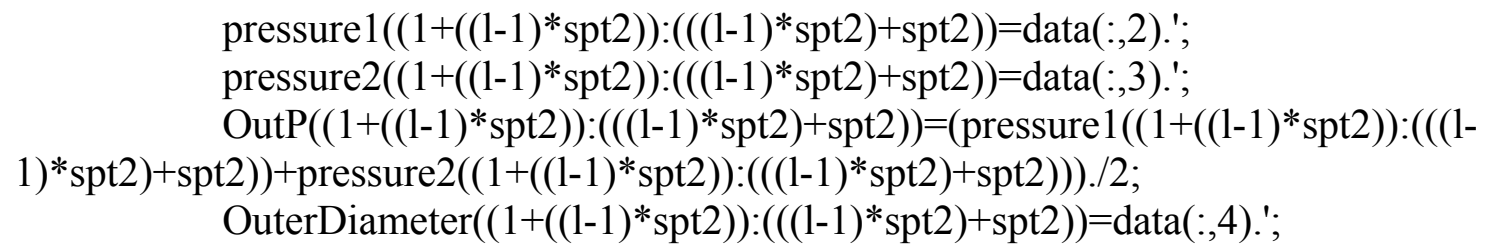

$\% \% \%$ Mean circumferential wall stress calculation variables $\mathrm{wtP} 1=0.0131 * 25.4 ; \%$ proximal thickness $\# 1$ of wrap in $\mathrm{mm}$ from histology measurements

widP $=0.1086 * 25.4 ; \%$ proximal unpressurized inner diameter of wrap in $\mathrm{mm}$ from histology measurements

wtP2 $=0.0133 * 25.4 ; \%$ proximal thickness \#2 of wrap in $\mathrm{mm}$ from histology measurements

$\mathrm{wtD} 1=0.0131 * 25.4 ; \%$ distal thickness $\# 1$ in of wrap $\mathrm{mm}$ from histology measurements

widD $=0.1086 * 25.4 ; \%$ distal unpressurized inner diameter of wrap in $\mathrm{mm}$ from histology measurements 
wtD2 $=0.0133 * 25.4 ; \%$ distal thickness $\# 2$ of wrap in $\mathrm{mm}$ from histology measurements

$\mathrm{wtP}=(\mathrm{wtP} 1+\mathrm{wtP} 2) / 2 ; \%$ proximnal thickness of wrap in $\mathrm{mm}$

$\mathrm{wtD}=(\mathrm{wtD} 1+\mathrm{wtD} 2) / 2 ; \%$ distal thickness of wrap in $\mathrm{mm}$

$\mathrm{wtu}=(\mathrm{wtP}+\mathrm{wtD}) / 2 ; \%$ unpressurized thickness of wrap in $\mathrm{mm}$

$\mathrm{wIDu}=($ widP+widD $) / 2 ; \%$ unpressurized inner diameter of wrap in $\mathrm{mm}$

$\mathrm{bu}=\mathrm{wIDu} / 2 ; \%$ unpressurized inner radius of wrap in $\mathrm{mm}$

$\mathrm{cu}=\mathrm{bu}+\mathrm{wtu}$

$\mathrm{vtP} 1=0.0131 * 25.4 ; \%$ proximal thickness $\# 1$ of vein in $\mathrm{mm}$ from histology

measurements

vidP $=0.1086 * 25.4 ; \%$ proximal unpressurized inner diameter of vein in $\mathrm{mm}$ from histology measurements

$\mathrm{vtP} 2=0.0133 * 25.4 ; \%$ proximal thickness $\# 2$ of vein in $\mathrm{mm}$ from histology measurements

$\mathrm{vtD} 1=0.0131 * 25.4 ; \%$ distal thickness $\# 1$ in of vein $\mathrm{mm}$ from histology measurements

vidD $=0.1086 * 25.4 ; \%$ distal unpressurized inner diameter of vein in $\mathrm{mm}$ from histology measurements

$\mathrm{vtD} 2=0.0133 * 25.4 ; \%$ distal thickness $\# 2$ of vein in $\mathrm{mm}$ from histology measurements

$\mathrm{vtP}=(\mathrm{vtP} 1+\mathrm{vtP} 2) / 2 ; \%$ proximnal thickness of vein in $\mathrm{mm}$

$\mathrm{vtD}=(\mathrm{vtD} 1+\mathrm{vtD} 2) / 2 ; \%$ distal thickness of vein in $\mathrm{mm}$

$\mathrm{vtu}=(\mathrm{vtP}+\mathrm{vtD}) / 2 ; \%$ unpressurized thickness of vein in $\mathrm{mm}$

$\mathrm{vIDu}=(\mathrm{vidP}+\mathrm{vidD}) / 2 ; \%$ unpressurized inner diameter of vein in $\mathrm{mm}$

$\mathrm{au}=\mathrm{vIDu} / 2 ; \%$ unpressurized inner radius of vein in $\mathrm{mm}$

$\mathrm{Lu}=50 ; \%$ unstretched length of vessel segment in $\mathrm{mm}$

$\mathrm{Lp}=1.3 * \mathrm{Lu} ; \%$ in-vivo length of vessel segment in $\mathrm{mm}$

$\mathrm{ODp}=\mathrm{ODf} ; \%$ pressurized outer diameter in $\mathrm{mm}$

$\mathrm{cp}=\mathrm{ODp} . / 2 ; \%$ pressurized outer radius in $\mathrm{mm}$

$\% \% \%$ calculate pressurized inner radius assuming incompressibility

$\operatorname{IRp}=\operatorname{zeros}(150,1)$

TEMPVAR1=zeros $(150,1)$;

TEMPVAR2 $=$ zeros $(150,1)$;

TEMPVAR1 $=\left(\left(\left(\mathrm{cp} \wedge^{\wedge} 2\right) \cdot{ }^{*} \mathrm{Lp} . *\left(\mathrm{cu}^{\wedge} 2-\mathrm{bu}{ }^{\wedge} 2\right)^{*} \mathrm{Lu}\right) . / \mathrm{Lp}\right.$;

$\mathrm{v}=1$;

for $\mathrm{v}=1$ :rate,

$\mathrm{bp}(\mathrm{v})=\operatorname{sqrt}(\operatorname{TEMPVAR} 1(\mathrm{v}))$;

end

bp $=$ bp.' \% pressurized interfacial radius in $\mathrm{mm}$

TEMPVAR2 $=\left(\left((c p . \wedge 2) \cdot{ }^{*} \mathrm{Lp} .{ }^{*}\left(\mathrm{cu}^{\wedge} 2-\mathrm{bu}^{\wedge} 2\right)^{*} \mathrm{Lu} / \mathrm{Lp}\right)-\left(\mathrm{bu} \wedge 2-\mathrm{au}{ }^{\wedge} 2\right) * \mathrm{Lu}\right) . / \mathrm{Lp}$;

$\mathrm{v}=1$;

for $\mathrm{v}=1$ :rate,

$\operatorname{ap}(v)=\operatorname{sqrt}(\operatorname{TEMPVAR} 2(\mathrm{v}))$;

end

ap=ap.' \% pressurized inner radius in $\mathrm{mm}$

$\% \% \%$ Interfacial pressure calculation 
$\mathrm{P}=1.333224 .{ }^{*}$ (OutPf.*46.76)./1e3; \% Convert intraluminal pressure from $\mathrm{mmHg}$ to $\mathrm{KPa}$

$\mathrm{Nu}=0.5 ; \%$ Poisson's ratio of wrap and vein

$\mathrm{Ew}=7500 ; \%$ Young's modulus of wrap in $\mathrm{KPa}$

$\mathrm{Ev}=600 ; \%$ Young's modulus of vein in $\mathrm{KPa}$

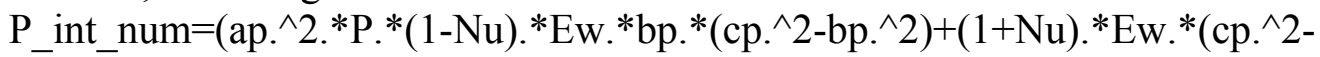

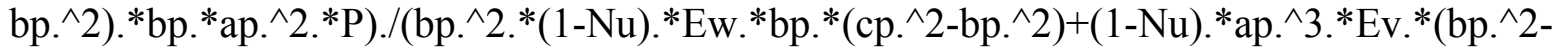

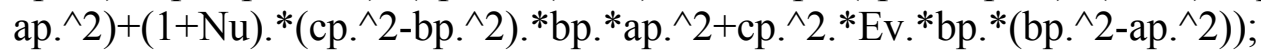

$\% \% \%$ Mid-vein-wall circumferential wall stress calculation

rho=(bp-ap). $/ 2 ; \%$ prescribes midwall cws calc

hoop_stress $=\left(\left(\right.\right.$ ap. ${ }^{\wedge} 2{ }^{*} \mathrm{P}-\mathrm{bp} .{ }^{\wedge} 2{ }^{*}{ }^{*} \mathrm{P} \_$int $\left.) . /(\mathrm{bp} . \wedge 2-\mathrm{ap}, \wedge 2)\right)+((\mathrm{P}-$

P_int).*ap.^2.*bp.^2)./((bp.^2-ap.^2)./rho);

$\% \% \%$ Lumenal shear stress calculation

$\mathrm{Mu}=1.02 ; \%$ viscosity of perfusate in dynes* ${ }^{*} / \mathrm{cm}^{\wedge} 2$

shear_stress $=(4 *$ Mu.*Flowf)./(pi.*ap.^3);

$\% \% \%$ Prescribe size of and calculate error vectors

Err1=zeros(rate, 1$)$;

Err2=Err1;

Err1(1:rate) $=$ mean(hoop_stress)-mean(CWSc); \% errl calc for direct action

$\% \% \%$ Eliminate phase shift $\mathrm{b} / \mathrm{w}$ i/o vectors

lag $=1$;

for $\mathrm{e}=1$ :rate-lag,

Err2(e)=hoop_stress(e)-CWSc(e+lag); \% err2 calc for direct action

end

$\% \% \%$ Append i/o vectors for plotting

SetPMasterPlot $((1+((\mathrm{k}-1) *$ rate $)):(((\mathrm{k}-1) *$ rate $)+$ rate $))=\mathrm{p} 1(:) ;$

SetCWSMasterPlot $((1+((\mathrm{k}-1) *$ rate $)):(((\mathrm{k}-1) *$ rate $)+$ rate $))=\operatorname{CWSc}(:)$;

MFlow $((1+((\mathrm{k}-1) *$ rate $)):(((\mathrm{k}-1) *$ rate $)+$ rate $))=$ Flowf(:).*330;

$\operatorname{MP}((1+((\mathrm{k}-1) *$ rate $)):(((\mathrm{k}-1) *$ rate $)+$ rate $))=\operatorname{OutPf}(:) * 46.76$;

MFlow $((1+((\mathrm{k}-1) *$ rate $)):(((\mathrm{k}-1) *$ rate $)+$ rate $))=$ Flowf $(:) . * 330$;

$\operatorname{MMP}((1+((\mathrm{k}-1) *$ rate $)):(((\mathrm{k}-1) *$ rate $)+$ rate $))=\operatorname{mean}(\operatorname{MP}((1+((\mathrm{k}-1) *$ rate $)):(((\mathrm{k}-$

$1)^{*}$ rate $)+$ rate $\left.)\right)$;

MMFlow $((1+((\mathrm{k}-1) *$ rate $)):(((\mathrm{k}-1) *$ rate $)+$ rate $))=\operatorname{mean}(\operatorname{MFlow}((1+((\mathrm{k}-1) *$ rate $)):((\mathrm{k}-$

$1)^{*}$ rate $)+$ rate $\left.)\right)$;

$\operatorname{MMR}((1+((\mathrm{k}-1) *$ rate $)):(((\mathrm{k}-1) *$ rate $)+$ rate $))=\operatorname{MMP}((1+((\mathrm{k}-1) *$ rate $)):(((\mathrm{k}-$

$1)^{*}$ rate $)+$ rate $\left.)\right) . / M M F l o w((1+((\mathrm{k}-1) *$ rate $)):(((\mathrm{k}-1) *$ rate $)+$ rate $))$;

$\operatorname{MOD}((1+((\mathrm{k}-1) *$ rate $)):(((\mathrm{k}-1) *$ rate $)+$ rate $))=\operatorname{ODf}(:)$;

$\operatorname{MCWS}((1+((\mathrm{k}-1) *$ rate $)):(((\mathrm{k}-1) *$ rate $)+$ rate $))=$ hoop_stress $(:)$;

$\operatorname{MSS}((1+((\mathrm{k}-1) *$ rate $)):(((\mathrm{k}-1) *$ rate $)+$ rate $))=$ shear_stress $(:) ;$

Error1 $((1+((\mathrm{k}-1) *$ rate $)):(((\mathrm{k}-1) *$ rate $)+$ rate $))=\operatorname{Err} \overline{1}(:)$

Error2 $((1+((\mathrm{k}-1) *$ rate $)):(((\mathrm{k}-1) *$ rate $)+$ rate $))=\operatorname{Err} 2(:)$; 
$\% \% \%$ Initialize variables for PID algorithm

ErrLast1 $=$ Err 1 ;

ErrLast2=Err2;

ErrLastLast $1=$ ErrLast 1 ;

ErrLastLast2=ErrLast2;

Input $1 \mathrm{a}=100$. SetP $1 ; \%$ input signal for simulation \#1

Input $2 \mathrm{a}=330 .{ }^{*} \mathrm{SetP} 2 ; \%$ input signal for simulation $\# 2$

$\% \% \%$ PID control variables for simulation \#1

$\mathrm{kd} 1=0$;

$\%$ derivative gain

$\mathrm{kp} 1=0$;

$\%$ proportional gain

ki1 $=0$;

$\%$ integral gain

numPID1=[kd1 kp1 ki1];

$\operatorname{denPID} 1=\left[\begin{array}{ll}1 & 0\end{array}\right]$;

numc $1=\operatorname{conv}($ num1, denPID1);

denc1=polyadd(conv(denPID1,den1), conv(numPID1,num1));

sys $1 \mathrm{a}=\mathrm{tf}($ numc $1, \mathrm{denc} 1, \mathrm{dt})$;

$\% \% \%$ PID control variables for simulation \#2

$\mathrm{kd} 2=0$;

$\%$ derivative gain

$\mathrm{kp} 2=0$;

$\%$ proportional gain

ki2 $=0$;

$\%$ integral gain

numPID2 $=[\mathrm{kd} 2 \mathrm{kp} 2 \mathrm{ki} 2]$;

denPID2 $=\left[\begin{array}{ll}1 & 0\end{array}\right]$;

numc $2=\operatorname{conv}($ num2, denPID2);

denc $2=$ polyadd(conv(denPID2, den2), $\operatorname{conv}($ numPID2,num2));

sys $2 \mathrm{a}=\mathrm{tf}($ numc $2, \mathrm{denc} 2, \mathrm{dt})$;

$\% \% \%$ Declare variables for PID control algorithm

$\mathrm{Kp}=1$;

$\%$ gain

$\mathrm{Ki}=20$;

$\%$ reset rate in repeats per minutes

$\mathrm{Kd}=1$;

$\%$ derivative time in minites

$\% \% \%$ Set controller tolerances

iteration $=0$;

iteration $\max =20$;

Err_tol=zeros(rate, 1$)$;

Err_tol( 1 :rate $)=1 ; \%$ tolerance in $\mathrm{KPa}$

for $\mathrm{p}=1$ :rate-q,

if $\left((0-\operatorname{Err} 2(p: p+q))>\operatorname{Err} \_t o l(p: p+q)\right) \mid((0-\operatorname{Err} 1(p: p+q))>\operatorname{Err}$ tol $(p: p+q))$,

Message $1=$ 'Error is -'

while

$\operatorname{Err1}(\mathrm{p}: \mathrm{p}+\mathrm{q}))>\operatorname{Err}$ tol $(\mathrm{p}: \mathrm{p}+\mathrm{q})) \&($ iteration $<=$ iteration_max $)$,

$((0-\operatorname{Err} 2(p: p+q))>$ Err_tol $(p: p+q)) \&((0-$

$\% \% \%$ simulate the system response to previous input

Input1_i=interp(Input1 $\mathrm{a}(\mathrm{p}: \mathrm{p}+\mathrm{q}), \mathrm{N}) ; \%$ resize input for siumulation 
Input $2 \_\mathrm{i}=$ interp $(\operatorname{Input} 2 \mathrm{a}(\mathrm{p}: \mathrm{p}+\mathrm{q}), \mathrm{N}) ; \%$ resize input for siumulation $\operatorname{IRp}=\operatorname{interp}(\operatorname{IRp}(\mathrm{p}: \mathrm{p}+\mathrm{q}), \mathrm{N}) ; \%$ resize input for siumulation $\mathrm{ORp}=\operatorname{interp}(\mathrm{ORp}(\mathrm{p}: \mathrm{p}+\mathrm{q}), \mathrm{N}) ; \%$ resize input for siumulation OutPS1a=lsim(sys1a,Input1_i,Time(1:length(Input1_i))); OutPS2a=lsim(sys2a,Input2_i,Time(1:1ength(Input2_i )) ;

$\% \% \%$ calculate the CWS from the simulated pressure output

OutPS1b=(OutPS1a.*(IRp.^2)+(((IRp.^2).*(ORp.^2))./(rho.^2)).*OutPS1a)./(ORp.^2-IRp.^2);

OutPS2b=(OutPS2a.*(IRp.^2)+(((IRp.^2).*(ORp.^2))./(rho.^2))**OutPS2a)./(ORp.^2-IRp.^2);

OutPS1 $(\mathrm{p}: \mathrm{p}+\mathrm{q})=$ resample(OutPS1b, $1, \mathrm{~N}) ; \%$ resisize input to system

OutPS2 $(p: p+q)=$ resample(OutPS2b, $1, N) ; \%$ resisize input to system

$\mathrm{IRp}=$ resample(IRp, $1, \mathrm{~N})$; \% resize input for siumulation

$\mathrm{ORp}=$ resample(ORp, $1, \mathrm{~N}) ; \%$ resize input for siumulation

$\% \% \%$ calculate the change in output using the derivative of the

$\% \% \%$ PID algorithm then add to the previous output

$\operatorname{Err1}(\mathrm{p}: \mathrm{p}+\mathrm{q})=\operatorname{mean}(\operatorname{OutPS} 1(\mathrm{p}: \mathrm{p}+\mathrm{q}))-\operatorname{mean}(\mathrm{CWSc}(\mathrm{p}: \mathrm{p}+\mathrm{q}))$;

$\operatorname{Err} 2(p: p+q)=\operatorname{OutPS} 2(p: p+q) \cdot-C W S c(p: p+q)$;

$\operatorname{dOutP} 1(p: p+q)=K p \cdot *(\operatorname{Err} 1(p: p+q)-$

$\operatorname{ErrLast} 1(\mathrm{p}: \mathrm{p}+\mathrm{q})+\mathrm{Ki} .{ }^{*} \operatorname{Err1}(\mathrm{p}: \mathrm{p}+\mathrm{q}) . / \mathrm{rate}+\mathrm{Kd} .{ }^{*}(\operatorname{Err} 1(\mathrm{p}: \mathrm{p}+\mathrm{q})-$

2. $\left.\left.{ }^{*} \operatorname{ErLast} 1(\mathrm{p}: \mathrm{p}+\mathrm{q})+\operatorname{ErrLastLast} 1(\mathrm{p}: \mathrm{p}+\mathrm{q})\right)\right)$;

dOutP2 $(p: p+q)=K p \cdot *(\operatorname{Err} 2(p: p+q)-$

$\operatorname{ErrLast} 2(p: p+q)+K i . * \operatorname{Err} 2(p: p+q) . / r a t e+K d . *(\operatorname{Err} 2(p: p+q)-$

2. $\left.\left.{ }^{*} \operatorname{ErrLast} 2(\mathrm{p}: \mathrm{p}+\mathrm{q})+\operatorname{ErrLastLast} 2(\mathrm{p}: \mathrm{p}+\mathrm{q})\right)\right)$;

$\% \% \%$ Update input - recaclculate pressure from simulated $\% \% \%$ stress calcs

$\operatorname{Input} 1(p: p+q)=\operatorname{OutPS} 1(p: p+q)+d O u t P 1(p: p+q)$;

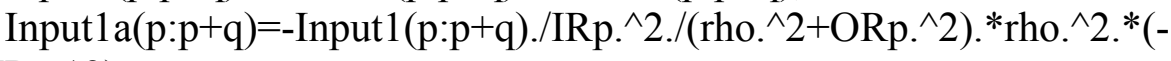

ORp.^2+IRp.^2);

Input $2(p: p+q)=\operatorname{OutPS} 2(p: p+q)+d O u t P 2(p: p+q)$;

Input $2 a(p: p+q)=-I n p u t 2(p: p+q) . / I R p . \wedge 2 . /\left(\right.$ rho. $\left.{ }^{\wedge}+O R p . \wedge 2\right) .^{*}$ rho.^2.*(-

ORp.^2+IRp.^2);

$\% \% \%$ Update variables for PID algorithm

$\operatorname{ErrLastLast} 1(\mathrm{p}: \mathrm{p}+\mathrm{q})=\operatorname{ErrLast} 1(\mathrm{p}: \mathrm{p}+\mathrm{q}) ;$

$\operatorname{ErrLastLast} 2(p: p+q)=\operatorname{ErrLast} 2(p: p+q)$;

$\operatorname{ErrLast} 1(p: p+q)=\operatorname{Err} 1(p: p+q)$;

ErrLast2 $(p: p+q)=E r r 2(p: p+q)$;

iteration $=$ iteration +1 ;

end

$\% \% \%$ Update setpoints 
$\operatorname{SetP} 1(p: p+q)=\operatorname{Input} 1 \mathrm{a}(\mathrm{p}: \mathrm{p}+\mathrm{q}) . / 100$

$\operatorname{SetP} 2(p: p+q)=\operatorname{Input} 2 a(p: p+q) . / 330$;

elseif $\left(\operatorname{Err} 2(p: p+q)>\operatorname{Err} \_t o l(p: p+q)\right) \mid\left(\operatorname{Err} 1(p: p+q)>\operatorname{Err} \_t o l(p: p+q)\right)$,

Message $1={ }^{\prime}$ 'Error is ${ }^{-}{ }^{\prime}$

while

$\left(\operatorname{Err} 2(p: p+q)>\operatorname{Err} \_t o l(p: p+q)\right) \&\left(\operatorname{Err1}(p: p+q)>\operatorname{Err} \_t o l(p: p+q)\right) \&($ iteration $<=$ iteration_max $)$, $\% \% \%$ simulate the system response to previous input

Input1_i $\mathrm{i}=$ interp(Input1 $\mathrm{a}(\mathrm{p}: \mathrm{p}+\mathrm{q}), \mathrm{N}) ; \%$ resize input for siumulation

Input2_i $\mathrm{i}$ interp(Input2a(p:p+q),N);\% resize input for siumulation

$\operatorname{IRp}=\operatorname{interp}(\operatorname{IRp}(\mathrm{p}: \mathrm{p}+\mathrm{q}), \mathrm{N}) ; \%$ resize input for siumulation

$\mathrm{ORp}=\operatorname{interp}(\mathrm{ORp}(\mathrm{p}: \mathrm{p}+\mathrm{q}), \mathrm{N}) ; \%$ resize input for siumulation

OutPS1a=1sim(sys1a,Input1_i,Time(1:length(Input1_i)));

OutPS2a=1sim(sys2a,Input2_i,Time(1:length(Input2_i)));

$\% \% \%$ calculate the CWS from the simulated pressure output

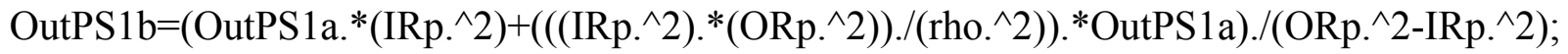

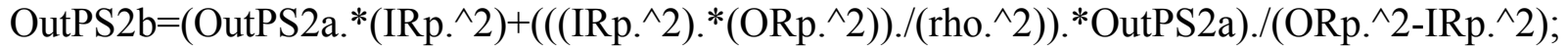

OutPS1 $(\mathrm{p}: \mathrm{p}+\mathrm{q})=$ resample(OutPS1b,1,N); \% resisize input to system

OutPS2 $(\mathrm{p}: \mathrm{p}+\mathrm{q})=$ resample $($ OutPS2b, $1, \mathrm{~N}) ; \%$ resisize input to system

IRp=resample(IRp, $1, \mathrm{~N}) ; \%$ resize input for siumulation

$\mathrm{ORp}=$ resample $(\mathrm{ORp}, 1, \mathrm{~N}) ; \%$ resize input for siumulation

$\% \% \%$ calculate the change in output using the derivative of the

$\% \% \%$ PID algorithm then add to the previous output

$\operatorname{Errl}(p: p+q)=\operatorname{mean}(\operatorname{OutPS} 1(p: p+q))-\operatorname{mean}(\operatorname{CWSc}(p: p+q))$;

$\operatorname{Err} 2(\mathrm{p}: \mathrm{p}+\mathrm{q})=\operatorname{OutPS} 2(\mathrm{p}: \mathrm{p}+\mathrm{q}) \cdot-\mathrm{CWSc}(\mathrm{p}: \mathrm{p}+\mathrm{q})$;

$\operatorname{dOutP} 1(p: p+q)=K p \cdot *(\operatorname{Err} 1(p: p+q)-$

$\operatorname{ErrLast} 1(\mathrm{p}: \mathrm{p}+\mathrm{q})+\mathrm{Ki} .{ }^{*} \operatorname{Err} 1(\mathrm{p}: \mathrm{p}+\mathrm{q}) . / \mathrm{rate}+\mathrm{Kd} .{ }^{*}(\operatorname{Err} 1(\mathrm{p}: \mathrm{p}+\mathrm{q})-$

2. $\left.\left.{ }^{*} \operatorname{ErLLast} 1(\mathrm{p}: \mathrm{p}+\mathrm{q})+\operatorname{ErrLastLast} 1(\mathrm{p}: \mathrm{p}+\mathrm{q})\right)\right)$;

$\operatorname{dOutP} 2(p: p+q)=K p \cdot *(\operatorname{Err} 2(p: p+q)-$

ErrLast2(p:p+q)+Ki.*Err2(p:p+q)./rate+Kd.*(Err2(p:p+q)-

2. $\left.\left.{ }^{*} \operatorname{ErLast} 2(\mathrm{p}: \mathrm{p}+\mathrm{q})+\operatorname{ErrLastLast} 2(\mathrm{p}: \mathrm{p}+\mathrm{q})\right)\right)$;

$\% \% \%$ Update input - recaclculate pressure from simulated

$\% \% \%$ stress calcs

Input $1(p: p+q)=\operatorname{OutPS} 1(p: p+q)-d O u t P 1(p: p+q)$

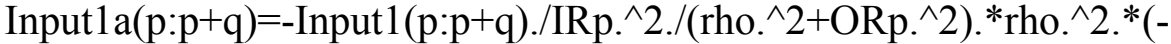

ORp.^2+IRp.^2);

Input $2(p: p+q)=\operatorname{OutPS} 2(p: p+q)-d O u t P 2(p: p+q)$

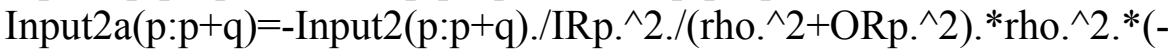

ORp.^2+IRp.^2);

$\% \% \%$ Update variables for PID algorithm 


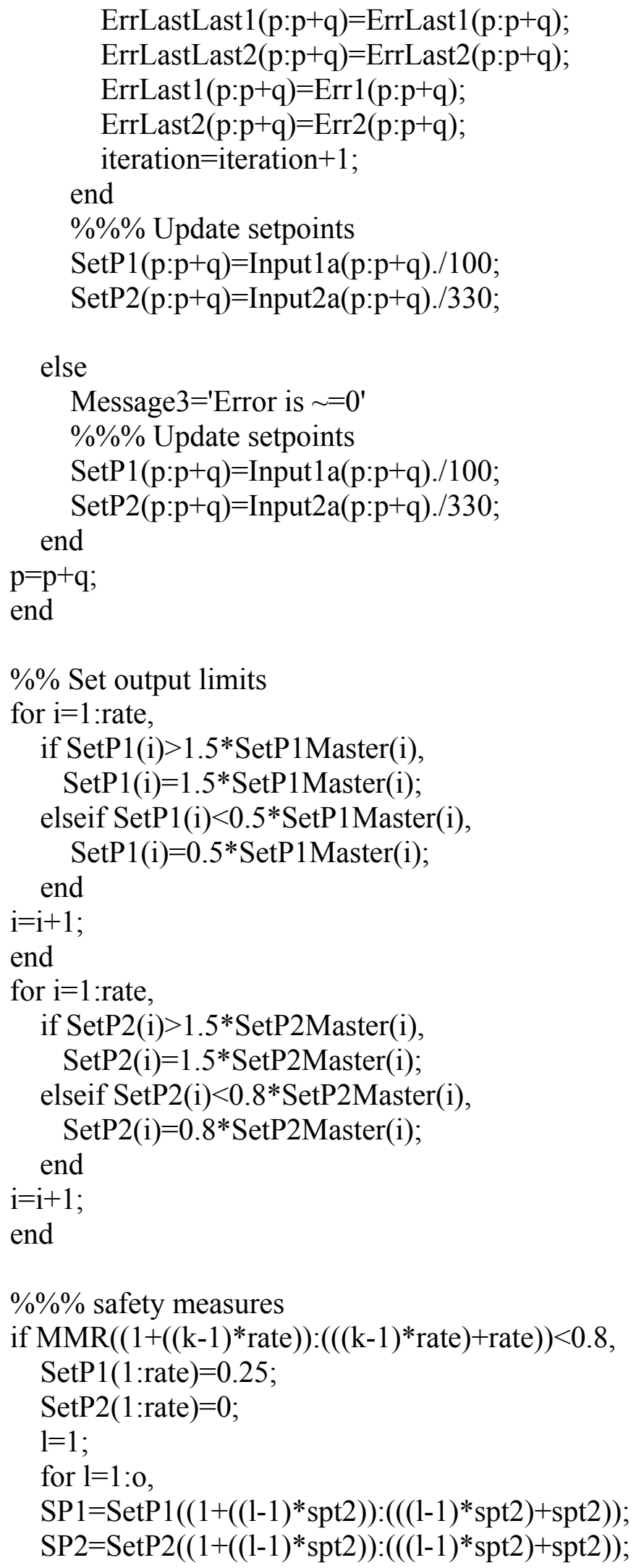




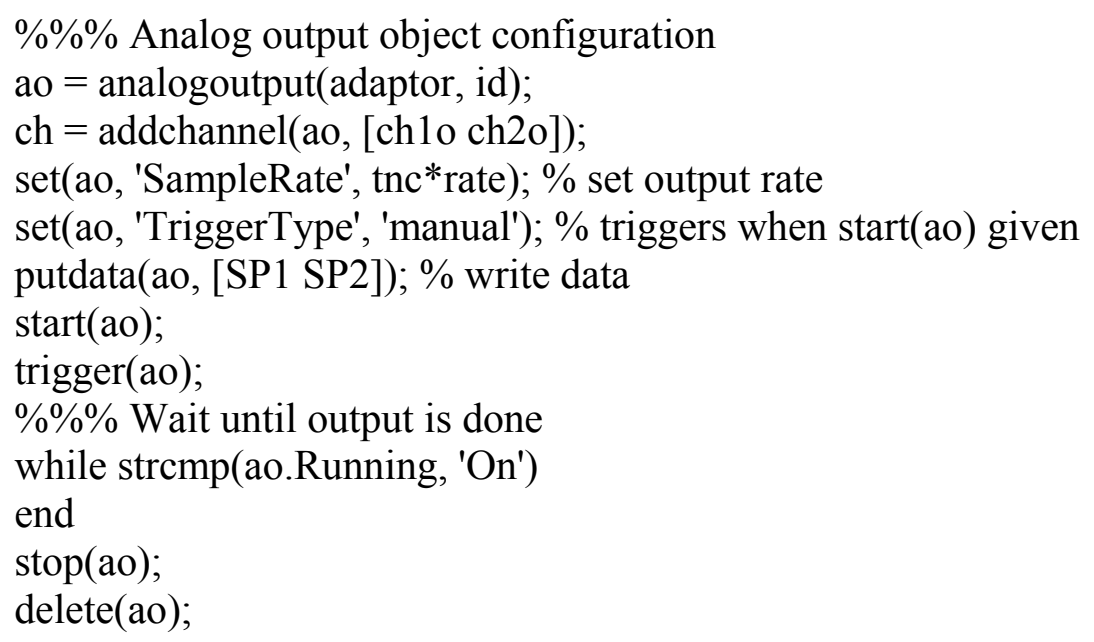


FlowSf=sgolayfilt(FlowS,n1,m2); \% filter flowrate data

ODSf=sgolayfilt(OuterDiameterS,n1,m3); \% filter outer diameter data

$\% \% \%$ Filter output again using a first order Savitzky-Golay filter

OutPSf=sgolayfilt(OutPSf,n1,m3); \% filter pressure data

FlowSf=sgolayfilt(FlowSf,n1,m2); \% filter flowrate data

ODSf=sgolayfilt(ODSf,n1,m3); \% filter outer diameter data

$\% \% \%$ Transpose acquired data to column vectors

OutPSf=OutPSf.';

FlowSf=FlowSf.';

ODSf $=$ ODSf.';

$\operatorname{MP}((1+((\mathrm{k}-1) *$ rate $)):(((\mathrm{k}-1) *$ rate $)+$ rate $))=\operatorname{OutPSf}(:) . * 46.76$;

$\operatorname{MFlow}((1+((\mathrm{k}-1) *$ rate $)):(((\mathrm{k}-1) *$ rate $)+$ rate $))=$ FlowSf(:).*330;

$\operatorname{MMP}((1+((\mathrm{k}-1) *$ rate $)):(((\mathrm{k}-1) *$ rate $)+$ rate $))=\operatorname{mean}(\mathrm{MP}((1+((\mathrm{k}-1) *$ rate $)):(((\mathrm{k}-$

$1)^{*}$ rate $)+$ rate $\left.)\right)$;

MMFlow $((1+((\mathrm{k}-1) *$ rate $)):(((\mathrm{k}-1) *$ rate $)+$ rate $))=\operatorname{mean}($ MFlow $((1+((\mathrm{k}-1) *$ rate $)):(((\mathrm{k}-$

1)*rate $)+$ rate $))$;

$\operatorname{MMR}((1+((\mathrm{k}-1) *$ rate $)):(((\mathrm{k}-1) *$ rate $)+$ rate $))=\operatorname{MMP}((1+((\mathrm{k}-1) *$ rate $)):(((\mathrm{k}-$

$1) *$ rate $)+$ rate $)) . / M M F l o w((1+((\mathrm{k}-1) *$ rate $)):(((\mathrm{k}-1) *$ rate $)+$ rate $))$;

else

end

$\mathrm{k}=\mathrm{k}+1$;

looper $2=\mathrm{k}$

end

$\% \%$ stop pump and piston

adaptor $=$ 'nidaq';

$\mathrm{id}=1$;

output $=0$;

ao = analogoutput(adaptor, id);

$\mathrm{ch}=\operatorname{addchannel}(\mathrm{ao},[\mathrm{ch} 1 \mathrm{o} \mathrm{ch} 2 \mathrm{o}])$;

set(ao, 'SampleRate', rate);

set(ao, 'TriggerType', 'manual');

putdata(ao, [output output]);

start(ao);

trigger(ao);

stop(ao);

delete(ao);

$\% \% \%$ Eliminate phase shift $\mathrm{b} / \mathrm{w}$ input and output CWS vectors

$\mathrm{i}=1$;

for $\mathrm{i}=1$ :length(SetCWSMasterPlot)-lag,

SetCWSMasterPlot(i)=SetCWSMasterPlot(i+lag);

end 
$\% \% \%$ Calculate Performance Metrics

$\mathrm{RSSp}=\mathrm{zeros}(\mathrm{n} *$ rate, 1$) ; \%$ set size of Residual Sum of Squares vector

npar $=2 ; \%$ \# of parameters

$\mathrm{np}=$ rate; \% size of input vector

$\mathrm{j}=10 ; \%$ for loop index

$\mathrm{h}=2 ; \%$ for loop index

for $\mathrm{k}=\mathrm{j}: \mathrm{n}$,

for $\mathrm{i}=\mathrm{h}: \mathrm{np}$,

$\operatorname{RSSp}(\mathrm{i})=\operatorname{RSSp}(\mathrm{i}-1)+((\operatorname{SetCWSMasterPlot}(\mathrm{i})-\mathrm{MCWS}(\mathrm{i})))^{\wedge} 2$;

end

$\operatorname{RSSc}(\mathrm{k})=$ mean $(\mathrm{RSSp})$;

end

$\mathrm{RSS}=\operatorname{mean}(\operatorname{RSSc}(\mathrm{j}: \mathrm{n}))$

$\%$ root mean square error calculation

RMSE $=\mathrm{sqrt}(\mathrm{RSS} /((\mathrm{np} * \mathrm{n}-\mathrm{j} *$ rate $)-$ npar $))$

$\% \% \%$ Plot results

$\mathrm{T}=\mathrm{T}(1:$ length$(\mathrm{MCWS}))$;

figure;

subplot $(2,2,1)$;

plot(T,MP,'b-');

$\operatorname{axis}([0 \mathrm{~T}($ length(MP) $) 0.95 *(\min (\mathrm{MP})) 1.05 *(\max (\mathrm{MP}))])$;

title('Measured Pressure vs Time')

xlabel('Time (s)');

ylabel('Pressure (mmHg)');

subplot $(2,2,2)$;

plot(T,MOD,'b-');

axis([0 T(length(MOD)) $0.95 *(\min (\mathrm{MOD})) 1.05 *(\max (\mathrm{MOD}))])$;

title('Outer Diameter vs Time')

xlabel('Time (s)');

ylabel('Outer Diameter $(\mathrm{mm})$ ');

subplot(2,2,3);

plot(T,MCWS,'b-');

hold on

plot(T,SetCWSMasterPlot,'r-');

axis([0 T(length(MCWS)) $0.95 *(\min (\operatorname{SetCWSMasterPlot})) 1.05 *(\max (\mathrm{MCWS}))])$;

title('Measured \& Set Circumferential Wall Stress')

xlabel('Time (s)');

ylabel('CWS (KPa)');

legend('Measured', 'SetPoint');

hold off

subplot $(2,2,4)$;

plot(T,MSS,'b-');

axis([0 T(length(MSS)) $0.95 *(\min (\mathrm{MSS})) 1.05 *(\max (\mathrm{MSS}))])$;

title('Shear Stress')

xlabel('Time (s)');

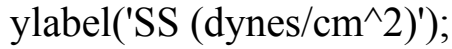


APPENDIX D

EX VIVO PERFUSION EXPERIMENTAL RECORDS 
Table D. 1 Perfusion experiment record for VEN vs. ART experiment performed on 04/23/2003

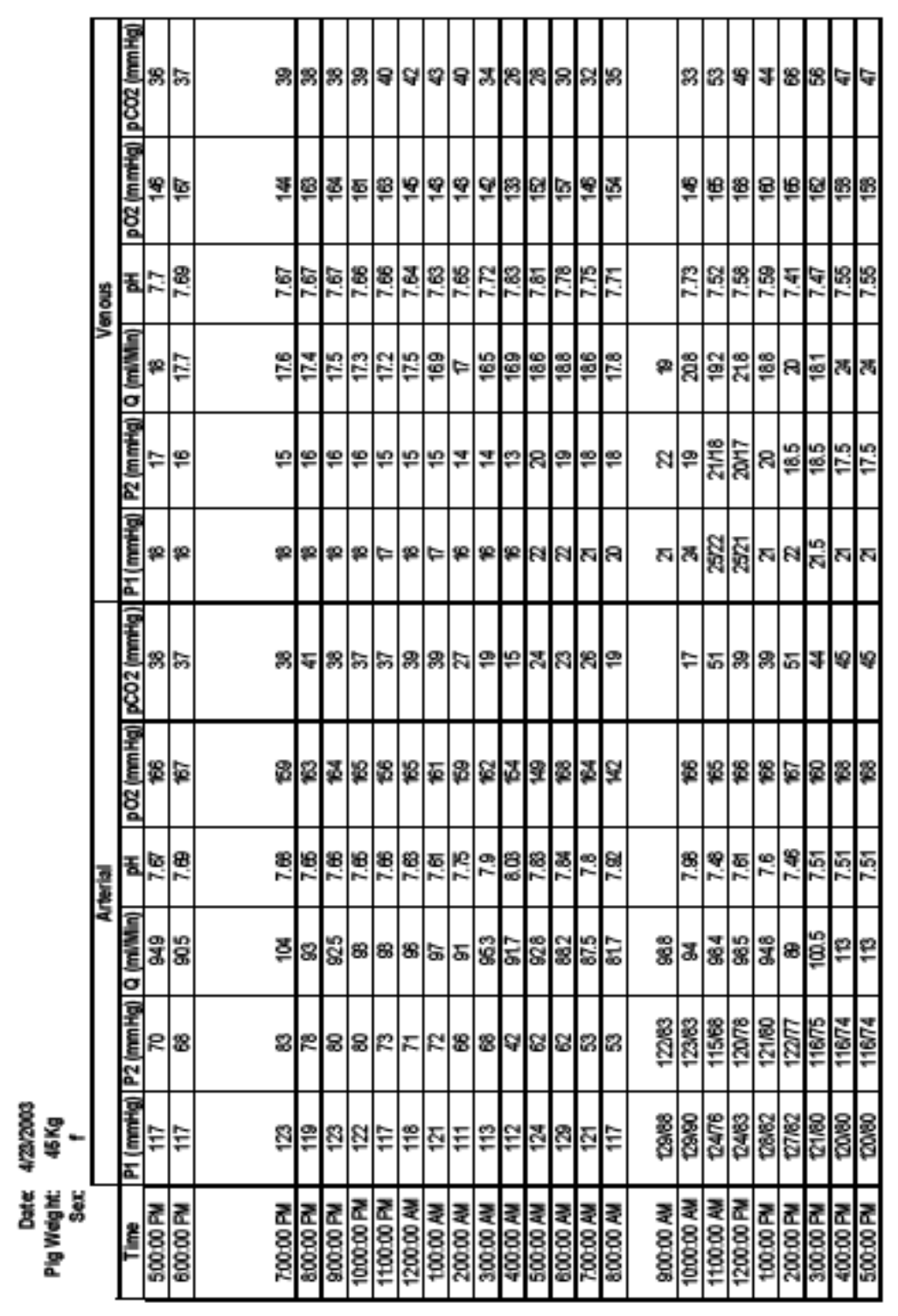


Table D. 2 Perfusion experiment record for VEN vs. ART experiment performed on 05/07/2003.

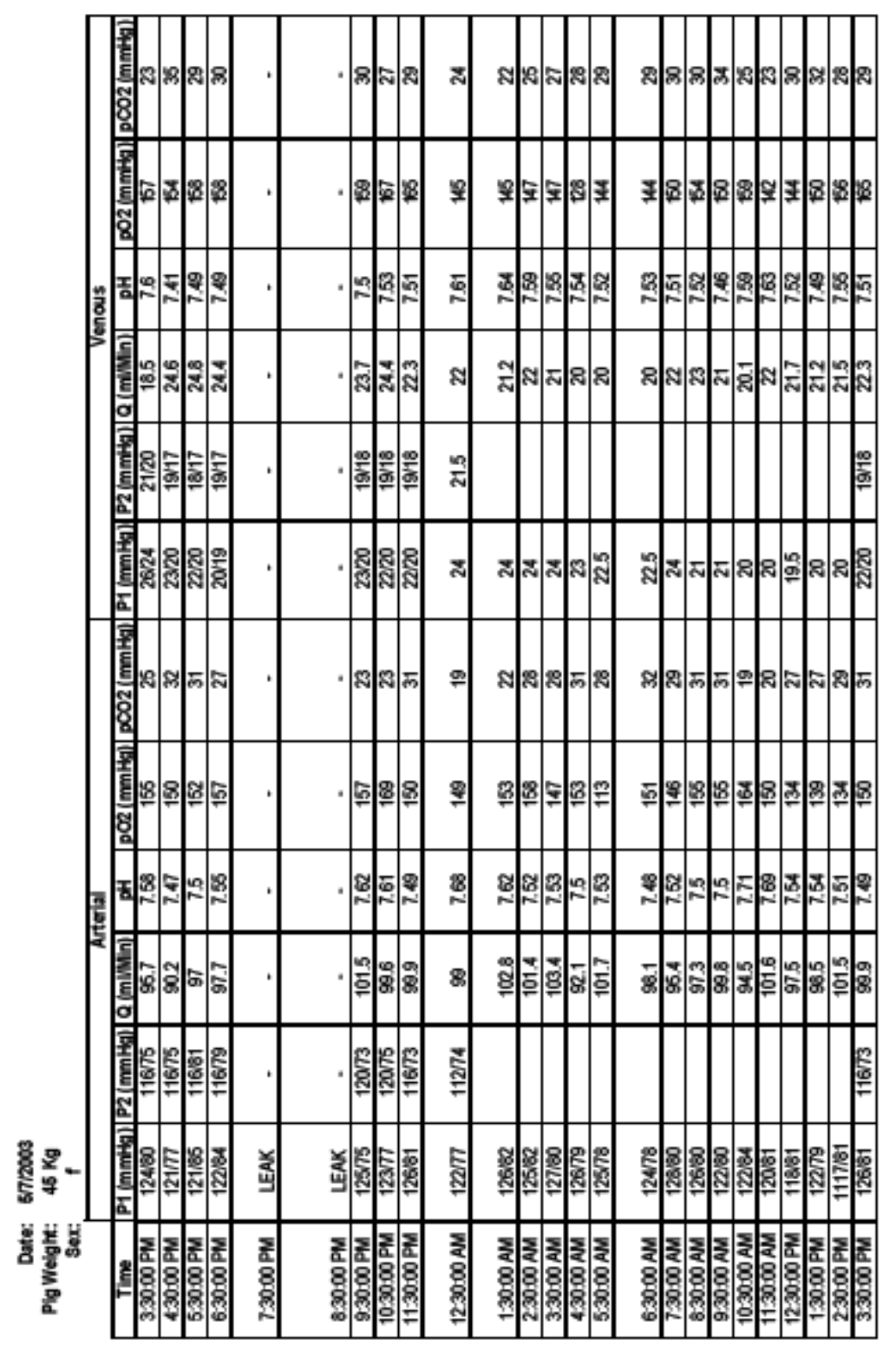


Table D. 3 Perfusion experiment record for VEN vs. ART experiment performed on 05/24/2006.

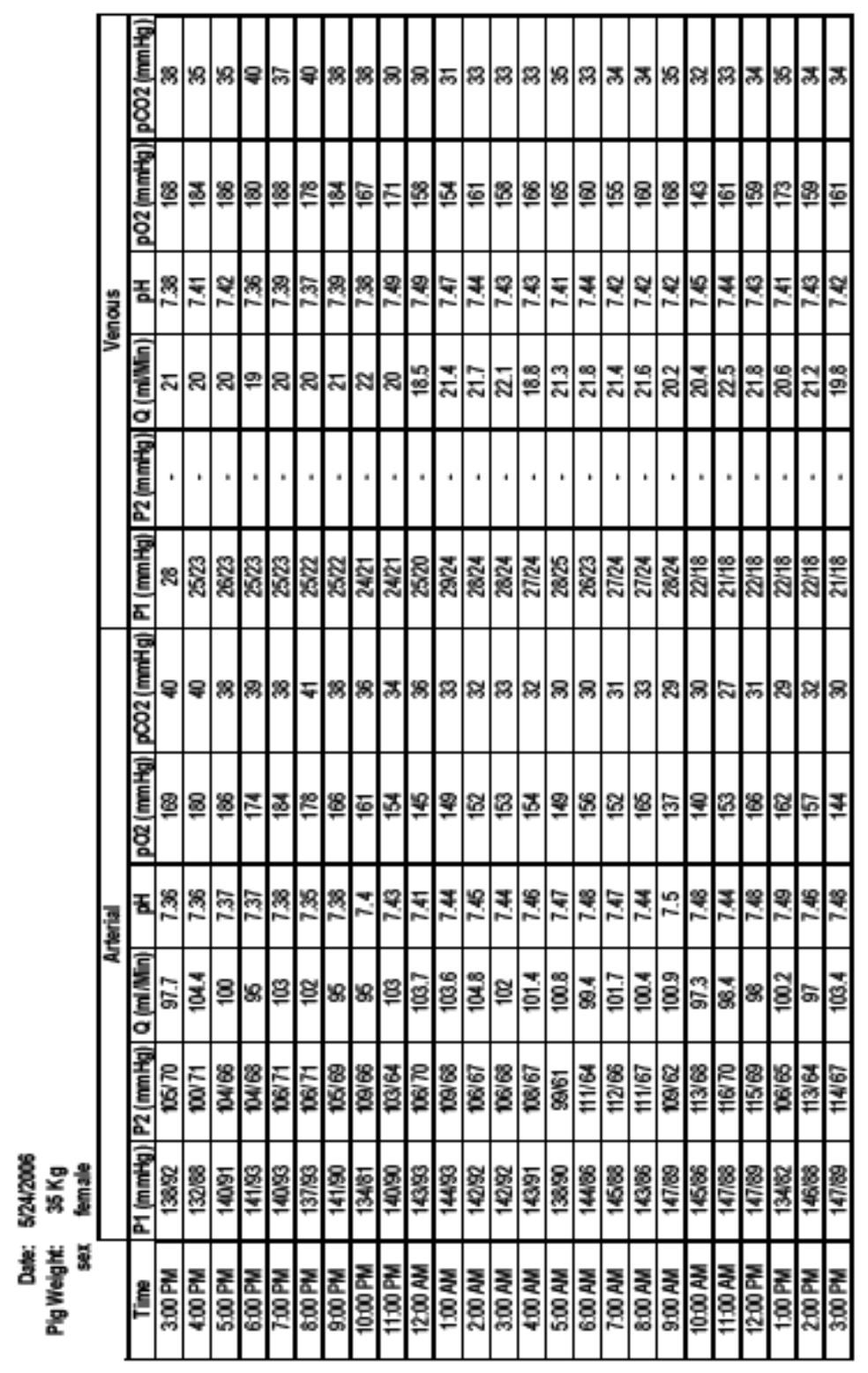


Table D. 4 Perfusion experiment record for VEN vs. ART experiment performed on 09/14/2006.

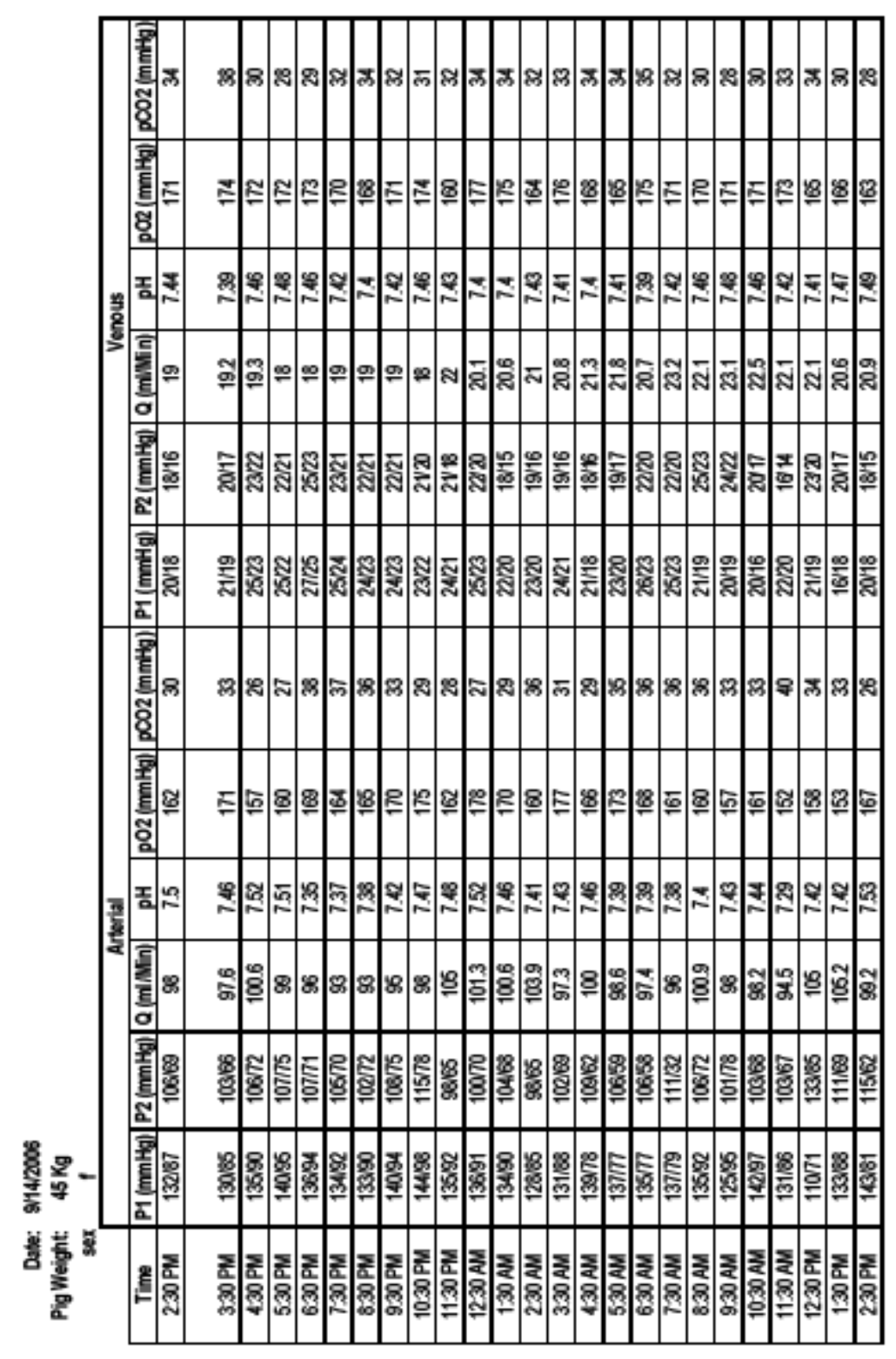


Table D. 5 Perfusion experiment record for VEN vs. ART experiment performed on 10/09/2004.

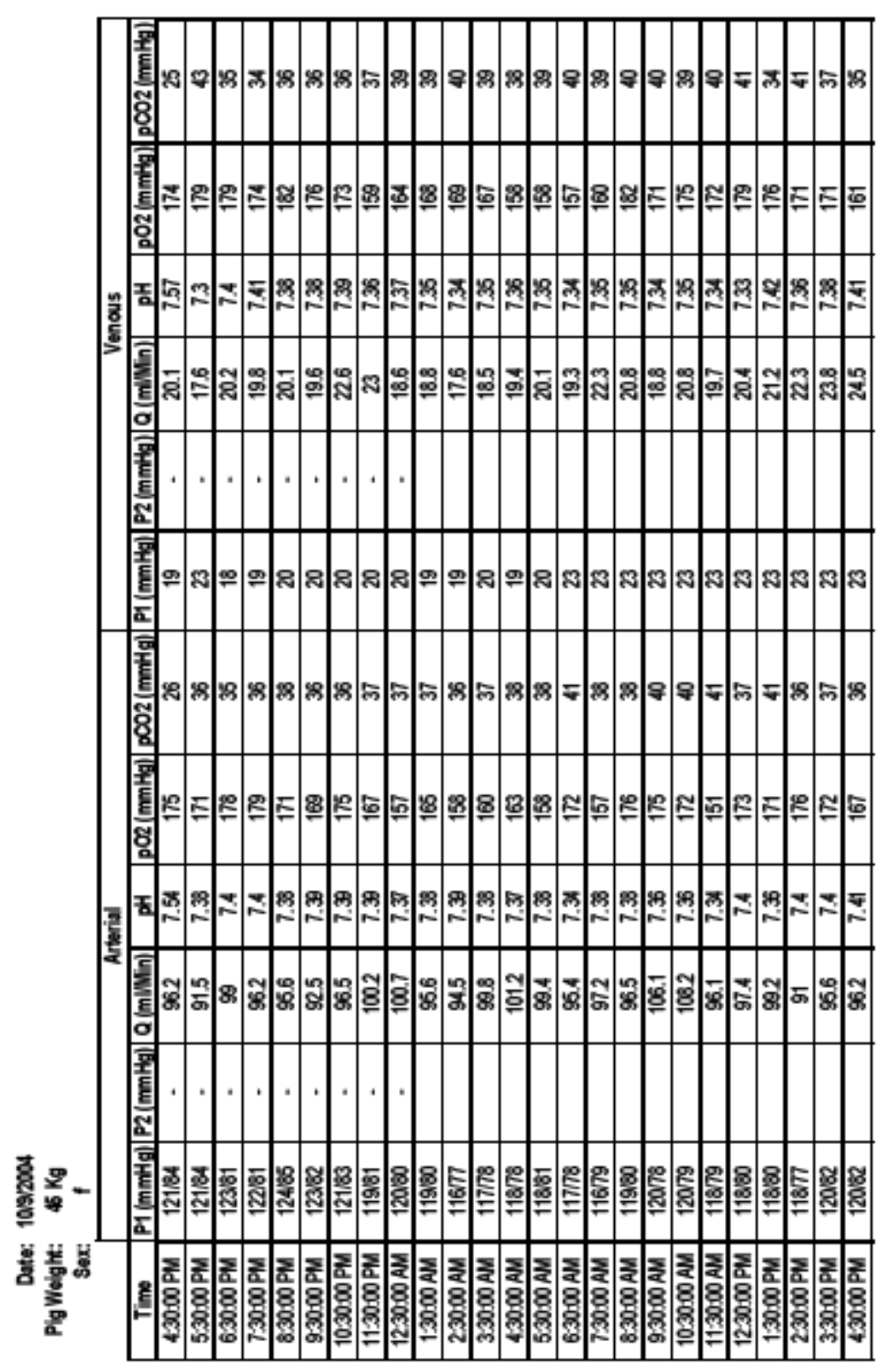


Table D. 6 Perfusion experiment record for VEN vs. ART experiment performed on 10/16/2004.

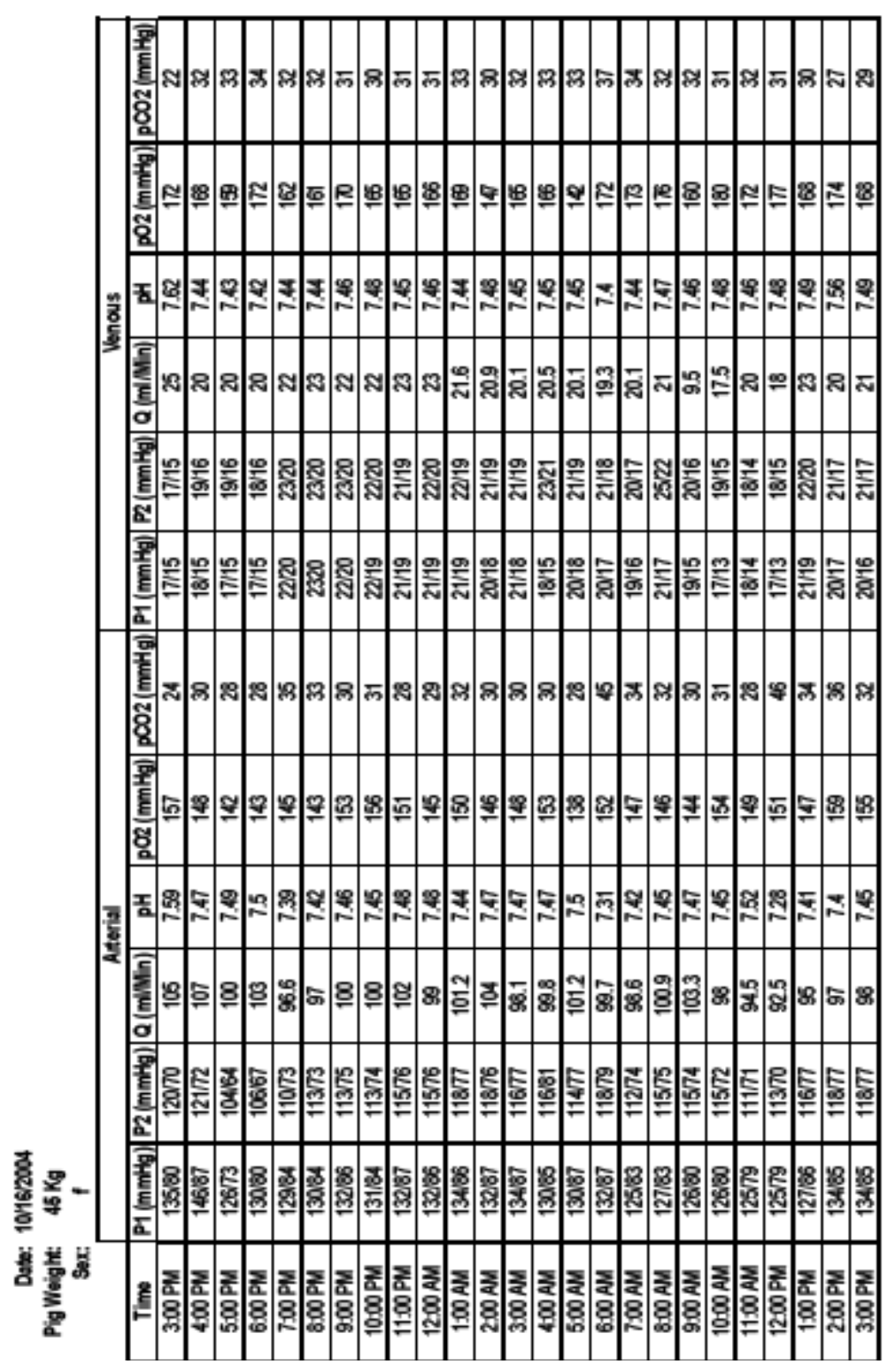



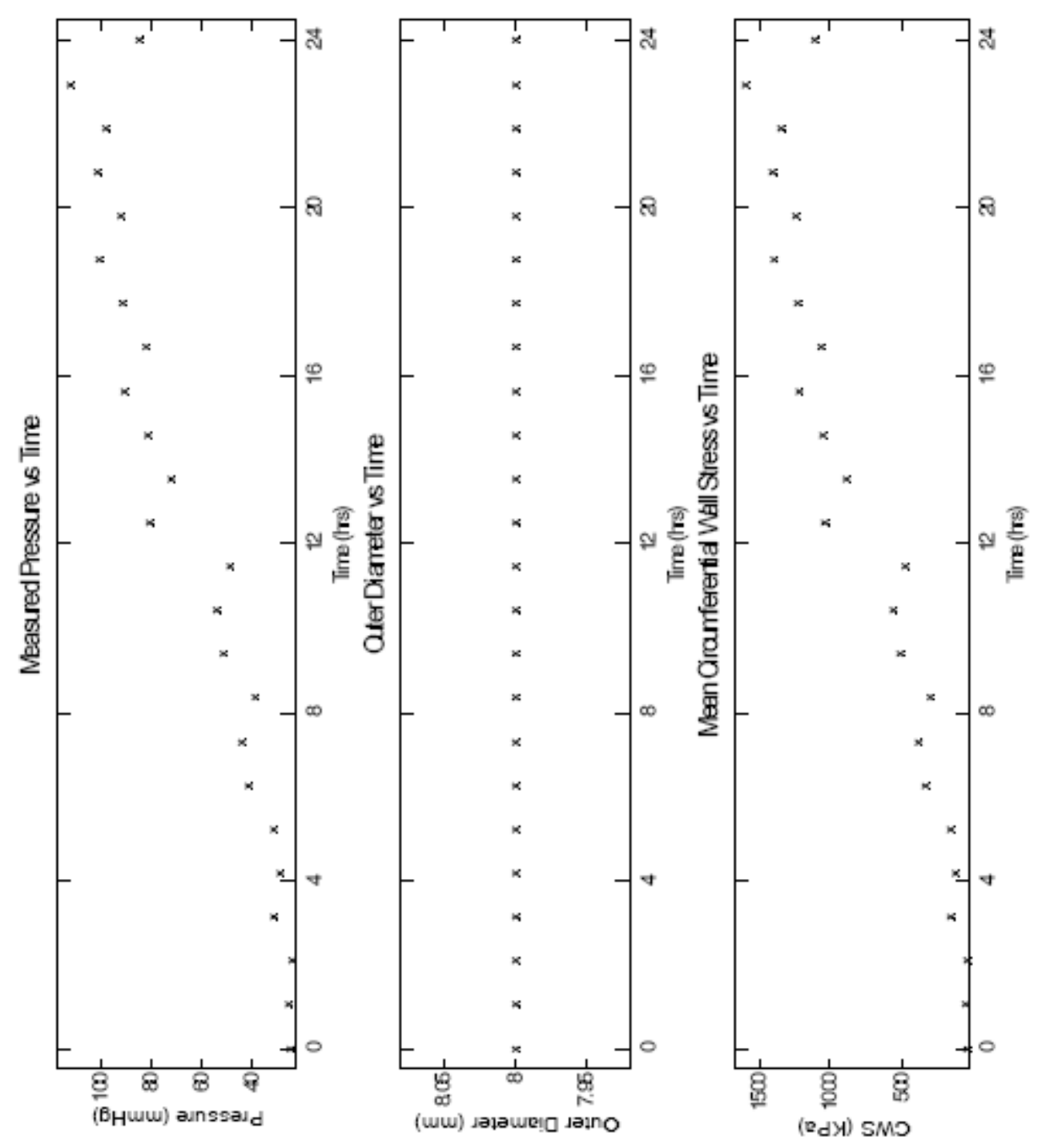

Figure D. 1 Mean intraluminal pressure, outer diameter, and CWS profiles for 24 hour ART vs. cART experiment performed on 10/22/2004. 


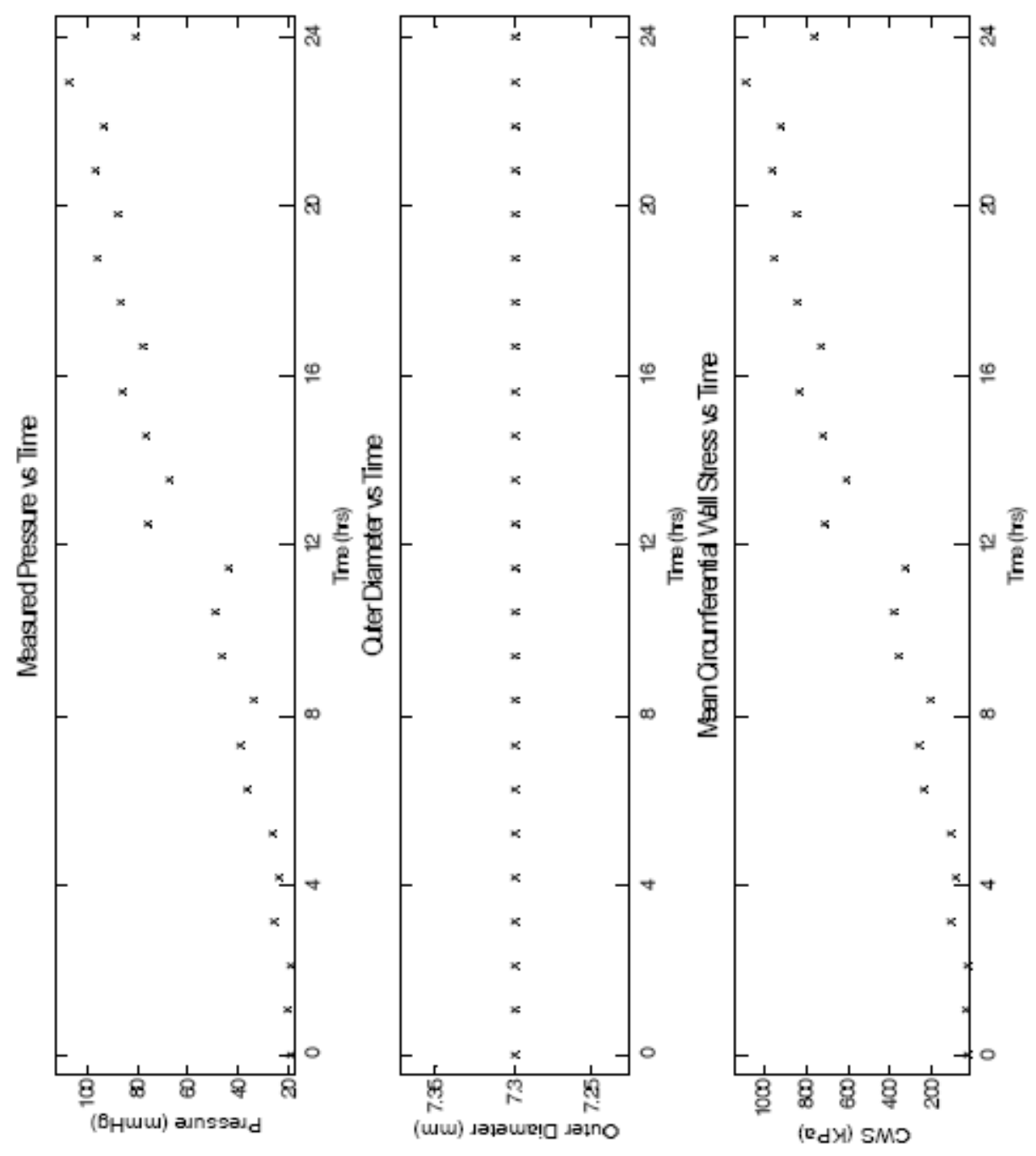

Figure D. 2 Mean intraluminal pressure, outer diameter, and CWS profiles for 24 hour ART vs. cART experiment performed on 11/01/2004. 


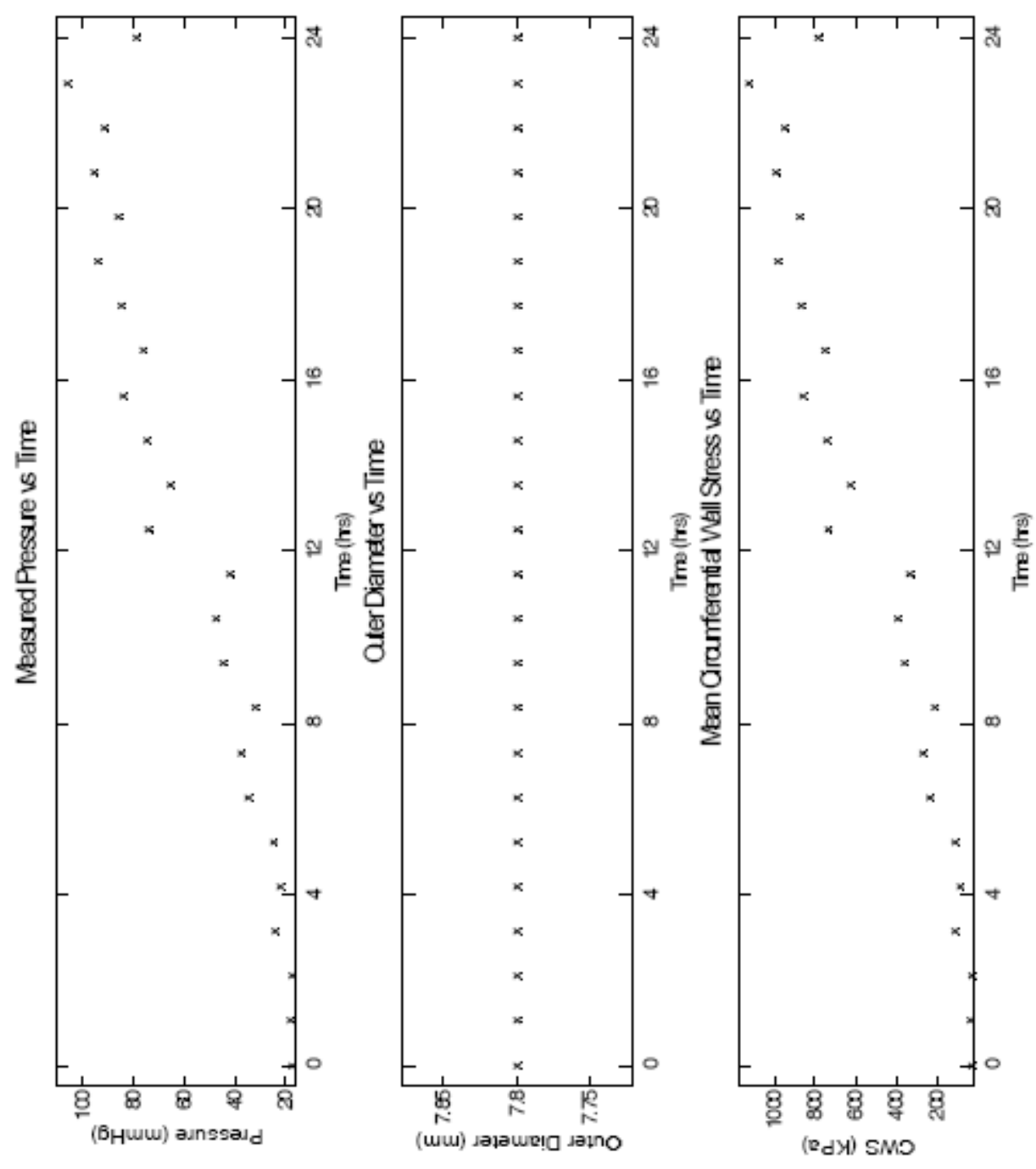

Figure D. 3 Mean intraluminal pressure, outer diameter, and CWS profiles for 24 hour ART vs. cART experiment performed on 11/11/2004. 

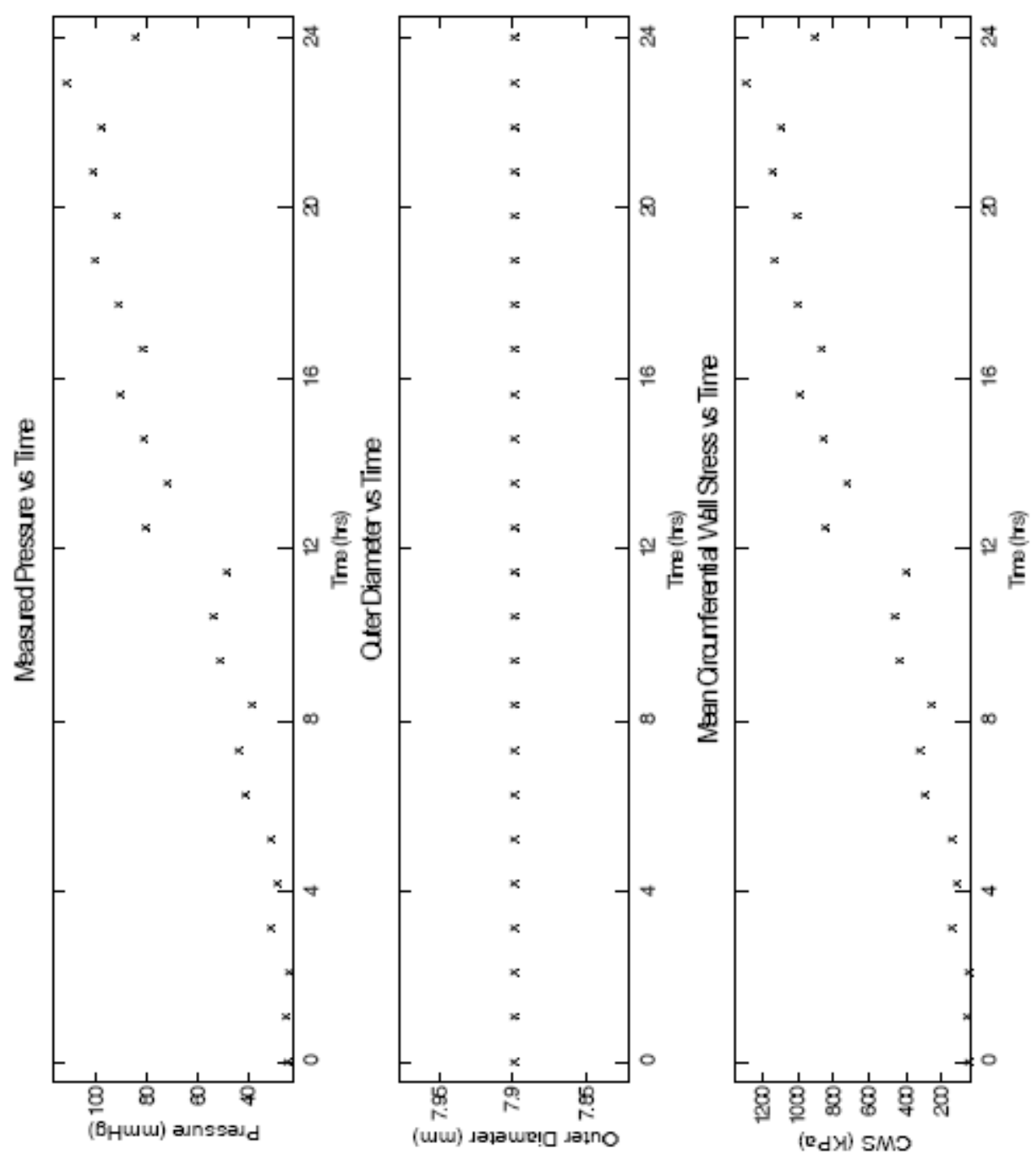

Figure D. 4 Mean intraluminal pressure, outer diameter, and CWS profiles for 24 hour ART vs. cART experiment performed on 09/21/2006. 


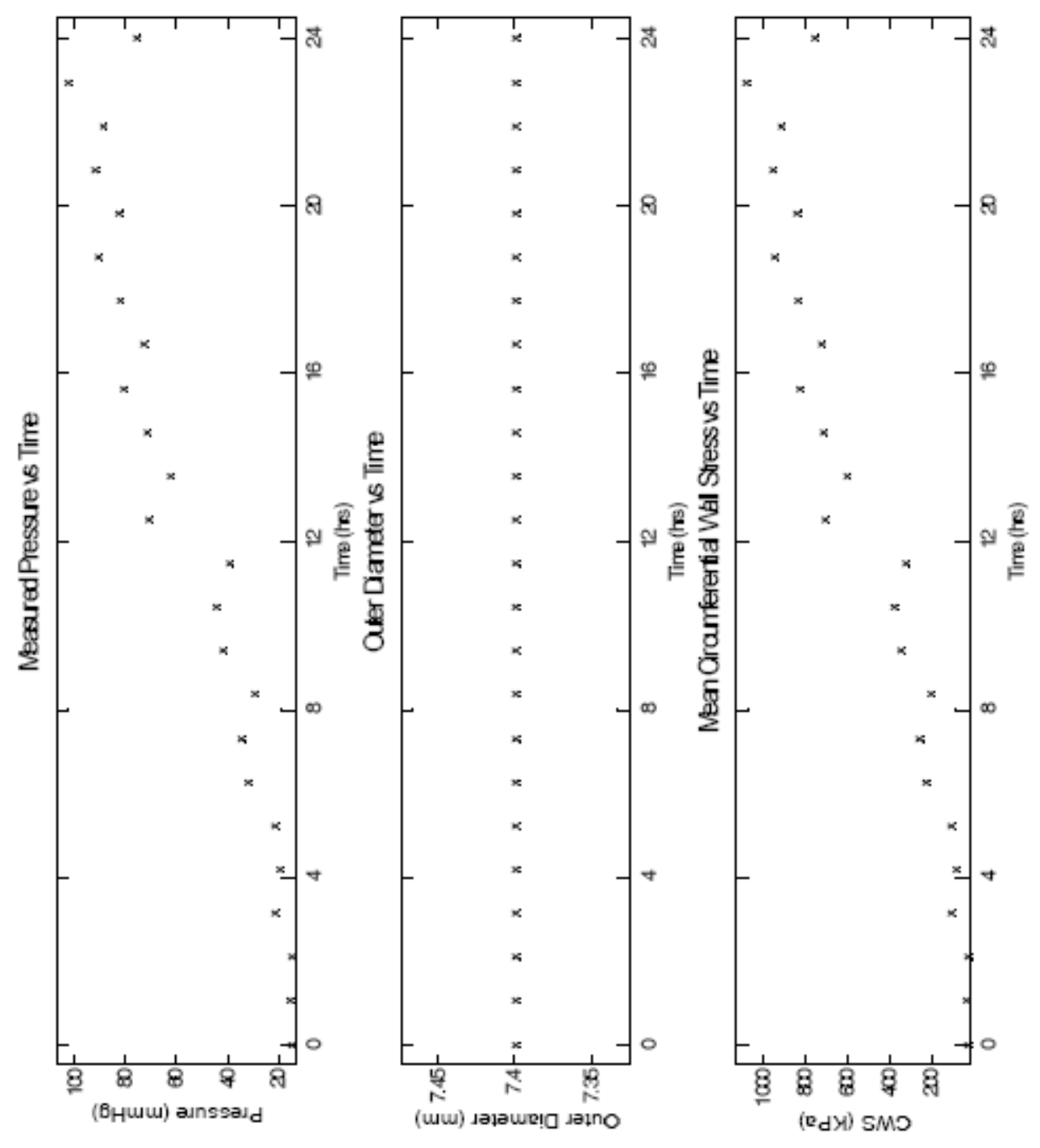

Figure D. 5 Mean intraluminal pressure, outer diameter, and CWS profiles for 24 hour ART vs. cART experiment performed on 10/16/2006. 

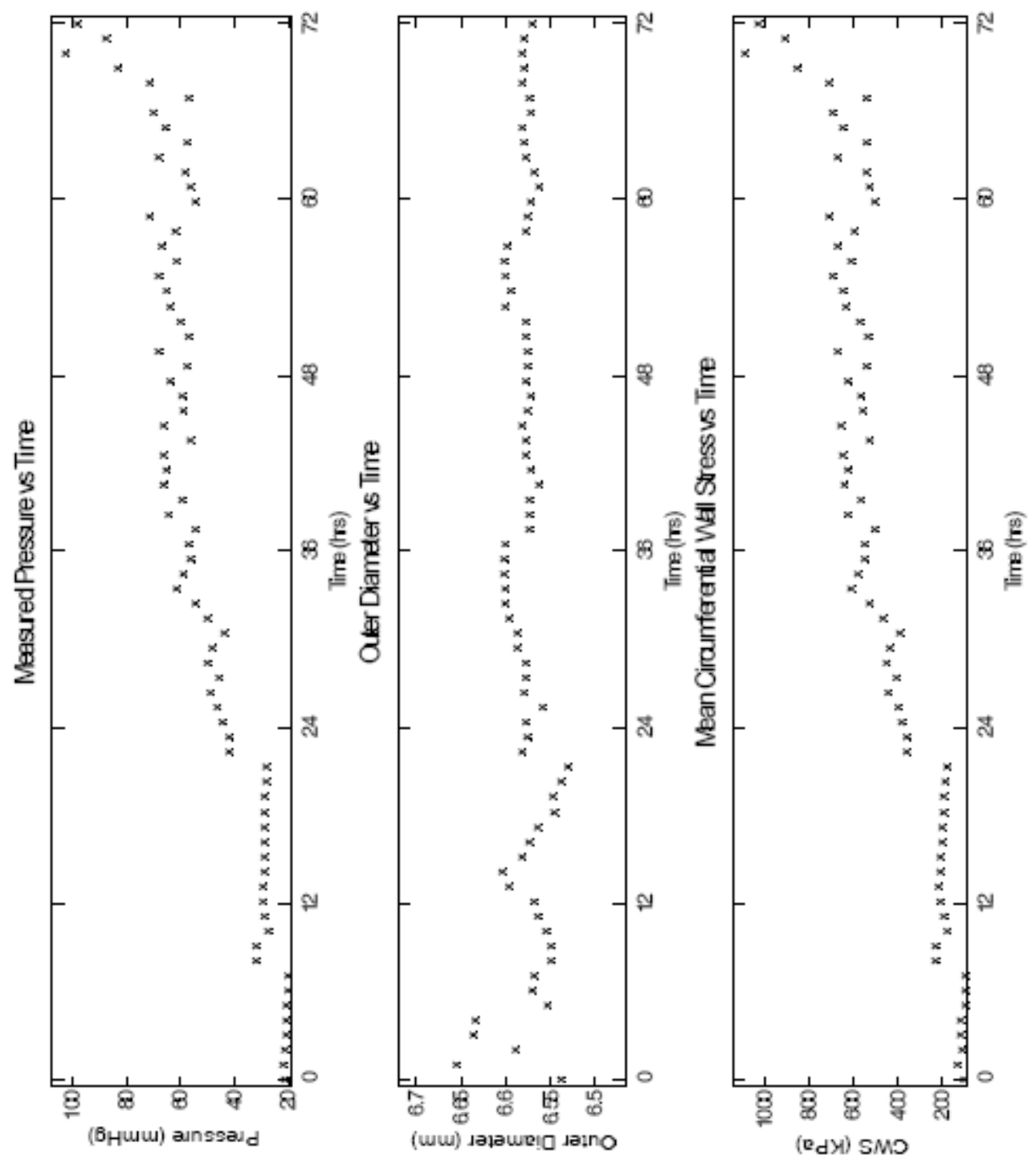

Figure D. 6 Mean intraluminal pressure, outer diameter, and CWS profiles for 72 hour ART vs. cART experiment performed on $02 / 16 / 2007$. 


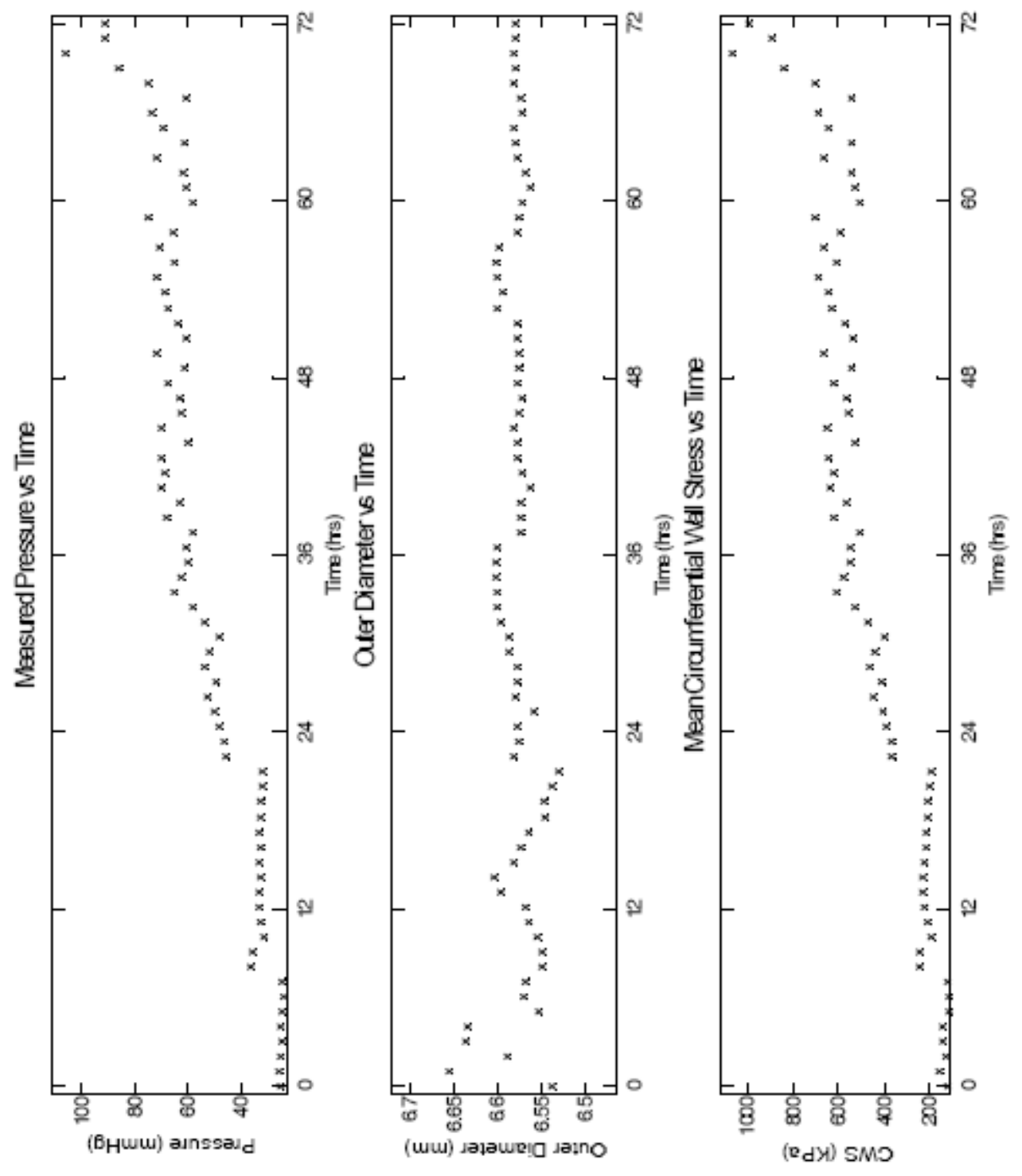

Figure D. 7 Mean intraluminal pressure, outer diameter, and CWS profiles for 72 hour ART vs. cART experiment performed on 03/07/2007. 


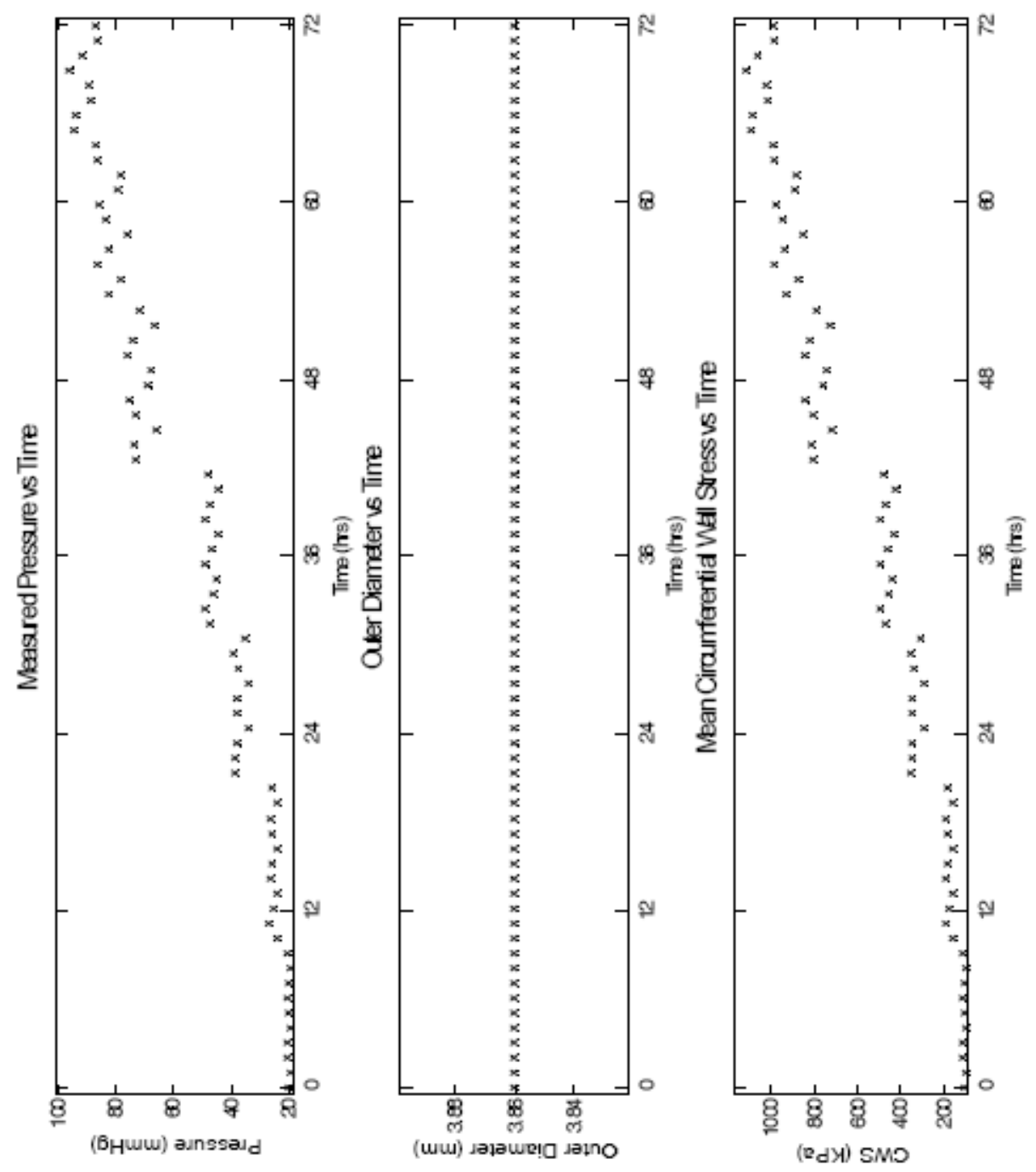

Figure D. 8 Mean intraluminal pressure, outer diameter, and CWS profiles for 72 hour ART vs. cART experiment performed on $03 / 15 / 2007$. 


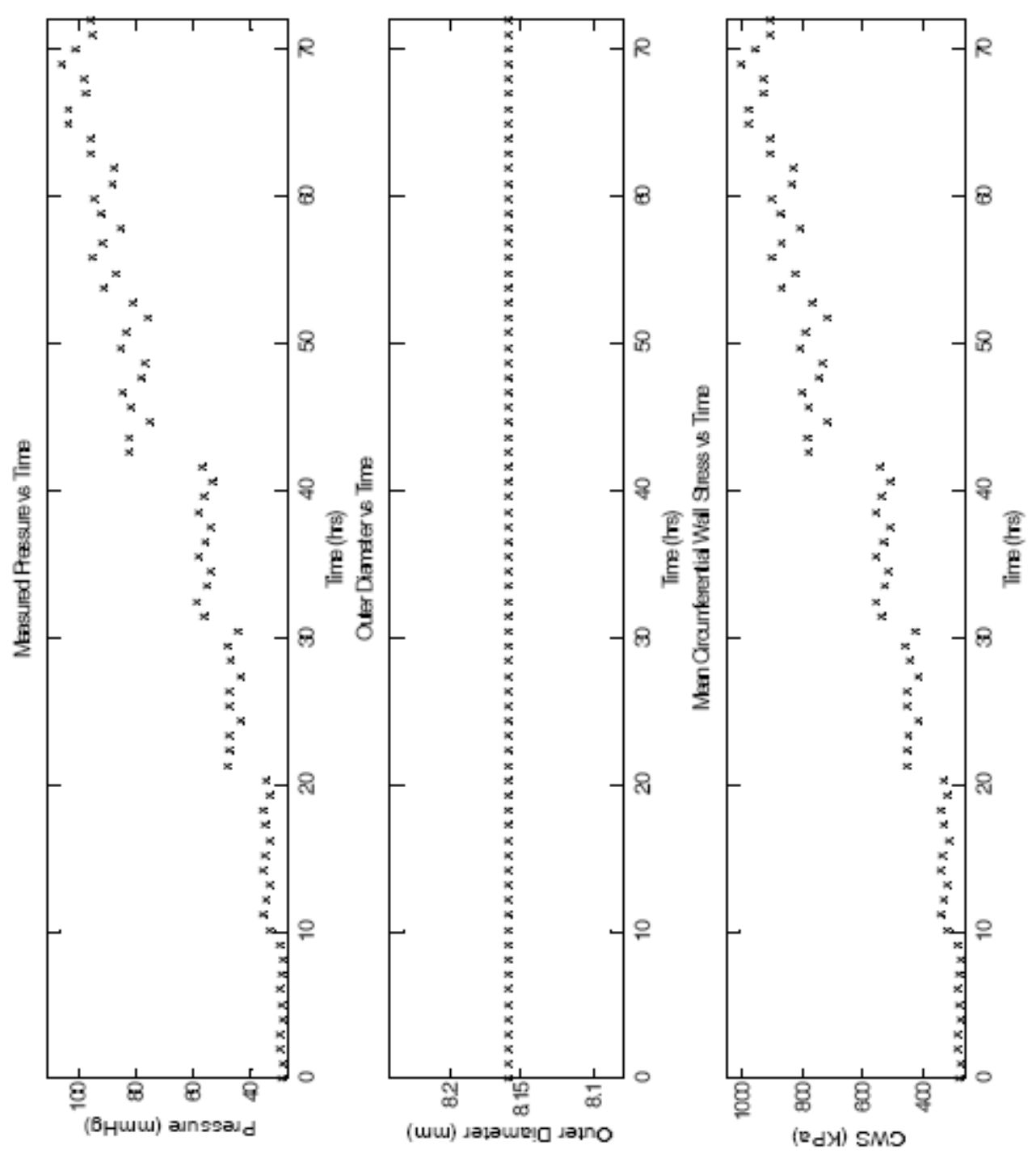

Figure D. 9 Mean intraluminal pressure, outer diameter, and CWS profiles for 72 hour ART vs. cART experiment performed on 04/02/2007. 
Vasomotor Challenge with EPI and SNP

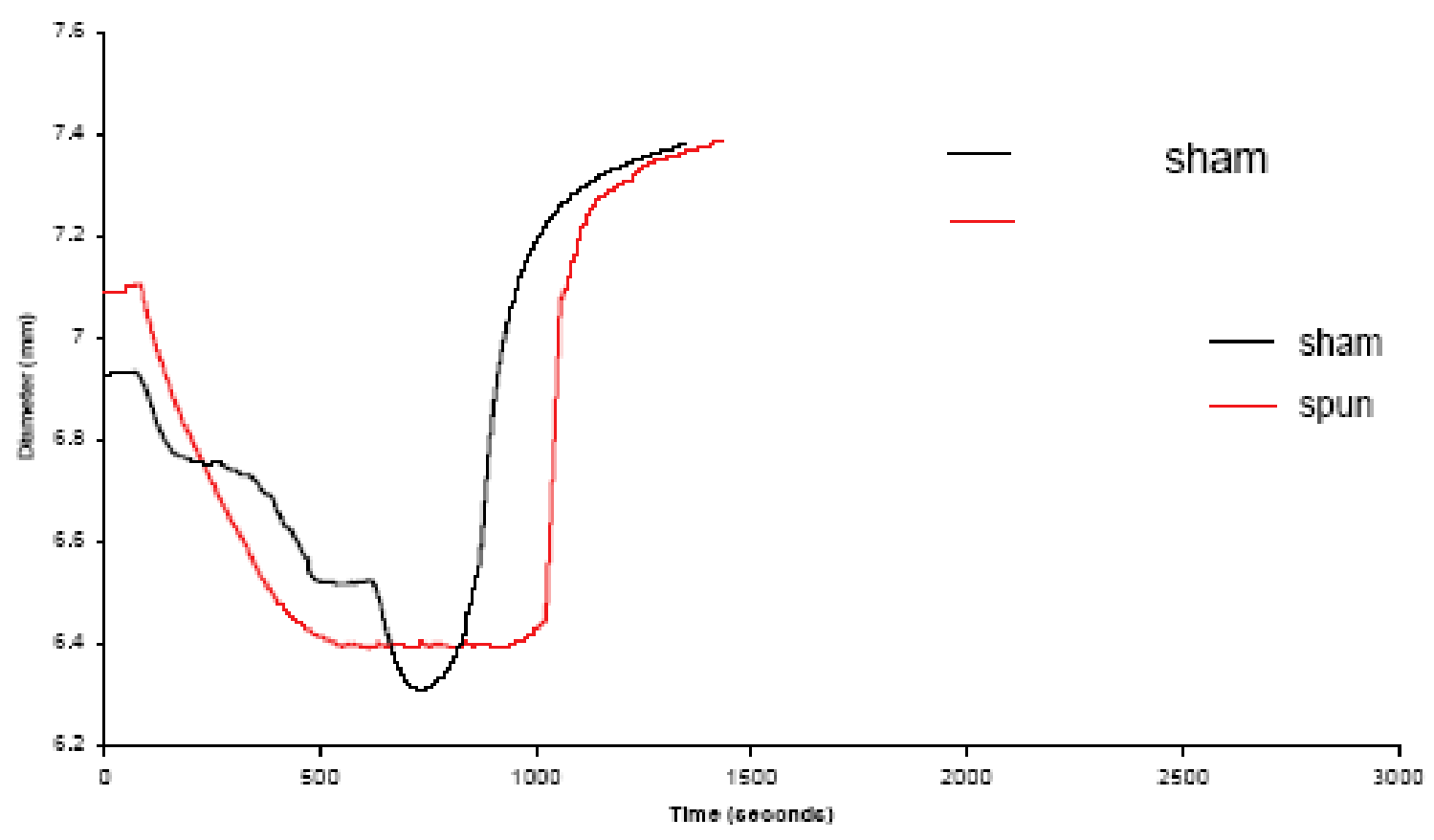

Figure D. 10 Vasomotor challenge outer diameter profiles for acute experiment performed on 03/22/2007. 
Vasomotor Challenge with EPI and SNP

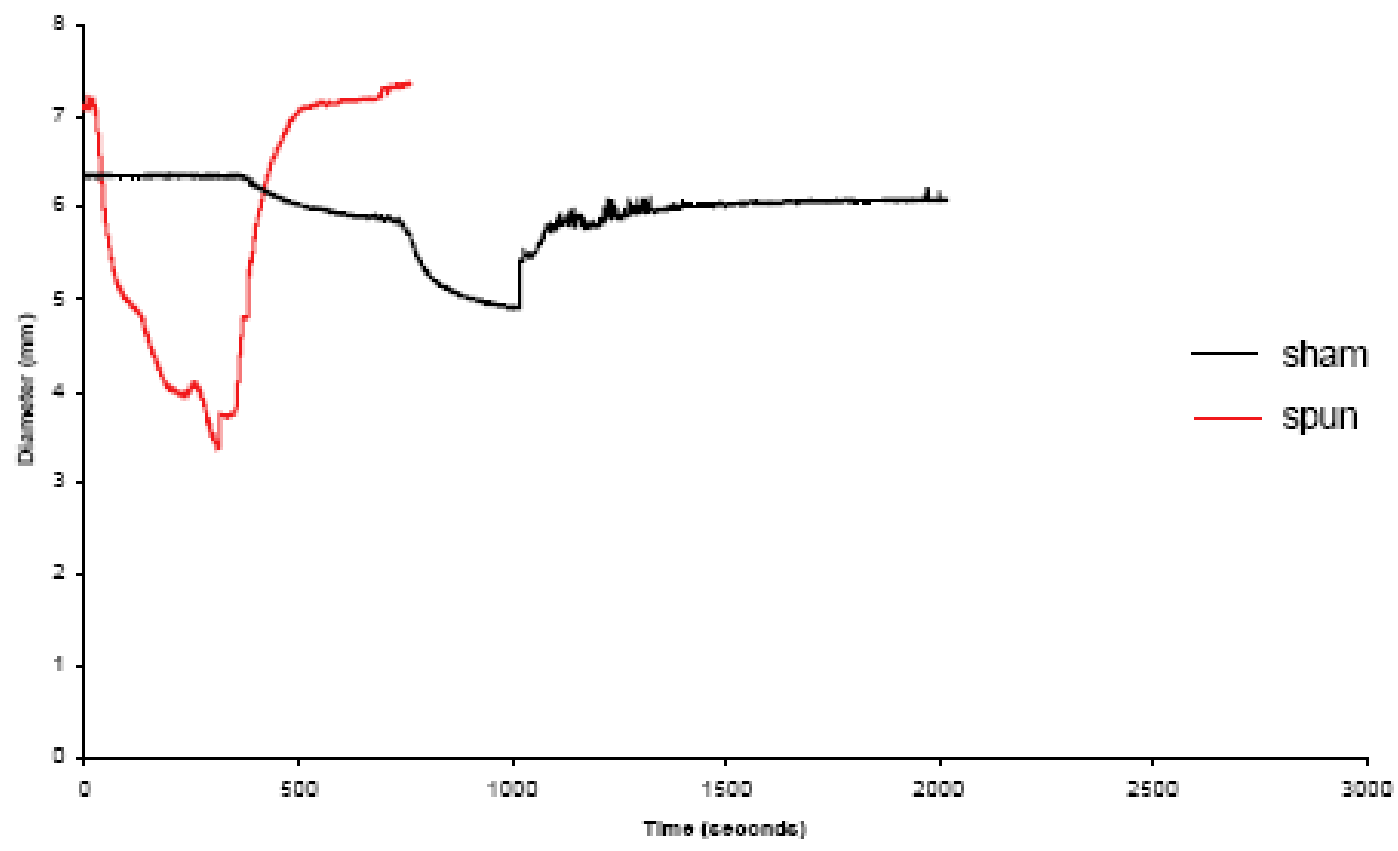

Figure D. 11 Vasomotor challenge outer diameter profiles for acute experiment performed on 05/17/2007. 


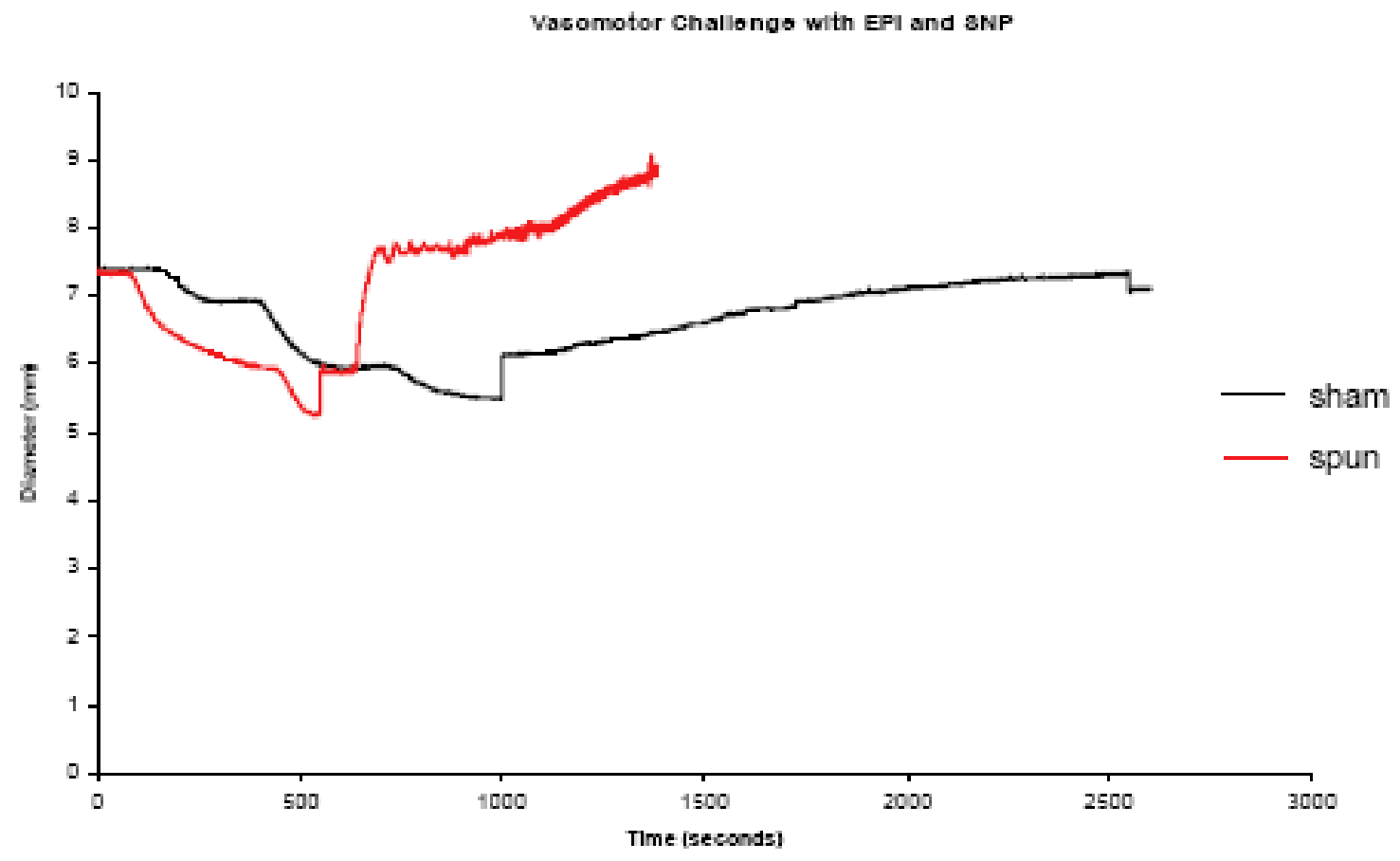

Figure D. 12 Vasomotor challenge outer diameter profiles for acute experiment performed on 05/23/2007. 


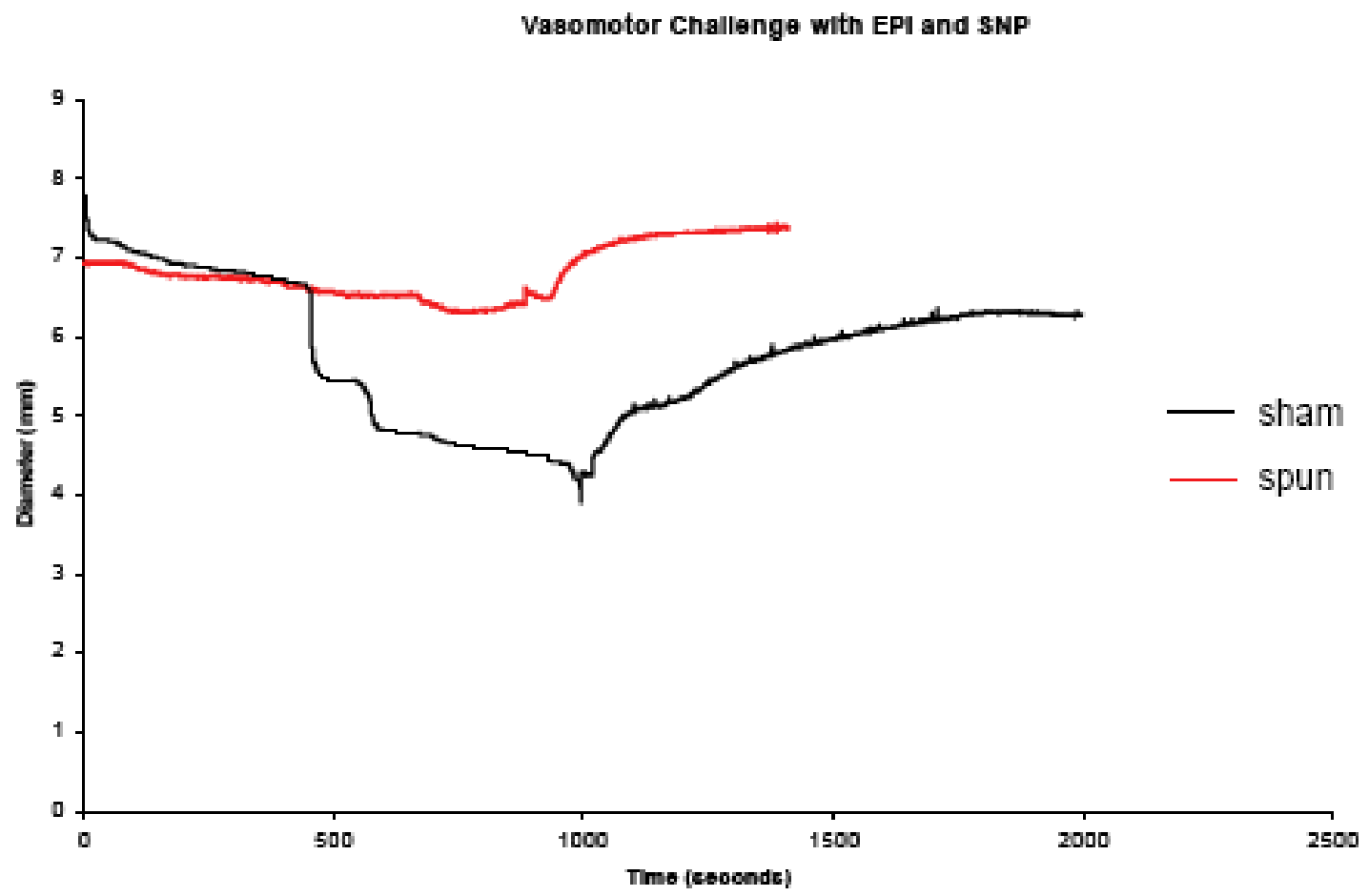

Figure D. 13 Vasomotor challenge outer diameter profiles for acute experiment performed on 06/07/2007.

Table D. 7 Mean values of percent constriction in response to epinephrine for all the acute ex vivo vasomotor challenge experiments.

\begin{tabular}{crr} 
& \multicolumn{2}{c}{$\%$ Constriction } \\
Expt \# & Sham & \multicolumn{1}{c}{ spun } \\
1 & 9.814105203 & 11.04252293 \\
2 & 29.52595014 & 77.34063745 \\
3 & 35.2919708 & 39.29791271 \\
4 & 70.991 & 10.238 \\
Mean & 36.40575654 & 34.47976827 \\
SD & 25.50703982 & 31.6082079 \\
SEM & 12.75351991 & 15.80410395
\end{tabular}


Table D. 8 Microsoft Excel output for student's t-test performed on the percent constriction data from the acute $e x$ vivo vasomotor challenge experiments.

\begin{tabular}{lrr} 
t-Test: Paired Two Sample for & & \\
Means & \multicolumn{1}{c}{ sham } & spun \\
\hline & 36.40576 & 34.47976827 \\
Mean & 650.6091 & 999.0788069 \\
Variance & 4 & 4 \\
Observations & -0.2131 & \\
Pearson Correlation & 0 & \\
Hypothesized Mean Difference & 3 & \\
Df & 0.086278 & \\
t Stat & 0.468341 & \\
$P(T<=t)$ one-tail & 2.353363 & \\
t Critical one-tail & 0.936682 & \\
$P(T<=t)$ two-tail & 3.182446 & \\
t Critical two-tail &
\end{tabular}

Table D. 9 Mean values of percent dilation in response to sodium nitroprusside for all the acute ex vivo vasomotor challenge experiments.

\begin{tabular}{crr} 
& \multicolumn{2}{c}{ \% Dilation } \\
Expt \# & sham & \multicolumn{1}{c}{ spun } \\
1 & 16.8572062 & 15.44797 \\
2 & 24.7445852 & 83.19223 \\
3 & 33.5036496 & 68.95636 \\
4 & 48.42 & 17.063 \\
Mean & 30.8813603 & 46.16489 \\
SD & 13.5254968 & 35.0282 \\
SEM & 6.7627484 & 17.5141
\end{tabular}


Table D. 10 Microsoft Excel output for student's t-test performed on the percent dilation data from the acute ex vivo vasomotor challenge experiments.

\begin{tabular}{lrr} 
t-Test: Paired Two Sample for \\
Means & & \\
\hline & \multicolumn{3}{c}{ sham } & spun \\
\hline Mean & 30.88136 & 46.16489 \\
Variance & 182.9391 & 1226.975 \\
Observations & 4 & 4 \\
Pearson Correlation & -0.17385 & \\
Hypothesized Mean Difference & 0 & \\
Df & 3 & \\
t Stat & -0.77031 & \\
P $(T<=t)$ one-tail & 0.24861 & \\
t Critical one-tail & 2.353363 & \\
P $(T<=t)$ two-tail & 0.49722 & \\
t Critical two-tail & 3.182446 & \\
\hline
\end{tabular}




\section{APPENDIX E}

\section{BIOLOGICAL ANALYSES PROTOCOLS}

Protocol E.1: Scanning Electron Microscopy Sample Work-up Procedure

\section{Procedure:}

1. Fix tissue in $2.5 \%$ gluteraldehyde in $0.1 \mathrm{M}$ PBS (pH 7.4) for 10 minutes.

2. Cut up tissue into small blocks $\left(8 \mathrm{~mm}^{3}\right)$ continue fixing for not more than 50 minutes.

3. Wash thoroughly in 3 changes $0.1 \mathrm{M}$ PBS for 15 minutes each.

4. Fix tissue in $1 \% \mathrm{OsO} 4$ in $0.1 \mathrm{M}$ PBS for 60 minutes.

5. Wash thoroughly in 3 changes $0.1 \mathrm{M}$ PBS for 15 minutes each.

6. Dehydrate in graded series of alcohol (in PBS) for 15 minutes each:

1. $30 \%$ ethanol

2. $50 \%$ ethanol

3. $70 \%$ ethanol

4. $90 \%$ ethanol

5. $\quad 100 \%$ ethanol $\times 3$

7. Critical point dry specimen.

8. Mount on studs.

9. Sputter coat specimen.

10. Store in desiccator. 
Protocol E.2: Hematoxylin and Eosin Histological Staining Procedure

\section{Equipment Required}

- Microscope slides

- Conklin jars

- Sink with small tubing/hose on faucet

- Slide covers (24 mm x $50 \mathrm{~mm})$

- VectaMount ${ }^{\mathrm{TM}}$ Permanent Mounting Medium (Vector Laboratories, Catalog\# H-5000)

\section{$\underline{\text { Reagents Required }}$}

95\% Ethanol (Pharmco or equivalent)

$100 \%$ Ethanol (Pharmco or equivalent)

Xylene (source: Thermo Electron Corporation Cat \#9990501 or equivalent)

Harris' Acidfied Hematoxylin (Thermo Electron Corporation Cat \#6765004)

Alcoholic Eosin Y (Thermo Electron Corporation Cat \#6766007)

Bluing Solution

Beibrich Scarlet (refer to Masson's Trichrome Staining SOP)

Aniline Blue 1\% Acetic Acid Water Phosphotungstic/Phosphomolbdic Acid

Solution (refer to Masson's Trichrome Staining SOP)

Wiegert's Hemetoxylin (refer to Masson's Trichrome Staining SOP) 
Solution Recipes

\begin{tabular}{|c|c|c|}
\hline \hline $\begin{array}{c}\text { Chemicals } \\
\text { Required }\end{array}$ & $\begin{array}{c}\text { Bluing } \\
\text { Solution }\end{array}$ & 95\% EtOH \\
\hline DI Water & $89 \mathrm{~mL}$ & $15 \mathrm{~mL}$ \\
\hline $\begin{array}{c}100 \% \\
\text { Ethanol }\end{array}$ & $207 \mathrm{~mL}$ & $285 \mathrm{~mL}$ \\
\hline $\begin{array}{c}30 \% \\
\mathrm{NH}_{4} \mathrm{OH}\end{array}$ & $4.5 \mathrm{~mL}$ & \\
\hline $\begin{array}{c}\text { Glacial } \\
\text { Acetic } \\
\text { Acid }\end{array}$ & $300 \mathrm{~mL}$ & $300 \mathrm{~mL}$ \\
\hline $\begin{array}{c}\text { Final } \\
\text { Volume }\end{array}$ & \\
\hline
\end{tabular}

\section{Procedure:}

$\begin{array}{cll}\text { Step } & \text { Reagent } & \text { Conc.\% Uses. } \\ 1 & \text { Dry Storage } & \\ 2 & \text { Heater Station } & \\ 3 & \text { Xylene } & \\ 4 & \text { Xylene } & \\ 5 & \text { Xylene } & \\ 6 & \text { Alcohol } & \\ 7 & \text { Alcohol } & \\ 8 & \text { Running Water Wash } & \\ 9 & \text { Harris Acidified } & \\ 10 & \text { Running Water Wash } \\ 11 & \text { Running Water Wash } \\ 12 & \text { Ammonia Water } & \\ 13 & \text { Running Water Wash } & \\ 14 & \text { Alcohol } & 95 \% \\ 15 & \text { Alcoholic Eosin } & \\ 16 & \text { Alcohol } & 95 \% \\ 17 & \text { Alcohol } & 95 \% \\ 18 & \text { Alcohol } & 100 \% \\ 19 & \text { Alcohol } & 100 \% \\ 20 & \text { Alcohol } & 100 \% \\ 21 & \text { Xylene } & \\ 22 & \text { Xylene } & \\ 23 & \text { Xylene } & \\ 24 & \text { Xylene } & \end{array}$

Time/ Limit

00:00 No Maximum

20:00 No Maximum

03:00 No Maximum

03:00 No Maximum

03:00 No Maximum

01:00 No Maximum

01:00 No Maximum

02:00 No Maximum

07:00 Standard

02:00 No Maximum

01:00 No Maximum

00:30 Standard

01:00 No Maximum

00:30 Standard

00:30 Standard

00:30 Standard

00:30 Standard

00:30 Standard

00:30 No Maximum

00:30 No Maximum

00:30 No Maximum

00:30 No Maximum

00:30 No Maximum

00:00 No Maximum 
Protocol E.3: Masson's Trichrome Histological Staining Procedure

\section{Equipment Required}

- Microscope slides

- Conklin jars

- Sink with small tubing/hose on faucet

- Slide covers (24 mm x $50 \mathrm{~mm})$

- VectaMount ${ }^{\mathrm{TM}}$ Permanent Mounting Medium(Vector Laboratories, Catalog\# H-5000) 


\section{$\underline{\text { Reagents Required }}$}

1. Bouin's Solution: (source-LabChem order $\# L C 111790-2$ or equivalent)

2. Weigert's Iron Hematoxylin:

Solution A:

Hematoxylin crystals (source - Fisher Scientific H345-25 or equivalent).............. $10.0 \mathrm{~g}$

$95 \%$ Ethanol (source - Pharmco or equivalent) (make up to total volume) ..................1.0 L

Solution B:

$29 \%$ Ferric Chloride, aqueous (source-Newcomer Supply 48206 or equivalent) ..............20.0 ml

Deionized water........................................................... $475.0 \mathrm{ml}$

Glacial acetic acid (source -JT Baker $9507-33$ or equivalent) $\ldots \ldots \ldots \ldots \ldots \ldots \ldots \ldots \ldots . .5 .0 \ldots \ldots$

Mix equal of solutions A and B just prior to use.

3. Biebrich Scarlet-Acid Fuchsin Solution:

$1 \%$ Biebrich scarlet stock (source - VWR 34172-156 or equivalent) in $100 \mathrm{ml}$ of $\mathrm{dH}_{2} \mathrm{O} \ldots \ldots .1 .0 \mathrm{~g}$

Deionized water.

$100.0 \mathrm{ml}$

$1 \%$ Acid fuchsin stock (source - EM Science 24172-024 or equivalent) .....

$1.0 \mathrm{~g}$

Deionized water.

$100.0 \mathrm{ml}$

Working solution:

1) $1 \%$ Biebrich scarlet stock

$90.0 \mathrm{ml}$

2) $1 \%$ Acid fuchsin stock.

$10.0 \mathrm{ml}$

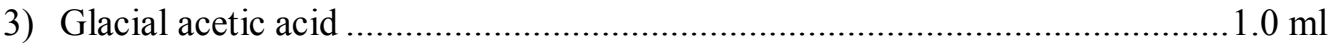

4. Phosphomolybdic-Phosphotungstic Acid Solution:

Phosphomolybdic Acid (source - Fisher Scientific A237-100 or equivalent) .......................... $5.0 \mathrm{~g}$

Phosphotungstic Acid (source - Fisher Scientific A248-100 or equivalent) ........ $5.0 \mathrm{~g}$

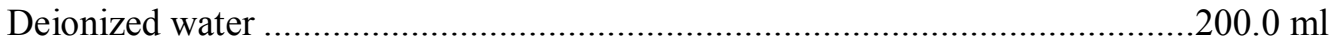

5. Aniline Blue Solution:

Aniline blue (source-Spectrum Al-170 or equivalent).......................................... $12.5 \mathrm{~g}$

Glacial acetic acid (source-JT Baker 9507-33 or equivalent)............................................10.0 10.0

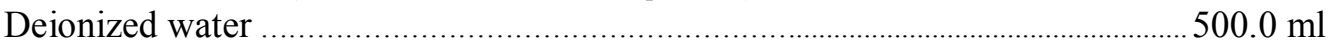

6. $1 \%$ Glacial Acetic Acid:

Glacial acetic acid (source-JT Baker 9507-33 or equivalent)................................. $10.0 \mathrm{ml}$

Deionized water

$.900 .0 \mathrm{ml}$ 


\section{Solution Recipes (if applicable)}

\begin{tabular}{|c|c|c|}
\hline \hline $\begin{array}{c}\text { Chemicals } \\
\text { Required }\end{array}$ & $\begin{array}{c}\text { Bluing } \\
\text { Solution }\end{array}$ & 95\% EtOH \\
\hline DI Water & $89 \mathrm{~mL}$ & $15 \mathrm{~mL}$ \\
\hline $\begin{array}{c}100 \% \\
\text { Ethanol }\end{array}$ & $207 \mathrm{~mL}$ & $285 \mathrm{~mL}$ \\
\hline $\begin{array}{c}30 \% \\
\mathrm{NH}_{4} \mathrm{OH}\end{array}$ & $4.5 \mathrm{~mL}$ & $300 \mathrm{~mL}$ \\
\hline $\begin{array}{c}\text { Glacial } \\
\text { Acetic } \\
\text { Acid }\end{array}$ \\
\hline $\begin{array}{c}\text { Final } \\
\text { Volume }\end{array}$ & $300 \mathrm{~mL}$ & \\
\hline
\end{tabular}

\section{Procedure:}

1. Check expiration date on all reagents. If expired, obtain or prepare new solution.

2. Place slides on the Gemini Stainer to deparaffinize.

\begin{tabular}{|c|c|c|c|}
\hline Step & Reagent & Conc. $\%$ & Time \\
\hline 1 & Dry Storage & 00:00 & \\
\hline 2 & Heater Station & $20: 00$ & \\
\hline 3 & Xylene & 05:00 & \\
\hline 4 & Xylene & 05:00 & \\
\hline 5 & Xylene & 05:00 & \\
\hline 6 & Alcohol & $100 \%$ & 01:00 \\
\hline 7 & Alcohol & $95 \%$ & 01:00 \\
\hline 8 & Running Water Wash & $02: 00$ & \\
\hline 9 & Running Water Wash & 02:00 & \\
\hline 10 & Running Water Wash & 02:00 & \\
\hline 11 & Tap Water & 00:00 & \\
\hline
\end{tabular}

3. Once the slides reach water, remove from the Gemini Stainer.

4. Place the Bouin's Solution in a general-purpose lab oven set $@ 60^{\circ} \mathrm{C}$ for 15 minutes to preheat the solution.

5. Place slides in the preheated Bouin's Solution. Incubate the slides for 1 hour at $60^{\circ} \mathrm{C}$.

6. Filter the Aniline Blue and the Biebrich Scarlet-Acid Fuchsin Solutions before use.

7. Remove the Bouin's solution from the oven and place in the fume hood to cool (approximately 5-10 minutes).

8. Once the slides are cooled, rinse in running tap water for 5 minutes. 
9. Place slides into staining rack and return to the Gemini Stainer and use the trichrome staining program.

\begin{tabular}{|c|c|c|}
\hline Step & Reagent & Conc. \\
\hline 1 & Dry Storage & $\ldots .00: 00$ \\
\hline 2 & Running Water Wash & $\ldots 10: 00$ \\
\hline 3 & WEIGERT'S HEMATOXYLIN & $\ldots 10: 00$ \\
\hline 4 & Running Water Wash & . \\
\hline 5 & BIEBRICH & $\ldots 2: 00$ \\
\hline 6 & Running Water Wash & ................................ \\
\hline 7 & P-P ACID & $\ldots 15: 00$ \\
\hline 8 & ANILINE BLUE & $\ldots . .05: 00$ \\
\hline 9 & Running Water Wash & $\ldots 00: 30$ \\
\hline 10 & Acid Water & $\ldots .01: 00$ \\
\hline 11 & Running Water Wash & .....00:30 \\
\hline 12 & Alcohol & 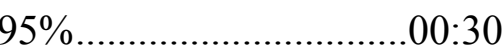 \\
\hline 13 & Alcohol & 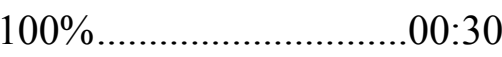 \\
\hline 14 & Alcohol & $100 \%$ \\
\hline 15 & Xylene & 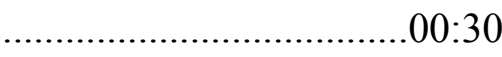 \\
\hline 16 & Xylene & $\ldots .00: 30$ \\
\hline 17 & Xylene & . \\
\hline 18 & Xylene & $\ldots .00: 00$ \\
\hline
\end{tabular}

10. When the staining is complete, coverslip.

Expected Results:

Nuclei ........................................Black

Collagen and mucus.........................Blue

Cytoplasm, Keratin and Muscle Fibers..........Red

Protocol E.4: Picro-Sirius Red (Puchtler's version)

\section{Equipment Required}

- Microscope slides

- Conklin jars

- Sink with small tubing/hose on faucet

- Slide covers (24mm x $50 \mathrm{~mm}$ )

- VectaMount ${ }^{\mathrm{TM}}$ Permanent Mounting Medium(Vector Laboratories, Catalog\# H-5000) 


\section{Reagents Required}

1. Weigert's Iron Hematoxylin:

Solution A:

Hemotoxylin crystals.................. $10 \mathrm{~g}$

(source-Fisher Scientific H345-25)

$95 \%$ Ethanol (make up to total volume) ............. $1 \mathrm{~L}$

(source-Pharmco)

Solution B:

29\% Ferric Chloride, aqueous................ $20 \mathrm{ml}$

(source-Newcomeer Supply 48206)

Distilled $\mathrm{H}_{2} \mathrm{O}$.

$475 \mathrm{ml}$

Glacial acetic acid......................... $5 \mathrm{ml}$

(source-JT Baker 9507-33)

Mix equal of solutions A and B just prior to use.

2. Picro-Sirius Red Solution:

Sirius Red F3B........................... $0.1 \mathrm{~g}$

(source: Spectrum Chemical S-1066)

Saturated Aqueous Picric Acid............... $\quad 500 \mathrm{ml}$

(source: Spectrum Chemical P-201)

3. Acetic Acid Water:

Glacial Acetic Acid........................... $35 \mathrm{ml}$

(source: JT Baker 9507-33)

Deionized water.

$75 \mathrm{ml}$

\section{Procedure:}

1. Check expiration date on all reagents. If expired, discard appropriately (refer to SOP\# R0020, Disposal of Hazardous Chemical Waste in Bridgeside Point) and obtain or prepare new solution.

2. Place slides on the Gemini Stainer to deparaffinize (refer to SOP\#E2013, Use and Care of the Varistain Gemini Stainer).

3. Once the slides reach water, remove from the Gemini Stainer (refer to SOP\#2013, Use and Care of the Varistain Gemini Stainer).

4. Stain slides in Weigert Iron Hematoxylin for 10 minutes.

5. Rinse in running tap water for 10 minutes.

6. Rinse in deionized water for 10 seconds.

7. Stain in Picro-Sirius Red for 1 hour at room temperature.

8. Decolorized in Acetic Acid water for 10 seconds.

9. Quickly rinse in two changes of $95 \%$ ethanol.

10. Quickly rinse in three changes of absolute alcohol.

11. Clear in three changes of xylene.

12. Coverslip. When the staining is complete, coverslip. (Refer to SOP\#E2007, Use and care of the Thermo Electron consul coverslipper).

\section{Expected Results:}

Nuclei-blue

Muscle, cytoplasm-yellow 
Collagen-red

Protocol E.5: Movat's Pentachrome Stain (Russell Modification)

\section{Equipment Required}

- Microscope slides

- Conklin jars

- Sink with small tubing/hose on faucet

- $\quad$ Slide covers $(24 \mathrm{~mm}$ x $50 \mathrm{~mm})$

- VectaMount ${ }^{\mathrm{TM}}$ Permanent Mounting Medium(Vector Laboratories, Catalog\# H-5000)

1. $\underline{1 \% \text { Alcian Blue }}$ 
Alcian blue-8GS (spectrum chemical AL-170 or equivalent) $\ldots \ldots \ldots \ldots \ldots \ldots \ldots \ldots 1.0 \mathrm{~g}$ Distilled water...................................................... $100 \mathrm{ml}$

Glacial Acetic acid (JT Baker 9507-33 or equivalent) ........................ ml

2. Alkaline Alcohol

Ammonium Hydroxide 28-30\% (Newcomer supply 1006A or equivalent)........10 ml

Alcohol, 95\% (source: Pharmco or equivalent)........................... $90 \mathrm{ml}$

3. Iodine-Iodide

Iodine (source: spectrum chemical I-1010 or equivalent) ..................... g g

Potassium Iodide (source: spectrum chemical P-1335 or equivalent)............. $\mathrm{g}$

Distilled Water ...................................................... $100 \mathrm{ml}$

4. Absolute Alcoholic Hematoxylin, 10\%

Hematoxylin (source: Spectrum Chemical HE1 15 or equivalent).........................10 g

Absolute Alcohol (source: Pharmco or equivalent).......................... $100 \mathrm{ml}$

5. Ferric Chloride, $10 \%$

Ferric Chloride (source: spectrum chemical F-1010 or equivalent)..................10 g

Distilled water $100 \mathrm{ml}$

6. Hematoxylin Solution

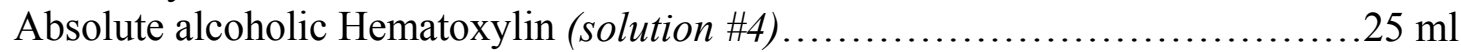

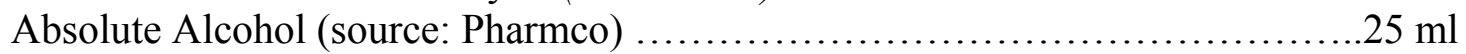

Ferric Chloride, 10\% aqueous (solution \#5)................................. $25 \mathrm{ml}$

Iodine-Iodide (solution \# 3)...............................................25 ml

Prepare just before use

7. Ferric Chloride, $2 \%$ (for differentiation)

Ferric Chloride (solution \# 5) .......................................... $10 \mathrm{ml}$

Distilled water. .........................................................40 ml

Prepare just before use

8. Sodium Thiosulfate, $5 \%$

Sodium Thiosulfate (source: spectrum chemical S-1498 or equivalent)............ $\mathrm{g}$

Distilled water $100 \mathrm{ml}$

9. Crocein Scarlet-Acid Fuchsin

Solution A (stock)

Crocein Scarlet (source: spectrum chemical C-3080 or equivalent) $\ldots \ldots \ldots \ldots \ldots \ldots 1 \mathrm{~g}$

Distilled water. $.99 .5 \mathrm{ml}$

Glacial Acetic Acid (source: JT Baker 9507-33 or equivalent).

$.0 .5 \mathrm{ml}$

Solution B (stock) 
Acid Fuchsin (source: Spectrum Chemical AC-155 or equivalent) $\ldots \ldots \ldots \ldots \ldots . .1 \mathrm{~g}$

Distilled water....................................................... $99.5 \mathrm{ml}$

Glacial acetic acid (source: JT Baker 9507-33 or equivalent) ..................

Working solution

Solution A........................................................... $80 \mathrm{ml}$

Solution B..........................................................20 ml

Prepare just before use.

10. Phosphotungstic Acid Solution, $5 \%$

Phosphotungstic Acid (source: Spectrum Chemical P1135 or equivalent).........5 g

Distilled water......................................................... $100 \mathrm{ml}$

\section{Alcoholic Safran}

Safran du Gatinais (source: spectrum chemical SA-110 or equivalent) $5 \mathrm{~g}$

Absolute alcohol (source: Pharmco or equivalent)....

$.83 .5 \mathrm{ml}$

\section{Procedure:}

1. Check expiration date on all reagents. If expired, discard appropriately (refer to SOP\# R0020 Disposal of Hazardous Chemical Waste in Bridgeside Point) and obtain or prepare new solution.

2. Place slides on the Gemini Stainer to deparaffinize (refer to SOP\#E2013, Use and Care of the Varistain Gemini Stainer).

3. Once the slides reach water, remove from the Gemini Stainer (refer to SOP\#2013 Use and Care of the Varistain Gemini Stainer).

4. Place the slides in Alcian blue for 20 minutes at room temperature.

5. Wash in running tap water 5 minutes.

6. Place slides in alkaline alcohol for 1 hour.

7. Wash in running tap water for 10 minutes.

8. Rinse once in distilled water.

9. Place the slides in Hematoxylin solution for 15 minutes.

10. Rinse in 5 changes of distilled water.

11. Place slides in $2 \%$ aqueous ferric chloride until the elastic fibers contrast sharply with the background.

12. Rinse in distilled water.

13. Place slides in sodium thiosulfate for 1 minute.

14. Wash in running tap water for 5 minutes, rinse in distilled water.

15. Place slides in crocein scarlet-acid fuchsin for 1 minute 30 seconds.

16. Rinse in 5 changes of distilled water.

17. Rinse in $0.5 \%$ Acetic Acid solution.

18. Place slides in 5\% phophotungstic acid solution, 2 changes five minutes each.

19 . Rinse in $0.5 \%$ acetic acid solution.

20 . Rinse in three changes of absolute alcohol.

21. Place slides in alcoholic safran solution for 15 minutes.

22. Rinse in three changes of absolute alcohol. 
23. Clear in three changes of xylene.

24. When the staining is complete, coverslip. (Refer to SOP\#E2007 Use and care of the Thermo Electron consul coverslipper).

\section{Expected Results:}

Nuclei and elastic fibers..........Black

Collagen........................ Yellow

Ground substance and mucin....Blue

Fibrinoid, fibrin..................Intense red

Muscle..........................Red

Protocol E.6: Immunohistochemical TUNEL Analysis Procedure 


\section{Equipment Required}

- $\quad$ 200uL Micropipette and tips

- Disposable transfer pipettes

- Vacuum

- Microscope slides

- Microscope slide covers

- Mounding medium (Gelvatol)

- Timer

- $\quad 37^{\circ} \mathrm{C}$ humidified incubator

\section{$\underline{\text { Reagents Required }}$}

- Fixation solution (4\% Paraformaldehyde)

- 1 xPBS

- In Situ Cell Death Kit, Fluorescine (Roche Applied Sciences)

- Label Solution (vial 2)

- Enzyme solution (vial 1)

- 3000 units/mL DNase I, Grade I in:

- $\quad 75$ mM Tris-HCl pH 7.5

- $1 \mathrm{mg} / \mathrm{mL}$ BSA

- Permeabilization solution:

- $\quad 0.1 \%$ sodium citrate

- $\quad 0.1 \%$ Triton X-100

- Protienase K solution:

- $\quad[50 \mathrm{ug} / \mathrm{mL}]$ Protienase K

- Gelvatol mounting solution

- Dapi nuclear stain

\section{Solution Recipes (if applicable)}

\begin{tabular}{|c|c|c|}
\hline \hline $\begin{array}{c}\text { Chemicals } \\
\text { Required }\end{array}$ & $\begin{array}{c}\text { Permeabilization } \\
\text { solution }\end{array}$ & $\begin{array}{c}\text { BSA/Tris- } \\
\text { HCl pH 7.5 } \\
(\mathbf{1 m g} / \mathbf{m L})\end{array}$ \\
\hline 1xPBS & BTV & BTV \\
\hline Triton-X 100 & $10 \mathrm{uL}$ & \\
\hline Sodium Citrate & $0.01 \mathrm{~g}$ & \\
\hline Tris-HCl pH 7.4 & & $750 \mathrm{uL}$ \\
\hline BSA & & $0.015 \mathrm{~g}$ \\
\hline Final Volume & $10 \mathrm{~mL}$ & $10 \mathrm{~mL}$ \\
\hline
\end{tabular}




\section{Procedure:}

1. Retrieve slides with samples attached. If slides were frozen, warm on slide warmer before use.

2. Fix slides in $4 \%$ Paraformaldehyde for 20 minutes at room temperature.

3. Rinse slides 3 times with $1 x P B S$ for 10 minutes each.

4. Incubate the slides in Proteinase-K solution for 10 minutes at room temp.

5. Incubate slides in Permeabilization solution for 10 minutes at room temp.

6. Acquire $0.010 \mathrm{ml}$ aliquot of DNase (300 units). Allow aliquot to thaw. Add $90 \mathrm{ml}$ $\mathrm{BSA} /$ Tris- $\mathrm{HCl}$ solution to the microfuge tube. Mix by pipetting.

7. Rinse slides $\mathrm{x} 3$ with $1 \mathrm{xPBS}$. Leave all samples and negative control in 1xPBS until steps 10 and beyond.

8. Apply DNase/BSA/Tris-HCl across positive control slide only. Incubate at room temperature for 10 minutes. ( $\sim 33 \mathrm{ml}$ per section).

9. Wash positive control slide $\mathrm{x} 3$ with $1 \mathrm{xPBS}$.

10. Remove $100 \mathrm{uL}$ of Label solution (vial 2) and apply it to the negative control sections only. ( $\sim 50 \mathrm{uL}$ per section).

11. Add 50uL of Enzyme solution (vial 1) to the remaining $450 \mathrm{uL}$ Label solution (vial 2) to make the TUNEL Reaction Mixture.

12. Add $50 \mathrm{uL}$ TUNEL Reaction Mixture to each section except the negative control sections. Incubate for 25 minutes in $37^{\circ} \mathrm{C}$ humidified incubator.

13. Rinse all sections $3 x$ in $1 x P B S$.

14. Apply one drop of DAPI nuclear stain for 1 minute to each sample.

15. Rinse $x 3$ with $1 \times$ PBS.

16. Mount using Gelvatol. 
Protocol E.7: Immunohistochemical PCNA Primary Antibody Incubation Procedure

\section{Equipment Required}

- Vacuum

- Microscope Slides

- Disposable Transfer Pipettes

- 200uL micropipette and tips

- Slide Box

\section{Reagents Required}

- 1 XPBS

- $99 \%$ ethanol

- DI Water

- 4\% Paraformaldehyde

- Permeabilization solution:

- $\quad 0.1 \%$ sodium citrate

- $\quad 0.1 \%$ Triton X-100

- $\mathrm{H}_{2} \mathrm{O}_{2}$ Solution $(0.3 \%)$

- Vectastain Kit

- Normal Horse Serum

- PCNA Primary Antibody (Dako, Clone PC-10)

Solution Recipes (if applicable)

\begin{tabular}{|c|c|c|c|c|}
\hline \hline $\begin{array}{c}\text { Chemicals } \\
\text { Required }\end{array}$ & $\begin{array}{c}\text { Permeabilizat } \\
\text { ion Solution }\end{array}$ & $\begin{array}{c}\mathrm{H}_{2} \mathrm{O}_{2} \\
\text { Solution }\end{array}$ & $\begin{array}{c}\text { Normal/ } \\
\text { Blocking } \\
\text { Serum }\end{array}$ & $\begin{array}{c}\text { Primary } \\
\text { Antibody }\end{array}$ \\
\hline $1 \times \mathrm{xBS}$ & $\mathrm{BTV}$ & & $5 \mathrm{~mL}$ & \\
\hline $\mathrm{DI} \mathrm{H}_{2} \mathrm{O}$ & & $9 \mathrm{~mL}$ & & \\
\hline $3 \% \mathrm{H}_{2} \mathrm{O}_{2}$ & & $1 \mathrm{~mL}$ & & \\
\hline $\begin{array}{c}\text { Normal } \\
\text { Horse } \\
\text { Serum }\end{array}$ & & $1 \mathrm{drop}$ & \\
\hline $\begin{array}{c}\text { Triton-X } \\
100\end{array}$ & $10 \mathrm{uL}$ & & & \\
\hline $\begin{array}{c}\text { Sodium } \\
\text { Citrate }\end{array}$ & $0.01 \mathrm{~g}$ & $10 \mathrm{~mL}$ & & \\
\hline $\begin{array}{c}\text { Final } \\
\text { Volume }\end{array}$ & $10 \mathrm{~mL}$ & & & \\
\hline
\end{tabular}

\section{Procedure:}

1. Retrieve slides and place in $37^{\circ} \mathrm{C}$ slide warmer for $30 \mathrm{~min}$.

2. Apply $4 \%$ Paraformaldehyde to all samples for $8 \mathrm{~min}$.

3. Apply $99 \%$ Ethanol for $2 \mathrm{~min}$.

4. Wash $\mathrm{x} 3$ with $1 \mathrm{xPBS}$, leaving third wash on for 30 minutes. 
5. Incubate in permeabilization solution for 15 minutes.

6. Wash $\times 2$ in $1 \times$ PBS.

7. Quench for $30 \mathrm{~min}$ in $0.3 \% \mathrm{H}_{2} \mathrm{O}_{2}$.

8. Wash $\mathrm{x} 2$ with $1 \times \mathrm{PBBS}$.

9. Block in Normal Serum for $30 \mathrm{~min}$.

10. Incubate in primary antibody solution for $60 \mathrm{~min}$ in humidified box at $37^{\circ} \mathrm{C}$. Be sure to leave Primary Delete samples (leftmost section on each slide), which should stay incubated in Normal Blocking Serum. 
Protocol E.8: VectaStain Elite TM Horseradish Peroxidase/Avidin-Biotin-Complex Detection System for Immunohistochemistry Staining

\section{Equipment Required}

- Microscope slides

- Vacuum

- $\quad 37^{\circ} \mathrm{C}$ humidified incubator

- Microscope 20x

- Conklin jars

\section{Reagents Required}

- 1 XPBS

- DI Water

- Vectastain Elite ${ }^{\mathrm{TM}}$ Kit (Vector Laboratories, Catalog\#PK-6200)

- Normal Horse Serum

- Solution A

- Solution B

- Biotinylated Universal Antibody

- Diaminobenzidine (DAB) Substrate Kit (Vector Laboratories, Catalog\#SK-4100)

- Hydrogen Peroxide Substrate Reagent

- DAB Substrate Reagent

- Solution Buffer

Solution Recipes (if applicable)

\begin{tabular}{|c|c|c|c|}
\hline \hline $\begin{array}{c}\text { Chemicals } \\
\text { Required }\end{array}$ & 2' Antibody & $\begin{array}{c}\text { ABC } \\
\text { Reagent }\end{array}$ & $\begin{array}{c}\text { DAB } \\
\text { Substrate }\end{array}$ \\
\hline 1xPBS & $5 \mathrm{~mL}$ & $5 \mathrm{~mL}$ & \\
\hline $\begin{array}{c}\text { Normal } \\
\text { Horse } \\
\text { Serum }\end{array}$ & 2 drops & & \\
\hline $\begin{array}{c}\text { Universal } \\
\text { antibody }\end{array}$ & 2 drops & 2 drops & \\
\hline Solution A & & 2 drops & $5 \mathrm{~mL}$ \\
\hline Solution B & & & 2 drops \\
\hline DI Water & & $5 \mathrm{~mL}$ & $5 \mathrm{~mL}$ \\
\hline $\begin{array}{c}\text { Solution } \\
\text { Buffer }\end{array}$ & & drops \\
\hline $\begin{array}{c}\mathrm{H}_{2} \mathrm{O}_{2} \\
\text { Solution }\end{array}$ & & & 4 drops \\
\hline DAB & & $\mathrm{mL}$ & \\
\hline $\begin{array}{c}\text { Final } \\
\text { Volume }\end{array}$ & & & \\
\hline
\end{tabular}




\section{Procedure:}

1. Wash away primary antibody with $1 x P B S 2 x$.

2. Incubate in secondary antibody for 60 minutes in $37^{\circ} \mathrm{C}$ humidified incubator.

3. Wash $\mathrm{x} 2$ with $1 \times \mathrm{xBS}$.

4. Incubate in Vectastain $\mathrm{ABC}$ solution for 30 minutes (ABC solution must sit for at least 30 minutes prior to use).

5. Wash $\mathrm{x} 2$ with $1 \times \mathrm{xPBS}$.

6. Under microscope, apply DAB substrate to all samples on one slide. Looking at one section, the DAB solution should be removed when the edges of the tissue begin to turn brown. Place the slide in DI water. 
Protocol E.9: Immunohistochemistry Staining Protocol for Golgi complex Primary Antibody Incubation

\section{Equipment Required}

- Vacuum

- Microscope Slides

- Disposable Transfer Pipettes

- 200uL micropipette and tips

- Slide Box

\section{Reagents Required}

- 1 XPBS

- DI Water

- 4\% Paraformaldehyde

- Permeabilization solution:

- $0.1 \%$ sodium citrate

- $\quad 0.1 \%$ Triton X-100

- $\mathrm{H}_{2} \mathrm{O}_{2}$ Solution $(0.3 \%)$

- Vectastain Elite ${ }^{\mathrm{TM}}$ Kit (Vector Laboratories, Catalog\# PK-6200)

- Normal Horse Serum

- Golgi Complex Primary Antibody (Abcam, Catalog\# ab14487) 
Solution Recipes (if applicable)

\begin{tabular}{|c|c|c|c|c|}
\hline $\begin{array}{l}\text { Chemicals } \\
\text { Required }\end{array}$ & $\begin{array}{c}\text { Permeabilizat } \\
\text { ion Solution }\end{array}$ & $\begin{array}{c}\mathrm{H}_{2} \mathrm{O}_{2} \\
\text { Solution }\end{array}$ & $\begin{array}{c}\text { Normal/ } \\
\text { Blocking } \\
\text { Serum }\end{array}$ & $\begin{array}{l}\text { Primary } \\
\text { Antibody }\end{array}$ \\
\hline $1 \times P B S$ & BTV & & $5 \mathrm{~mL}$ & \\
\hline $\mathrm{DI} \mathrm{H}_{2} \mathrm{O}$ & & $9 \mathrm{~mL}$ & & \\
\hline $3 \% \mathrm{H}_{2} \mathrm{O}_{2}$ & & $1 \mathrm{~mL}$ & & \\
\hline $\begin{array}{l}\text { Normal } \\
\text { Horse } \\
\text { Serum } \\
\end{array}$ & & & 1 drop & \\
\hline $\begin{array}{c}\text { Triton-X } \\
100\end{array}$ & $10 \mathrm{uL}$ & & & \\
\hline $\begin{array}{l}\text { Sodium } \\
\text { Citrate }\end{array}$ & $0.01 \mathrm{~g}$ & & & \\
\hline $\begin{array}{c}\text { Final } \\
\text { Volume }\end{array}$ & $10 \mathrm{~mL}$ & $10 \mathrm{~mL}$ & $5 \mathrm{~mL}$ & \\
\hline $\begin{array}{l}\text { Chemicals } \\
\text { Required }\end{array}$ & $\begin{array}{c}\text { Permeabilizat } \\
\text { ion Solution }\end{array}$ & $\begin{array}{c}\mathrm{H}_{2} \mathrm{O}_{2} \\
\text { Solution }\end{array}$ & $\begin{array}{c}\text { Normal/ } \\
\text { Blocking } \\
\text { Serum }\end{array}$ & $\begin{array}{c}\text { Primary } \\
\text { Antibody }\end{array}$ \\
\hline $1 \times \mathrm{PBS}$ & BTV & & $5 \mathrm{~mL}$ & \\
\hline $\mathrm{H}_{2} \mathrm{O}$ & & & & \\
\hline $\begin{array}{l}\text { Normal } \\
\text { Horse } \\
\text { Serum }\end{array}$ & & & 1 drop & \\
\hline $\begin{array}{c}\text { Triton-X } \\
100\end{array}$ & $10 \mathrm{uL}$ & & & \\
\hline $\begin{array}{l}\text { Sodium } \\
\text { Citrate }\end{array}$ & $0.01 \mathrm{~g}$ & & & \\
\hline $\begin{array}{c}\text { Final } \\
\text { Volume }\end{array}$ & $10 \mathrm{~mL}$ & & & \\
\hline
\end{tabular}

\section{Procedure:}

1. Retrieve slides and place in $37^{\circ} \mathrm{C}$ slide warmer for $30 \mathrm{~min}$.

2. Apply 4\% Paraformaldehyde to all samples for $30 \mathrm{~min}$.

3. Was $\mathrm{x} 3$ with $1 \mathrm{xPBS}$, leaving third wash on for 30 minutes.

4. Incubate in permeabilization solution for 15 minutes.

5. Wash $\mathrm{x} 2$ in $1 \times \mathrm{xBS}$.

6. Quench for $30 \mathrm{~min}$ in $0.3 \% \mathrm{H}_{2} \mathrm{O}_{2}$.

7. Wash $\mathrm{x} 2$ with $1 \times \mathrm{PBS}$.

8. Block in Normal Serum for $30 \mathrm{~min}$.

9. Incubate in primary antibody solution for $60 \mathrm{~min}$ in humidified box at $37^{\circ} \mathrm{C}$. Be sure to leave Primary Delete samples (leftmost section on each slide), which should stay incubated in Normal Blocking Serum. 


\section{Protocol E.10: Transmission Electron Microscopy Sample Work-up Procedure}

\section{Procedure:}

1. Fix tissue in $2.5 \%$ glutaraldehyde in $0.1 \mathrm{M} \mathrm{PBS}(\mathrm{pH} 7.4)$ for 10 minutes.

2. Cut up tissue into small blocks $\left(1 \mathrm{~mm}^{3}\right)$ and continue fixing for at least 50 minutes.

3. Wash in 3 changes $0.1 \mathrm{M}$ PBS for 15 minutes each.

4. Post fix specimens in $1 \%$ Osmium tetroxide containing $1 \%$ potassium ferricyanide for $1 \mathrm{hr}$.

5. Wash in 3 changes $0.1 \mathrm{M}$ PBS for 15 minutes each.

6. Dehydrate in graded series of alcohol (in PBS) for 15 minutes each:

1. $30 \%$ ethanol

2. $50 \%$ ethanol

3. $70 \%$ ethanol

4. $90 \%$ ethanol

5. $100 \%$ ethanol $\times 3$

7. Dehydrate further in two 10 minute changes of propylene oxide. *

8. Infiltrate with a 1:1 mix of propylene oxide and epon for 1-3 hrs or overnight.

9. Infiltrate with pure epon overnight at $4^{\circ} \mathrm{C}$ or 1 hour at room temperature.

10. Continue infiltration with three 1 hour changes of epon.

11. Embed in pure epon at $37^{\circ} \mathrm{C}$ for 24 hours.

12. Cure for 48 hours at $60^{\circ} \mathrm{C}$. 


\section{Protocol E.11: Tissue Homogenization And Protein Extraction}

\section{Equipment Required}

$-\quad-80^{\circ} \mathrm{C}$

- Analytical Balance w/ weigh boats

- Homogenizer

- $150 \mathrm{ml}$ conical tube

- $\quad 15 \mathrm{ml}$ test tube per tissue section to be homogenized

- $\quad$ Several $1.5 \mathrm{ml}$ microfuge tubes

- Vortex genie fitted with microfuge tube rack

- Microcentrifuge

\section{Reagents Required}

- T-PER (Pierce Cat\# 78510)

- 0.1M PMSF

- 5mM Leupeptin

- $1 \mathrm{mM}$ Antipain

- $2 \mathrm{mM}$ Pepstatin A

- 2mM Chymostatin

\section{Solution Recipes (if applicable)}

\begin{tabular}{|c|c|c|c|c|c|c|}
\hline $\begin{array}{c}\text { Chemicals } \\
\text { Required }\end{array}$ & T-PER & PMSF & Leupeptin & Antipain & Pepstatin A & $\begin{array}{c}\text { Chymost } \\
\text { atin }\end{array}$ \\
\hline (Amount) & & & & & & \\
\hline $\begin{array}{c}\text { Extraction } \\
\text { Solution } \dagger\end{array}$ & $20 \mathrm{ml}$ & $240 \mu \mathrm{l}$ & $10 \mu \mathrm{l}$ & $10 \mu \mathrm{l}$ & $2.5 \mu \mathrm{l}$ & $10 \mu \mathrm{l}$ \\
\hline $\begin{array}{c}\text { Final } \\
\text { Volume }\end{array}$ & $20 \mathrm{ml}$ & $240 \mu \mathrm{l}$ & $10 \mu \mathrm{l}$ & $10 \mu \mathrm{l}$ & $2.5 \mu \mathrm{l}$ & $10 \mu \mathrm{l}$ \\
\hline
\end{tabular}

$\uparrow$ Volumes based on homogenization of 1 gram of tissue

\section{Procedure:}

1. Thaw samples from the $-80 \mathrm{C}$ freezer on ice.

2. Prepare Extraction Solution according to recipe above.

3. Once thawed remove the samples from tubes, weigh them separately and place them in fresh $5 \mathrm{ml}$ test tubes.

4. Add Extraction Solution based on the following ratio: $20 \mathrm{mls}$ per gram of tissue.

5. If necessary cut tissue into smaller pieces so that a volume of less than $2.5 \mathrm{mls}$ is used in a single tube. 
6. Homogenize tissue on ice until well blended.

7. Perform 3 cycles of freeze/thaw:

a. $5 \mathrm{~min}$ freeze in $-80 \mathrm{C}$

b. 20 min thaw on ice

8. Transfer cell lysates to microfuge tubes.

9. Place tubes on vortex genie and shake for $30 \mathrm{~min}$ at $4 \mathrm{C}$.

10. Spin tubes at $12,000 \mathrm{RPM}$ for $30 \mathrm{~min}$ at $4 \mathrm{C}$.

11. Remove supernatant to separate microfuge tubes

12. Store the pellets and supernatants in the $-80 \mathrm{C}$.

13. Quantify protein using BCA protein concentration assay

14. If insufficient protein is obtained resuspend pellets in Extraction Solution and repeat steps 6-13. 


\section{Protocol E.12: RNA/DNA/Protein Isolation}

\section{Equipment Required}

- Ice bucket w/ ice

- Pipet aide

- Homogenizer

- $50 \mathrm{ml}$ VWR high speed centrifuge tubes (Sterile-RNase free)

- $15 \mathrm{ml}$ conical tubes

- Sterile pipets

$-5 \mathrm{ml}$

$-10 \mathrm{ml}$

$-25 \mathrm{ml}$

- Sorvall Legend RT centrifuge w/ HIGHconic rotor $75003046 / 3057$ rotor

- Water bath

- Kim wipes

- Gloves (RNase free)

- Vacuum apparatus:

- $500 \mathrm{ml}$ side arm flask

- \#8 Stopper w/ hole

- Tubing

- Micropipettors (RNA work only):

- P1000

- P200

- Qiagen RNeasy mini kit:

- $2 \mathrm{ml}$ collecting tubes

- $1.5 \mathrm{ml}$ collecting tubes

- RNeasy mini columns

- $3 \mathrm{ml}$ sterile syringes

- 1.5", 18 gauge sterile needles

- Spectrafuge 16M

- RNase free pipet tips:

- P1000

- P200

- Vacuum desicator 


\section{$\underline{\text { Reagents Required }}$}

- Trizol

- Cholorform

- Ethanol - RNA Grade (EtOH)

- Ethanol - Not RNA Grade

- $\beta$-mercaptoethanol ( $\beta$-Me)

- RLT buffer (Qiagen)

- RPE Buffer (Qiagen)

- RNase free water (Qiagen)

- RNASEZAP ${ }^{\mathrm{TM}}$

- Isopropanol

- Guanadine hydrochloride

- Sodium dodecyl sulfate (SDS)

- Sarkosyl

- Glycogen

- Tris-HCl

\section{Solution Recipes (if applicable)}

\begin{tabular}{|c|c|c|c|c|c|}
\hline \hline $\begin{array}{c}\text { Chemicals } \\
\text { Required }\end{array}$ & $\begin{array}{c}\text { Guanadine } \\
\text { Hydrochloride }\end{array}$ & Ethanol & Tris-HCl & SDS & Sarkosyl \\
\hline $\begin{array}{c}\text { Washing Solution } \\
\text { QC 150mL }\end{array}$ & $4.29 \mathrm{~g}$ & $\begin{array}{c}142.5 \mathrm{~m} \\
\mathrm{~L}\end{array}$ & & $0.050 \mathrm{~g}$ & $0.917 \mathrm{~g}$ \\
\hline $\begin{array}{c}\text { Resuspension } \\
\text { Solution } \\
\text { QC 50mL }\end{array}$ & $150 \mathrm{~mL}$ & $\begin{array}{c}2.5 \mathrm{~mL} \\
\mathrm{pH} 8\end{array}$ & $50 \mathrm{~mL}$ & \\
\hline Final Volume & \multicolumn{2}{|c|}{} & & \\
\hline
\end{tabular}

\section{Procedure:}

This procedure isolates RNA, DNA and protein from total lysate of tissue that has been embedded in OCT and then cut into section of thickness $\leq 60 \mu \mathrm{m}$. Since one of the goals is to isolate quality, intact RNA it is important to use excellent sterile molecular biology technique. Everything that comes into contact with the samples in anyway should be handled with RNase 
free gloves. All work surfaces should be treated with RNase ZAP and then covered with sterile

RNase free blue pads. Ensure that equipment that has not been treated with RNase ZAP does not come in contact with the prepared surfaces until proper decontamination procedures have been taken. All pipets, tubes, and tips should be sterile, fresh, and designated as RNA work only, to prevent contamination by RNase. Do NOT perform this procedure until you have been properly instructed by Doug Chew on proper RNA handling technique!

\section{RNA Isolation:}

1. Prepare the work area for RNase free work by treating the bench top, pipet aides, pipettors, homogenzier, tube racks, and centrifuge with RNase ZAP then cover all work surfaces with blue pads to ensure an RNase free workspace. Also, make sure the ice used is well packed to prevent contamination.

2. Remove one sample from the $-80^{\circ} \mathrm{C}$ freezer and place on ice. Samples should be in VWR high speed centrifuge tubes. If they are not, or if you are not sure the samples MUST be transferred into high speed centrifuge tubes prior to continuing with this protocol. Immediately add $10 \mathrm{mls}$ of Trizol and homogenize the sample for $90 \mathrm{sec}$ then place back on ice for $90 \mathrm{sec}$. Perform one more homogenize for $60 \mathrm{sec}$ ensuring homogenous solution.

3. Clean the homogenization probe by preparing the following volumes and then rinsing with the following solutions in order: (prepare all volumes in $50 \mathrm{~mL}$ centrifuge tubes)

- Trizol $(15 \mathrm{~mL})$

- Cholorform $(50 \mathrm{~mL})$

- Ethanol $(50 \mathrm{~mL})$

Then wipe the probe with RNase free Kim wipes.

4. Repeat steps 2 and 3 for each sample to be fractionated.

5. Centrifuge samples in Sorvall Legend RT with HIGHconic rotor 75003046/3057 (a) $11,500 \mathrm{xg}$ and $4^{\circ} \mathrm{C}$ for $30 \mathrm{~min}$.

6. Remove the supernatant to fresh VWR high speed $50 \mathrm{ml}$ centrifuge tubes. Save the pellets for the Protein Isolation Protocol (see Fig. 1). At this point the tubes can be frozen in the $80^{\circ} \mathrm{C}$ for storage and then thawed in a $30^{\circ} \mathrm{C}$ water bath with gentle agitation every 3-4 min.

7. Incubate all samples in a $30^{\circ} \mathrm{C}$ water bath for at least 20 minutes with gentle agitation every 3-4 min.

8. Remove samples from the water bath and wipe all condensation and water off of them with an RNase free Kim wipe.

9. Add $3 \mathrm{ml}$ of chloroform to each tube using either glass or polypropylene pipets (Polystyrene reacts with the chloroform!).

10. Shake samples by hand for $15 \mathrm{sec}$ and incubate in a $30^{\circ} \mathrm{C}$ water bath for 10 minutes with gentle agitation every 3-4 minutes. 
11. After the incubation centrifuge the samples according to step 6 .

12. When removing the samples from the centrifuge take care not to disturb the interface between the aqueous, interphase, and organic phases. Transfer the aqueous phase (on top) to fresh $15 \mathrm{ml}$ conical tubes for subsequent RNA Cleanup.

\section{RNA Cleanup:}

This protocol is a minor variation on the Qiagen RNA Cleanup kit protocol.

1. Connect a 500ml side arm flask fitted with a \#8 stopper with 1 hole to the lab vacuum. Use RNase ZAP to clean the flask, benchtop, stopper and tubing.

2. Attach a sterile $1.5^{\prime \prime} 18$ gauge needle to the outflow and a sterile $3 \mathrm{ml}$ syringe to the inflow of a Qiagen spin column. NOTE: It is important that the column stay RNase free - take extra care to use good sterile technique and not allow the column to touch any non-sterile or nonRNase free surface (e.g. flask, stoppper, bench top, ungloved hands, etc).

3. Place the entire needle, column, syringe construct into the hole of the stopper, needle side down.

4. Add $17.5 \mathrm{ml}$ of $\mathrm{RLT}+\beta-\mathrm{Me}$ and $12.5 \mathrm{ml}$ of $\mathrm{EtOH}$ to one sample, and pipet up and down several times to mix well.

5. Add $3 \mathrm{mls}$ of sample to the syringe in the vacuum flask and then turn the vacuum on for $2 \mathrm{sec}$ and immediately turn off.

6. As the sample is sucked onto the column continue to add sample from the tube to the syringe until the entire volume is loaded. It should take between 3-5 minutes.

7. Once the entire sample is loaded onto the column disassemble the needle, column, syringe construct and place the column in a fresh $2 \mathrm{ml}$ collecting tube.

8. Repeat steps $18-23$ for the remaining samples.

9. Add $500 \mu \mathrm{l}$ of RPE $+\mathrm{EtOH}$ to each column and place each column-collecting tube pair in the Spectrafuge 16M. Spin at max speed for $15 \mathrm{sec}$.

10. Remove the column collecting tube pairs and discard the flow-throughs. This should be done using a micropipettor, NOT by pouring!

11. Repeat steps 9 and 10 for a total of 3 times.

12. Transfer the columns to $2 \mathrm{ml}$ fresh collecting tubes and centrifuge in the Spectrafuge $16 \mathrm{M}$ for 1 min at max speed.

13. Transfer the columns to fresh $1.5 \mathrm{ml}$ collecting tubes.

14. Add $30 \mu \mathrm{l}$ of RNase free water to each column and centrifuge in the Spectrafuge 16M for $1 \mathrm{~min}$ at max speed.

15. Repeat step 14.

16. Discard the columns and cap the $1.5 \mathrm{ml}$ collecting tubes. These samples should either be processed immediately or stored at $-80^{\circ} \mathrm{C}$. 


\section{DNA Isolation:}

1. Remove and discard remaining aqueous layer (top) from the tubes. It is important to completely remove the aqueous layer to ensure quality DNA.

2. Add $3 \mathrm{ml}$ of $100 \%$ ethanol to each sample and mix by inverting several times.

3. Incubate the samples $@ 30^{\circ} \mathrm{C}$ for 3 minutes.

4. Centrifuge in Sorvall Legend RT with HIGHconic rotor 75003046/3057 no more than 2000xg@4 ${ }^{\circ} \mathrm{C}$ for $5 \mathrm{~min}$.

5. *Remove existing "solid" layer from the top of the sample into another $50 \mathrm{ml}$ centrifuge tube. This is the OCT layer, in order to accomplish this step the layer will need to be extracted by pipet or scooped off using some sort of spatula.

6. Repeat centrifuge spin in Sorvall Legend RT with HIGHconic rotor 75003046/3057 no more than 2000xg@4 $9{ }^{\circ} \mathrm{C}$ for 5 min

7. Remove any remaining layer from the top of the solution and repeat step 6 . If no layer seems to be present continue with step 7. It is important to make sure there is absolutely no layer on top of the solution at this point. Any OCT left in the solution will effect your protein isolation in the next step of this protocol.

8. Remove the phenol-ethanol supernatant to a fresh $50 \mathrm{ml}$ tube and save for the Protein Isolation Protocol. Save the DNA pellet for the DNA Cleanup Protocol.

\section{Protein Isolation:}

1. Add $15 \mathrm{ml}$ of isopropanol and $20 \mu \mathrm{g}$ of glycogen to the freshly collected supernatant in step 7 of the DNA Isoloation Protocol.

2. Incubate samples $@ 30^{\circ} \mathrm{C}$ for 10 minutes.

3. Centrifuge samples in Sorvall Legend RT with HIGHconic rotor 75003046/3057 (a) $11,500 x g$ and $4{ }^{\circ} \mathrm{C}$ for $30 \mathrm{~min}$.

4. Discard the supernatant. (collect into $50 \mathrm{~mL}$ centrifuge tube, Non high speed)

5. Add $20 \mathrm{ml}$ of Wash Solution and incubate at $30^{\circ} \mathrm{C}$ for 20 minutes.

6. Centrifuge in Sorvall Legend RT with HIGHconic rotor $75003046 / 3057$ at $7500 \times \mathrm{xg}$ at $4^{\circ} \mathrm{C}$ for $5 \mathrm{~min}$.

7. Repeat steps 4-6 for a total of 3 times, each time collecting the solution in a non high speed centrifuge tube to be evaluated and run in an electrophoresis gel at a later time.

8. Discard the supernatant, add $2 \mathrm{ml}$ of $100 \%$ ethanol and incubate at $30^{\circ} \mathrm{C}$ for 20 minutes.

9. Repeat step 6

10. Vacuum dry the pellet for 5-10 minutes: Poke a small hole in the top of the tube and place in a vacuum desicator under lab vacuum.

11. Resuspend protein pellet in resuspension buffer at $50^{\circ} \mathrm{C}$.

12. Add this solution to the pellet saved in step 7 of the RNA Isolation Protocol.

13. Resuspend that pellet as in step 11.

14. Centrifuge in Sorvall Legend RT with HIGHconic rotor $75003046 / 3057$ at $10000 x g$ at $4{ }^{\circ} \mathrm{C}$ for 10 min. NOTE: This will change when we purchase a new rotor.

15. Transfer the supernatant to a fresh tube. This sample can be stored at $-20^{\circ} \mathrm{C}$ for future analysis. Save the pellet to make sure that all of the protein was resuspended from step 11. 


\section{Protocol E.13: SDS Page}

\section{Equipment Required}

- $\quad$ Mini-PROTEAN 3 cell (Figure E.1)

- Spacer plate

- Short plate

- Casting frame

- Casting stand

- Gel cassette sandwich

- Combs

- Buffer dam

- Electrode assembly

- Clamping frame

- Inner chanber

- Mini Tank and Lid

- Sample loading guide

- Pipet Aide

- $\quad 5 \mathrm{ml}$ disposable pipets

- Micropippetors

- P20

- P200

- P1000

- $\quad 50 \mathrm{ml}$ Conical tubes

- $\quad 0.5 \mathrm{ml}$ Microfuge tubes

- Heating block, vortex genie, benchtop microcentrifuge.

\section{$\underline{\text { Reagents Required }}$}

- $\quad$ Acrylamide/Bis, 37.5:1 mixture

- $\quad 1.5 \mathrm{M}$ Tris-HCl, $\mathrm{pH} 8.8$

- $\quad 0.5 \mathrm{M}$ Tris-HCl, $\mathrm{pH} 6.8$

- $\quad 10 \%$ SDS solution

- TEMED

- $10 \%$ APS

- Laemmli Buffer

- 2-mercaptoethanol

- 10x Tris/Glycine/SDS Buffer 

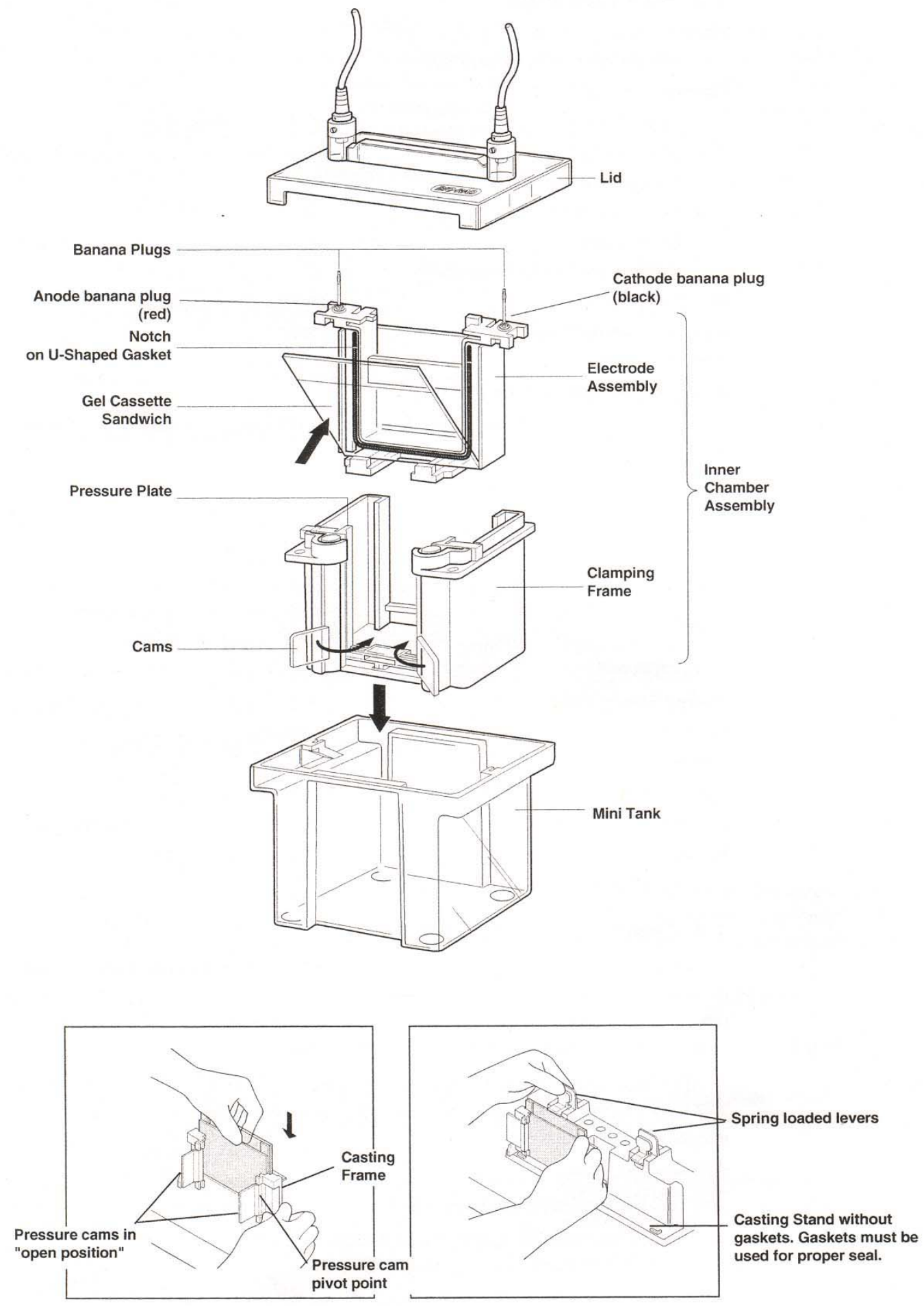

Figure E. 1 Diagram of the Mini-PROTEAN 3 cell from Bio-Rad. Image adapted from the Mini-PROTEAN 3 Cell Instruction Manual. 


\begin{tabular}{|c|c|c|c|c|c|c|c|c|c|c|}
\hline \multicolumn{11}{|c|}{ Solution Recipes } \\
\hline $\begin{array}{c}\text { Chemicals } \\
\text { Require } \\
d\end{array}$ & $\begin{array}{c}10 \mathrm{x} \\
\text { Tris/Gl } \\
\text { ycine/S } \\
\text { DS } \\
\text { Buffer } \\
\end{array}$ & $\begin{array}{l}\text { Acrylami } \\
\text { de/Bis }\end{array}$ & $\begin{array}{c}1.5 \mathrm{M} \\
\text { Tris-HCl } \\
\mathrm{pH} 8.8\end{array}$ & $\begin{array}{c}0.5 \mathrm{M} \\
\text { Tris-HCl } \\
\text { pH } 6.8\end{array}$ & $\begin{array}{l}10 \% \\
\text { SDS }\end{array}$ & $\begin{array}{l}10 \% \\
\text { APS }\end{array}$ & TEMED & $\begin{array}{l}\text { Laemmli } \\
\text { Buffer }\end{array}$ & $\begin{array}{c}2- \\
\text { Mercapt } \\
\text { oethanol }\end{array}$ & Water \\
\hline $\begin{array}{l}\text { Running } \\
\text { buffer }\end{array}$ & $\begin{array}{c}100 \\
\mathrm{ml}\end{array}$ & & & & & & & & & $\begin{array}{c}900 \mathrm{~m} \\
1\end{array}$ \\
\hline $\begin{array}{l}\text { Sample } \\
\text { Buffer }\end{array}$ & & & & & & & & $950 \mu \mathrm{l}$ & $50 \mu \mathrm{l}$ & \\
\hline $\begin{array}{c}7.5 \% \\
\text { Resolving } \\
\text { Gel }\end{array}$ & & $2.5 \mathrm{ml}$ & $2.5 \mathrm{ml}$ & & $100 \mu \mathrm{l}$ & $50 \mu 1$ & $5 \mu l$ & & & $\begin{array}{c}4.85 \mathrm{~m} \\
1\end{array}$ \\
\hline $\begin{array}{c}10 \% \\
\text { Resolving } \\
\text { Gel }\end{array}$ & & $3.3 \mathrm{ml}$ & $2.5 \mathrm{ml}$ & & $100 \mu \mathrm{l}$ & $50 \mu 1$ & $5 \mu l$ & & & $4.1 \mathrm{ml}$ \\
\hline $\begin{array}{c}12 \% \\
\text {.Resolving } \\
\text { Gel }\end{array}$ & & $4 \mathrm{ml}$ & $2.5 \mathrm{ml}$ & & $100 \mu \mathrm{l}$ & $50 \mu 1$ & $5 \mu l$ & & & $3.4 \mathrm{ml}$ \\
\hline $\begin{array}{c}15 \% \\
\text { Resolving } \\
\text { Gel } \\
\end{array}$ & & $5 \mathrm{ml}$ & $2.5 \mathrm{ml}$ & & $100 \mu \mathrm{l}$ & $50 \mu 1$ & $5 \mu l$ & & & $2.5 \mathrm{ml}$ \\
\hline $\begin{array}{l}\text { Stacking } \\
\text { Gel }\end{array}$ & & $1.3 \mathrm{ml}$ & & $2.5 \mathrm{ml}$ & $100 \mu \mathrm{l}$ & $50 \mu \mathrm{l}$ & $10 \mu \mathrm{l}$ & & & $6.1 \mathrm{ml}$ \\
\hline
\end{tabular}

\section{Procedure:}

1. Place the casting frame on a flat surface with the pressure cams in the open position (level arms turned forward) and facing forward.

2. Place a short plate on top of a spacer plate (there should be a space between the two plates).

3. With the label on the spacer plate facing up and forward, slide the two glass plates into the casting frame.

4. Make sure the bottom edges of the glass plates are level with the surface of the bench top and close the pressure cams by swinging the lever arms outward.

5. On the casting stand engage the spring-loaded lever and place the gel cassette assembly onto the gray casting gasket. Release the lever so the clamp is pushing spacer plate down into the casting gasket.

6. If running two gels repeat steps 1-5 for the other gel. The casting stand has two places for gel sandwich assemblies.

7. To cast a gel place a comb completely into the assembled gel cassette and mark the glass plate $1 \mathrm{~cm}$ below the comb teeth. After marking the level remove the comb.

8. Prepare the Resolving Gel solution by combining all reagents except the APS and TEMED according to recipe above in a $50 \mathrm{ml}$ conical tube. The percent gel depends on the molecular weight of the protein(s) of interest.

9. Add the APS and TEMED and then immediately pour into the gel cassette using a $5 \mathrm{ml}$ pipette. Fill to the line drawn in step 7.

10. Overlay the monomer resolving solution with 2-propanol and allow the gel to polymerize for 45min - 1 hour.

11. Prepare the Stacking Gel solution by combining all reagents except the APS and TEMED according to the recipe above in a $50 \mathrm{ml}$ conical tube. 
12. Before casting the stacking gel, use a piece of filter paper to dry the gel area $b / w$ the glass plates above the resolving gel taking care not to touch the surface of the gel.

13. Add the APS and TEMED and immediately pour on top of the resolving gel, completely filling the cassette.

14. Insert the comb slowing to prevent trapping of bubbles in the gel.

15. Allow the stacking gel to polymerize for $30-45 \mathrm{~min}$.

16. Once polymerized the gel cassette sandwiches can be removed from the casting frames. The combs should then be carefully removed and the wells of the gel rinsed with deionized water.

17. Place a gel cassette sandwich into the slots at the bottom of each side of the electrode assembly. Be sure the short glass plate faces inward toward the green U-shaped gasket. If only use one gel add the buffer dam to the other side of the electrode assembly.

18. While holding the gel cassette sandwiches to the electrode assembly place the assembly into the clamping frame and close the two cam levers to seal the inner chamber formed by the two gel cassettes or gel cassette and buffer dam.

19. Lower the inner chamber assembly into the mini tank and fill the inner chamber with about $125 \mathrm{ml}$ of Running buffer. The level should be about half way between the top of the short glass plate and the top of the spacer plate.

20. Add about $200 \mathrm{ml}$ of Running buffer to the mini tank.

21. Prepare protein samples in $0.5 \mathrm{ml}$ microfuge tubes according to the following subprotocol:

a. Mix equal volumes of protein sample and sample buffer prepared according to recipe above.

b. Vortex and pulse spin the samples.

c. Place the sample in a $95 \mathrm{C}$ heat block for 10 minutes.

d. Vortex and pulse spin the samples again

22. Place sample loading guide on top of the inner chamber and load protein samples using a micropipettor (P20 or P200) and gel loading tips.

23. After all samples have been loaded including molecular weight and other appropriate standards place the lid on the mini tank making sure the color coded bananna plugs on the electrode assembly match with the appropriate jacks on the lid.

24. Run the gel at 180 constant volts for 45 minutes. The dye front should run all the way to the bottle of the gel. These parameters may need to be adjusted in order to optimize the separation achieved.

25. When the electrophoresis is complete turn the power supply off, and disconnect all the leads. Remove the lid and the inner chamber assembly. Pour off the running buffer and discard.

26. Open the cams on the clamping frame and remove the electrode assembly and gel cassette sandwiches.

27. To remove the gel from the sandwich, separate the two plates (Spacer plate and Short plate). The gel will usually stick to one of these plates. If the gel sticks to the spacer plate you may have to release it by running a razor blade on the spacer edge. The gel can be then floated off the plate by inverting the gel and plate under solution and gently agitating under the gel separates from the plate. The solution used here depends on how the gel will be analyzed.

28. To clean glass plates refer to SDS-PAGE plate cleaning protocol.

29. All other components should be thoroughly rinsed in deionized water after each use. 


\section{Protocol E.14: Western Blot}

\section{Equipment Required}

- 2-Ready Gel Blotting Sandwiches

- 2 Filter papers (Each)

- 1 PVDF membrane (Each)

- Mini Trans-Blot Electrophoretic Transfer Cell (Figures E.2 and E.3)

- 2-Gel cassette holders

- 4-Fiber pads

- Electrode module

- Bio-Ice cooling unit

- Buffer tank

- Lid

- Small stir bar

- Magnetic stir plate

- Rocker table

- Kapak pouches

- Impulse sealer

- Rotary platform

\section{$\underline{\text { Reagents Required }}$}

- 10x Tris/Glycine/SDS Buffer

- $\quad 100 \%$ Methanol

- $10 x$ TBS

- Tween 20

- BSA (Blot-Qualified, Promega \#W384A)

- Promega anti-IgG AP conjugated antibody

- Western Blue Stabilized Substrate for Alkaline Phosphatase (Promega, \#S3841 


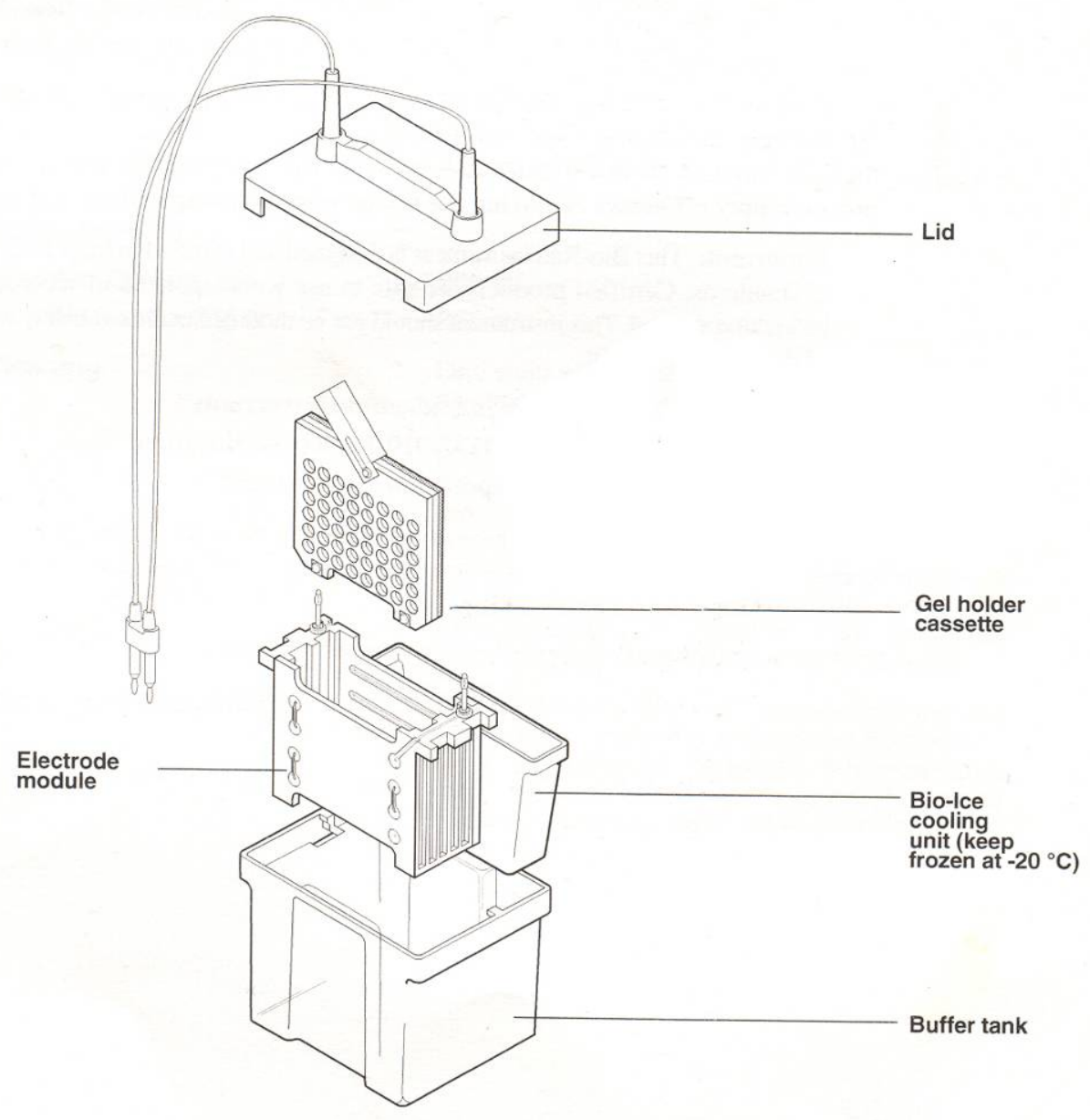

Figure E. 2 Diagram of Mini Trans-Blot Electrophoretic Transfer Cell from Bio-Rad. Image adapted from the Mini-PROTEAN 3 Cell Instruction Manual. 


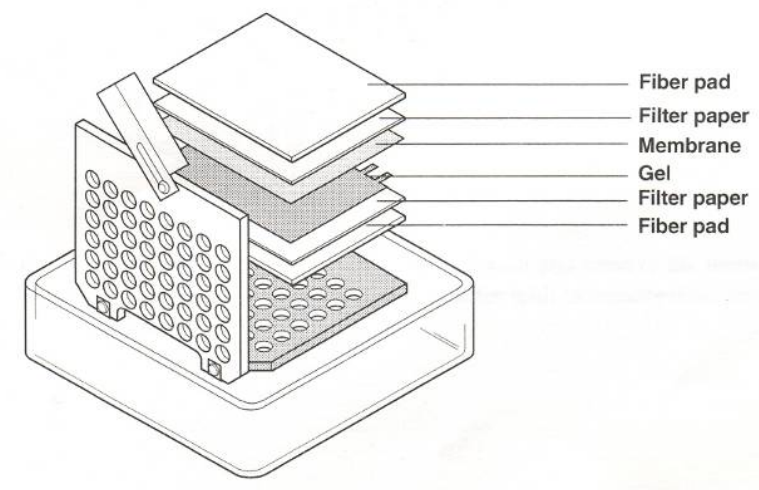

Figure E. 3 Diagram of the assembly of the gel transfer cassettes. Image adapted from the Mini-PROTEAN 3 Cell Instruction Manual.

Solution Recipes (if applicable)

\begin{tabular}{|c|c|c|c|c|c|c|}
\hline \hline $\begin{array}{c}\text { Chemicals } \\
\text { Required }\end{array}$ & $\begin{array}{c}10 \mathrm{x} \\
\text { Tris/Glycine/ } \\
\text { SDS }\end{array}$ & $\begin{array}{c}10 \mathrm{x} \\
\text { TBS }\end{array}$ & BSA & Tween 20 & $\begin{array}{c}\text { Methan } \\
\text { ol }\end{array}$ & $\begin{array}{c}\text { Total } \\
\text { Volume } \\
\text { (Balance } \\
\text { Water) }\end{array}$ \\
\hline $\begin{array}{c}\text { Transfer } \\
\text { Buffer }\end{array}$ & $100 \mathrm{ml}$ & & & & $200 \mathrm{ml}$ & $1 \mathrm{~L}$ \\
\hline $\begin{array}{c}\text { TBST } \\
\text { Blocking } \\
\text { Solution }\end{array}$ & & $100 \mathrm{ml}$ & & $750 \mu l$ & & $1 \mathrm{~L}$ \\
\hline TBS & & $10 \mathrm{ml}$ & $1 \mathrm{~g}$ & & & $100 \mathrm{ml}$ \\
\hline
\end{tabular}

\section{Procedure:}

1. Run samples of protein extract from cell or tissue lysates on an SDS-PAGE gel appropriate for the molecular weight of the protein of interest. Samples amounts

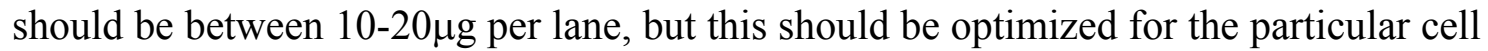
or tissue. (See SDS-PAGE and Tissue Homogenization And Protein Extraction protocols)

2. At the conclusion of the electrophoresis remove the gels and incubate them in Transfer Buffer for at least 15 minutes.

3. Fill the Bio-Ice cooling unit with water and place in the $-80 \mathrm{C}$ freezer.

4. While gels are equilibrating in the Transfer Buffer take two Ready Gel Blotting Sandwiches (Bio-Rad 162-0218) and remove the PVDF membrane from each. The membranes should be wetted in $100 \%$ methanol for 1 minute and then equilibrated in 
Transfer Buffer for 15 minutes. The filter papers can be placed directly into the Transfer Buffer and equilibrated for 15 minutes also. Take care to wear gloves so as to not contaminate the membrane.

5. Once gels and membranes are equilibrated build gel transfer cassettes as follows (Figure 2).

a. Open gel holder cassette.

b. Dip fiber pad in transfer buffer and lay on top of the black side of the holder.

c. Center one of the filter papers from the Ready Gel Blotting Sandwiches on the fiber pad.

d. Next layer the gel on top of the filter paper making sure there are no bubbles between the filter paper and the gel. The gel should be square with no uneven stretching.

e. Place the PVDF membrane on top of the gel again making sure there are no bubbles between the membrane and the gel.

f. Place the second filter paper on the membrane again eliminating any bubbles.

g. Finally dip the second fiber pad in Transfer Buffer and add that to the top.

h. Fold the white side of the cassette holder over and close and lock it.

i. Repeat this for the steps a-h for the second gel.

6. Place gel transfer cassettes into electrode module with the locking mechanism facing up and the black sides facing the black side of the electrode module.

7. Add a small stir bar to the buffer tank.

8. Place the electrode module with cassettes into the buffer tank so the electrodes are in the middle of the tank. Add the Bio-Ice cooling unit from the freezer.

9. Fill the tank with Transfer Buffer and place the whole unit on a magnetic stir plate in the refrigerator.

10. Add the lid and connect it to the power source.

11. Transfer for $18-22$ hours at $90 \mathrm{~mA} / 30 \mathrm{~V}$ with moderate stirring.

12. When the transfer is complete disassemble transfer cell and gel transfer sandwiches and place the membranes in Blocking Solution.

13. Incubate membranes in Blocking Solution on rocker table for at least 1 hour. May need to increase the blocking time to reduce background.

14. While incubating prepare antibody incubation pouches using Kapak pouches and impulse sealer. The pouches should be just big enough for the membrane plus $2-3 \mathrm{ml}$ of solution.

15. When membranes have been blocked for at least 1 hour transfer the membranes to the antibody incubation pouches. Add $2.5 \mathrm{ml}$ of primary antibody solution to the pouch, seal, and place on a rotary platform. Primary antibody incubation should be for at least 1 hour at room temperature but can also be performed overnight at $4^{\circ} \mathrm{C}$.

Note: Primary antibody solution is composed of the primary antibody diluted into

TBST. The appropriate dilution depends on the specific antibody and the tissue sample being analyzed. The dilution should be optimized for each experimental setup.

16. After incubation in primary antibody remove the membranes from the pouches and wash them three times in TBST for 10 minutes on rocker table per wash.

17. During the washing steps prepare antibody incubation pouches as in step 14. At the conclusion of step 16, transfer the membranes to the antibody incubation pouches. Add $2.5 \mathrm{ml}$ of secondary antibody solution to the pouch, seal, and place on a rotary platform. 
Note: Secondary antibody solution consists of Promega anti-IgG AP conjugated antibody diluted 1:5000 in TBST.

18. Incubate the membranes in secondary antibody for 30-60 minutes.

19. Remove the membranes from the incubation pouches and wash them three times in TBST for 10 minutes on rocker table per wash.

20. Rinse the membranes in TBS (w/o Tween) three times for 10 minutes per wash.

21. Add $15 \mathrm{ml}$ of Western Blue Stabilized Substrate for Alkaline Phosphatase to the membranes and agitate on the rocker table to allow bands to appear. Development of bands can take 1 minute to several hours. Most take place in less than 15 minutes. Development times will vary depending on the protein, tissue, and antibodies of interest.

22. Once desired band intensity is reached exchanging the substrate for deionized water can stop the reaction. Membranes should be visualized wet since bands and background will fade upon drying. The original intensity will return upon rewetting in methanol first...then deionized water.

\section{Protocol E.15: RNA Isolation}




\section{Equipment Required}

- Ice bucket w/ ice

- Pipet aide

- Homogenizer

- $50 \mathrm{ml}$ conical tubes (Sterile-RNase free)

- Sterile pipets

$-5 \mathrm{ml}$

$-10 \mathrm{ml}$

$-25 \mathrm{ml}$

- Sorvall Legend RT centrifuge w/ Sorvall 75006466 rotor

- Water bath

- Kim wipes

- Gloves (RNase free)

- Vacuum apparatus:

- $500 \mathrm{ml}$ side arm flask

- \#8 Stopper w/ hole

- Tubing

- Micropipettors (RNA work only):

- P1000

- P200

- Qiagen RNeasy mini kit

- $2 \mathrm{ml}$ collecting tubes

- $1.5 \mathrm{ml}$ collecting tubes

- RNeasy mini columns

- $3 \mathrm{ml}$ sterile syringes

- $1.5^{\prime \prime}, 18$ gauge sterile needles

- Spectrafuge 16M

- RNase free pipet tips:

- P1000

- P200 


\section{$\underline{\text { Reagents Required }}$}

- $180 \mathrm{ml}$ Trizol

- 54ml Cholorform

- $250 \mathrm{ml}$ Ethanol - RNA Grade (EtOH

- $4 \mathrm{ml} \beta$-mercaptoethanol ( $\beta$-Me)

- $400 \mathrm{ml}$ RLT buffer (Qiagen)

- $10 \mathrm{ml}$ RPE Buffer (Qiagen)

- $1.2 \mathrm{ml}$ RNase free water (Qiagen)

- RNase ZAP

\section{Solution Recipes (if applicable)}

\begin{tabular}{|c|c|c|c|c|c|l|}
\hline $\begin{array}{c}\text { Chemicals } \\
\text { Required }\end{array}$ & RLT & $\beta-\mathrm{Me}$ & $\mathrm{EtOH}$ & $\mathrm{RPE}$ & & \\
\hline (Amount) & & & & & & \\
\hline RLT+ $\beta-M e$ & $400 \mathrm{ml}$ & $4 \mathrm{ml}$ & & & & \\
\hline RPE+EtOH & & & $44 \mathrm{ml}$ & $10 \mathrm{ml}$ & & \\
\hline $\begin{array}{c}\text { Final } \\
\text { Volume }\end{array}$ & 400 & 4 & 44 & 10 & & \\
\hline
\end{tabular}

\section{Procedure:}

This procedure isolates RNA from tissue that has been embedded in OCT and then cut into section of thickness $\leq 60 \mu \mathrm{m}$. It is written for a batch of 18 samples, the maximum allowed by this laboratory's resources. Since the goal is to isolate quality, intact RNA it is important to use excellent sterile molecular biology technique. Everything that comes into contact with the samples in anyway should be handled with RNase free gloves. All work surfaces should be treated with RNase ZAP. All pipettes, tubes, and tips should be sterile, fresh, and designated as RNA work only, to prevent contamination by RNase.

1. Prepare the work area for RNase free work by treating the bench top, pipette aides, pipettors, homogenizer, tube racks, and centrifuge with RNase ZAP. Also, make sure the ice used is well backed to prevent contamination.

2. Remove one (of 18) sample from the $-80^{\circ} \mathrm{C}$ freezer and place on ice. Immediately add $10 \mathrm{mls}$ of Trizol and homogenize the sample for $90 \mathrm{sec}$ on ice.

3. Clean the homogenization probe by rinsing with the following solutions in order:

- Trizol 
- Chloroform

- Ethanol

Then wipe the probe with RNase free Kim wipes.

4. Repeat steps 2 and 3 for a total of 10 samples.

5. Homogenize each sample again for $90 \mathrm{sec}$ on ice.

6. Centrifuge those samples in Sorvall Legend RT with Sorvall rotor 75006446 at 3000xg at $2^{\circ} \mathrm{C}$ for $45 \mathrm{~min}$.

7. While those 10 samples are spinning repeat steps $2-5$ for the remaining 8 samples.

8. Remove the first 10 samples from the centrifuge and replace with the remaining 8 . Repeat step 6 for the those samples.

9. For the samples just removed from the centrifuge remove the supernatant to fresh $50 \mathrm{ml}$ conical tubes. At this point the tubes can be frozen in the $-80^{\circ} \mathrm{C}$ for storage and then thawed in a $30^{\circ} \mathrm{C}$ water bath with gentle agitation every 3-4 min.

10. Repeat step 9 for the remaining 8 samples when the centrifuge spin is finished.

11. Incubate all samples in a $30^{\circ} \mathrm{C}$ water bath for at least 20 minutes.

12. Remove samples from the water bath and wipe all condensation and water off of them with an RNase free Kim wipe.

13. Add $3 \mathrm{ml}$ of chloroform to each tube using either glass or polypropylene pipettes (Polystyrene reacts with the chloroform!).

14. Shake samples by hand for $15 \mathrm{sec}$ and incubate in a $30^{\circ} \mathrm{C}$ water bath for 10 minutes with gentle agitation every 3-4 minutes.

15. After the incubation centrifuge the samples according to step 6 .

16. When removing the samples from the centrifuge take care not to disturb the interface between the aqueous and organic phases. Transfer the aqueous phase (on top) to fresh $50 \mathrm{ml}$ conical tubes.

17. The remaining steps are a minor variation on the Qiagen RNA Cleanup kit protocol. Connect a $500 \mathrm{ml}$ side arm flask fitted with a $\# 8$ stopper with 1 hole to the lab vacuum. Use RNase ZAP to clean the flask, bench top, stopper and tubing.

18. Attach a sterile $1.5 " 18$ gauge needle to the outflow and a sterile $3 \mathrm{ml}$ syringe to the inflow of a Qiagen spin column. NOTE: It is important that the column stay RNase free - take extra care to use good sterile technique and not allow the column to touch any non-sterile or RNase free surface (e.g. flask, stopper, bench top, ungloved hands, etc).

19. Place the entire needle, column, syringe construct into the hole of the stopper, needle side down.

20. Add $17.5 \mathrm{ml}$ of RLT $+\beta-\mathrm{Me}$ and $12.5 \mathrm{ml}$ of EtOH to one sample, and pipette up and down several times to mix well.

21. Add $3 \mathrm{mls}$ of sample to the syringe in the vacuum flask and then turn on the vacuum on for $2 \mathrm{sec}$ and immediately turn off.

22. As the sample is sucked onto the column continue to add sample from the tube to the syringe until the entire volume is loaded. It should take between 3-5 minutes.

23. Once the entire sample is loaded onto the column disassemble the needle, column, syringe construct and place the column in a fresh $2 \mathrm{ml}$ collecting tube.

24. Repeat steps $18-23$ for the remaining samples (18 total). 
25. Add $500 \mu \mathrm{l}$ of RPE+EtOH to each column and place each column-collecting tube pair in the Spectrafuge 16M. Spin at max speed for $15 \mathrm{sec}$.

26. Remove the column collecting tube pairs and discard the flow-throughs. This should be done using a micropipettor, NOT by pouring!

27. Repeat steps 25 and 26 for a total of 3 times.

28. Transfer the columns to $2 \mathrm{ml}$ fresh collecting tubes and centrifuge in the Spectrafuge $16 \mathrm{M}$ for $1 \mathrm{~min}$ at max speed.

29. Transfer the columns to fresh $1.5 \mathrm{ml}$ collecting tubes.

30. Add $30 \mu \mathrm{l}$ of RNase free water to each column and centrifuge in the Spectrafuge 16M for $1 \mathrm{~min}$ at max speed.

31. Repeat step 30.

32. Discard the columns cap the $1.5 \mathrm{ml}$ collecting tubes. These samples should either be processed immediately for stored at $-80^{\circ} \mathrm{C}$. 


\section{APPENDIX F}

\section{IMMUNOHISTOCHEMISTRY DATA}

Figure F. 1 shows representative Live/Dead ${ }^{\mathrm{TM}}$ images from each experimental group from the 92 hour timepoint of the single $(\mathrm{N}=1)$ experiment performed to assess tissue necrosis within electrospun PIJV segments. There were three IH related endpoints that were quantified for the PIJVs from the ex vivo perfusion experiments. Figure F. 2 to Figure F. 67 show representative images for each endpoint for each PIJV segment from all the ex vivo perfusion experiments. Following each set of images, the values for the mean percent positive cells for each PIJV segment are tabulated. The corresponding statistical software output tables are also provided for each student's t-test that was performed. 


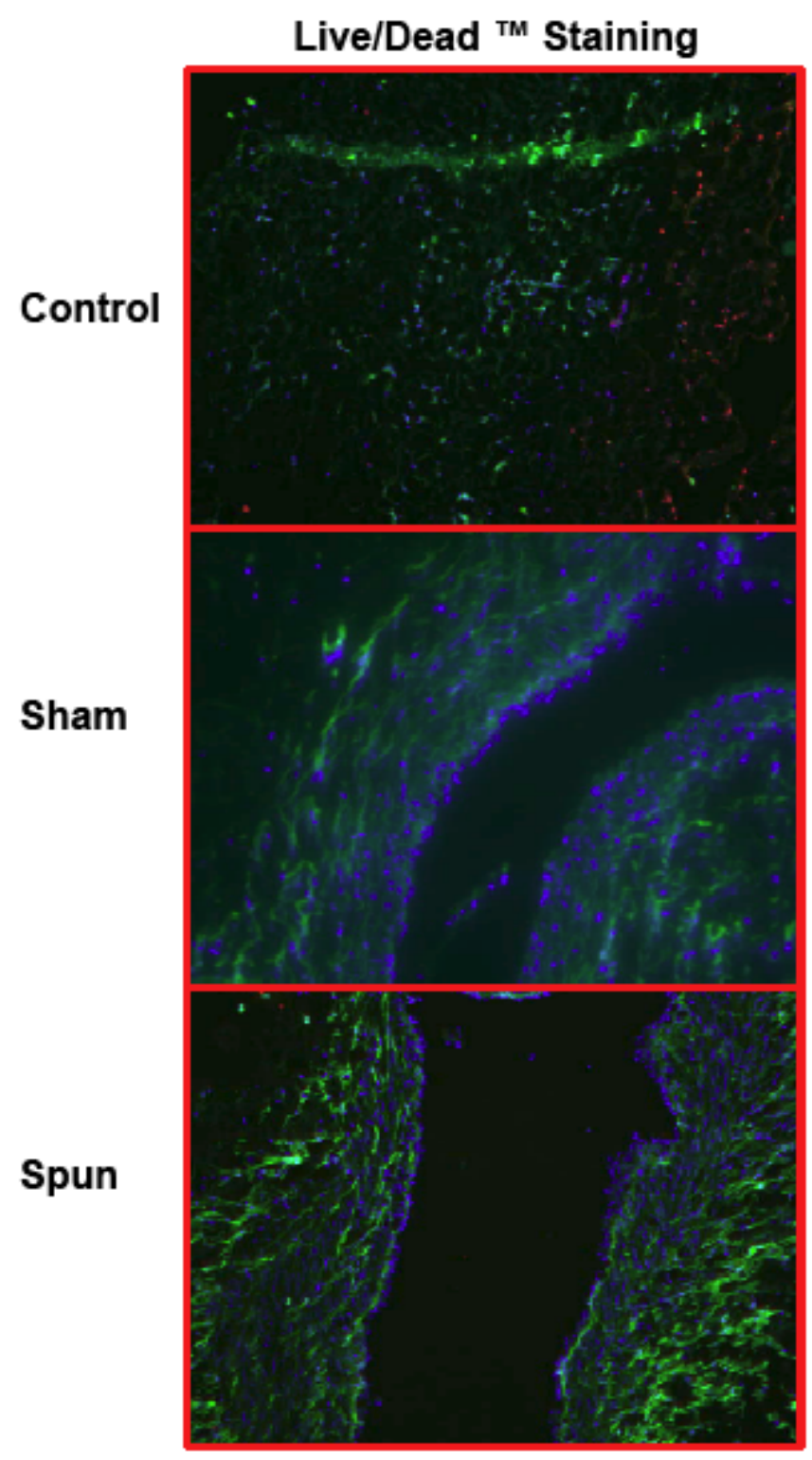

Figure F. 1 Representative Live/Dead ${ }^{\mathrm{TM}}$ images from all three conditions ( 92 hour timepoint) of the experiment to assess tissue necrosis in electrospun PIJVs. Note: blue, nuclei; green, live cells; red, necrotic cells. All images were taken at $100 \mathrm{x}$ magnification. 
VEN

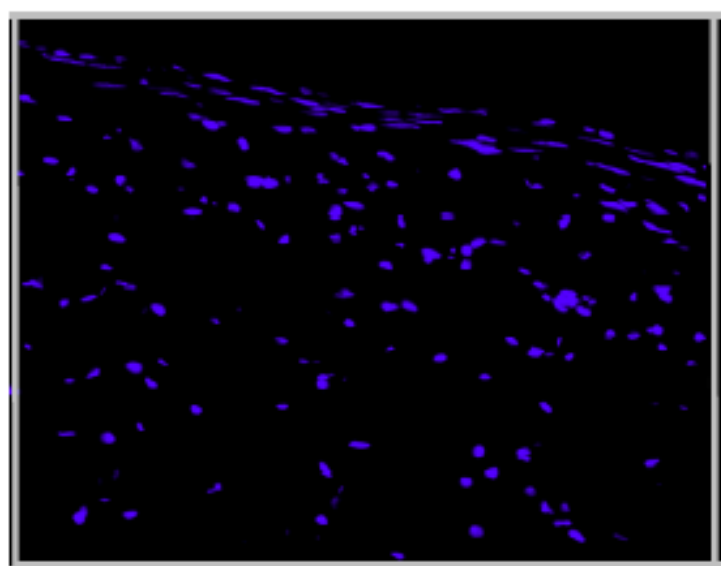

ART

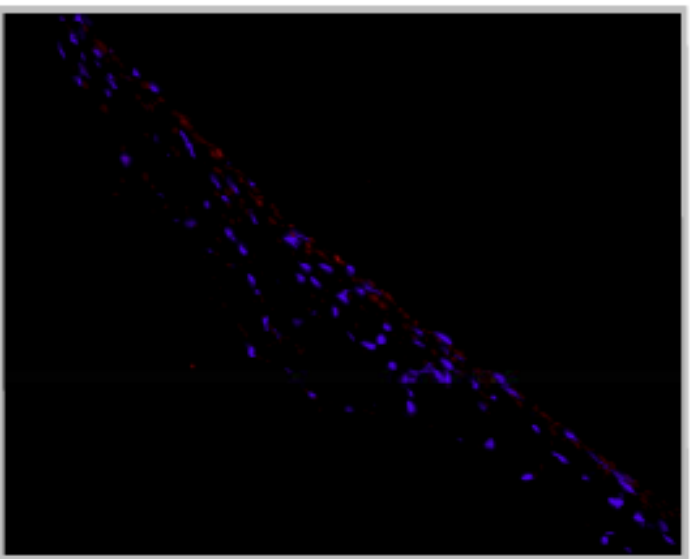

Figure F. 2Representative TUNEL images from 24 hour VEN vs. ART experiment performed on 04/23/2003. Nuclei are stained blue and TUNEL positive cells are stained red.

VEN

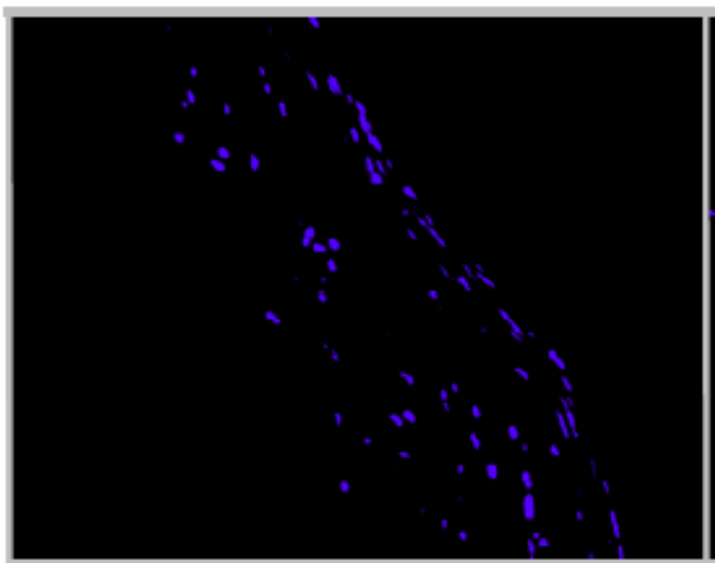

ART

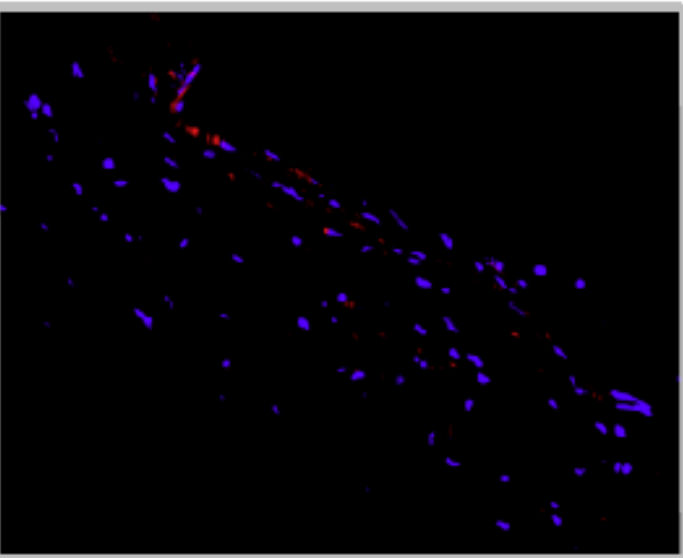

Figure F. 3 Representative TUNEL images from 24 hour VEN vs. ART experiment performed on 05/07/2003. Nuclei are stained blue and TUNEL positive cells are stained red. 
VEN

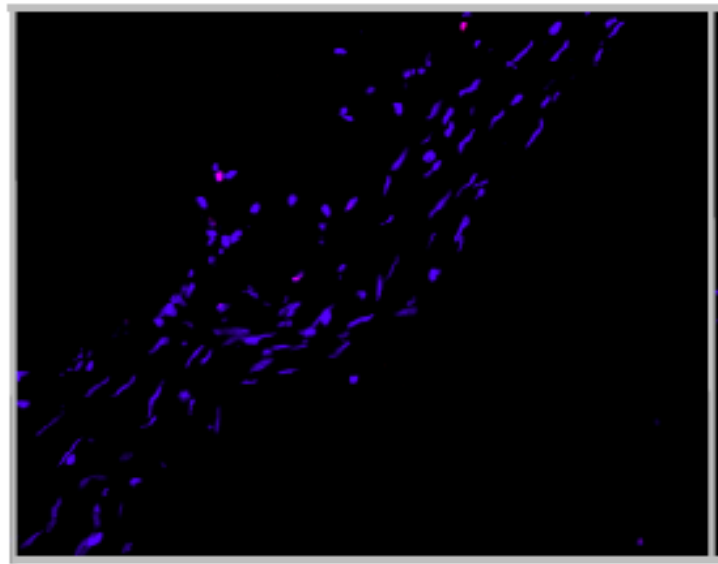

ART

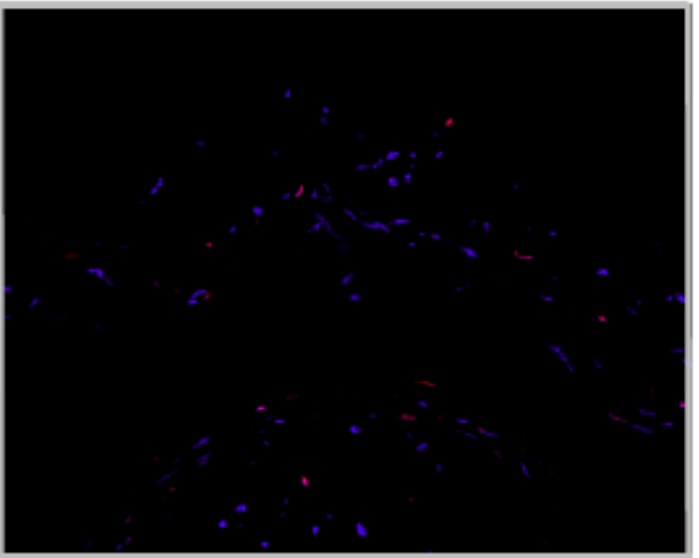

Figure F. 4 Representative TUNEL images from 24 hour VEN vs. ART experiment performed on 10/09/2004. Nuclei are stained blue and TUNEL positive cells are stained red.

VEN

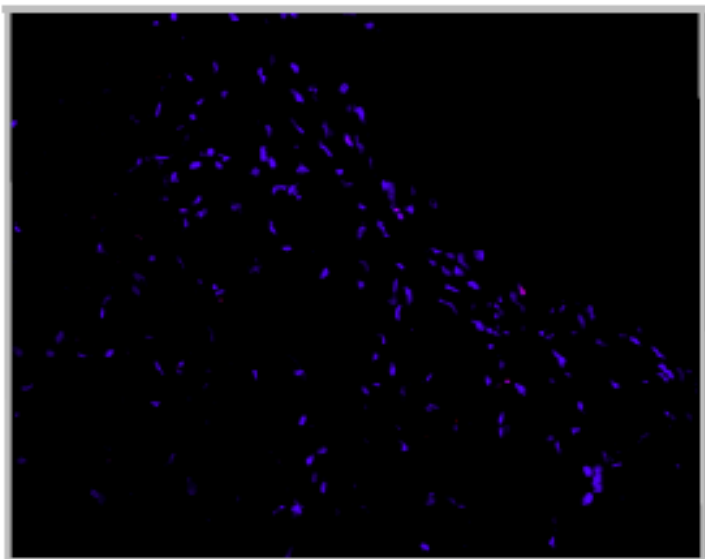

ART

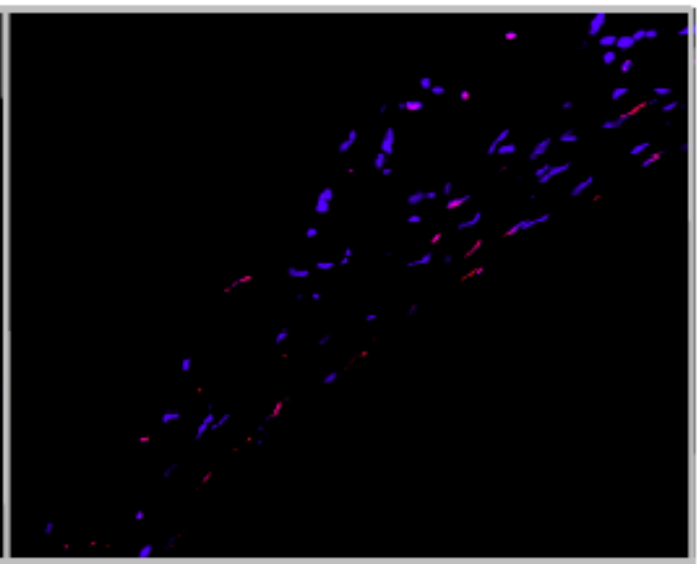

Figure F. 5 Representative TUNEL images from 24 hour VEN vs. ART experiment performed on 10/16/2004. Nuclei are stained blue and TUNEL positive cells are stained red. 


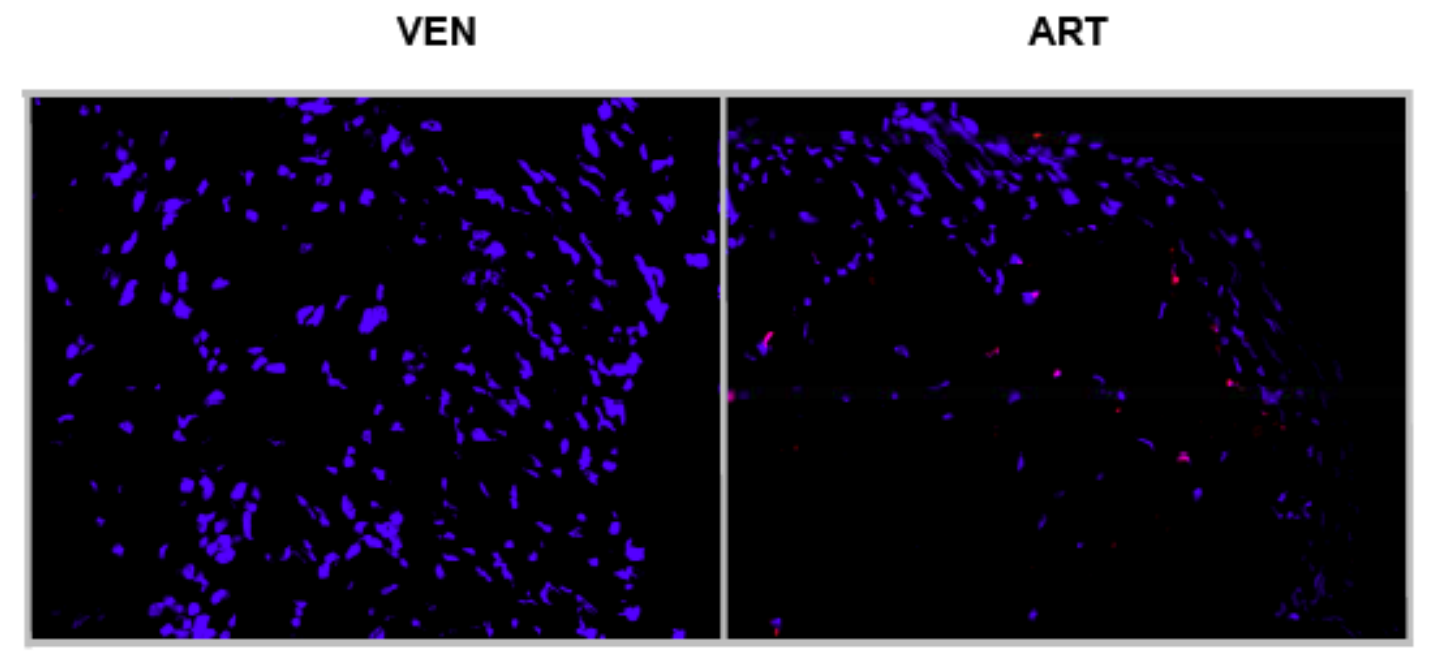

Figure F. 6 Representative TUNEL images from 24 hour VEN vs. ART experiment performed on 05/24/2006. Nuclei are stained blue and TUNEL positive cells are stained red.

VEN

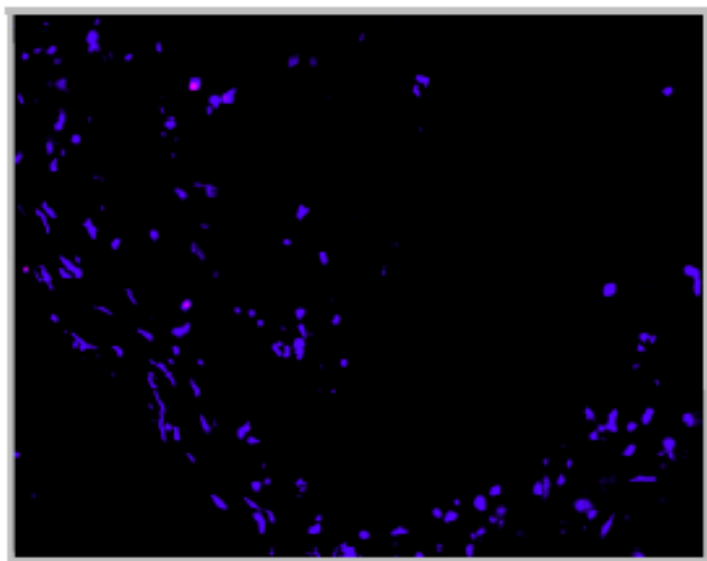

ART

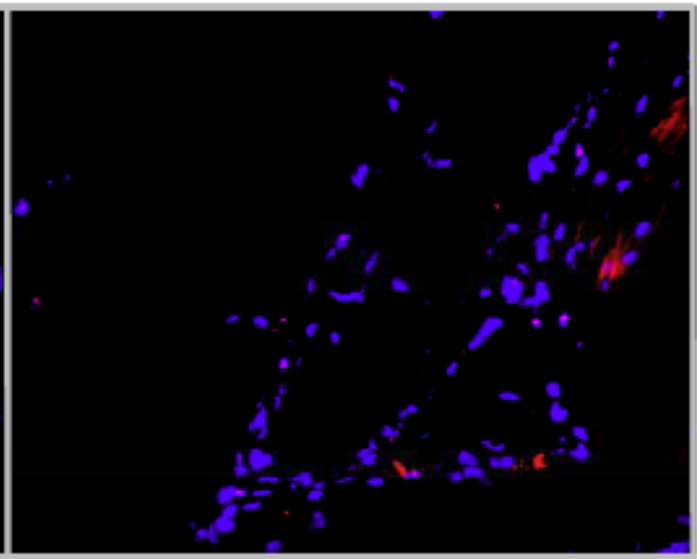

Figure F. 7 Representative TUNEL images from 24 hour VEN vs. ART experiment performed on 09/14/2006. Nuclei are stained blue and TUNEL positive cells are stained red. 


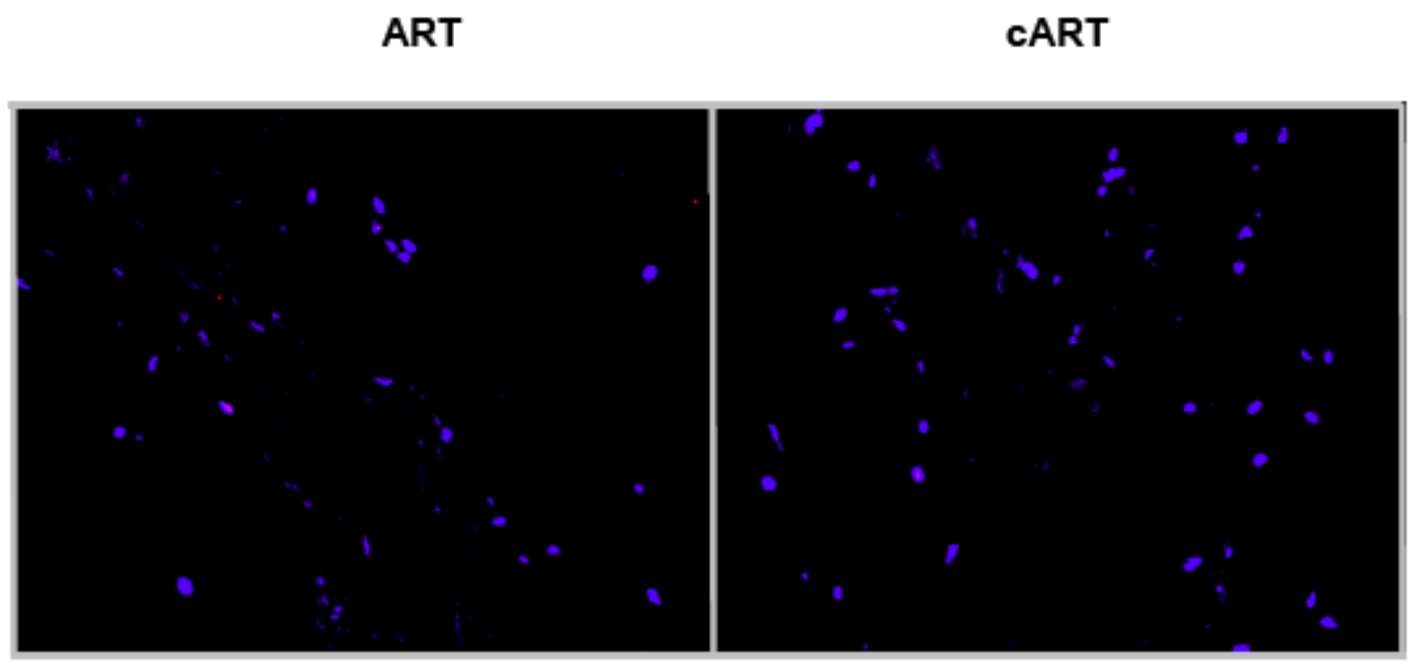

Figure F. 8 Representative TUNEL images from 24 hour ART vs. cART experiment performed on 10/22/2004. Nuclei are stained blue and TUNEL positive cells are stained red.

ART

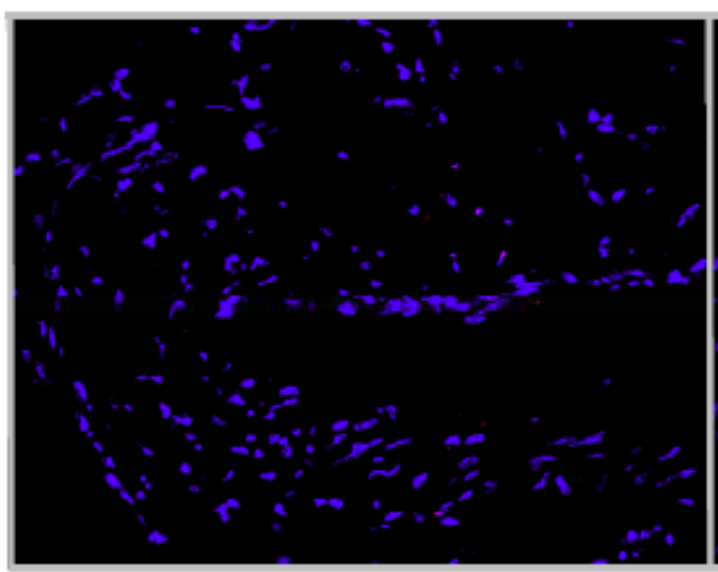

\section{cART}

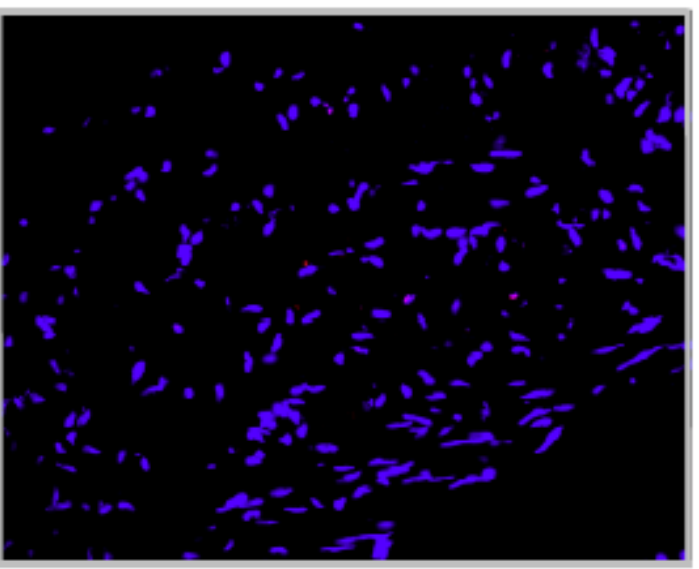

Figure F. 9 Representative TUNEL images from 24 hour ART vs. cART experiment performed on 11/01/2004. Nuclei are stained blue and TUNEL positive cells are stained red. 


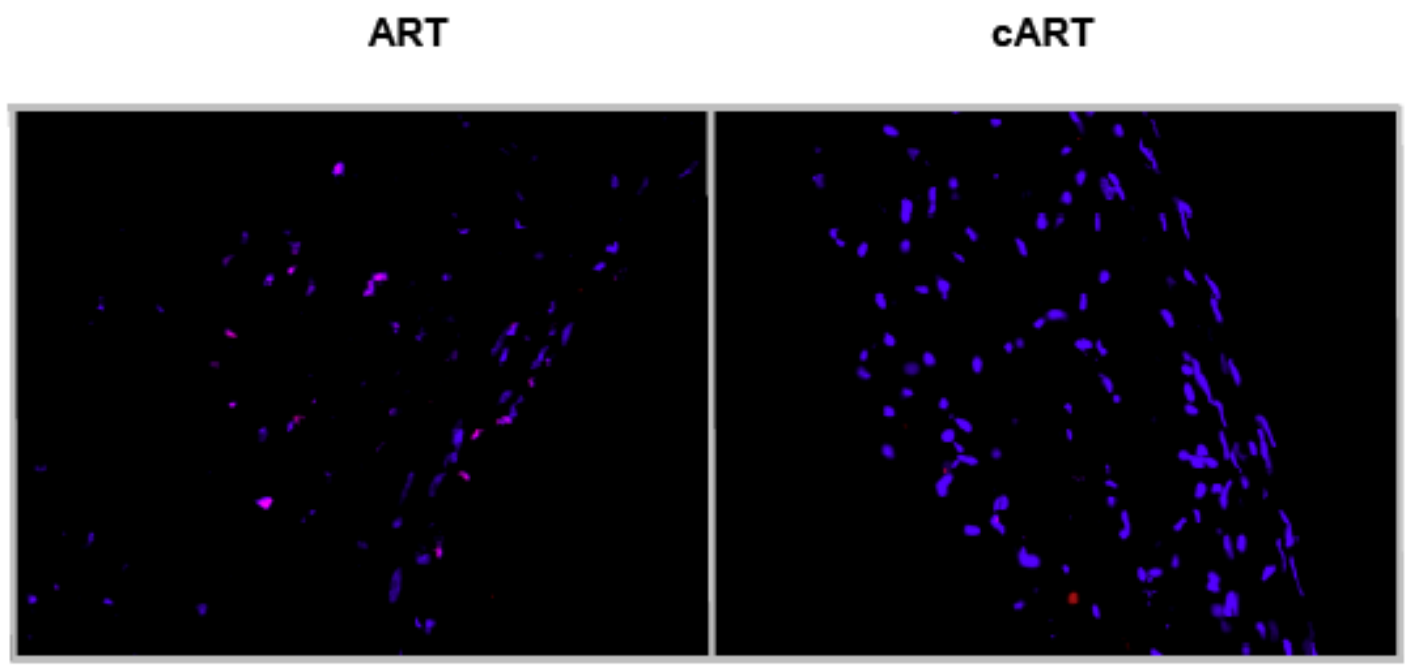

Figure F. 10 Representative TUNEL images from 24 hour ART vs. cART experiment performed on 11/11/2004. Nuclei are stained blue and TUNEL positive cells are stained red.

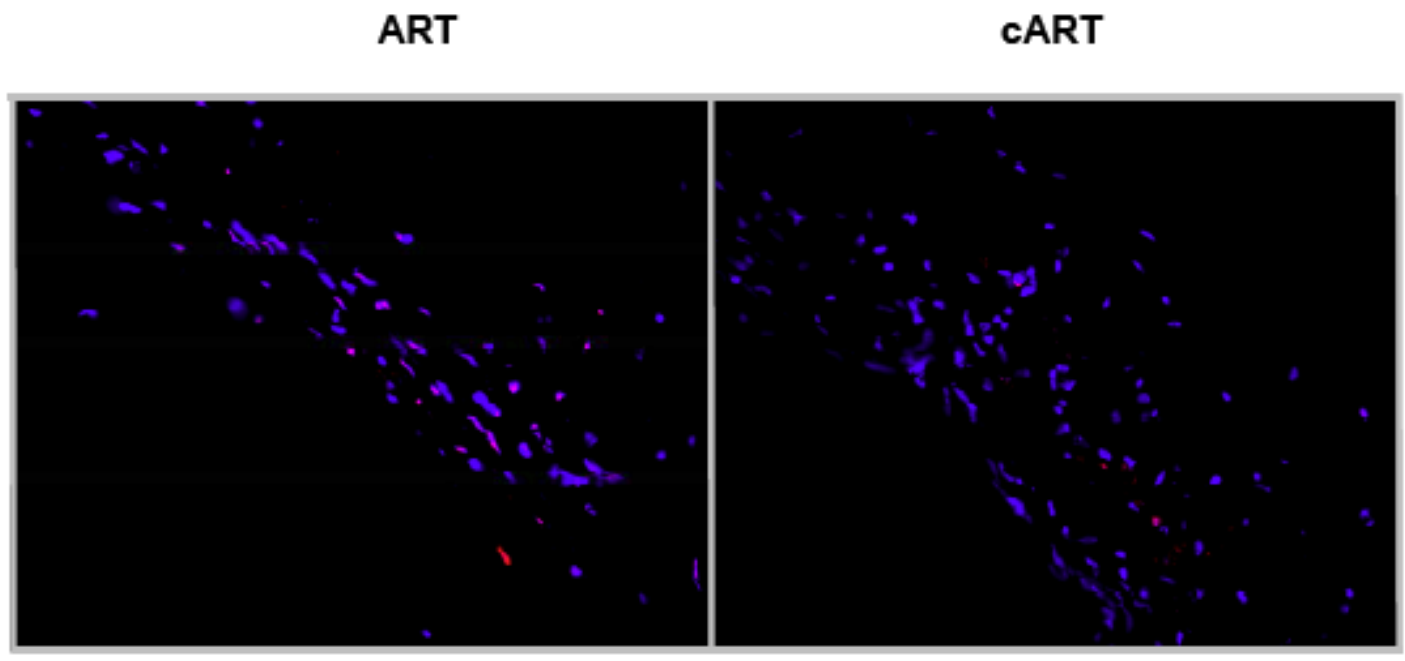

Figure F. 11 Representative TUNEL images from 24 hour ART vs. cART experiment performed on 09/21/2006. Nuclei are stained blue and TUNEL positive cells are stained red. 


\section{ART}

\section{CART}
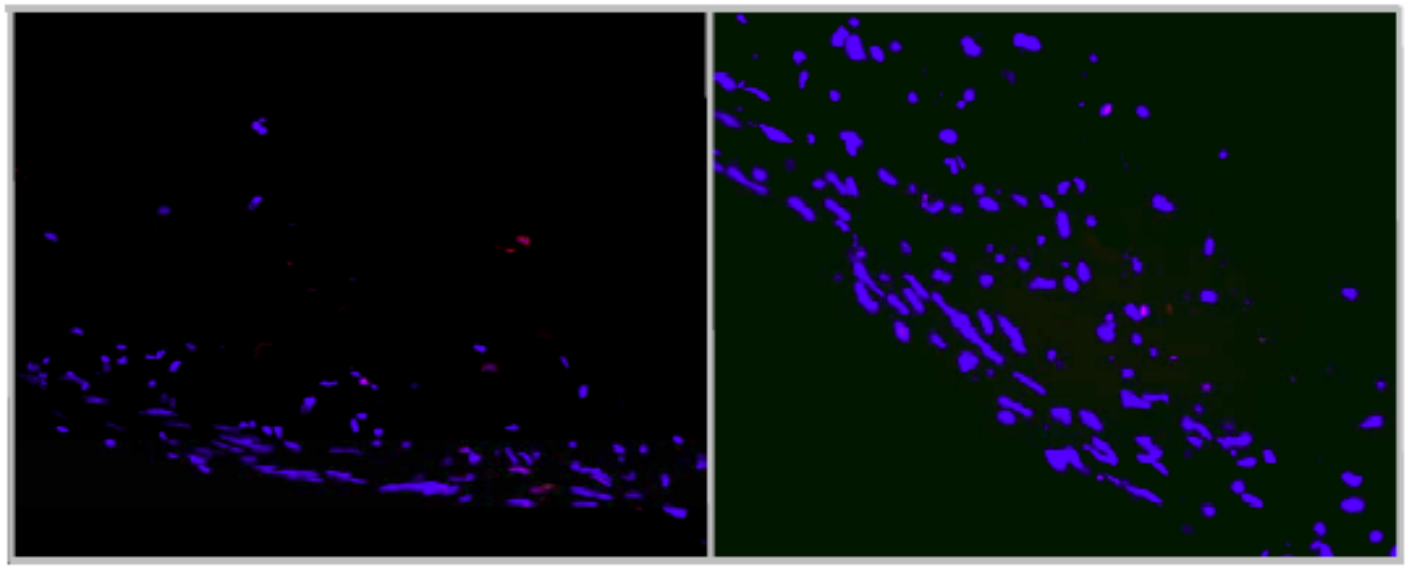

Figure F. 12 Representative TUNEL images from 24 hour ART vs. cART experiment performed on 10/16/2006. Nuclei are stained blue and TUNEL positive cells are stained red.

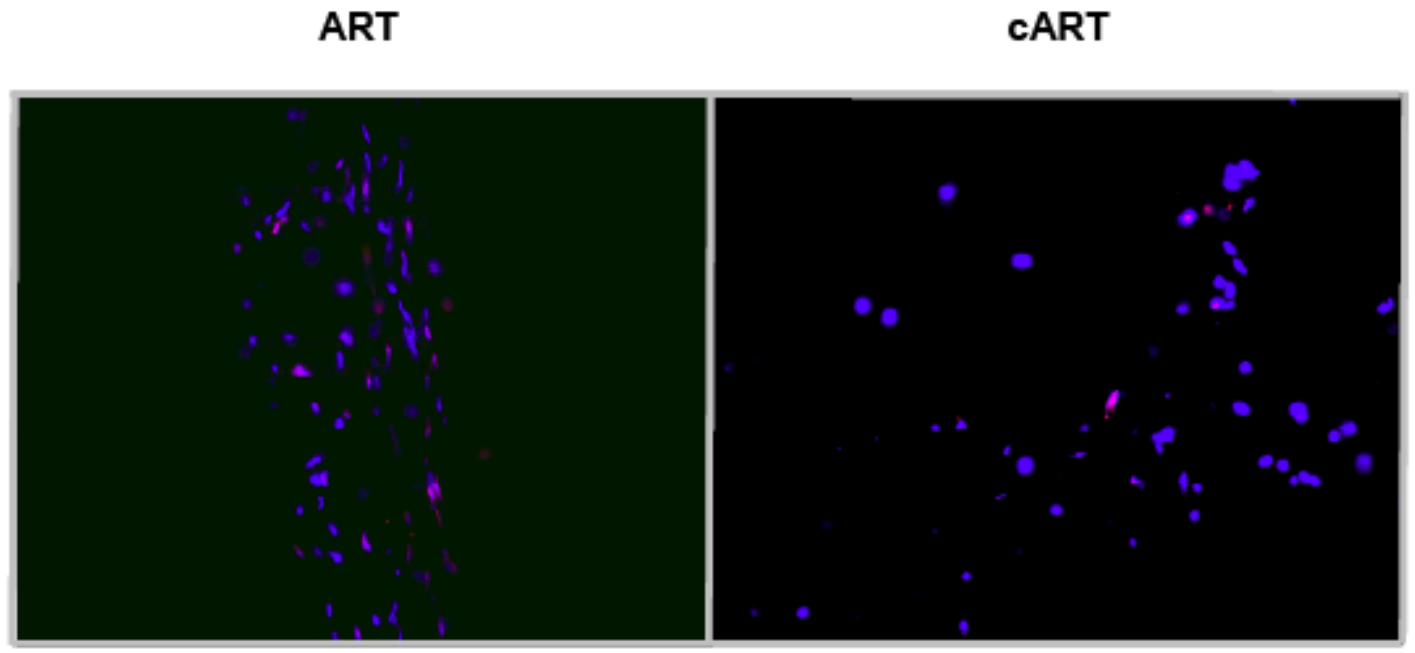

Figure F. 13 Representative TUNEL images from 72 hour ART vs. cART experiment performed on 02/16/2007. Nuclei are stained blue and TUNEL positive cells are stained red. 


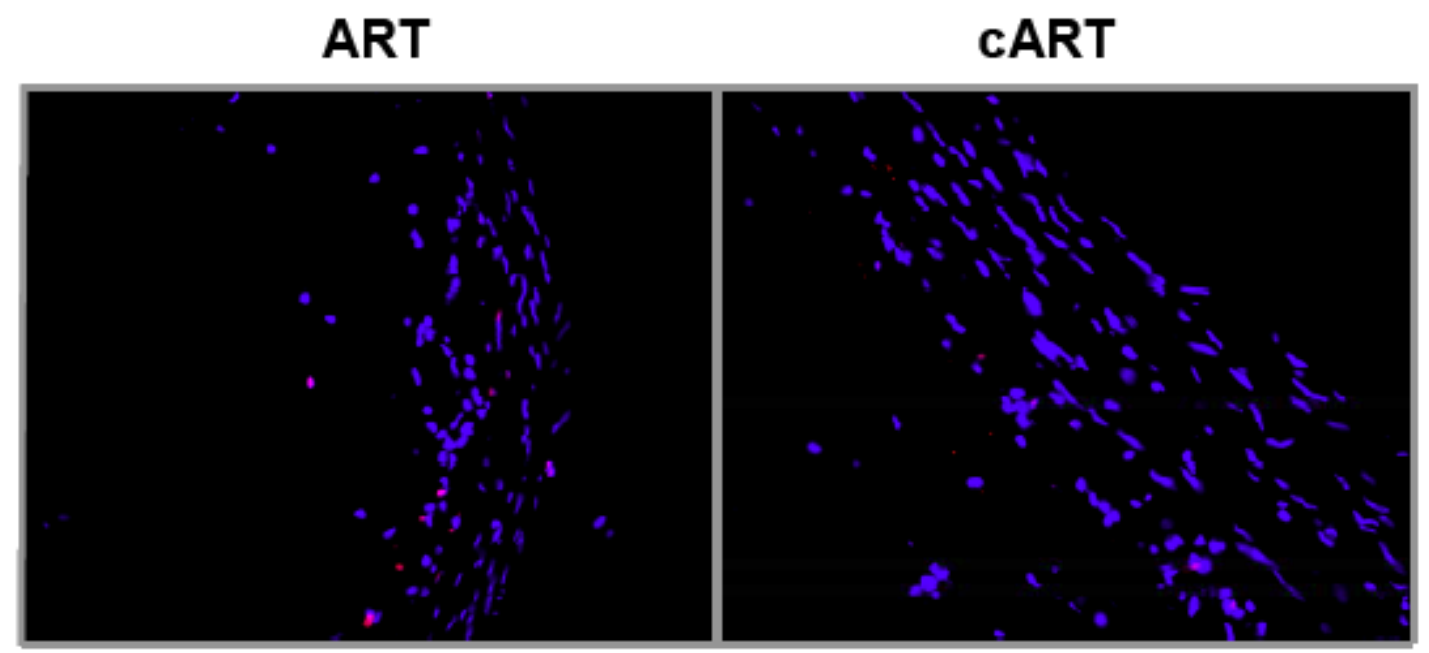

Figure F. 14 Representative TUNEL images from 72 hour ART vs. cART experiment performed on 03/07/2007. Nuclei are stained blue and TUNEL positive cells are stained red.

ART

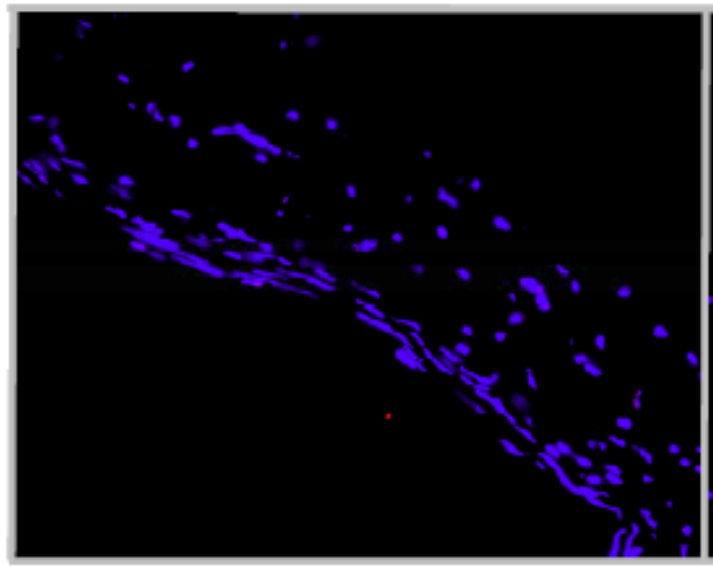

\section{cART}

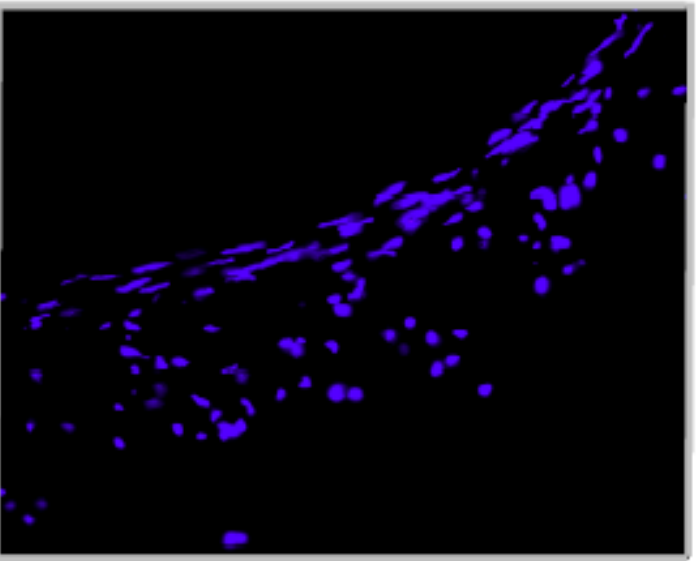

Figure F. 15 Representative TUNEL images from 72 hour ART vs. cART experiment performed on 03/15/2007. Nuclei are stained blue and TUNEL positive cells are stained red. 
ART

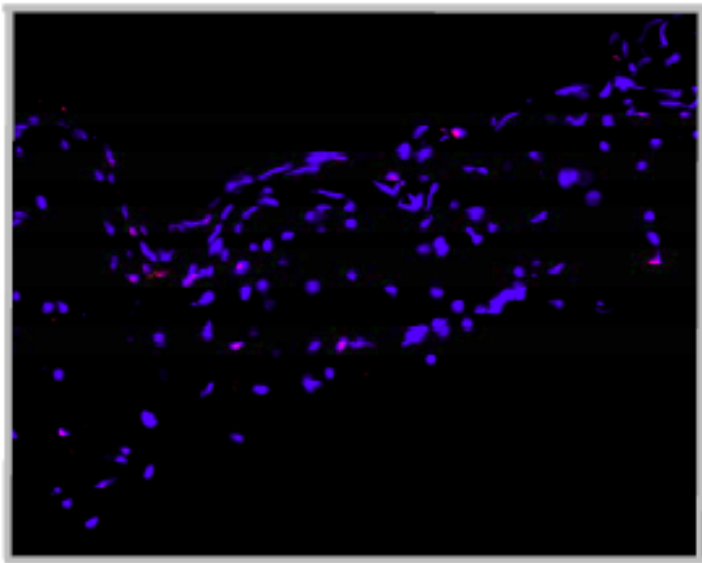

CART

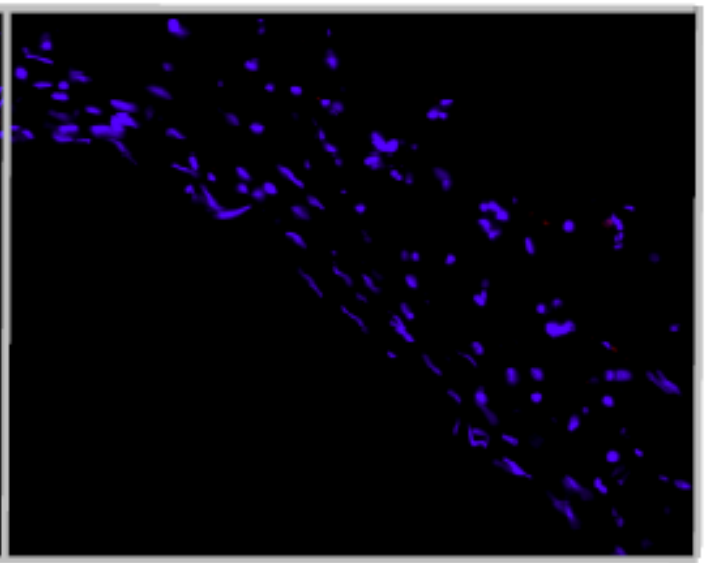

Figure F. 16 Representative TUNEL images from 72 hour ART vs. cART experiment performed on 04/02/2007. Nuclei are stained blue and TUNEL positive cells are stained red.

ART

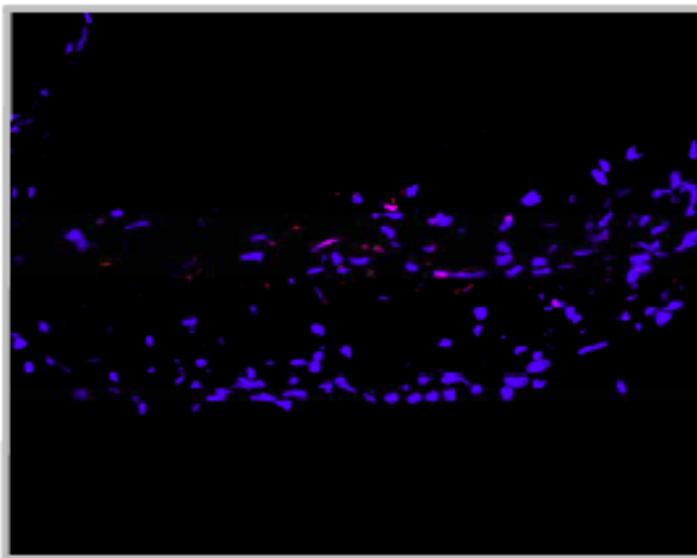

WART

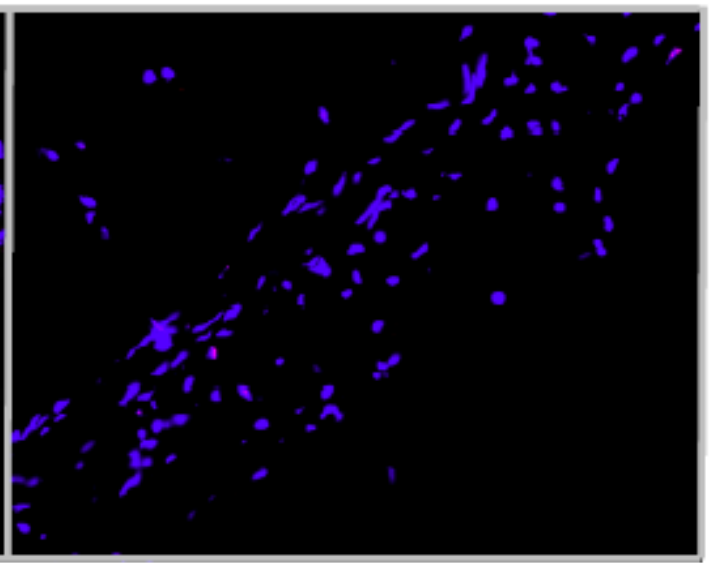

Figure F. 17 Representative TUNEL images from 72 hour ART vs. cART experiment performed on 04/26/2007. Nuclei are stained blue and TUNEL positive cells are stained red. 
ART

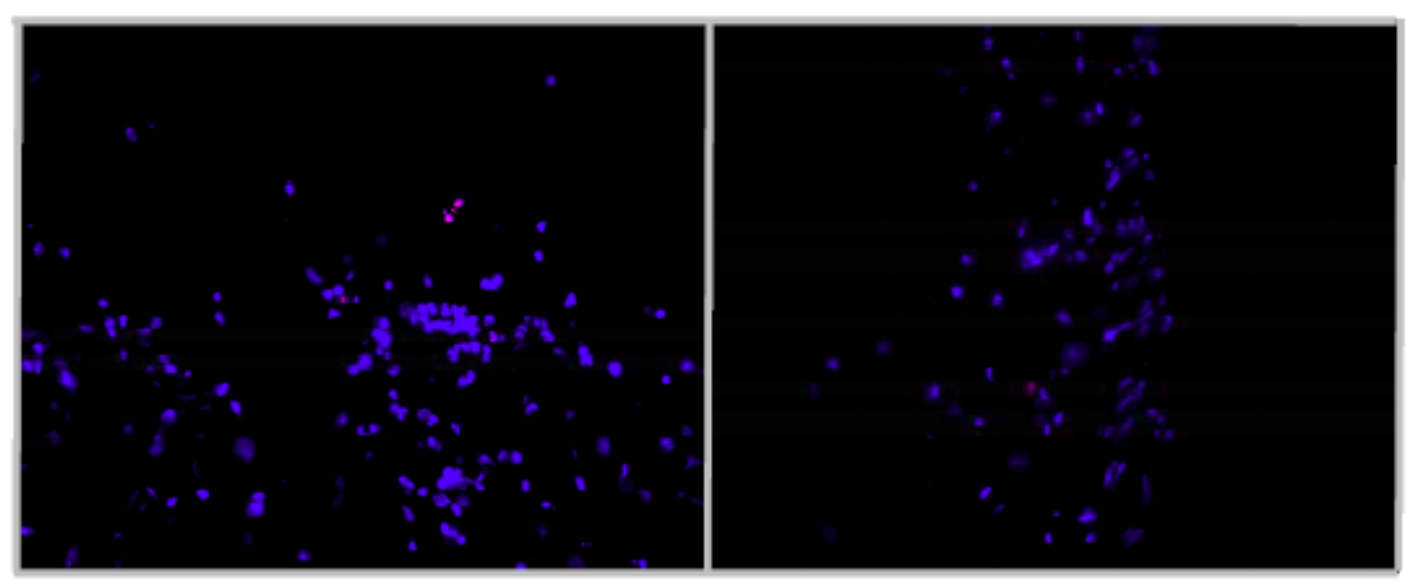

Figure F. 18 Representative TUNEL images from 24 hour ART vs. wART experiment performed on 10/23/2006. Nuclei are stained blue and TUNEL positive cells are stained red.

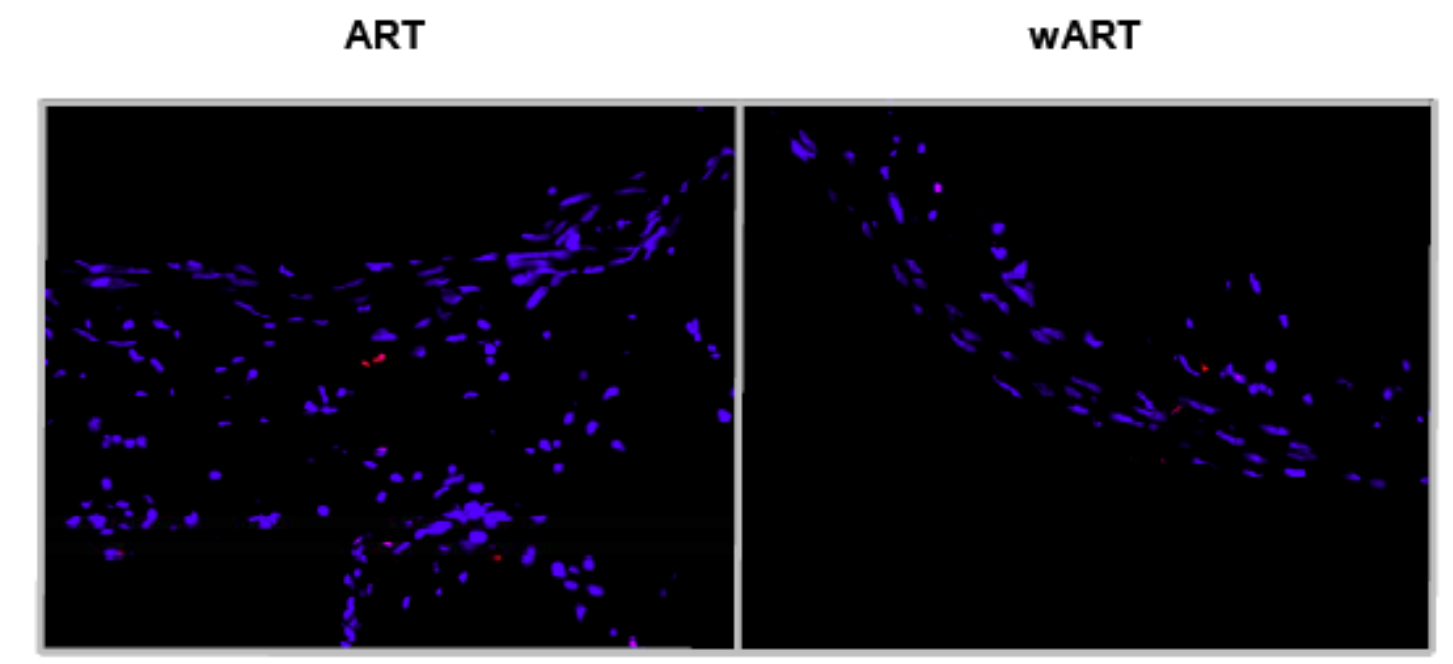

Figure F. 19 Representative TUNEL images from 24 hour ART vs. wART experiment performed on 10/30/2006. Nuclei are stained blue and TUNEL positive cells are stained red. 


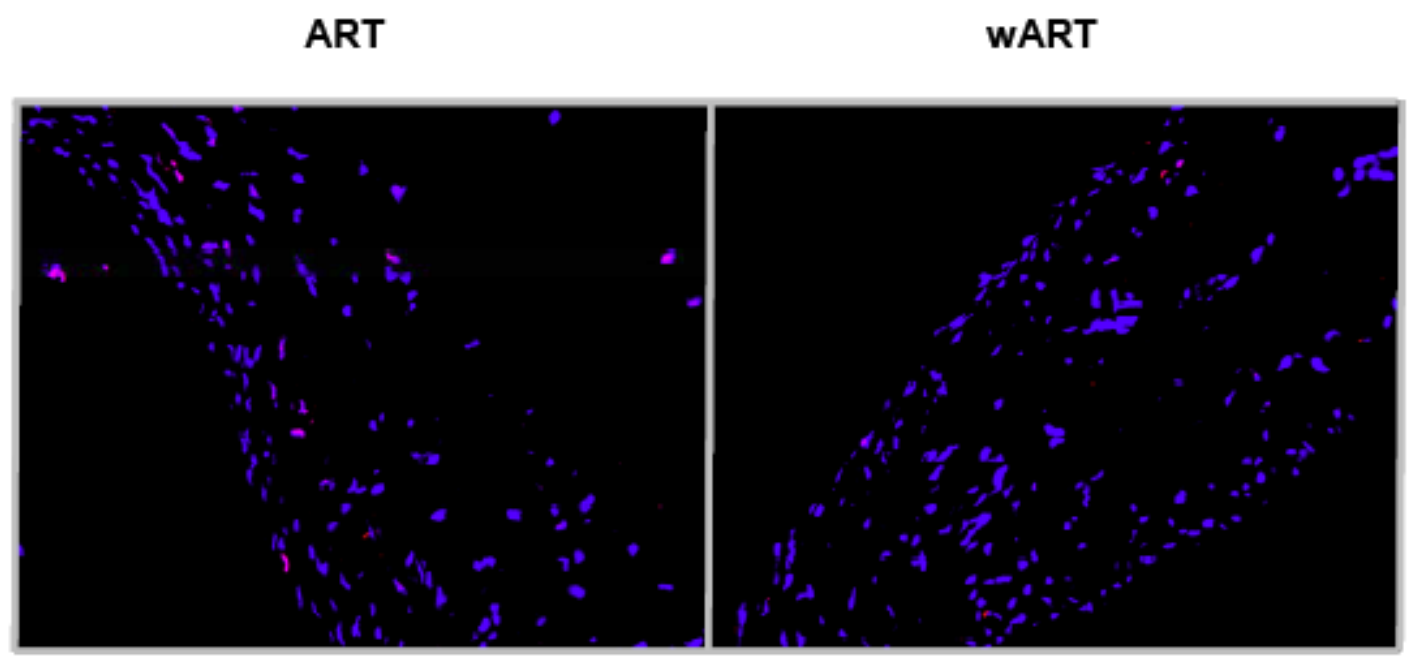

Figure F. 20 Representative TUNEL images from 24 hour ART vs. wART experiment performed on 11/06/2006. Nuclei are stained blue and TUNEL positive cells are stained red.

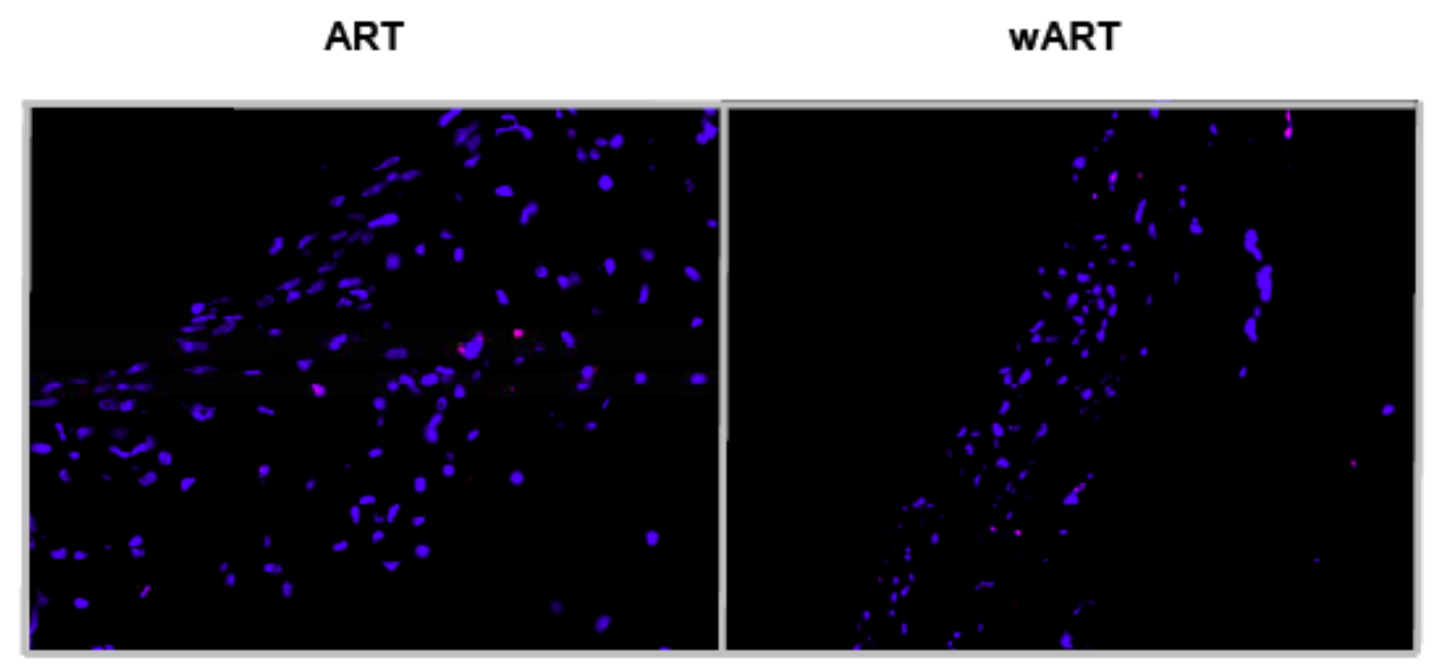

Figure F. 21 Representative TUNEL images from 24 hour ART vs. wART experiment performed on 11/13/2006. Nuclei are stained blue and TUNEL positive cells are stained red. 
ART

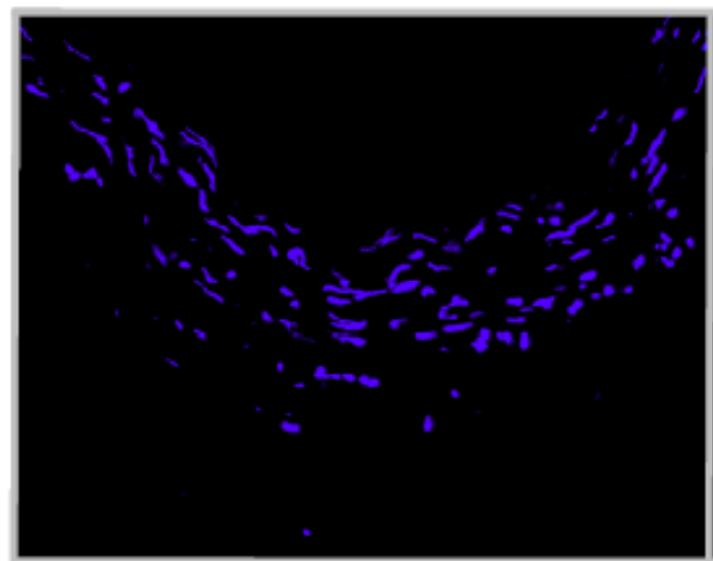

wART

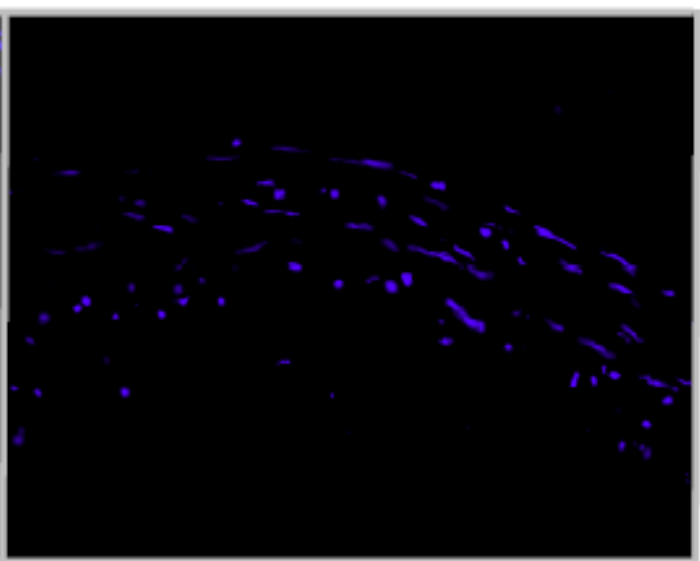

Figure F. 22 Representative TUNEL images from 24 hour ART vs. wART experiment performed on 11/20/2006. Nuclei are stained blue and TUNEL positive cells are stained red.

ART

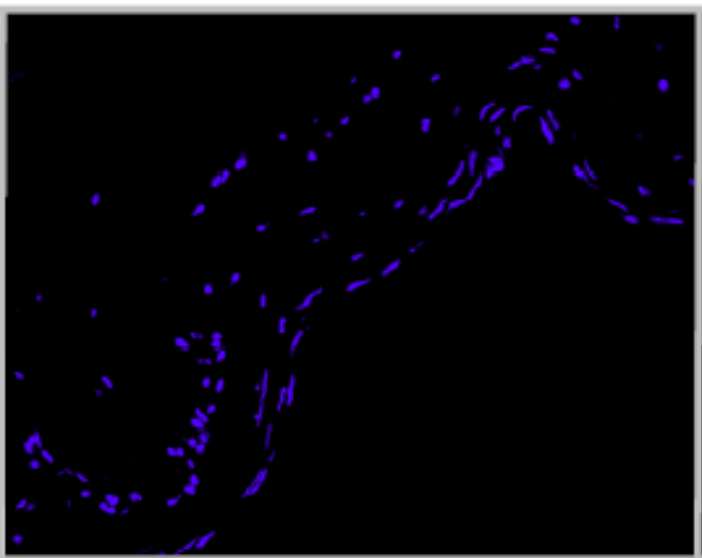

WART

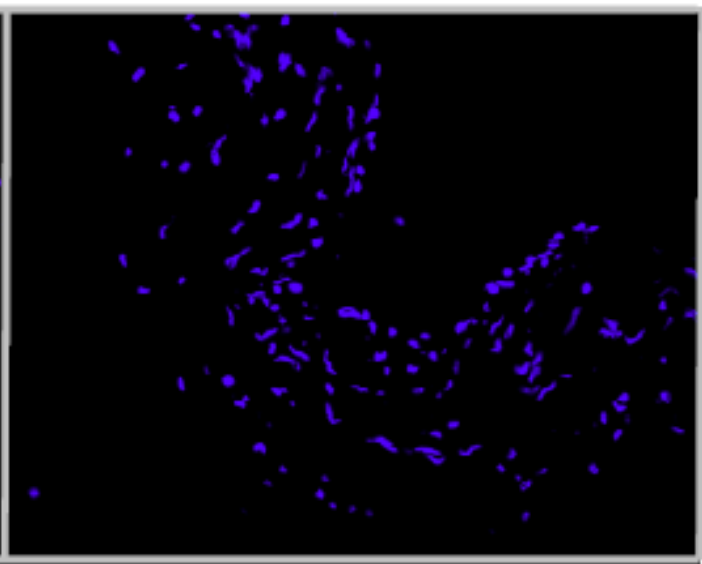

Figure F. 23 Representative TUNEL images from 24 hour ART vs. wART experiment performed on 11/27/2006. Nuclei are stained blue and TUNEL positive cells are stained red. 
Table F. 1 Mean values of percent TUNEL positive cells for all ex vivo experiments.

\begin{tabular}{ccccccccc} 
& \multicolumn{2}{c}{ VEN vs. ART 24 hrs } & \multicolumn{2}{c}{ ART vs. cART 24 hrs } & \multicolumn{2}{c}{ ART vs. cART 72 hrs } & \multicolumn{2}{c}{ ART vs. wART 24 hrs } \\
Expt \# & VEN & ART & ART & cART & ART & CART & ART & wART \\
$\mathbf{1}$ & 0.3756 & 0.1639 & 4.2805 & 0.0287 & 55.67992 & 26.82632 & 7.7482 & 5.5986 \\
$\mathbf{2}$ & 0 & 1.1875 & 8.3637 & 1.443 & 20.65321 & 6.5384 & 17.0628 & 3.8261 \\
$\mathbf{3}$ & 8.0367 & 17.1644 & 25.273 & 3.0815 & 7.6482 & 2.6909 & 21.1093 & 4.0694 \\
$\mathbf{4}$ & 3.3966 & 25.4254 & 30.8913 & 3.3698 & 17.6988 & 1.629 & 10.2532 & 5.6982 \\
$\mathbf{5}$ & 0 & 8.3566 & 10.8214 & 3.6655 & 2.1423 & 0.3762 & 0 & 0 \\
$\mathbf{6}$ & 1.9673 & 6.7687 & - & - & - & - & 0 & 0 \\
& & & & & & & & \\
mean & 2.296033 & 9.844417 & 15.92598 & 2.3177 & 20.76449 & 7.612163 & 9.36225 & 3.198717 \\
SE & 1.271563 & 3.986132 & 5.148736 & 0.689619 & 9.346981 & 4.912804 & 3.540217 & 1.058649
\end{tabular}

Table F. 2 Microsoft Excel output for student's t-test performed on the mean percent TUNEL positive cells from 24 hour VEN vs. ART ex vivo experiments.

t-Test: Paired Two Sample for Means

\begin{tabular}{lrr}
\hline & VEN & \multicolumn{1}{c}{ ART } \\
\hline Mean & 2.296033 & 9.844417 \\
Variance & 9.701234 & 95.33549 \\
Observations & 6 & 6 \\
Pearson Correlation & 0.671215 & \\
Hypothesized Mean Difference & 0 & \\
df & 5 & \\
t Stat & -2.30741 & \\
$\mathrm{P}(T<=t)$ one-tail & 0.034565 & \\
t Critical one-tail & 2.015048 & \\
$\mathrm{P}(T<=t)$ two-tail & 0.06913 & \\
t Critical two-tail & 2.570582 & \\
\hline
\end{tabular}


Table F. 3 Microsoft Excel output for student's t-test performed on the mean percent TUNEL positive cells from 24 hour ART vs. cART ex vivo experiments.

t-Test: Paired Two Sample for Means

\begin{tabular}{lrr}
\hline & \multicolumn{1}{c}{ ART } & \multicolumn{1}{c}{ cART } \\
\hline Mean & 15.92598 & 2.3177 \\
Variance & 132.5474 & 2.377873 \\
Observations & 5 & 5 \\
Pearson Correlation & 0.693891 & \\
Hypothesized Mean Difference & 0 & \\
df & 4 & \\
t Stat & 2.897511 & \\
P $(T<=t)$ one-tail & 0.022114 & \\
t Critical one-tail & 2.131847 & \\
$\mathrm{P}(T<=t)$ two-tail & 0.044228 & \\
t Critical two-tail & 2.776445 & \\
\hline
\end{tabular}

Table F. 4 Microsoft Excel output for student's t-test performed on the mean percent TUNEL positive cells from 72 hour ART vs. cART ex vivo experiments.

t-Test: Paired Two Sample for Means

\begin{tabular}{lrr}
\hline & \multicolumn{1}{c}{ ART } & \multicolumn{1}{c}{ cART } \\
\hline Mean & 20.76449 & 7.612163 \\
Variance & 436.8303 & 120.6782 \\
Observations & 5 & 5 \\
Pearson Correlation & 0.967588 & \\
Hypothesized Mean Difference & 0 & \\
df & 4 & \\
t Stat & 2.76425 & \\
P $(T<=t)$ one-tail & 0.025314 & \\
t Critical one-tail & 2.131847 & \\
$P(T<=t)$ two-tail & 0.050628 & \\
t Critical two-tail & 2.776445 & \\
\hline
\end{tabular}


Table F. 5 Microsoft Excel output for student's t-test performed on the mean percent TUNEL positive cells from 24 hour ART vs. WART ex vivo experiments.

t-Test: Paired Two Sample for Means

\begin{tabular}{lrr}
\hline & \multicolumn{1}{c}{$A R T$} & WART \\
\hline Mean & 9.36225 & 3.198717 \\
Variance & 75.19881 & 6.724427 \\
Observations & 6 & 6 \\
Pearson Correlation & 0.651991 & \\
Hypothesized Mean Difference & \multicolumn{2}{c}{0} \\
df & 5 & \\
t Stat & 2.081664 & \\
$\mathrm{P}(T<=\mathrm{t})$ one-tail & 0.045934 & \\
t Critical one-tail & 2.015048 & \\
$\mathrm{P}(\mathrm{T}<=\mathrm{t})$ two-tail & 0.091868 & \\
$\mathrm{t}$ Critical two-tail & 2.570582 & \\
\hline
\end{tabular}




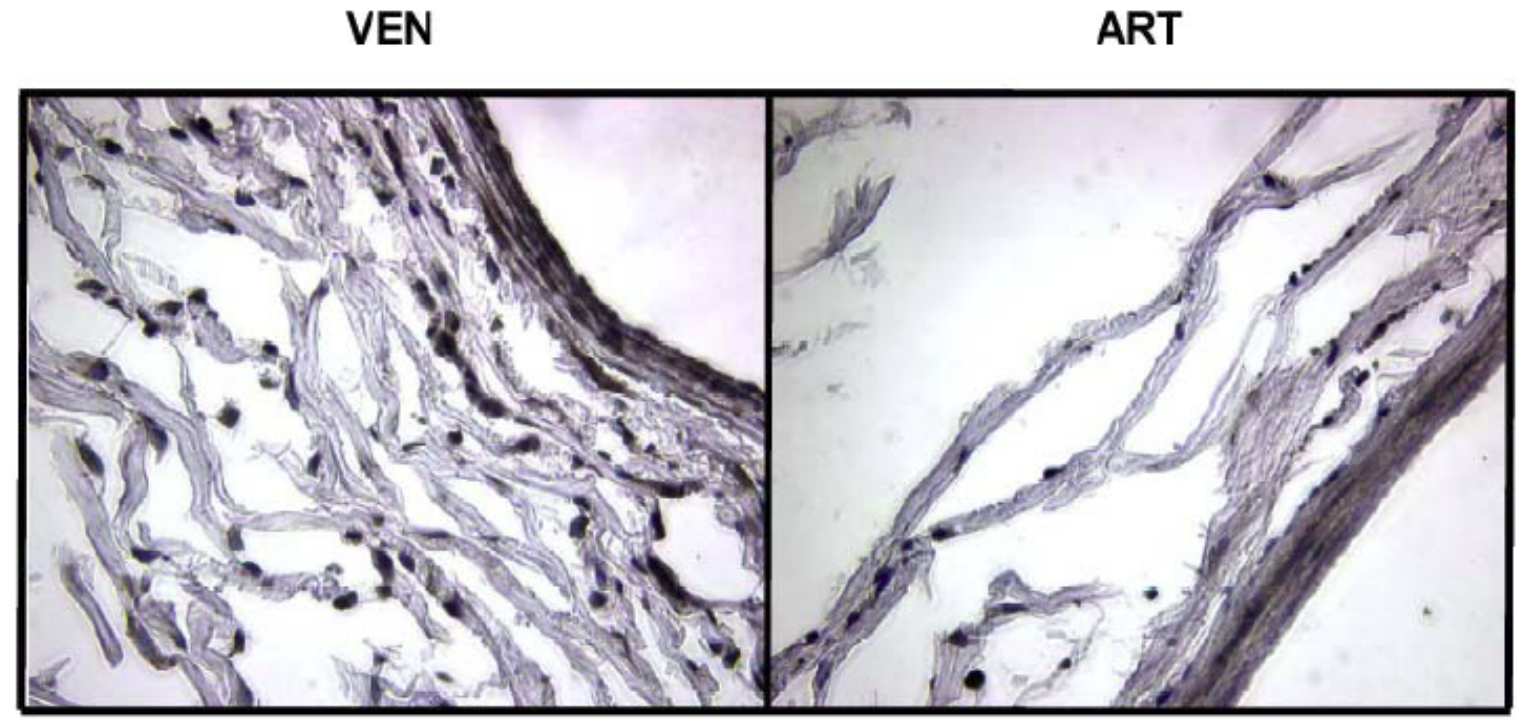

Figure F. 24 Representative PCNA images from 24 hour VEN vs. ART experiment performed on 04/23/2003. Nuclei are stained blue and PCNA positive cells are stained brown.

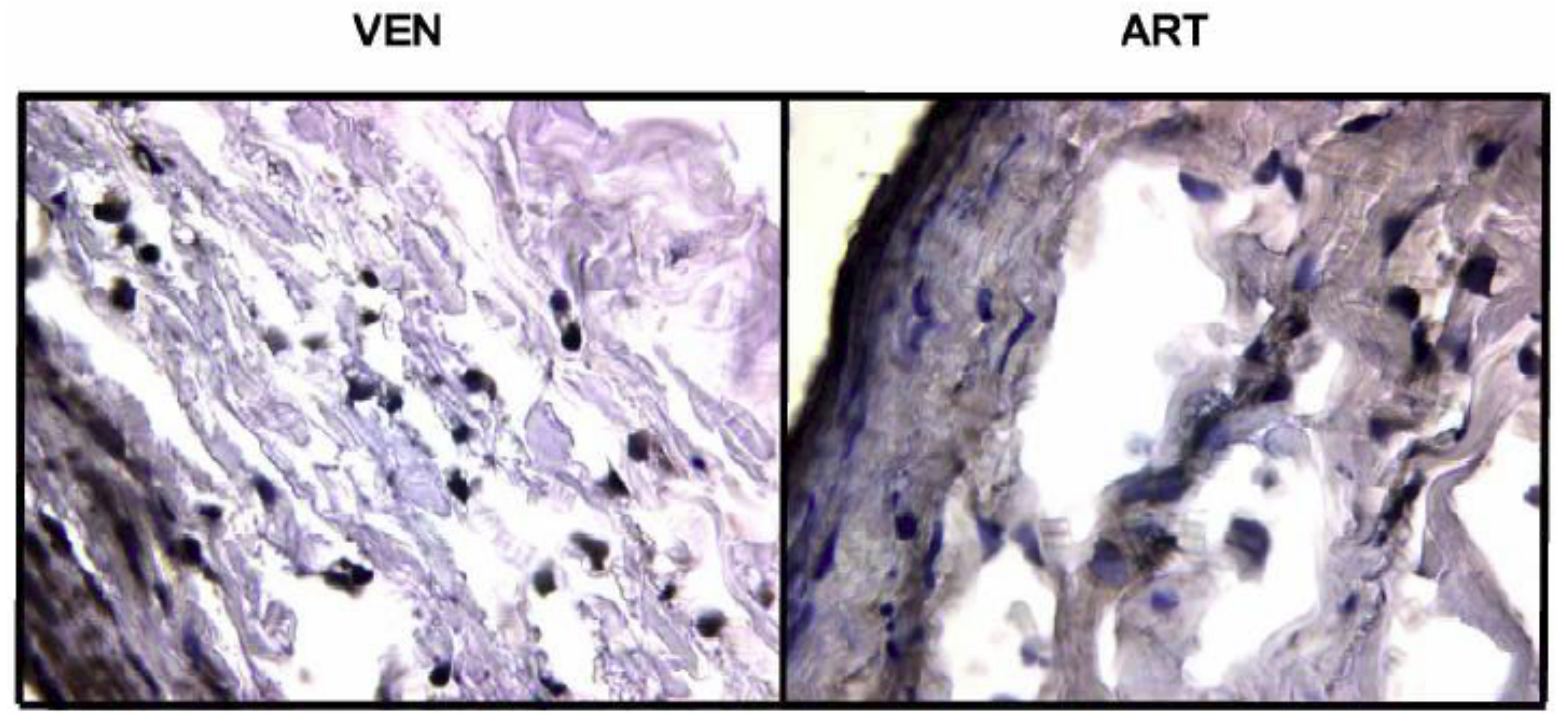

Figure F. 25 Representative PCNA images from 24 hour VEN vs. ART experiment performed on 05/07/2003. Nuclei are stained blue and PCNA positive cells are stained brown. 


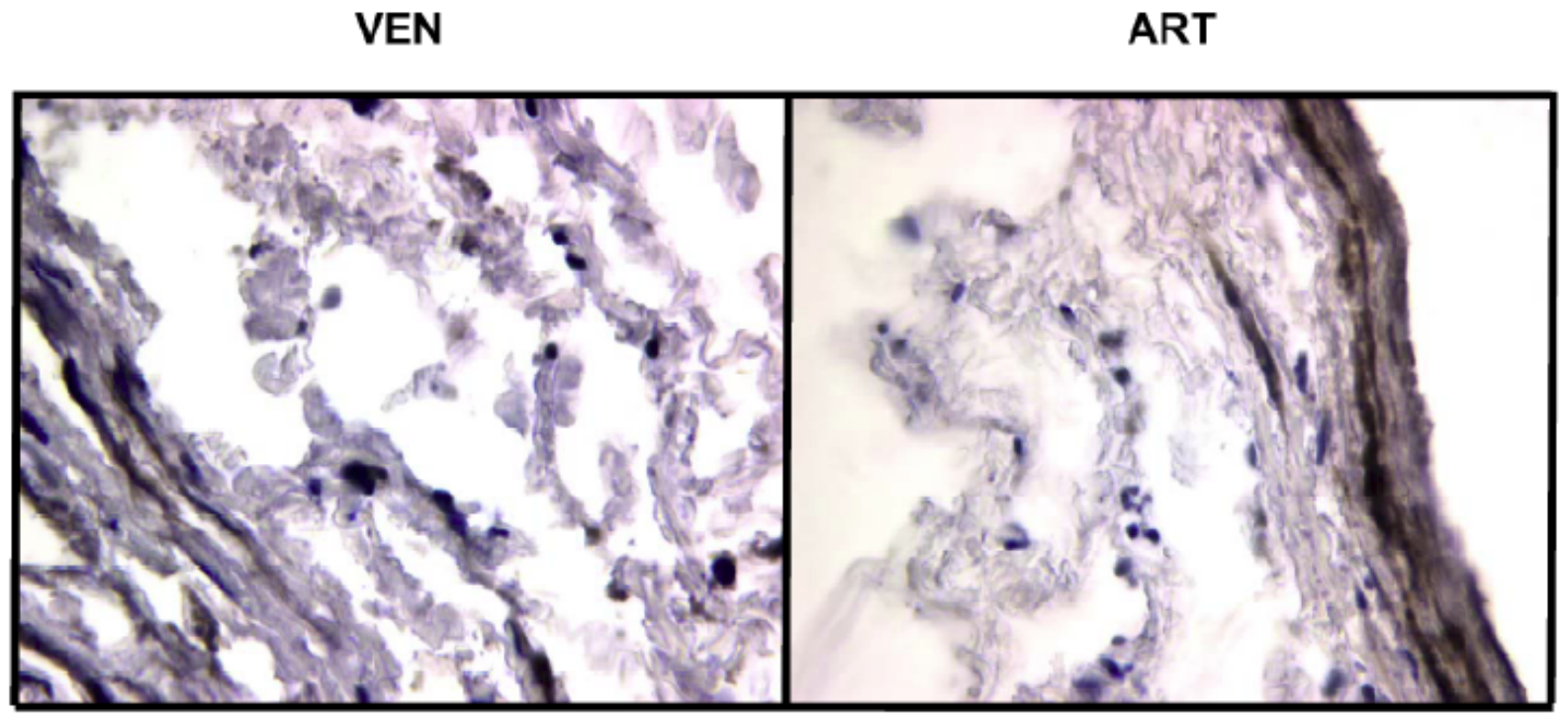

Figure F. 26 Representative PCNA images from 24 hour VEN vs. ART experiment performed on 10/09/2004. Nuclei are stained blue and PCNA positive cells are stained brown.

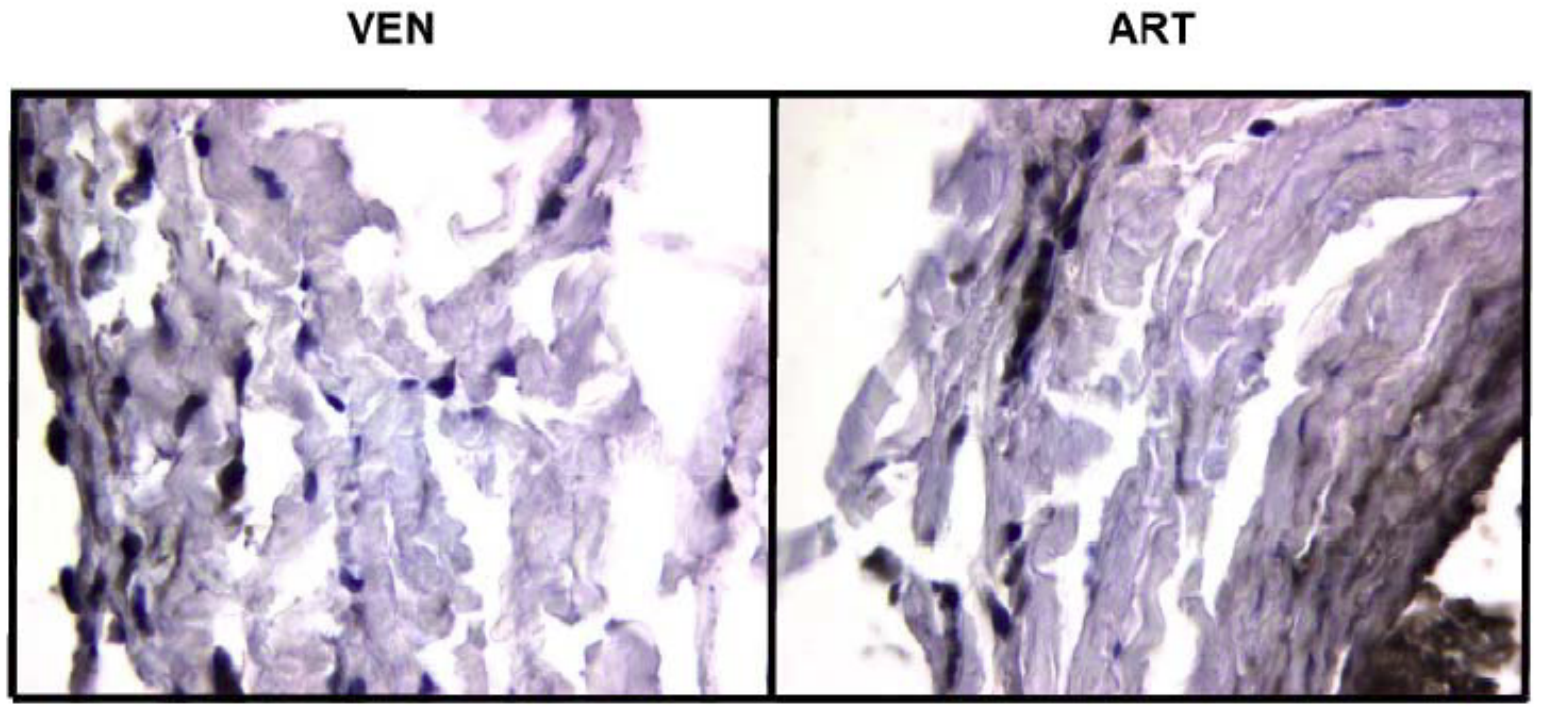

Figure F. 27 Representative PCNA images from 24 hour VEN vs. ART experiment performed on 10/16/2004. Nuclei are stained blue and PCNA positive cells are stained brown. 


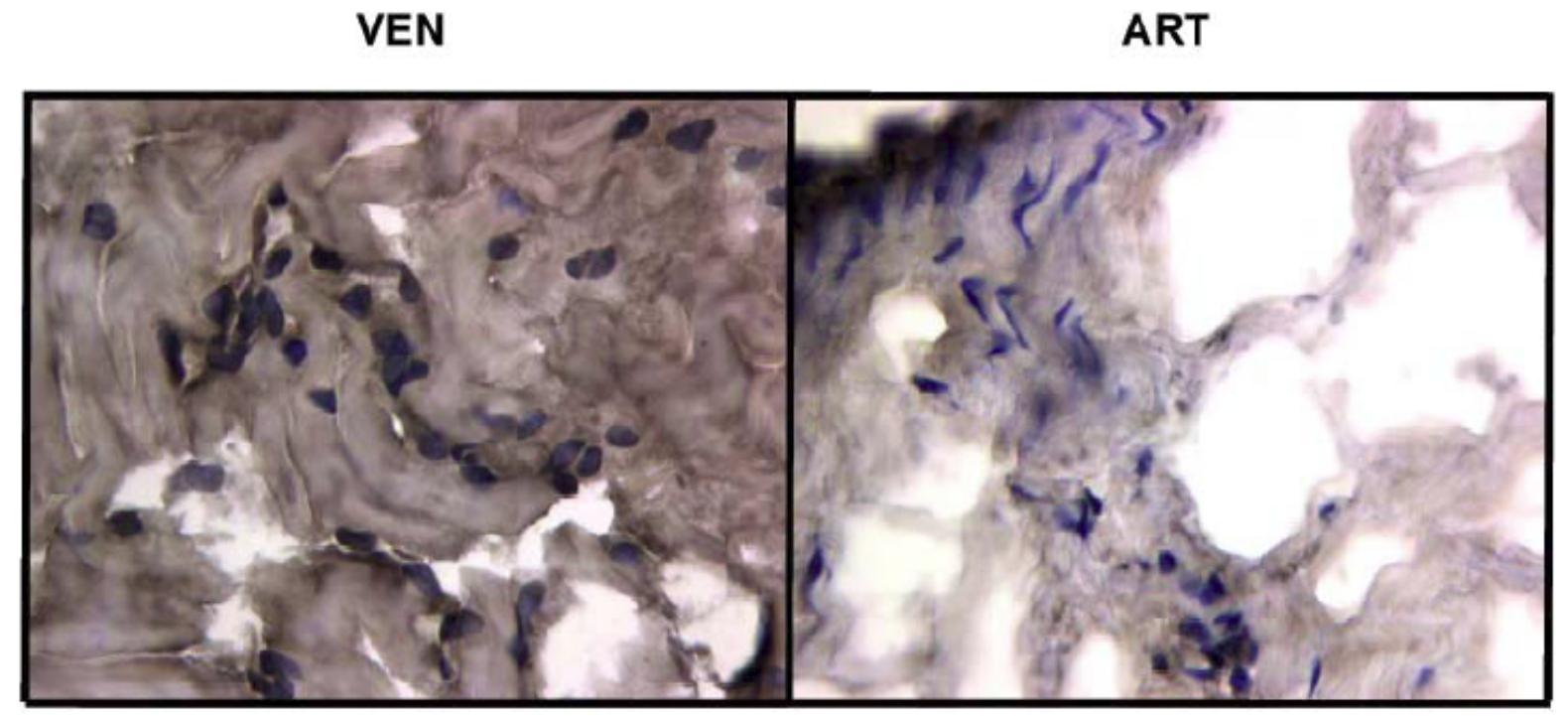

Figure F. 28 Representative PCNA images from 24 hour VEN vs. ART experiment performed on 05/24/2006. Nuclei are stained blue and PCNA positive cells are stained brown.

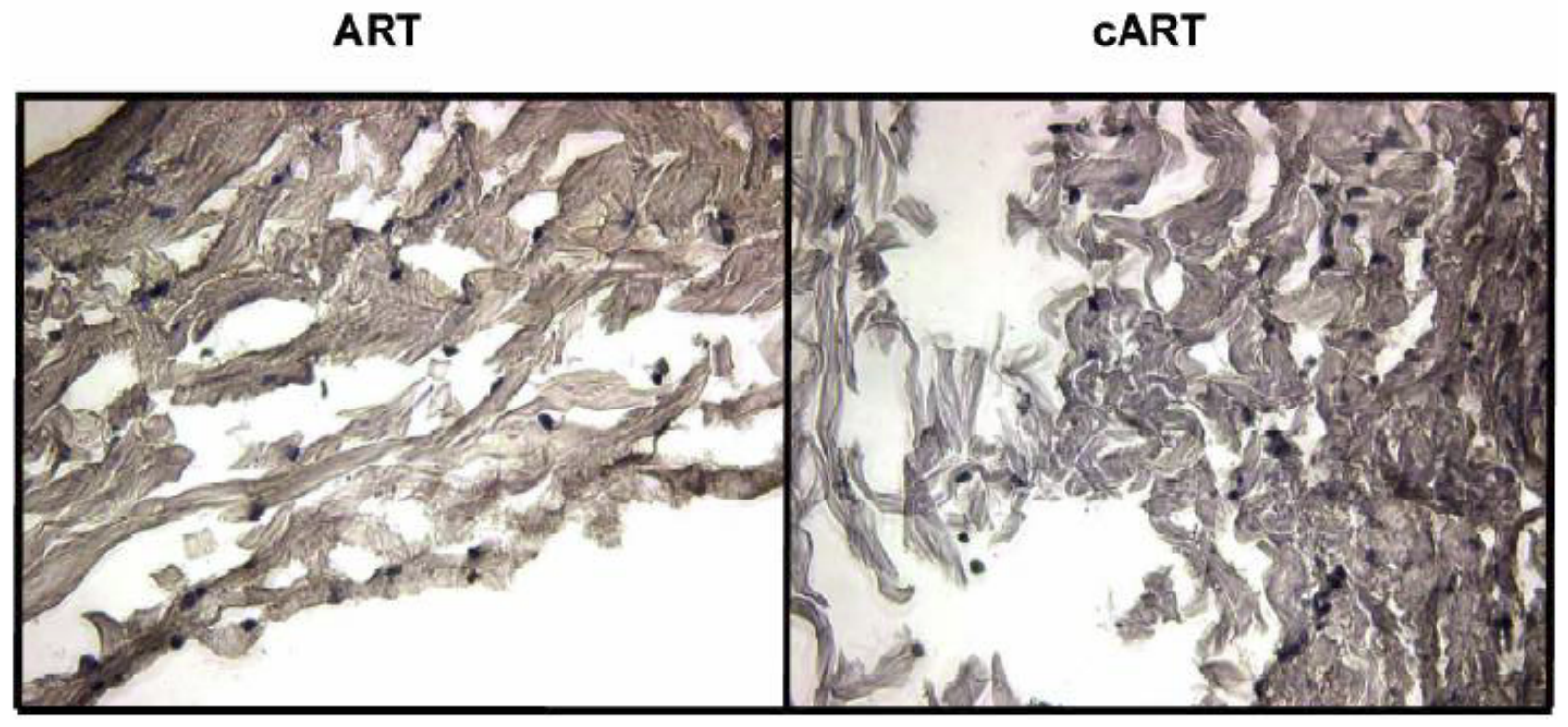

Figure F. 29 Representative PCNA images from 24 hour VEN vs. ART experiment performed on 09/14/2006. Nuclei are stained blue and PCNA positive cells are stained brown. 


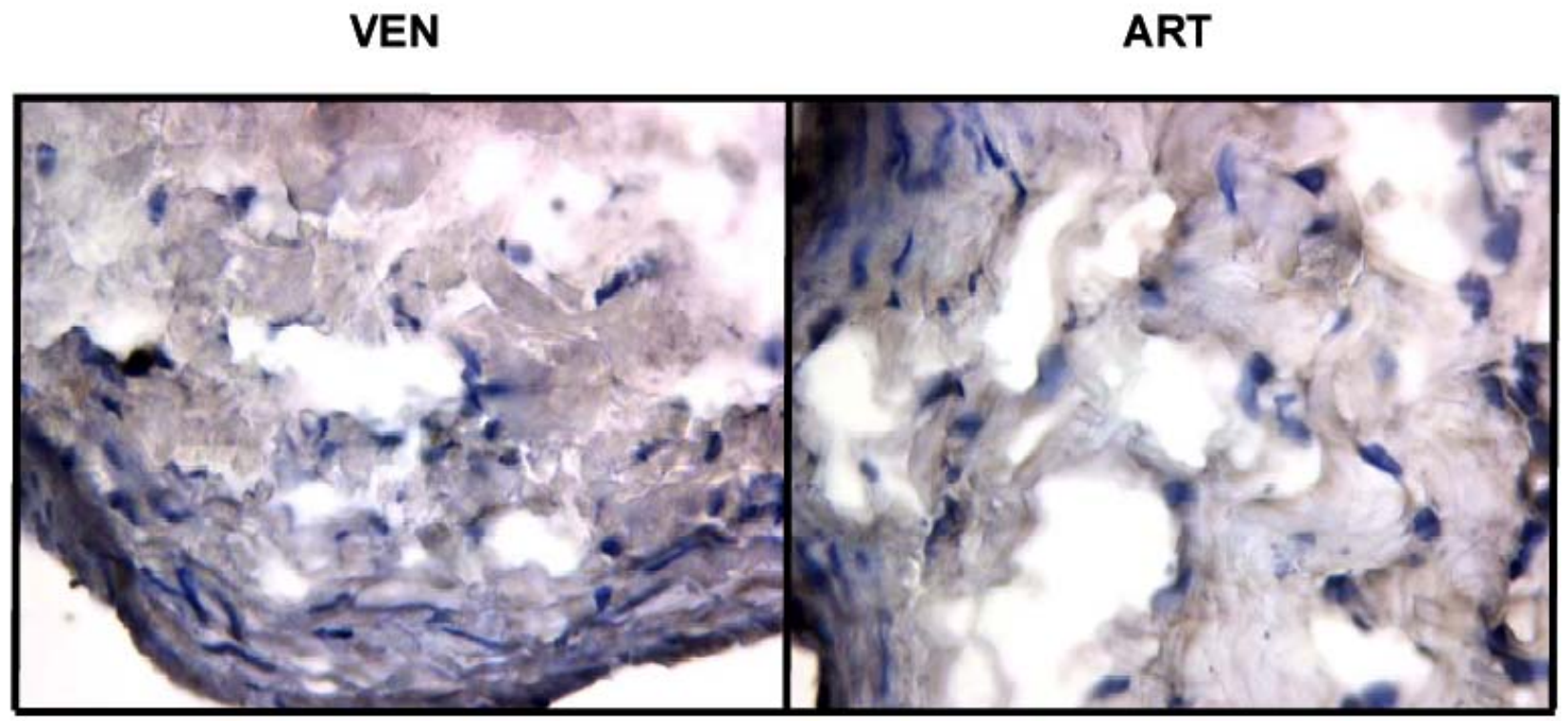

Figure F. 30 Representative PCNA images from 24 hour ART vs. cART experiment performed on 10/22/2004. Nuclei are stained blue and PCNA positive cells are stained brown.

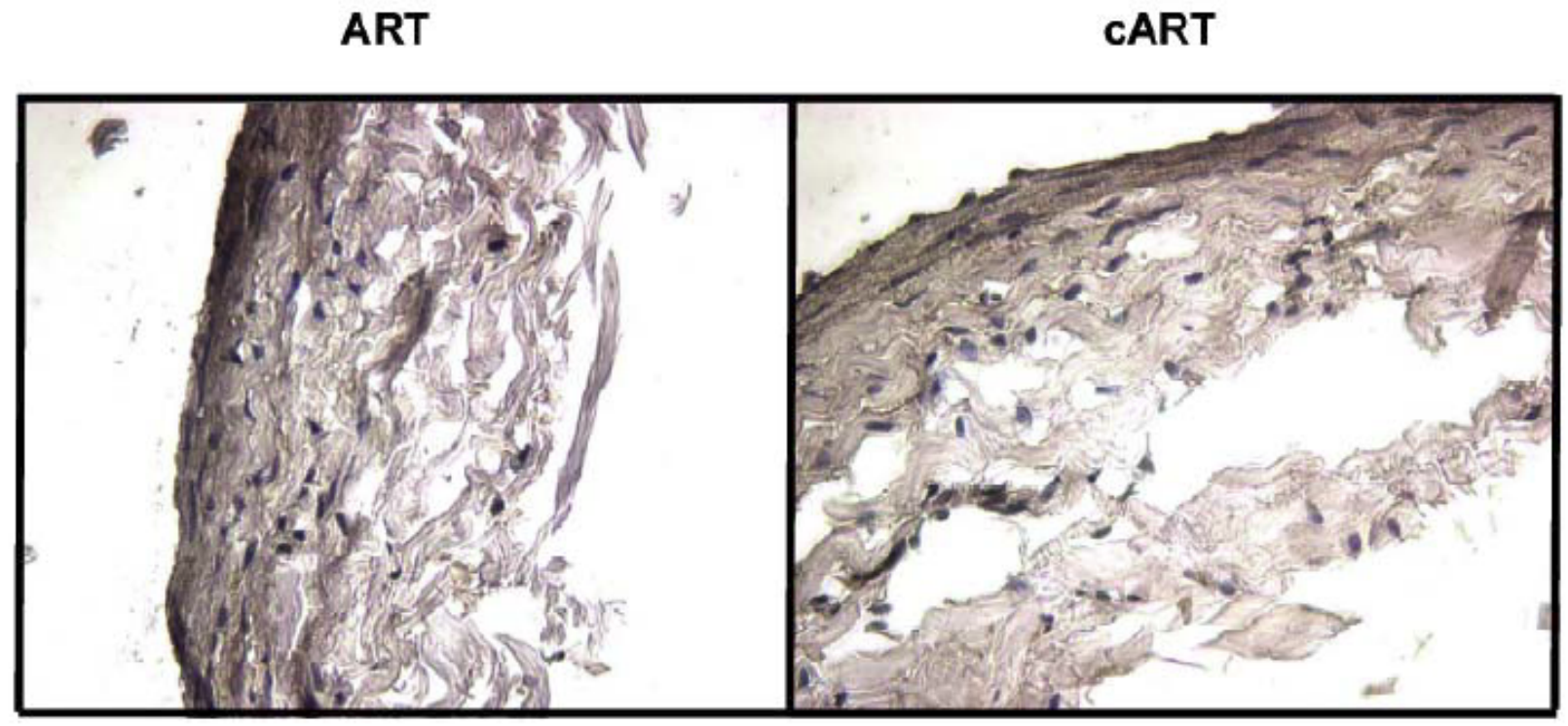

Figure F. 31 Representative PCNA images from 24 hour ART vs. cART experiment performed on 11/01/2004. Nuclei are stained blue and PCNA positive cells are stained brown. 


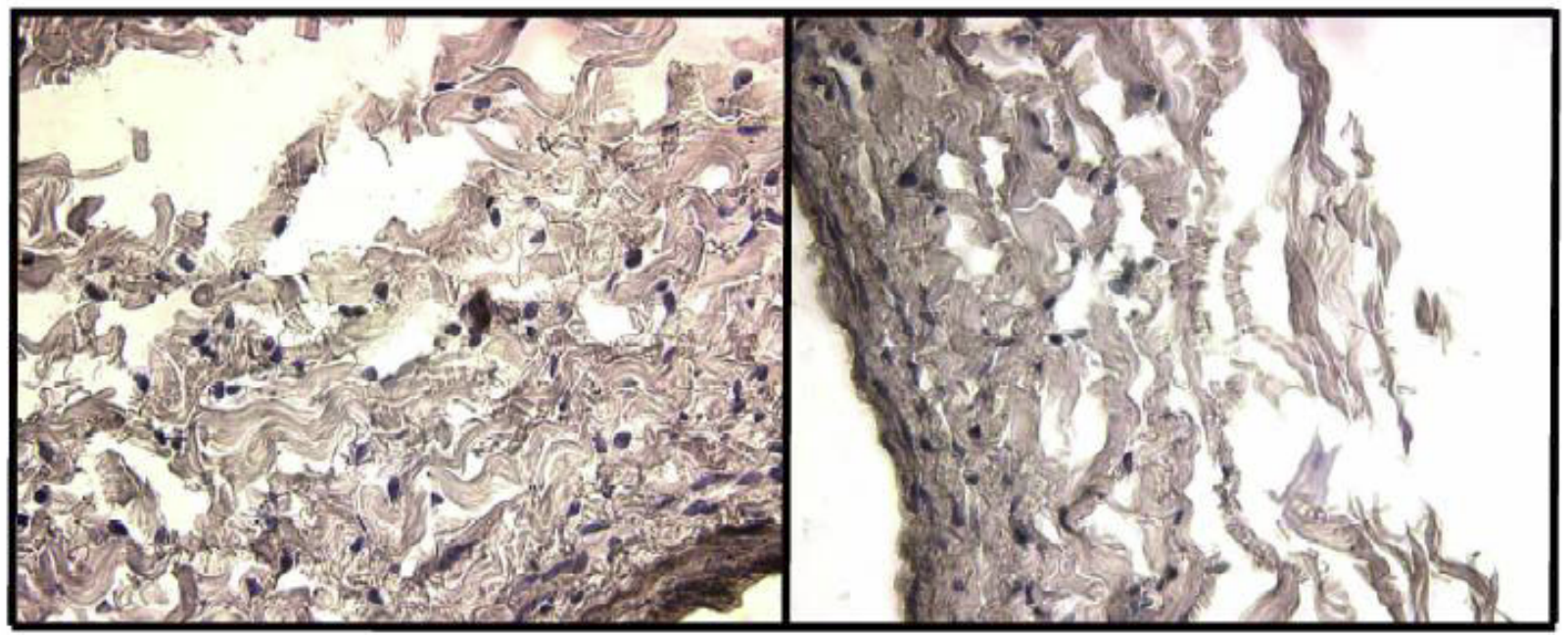

Figure F. 32 Representative PCNA images from 24 hour ART vs. cART experiment performed on 11/11/2004. Nuclei are stained blue and PCNA positive cells are stained brown.

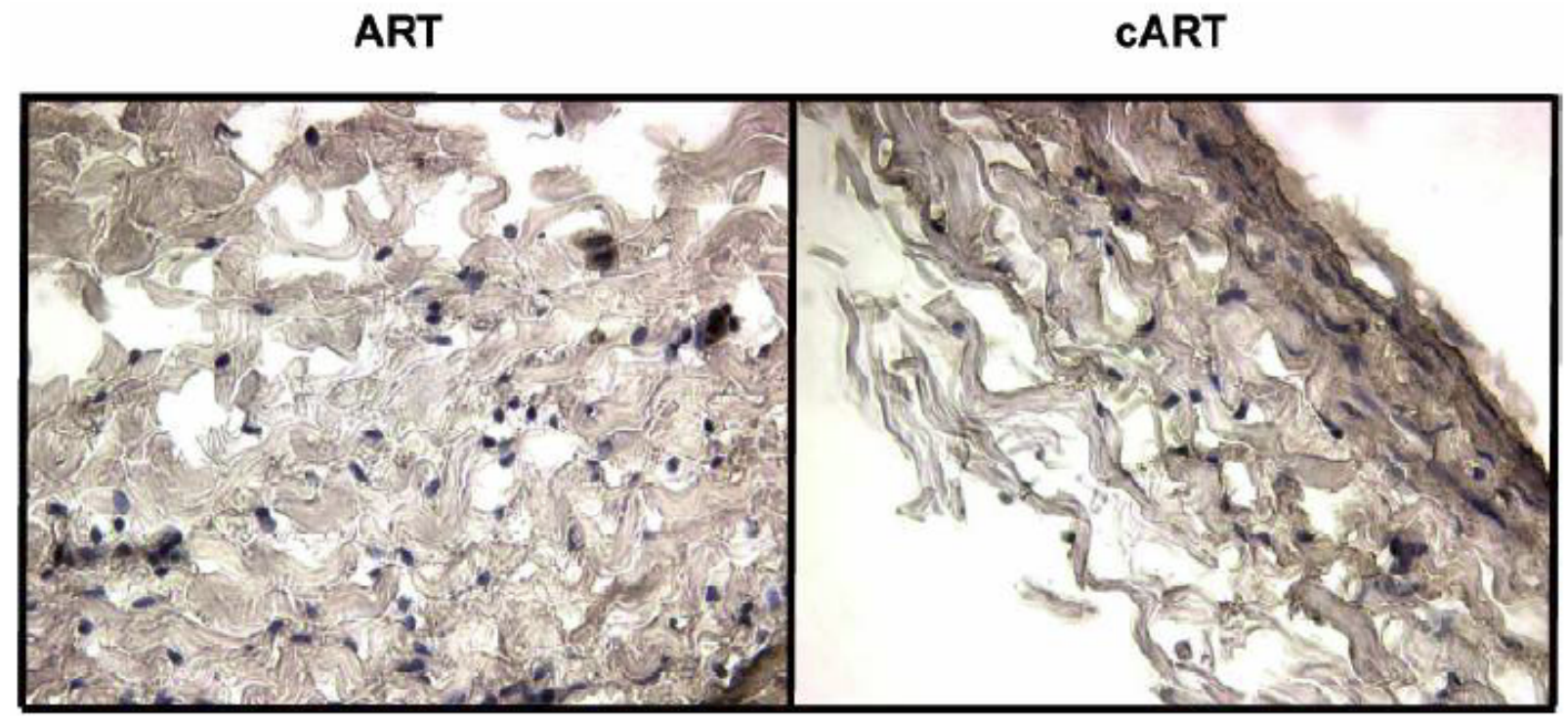

Figure F. 33 Representative PCNA images from 24 hour ART vs. cART experiment performed on 09/21/2006. Nuclei are stained blue and PCNA positive cells are stained brown. 


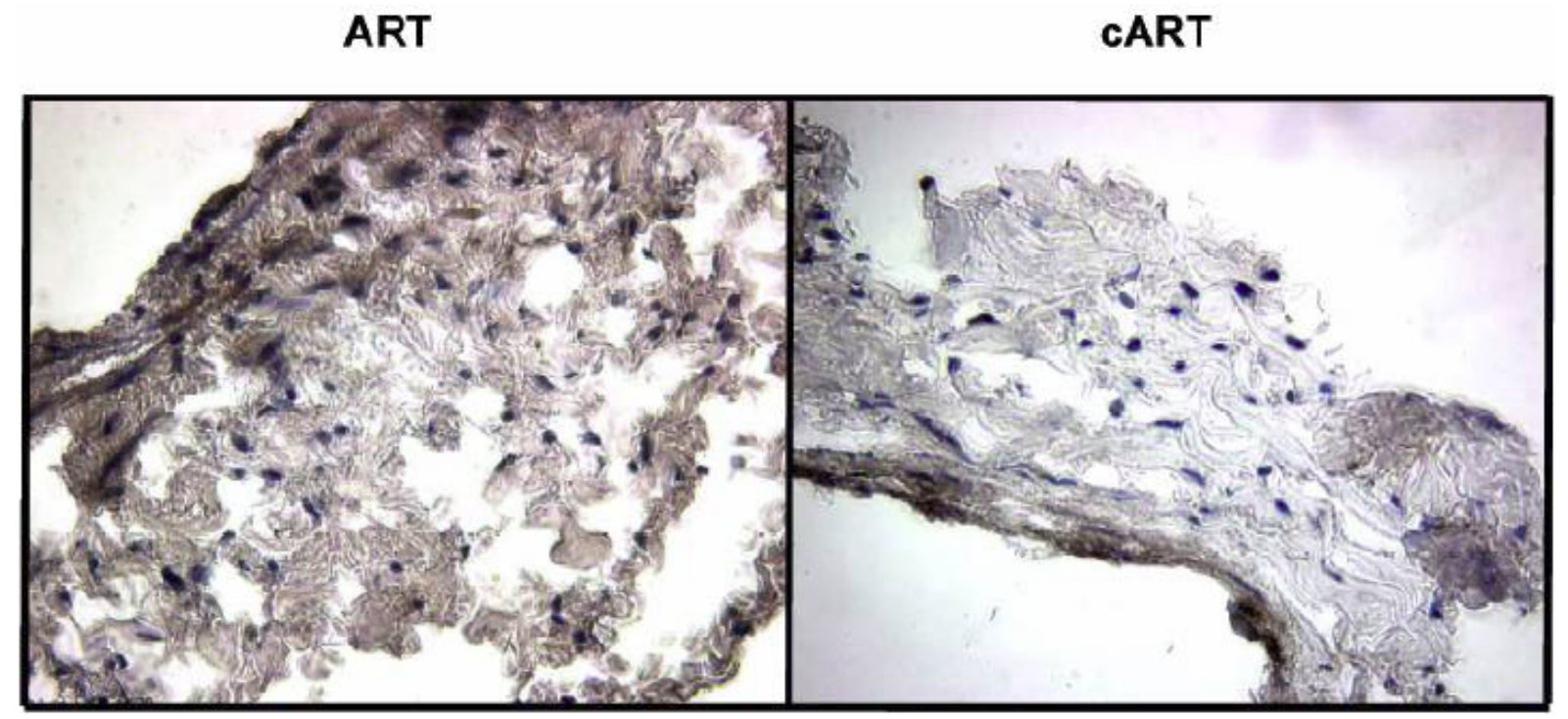

Figure F. 34 Representative PCNA images from 24 hour ART vs. cART experiment performed on 10/16/2006. Nuclei are stained blue and PCNA positive cells are stained brown.

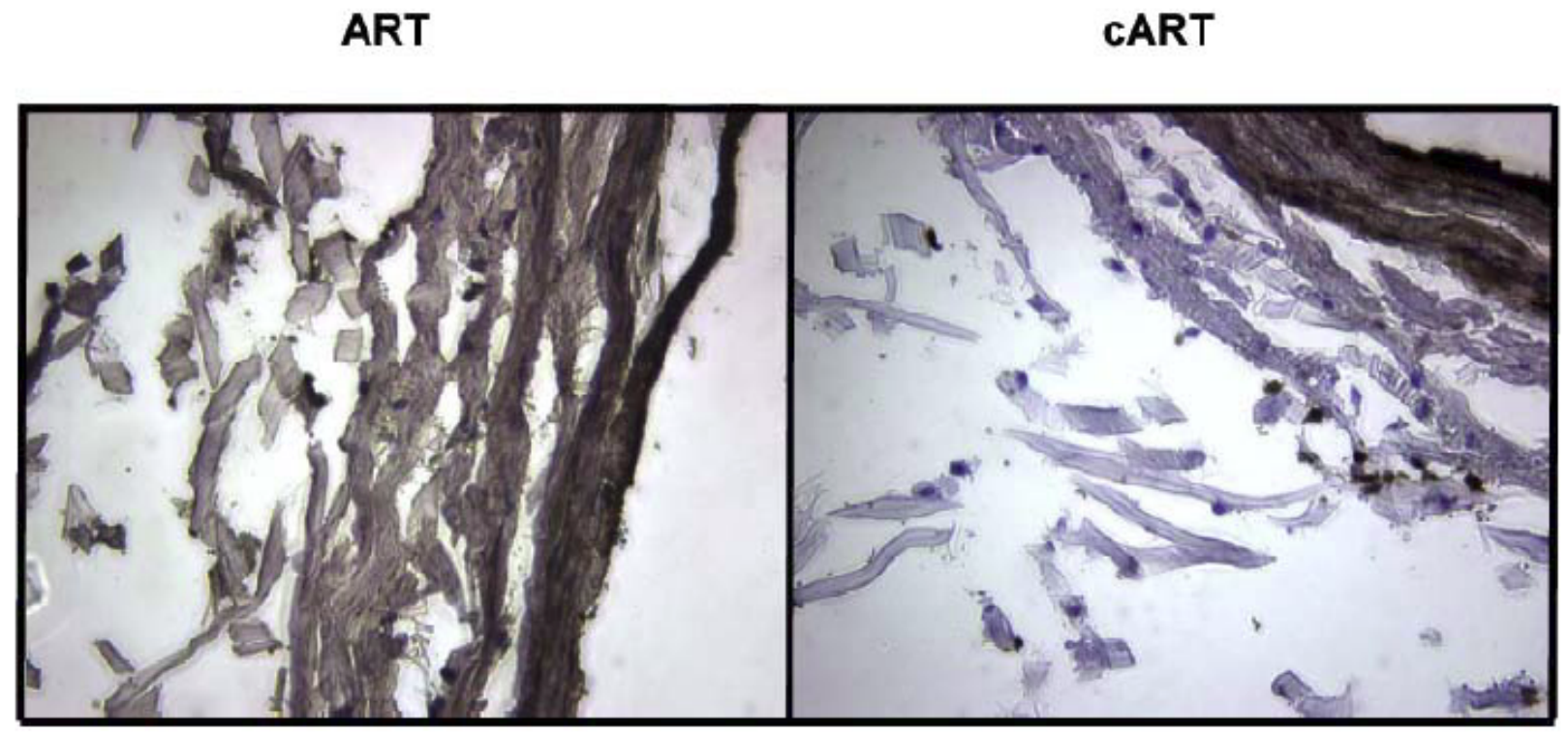

Figure F. 35 Representative PCNA images from 72 hour ART vs. cART experiment performed on 02/16/2007. Nuclei are stained blue and PCNA positive cells are stained brown. 


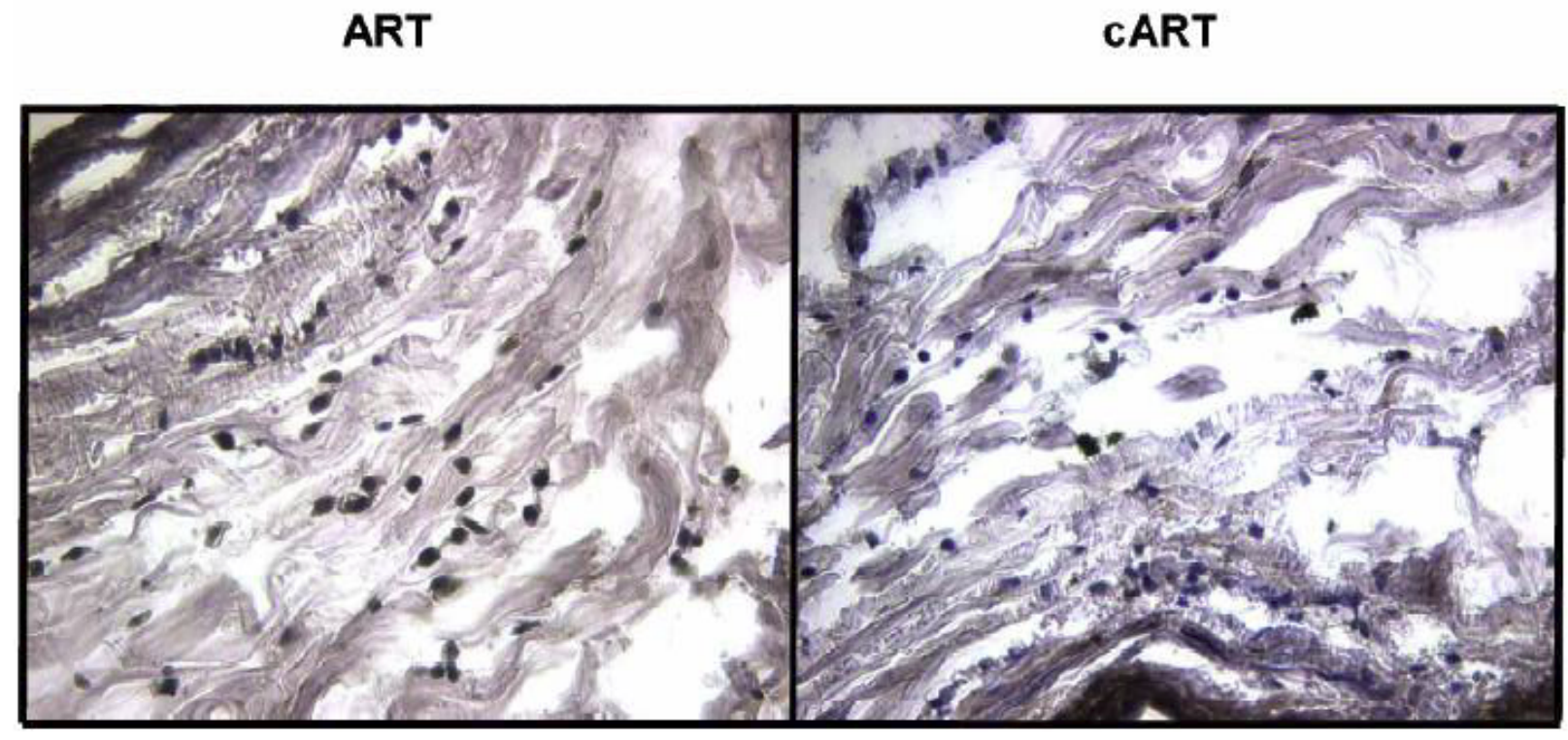

Figure F. 36 Representative PCNA images from 72 hour ART vs. cART experiment performed on 03/07/2007. Nuclei are stained blue and PCNA positive cells are stained brown.

ART

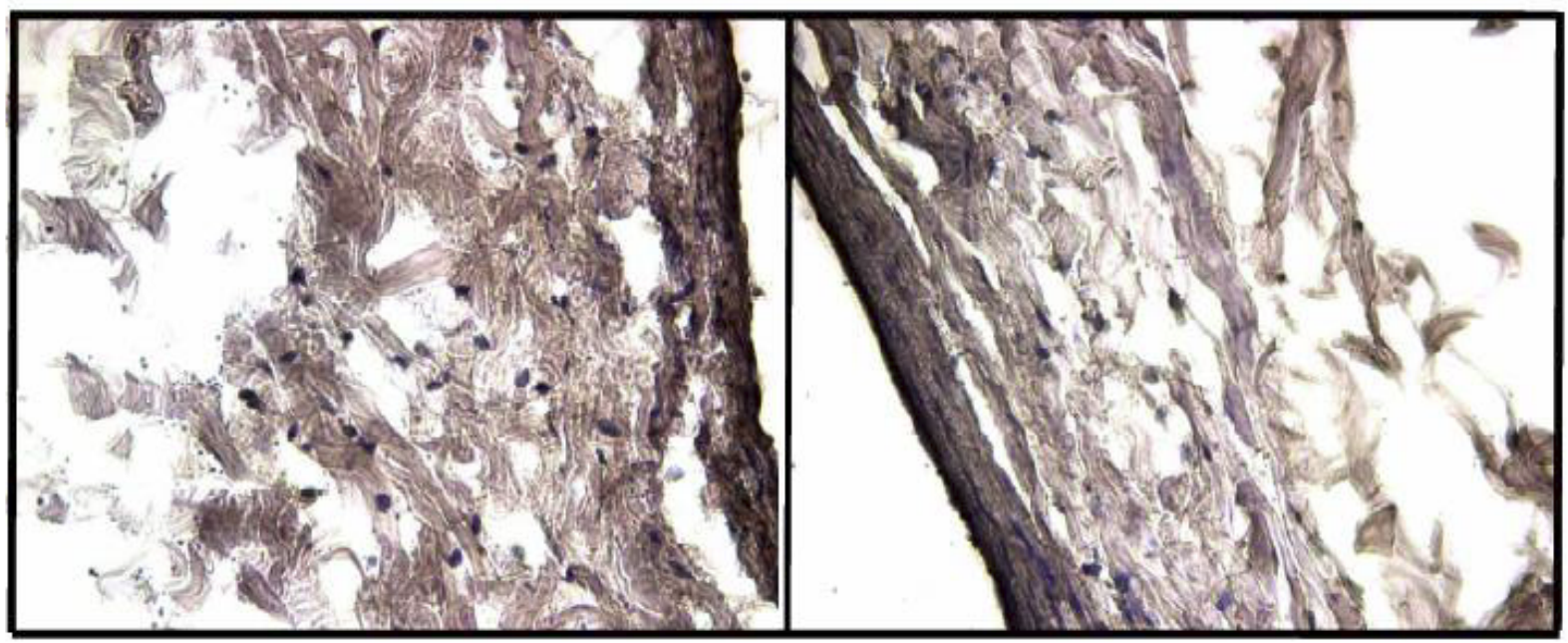

Figure F. 37 Representative PCNA images from 72 hour ART vs. cART experiment performed on 03/15/2007. Nuclei are stained blue and PCNA positive cells are stained brown. 


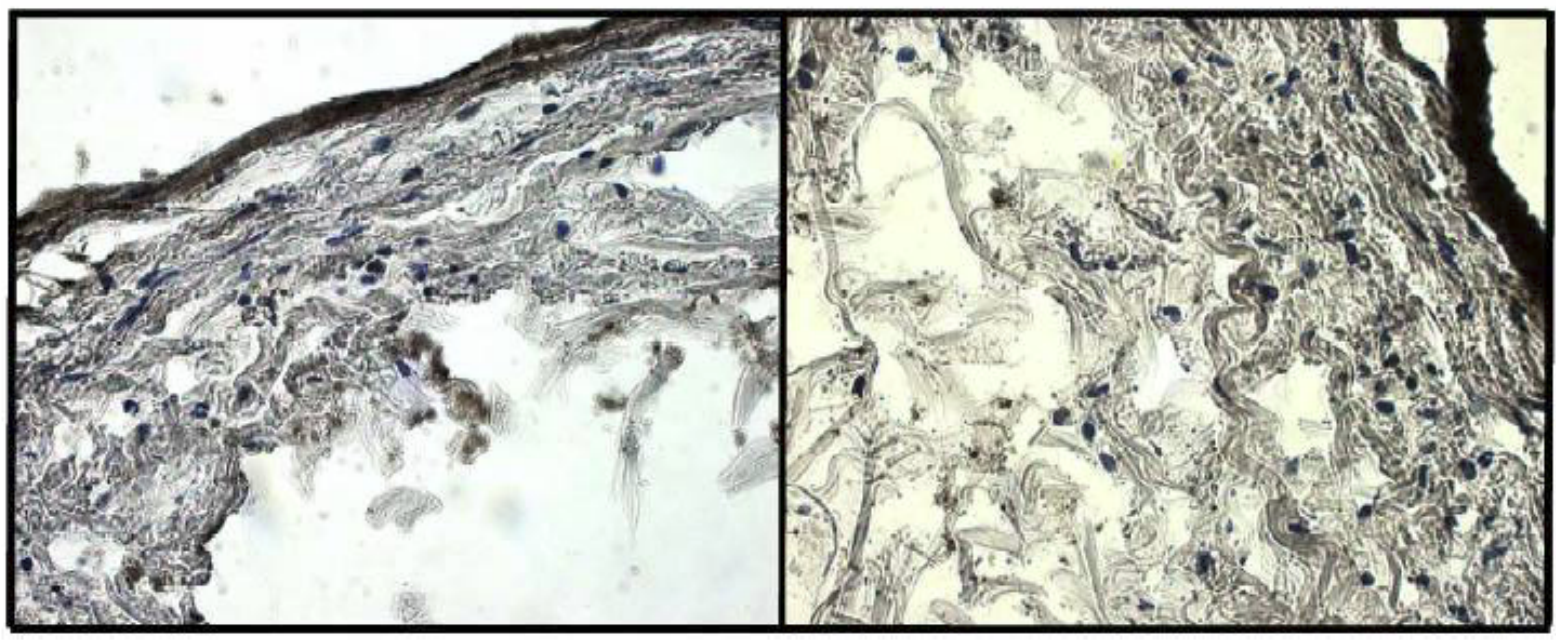

Figure F. 38 Representative PCNA images from 72 hour ART vs. cART experiment performed on 04/02/2007. Nuclei are stained blue and PCNA positive cells are stained brown.

\section{ART}

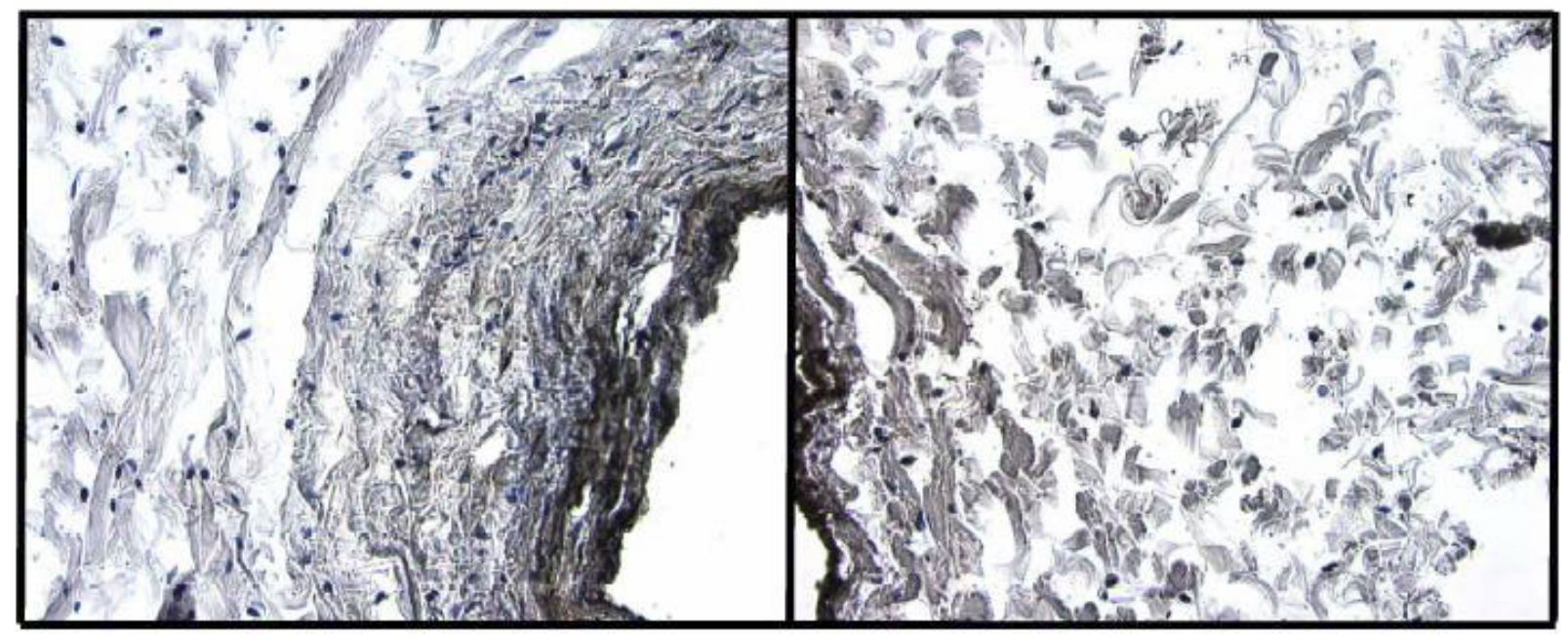

Figure F. 39 Representative PCNA images from 72 hour ART vs. cART experiment performed on 04/26/2007. Nuclei are stained blue and PCNA positive cells are stained brown. 


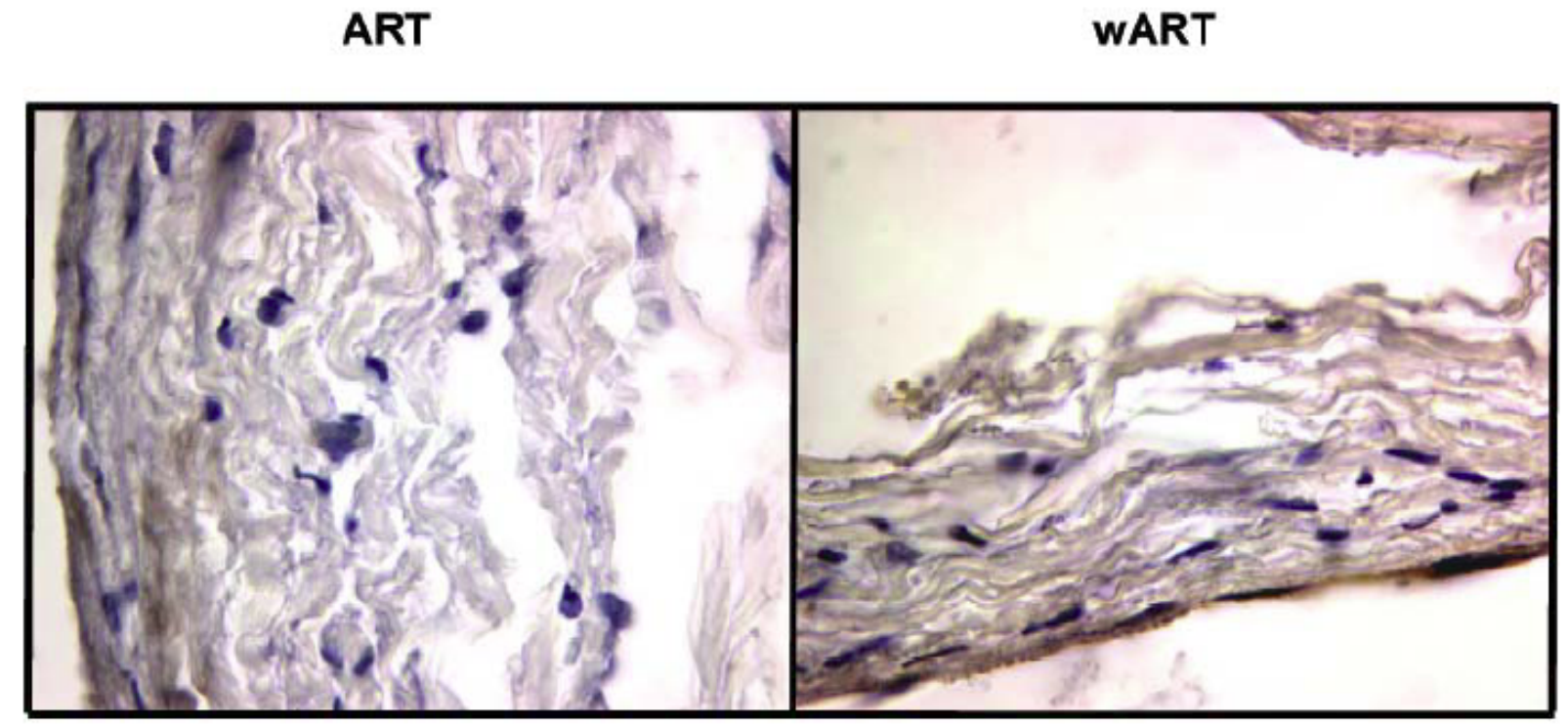

Figure F. 40 Representative PCNA images from 24 hour ART vs. wART experiment performed on 10/23/2006. Nuclei are stained blue and PCNA positive cells are stained brown.

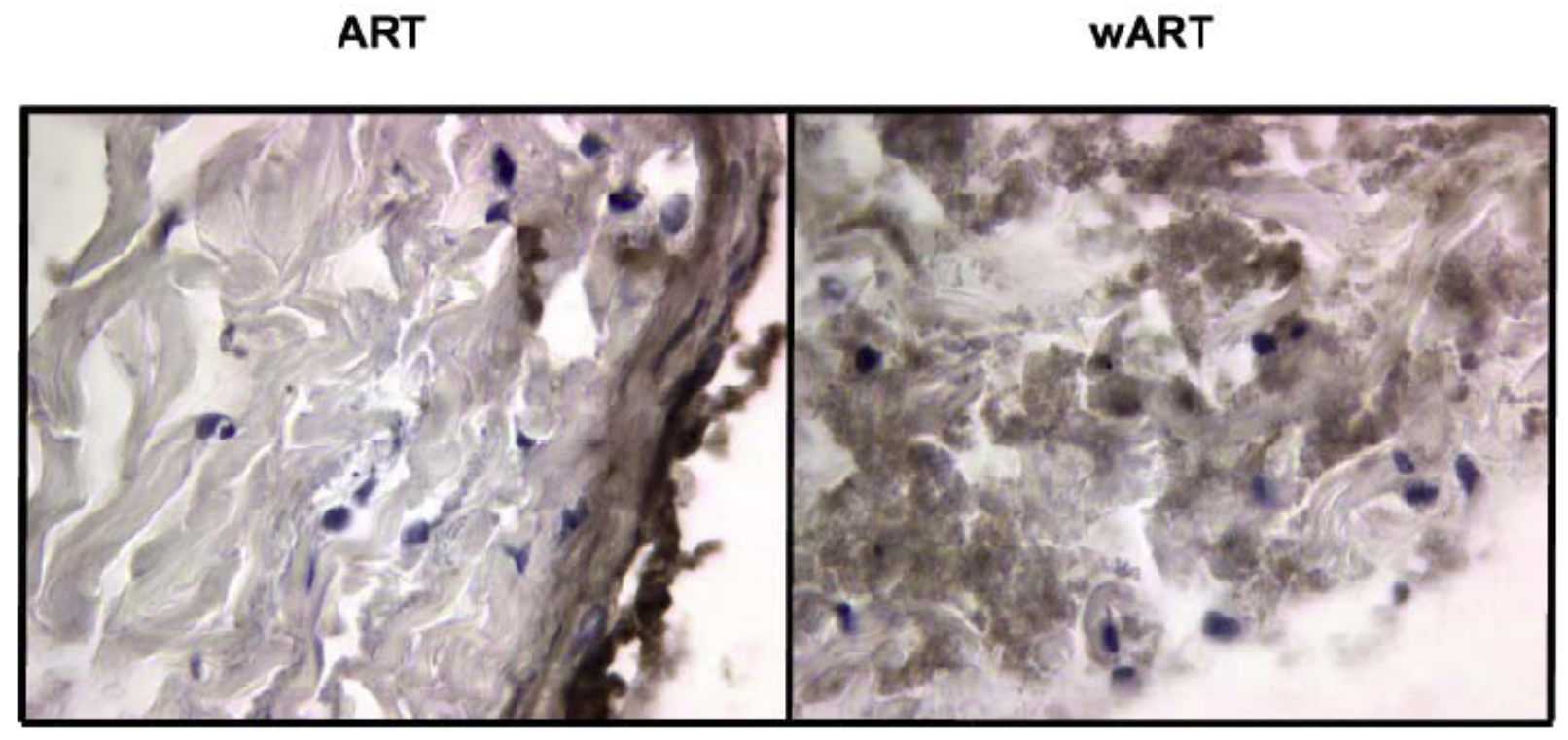

Figure F. 41 Representative PCNA images from 24 hour ART vs. wART experiment performed on 10/30/2006. Nuclei are stained blue and PCNA positive cells are stained brown. 


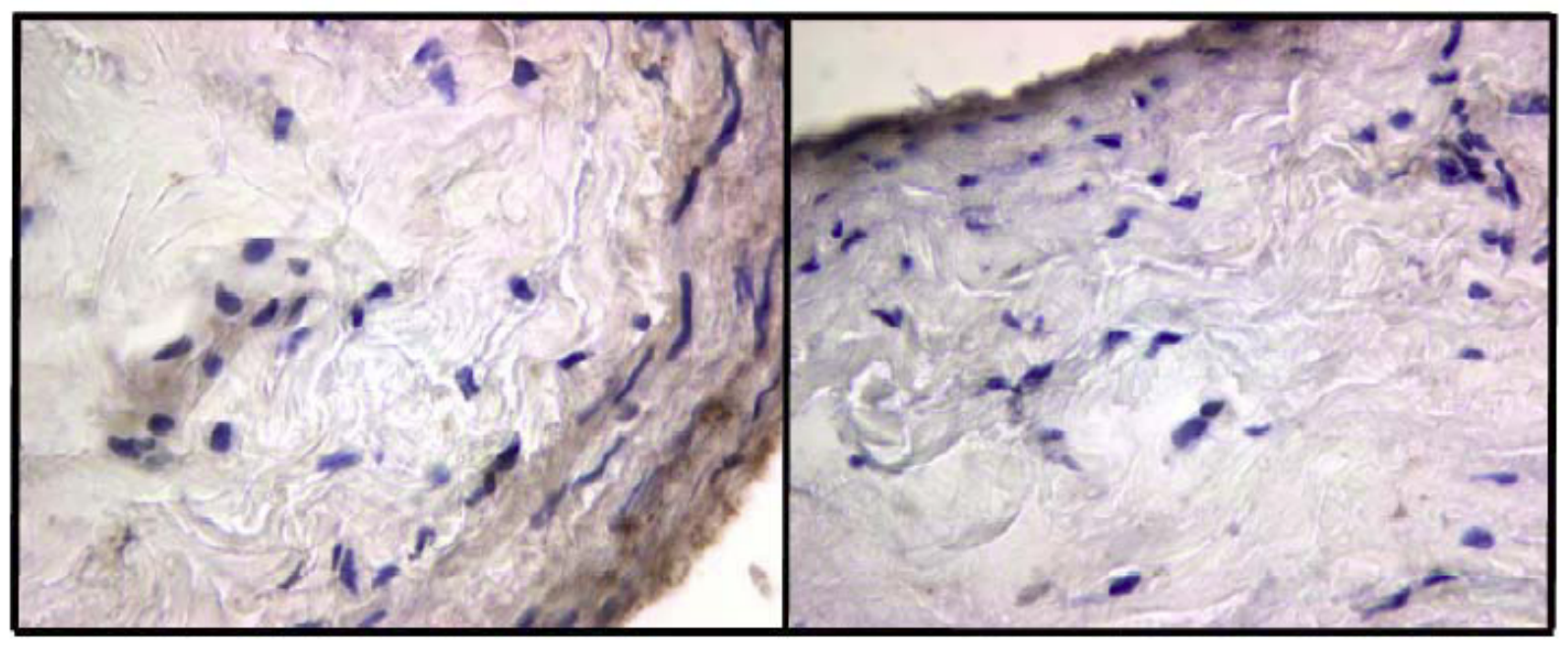

Figure F. 42 Representative PCNA images from 24 hour ART vs. wART experiment performed on 11/06/2006. Nuclei are stained blue and PCNA positive cells are stained brown.

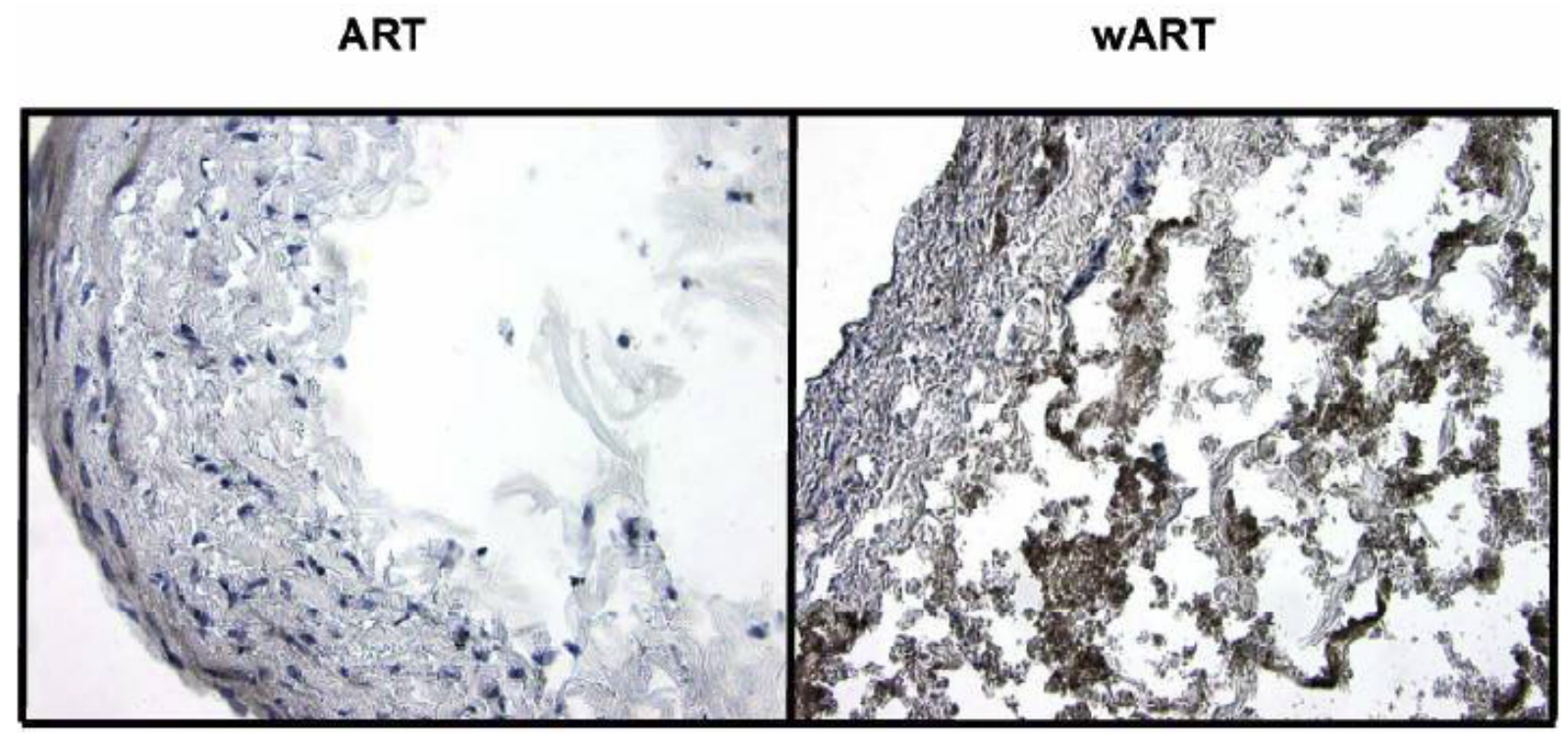

Figure F. 43 Representative PCNA images from 24 hour ART vs. wART experiment performed on 11/13/2006. Nuclei are stained blue and PCNA positive cells are stained brown. 


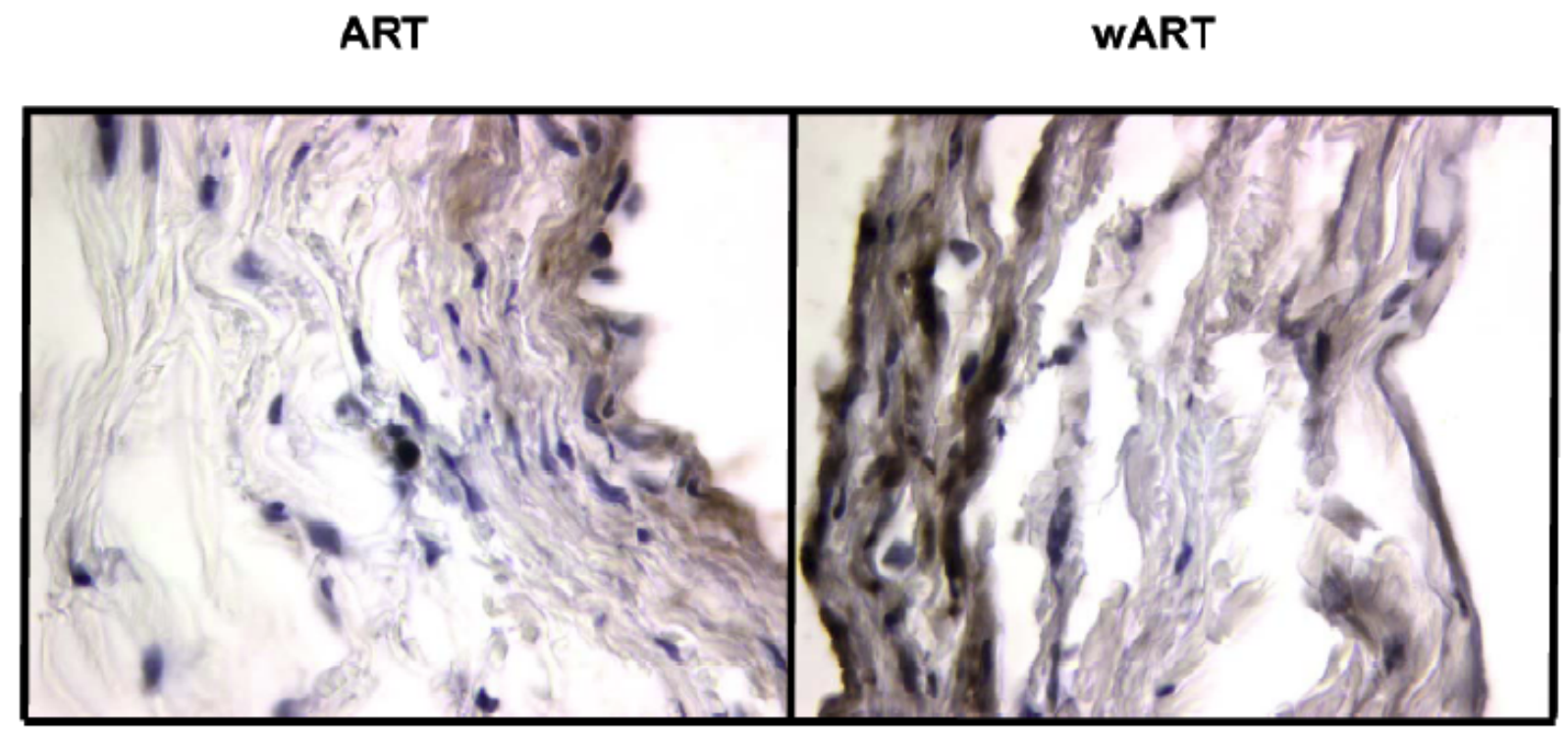

Figure F. 44 Representative PCNA images from 24 hour ART vs. wART experiment performed on 11/20/2006. Nuclei are stained blue and PCNA positive cells are stained brown.

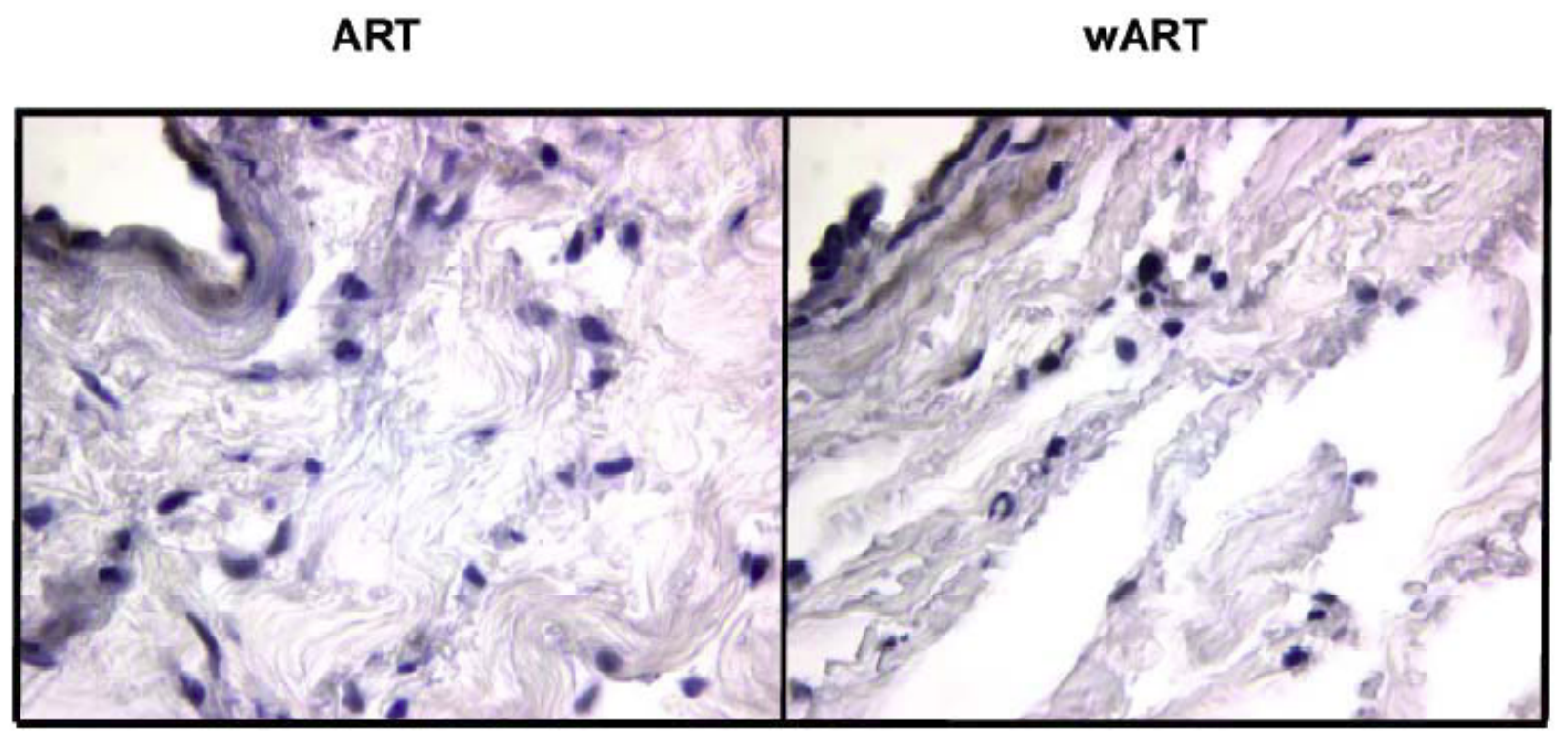

Figure F. 45 Representative PCNA images from 24 hour ART vs. wART experiment performed on 11/27/2006. Nuclei are stained blue and PCNA positive cells are stained brown. 
Table F. 6 Mean values of percent PCNA positive cells for all ex vivo experiments.

\begin{tabular}{ccccccccc} 
& \multicolumn{2}{c}{ VEN vs. ART 24 hrs } & \multicolumn{2}{c}{ ART vs. cART 24 hrs } & \multicolumn{2}{c}{ ART vs. cART 72 hrs } & \multicolumn{2}{c}{ ART vs. wART 24 hrs } \\
Expt \# & VEN & ART & ART & cART & ART & cART & ART & wART \\
$\mathbf{1}$ & 57.5622 & 46.6891 & 29.1187 & 61.2948 & 27.8515 & 43.9677 & 17.135 & 46.6065 \\
$\mathbf{2}$ & 72.3643 & 46.3175 & 31.104 & 41.1294 & 45.2836 & 41.8688 & 30.6972 & 59.596 \\
$\mathbf{3}$ & 70.6504 & 46.9431 & 20.5876 & 37.788 & 47.7593 & 46.0034 & 26.1277 & 26.0411 \\
$\mathbf{4}$ & 59.7265 & 47.1527 & 17.2865 & 42.9652 & 40.7072 & 41.4621 & 20.8188 & 71.5858 \\
$\mathbf{5}$ & 68.5151 & 26.0892 & 22.4641 & 41.9854 & 31.8016 & 44.9698 & 17.3281 & 64.3445 \\
$\mathbf{6}$ & - & - & - & - & - & - & 14.9008 & 41.953 \\
& & & & & & & & \\
mean & 65.7637 & 42.63832 & 24.5242 & 45.79435 & 38.68064 & 43.65436 & 21.16793 & 51.68782 \\
SE & 2.989401 & 4.139621 & 3.320195 & 5.276769 & 3.838747 & 0.875808 & 2.488734 & 6.82398
\end{tabular}

Table F. 7 Microsoft Excel output for student's t-test performed on the mean percent PCNA positive cells from 24 hour VEN vs. ART ex vivo experiments.

t-Test: Paired Two Sample for Means

\begin{tabular}{lrr}
\hline & VEN & \multicolumn{1}{c}{ ART } \\
\hline Mean & 65.7637 & 42.63832 \\
Variance & 44.68259 & 85.68233 \\
Observations & 5 & 5 \\
Pearson Correlation & -0.24521 & \\
Hypothesized Mean Difference & 0 & \\
df & 4 & \\
t Stat & 4.078996 & \\
$\mathrm{P}(T<=t)$ one-tail & 0.007555 & \\
t Critical one-tail & 2.131847 & \\
$\mathrm{P}(T<=t)$ two-tail & 0.015111 & \\
t Critical two-tail & 2.776445 & \\
\hline
\end{tabular}


Table F. 8 Microsoft Excel output for student's t-test performed on the mean percent PCNA positive cells from 24 hour ART vs. cART ex vivo experiments.

t-Test: Paired Two Sample for

Means

\begin{tabular}{lrr}
\hline & \multicolumn{1}{c}{ ART } & \multicolumn{1}{c}{ cART } \\
\hline Mean & 24.5242 & 45.79435 \\
Variance & 44.09477 & 111.3771 \\
Observations & 5 & 5 \\
Pearson Correlation & 0.440055 & \\
Hypothesized Mean Difference & 0 & \\
df & 3 & \\
t Stat & -4.39251 & \\
$\mathrm{P}(T<=\mathrm{t})$ one-tail & 0.010931 & \\
t Critical one-tail & 2.353363 & \\
$\mathrm{P}(\mathrm{T}<=\mathrm{t})$ two-tail & 0.021863 & \\
$\mathrm{t}$ Critical two-tail & 3.182446 & \\
\hline
\end{tabular}

Table F. 9 Microsoft Excel output for student's t-test performed on the mean percent PCNA positive cells from 72 hour ART vs. cART ex vivo experiments.

t-Test: Paired Two Sample for Means

\begin{tabular}{lrr}
\hline & \multicolumn{1}{c}{ ART } & \multicolumn{1}{c}{ CART } \\
\hline Mean & 38.68064 & 43.65436 \\
Variance & 73.67989 & 3.835195 \\
Observations & 5 & 5 \\
Pearson Correlation & -0.10929 & \\
Hypothesized Mean Difference & 0 & \\
df & 4 & \\
t Stat & -1.23429 & \\
P $(T<=t)$ one-tail & 0.142326 & \\
t Critical one-tail & 2.131847 & \\
P $(T<=t)$ two-tail & 0.284653 & \\
t Critical two-tail & 2.776445 & \\
\hline
\end{tabular}


Table F. 10 Microsoft Excel output for student's t-test performed on the mean percent PCNA positive cells from 24 hour ART vs. WART ex vivo experiments.

t-Test: Paired Two Sample for

Means

\begin{tabular}{lrr}
\hline & \multicolumn{1}{c}{ ART } & WART \\
\hline Mean & 21.16793 & 51.68782 \\
Variance & 37.16279 & 279.4002 \\
Observations & 6 & 6 \\
Pearson Correlation & -0.05081 & \\
Hypothesized Mean Difference & \multicolumn{2}{c}{0} \\
df & 5 & \\
t Stat & -4.13465 & \\
$\mathrm{P}(\mathrm{T}<=\mathrm{t})$ one-tail & 0.004522 & \\
t Critical one-tail & 2.015048 & \\
$\mathrm{P}(\mathrm{T}<=\mathrm{t})$ two-tail & 0.009044 & \\
t Critical two-tail & 2.570582 & \\
\hline
\end{tabular}




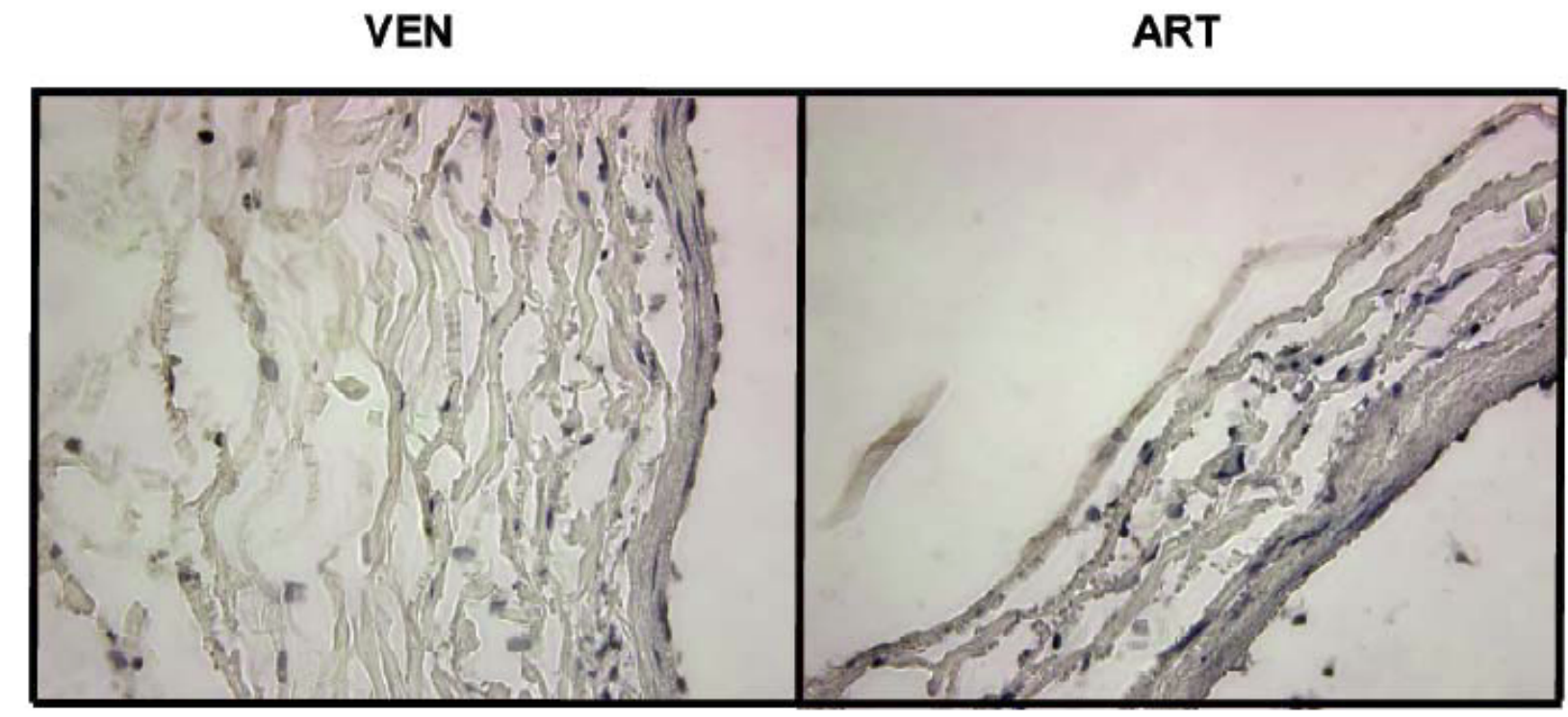

Figure F. 46 Representative Golgi complex images from 24 hour VEN vs. ART experiment performed on 04/23/2003. Nuclei are stained blue and Golgi complex positive cells are stained brown.

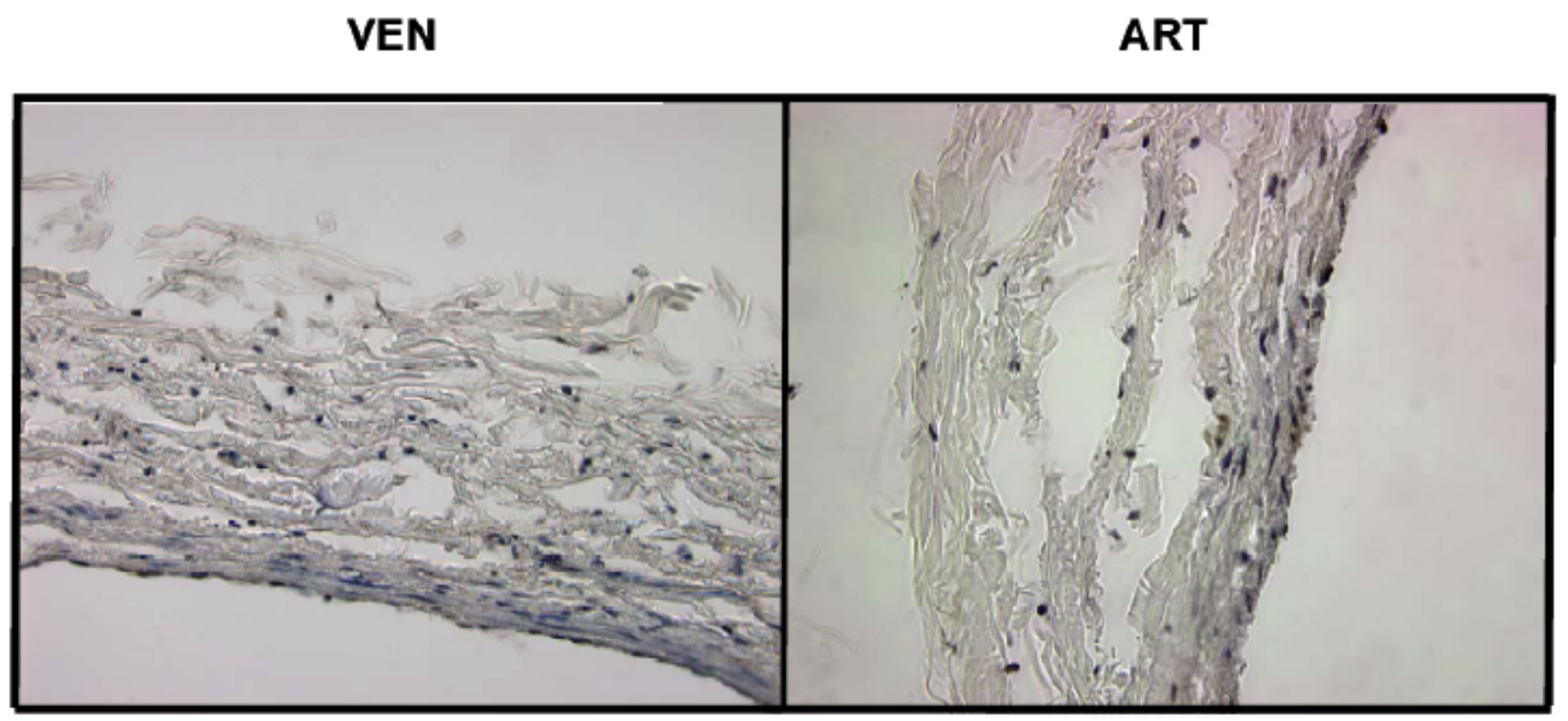

Figure F. 47 Representative Golgi complex images from 24 hour VEN vs. ART experiment performed on 05/07/2003. Nuclei are stained blue and Golgi complex positive cells are stained brown. 


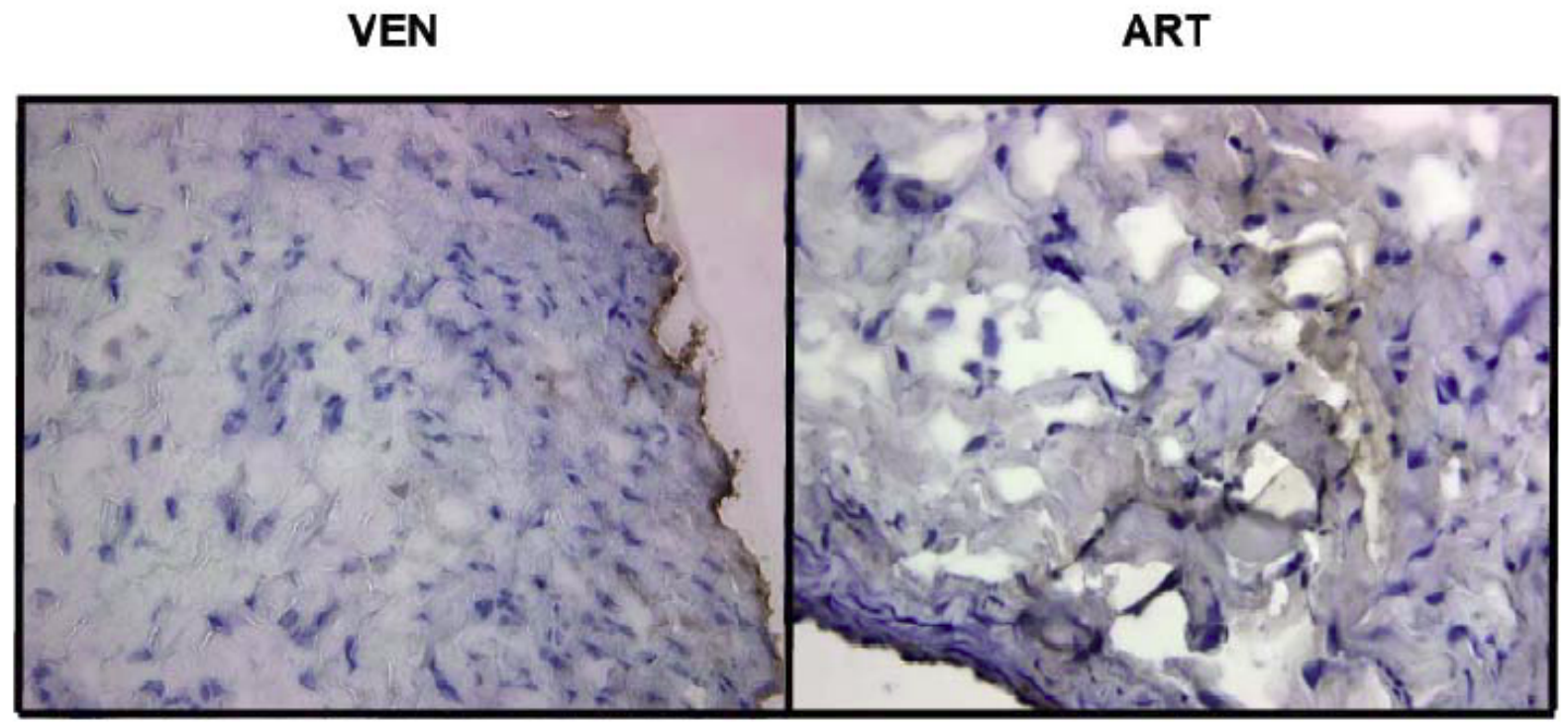

Figure F. 48 Representative Golgi complex images from 24 hour VEN vs. ART experiment performed on 10/09/2004. Nuclei are stained blue and Golgi complex positive cells are stained brown.

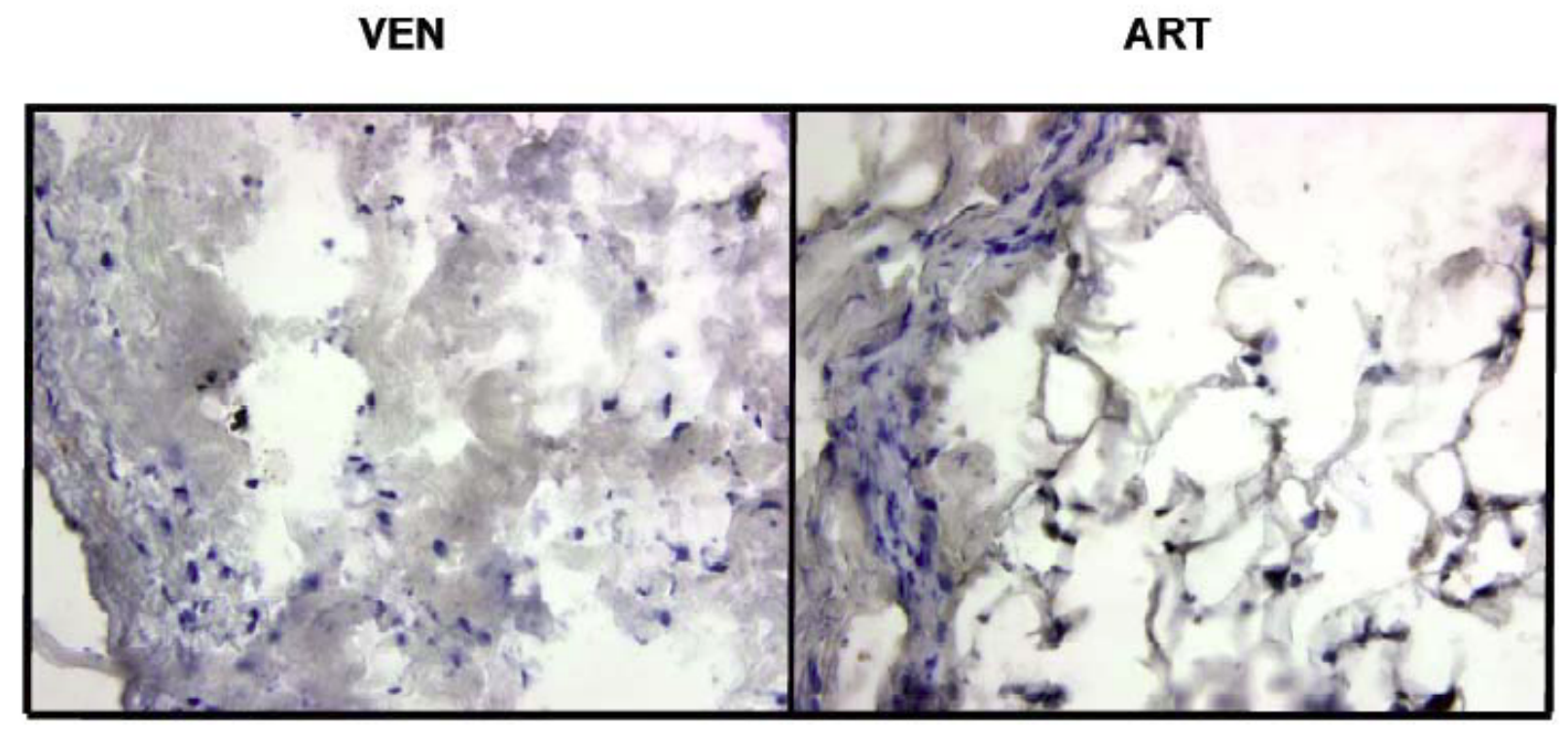

Figure F. 49 Representative Golgi complex images from 24 hour VEN vs. ART experiment performed on 10/16/2004. Nuclei are stained blue and Golgi complex positive cells are stained brown. 


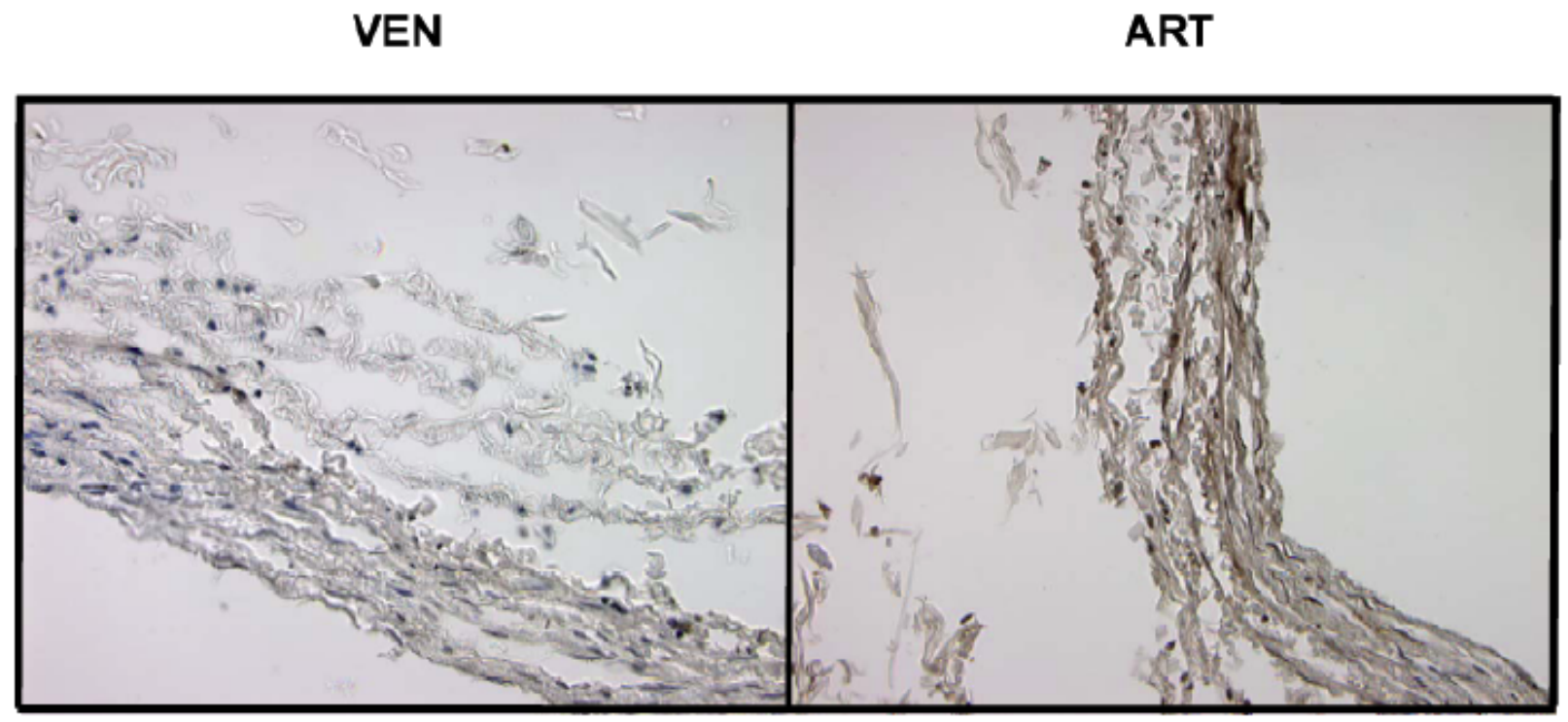

Figure F. 50 Representative Golgi complex images from 24 hour VEN vs. ART experiment performed on 05/24/2006. Nuclei are stained blue and Golgi complex positive cells are stained brown.

VEN

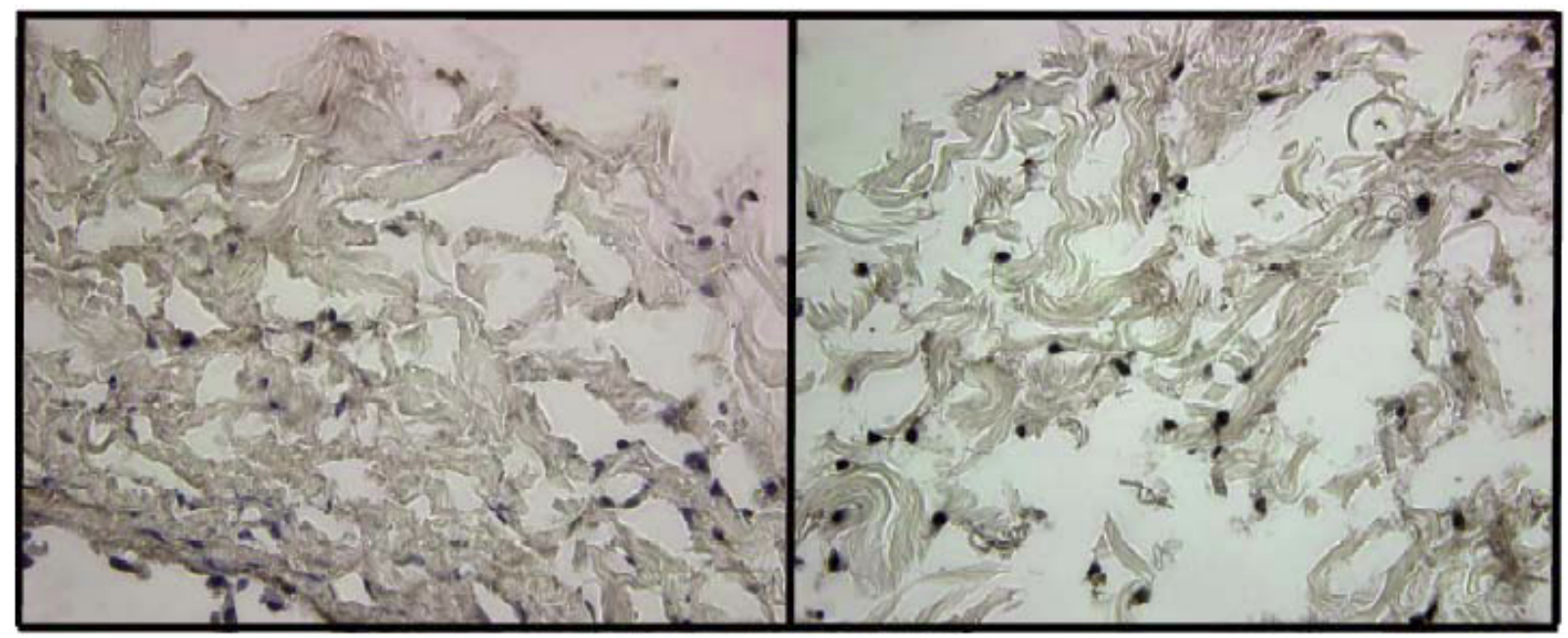

Figure F. 51 Representative Golgi complex images from 24 hour VEN vs. ART experiment performed on 09/14/2006. Nuclei are stained blue and Golgi complex positive cells are stained brown. 


\section{ART}

CART

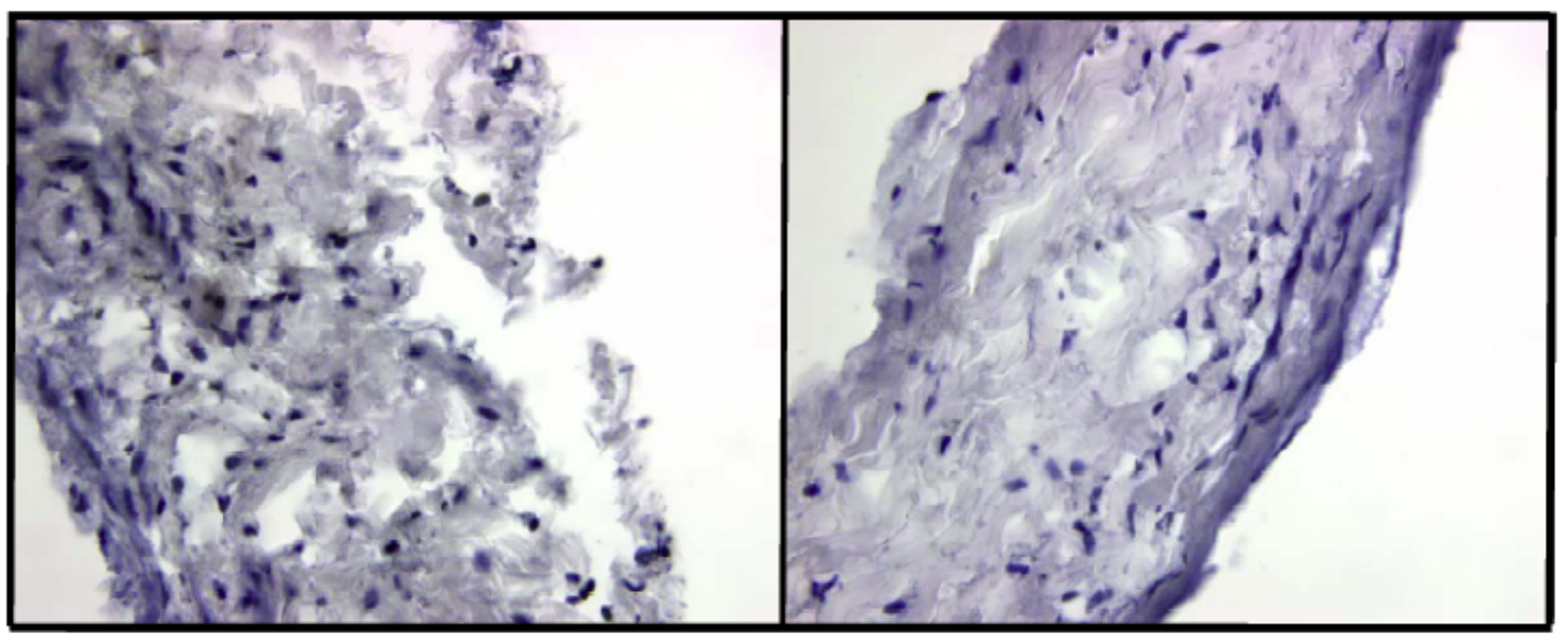

Figure F. 52 Representative Golgi complex images from 24 hour ART vs. cART experiment performed on 10/22/2004. Nuclei are stained blue and Golgi complex positive cells are stained brown.

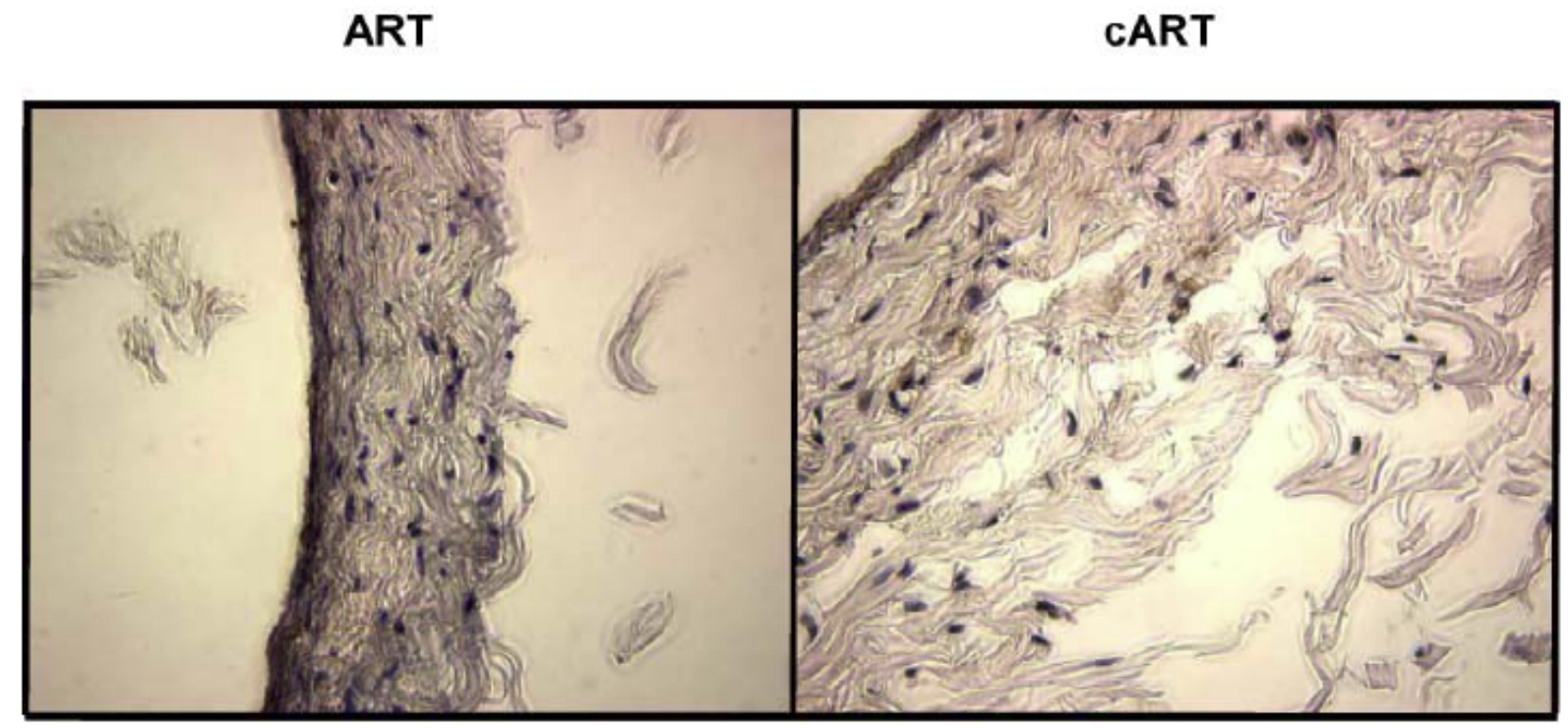

Figure F. 53 Representative Golgi complex images from 24 hour ART vs. cART experiment performed on 11/01/2004. Nuclei are stained blue and Golgi complex positive cells are stained brown. 


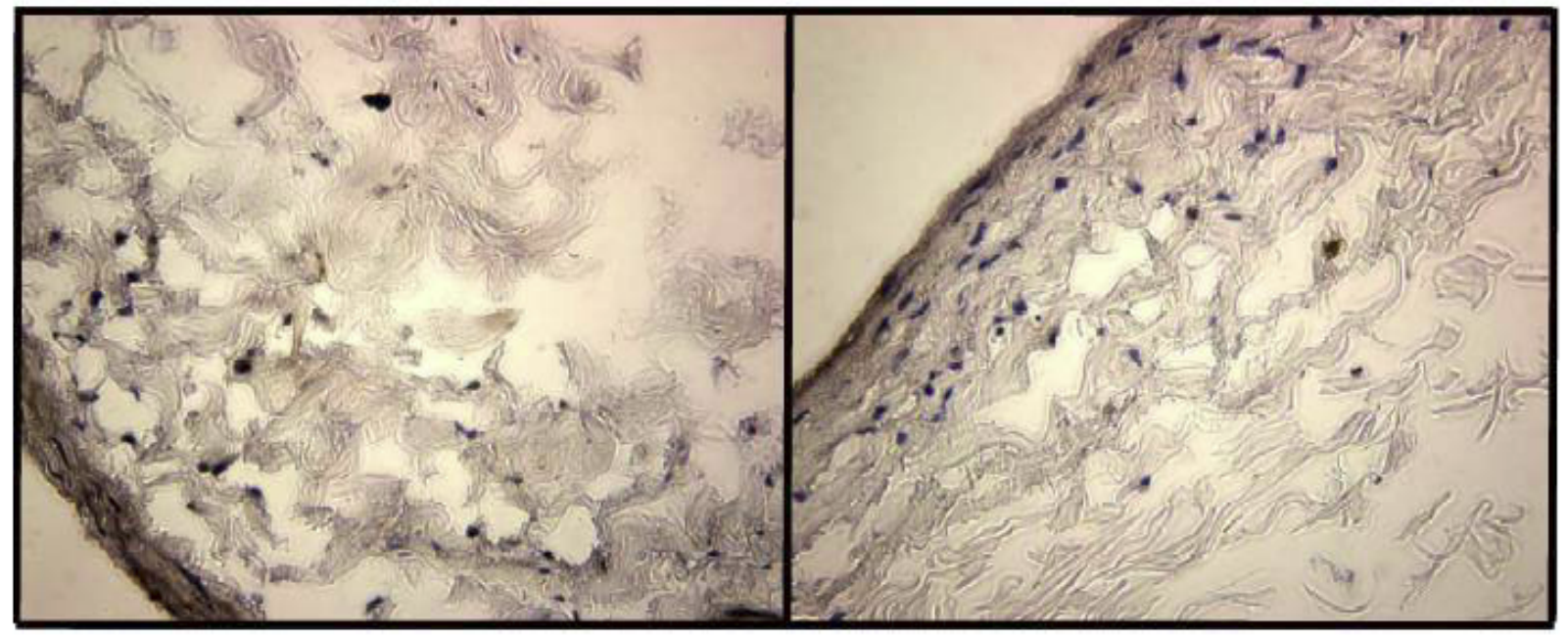

Figure F. 54 Representative Golgi complex images from 24 hour ART vs. cART experiment performed on 11/11/2004. Nuclei are stained blue and Golgi complex positive cells are stained brown.

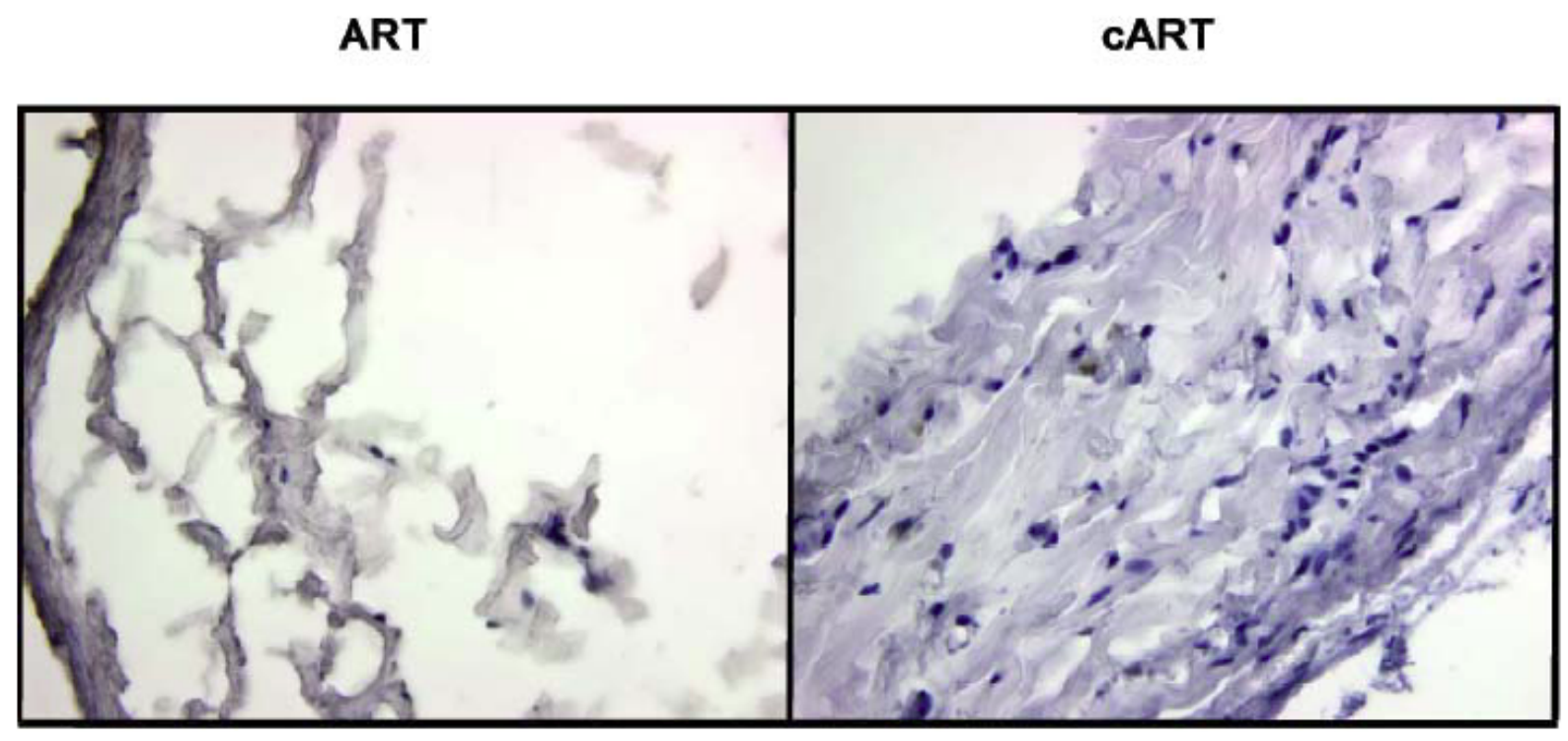

Figure F. 55 Representative Golgi complex images from 24 hour ART vs. cART experiment performed on 09/21/2006. Nuclei are stained blue and Golgi complex positive cells are stained brown. 


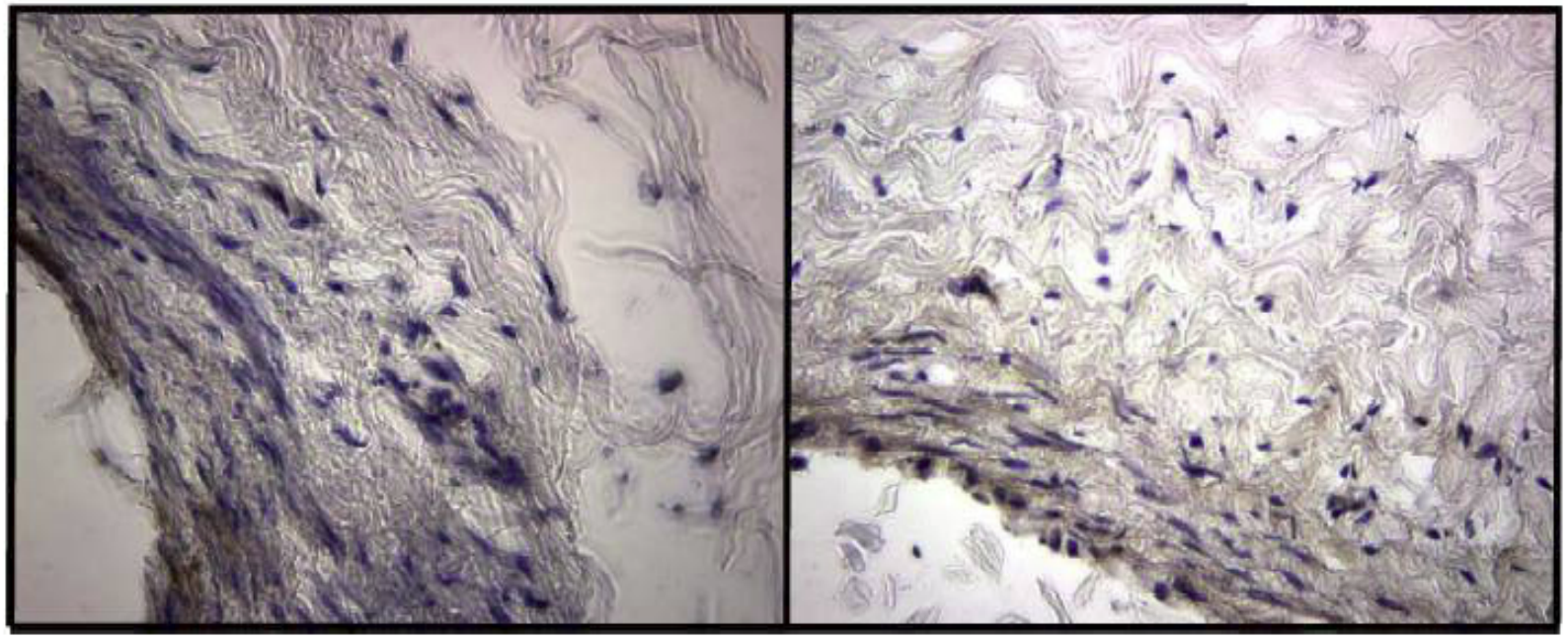

Figure F. 56 Representative Golgi complex images from 24 hour ART vs. cART experiment performed on 10/16/2006. Nuclei are stained blue and Golgi complex positive cells are stained brown.

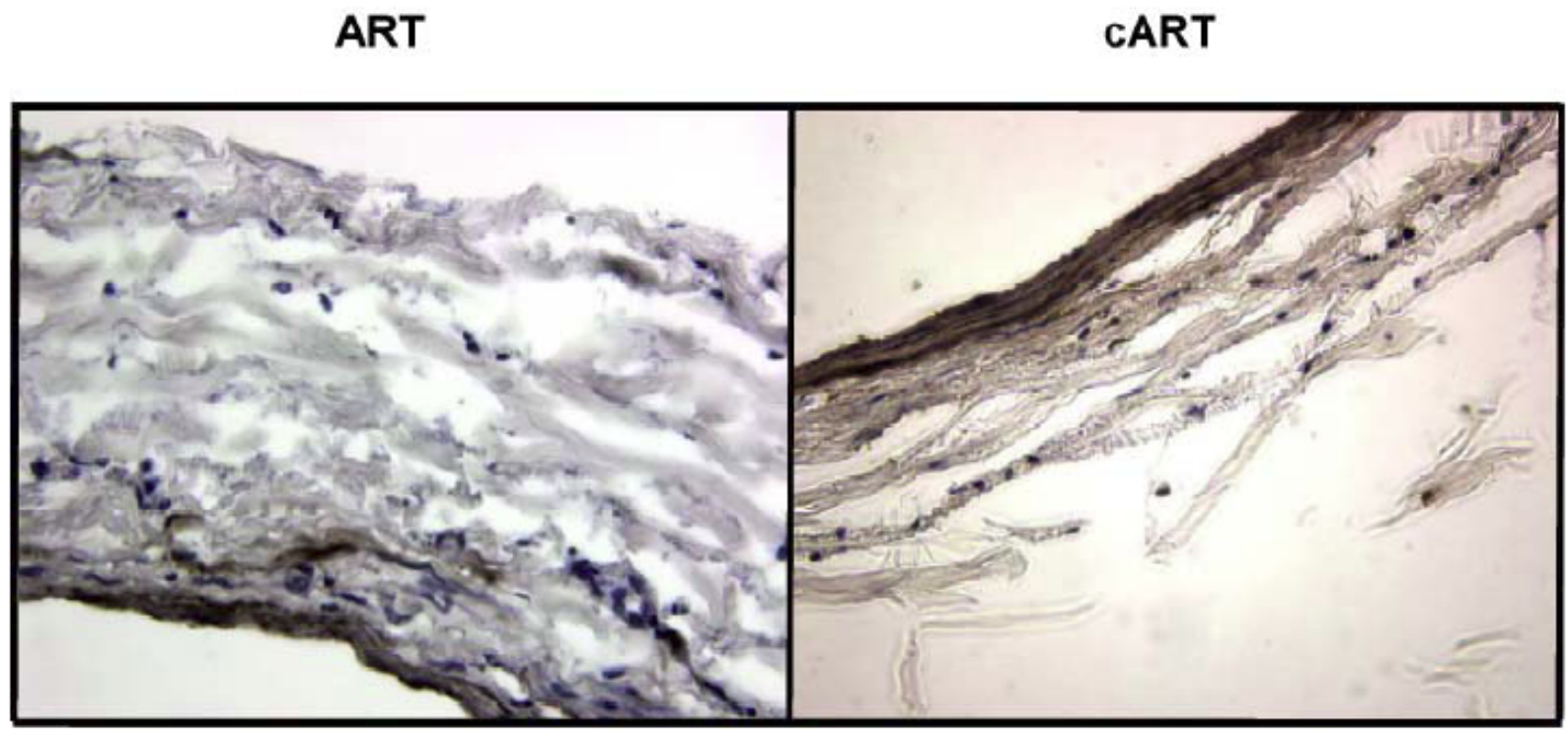

Figure F. 57 Representative Golgi complex images from 72 hour ART vs. cART experiment performed on 02/16/2007. Nuclei are stained blue and Golgi complex positive cells are stained brown. 


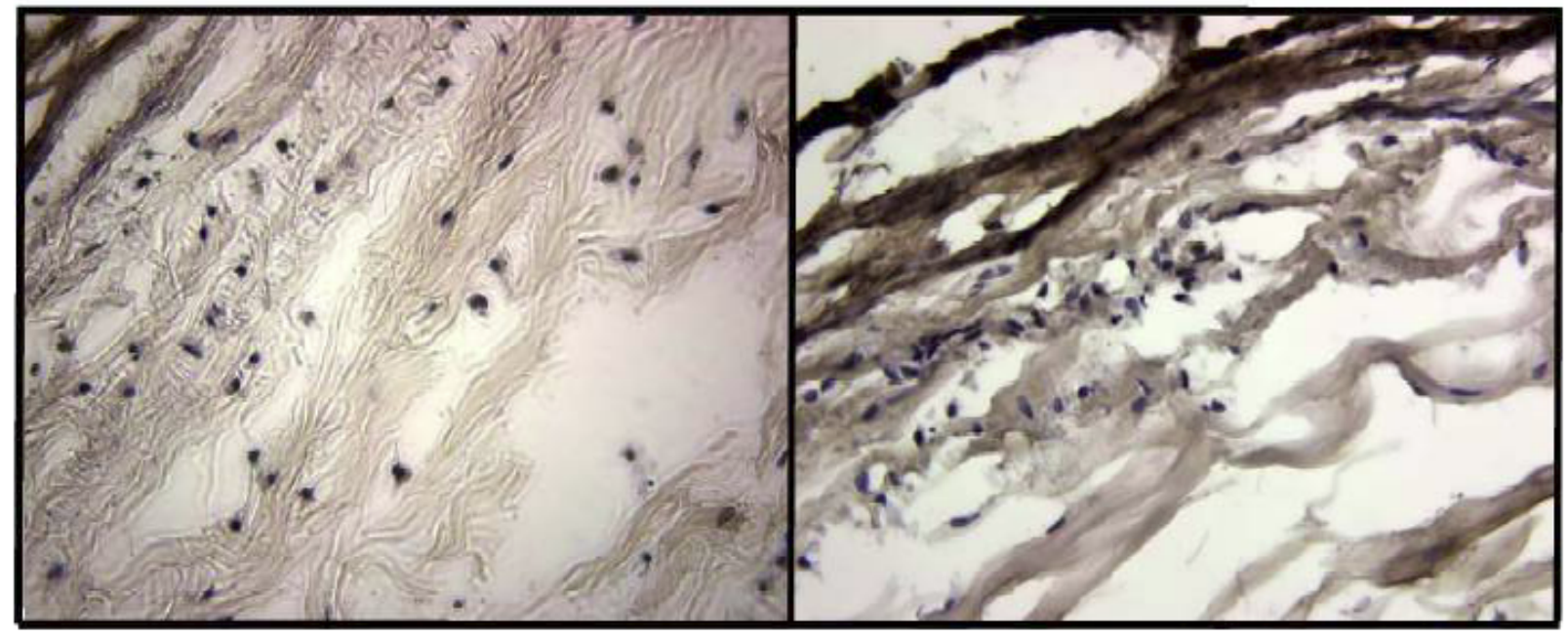

Figure F. 58 Representative Golgi complex images from 72 hour ART vs. cART experiment performed on 03/07/2007. Nuclei are stained blue and Golgi complex positive cells are stained brown.

\section{ART}

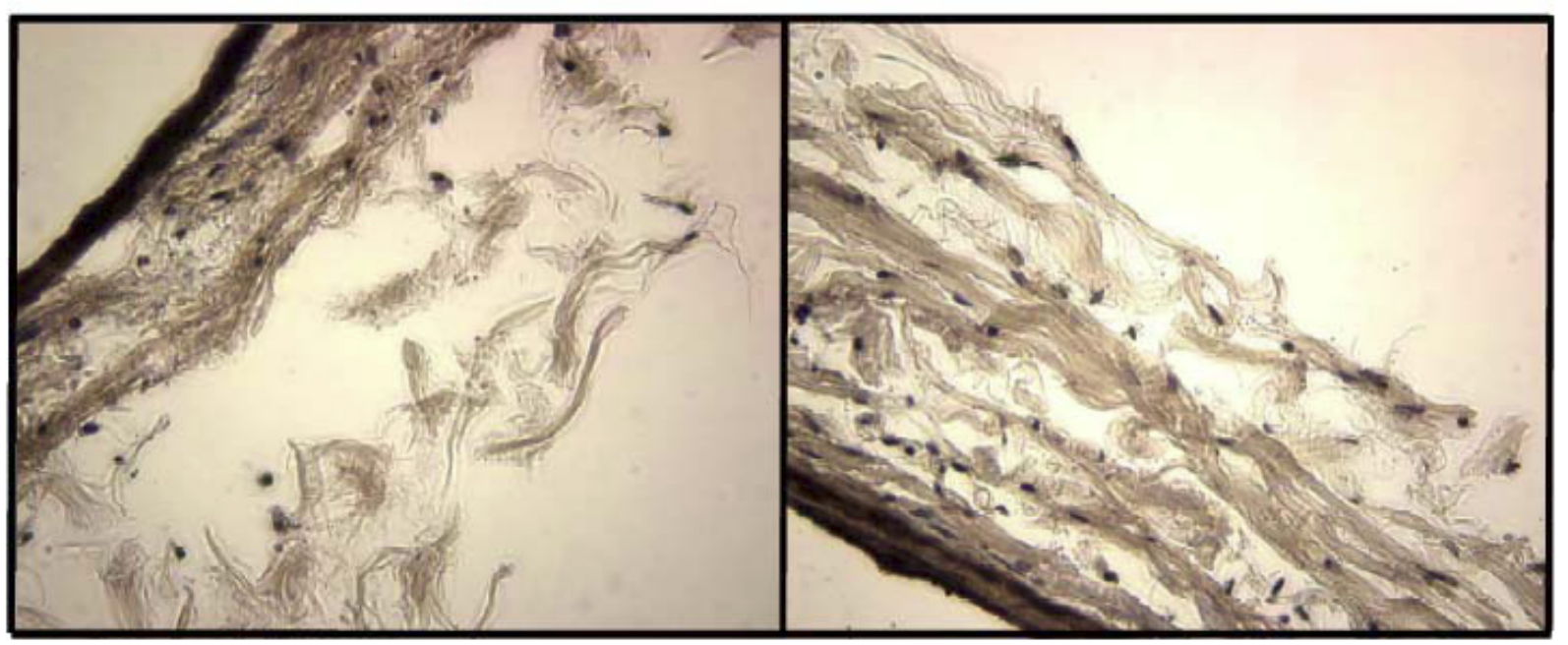

Figure F. 59 Representative Golgi complex images from 72 hour ART vs. cART experiment performed on 03/15/2007. Nuclei are stained blue and Golgi complex positive cells are stained brown. 


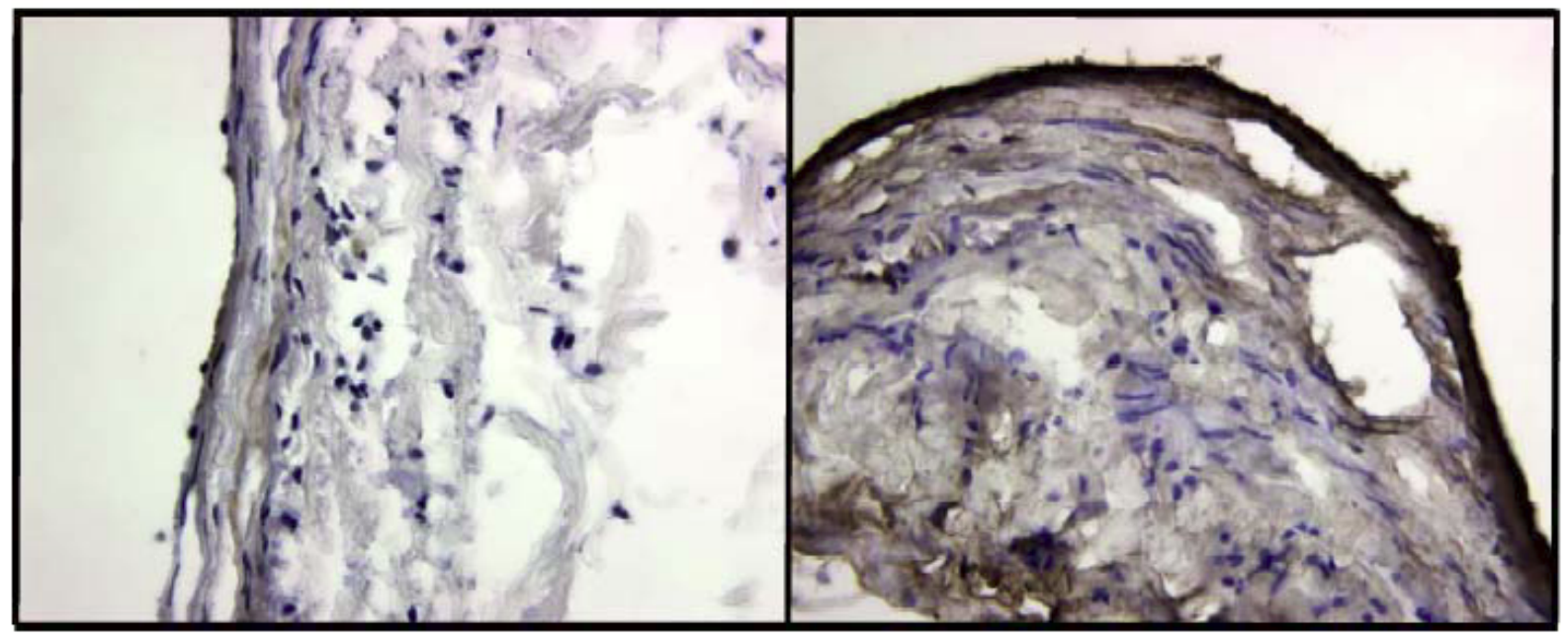

Figure F. 60 Representative Golgi complex images from 72 hour ART vs. cART experiment performed on 04/02/2007. Nuclei are stained blue and Golgi complex positive cells are stained brown.

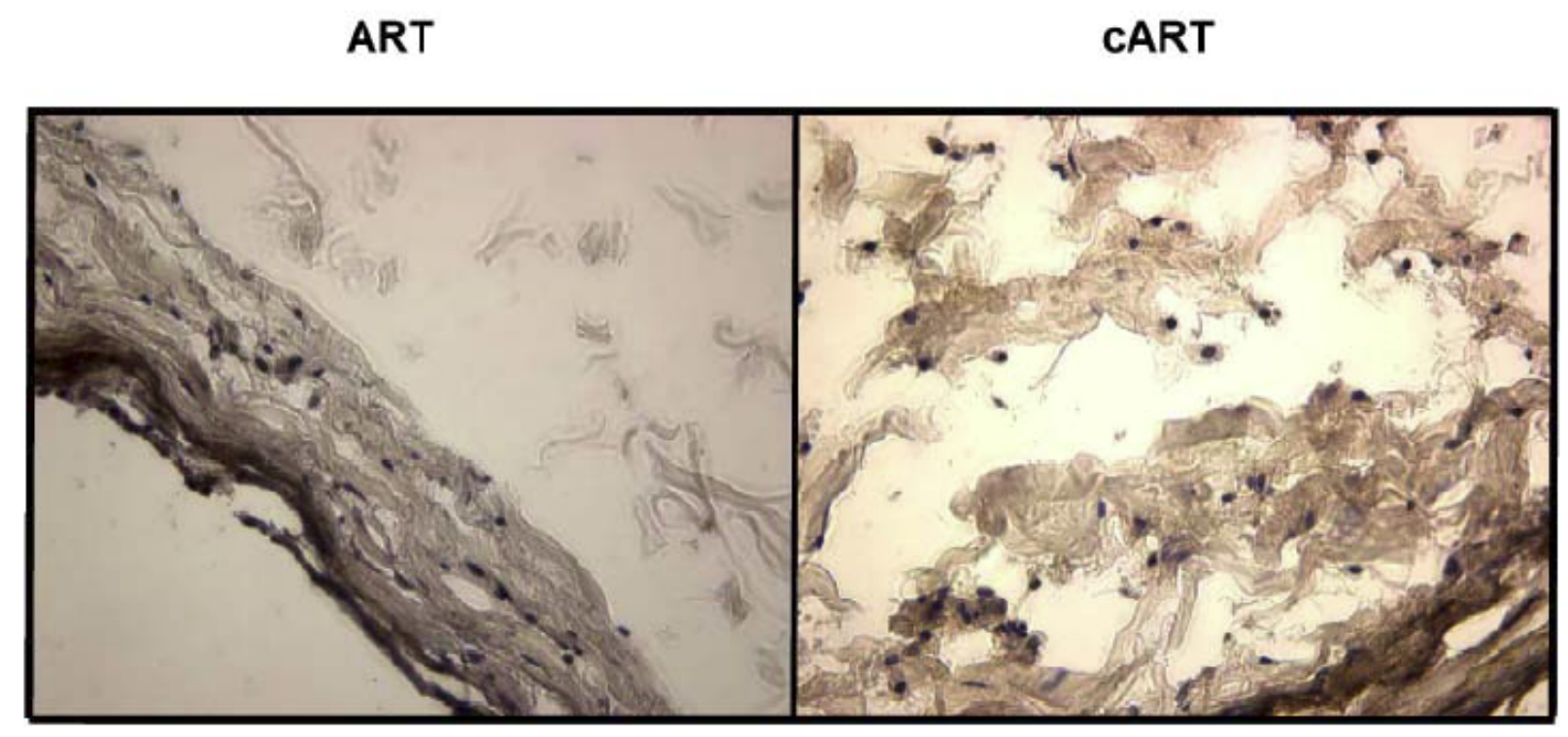

Figure F. 61 Representative Golgi complex images from 72 hour ART vs. cART experiment performed on 04/26/2007. Nuclei are stained blue and Golgi complex positive cells are stained brown. 


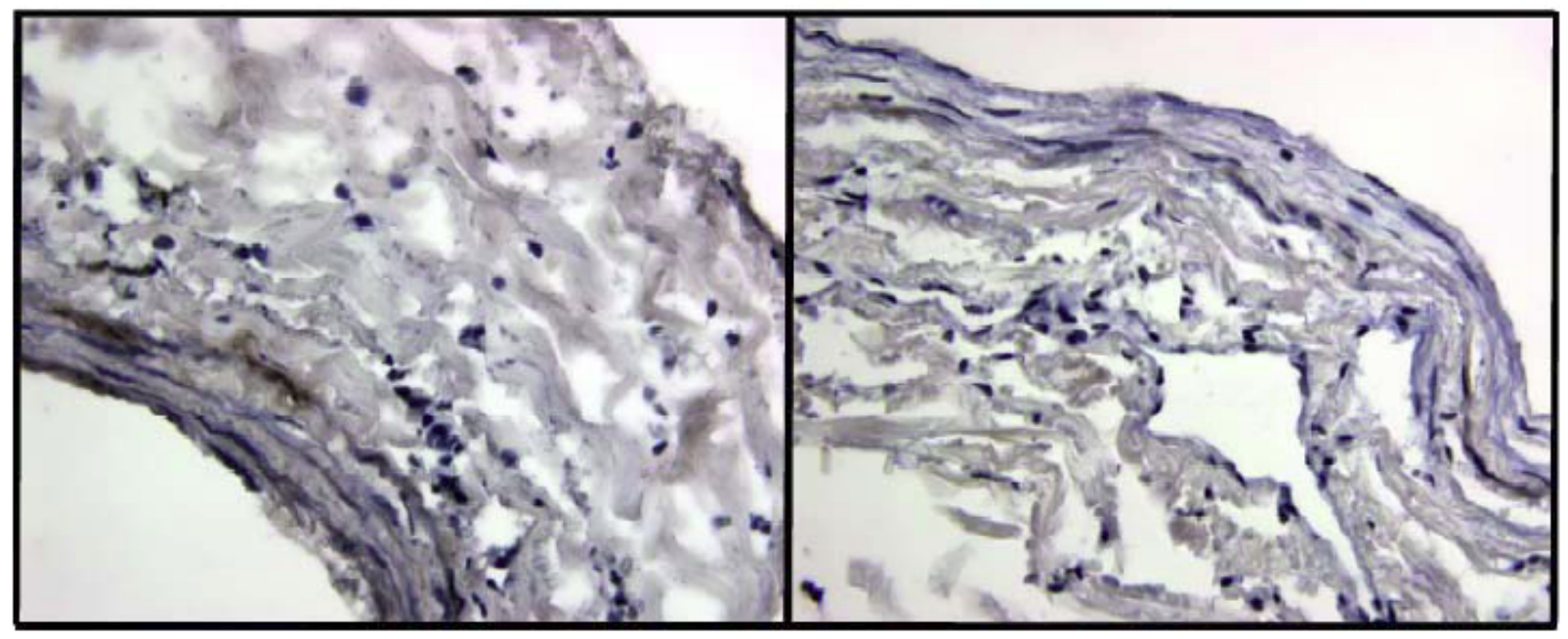

Figure F. 62 Representative Golgi complex images from 24 hour ART vs. wART experiment performed on 10/23/2006. Nuclei are stained blue and Golgi complex positive cells are stained brown.

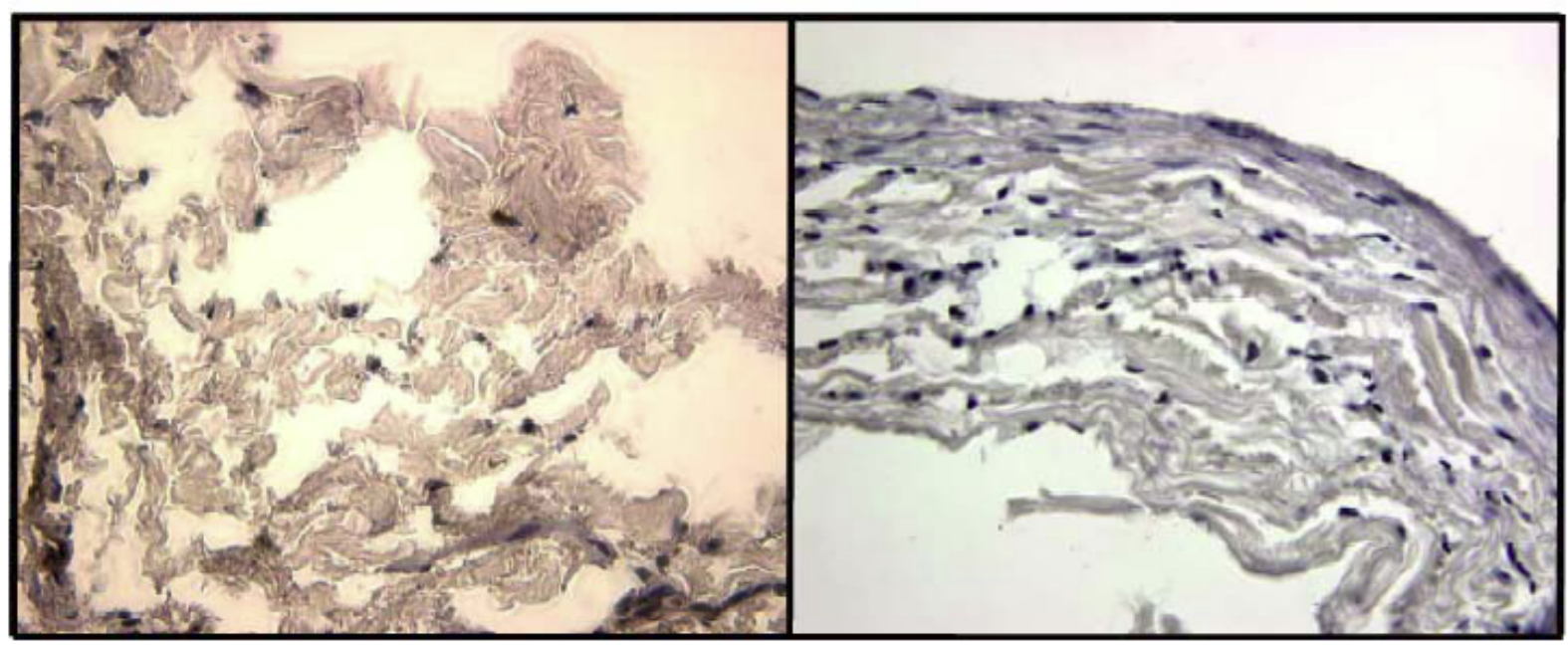

Figure F. 63 Representative Golgi complex images from 24 hour ART vs. wART experiment performed on 10/30/2006. Nuclei are stained blue and Golgi complex positive cells are stained brown. 


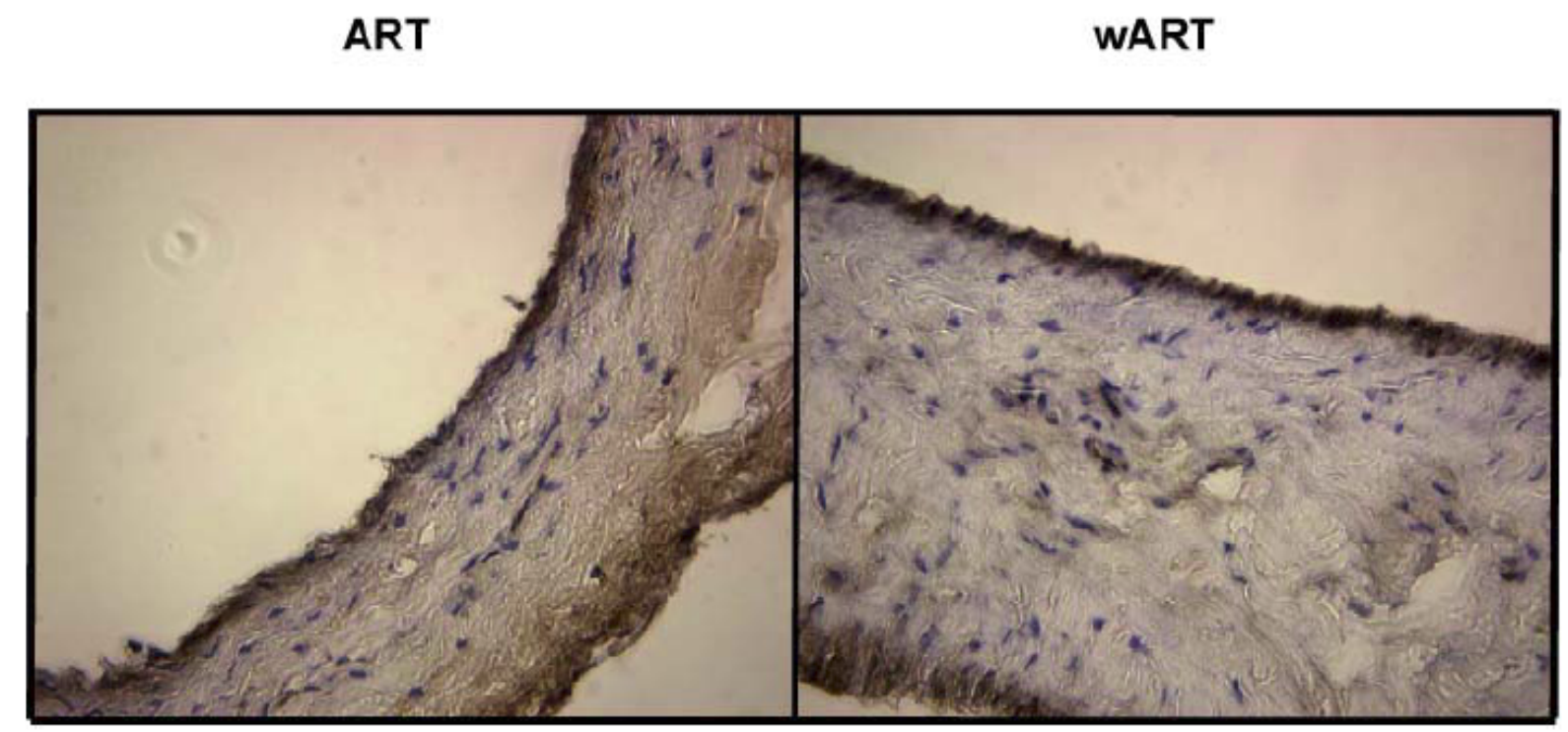

Figure F. 64 Representative Golgi complex images from 24 hour ART vs. wART experiment performed on 11/06/2006. Nuclei are stained blue and Golgi complex positive cells are stained brown.

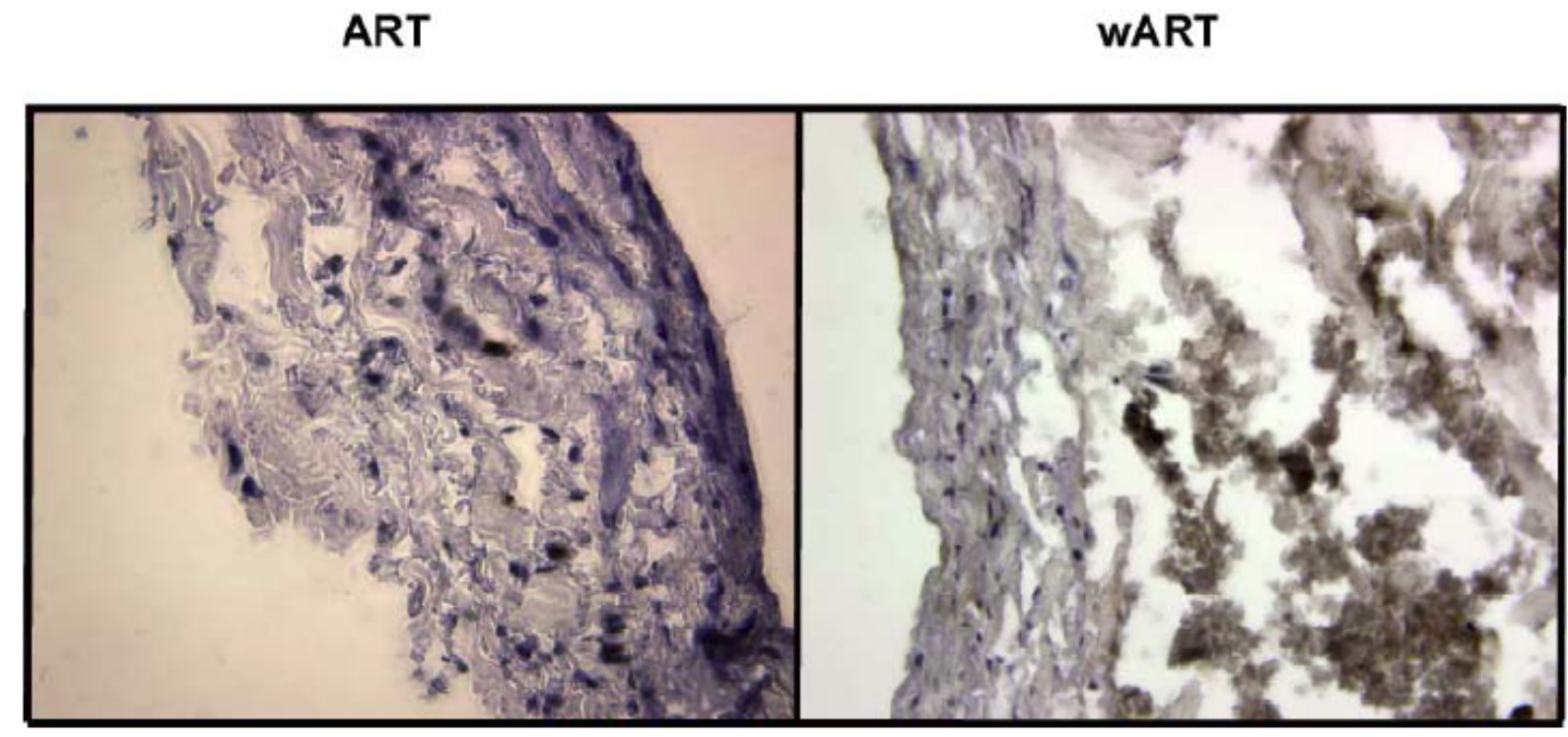

Figure F. 65 Representative Golgi complex images from 24 hour ART vs. wART experiment performed on 11/13/2006. Nuclei are stained blue and Golgi complex positive cells are stained brown. 


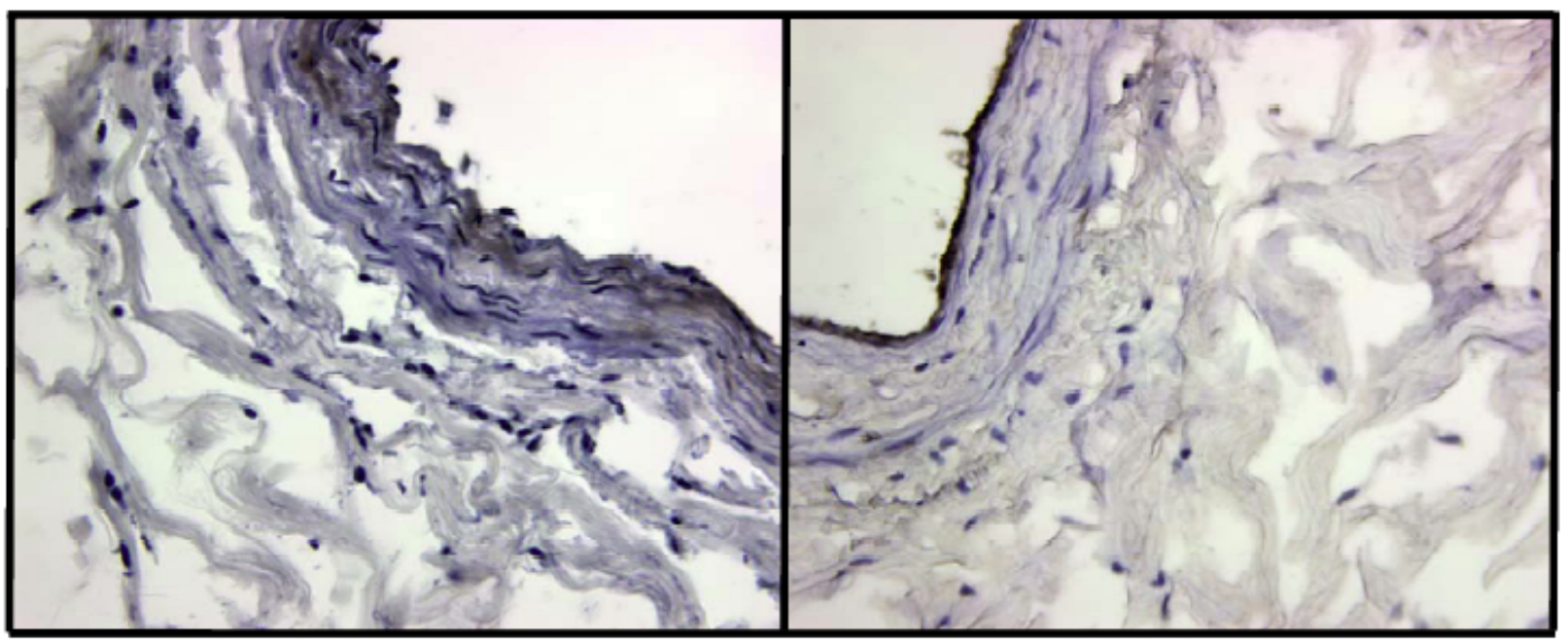

Figure F. 66 Representative Golgi complex images from 24 hour ART vs. wART experiment performed on 11/20/2006. Nuclei are stained blue and Golgi complex positive cells are stained brown.

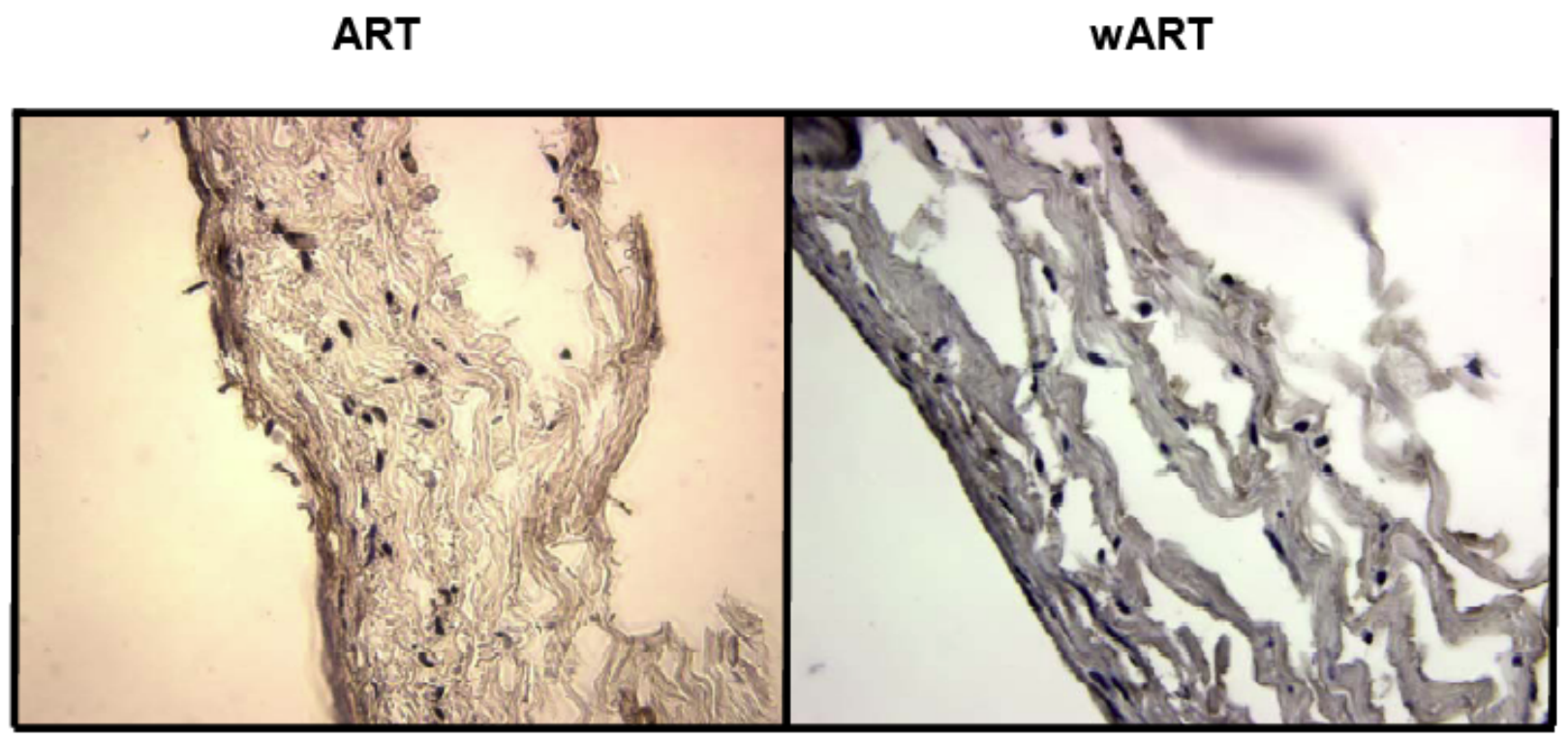

Figure F. 67 Representative Golgi complex images from 24 hour ART vs. wART experiment performed on 11/27/2006. Nuclei are stained blue and Golgi complex positive cells are stained brown. 
Table F. 11 Mean values of percent Golgi complex positive cells for all ex vivo experiments.

\begin{tabular}{ccccccccc} 
& \multicolumn{2}{c}{ VEN vs. ART 24 hrs } & \multicolumn{2}{c}{ ART vs. cART 24 hrs } & \multicolumn{2}{c}{ ART vs. cART 72 hrs } & \multicolumn{2}{c}{ ART vs. wART 24 hrs } \\
Expt \# & VEN & ART & ART & CART & ART & cART & ART & wART \\
$\mathbf{1}$ & 51.4446 & 40.8359 & 40.9899 & 25 & 47 & 41.0011 & 47.8893 & 47.1086 \\
$\mathbf{2}$ & 21.81 & 43.2144 & 28.1056 & 20.6703 & 51.4639 & 40.5604 & 46.2221 & 32 \\
$\mathbf{3}$ & 19.8375 & 78.6002 & 33.4402 & 29.5534 & 54.7943 & 43.9356 & 28.715 & 24.176 \\
$\mathbf{4}$ & 27.2568 & 55.8537 & 23.5913 & 30.6032 & 50 & 42.4608 & 36.7115 & 37.3016 \\
$\mathbf{5}$ & 11.121 & 39.5646 & 51.1614 & 39.3182 & 45.4987 & 53.0201 & 31.8049 & 26.6867 \\
$\mathbf{6}$ & - & - & - & - & - & - & 33.1479 & 37.1068 \\
& & & & & & & & \\
mean & 26.29398 & 51.61376 & 35.45768 & 29.02902 & 49.75138 & 44.1956 & 37.41512 & 34.0632833 \\
SE & 6.802545 & 7.3421346 & 4.879275 & 3.120268 & 1.644399 & 2.28438658 & 3.230801 & 3.39783984
\end{tabular}

Table F. 12 Microsoft Excel output for student's t-test performed on the mean percent Golgi complex positive cells from 24 hour VEN vs. ART ex vivo experiments.

t-Test: Paired Two Sample for Means

\begin{tabular}{lrr}
\hline & VEN & \multicolumn{1}{c}{ ART } \\
\hline Mean & 26.29398 & 51.61376 \\
Variance & 231.3731 & 269.5347 \\
Observations & 5 & 5 \\
Pearson Correlation & -0.22098 & \\
Hypothesized Mean Difference & 0 & \\
df & 4 & \\
t Stat & -2.28995 & \\
P(T<=t) one-tail & 0.041931 & \\
t Critical one-tail & 2.131847 & \\
P(T<=t) two-tail & 0.083862 & \\
t Critical two-tail & 2.776445 & \\
\hline
\end{tabular}


Table F. 13 Microsoft Excel output for student's t-test performed on the mean percent Golgi complex positive cells from 24 hour ART vs. cART ex vivo experiments.

t-Test: Paired Two Sample for

Means

\begin{tabular}{lrr}
\hline & \multicolumn{1}{c}{ ART } & \multicolumn{1}{c}{ cART } \\
\hline Mean & 35.45768 & 29.02902 \\
Variance & 119.0366 & 48.68036 \\
Observations & 5 & 5 \\
Pearson Correlation & 0.594448 & \\
Hypothesized Mean Difference & 0 & \\
df & 4 & \\
t Stat & 1.635898 & \\
P(T<=t) one-tail & 0.088601 & \\
t Critical one-tail & 2.131847 & \\
$\mathrm{P}(T<=t)$ two-tail & 0.177201 & \\
t Critical two-tail & 2.776445 & \\
\hline
\end{tabular}

Table F. 14 Microsoft Excel output for student's t-test performed on the mean percent Golgi complex positive cells from 72 hour ART vs. cART ex vivo experiments.

t-Test: Paired Two Sample for Means

\begin{tabular}{lrr}
\hline & \multicolumn{1}{c}{ ART } & \multicolumn{1}{c}{ CART } \\
\hline Mean & 49.75138 & 44.1956 \\
Variance & 13.52024 & 26.09211 \\
Observations & 5 & 5 \\
Pearson Correlation & -0.48858 & \\
Hypothesized Mean Difference & 0 & \\
df & 4 & \\
t Stat & 1.631718 & \\
P(T<=t) one-tail & 0.089037 & \\
t Critical one-tail & 2.131847 & \\
P(T<=t) two-tail & 0.178075 & \\
t Critical two-tail & 2.776445 & \\
\hline
\end{tabular}


Table F. 15 Microsoft Excel output for student's t-test performed on the mean percent Golgi complex positive cells from 24 hour ART vs. WART ex vivo experiments.

t-Test: Paired Two Sample for

Means

\begin{tabular}{lrr}
\hline & \multicolumn{1}{c}{ ART } & WART \\
\hline Mean & 37.41512 & 34.06328 \\
Variance & 62.62845 & 69.27189 \\
Observations & 6 & 6 \\
Pearson Correlation & 0.700225 & \\
Hypothesized Mean Difference & \multicolumn{2}{c}{0} \\
df & 5 & \\
t Stat & 1.30375 & \\
P $(T<=t)$ one-tail & 0.124557 & \\
t Critical one-tail & 2.015048 & \\
P $(T<=t)$ two-tail & 0.249115 & \\
t Critical two-tail & 2.570582 & \\
\hline
\end{tabular}




\section{APPENDIX G}

\section{IN VIVO EXPERIMENTAL DATA}

In this Appendix an image is provided for each implanted AVG from the last 4 (see Table 4.1) in vivo experiments. These images were taken immediately upon re-establishing flow in the AVG after implantation. A representative MPC staining image for each of the AVGs included in the morphometric analysis (sham: $\mathrm{N}=6$; spun: $\mathrm{N}=4$ ) is also provided in this Appendix. Following the MPC images, the measured intimal-to-medial thickness ratios are tabulated. The corresponding statistical software output table is also provided for the student's t-test that was performed. 


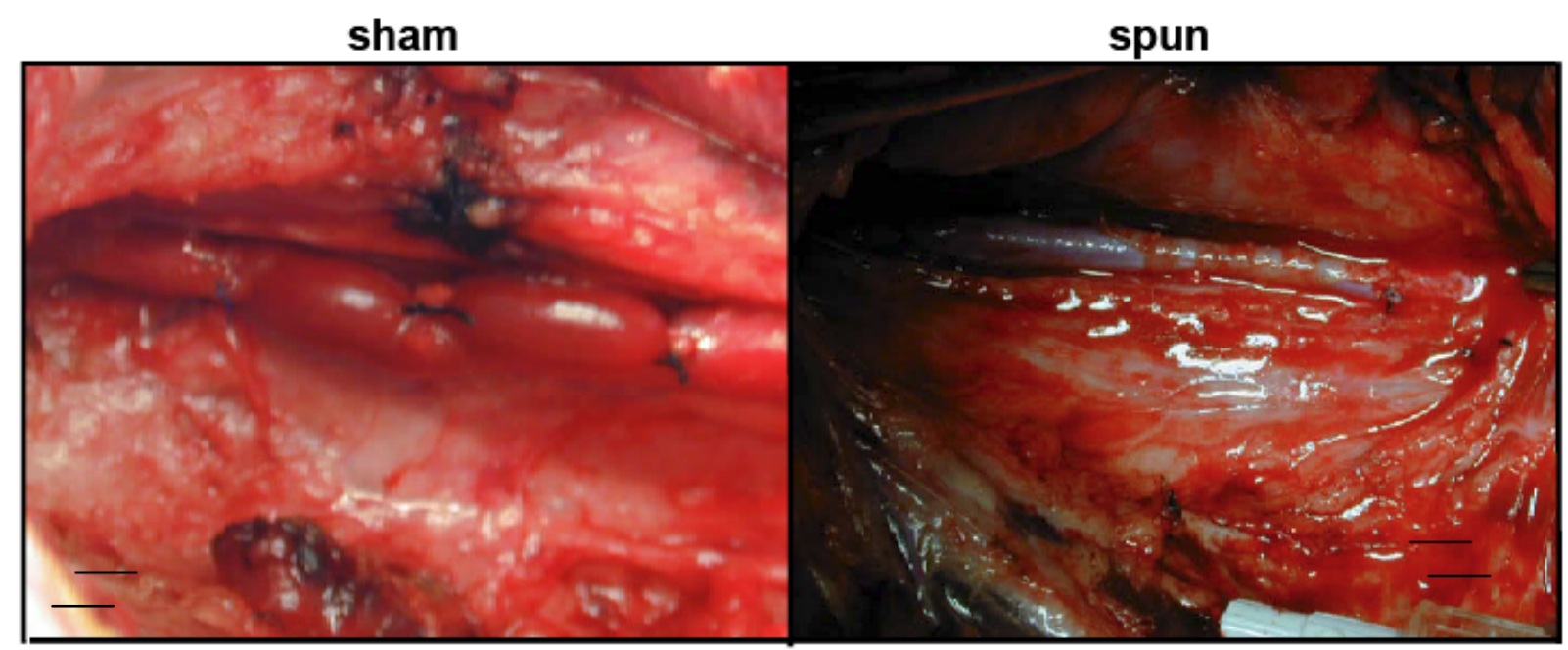

Figure G. 1 Image of both sham and spun AVGs implanted as carotid interposition grafts on 06/11/2007. Scale bar is $1 \mathrm{~cm}$.

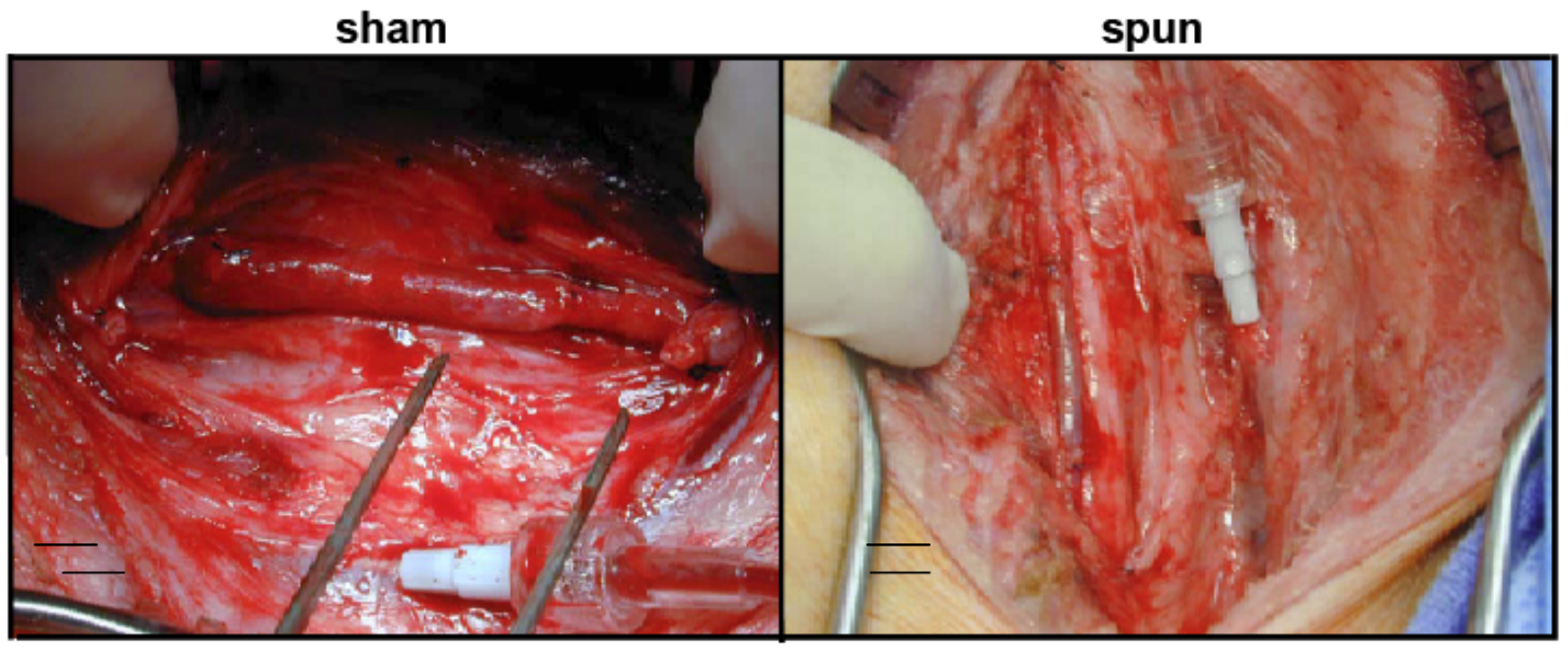

Figure G. 2 Image of both sham and spun AVGs implanted as carotid interposition grafts on 06/26/2007. Scale bar is $1 \mathrm{~cm}$. 


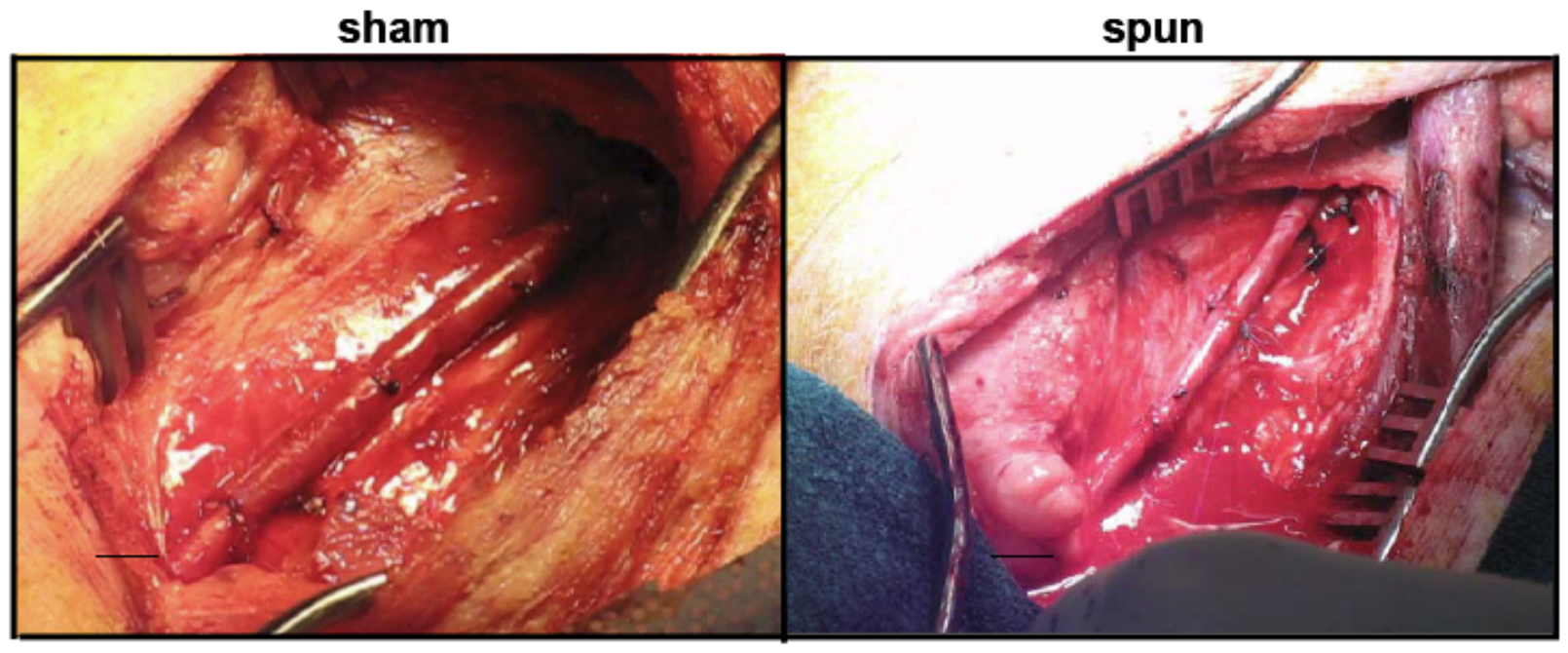

Figure G. 3 Image of both sham and spun AVGs implanted as carotid interposition grafts on 06/27/2007. Scale bar is $1 \mathrm{~cm}$.

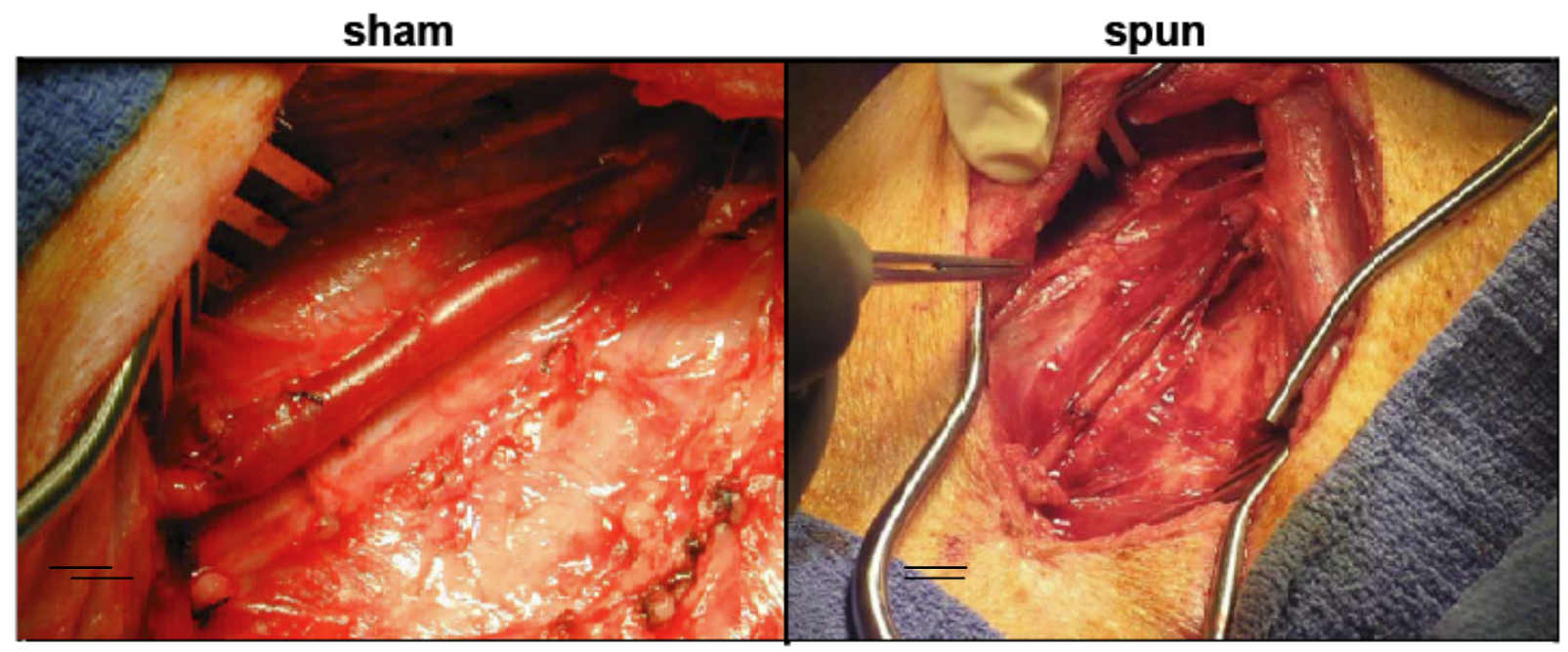

Figure G. 4 Image of both sham and spun AVGs implanted as carotid interposition grafts on 06/28/2007. Scale bar is $1 \mathrm{~cm}$. 


\section{sham}

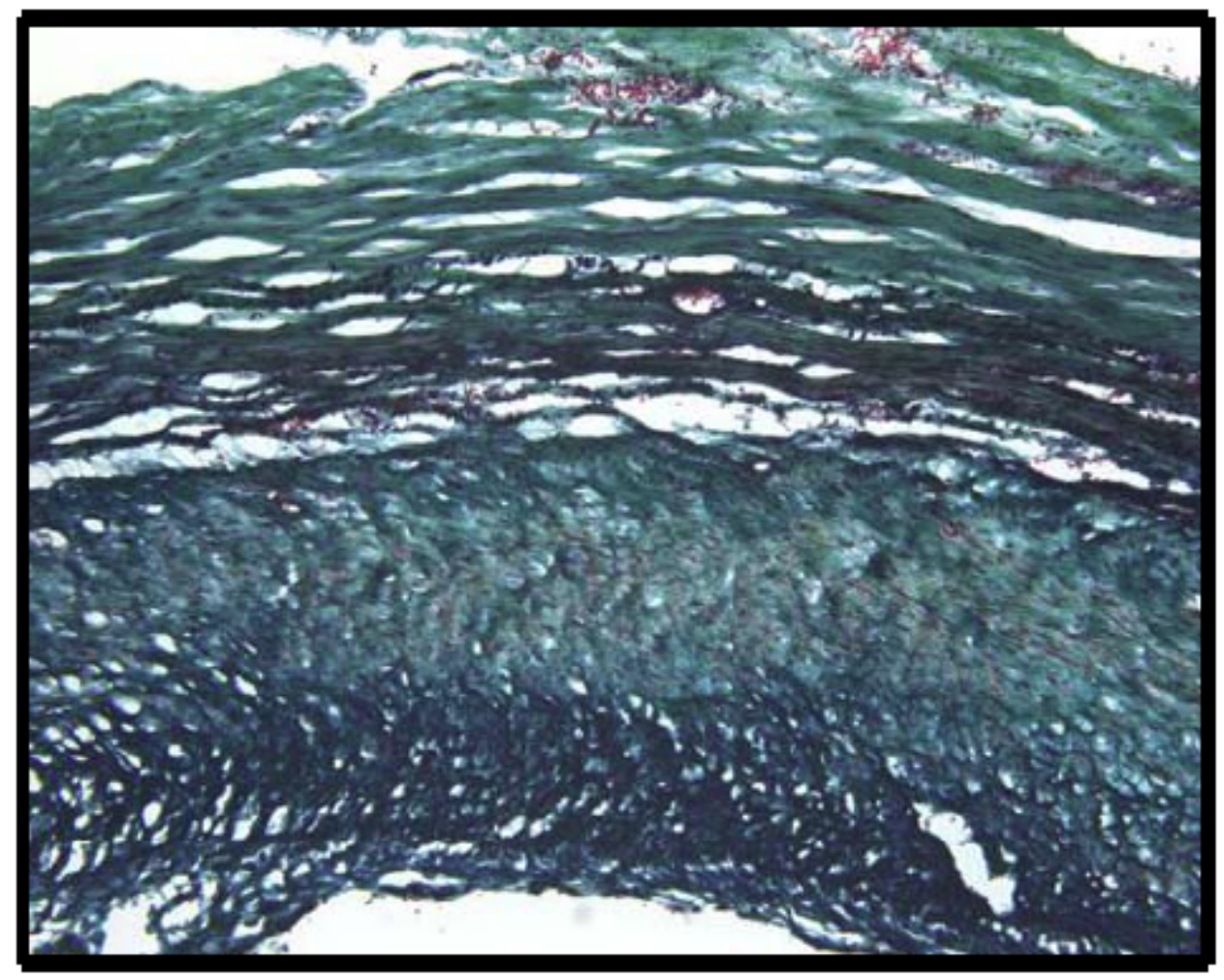

Figure G. 5 Representative image of MPC stained section from sham AVG implanted as carotid interposition graft on $12 / 04 / 2006$.

\section{sham}

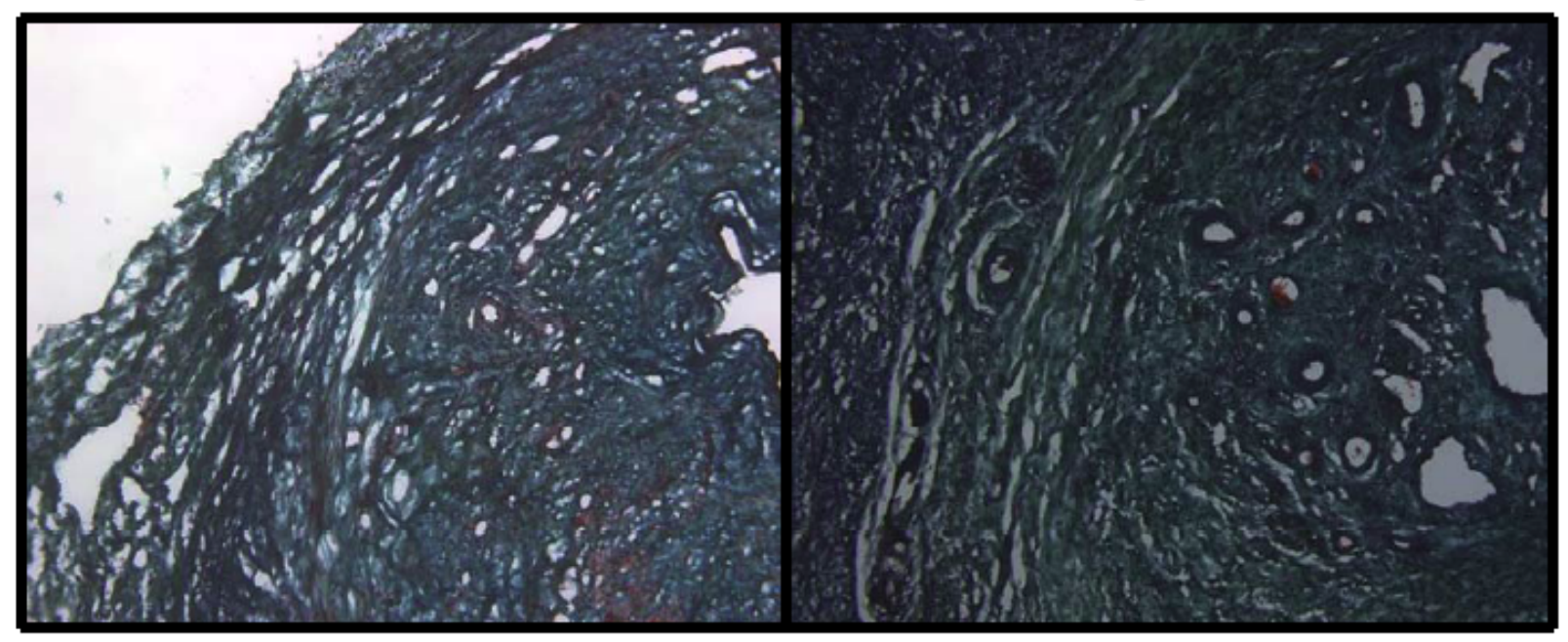

Figure G. 6 Representative images of MPC stained sections from both sham and spun AVGs implanted as carotid interposition grafts on 12/05/2006. 


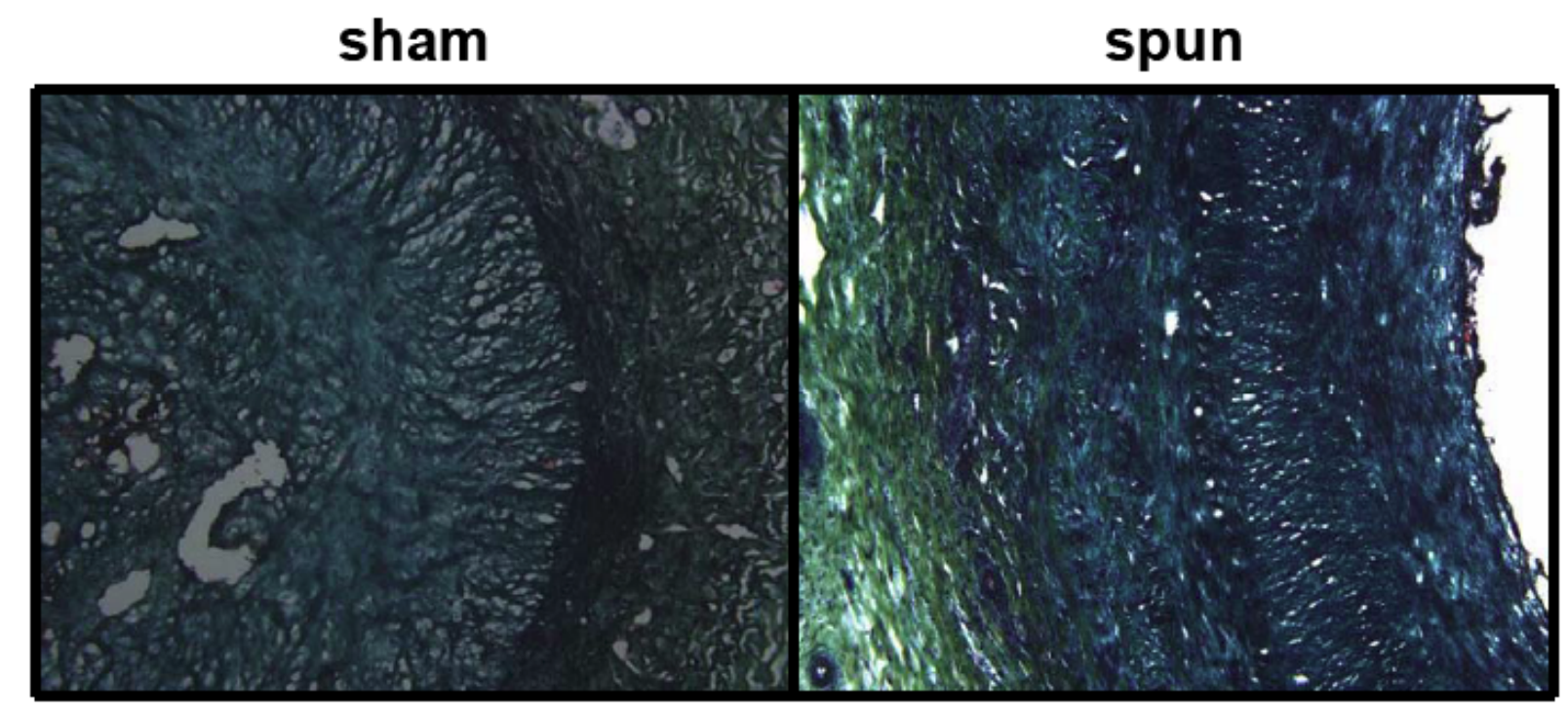

Figure G. 7 Representative images of MPC stained sections from both sham and spun AVGs implanted as carotid interposition grafts on 12/06/2006.

\section{sham spun}

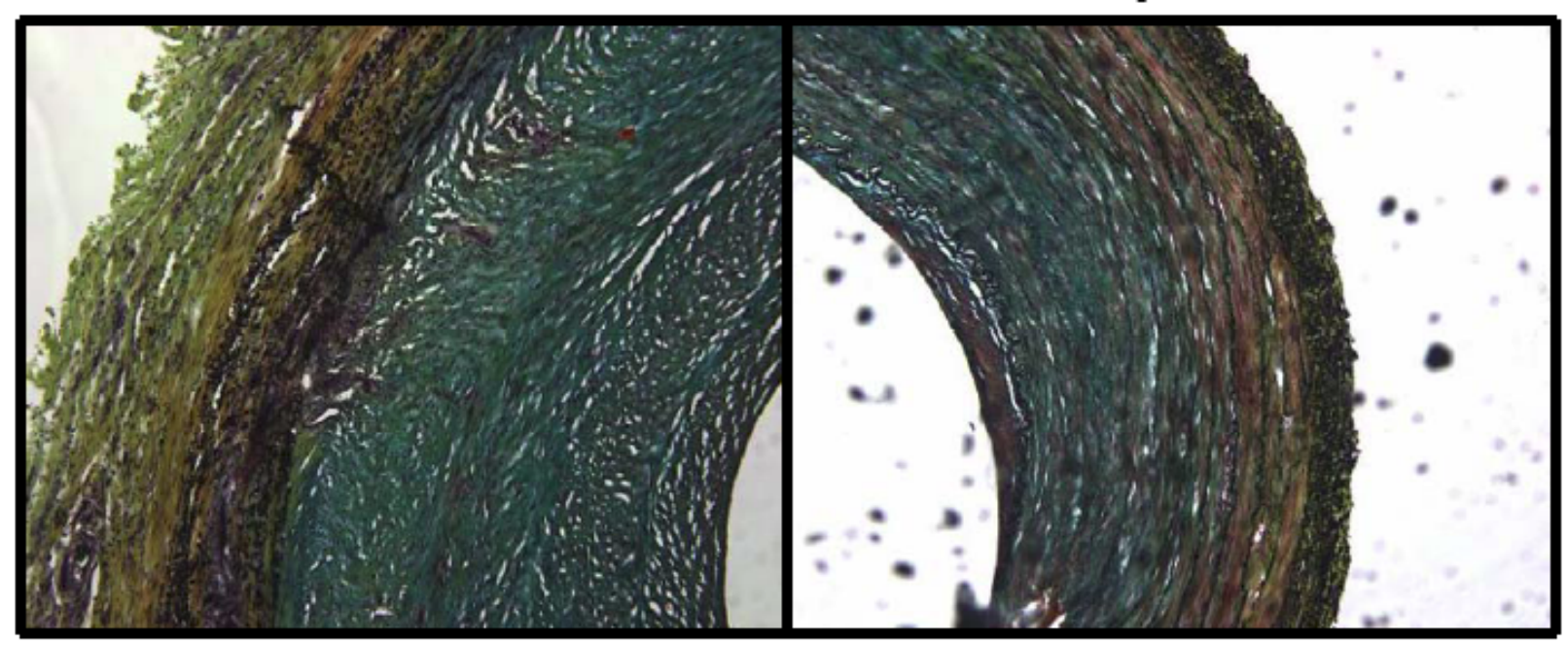

Figure G. 8 Representative images of MPC stained sections from both sham and spun AVGs implanted as carotid interposition grafts on 06/11/2007. 


\section{sham}

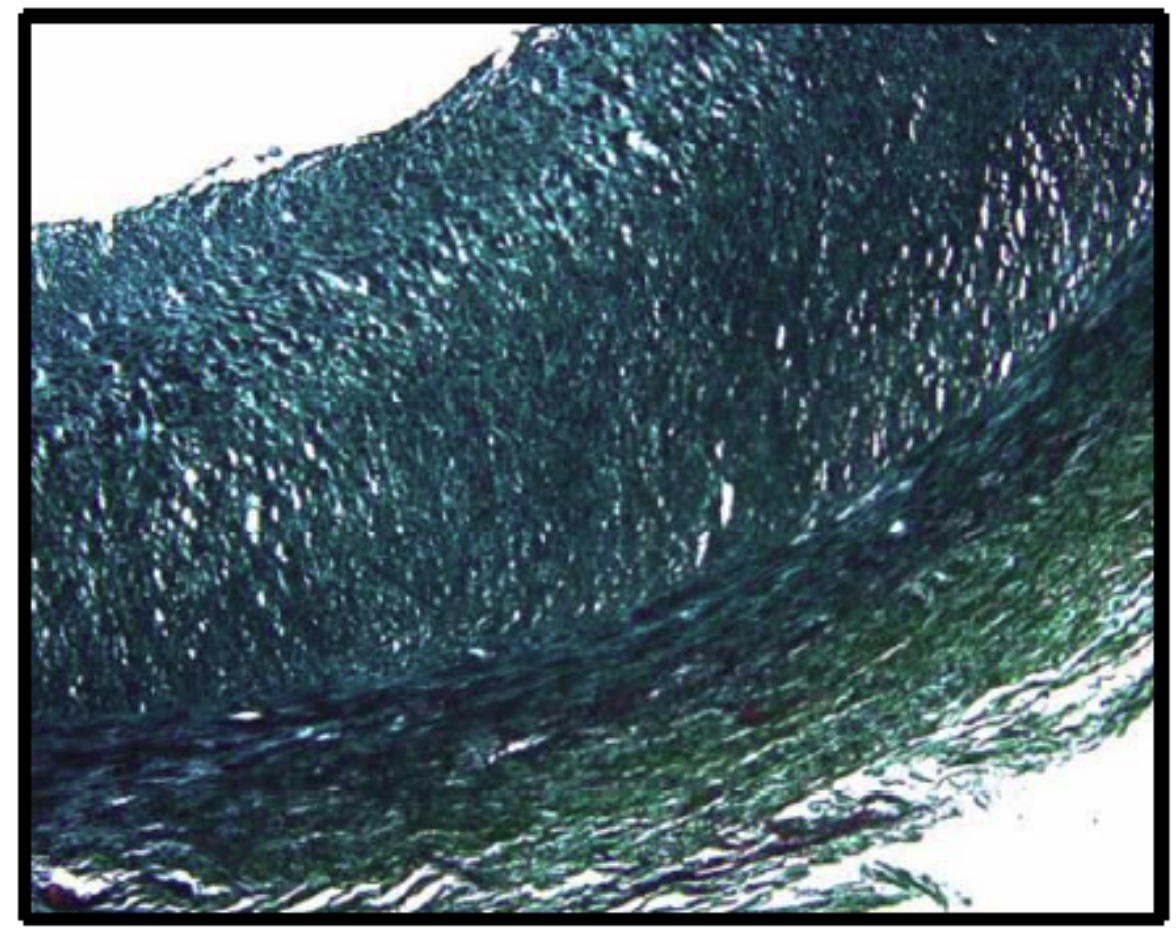

Figure G. 9 Representative image of MPC stained sections from sham AVG implanted as carotid interposition graft on $06 / 26 / 2007$.

\section{sham}

spun

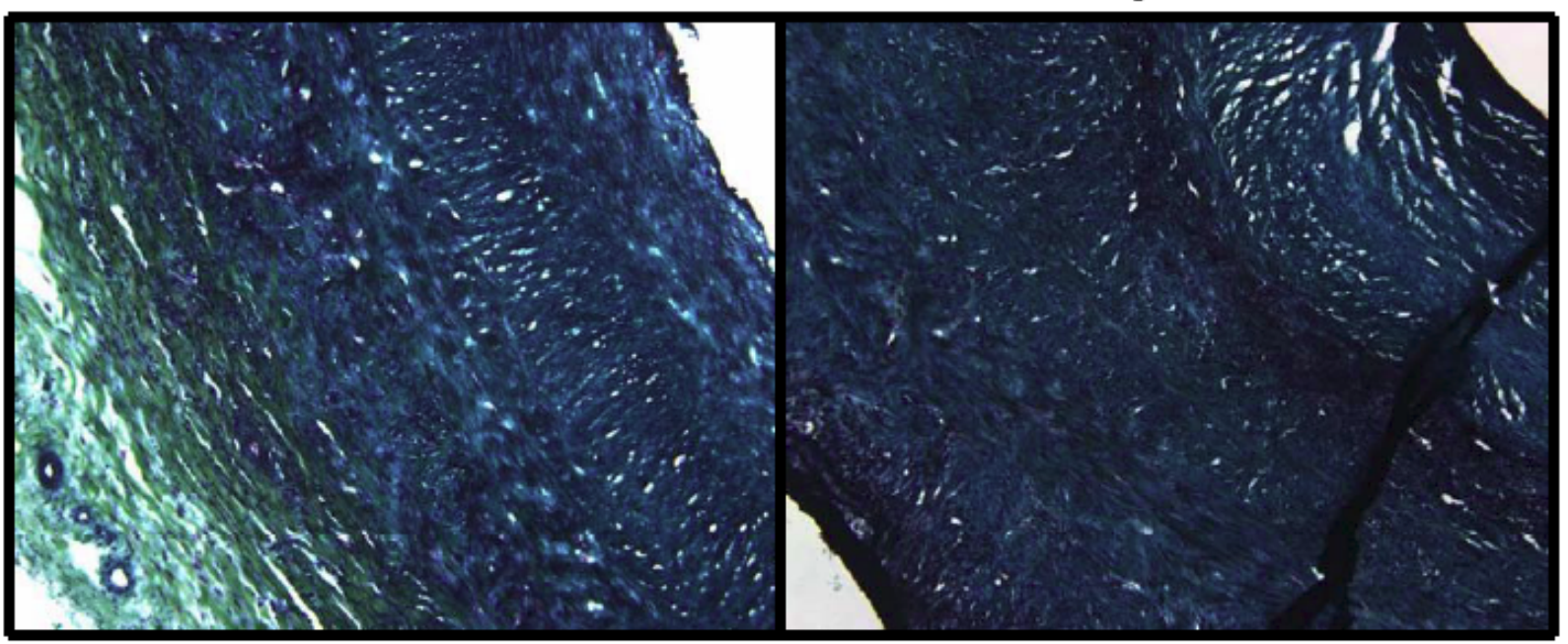

Figure G. 10 Representative images of MPC stained sections from both sham and spun AVGs implanted as carotid interposition grafts on 06/27/2007. 
Table G. 1 Mean intimal-to-medial thickness ratios from morphometric analysis of MPC stained sections from in vivo experiments.

\begin{tabular}{|c|c|c|}
\hline Expt \# & Sham & Spun \\
\hline 1 & 2.5625 & 2.8951 \\
\hline 2 & 3.0476 & 2.4086 \\
\hline 3 & 4.2222 & 3.4722 \\
\hline 4 & 3.5417 & 2.2458 \\
\hline 5 & 3.2083 & \\
\hline 6 & 3.1667 & \\
\hline Mean & 3.2915 & 2.755425 \\
\hline SEM & 0.555379 & 0.551749 \\
\hline
\end{tabular}

Table G. 2 Microsoft Excel output for student's t-test performed on the mean intimal-to-medial thickness ratio data from the in vivo experiments.

t-Test: Two-Sample Assuming Equal

Variances

\begin{tabular}{lrr}
\hline & \multicolumn{1}{c}{ sham } & \multicolumn{1}{c}{ spun } \\
\hline Mean & 3.2915 & 2.755425 \\
Variance & 0.308446 & 0.304426909 \\
Observations & 6 & 4 \\
Pooled Variance & 0.306939 & \\
Hypothesized Mean Difference & 0 & \\
df & 8 & \\
t Stat & 1.499013 & \\
$\mathrm{P}(\mathrm{T}<=\mathrm{t})$ one-tail & 0.086127 & \\
t Critical one-tail & 1.859548 & \\
$\mathrm{P}(\mathrm{T}<=\mathrm{t})$ two-tail & 0.172254 & \\
t Critical two-tail & 2.306004 & \\
\hline
\end{tabular}




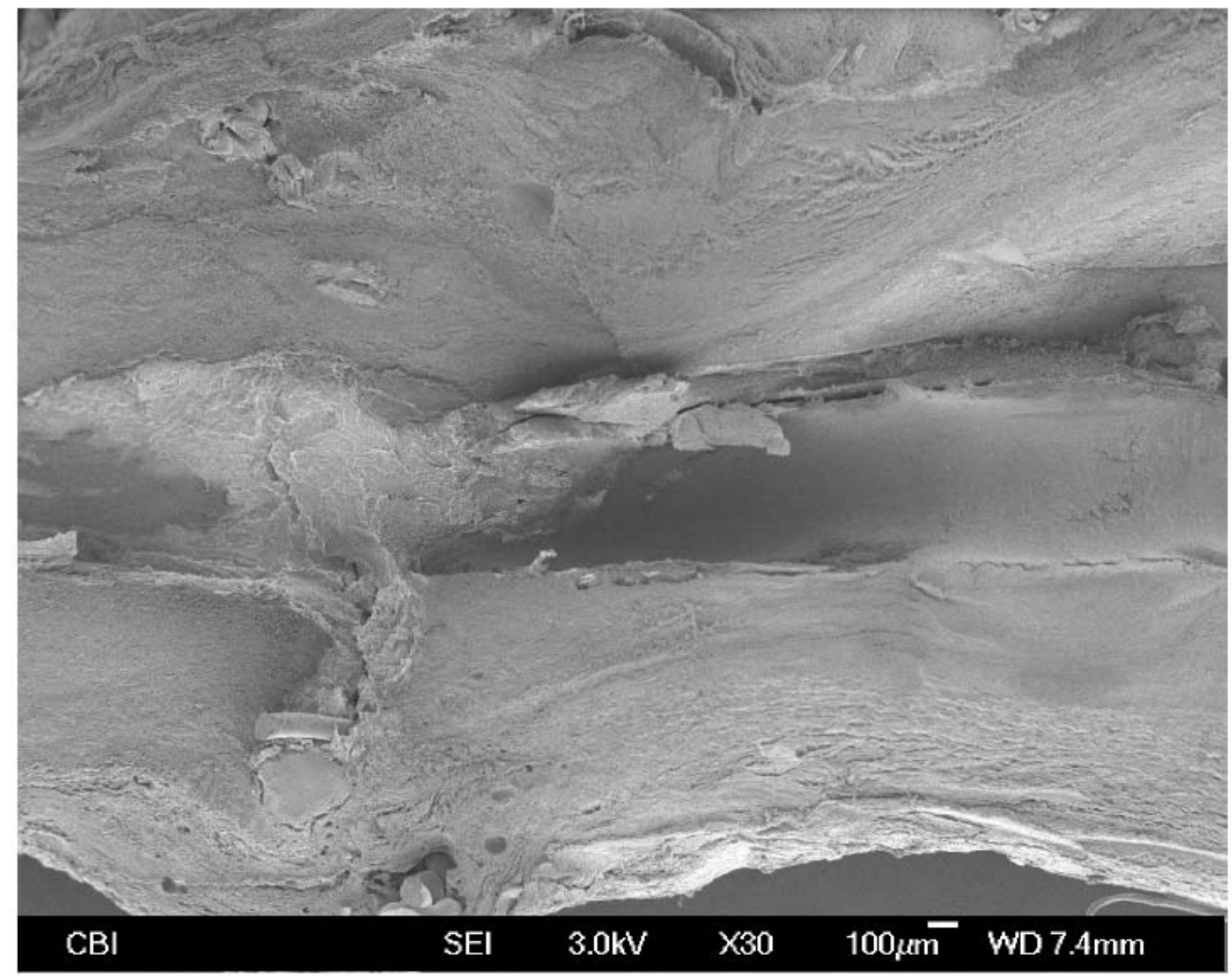

Figure G. 11 Low magnification SEM image of sham AVG implanted as a carotid interposition graft on $06 / 11 / 2007$. 


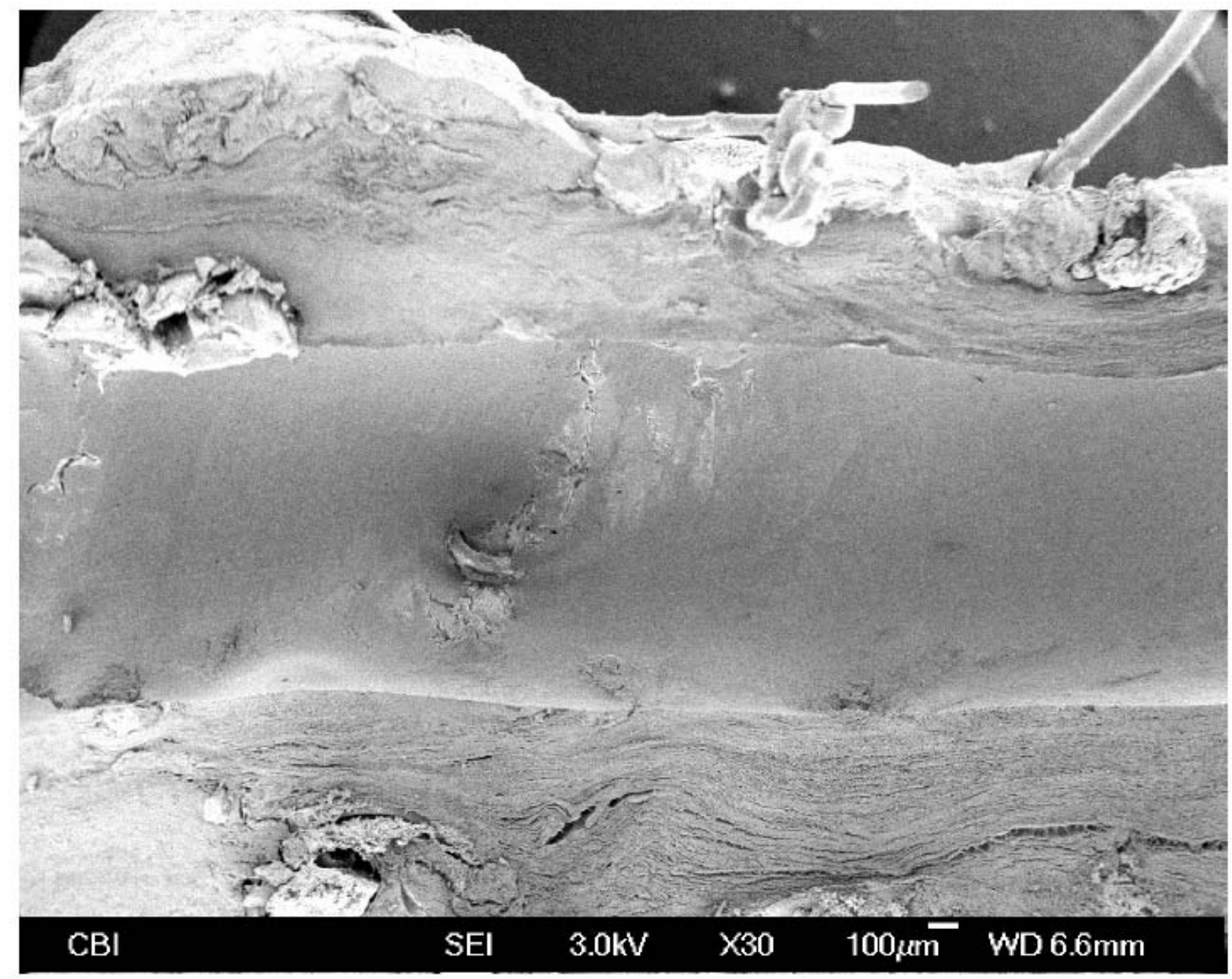

Figure G. 12 Low magnification SEM image of spun AVG implanted as a carotid interposition graft on 06/11/2007. 


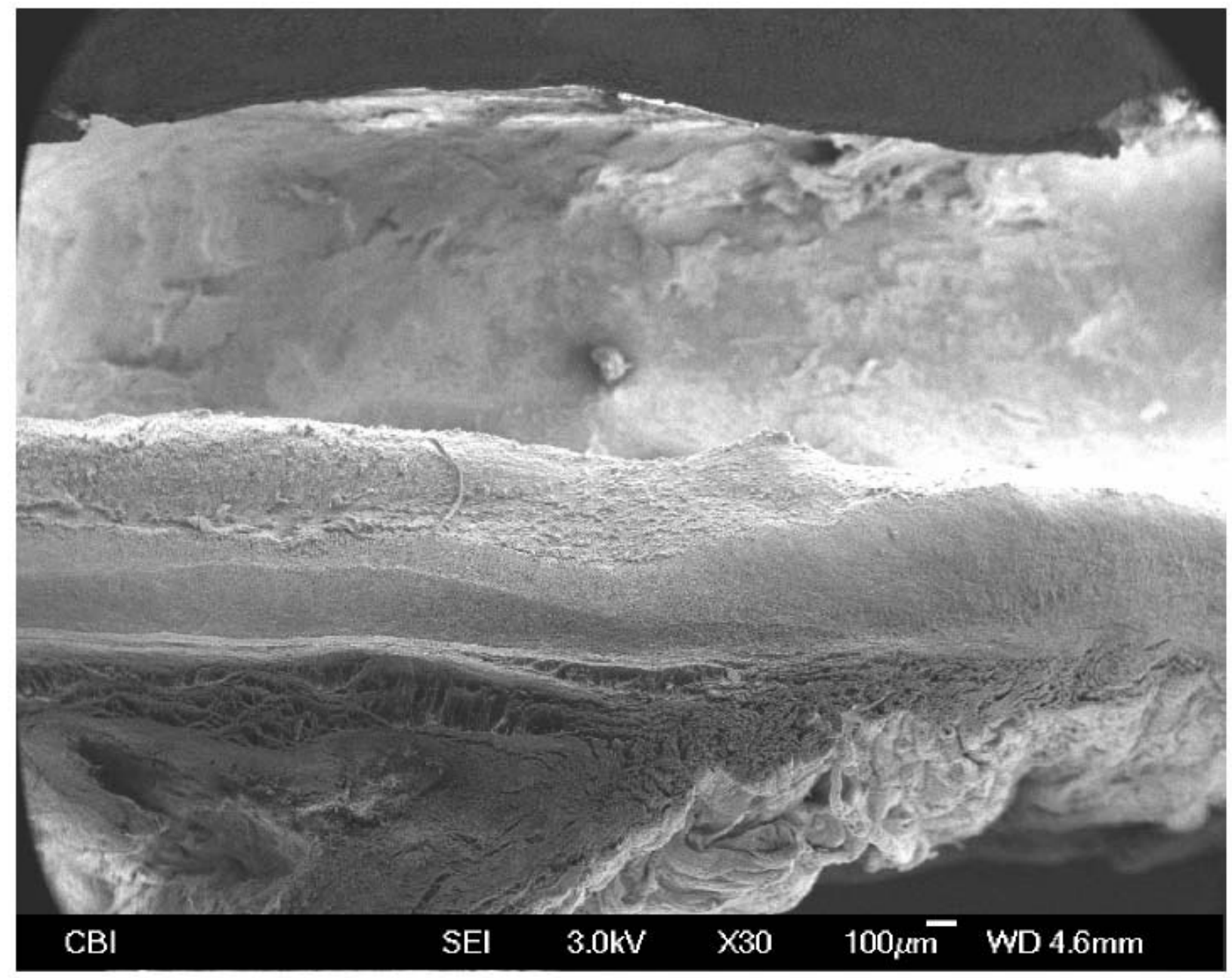

Figure G. 13 Low magnification SEM image of sham AVG implanted as a carotid interposition graft on 06/26/2007. 


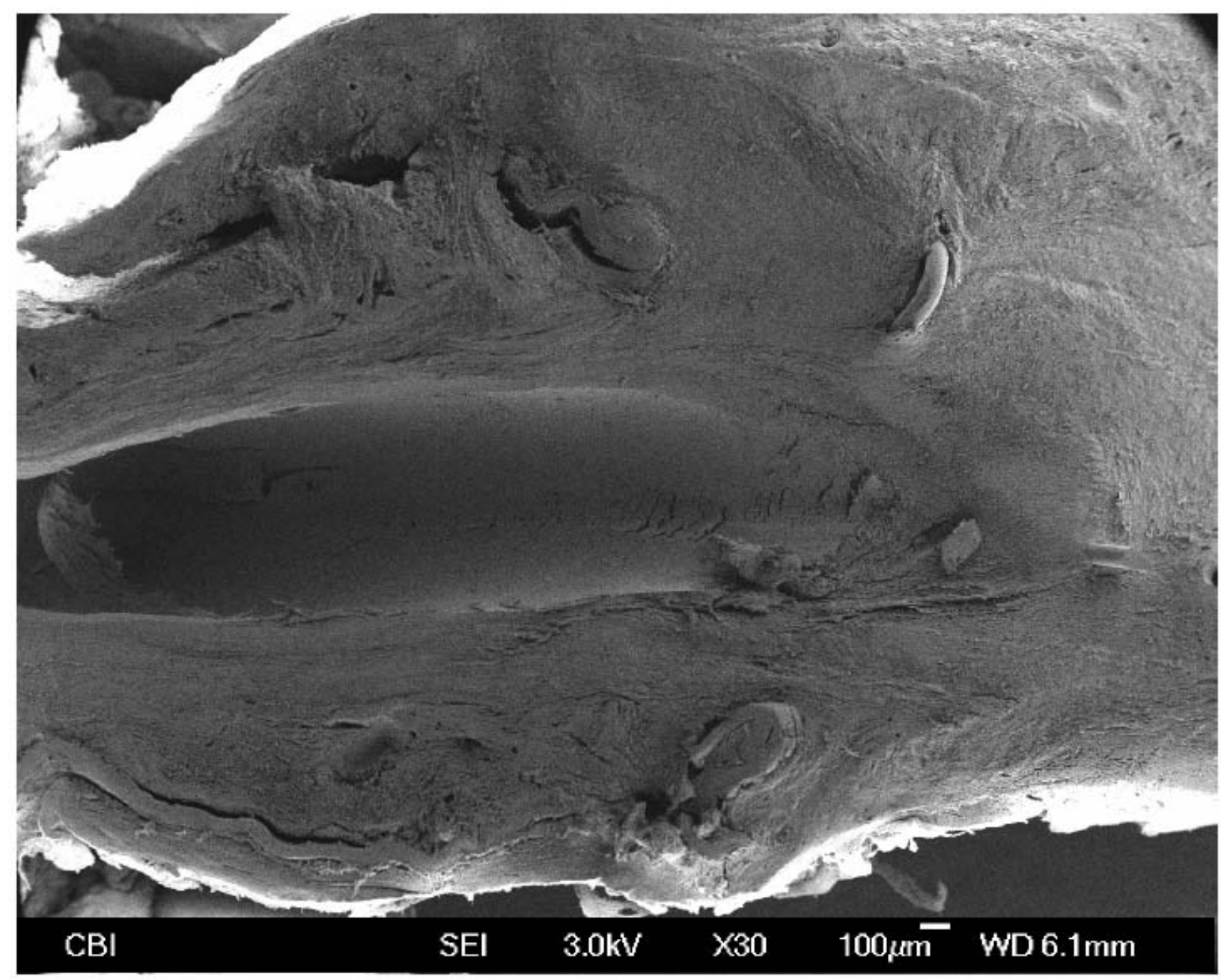

Figure G. 14 Low magnification SEM image of spun AVG implanted as a carotid interposition graft on 06/26/2007. 


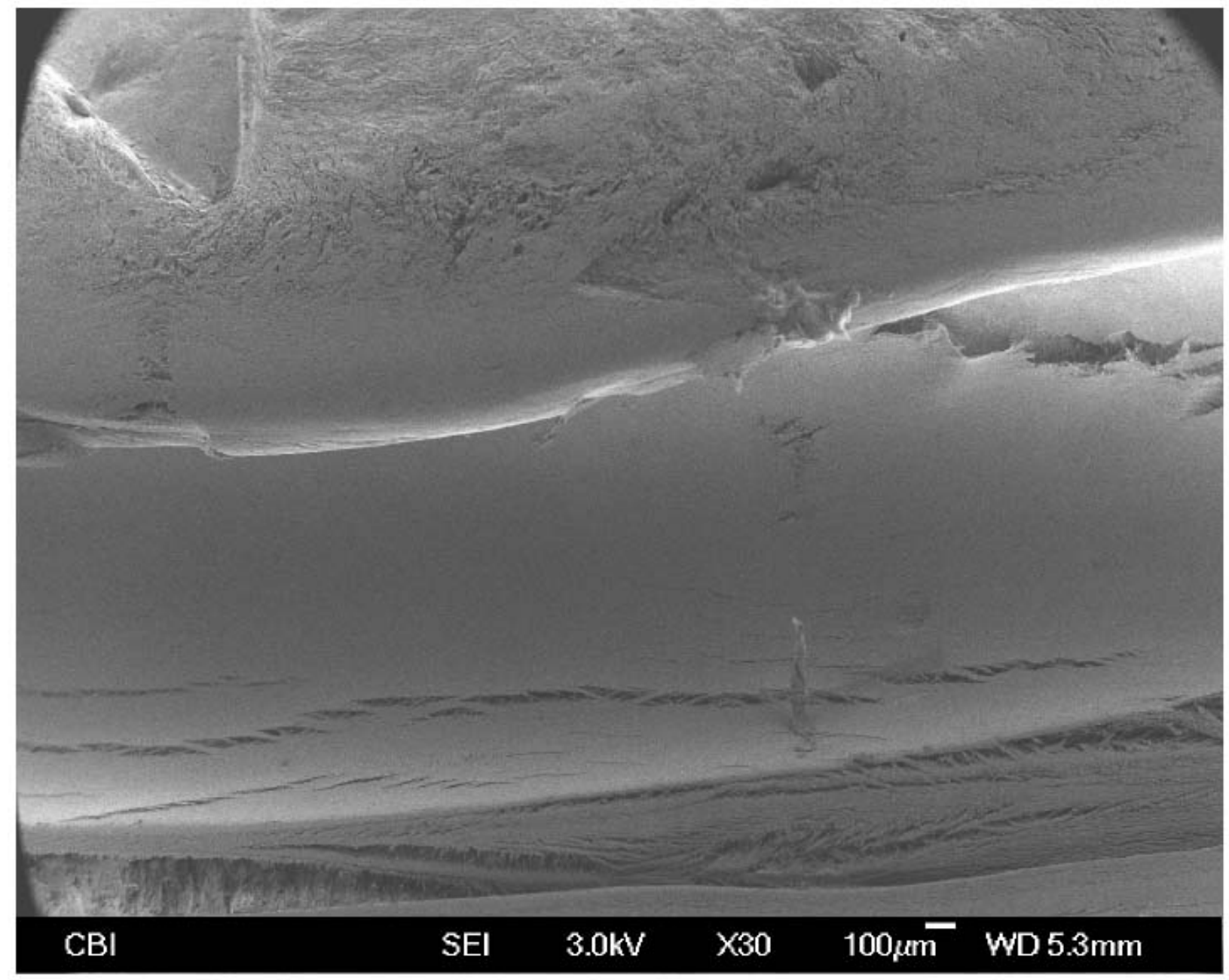

Figure G. 15 Low magnification SEM image of spun AVG implanted as a carotid interposition graft on 06/28/2007. 


\section{APPENDIX H}

\section{TROUBLESHOOTING MOLECULAR BIOLOGICAL ANALYSES}

The endpoints originally proposed in Section 1.2 as early markers of IH included many proteins that we wished to quantitatively assess at the gene expression and the protein translation levels via quantitative polymerase chain reaction (Q-PCR) and western blotting techniques, respectively. However, these assays were found to be extremely difficult and troubleshooting them was beyond the scope of this dissertation. It was then decided, and agreed upon by all committee members, that immunohistochemistry (IHC) was an acceptable technique for detecting the expression of some of the proteins of interest. In order to obtain quantifiable results via IHC, we chose only endpoints that were expressed intracellularly or matricellularly. That is, we chose only endpoints that would allow positively stained cells to be counted either manually or with an appropriate software application. For this we focused our attention on detecting the expression of: TNC, TSP, SPARC, Mig-2, ILK, TUNEL, PCNA, and Golgi complex. Figure H. 1 shows a flowchart of the techniques that were attempted in order to troubleshoot the IHC, western blotting, and Q-PCR techniques for these endpoints. The analyses that were successful, as well as the rationale for terminating the methods that were not successful are also outlined in Figure H. 1.

The first major hurdle that was encountered with the fluorescent IHC analysis stems from the fact that vascular tissue is inherently very auto-fluorescent, mainly due to the high elastin content. Auto-fluorescence prevented the detection of most proteins of interest because they 
were expressed in very small levels. In fact the only endpoint that was successfully evaluated with fluorescent IHC techniques was TUNEL. This was because the signal generated by the commercially available kit was stronger than the auto-fluorescent signal which allowed the exposure time to be reduced to the point that the auto-fluorescence was no longer visible. Fluorescent IHC images from several of the proteins that we attempted to detect are shown in Figure H. 2 to Figure H. 6. The very diffuse fluorescent signal seen in these images was not what we expected to see. Instead the positive signal should only co-localize with the nuclear staining. This was not the case, so this technique was abandoned for all other endpoints except TUNEL.

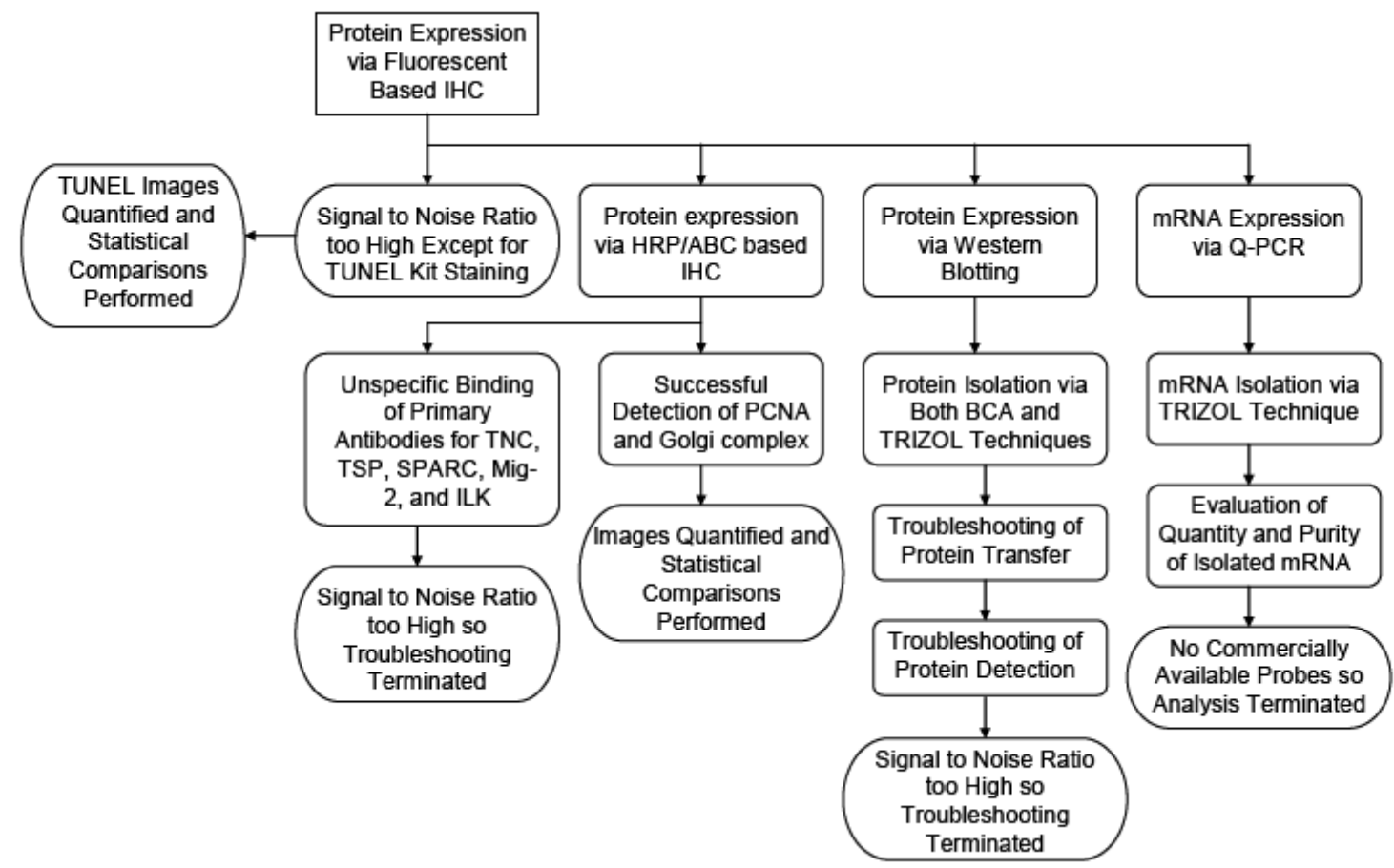

Figure H. 1 Flowchart depicting the molecular biological analyses that were either successfully evaluated, or attempted but discontinued because troubleshooting them was beyond the scope of this dissertation. 


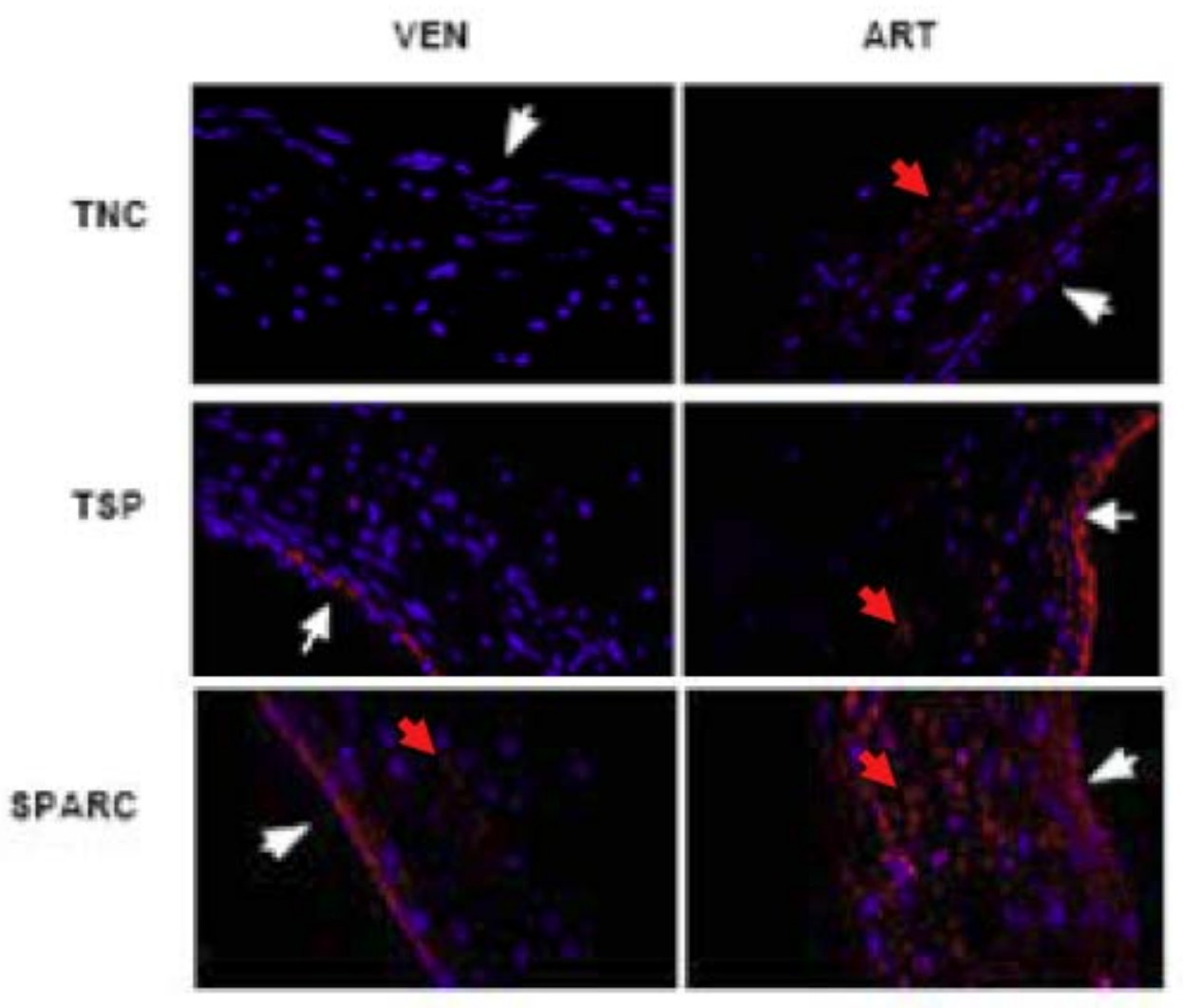

Figure H. 2 Representative immunohistochemistry images taken from a VEN vs. ART 24 hour experiment. In all images, red represents positive staining, and the nuclei label blue. Images were taken at 400x magnification under immersion oil. In all panels the white arrow designates the vessel lumen, and the red arrow indicates the observed autofluorescence. 
PCNA Analysis: PIJV ART 24 hours

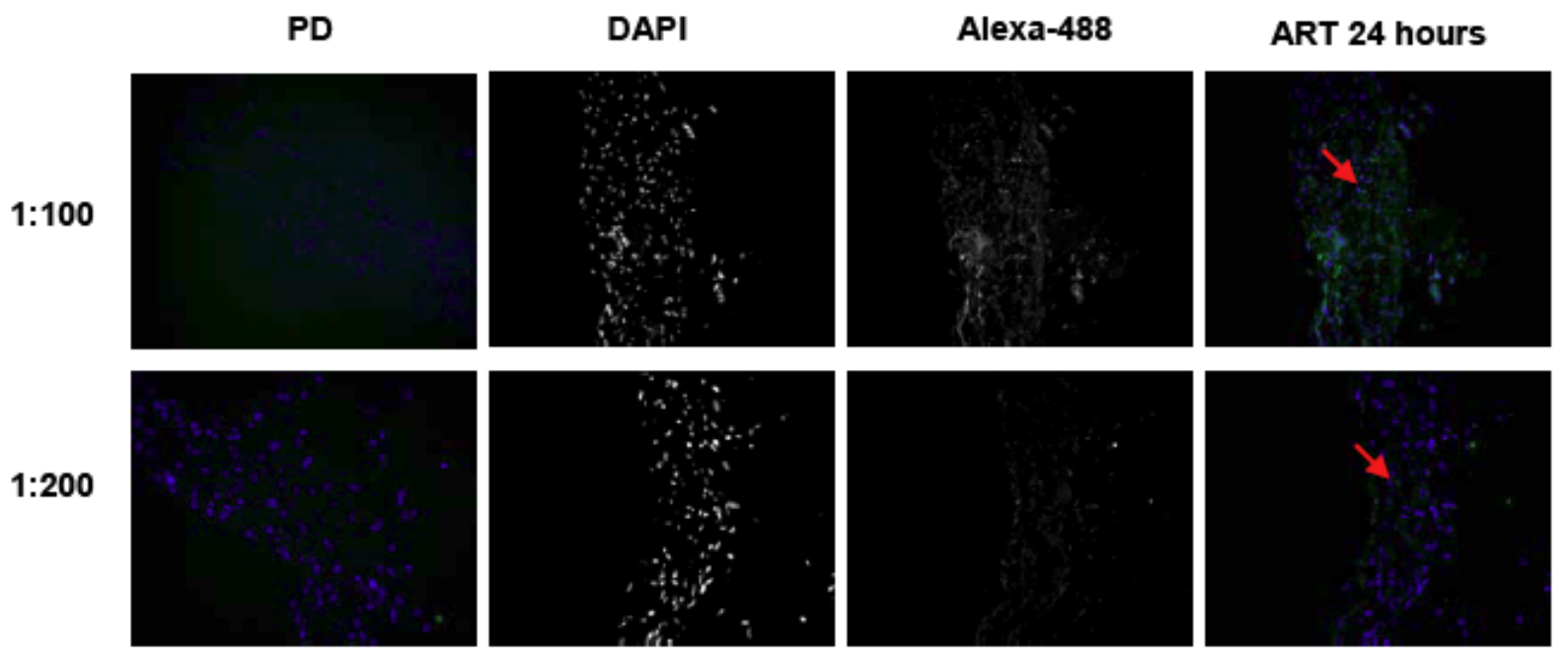

Figure H. 3 Fluorescent microscopy images of PIJV tissue perfused ex vivo under ART conditions for 24 hours stained with two dilutions of mouse-anti-human primary PCNA antibody. Note: PD, primary delete; DAPI, nuclear stain; Alexa-488, donkey-anti-mouse secondary antibody. The red arrow indicates the observed autofluorescence.

PCNA Analysis: PIJV cART 24 hours

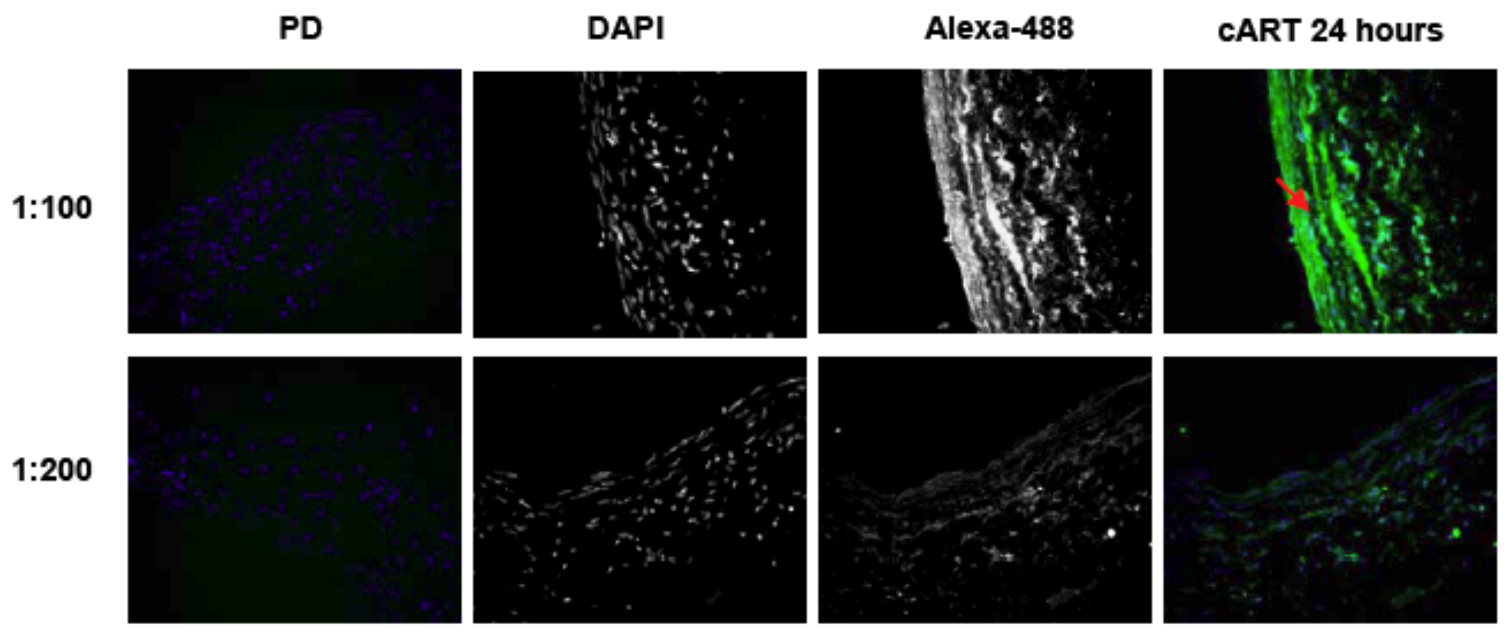

Figure H. 4 Fluorescent microscopy images of PIJV tissue perfused ex vivo under cART conditions for 24 hours stained with two dilutions of mouse-anti-human primary PCNA antibody. Note: PD, primary delete; DAPI, nuclear stain; Alexa-488, donkey-anti-mouse secondary antibody. The red arrow indicates the observed autofluorescence. 


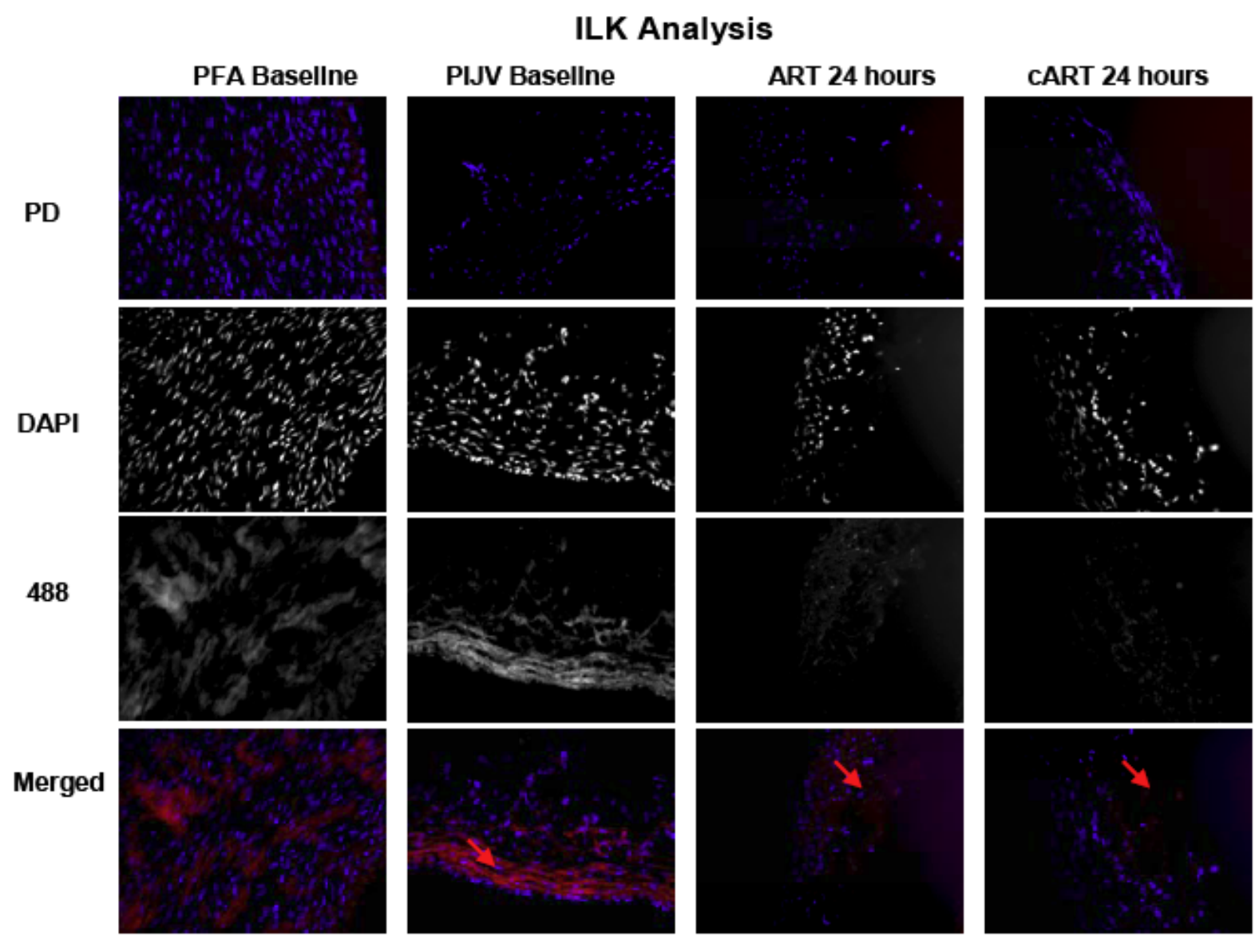

Figure H. 5 Fluorescent microscopy images of PIJV tissue: PFA Baseline, porcine femoral artery fixed immediately after harvest; PIJV Baseline, fixed immediately after harvest; and either perfused ex vivo under ART or cART conditions for 24 hours stained with mouse-anti-human primary ILK antibody. Note: PD, primary delete; DAPI, nuclear stain; 488, donkey-anti-mouse secondary antibody. The red arrow indicates the observed autofluorescence. 


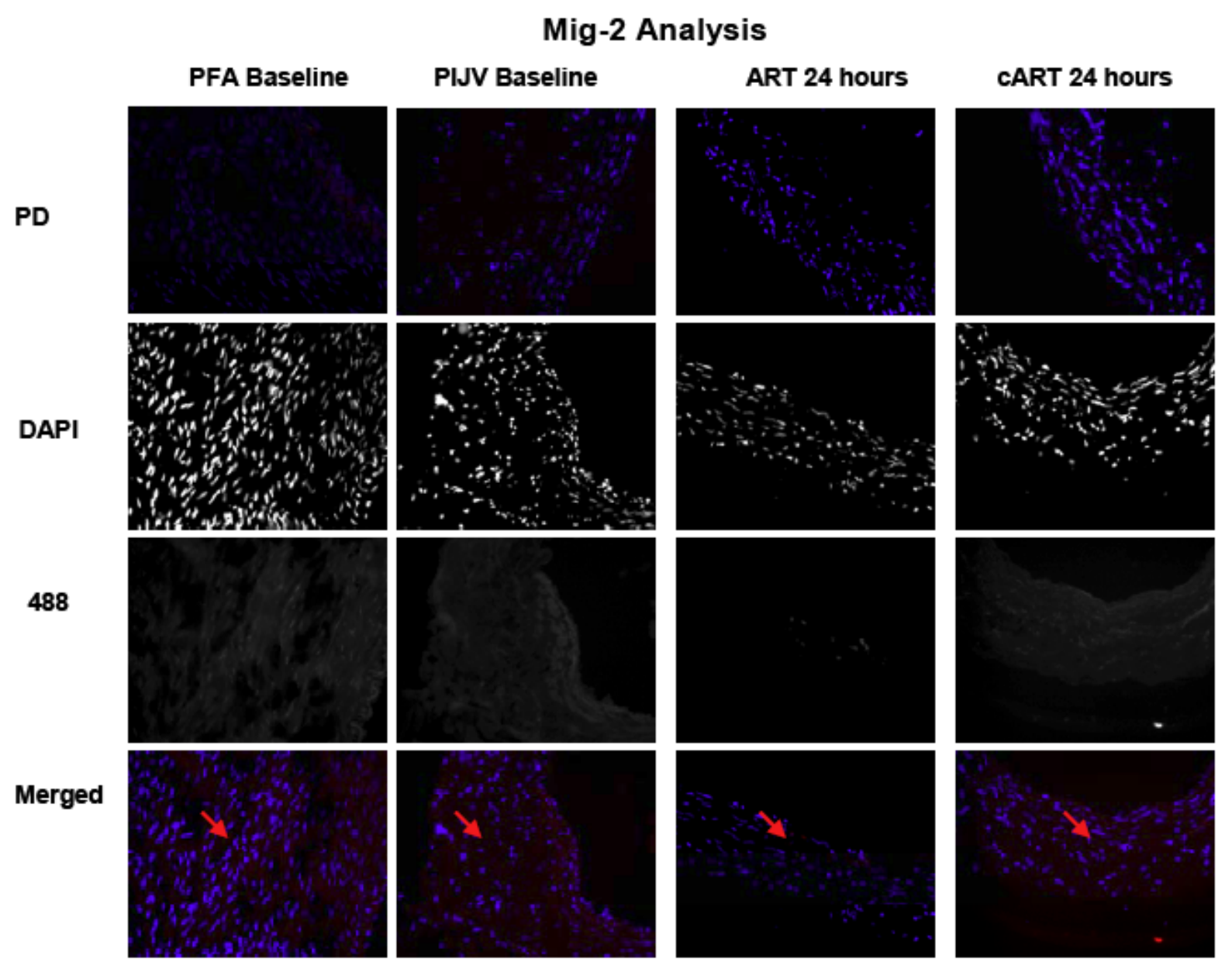

Figure H. 6 Fluorescent microscopy images of PIJV tissue: PFA Baseline, porcine femoral artery fixed immediately after harvest; PIJV Baseline, fixed immediately after harvest; and either perfused ex vivo under ART or cART conditions for 24 hours stained with mouse-anti-human primary Mig-2 antibody. Note: PD, primary delete; DAPI, nuclear stain; 488, donkey-anti-mouse secondary antibody. The red arrow indicates the observed autofluorescence.

The next major hurdle that was encountered with these proposed endpoints was with troubleshooting the HRP/ABC based IHC detection techniques. A point of major difficulty with molecular analysis of porcine tissue is the lack of availability of pig antibodies. No pig antibodies were available for any of our proteins of interest. Most of the primary antibodies that are available for these proteins are raised in a mouse or rabbit host against the human gene. 
Several primary and secondary antibody combinations were attempted while troubleshooting these IHC endpoints. Some of the results obtained with mouse primary antibodies for SPARC (rabbit anti-human: Santa Cruz Biotechnology, Inc.), ILK and Mig-2 (Lab of Dr. Chuanyue Wu, Deptartment of Pathology, University of Pittsburgh) are shown in Figure H. 7 and Figure H. 8. Note the un-specific binding of the primary antibodies which can be the only explanation when we consider the very clear primary delete images.
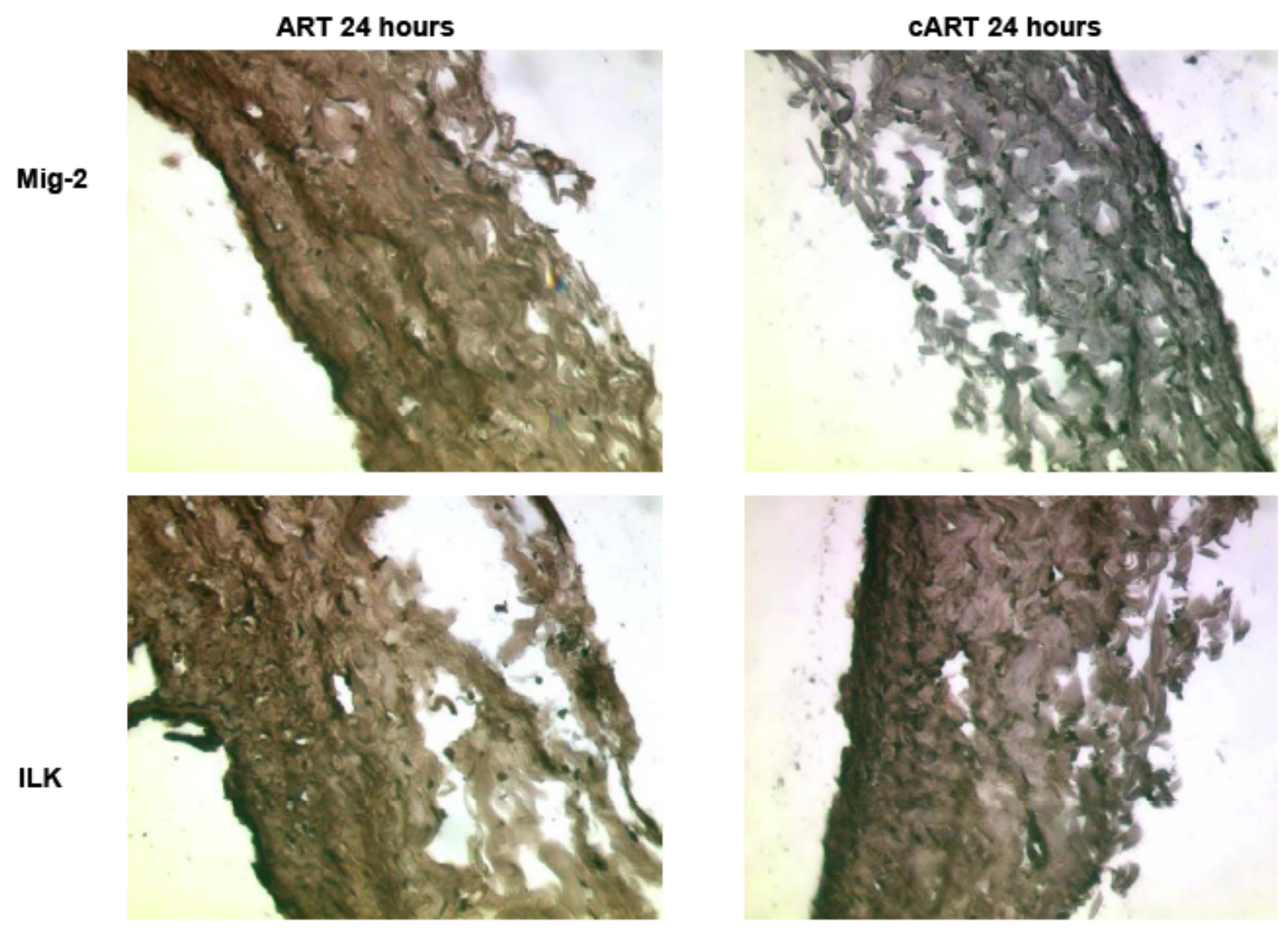

Figure H. 7 HRP/ABC based IHC images of PIJV tissue perfused ex vivo for 24 hours either under ART or cART conditions and stained with ILK and Mig-2 mouse-anti-human primary antibodies. Unspecific binding of primary antibodies results in diffuse brown staining throughout the tissue. 


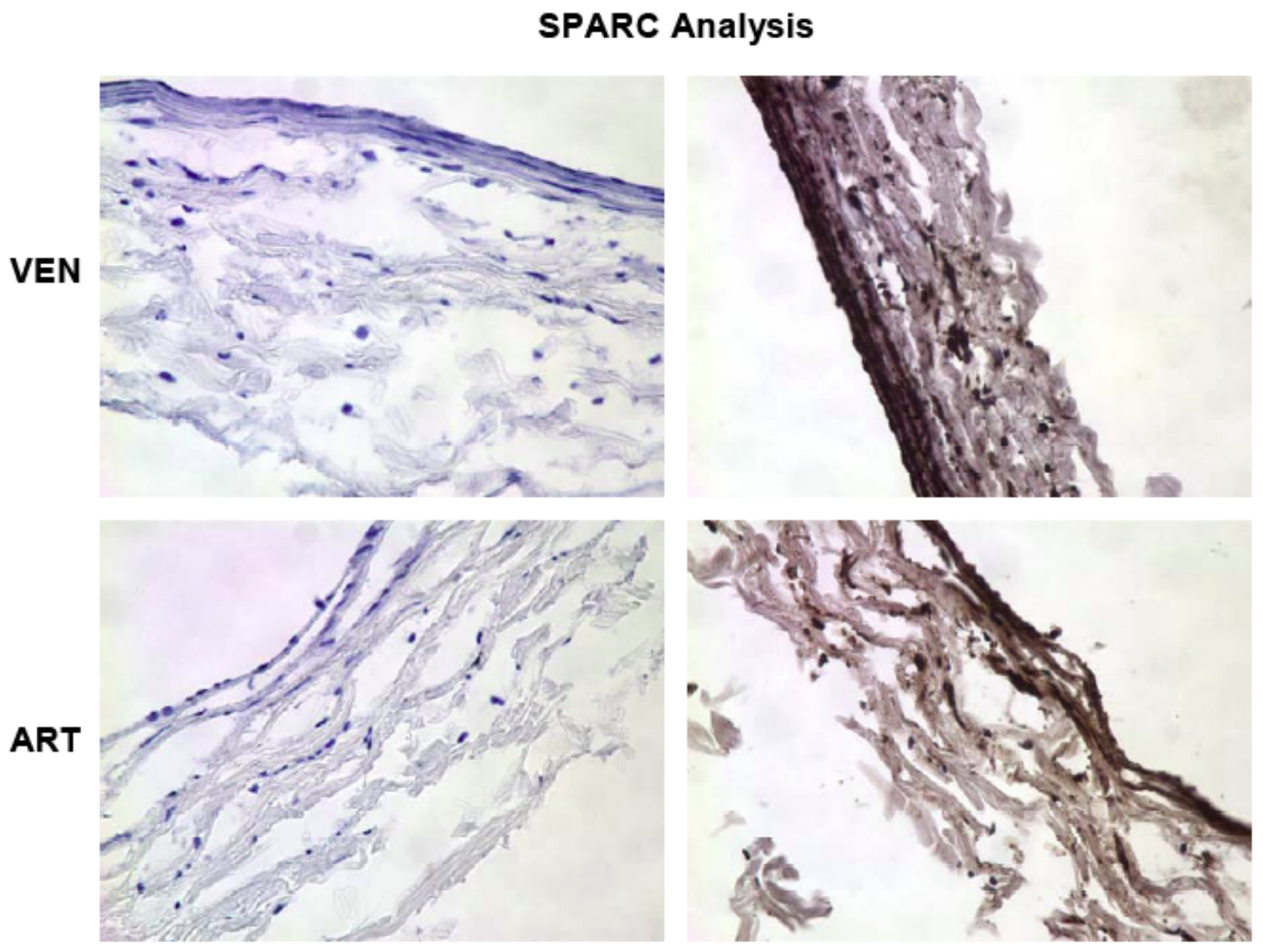

Figure H. 8 HRP/ABC based IHC images of PIJV tissue perfused ex vivo for 24 hours either under VEN or ART conditions and stained with a SPARC rabbit-anti-human primary antibody. The panels on the left are primary deletes. Unspecific binding of primary antibodies results in diffuse brown staining throughout the tissue.

The next molecular analysis technique we attempted unsuccessfully to perform was western blotting. Specifically, in all blots that were run there were always bands detected above and/or below the correct molecular weight for all proposed proteins. Western blots were run using total protein isolated from tissue homogenates. The two separate protocols that were used for protein isolation are provided in Appendix E (protocols E.11 and E.12). The standard protocols that were used for protein separation (SDS PAGE) and western blotting are also provided in Appendix E (protocols E.13 and E.14, respectively) It was determined that the 
"extra" bands were detected due to unspecific binding of the primary antibodies. The consistency with which this was observed between many different proteins that we attempted to evaluate led us to believe that there was some intrinsic property of pig vascular tissue that caused the extra bands in all our gels. The culprit was hypothesized to be "sticky" proteins that are constitutively expressed within blood vessels perhaps as part of the immune response. The vasculature being one of the first lines of defense against infection may express proteins that have a high affinity for binding to foreign antigens that are presented via the circulation. Several different secondary detection systems, primary and secondary antibody concentrations, and blotting conditions were attempted. Also two different protein isolation techniques were attempted and compared. Trizol (Invitrogen) and T-PER (Pierce) were both used and yielded similar results. At this point, all western blot trouble shooting was ceased and focus was placed on detection of the expression of intracellular proteins via immunohistochemistry techniques.

Some western blots that were attempted for a few of the proposed endpoints in Section 1.2 are shown in Figure H. 9. Blots for ILK (Figure H. 9(A)), Mig-2 (Figure H. 9(B)), SPARC (Figure H. 9(C)), and TN-C (Figure H. 9(D)) are shown and it can be seen that in all cases, there were nonspecific bands detected. In each case the unspecific bands are both above and below the actual molecular weight of the protein of interest which is indicated by the arrow in each panel. 


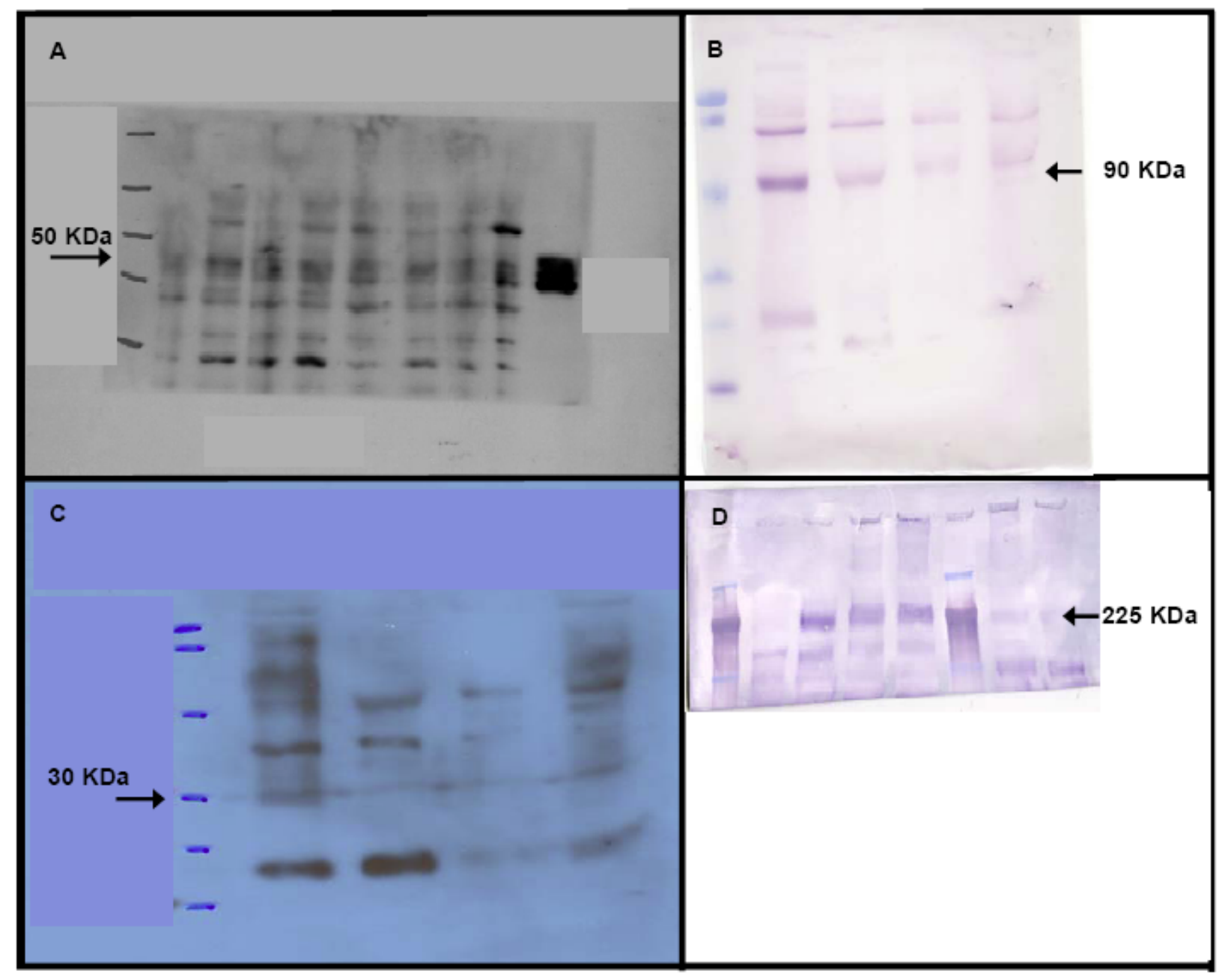

Figure H. 9 Representative western blots performed on total protein isolates from various ex vivo vascular perfusion experiments. The western blots shown were probed for ILK (A), Mig-2 (B), SPARC (C), and TN-C (D). Both chemiluminescent $(\mathrm{A} \& \mathrm{C})$ and alkaline-phosphatase $(\mathrm{B} \& \mathrm{D})$ detection techniques were attempted. Note the extra bands, both above and below the correct respective molecular weights, detected in each blot for each protein of interest.

In order to perform the mRNA analysis as originally proposed, the homologies between the human and porcine gene sequences of interest were compared. It was decided that all the porcine primer-probe sets for RT-PCR would have to be designed since none were readily commercially available. Applied Biosystems, Inc. suggested that they would design and manufacture custom primer-probe sets for any known gene sequence. The problem is that they 
could not guarantee that their probes would work in our hands since they would not perform all of the quality assurance checks (to ensure the primers amplify the single gene of interest) on the custom primers. In addition, the porcine genome is so poorly characterized that they could not be as confident in the quality of custom porcine primers as they are for other species where the genome is more fully annotated. Since we were not equipped to perform the genomics studies and quality control analyses to ensure reliable, accurate results, we felt it was beyond the scope of this dissertation to pursue the mRNA analysis. However in preparation to perform the RTPCR analysis, total RNA was isolated from all the ex vivo experimental tissue as follows: Tissue samples were ground to a fine powder with a mortar and pestle in liquid nitrogen. The powder was transferred to $3 \mathrm{mLs}$ of Trizol (Invitrogen, Carlsbad, CA) isolated as directed by Invitrogen. The isolation was followed by Qiagen clean-up (Qiagen, Valencia, CA). After washing away the undesired macromolecules from the column, the RNA was eluted into water. The specific protocol that was used is provided in Appendix E (protocol E.15). The samples were then taken to the Genomic and Proteomics Core Laboratory at the University of Pittsburgh to determine the quantity and purity of the mRNA. A sample gel is shown in Figure H. 10.

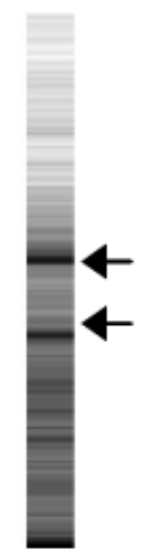

Figure H. 10 Gel representation of capillary electrophoresis result from a representative sample. The two major bands represent the $28 \mathrm{~s}$ and $18 \mathrm{~s}$ ribosomal subunits. Note the clean bands with little or no degradation. 
As mentioned in Section 3.2.6.6.1, TEM was performed in order to visualize Golgi complex and rough endoplasmic reticulum as markers of a synthetic SMC phenotype. However, we realized that quantification of the relative amounts of these organelles was not feasible via TEM imaging due to the extremely high magnification that was required to visualize them. Figure H. 11 to Figure H. 13 show representative TEM images from some ex vivo perfusion experiments (see Table 3.2). 


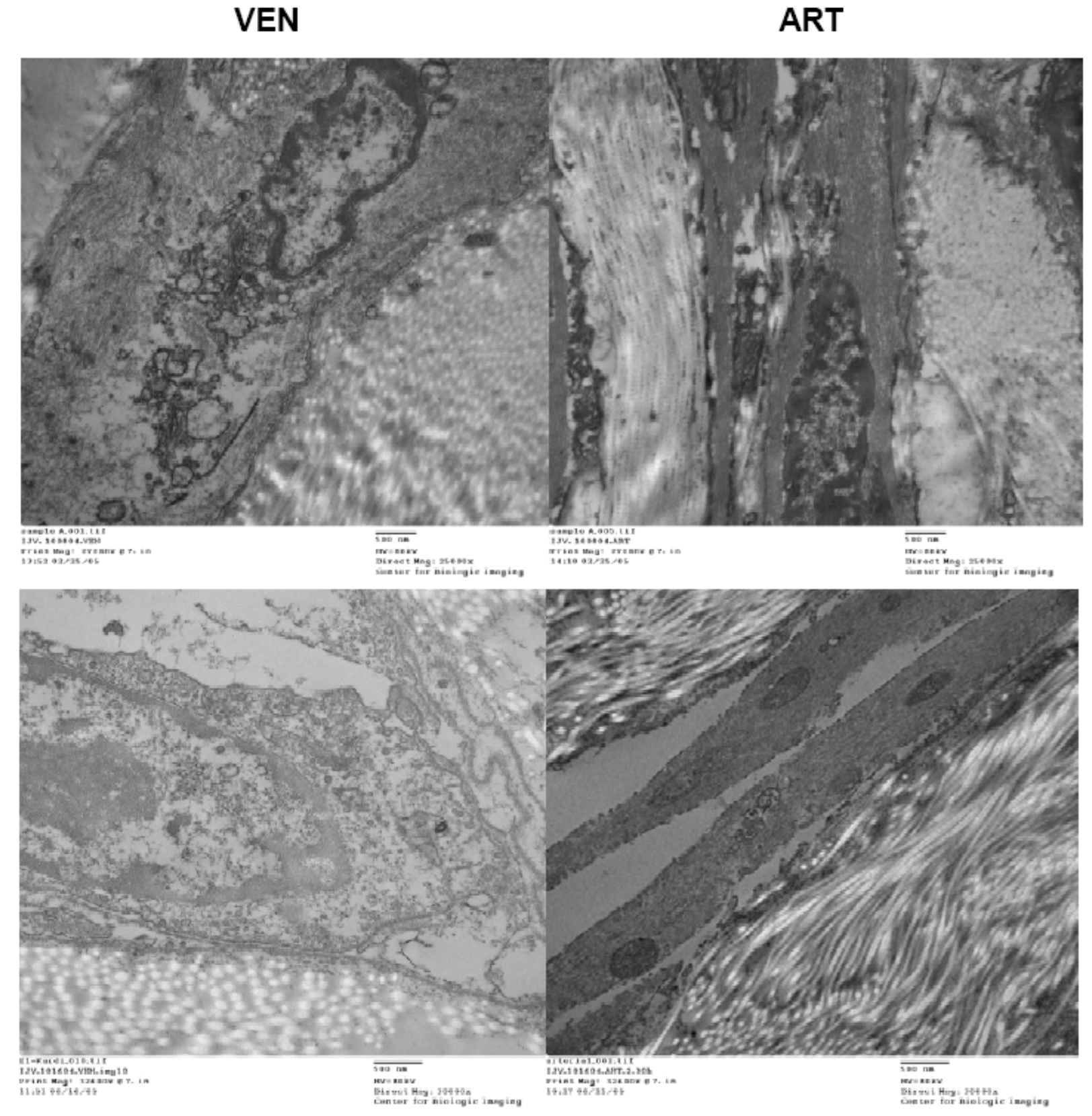

Figure H. 11 Transmission electron microscopy (TEM) images of sections prepared from vein segments perfused, ex vivo, for 24 hours under venous VEN vs. ART conditions. The top two panels are from an experiment performed on $05 / 07 / 2003$. The bottom two panels are from an experiment performed on 10/09/2004. Images were taken at a magnification of 30,000x. 


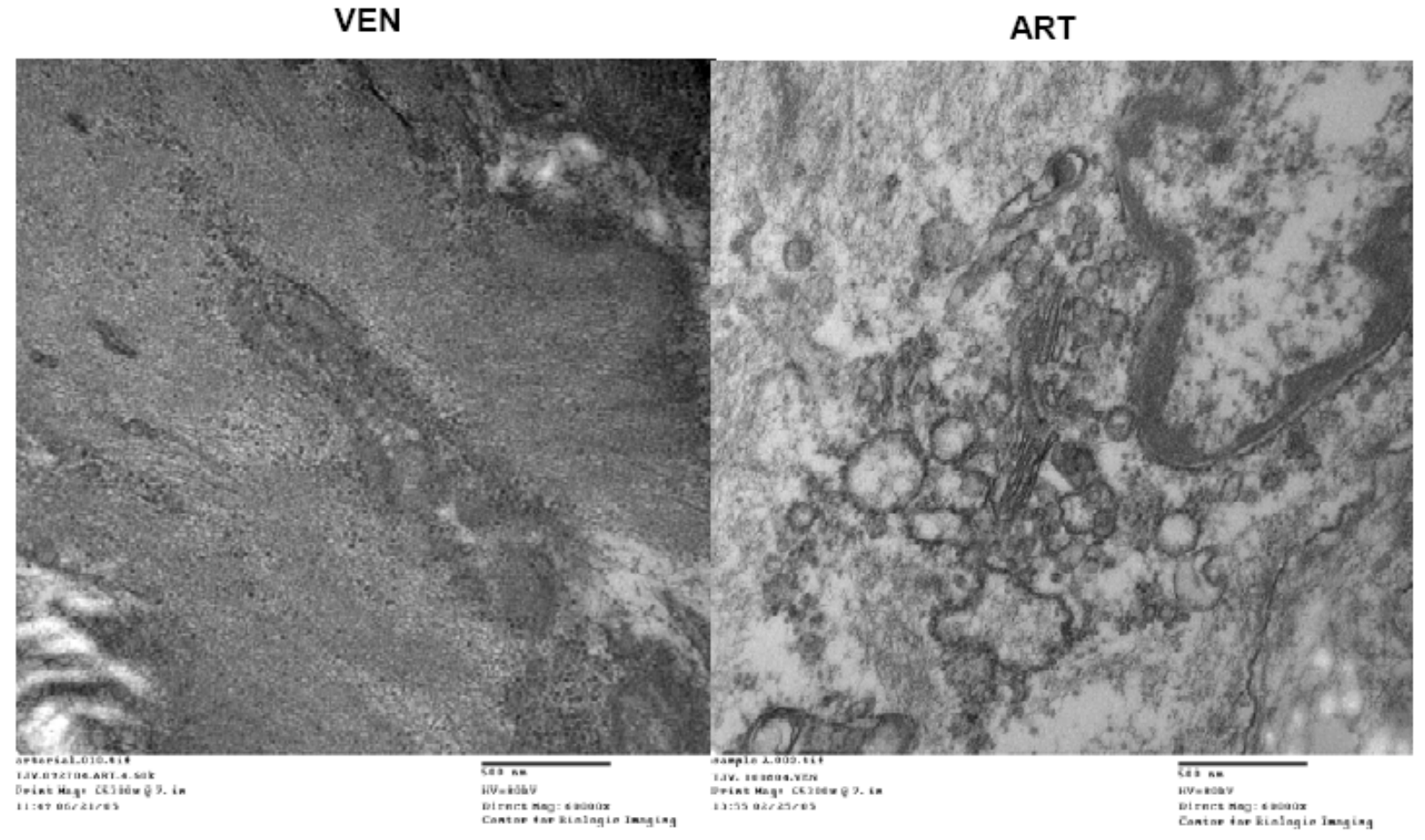

Figure H. 12 Transmission electron microscopy (TEM) images of sections prepared from vein segments perfused, ex vivo, for 24 hours under venous VEN vs. ART conditions. The images are from an experiment performed on 10/16/2004. Images were taken at a magnification of 30,000x. 


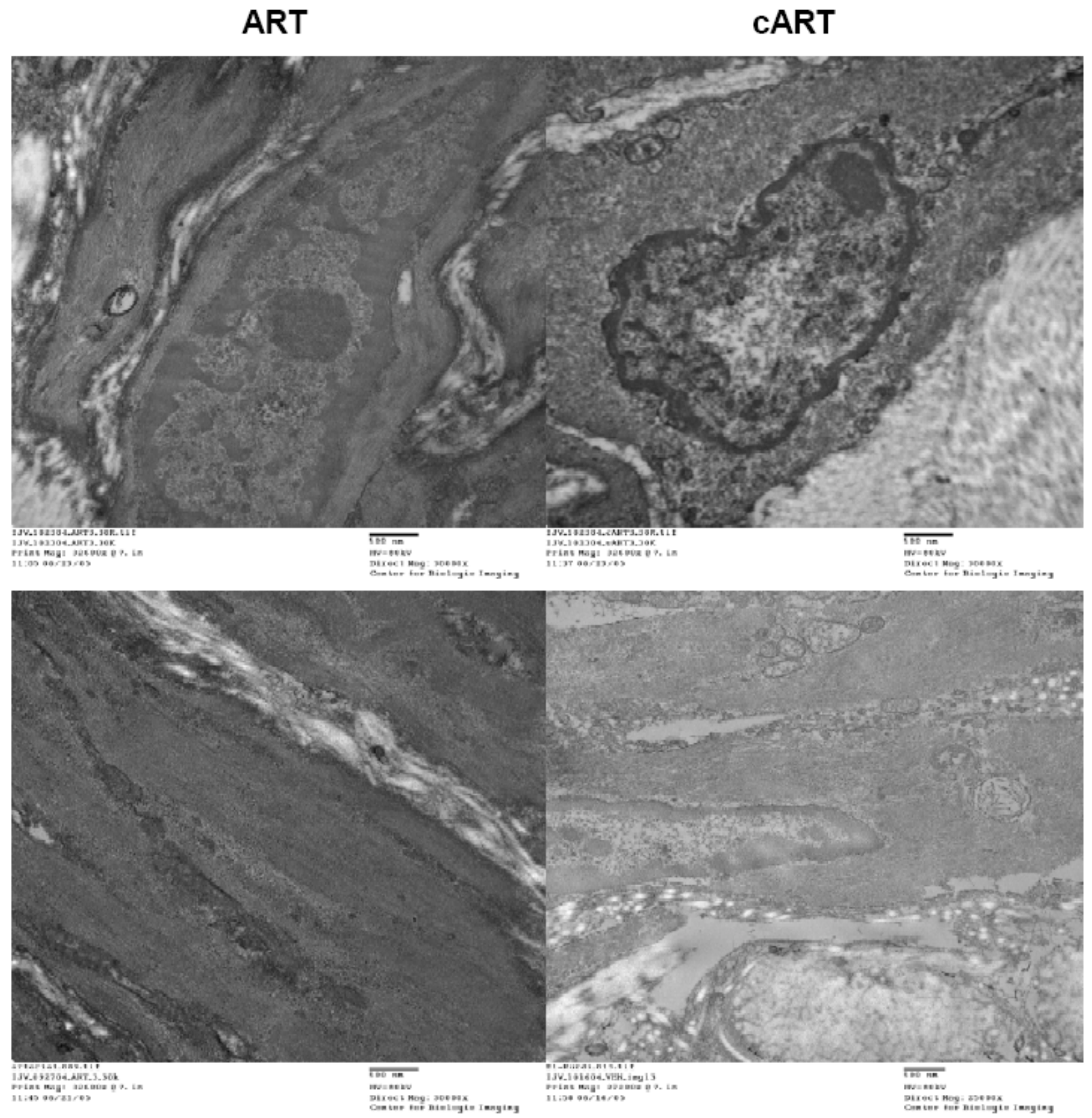

Figure H. 13 Transmission electron microscopy (TEM) images of sections prepared from vein segments perfused, ex vivo, for 24 hours under venous ART vs. cART conditions. The top two panels are from an experiment performed on 10/22/2004. The bottom two panels are from an experiment performed on 11/11/2004. Images were taken at a magnification of $30,000 x$. 


\section{BIBLIOGRAPHY}

1. Mitra, AK, Gangahar DM, and Agrawal DK. Cellular, molecular and immunological mechanisms in the pathophysiology of vein graft intimal hyperplasia. Immunol Cell Biol. 2006. 84(2): p. 115-24.

2. Kumar, V, Fausto N, and Abbas A. Robbins \& coltran pathologic basis of disease. 7th ed. 2004. Saunders.

3. Labadie, RF, Antaki JF, Williams JL, Katyal S, Ligush J, Watkins SC, Pham SM, and Borovetz HS. Pulsatile perfusion system for ex vivo investigation of biochemical pathways in intact vascular tissue. Am J Physiol. 1996. 270(2 Pt 2): p. H760-8.

4. Holzapfel, GA, Sommer G, Gasser CT, and Regitnig P. Determination of layer-specific mechanical properties of human coronary arteries with nonatherosclerotic intimal thickening and related constitutive modeling. Am J Physiol Heart Circ Physiol. 2005. 289(5): p. H2048-58.

5. AHA, Heart disease and stroke statistics: 2006 update. 2006, American Heart Association: Dallas, TX.

6. Feinglass, J, Kaushik S, Handel D, Kosifas A, Martin G, and Pearce WH. Peripheral bypass surgery and amputation. Arch. Surg. 2000. 135: p. 75.

7. Shears, LL, 2nd, Kibbe MR, Murdock AD, Billiar TR, Lizonova A, Kovesdi I, Watkins SC, and Tzeng E. Efficient inhibition of intimal hyperplasia by adenovirus-mediated inducible nitric oxide synthase gene transfer to rats and pigs in vivo. J Am Coll Surg. 1998. 187(3): p. 295-306.

8. Wan, S, Shukla N, Angelini GD, Yim AP, Johnson JL, and Jeremy JY. Nitric oxidedonating aspirin (ncx 4016) inhibits neointimal thickening in a pig model of saphenous vein-carotid artery interposition grafting: A comparison with aspirin and morpholinosydnonimine (sin-1). J Thorac Cardiovasc Surg. 2007. 134(4): p. 1033-9.

9. Statistics, $\mathrm{NCfH}$, Quarterly fact sheet: Monitoring health care in america. 1996, National Center for Health Statistics: Hyattsville, MD. 
10. Pelletier, LC, The saphenous vein graft: What have we learned from the past 25 year?, in Conduits for myocardial revascularization, Carrier M and Pelletier LC, Editors. 1993, RG Landes, Co.: Austin.

11. Von Segesser, LK. Arterial grafting for myocardial revascularization. 1991, New York: Springer-Verlag.

12. Weintraub, WS, Jones EL, Craver JM, and Guyton RA. Frequency of repeat coronary bypass or coronary angioplasty after coronary artery bypass surgery using saphenous venous grafts. Am J Cardiol. 1994. 73(2): p. 103-12.

13. Acar, C, Jebara VA, Portoghese M, Beyssen B, Pagny JY, Grare P, Chachques JC, Fabiani JN, Deloche A, and Guermonprez JL. Revival of the radial artery for coronary artery bypass grafting. Ann of Thorac Surg. 1992. 54(4): p. 652-9; discussion 659-60.

14. Dalman, RL and Taylor LM, Jr. Basic data related to infrainguinal revascularization procedures. Ann Vasc Surg. 1990. 4(3): p. 309-12.

15. Davies, MG and Hagen PO. Pathophysiology of vein graft failure: A review. European J Vasc Endovas Sur. 1995. 9(1): p. 7-18.

16. FitzGibbon, GM, Leach AJ, Keon WJ, Burton JR, and Kafka HP. Coronary bypass graft fate. Angiographic study of 1,179 vein grafts early, one year, and five years after operation. J Thorac Cardiovasc Surg. 1986. 91(5): p. 773-8.

17. Grondin, CM, Campeau L, Lesperance J, Solymoss BC, Vouhe P, Castonguay YR, Meere $\mathrm{C}$, and Bourassa MG. Atherosclerotic changes in coronary vein grafts six years after operation. Angiographic aspect in 110 patients. J Thorac Cardiovasc Surg. 1979. 77(1): p. 24-31.

18. Bandyk, DF and Mills JL. The failing graft: An evolving concept. Semin Vasc Surg. 1993. 6(2): p. 75-7.

19. Butany, JW, David TE, and Ojha M. Histological and morphometric analyses of early and late aortocoronary vein grafts and distal anastomoses. Can J Cardiol. 1998. 14(5): p. 671-7.

20. Cable, DG, Caccitolo JA, Caplice N, O_Brien T, Simari RD, Daly RC, Dearani JA, Mullany CJ, Orszulak TA, and Schaff HV. The role of gene therapy for intimal hyperplasia of bypass grafts. Circulation. 1999. 100(19 Suppl): p. II392-6.

21. Dilley, RJ, McGeachie JK, and Prendergast FJ. A review of the histologic changes in vein-to-artery grafts, with particular reference to intimal hyperplasia. Arch Surg. 1988. 123(6): p. 691-6. 
22. Zhang, L, Sivashanmugam P, Wu JH, Brian L, Exum ST, Freedman NJ, and Peppel K. Tumor necrosis factor receptor-2 signaling attenuates vein graft neointima formation by promoting endothelial recovery. Arterioscler Thromb Vasc Biol. 2008. 28(2): p. 284-9.

23. Horstick, G, Bierbach B, Schlindwein P, Abegunewardene N, Vosseler M, Bittinger F, Becker D, Lauterbach M, Lehr HA, and Kempski O. Resistance of the internal mammary artery to restenosis: A histomorphologic study of various porcine arteries. J Vasc Res. 2008. 45(1): p. 45-53.

24. Wong, AP, Nili N, Jackson ZS, Qiang B, Leong-Poi H, Jaffe R, Raanani E, Connelly PW, Sparkes JD, and Strauss BH. Expansive remodeling in venous bypass grafts: Novel implications for vein graft disease. Atherosclerosis. 2008. 196(2): p. 580-9.

25. Deng, YZ, Liu SJ, Ma L, Li HF, Li YF, Sun ZQ, and Chen JJ. [origin of neointimal cells in autologous vein graft in rat model]. Zhonghua Wai Ke Za Zhi. 2007. 45(20): p. 14247.

26. Jeremy, JY, Gadsdon P, Shukla N, Vijayan V, Wyatt M, Newby AC, and Angelini GD. On the biology of saphenous vein grafts fitted with external synthetic sheaths and stents. Biomaterials. 2007. 28(6): p. 895-908.

27. Angelini, GD, Bryan AJ, Williams HM, Morgan R, and Newby AC. Distention promotes platelet and leukocyte adhesion and reduces short-term patency in pig arteriovenous bypass grafts. J Thorac Cardiovas Surg. 1990. 99(3): p. 433-9.

28. Campbell, PA, McGeachie JK, and Prendergast FJ. Vein grafts for arterial repair: Their success and reasons for failure. Annals of the Royal College of Surgeons of England. 1981. 63(4): p. 257-60.

29. Campeau L, LJ, Bourasa MG. Natural history of saphenous vein aortocoronary bypass grafts. Mod Concepts Cardiovasc Dis. 1984. 53: p. 59-63.

30. Fuchs, JC, Mitchener JS, and Hagen PO. Postoperative changes in autologous vein grafts. Annals of Surgery. 1978. 188(1): p. 1-15.

31. Huynh, TT, Davies MG, Trovato MJ, Svendsen E, and Hagen PO. Alterations in wall tension and shear stress modulate tyrosine kinase signaling and wall remodeling in experimental vein grafts. J Vasc Surg. 1999. 29(2): p. 334-44.

32. Liu, SQ and Fung YC. Changes in the organization of the smooth muscle cells in rat vein grafts. Ann Biomed Eng. 1998. 26(1): p. 86-95.

33. Ramos, JR, Berger K, Mansfield PB, and Sauvage LR. Histologic fate and endothelial changes of distended and nondistended vein grafts. Annals of Surgery. 1976. 183(3): p. 205-28. 
34. Resnick, $\mathrm{N}$ and Gimbrone MA. Hemodynamic forces are complex regulators of endothelial gene expression. FASEB J. 1995. 9(10): p. 874-82.

35. Sumpio, B. Hemodynamic forces and vascular cell biology. 1993: Austin: R.G. Landes Company.

36. Szilagyi, DE, Elliott JP, Hageman JH, Smith RF, and Dall_olmo CA. Biologic fate of autogenous vein implants as arterial substitutes: Clinical, angiographic and histopathologic observations in femoro-popliteal operations for atherosclerosis. Annals of Surgery. 1973. 178(3): p. 232-46.

37. Zwolak, RM, Adams MC, and Clowes AW. Kinetics of vein graft hyperplasia: Association with tangential stress. J Vasc Surg. 1987. 5(1): p. 126-36.

38. Porter, KE, Nydahl S, Dunlop P, Varty K, Thrush AJ, and London NJ. The development of an in vitro flow model of human saphenous vein graft intimal hyperplasia. Cardiovase Res. 1996. 31(4): p. 607-14.

39. Vorp, DA, Severyn DA, Steed DL, and Webster MW. A device for the application of cyclic twist and extension on perfused vascular segments. Am J Physiol. 1996. 270(2 Pt 2): p. H787-95.

40. Cabrera Fischer, EI, Bia Santana D, Cassanello GL, Zocalo Y, Crawford EV, Casas RF, and Armentano RL. Reduced elastic mismatch achieved by interposing vein cuff in expanded polytetrafluoroethylene femoral bypass decreases intimal hyperplasia. Artif Organs. 2005. 29(2): p. 122-30.

41. Ducasse, E, Fleurisse L, Vernier G, Speziale F, Fiorani P, Puppinck P, and Creusy C. Interposition vein cuff and intimal hyperplasia: An experimental study. Eur J Vasc Endovasc Surg. 2004. 27(6): p. 617-21.

42. Huynh, TT, Iaccarino G, Davies MG, Safi HJ, Koch WJ, and Hagen PO. External support modulates $\mathrm{g}$ protein expression and receptor coupling in experimental vein grafts. Surgery. 1999. 126(2): p. 127-34.

43. Jeremy, JY, Bulbulia R, Johnson JL, Gadsdon P, Vijayan V, Shukla N, Smith FC, and Angelini GD. A bioabsorbable (polyglactin), nonrestrictive, external sheath inhibits porcine saphenous vein graft thickening. J Thorac Cardiovasc Surg. 2004. 127(6): p. $1766-72$.

44. Karayannacos, PE, Hostetler JR, Bond MG, Kakos GS, Williams RA, Kilman JW, and Vasko JS. Late failure in vein grafts: Mediating factors in subendothelial fibromuscular hyperplasia. Ann Surg. 1978. 187(2): p. 183-8.

45. Kohler, TR, Kirkman TR, and Clowes AW. The effect of rigid external support on vein graft adaptation to the arterial circulation. J Vasc Surg. 1989. 9(2): p. 277-85. 
46. Kohler, TR, Toleikis PM, Gravett DM, and Avelar RL. Inhibition of neointimal hyperplasia in a sheep model of dialysis access failure with the bioabsorbable vascular wrap paclitaxel-eluting mesh. J Vasc Surg. 2007. 45(5): p. 1029-1037; discussion 1037-8.

47. Liu, SQ, Moore MM, Glucksberg MR, Mockros LF, Grotberg JB, and Mok AP. Partial prevention of monocyte and granulocyte activation in experimental vein grafts by using a biomechanical engineering approach. J Biomech. 1999. 32(11): p. 1165-75.

48. Liu, SQ, Ruan YY, Tang D, Li YC, Goldman J, and Zhong L. A possible role of initial cell death due to mechanical stretch in the regulation of subsequent cell proliferation in experimental vein grafts. Biomech Model Mechanobiol. 2002. 1(1): p. 17-27.

49. Mehta, D, George SJ, Jeremy JY, Izzat MB, Southgate KM, Bryan AJ, Newby AC, and Angelini GD. External stenting reduces long-term medial and neointimal thickening and platelet derived growth factor expression in a pig model of arteriovenous bypass grafting. Nat Med. 1998. 4(2): p. 235-9.

50. Parsonnet, V, Lari AA, and Shah IH. New stent for support of veins in arterial grafts. Arch Surg. 1963. 87: p. 696-702.

51. Vijayan, V, Shukla N, Johnson JL, Gadsdon P, Angelini GD, Smith FC, Baird R, and Jeremy JY. Long-term reduction of medial and intimal thickening in porcine saphenous vein grafts with a polyglactin biodegradable external sheath. J Vasc Surg. 2004. 40(5): p. 1011-9.

52. Vijayan, V, Smith FC, Angelini GD, Bulbulia RA, and Jeremy JY. External supports and the prevention of neointima formation in vein grafts. Eur J Vasc Endovasc Surg. 2002. 24(1): p. 13-22.

53. Murphy, GJ, Newby AC, Jeremy JY, Baumbach A, and Angelini GD. A randomized trial of an external dacron sheath for the prevention of vein graft disease: The extent study. $\mathrm{J}$ Thorac Cardiovasc Surg. 2007. 134(2): p. 504-5.

54. Zou, RJ, Zou LJ, Huang SD, Wang Y, Han L, Ji GY, and Xu ZY. Effect of external stents on prevention of intimal hyperplasia in a canine vein graft model. Chin Med J (Engl). 2007. 120(24): p. 2264-7.

55. Liao, SW, Lu X, Putnam AJ, and Kassab GS. A novel time-varying poly lactic-co glycolic acid external sheath for vein grafts designed under physiological loading. Tissue Eng. 2007. 13(12): p. 2855-62.

56. Taylor, GI. Disintegration of water drops in an electric filed. . Proc R Soc Lond. 1964. 280: p. 383-397. 
57. Stankus, JJ, Guan J, and Wagner WR. Fabrication of biodegradable elastomeric scaffolds with sub-micron morphologies. J Biomed Mater Res A. 2004. 70(4): p. 603-14.

58. Slomp, J, van_Munsteren JC, Poelmann RE, de_Reeder EG, Bogers AJ, and Gittenberger_de_Groot AC. Formation of intimal cushions in the ductus arteriosus as a model for vascular intimal thickening. An immunohistochemical study of changes in extracellular matrix components. Atherosclerosis. 1992. 93(1-2): p. 25-39.

59. Wallitt, EJ, Jevon M, and Hornick PI. Therapeutics of vein graft intimal hyperplasia: 100 years on. Ann Thorac Surg. 2007. 84(1): p. 317-23.

60. Kreienberg, P, Cheema M, Chang BB, Paty PS, Roddy SP, and Darling RC, 3rd. Primary revision of mid-vein stenoses in venous bypass conduits: Venous patch versus interposition vein. J Vasc Surg. 2007. 45(5): p. 929-34; discussion 934-5.

61. Alp, NJ, West NE, Arnold N, Gunn J, Banning AP, and Channon KM. Increased intimal hyperplasia in experimental vein graft stenting compared to arterial stenting: Comparisons in a new rabbit model of stent injury. Cardiovasc Res. 2002. 56(1): p. 16472 .

62. Chan, AK, Kalmes A, Hawkins S, Daum G, and Clowes AW. Blockade of the epidermal growth factor receptor decreases intimal hyperplasia in balloon-injured rat carotid artery. J Vasc Surg. 2003. 37(3): p. 644-9.

63. Clowes, AW, Clowes MM, Fingerle J, and Reidy MA. Kinetics of cellular proliferation after arterial injury. V. Role of acute distension in the induction of smooth muscle proliferation. Laboratory Investigation; a Journal of Technical Methods and Pathology. 1989. 60(3): p. 360-4.

64. Davies, MG, Mason DP, Tran PK, Deou J, Hawkins S, and Clowes AW. G-protein expression and intimal hyperplasia after arterial injury: A role for galpha(i) proteins. J Vasc Surg. 2001. 33(2): p. 408-18.

65. Fingerle, J, Au YP, Clowes AW, and Reidy MA. Intimal lesion formation in rat carotid arteries after endothelial denudation in absence of medial injury. Arteriosclerosis (Dallas, Tex.). 1992. 10(6): p. 1082-7.

66. Kumar, B, Dreja K, Shah SS, Cheong A, Xu SZ, Sukumar P, Naylor J, Forte A, Cipollaro M, McHugh D, Kingston PA, Heagerty AM, Munsch CM, Bergdahl A, HultgardhNilsson A, Gomez MF, Porter KE, Hellstrand P, and Beech DJ. Upregulated trpc1 channel in vascular injury in vivo and its role in human neointimal hyperplasia. Circ Res. 2006. 98(4): p. 557-63.

67. Nanobashvili, J, Prager M, Jozkowicz A, Neumayer C, Fugl A, Blumer R, Cabaj A, Wrba F, Polterauer P, and Huk I. Positive effect of treatment with synthetic steroid hormone 
tibolon on intimal hyperplasia and restenosis after experimental endothelial injury of rabbit carotid artery. Eur Surg Res. 2004. 36(2): p. 74-82.

68. Noiseux, N, Boucher CH, Cartier R, and Sirois MG. Bolus endovascular pdgfr-beta antisense treatment suppressed intimal hyperplasia in a rat carotid injury model. Circulation. 2000. 102(11): p. 1330-6.

69. Rosenthal, D, Stevens SL, Skillern CS, Wellons ED, Robinson K, Matsuura JH, and Gannon BJ. Topical application of beta-radiation to reduce intimal hyperplasia after carotid artery balloon injury in rabbit. A possible application for brachytherapy in vascular surgery. Cardiovasc Radiat Med. 2002. 3(1): p. 16-9.

70. Shi, Y, O_Brien JE, Fard A, Mannion JD, Wang D, and Zalewski A. Adventitial myofibroblasts contribute to neointimal formation in injured porcine coronary arteries. Circulation. 1996. 94(7): p. 1655-64.

71. Simosa, HF, Wang G, Sui X, Peterson T, Narra V, Altieri DC, and Conte MS. Survivin expression is up-regulated in vascular injury and identifies a distinct cellular phenotype. $\mathrm{J}$ Vasc Surg. 2005. 41(4): p. 682-90.

72. Usui, M, Asahara T, Naitoh Y, Katoh T, and Ibukiyama C. Photodynamic therapy for the prevention of intimal hyperplasia in balloon-injured rabbit arteries. Jpn Circ J. 1999. 63(5): p. 387-93.

73. Wan, L, Li DY, Yang B, and Wu QY. Perivenous application of fibrin glue prevents the early injury of jugular vein graft to arterial circulation in rabbits. Chin Med J (Engl). 2006. 119(4): p. 300-4.

74. $\mathrm{Wu}, \mathrm{KJ}$, Yee A, Zhu NL, Gordon EM, and Hall FL. Characterization of differential gene expression in monkey arterial neointima following balloon catheter injury. Int $\mathrm{J}$ Mol Med. 2000. 6(4): p. 433-40.

75. Zeng, R, Liu X, and Song L. [artery denuded model and effects of three kinds of antisense c-myc rna]. Zhonghua Yi Xue Za Zhi. 2000. 80(2): p. 140-4.

76. Zubilewicz, T, Wronski J, Bourriez A, Terlecki P, Guinault AM, Muscatelli-Groux B, Michalak J, Melliere D, Becquemin JP, and Allaire E. Injury in vascular surgery--the intimal hyperplastic response. Med Sci Monit. 2001. 7(2): p. 316-24.

77. Goldman, J, Zhong L, and Liu SQ. Negative regulation of vascular smooth muscle cell migration by blood shear stress. Am J Physiol Heart Circ Physiol. 2007. 292(2): p. H92838.

78. Yamamoto, K, Onoda K, Sawada Y, Fujinaga K, Imanaka-Yoshida K, Yoshida T, and Shimpo H. Locally applied cilostazol suppresses neointimal hyperplasia and medial thickening in a vein graft model. Ann Thorac Cardiovasc Surg. 2007. 13(5): p. 322-30. 
79. Ramlawi, B, Scott JR, Feng J, Mieno S, Raman KG, Gallo D, Csizmadia E, Yoke Chin B, Bach FH, Otterbein LE, and Sellke FW. Inhaled carbon monoxide prevents graftinduced intimal hyperplasia in swine. J Surg Res. 2007. 138(1): p. 121-7.

80. Dobrin, PB, Littooy FN, and Endean ED. Mechanical factors predisposing to intimal hyperplasia and medial thickening in autogenous vein grafts. Surgery. 1989. 105(3): p. 393-400.

81. Gusic, RJ, Myung R, Petko M, Gaynor JW, and Gooch KJ. Shear stress and pressure modulate saphenous vein remodeling ex vivo. J Biomech. 2005. 38(9): p. 1760-9.

82. Goldman, J, Zhong L, and Liu SQ. Negative regulation of vascular smooth muscle cell migration by blood shear stress. Am J Physiol Heart Circ Physiol. 2006.

83. Jiang, Z, Berceli SA, Pfahnl CL, Wu L, Goldman D, Tao M, Kagayama M, Matsukawa A, and Ozaki CK. Wall shear modulation of cytokines in early vein grafts. J Vasc Surg. 2004. 40(2): p. 345-50.

84. Jiang, Z, Wu L, Miller BL, Goldman DR, Fernandez CM, Abouhamze ZS, Ozaki CK, and Berceli SA. A novel vein graft model: Adaptation to differential flow environments. Am J Phys. Heart Circ Phys. 2004. 286(1): p. H240-5.

85. Morinaga, K, Okadome K, Kuroki M, Miyazaki T, Muto Y, and Inokuchi K. Effect of wall shear stress on intimal thickening of arterially transplanted autogenous veins in dogs. J Vasc Surg. 1985. 2(3): p. 430-3.

86. Nelson, PR, O'Malley KA, Feezor RJ, Moldawer LL, and Seeger JM. Genomic and proteomic determinants of lower extremity revascularization failure: Rationale and study design. J Vasc Surg. 2007. 45 Suppl A: p. A82-91.

87. Campbell, GR and Campbell JH. Smooth muscle phenotypic changes in arterial wall homeostasis: Implications for the pathogenesis of atherosclerosis. Exp Molec Path. 1985. 42(2): p. 139-62.

88. Chamley-Campbell, JH and Campbell GR. What controls smooth muscle phenotype? Atherosclerosis. 1981. 40(3-4): p. 347-57.

89. Clowes, AW, Reidy MA, and Clowes MM. Kinetics of cellular proliferation after arterial injury. I. Smooth muscle growth in the absence of endothelium. Laboratory Investigation; a Journal of Technical Methods and Pathology. 1983. 49(3): p. 327-33.

90. Francis, DJ, Parish CR, McGarry M, Santiago FS, Lowe HC, Brown KJ, Bingley JA, Hayward IP, Cowden WB, Campbell JH, Campbell GR, Chesterman CN, and Khachigian LM. Blockade of vascular smooth muscle cell proliferation and intimal thickening after balloon injury by the sulfated oligosaccharide pi-88: Phosphomannopentaose sulfate 
directly binds fgf-2, blocks cellular signaling, and inhibits proliferation. Circ Res. 2003. 92(8): p. e70-7.

91. Gidlof, AC, Ocaya P, Olofsson PS, Torma H, and Sirsjo A. Differences in retinol metabolism and proliferative response between neointimal and medial smooth muscle cells. J Vasc Res. 2006. 43(4): p. 392-398.

92. Neuville, P, Yan Z, Gidlof A, Pepper MS, Hansson GK, Gabbiani G, and Sirsjo A. Retinoic acid regulates arterial smooth muscle cell proliferation and phenotypic features in vivo and in vitro through an raralpha-dependent signaling pathway. Arterioscler Thromb Vasc Biol. 1999. 19(6): p. 1430-6.

93. O'Brien, ER, Alpers CE, Stewart DK, Ferguson M, Tran N, Gordon D, Benditt EP, Hinohara T, Simpson JB, and Schwartz SM. Proliferation in primary and restenotic coronary atherectomy tissue. Implications for antiproliferative therapy. Circ Res (Online). 1993. 73(2): p. 223-31.

94. Okamoto, E, Suzuki T, Aikawa M, Imataka K, Fujii J, Kuro_o M, Nakahara K, Hasegawa A, Yazaki Y, and Nagai R. Diversity of the synthetic-state smooth-muscle cells proliferating in mechanically and hemodynamically injured rabbit arteries. Laboratory Investigation; a Journal of Technical Methods and Pathology. 1996. 74(1): p. $120-8$.

95. Schwartz SM, MR. The vascular smooth muscle cell: Molecular and biological responses to the extracellular matrix. 1995: Academic Press. 1-37.

96. Thyberg, J. Differentiated properties and proliferation of arterial smooth muscle cells in culture. International Review of Cytology. 1996. 169: p. 183-265.

97. Thyberg, J. Phenotypic modulation of smooth muscle cells during formation of neointimal thickenings following vascular injury. Histology and Histopathology. 1998. 13(3): p. 871-91.

98. Tran, PK, Tran-Lundmark K, Soininen R, Tryggvason K, Thyberg J, and Hedin U. Increased intimal hyperplasia and smooth muscle cell proliferation in transgenic mice with heparan sulfate-deficient perlecan. Circ Res. 2004. 94(4): p. 550-8.

99. Wakino, S, Kintscher U, Kim S, Jackson S, Yin F, Nagpal S, Chandraratna RA, Hsueh WA, and Law RE. Retinoids inhibit proliferation of human coronary smooth muscle cells by modulating cell cycle regulators. Arterioscler Thromb Vasc Biol. 2001. 21(5): p. 74651.

100. Walker, T, Wendel HP, Tetzloff L, Raabe C, Heidenreich O, Simon P, Scheule AM, and Ziemer G. Inhibition of adhesion molecule expression on human venous endothelial cells by non-viral sirna transfection. J Cell Mol Med. 2007. 11(1): p. 139-47. 
101. Amabile, PG, Wong H, Uy M, Boroumand S, Elkins CJ, Yuksel E, Waugh JM, and Dake MD. In vivo vascular engineering of vein grafts: Directed migration of smooth muscle cells by perivascular release of elastase limits neointimal proliferation. J Vasc Interv Radiol. 2002. 13(7): p. 709-15.

102. Craig, SW and Johnson RP. Assembly of focal adhesions: Progress, paradigms, and portents. Current Opinion in Cell Biology. 1996. 8(1): p. 74-85.

103. Garanich, JS, Pahakis M, and Tarbell JM. Shear stress inhibits smooth muscle cell migration via nitric oxide-mediated downregulation of matrix metalloproteinase-2 activity. Am J Physiol Heart Circ Physiol. 2005. 288(5): p. H2244-52.

104. Jockusch, BM, Bubeck P, Giehl K, Kroemker M, Moschner J, Rothkegel M, Rudiger M, Schluter K, Stanke G, and Winkler J. The molecular architecture of focal adhesions. Annual Review of Cell and Developmental Biology. 1995. 11: p. 379-416.

105. Johnson, C and Galis ZS. Matrix metalloproteinase-2 and -9 differentially regulate smooth muscle cell migration and cell-mediated collagen organization. Arterioscler Thromb Vasc Biol. 2004. 24(1): p. 54-60.

106. Kallenbach, K, Fernandez HA, Seghezzi G, Baumann FG, Patel S, Grossi EA, Galloway $\mathrm{AC}$, and Mignatti P. A quantitative in vitro model of smooth muscle cell migration through the arterial wall using the human amniotic membrane. Arterioscler Thromb Vasc Biol. 2003. 23(6): p. 1008-13.

107. Kenagy, RD and Clowes AW. Blockade of smooth muscle cell migration and proliferation in baboon aortic explants by interleukin-lbeta and tumor necrosis factoralpha is nitric oxide-dependent and nitric oxide-independent. J Vasc Res. 2000. 37(5): p. 381-9.

108. Liu, B, Itoh H, Louie O, Kubota K, and Kent KC. The signaling protein rho is necessary for vascular smooth muscle migration and survival but not for proliferation. Surgery. 2002. 132(2): p. 317-25.

109. Miyata, K, Oike Y, Hoshii T, Maekawa H, Ogawa H, Suda T, Araki K, and Yamamura $\mathrm{K}$. Increase of smooth muscle cell migration and of intimal hyperplasia in mice lacking the alpha/beta hydrolase domain containing 2 gene. Biochem Biophys Res Commun. 2005. 329(1): p. 296-304.

110. Okazaki, J, Mawatari K, Liu B, and Kent KC. The effect of protein kinase $\mathrm{c}$ and its alpha subtype on human vascular smooth muscle cell proliferation, migration and fibronectin production. Surgery. 2000. 128(2): p. 192-7.

111. Willis, AI, Pierre-Paul D, Sumpio BE, and Gahtan V. Vascular smooth muscle cell migration: Current research and clinical implications. Vasc Endovascular Surg. 2004. 38(1): p. 11-23. 
112. Schachner, T, Steger C, Heiss S, Bonaros N, Sterlacci W, Laufer G, and Bonatti J. Paclitaxel treatment reduces neointimal hyperplasia in cultured human saphenous veins. Eur J Cardiothorac Surg. 2007. 32(6): p. 906-11.

113. Conte, MS. Molecular engineering of vein bypass grafts. J Vasc Surg. 2007. 45 Suppl A: p. A74-81.

114. Liu, YP, Wen SJ, Liu Y, Zhao LM, Guo YH, Chen XJ, Wang ZG, Liu JL, Wen J, Wang $\mathrm{SQ}$, and Tang J. [hyperplasia suppressor gene expression in vascular smooth muscle cells derived from normotentive and hypertensive patients underwent bypass surgery]. Zhonghua Xin Xue Guan Bing Za Zhi. 2007. 35(10): p. 914-8.

115. Chung, AW, Wong J, Luo H, Hsiang YN, van Breemen C, and Okon EB. Arterialization of a vein graft promotes cell cycle progression through akt and p38 mitogen-activated protein kinase pathways: Impact of the preparation procedure. Can J Cardiol. 2007. 23(14): p. 1147-54.

116. Chung, CY, Murphy_Ullrich JE, and Erickson HP. Mitogenesis, cell migration, and loss of focal adhesions induced by tenascin-c interacting with its cell surface receptor, annexin ii. Molecular Biology of the Cell. 1996. 7(6): p. 883-92.

117. Chung, JK, Lee T, Jung IM, Kim YK, Min SK, Suh JW, and Kim SJ. Expression of cell cycle regulators during smooth muscle cell proliferation after balloon catheter injury of rat artery. J Korean Med Sci. 2004. 19(3): p. 327-32.

118. Corpataux, JM, Naik J, Porter KE, and London NJ. The effect of six different statins on the proliferation, migration, and invasion of human smooth muscle cells. J Surg Res. 2005. 129(1): p. 52-6.

119. Corpataux, JM, Naik J, Porter KE, and London NJ. A comparison of six statins on the development of intimal hyperplasia in a human vein culture model. Eur J Vasc Endovasc Surg. 2005. 29(2): p. 177-81.

120. Fasciano, S, Patel RC, Handy I, and Patel CV. Regulation of vascular smooth muscle proliferation by heparin: Inhibition of cyclin-dependent kinase 2 activity by p27(kip1). J Biol Chem. 2005. 280(16): p. 15682-9.

121. Fletcher, JP, Ao PY, and Hawthorne WJ. Antiproliferative effects of low molecular weight heparin. ANZ J Surg. 2004. 74(9): p. 793-6.

122. Mekontso-Dessap, A, Kirsch M, Guignambert C, Zadigue P, Adnot S, Loisance D, and Eddahibi S. Vascular-wall remodeling of 3 human bypass vessels: Organ culture and smooth muscle cell properties. J Thorac Cardiovasc Surg. 2006. 131(3): p. 651-8. 
123. Nicholl, SM, Roztocil E, Galaria, II, and Davies MG. Plasmin induces smooth muscle cell proliferation. J Surg Res. 2005. 127(1): p. 39-45.

124. Pintucci, G, Saunders PC, Gulkarov I, Sharony R, Kadian-Dodov DL, Bohmann K, Baumann FG, Galloway AC, and Mignatti P. Anti-proliferative and anti-inflammatory effects of topical mapk inhibition in arterialized vein grafts. Faseb J. 2006. 20(2): p. 398400.

125. Uglow, EB, Slater S, Sala_Newby GB, Aguilera_Garcia CM, Angelini GD, Newby AC, and George SJ. Dismantling of cadherin-mediated cell-cell contacts modulates smooth muscle cell proliferation. Circ Res (Online). 2003. 92(12): p. 1314-21.

126. Kon, ZN, White C, Kwon MH, Judy J, Brown EN, Gu J, Burris NS, Laird PC, Brown T, Brazio PS, Gammie J, Brown J, Griffith BP, and Poston RS. The role of preexisting pathology in the development of neointimal hyperplasia in coronary artery bypass grafts. J Surg Res. 2007. 142(2): p. 351-6.

127. Kaiura, TL, Itoh $\mathrm{H}$, and Kent $\mathrm{KC}$. The role of mitogen-activated protein kinase and protein kinase $\mathrm{c}$ in fibronectin production in human vascular smooth muscle cells. J Surg Res. 1999. 84(2): p. 212-7.

128. Murakami, S, Toda Y, Seki T, Munetomo E, Kondo Y, Sakurai T, Furukawa Y, Matsuyama M, Nagate T, Hosokawa N, and Nagata K. Heat shock protein (hsp) 47 and collagen are upregulated during neointimal formation in the balloon-injured rat carotid artery. Atherosclerosis. 2001. 157(2): p. 361-8.

129. Rodriguez-Feo, JA, Sluijter JP, de Kleijn DP, and Pasterkamp G. Modulation of collagen turnover in cardiovascular disease. Curr Pharm Des. 2005. 11(19): p. 2501-14.

130. Thyberg, J, Blomgren K, Roy J, Tran PK, and Hedin U. Phenotypic modulation of smooth muscle cells after arterial injury is associated with changes in the distribution of laminin and fibronectin. J Histochem Cytochem. 1997. 45(6): p. 837-46.

131. Zhang, WD, Bai HZ, Sawa Y, Yamakawa T, Kadoba K, Taniguchi K, Masuda J, Ogata J, Shirakura R, and Matsuda H. Association of smooth muscle cell phenotypic modulation with extracellular matrix alterations during neointima formation in rabbit vein grafts. J Vasc Surg. 1999. 30(1): p. 169-83.

132. Ye, ZX, Leu HB, Wu TC, Lin SJ, and Chen JW. Baseline serum matrix metalloproteinase-9 level predicts long-term prognosis after coronary revascularizations in stable coronary artery disease. Clin Biochem. 2008. 41(4-5): p. 292-298.

133. Turner, NA, Ho S, Warburton P, O'Regan DJ, and Porter KE. Smooth muscle cells cultured from human saphenous vein exhibit increased proliferation, invasion, and mitogen-activated protein kinase activation in vitro compared with paired internal mammary artery cells. J Vasc Surg. 2007. 45(5): p. 1022-8. 
134. Turner, NA, Hall KT, Ball SG, and Porter KE. Selective gene silencing of either mmp-2 or mmp-9 inhibits invasion of human saphenous vein smooth muscle cells. Atherosclerosis. 2007. 193(1): p. 36-43.

135. Feldman, LJ, Mazighi M, Scheuble A, Deux JF, De Benedetti E, Badier-Commander C, Brambilla E, Henin D, Steg PG, and Jacob MP. Differential expression of matrix metalloproteinases after stent implantation and balloon angioplasty in the hypercholesterolemic rabbit. Circulation. 2001. 103(25): p. 3117-22.

136. Furman, C, Luo Z, Walsh K, Duverger N, Copin C, Fruchart JC, and Rouis M. Systemic tissue inhibitor of metalloproteinase-1 gene delivery reduces neointimal hyperplasia in balloon-injured rat carotid artery. FEBS Lett. 2002. 531(2): p. 122-6.

137. Galis, ZS, Muszynski M, Sukhova GK, Simon_Morrissey E, Unemori EN, Lark MW, Amento E, and Libby P. Cytokine-stimulated human vascular smooth muscle cells synthesize a complement of enzymes required for extracellular matrix digestion. C Res (Online). 1994. 75(1): p. 181-9.

138. Godin, D, Ivan E, Johnson C, Magid R, and Galis ZS. Remodeling of carotid artery is associated with increased expression of matrix metalloproteinases in mouse blood flow cessation model. Circulation. 2000. 102(23): p. 2861-6.

139. Kishi, J and Hayakawa T. Synthesis of latent collagenase and collagenase inhibitor by bovine aortic medial explants and cultured medial smooth muscle cells. Connective Tissue Research. 1989. 19(1): p. 63-76.

140. Loftus, IM, Porter K, Peterson M, Boyle J, London NJ, Bell PR, and Thompson MM. Mmp inhibition reduces intimal hyperplasia in a human vein graft stenosis model. Ann N Y Acad Sci. 1999. 878: p. 547-50.

141. Loftus, IM and Thompson MM. The role of matrix metalloproteinases in vascular disease. Vasc Med. 2002. 7(2): p. 117-33.

142. Mavromatis, K, Fukai T, Tate M, Chesler N, Ku DN, and Galis ZS. Early effects of arterial hemodynamic conditions on human saphenous veins perfused ex vivo. Arterioscler Thromb Vasc Biol. 2000. 20(8): p. 1889-95.

143. Meng, X, Mavromatis K, and Galis ZS. Mechanical stretching of human saphenous vein grafts induces expression and activation of matrix-degrading enzymes associated with vascular tissue injury and repair. Exp Mol Pathol. 1999. 66(3): p. 227-37.

144. Patterson, MA, Leville CD, Hower CD, Jean-Claude JM, Seabrook GR, Towne JB, and Cambria RA. Shear force regulates matrix metalloproteinase activity in human saphenous vein organ culture. J Surg Res. 2001. 95(1): p. 67-72. 
145. Porter, KE, Thompson MM, Loftus IM, McDermott E, Jones L, Crowther M, Bell PR, and London NJ. Production and inhibition of the gelatinolytic matrix metalloproteinases in a human model of vein graft stenosis. Eur J Vasc Endovasc Surg. 1999. 17(5): p. 40412.

146. Sukhova, GK, Shi GP, Simon DI, Chapman HA, and Libby P. Expression of the elastolytic cathepsins $\mathrm{s}$ and $\mathrm{k}$ in human atheroma and regulation of their production in smooth muscle cells. J Clin Invest. 1998. 102(3): p. 576-83.

147. West, NE, Qian H, Guzik TJ, Black E, Cai S, George SE, and Channon KM. Nitric oxide synthase (nnos) gene transfer modifies venous bypass graft remodeling: Effects on vascular smooth muscle cell differentiation and superoxide production. Circulation. 2001. 104(13): p. 1526-32.

148. Yamada, T, Shiraishi R, Taki K, Nakano S, Tokunaga O, and Itoh $T$. Immunohistochemical and ultrastructural examination of smooth muscle cells in aortocoronary saphenous vein grafts. Angiology. 1997. 48(5): p. 381-90.

149. Jiang, Z, Yu P, Tao M, Fernandez C, Ifantides C, Moloye O, Schultz GS, Ozaki CK, and Berceli SA. Tgf-beta- and ctgf-mediated fibroblast recruitment influences early outward vein graft remodeling. Am J Physiol Heart Circ Physiol. 2007. 293(1): p. H482-8.

150. Brody, WR, Angeli WW, and Kosek JC. Histologic fate of the venous coronary artery bypass in dogs. Am J Path. 1972. 66(1): p. 111-30.

151. Schwartz, SM, Campbell GR, and Campbell JH. Replication of smooth muscle cells in vascular disease. Circ Res (Online). 1986. 58(4): p. 427-44.

152. Allaire, E and Clowes AW. Endothelial cell injury in cardiovascular surgery: The intimal hyperplastic response. Ann Thorac Surg. 1997. 63: p. 582-591.

153. Libby, P, Warner SJ, Salomon RN, and Birinyi LK. Production of platelet-derived growth factor-like mitogen by smooth-muscle cells from human atheroma. New Eng J Med. 1988. 318(23): p. 1493-8.

154. Ross, R. The pathogenesis of atherosclerosis: A perspective for the 1990s. Nature. 1993. 362(6423): p. 801-9.

155. Sjolund, M, Hedin U, Sejersen T, Heldin $\mathrm{CH}$, and Thyberg J. Arterial smooth muscle cells express platelet-derived growth factor (pdgf) a chain mrna, secrete a pdgf-like mitogen, and bind exogenous pdgf in a phenotype- and growth state-dependent manner. $\mathrm{J}$ Cell Biol. 1988. 106(2): p. 403-13.

156. Morisaki, N, Kanzaki T, Motoyama N, Saito Y, and Yoshida S. Cell cycle-dependent inhibition of DNA synthesis by prostaglandin i2 in cultured rabbit aortic smooth muscle cells. Atherosclerosis. 1988. 71(2-3): p. 165-71. 
157. Nilsson, J, von-Euler AM, and Dalsgaard CJ. Stimulation of connective tissue cell growth by substance $p$ and substance $k$. Nature. 1985. 315(6014): p. 61-3.

158. Owen, NE. Effect of prostaglandin e1 on DNA synthesis in vascular smooth muscle cells. Am J Phys. 1986. 250(4 Pt 1): p. C584-8.

159. Palmberg, L, Claesson HE, and Thyberg J. Leukotrienes stimulate initiation of DNA synthesis in cultured arterial smooth muscle cells. J Cell Sci. 1987. 88 ( Pt 2): p. 151-9.

160. Taubman, MB, Berk BC, Izumo S, Tsuda T, Alexander RW, and Nadal_Ginard B. Angiotensin II induces c-fos mrna in aortic smooth muscle. Role of ca2+ mobilization and protein kinase c activation. J Biol Chem. 1989. 264(1): p. 526-30.

161. Birukov, KG, Shirinsky VP, Stepanova OV, Tkachuk VA, Hahn AW, Resink TJ, and Smirnov VN. Stretch affects phenotype and proliferation of vascular smooth muscle cells. Mol Cell Biochem. 1995. 144(2): p. 131-9.

162. Dufourcq, P, Louis H, Dandre F, Lavie J, Bonnet J, and Lamaziere JM. Phenotypic modification of arterial smooth muscle cells in response to medial dissection. Coronary Artery Disease. 1997. 8(3-4): p. 163-70.

163. Wolff, RA, Malinowski RL, Heaton NS, Hullett DA, and Hoch JR. Transforming growth factor-betal antisense treatment of rat vein grafts reduces the accumulation of collagen and increases the accumulation of h-caldesmon. J Vasc Surg. 2006. 43(5): p. 1028-36.

164. Zeidan, A, Nordstrom I, Dreja K, Malmqvist U, and Hellstrand P. Stretch-dependent modulation of contractility and growth in smooth muscle of rat portal vein. Circ Res (Online). 2000. 87(3): p. 228-34.

165. Goldman, J, Zhong L, and Liu SQ. Degradation of alpha-actin filaments in venous smooth muscle cells in response to mechanical stretch. Am J Phys. Heart and Circ Phys. 2003. 284(5): p. H1839-47.

166. Campbell, GR, Campbell JH, Manderson JA, Horrigan S, and Rennick RE. Arterial smooth muscle. A multifunctional mesenchymal cell. Archives of Pathology \& Laboratory Medicine. 1988. 112(10): p. 977-86.

167. Nagai, R, Kuro_o M, Babij P, and Periasamy M. Identification of two types of smooth muscle myosin heavy chain isoforms by cdna cloning and immunoblot analysis. J Biol Chem. 1989. 264(17): p. 9734-7.

168. Frid, MG, Shekhonin BV, Koteliansky VE, and Glukhova MA. Phenotypic changes of human smooth muscle cells during development: Late expression of heavy caldesmon and calponin. Dev Biol. 1992. 153(2): p. 185-93. 
169. van der Loop, FT, Gabbiani G, Kohnen G, Ramaekers FC, and van_Eys GJ. Differentiation of smooth muscle cells in human blood vessels as defined by smoothelin, a novel marker for the contractile phenotype. ATVB (Online). 1997. 17(4): p. 665-71.

170. Thyberg, J, Blomgren K, Hedin U, and Dryjski M. Phenotypic modulation of smooth muscle cells during the formation of neointimal thickenings in the rat carotid artery after balloon injury: An electron-microscopic and stereological study. Cell Tissue Res. 1995. 281(3): p. 421-33.

171. Kalra, M and Miller VM. Early remodeling of saphenous vein grafts: Proliferation, migration and apoptosis of adventitial and medial cells occur simultaneously with changes in graft diameter and blood flow. J Vasc Res. 2000. 37(6): p. 576-84.

172. Bornstein, P. Diversity of function is inherent in matricellular proteins: An appraisal of thrombospondin 1. J Cell Biol. 1995. 130(3): p. 503-6.

173. Sage, EH and Bornstein P. Extracellular proteins that modulate cell-matrix interactions. Sparc, tenascin, and thrombospondin. J Biol Chem. 1991. 266(23): p. 14831-4.

174. Nikolopoulos, SN and Turner CE. Integrin-linked kinase (ilk) binding to paxillin ld1 motif regulates ilk localization to focal adhesions. J Biol Chem. 2001. 276(26): p. 23499505.

175. Tu, Y, Wu S, Shi X, Chen K, and Wu C. Migfilin and mig-2 link focal adhesions to filamin and the actin cytoskeleton and function in cell shape modulation. Cell. 2003. 113: p. 37-47.

176. Murphy-Ullrich, JE. The de-adhesive activity of matricellular proteins: Is intermediate cell adhesion an adaptive state? J Clin Invest. 2001. 107(7): p. 785-90.

177. $\mathrm{Wu}, \mathrm{C}$ and Dedhar S. Integrin-linked kinase (ilk) and its interactors: A new paradigm for the coupling of extracellular matrix to actin cytoskeleton and signaling complexes. J Cell Biol. 2001. 155(4): p. 505-10.

178. Greenwood, JA, Theibert AB, Prestwich GD, and Murphy_Ullrich JE. Restructuring of focal adhesion plaques by pi 3-kinase. Regulation by ptdins $(3,4,5)$-p(3) binding to alphaactinin. J Cell Biol. 2000. 150(3): p. 627-42.

179. Murphy-Ullrich, JE, Lightner VA, Aukhil I, Yan YZ, Erickson HP, and Hook M. Focal adhesion integrity is downregulated by the alternatively spliced domain of human tenascin. J Cell Biol. 1991. 115(4): p. 1127-36.

180. $\mathrm{Wu}, \mathrm{C}$. Integrin-linked kinase and pinch: Partners in regulation of cell-extracellular matrix interaction and signal transduction. J Cell Sci. 1999. 112 ( Pt 24): p. 4485-9. 
181. Newby, AC, Southgate KM, and Davies MG. Extracellular matrix degrading metalloproteinases in the pathogensis of arteriosclerosis. Basic Res Cardiol. 1994. 89(Suppl 1): p. 59-70.

182. Porter, KE, Naik J, Turner NA, Dickison T, Thompson MM, and London JM. Simvastatin inhibits human saphenous vein neointima formation via inhibition of smooth muscle cell proliferation and migration. J. Vasc. Surg. 2002. 36: p. 150-7.

183. Southgate, KM, Davies M, Booth RF, and Newby AC. Involvement of extracellularmatrix-degrading metalloproteinases in rabbit aortic smooth-muscle cell proliferation. Biochem J. 1992. 288 ( Pt 1): p. 93-9.

184. Lafleur, MA, Hollenberg MD, Atkinson SJ, Knauper V, Murphy G, and Edwards DR. Activation of pro-(matrix metalloproteinase-2) (pro-mmp-2) by thrombin is membranetype-mmp-dependent in human umbilical vein endothelial cells and generates a distinct $63 \mathrm{kda}$ active species. Biochem J. 2001. 357(Pt 1): p. 107-15.

185. George, SJ, Baker AH, Angelini GD, and Newby AC. Gene transfer of tissue inhibitor of metalloproteinase-2 inhibits metalloproteinase activity and neointima formation in human saphenous veins. Gene Ther. 1998. 5(11): p. 1552-60.

186. George, SJ, Johnson JL, Angelini GD, Newby AC, and Baker AH. Adenovirus-mediated gene transfer of the human timp-1 gene inhibits smooth muscle cell migration and neointimal formation in human saphenous vein. Hum Gene Ther. 1998. 9(6): p. 867-77.

187. Lijnen, HR, Soloway P, and Collen D. Tissue inhibitor of matrix metalloproteinases-1 impairs arterial neointima formation after vascular injury in mice. Circ Res. 1999. 85(12): p. 1186-91.

188. George, SJ, Zaltsman AB, and Newby AC. Surgical preparative injury and neointima formation increase mmp-9 expression and $\mathrm{mmp}-2$ activation in human saphenous vein. Cardiovasc Res. 1997. 33(2): p. 447-59.

189. Porter, KE, Loftus IM, Peterson M, Bell PR, London NJ, and Thompson MM. Marimastat inhibits neointimal thickening in a model of human vein graft stenosis. $\mathrm{Br} \mathbf{J}$ Surg. 1998. 85(10): p. 1373-7.

190. Redmond, EM, Cahill PA, Hirsch M, Wang YN, Sitzmann JV, and Okada SS. Effect of pulse pressure on vascular smooth muscle cell migration: The role of urokinase and matrix metalloproteinase. Thrombosis \& Haemostasis. 1999. 81(2): p. 293-300.

191. Grote, K, Flach I, Luchtefeld M, Akin E, Holland SM, Drexler H, and Schieffer B. Mechanical stretch enhances mrna expression and proenzyme release of matrix metalloproteinase-2 (mmp-2) via nad(p)h oxidase-derived reactive oxygen species. Circ Res. 2003. 92(11): p. 80-6. 
192. Asanuma, K, Magid R, Johnson C, Nerem RM, and Galis ZS. Uniaxial strain upregulates matrix-degrading enzymes produced by human vascular smooth muscle cells. Am J Physiol Heart Circ Physiol. 2003. 284(5): p. H1778-84.

193. Tyagi, SC, Lewis K, Pikes D, Marcello A, Mujumdar VS, Smiley LM, and Moore CK. Stretch-induced membrane type matrix metalloproteinase and tissue plasminogen activator in cardiac fibroblast cells. J Cell Physiol. 1998. 176(2): p. 374-82.

194. Yamaoka, A, Matsuo T, Shiraga F, and Ohtsuki H. Timp-1 production by human scleral fibroblast decreases in response to cyclic mechanical stretching. Opthal Res. 2001. 33(2): p. $98-101$.

195. Bassiouny, HS, Song RH, Kocharyan H, Kins E, and Glagov S. Low flow enhances platelet activation after acute experimental arterial injury. J Vasc Surg. 1998. 27(5): p. 910-8.

196. Nakazawa, T, Yasuhara H, Shigematsu K, and Shigematsu H. Smooth muscle cell migration induced by shear-loaded platelets and endothelial cells. Enhanced plateletderived growth factor production by shear-loaded platelets. International Angiology : a Journal of the International Union of Angiology. 2000. 19(2): p. 142-6.

197. Powell, RJ, Carruth JA, Basson MD, Bloodgood R, and Sumpio BE. Matrix-specific effect of endothelial control of smooth muscle cell migration. J Vasc Surg. 1996. 24(1): p. 51-7.

198. Shigematsu, K, Yasuhara H, Shigematsu H, and Muto T. Direct and indirect effects of pulsatile shear stress on the smooth muscle cell. International Angiology : a Journal of the International Union of Angiology. 2000. 19(1): p. 39-46.

199. Sho, M, Sho E, Singh TM, Komatsu M, Sugita A, Xu C, Nanjo H, Zarins CK, and Masuda H. Subnormal shear stress-induced intimal thickening requires medial smooth muscle cell proliferation and migration. Exper Molec Path. 2002. 72(2): p. 150-60.

200. Westerband, A, Gentile AT, Hunter GC, Gooden MA, Aguirre ML, Berman SS, and Mills JL. Intimal growth and neovascularization in human stenotic vein grafts. J Am Col Surg. 2000. 191(3): p. 264-71.

201. Cheng, J and Du J. Mechanical stretch simulates proliferation of venous smooth muscle cells through activation of the insulin-like growth factor-1 receptor. ATVB. 2007. 27(8): p. $1744-51$.

202. Tineli, RA, Viaro F, Dalio MB, Reis GS, Basseto S, Vicente WV, Rodrigues AJ, and Evora PR. Mechanical forces and human saphenous veins: Coronary artery bypass graft implications. Rev Bras Cir Cardiovasc. 2007. 22(1): p. 87-95. 
203. Alcocer, F, Whitley D, Salazar J, Jordan W, and Bland KI. Mutual exclusion of apoptosis and hsp70 in human vein intimal hyperplasia in vitro. J Surg Res. 2001. 96(1): p. 75-80.

204. Igase, M, Okura T, Kitami Y, and Hiwada K. Apoptosis and bcl-xs in the intimal thickening of balloon-injured carotid arteries. Clin Sci (Lond). 1999. 96(6): p. 605-12.

205. Kamenz, J, Seibold W, Wohlfrom M, Hanke S, Heise N, Lenz C, and Hanke H. Incidence of intimal proliferation and apoptosis following balloon angioplasty in an atherosclerotic rabbit model. Cardiovasc Res. 2000. 45(3): p. 766-76.

206. Wang, GJ, Sui XX, Simosa HF, Jain MK, Altieri DC, and Conte MS. Regulation of vein graft hyperplasia by survivin, an inhibitor of apoptosis protein. ATVB. 2005. 25(10): p. 2081-7.

207. Hoel, AW, Wang GJ, Simosa HF, and Conte MS. Regulation of vascular smooth muscle cell growth by survivin. Vascular. 2007. 15(6): p. 344-9.

208. Jiang, Z, Shukla A, Miller BL, Espino DR, Tao M, Berceli SA, and Ozaki CK. Tumor necrosis factor-alpha and the early vein graft. J Vasc Surg. 2007. 45(1): p. 169-76.

209. Nishibe, T, Miyazaki K, Kudo F, Flores J, Nagato M, Kumada T, and Yasuda K. Induction of angiotensin converting enzyme in neointima after intravascular stent placement. Int Angiol. 2002. 21(3): p. 250-5.

210. Zuckerbraun, BS, McCloskey CA, Mahidhara RS, Kim PK, Taylor BS, and Tzeng E. Overexpression of mutated ikappabalpha inhibits vascular smooth muscle cell proliferation and intimal hyperplasia formation. J Vasc Surg. 2003. 38(4): p. 812-9.

211. Hilker, M, Buerke M, Lehr HA, Oelert H, and Hake U. Bypass graft disease: Analysis of proliferative activity in human aorto-coronary bypass grafts. Heart Surg Forum. 2002. 5 Suppl 4: p. S331-41.

212. Predel, HG, Yang Z, von_Segesser L, Turina M, Buhler FR, and Luscher TF. Implications of pulsatile stretch on growth of saphenous vein and mammary artery smooth muscle. Lancet. 1992. 340(8824): p. 878-9.

213. Dethlefsen, SM, Shepro D, and D'Amore PA. Comparison of the effects of mechanical stimulation on venous and arterial smooth muscle cells in vitro. J Vasc Res. 1996. 33(5): p. 405-13.

214. $\mathrm{Hu}, \mathrm{Y}$, Bock G, Wick G, and Xu Q. Activation of pdgf receptor alpha in vascular smooth muscle cells by mechanical stress. Faseb J. 1998. 12(12): p. 1135-42.

215. Wolf, YG, Rasmussen LM, and Ruoslahti E. Antibodies against transforming growth factor-beta 1 suppress intimal hyperplasia in a rat model. J Clin Invest. 1994. 93(3): p. 1172-8. 
216. Li, Z, Moore S, and Alavi MZ. Mitogenic factors released from smooth muscle cells are responsible for neointimal cell proliferation after balloon catheter deendothelialization. Exp Mol Pathol. 1995. 63(2): p. 77-86.

217. Miano, JM, Vlasic N, Tota RR, and Stemerman MB. Smooth muscle cell immediateearly gene and growth factor activation follows vascular injury. A putative in vivo mechanism for autocrine growth. Arterioscler Thromb. 1993. 13(2): p. 211-9.

218. Uzui, H, Lee JD, Shimizu H, Tsutani H, and Ueda T. The role of protein-tyrosine phosphorylation and gelatinase production in the migration and proliferation of smooth muscle cells. Atherosclerosis. 2000. 149(1): p. 51-9.

219. Mii, S, Ware JA, and Kent KC. Transforming growth factor-beta inhibits human vascular smooth muscle cell growth and migration. Surgery. 1993. 114(2): p. 464-70.

220. Qian, H, Zhang B, and Zhao $\mathrm{H}$. [gene expression of bfgf and intimal hyperplasia of autologous vein grafts in rats]. Zhonghua Yi Xue Za Zhi. 1996. 76(11): p. 826-8.

221. Francis, SE, Hunter S, Holt CM, Gadsdon PA, Rogers S, Duff GW, Newby AC, and Angelini GD. Release of platelet-derived growth factor activity from pig venous arterial grafts. J Thorac Cardiovasc Surg. 1994. 108(3): p. 540-8.

222. Cho, A and Reidy MA. Matrix metalloproteinase-9 is necessary for the regulation of smooth muscle cell replication and migration after arterial injury. Circ Res. 2002. 91(9): p. $845-51$.

223. Annabi, B, Shedid D, Ghosn P, Kenigsberg RL, Desrosiers RR, Bojanowski MW, Beaulieu E, Nassif E, Moumdjian R, and Beliveau R. Differential regulation of matrix metalloproteinase activities in abdominal aortic aneurysms. J Vasc Surg. 2002. 35(3): p. $539-46$.

224. Cowan, DB and Langille BL. Cellular and molecular biology of vascular remodeling. Current Opinion in Lipidology. 1996. 7(2): p. 94-100.

225. Fulton, GJ, Davies MG, Barber L, Gray JL, Svendsen E, and Hagen PO. Local effects of nitric oxide supplementation and suppression in the development of intimal hyperplasia in experimental vein grafts. Eur J Vasc Endovasc Surg. 1998. 15(4): p. 279-89.

226. Cox, JL, Chiasson DA, and Gotlieb AI. Stranger in a strange land: The pathogenesis of saphenous vein graft stenosis with emphasis on structural and functional differences between veins and arteries. Progress in Cardiovascular Diseases. 1991. 34(1): p. 45-68.

227. Guarda, E, Katwa LC, Campbell SE, Tanner MA, Webel RM, Laughlin H, Jenkins S, and Myers PR. Extracellular matrix collagen synthesis and degradation following coronary 
balloon angioplasty. Journal of Molecular and Cellular Cardiology. 1996. 28(4): p. 699706.

228. Wesly, RL, Vaishnav RN, Fuchs JC, Patel DJ, and Greenfield JC. Static linear and nonlinear elastic properties of normal and arterialized venous tissue in dog and man. C Res (Online). 1975. 37(4): p. 509-20.

229. Davies, AH, Magee TR, Baird RN, and Horrocks M. Prevention of malalignment during non-reversed femorodistal bypass. Ann R Coll Surg Engl. 1992. 74(6): p. 434-5.

230. Davies, AH, Magee TR, Baird RN, Sheffield E, and Horrocks M. Pre-bypass morphological changes in vein grafts. Eur J Vasc Surg. 1993. 7(6): p. 642-7.

231. Gusic, RJ, Petko M, Myung R, William Gaynor J, and Gooch KJ. Mechanical properties of native and ex vivo remodeled porcine saphenous veins. J Biomech. 2005. 38(9): p. $1770-9$.

232. Bassiouny, HS, White S, Glagov S, Choi E, Giddens DP, and Zarins CK. Anastomotic intimal hyperplasia: Mechanical injury or flow induced. J Vasc Surg. 1992. 15(4): p. 70816; discussion 716-7.

233. Berkowitz, HD, Fox AD, and Deaton $\mathrm{DH}$. Reversed vein graft stenosis: Early diagnosis and management. J Vasc Surg. 1992. 15(1): p. 130-41; discussion 141-2.

234. Tai, NR, Salacinski HJ, Edwards A, Hamilton G, and Seifalian AM. Compliance properties of conduits used in vascular reconstruction. Br J Surg. 2000. 87(11): p. 151624.

235. Jacot, JG, Abdullah I, Belkin M, Gerhard-Herman M, Gaccione P, Polak JF, Donaldson $\mathrm{MC}$, Whittemore AD, and Conte MS. Early adaptation of human lower extremity vein grafts: Wall stiffness changes accompany geometric remodeling. J Vasc Surg. 2004. 39(3): p. 547-55.

236. Stooker, W, Gok M, Sipkema P, Niessen HW, Baidoshvili A, Westerhof N, Jansen EK, Wildevuur CR, and Eijsman L. Pressure-diameter relationship in the human greater saphenous vein. Ann Thorac Surg. 2003. 76(5): p. 1533-8.

237. Bunt, TJ. Synthetic vascular graft infections. I. Graft infections. Surgery. 1983. 93(6): p. $733-46$.

238. Edwards, WH, Jr., Martin RS, 3rd, Jenkins JM, Edwards WH, Sr., and Mulherin JL, Jr. Primary graft infections. J Vasc Surg. 1987. 6(3): p. 235-9.

239. Rho, KS, Jeong L, Lee G, Seo BM, Park YJ, Hong SD, Roh S, Cho JJ, Park WH, and Min BM. Electrospinning of collagen nanofibers: Effects on the behavior of normal 
human keratinocytes and early-stage wound healing. Biomaterials. 2006. 27(8): p. 145261.

240. Kanjickal, D, Lopina S, Evancho-Chapman MM, Schmidt S, Donovan D, and Springhetti S. Polymeric sustained local drug delivery system for the prevention of vascular intimal hyperplasia. J Biomed Mater Res A. 2004. 68(3): p. 489-95.

241. Cagiannos, C, Abul-Khoudoud OR, DeRijk W, Shell DHt, Jennings LK, Tolley EA, Handorf CR, and Fabian TC. Rapamycin-coated expanded polytetrafluoroethylene bypass grafts exhibit decreased anastomotic neointimal hyperplasia in a porcine model. $\mathrm{J}$ Vasc Surg. 2005. 42(5): p. 980-8.

242. Stankus, JJ, Guan J, Fujimoto K, and Wagner WR. Microintegrating smooth muscle cells into a biodegradable, elastomeric fiber matrix. Biomaterials. 2006. 27(5): p. 735-44.

243. Wang, AY, Bobryshev YV, Cherian SM, Liang H, Tran D, Inder SJ, Lord RS, Ashwell $\mathrm{KW}$, and Farnsworth AE. Expression of apoptosis-related proteins and structural features of cell death in explanted aortocoronary saphenous vein bypass grafts. Cardiovase Surg. 2001. 9(4): p. 319-28.

244. Brant, AM, Chmielewski JF, Hung TK, and Borovetz HS. Simulation in vitro of pulsatile vascular hemodynamics using a cad/cam-designed cam disc and roller follower. Artif Organs. 1986. 10(5): p. 419-21.

245. Brant, AM, Rodgers GJ, and Borovetz HS. Measurement in vitro of pulsatile arterial diameter using a helium-neon laser. J Appl Physiol. 1987. 62(2): p. 679-83.

246. Brant, AM, Shah SS, Rodgers VG, Hoffmeister J, Herman IM, Kormos RL, and Borovetz HS. Biomechanics of the arterial wall under simulated flow conditions. J Biomech. 1988. 21(2): p. 107-13.

247. Brant, AM, Teodori MF, Kormos RL, and Borovetz HS. Effect of variations in pressure and flow on the geometry of isolated canine carotid arteries. J Biomech. 1987. 20(9): p. $831-8$.

248. Severyn, DA, Muluk SC, and Vorp DA. The influence of hemodynamics and wall biomechanics on the thrombogenicity of vein segments perfused in vitro. J Surg Res. 2004. 121(1): p. 31-7.

249. Vorp, DA, Peters DG, and Webster MW. Gene expression is altered in perfused arterial segments exposed to cyclic flexure ex vivo. Ann Biomed Eng. 1999. 27(3): p. 366-71.

250. Carrel, A and Lindbergh C. The culture of organs. 1938, New York: Paul B. Hoeber, Inc. 
251. Badimon, L, Badimon JJ, Galvez A, Chesebro JH, and Fuster V. Influence of arterial damage and wall shear rate on platelet deposition. Ex vivo study in a swine model. Arteriosclerosis. 1986. 6(3): p. 312-20.

252. Badimon, L, Badimon JJ, Turitto VT, and Fuster V. Role of von willebrand factor in mediating platelet-vessel wall interaction at low shear rate; the importance of perfusion conditions. Blood. 1989. 73(4): p. 961-7.

253. Badimon, L, Badimon JJ, Turitto VT, Rand J, and Fuster V. Functional behavior of vessels from pigs with von willebrand disease. Values of platelet deposition are identical to those obtained on normal vessels. Arteriosclerosis. 1989. 9(2): p. 184-8.

254. Badimon, L, Martinez-Gonzalez J, Royo T, Lassila R, and Badimon JJ. A sudden increase in plasma epinephrine levels transiently enhances platelet deposition on severely damaged arterial wall--studies in a porcine model. Thromb Haemost. 1999. 82(6): p. $1736-42$.

255. Vilahur, G, Baldellou MI, Segales E, Salas E, and Badimon L. Inhibition of thrombosis by a novel platelet selective s-nitrosothiol compound without hemodynamic side effects. Cardiovasc Res. 2004. 61(4): p. 806-16.

256. Clerin, V, Gusic RJ, O'Brien J, Kirshbom PM, Myung RJ, Gaynor JW, and Gooch KJ. Mechanical environment, donor age, and presence of endothelium interact to modulate porcine artery viability ex vivo. Ann Biomed Eng. 2002. 30(9): p. 1117-27.

257. Clerin, V, Nichol JW, Petko M, Myung RJ, Gaynor JW, and Gooch KJ. Tissue engineering of arteries by directed remodeling of intact arterial segments. Tissue Eng. 2003. 9(3): p. 461-72.

258. Jerius, H, Bagwell CA, Beall A, Karolyi D, and Brophy C. Vascular smooth muscle mechanics in isolated perfused segments of carotid arteries. Surgery. 2000. 127(2): p. $148-54$.

259. Paroz, A, Probst H, Saucy F, Mazzolai L, Rizzo E, Ris HB, and Corpataux JM. Comparison of morphological and functional alterations of human saphenous veins after seven and fourteen days of ex vivo perfusion. Eur Surg Res. 2004. 36(5): p. 274-81.

260. Rey, J, Probst H, Mazzolai L, Bosman FT, Pusztaszeri M, Stergiopulos N, Ris HB, Hayoz D, Saucy F, and Corpataux JM. Comparative assessment of intimal hyperplasia development after 14 days in two different experimental settings: Tissue culture versus ex vivo continuous perfusion of human saphenous vein. J Surg Res. 2004. 121(1): p. 42-9.

261. Hahn, MS, McHale MK, Wang E, Schmedlen RH, and West JL. Physiologic pulsatile flow bioreactor conditioning of poly(ethylene glycol)-based tissue engineered vascular grafts. Ann Biomed Eng. 2007. 35(2): p. 190-200. 
262. Bergh, N, Ekman M, Ulfhammer E, Andersson M, Karlsson L, and Jern S. A new biomechanical perfusion system for ex vivo study of small biological intact vessels. Ann Biomed Eng. 2005. 33(12): p. 1808-18.

263. Chesler, NC and Enyinna OC. Particle deposition in arteries ex vivo: Effects of pressure, flow, and waveform. J Biomech Eng. 2003. 125(3): p. 389-94.

264. Chesler, NC, Thompson-Figueroa J, and Millburne K. Measurements of mouse pulmonary artery biomechanics. J Biomech Eng. 2004. 126(2): p. 309-14.

265. Gan, L, Sjogren LS, Doroudi R, and Jern S. A new computerized biomechanical perfusion model for ex vivo study of fluid mechanical forces in intact conduit vessels. $\mathrm{J}$ Vasc Res. 1999. 36(1): p. 68-78.

266. Gosselin, C, Vorp DA, Warty V, Severyn DA, Dick EK, Borovetz HS, and Greisler HP. Eptfe coating with fibrin glue, fgf-1, and heparin: Effect on retention of seeded endothelial cells. J Surg Res. 1996. 60(2): p. 327-32.

267. Han, $\mathrm{HC}$ and $\mathrm{Ku} \mathrm{DN}$. Contractile responses in arteries subjected to hypertensive pressure in seven-day organ culture. Ann Biomed Eng. 2001. 29(6): p. 467-75.

268. Maruta, F, Parker AL, Fisher KD, Murray PG, Kerr DJ, and Seymour LW. Use of a phage display library to identify oligopeptides binding to the lumenal surface of polarized endothelium by ex vivo perfusion of human umbilical veins. J Drug Target. 2003. 11(1): p. 53-9.

269. McFetridge, PS, Bodamyali T, Horrocks M, and Chaudhuri JB. Endothelial and smooth muscle cell seeding onto processed ex vivo arterial scaffolds using $3 \mathrm{~d}$ vascular bioreactors. ASAIO J. 2004. 50(6): p. 591-600.

270. McFetridge, PS, Abe K, Horrocks M, and Chaudhuri JB. Vascular tissue engineering: Bioreactor design considerations for extended culture of primary human vascular smooth muscle cells. ASAIO J. 2007. 53(5): p. 623-30.

271. Aerospace, MME. Entrance length. 2005. URL: http://wwwmdp.eng.cam.ac.uk/library/enginfo/aerothermal_dvd_only/aero/fprops/pipeflow/node9.ht $\mathrm{ml}$.

272. Muluk, SC, Kibbe MR, Gleixner SL, Vorp DA, and Billiar TR. Effect of nitric oxide on tissue factor expression by vascular tissue: Studies in cell culture and with perfused vein segments. Surg Forum. 1998. 49: p. 288-290.

273. Muluk, SC, Vorp DA, Severyn DA, Gleixner S, Johnson PC, and Webster MW. Enhancement of tissue factor expression by vein segments exposed to coronary arterial hemodynamics. J Vasc Surg. 1998. 27(3): p. 521-7. 
274. Vorp, DA, Severyn DA, and Mears JR. An experimental system to expose perfused vascular segments to cyclic bending ex-vivo. Adv in Bioeng. 1997. 36: p. 115-116.

275. Ligush, J, Labadie RF, Berceli SA, Ochoa JB, and Borovetz HS. Evaluation of endothelium-derived nitric oxide mediated vasodilation utilizing ex vivo perfusion of an intact vessel. J Surg Res. 1992. 52(5): p. 416-21.

276. Ljung, L. System identification - theory for the user. 2nd ed. 1999, Upper Saddle River, N.J.: Prentice Hall.

277. Zadeh, L. From circuit thoery to sytem theory. Proc IRE. 1962. 50: p. 856-865.

278. Favoreel, W, De Moor B, and Van Overschee P. Subsapce state space sytem identification for industrial processes. Proc of the 5th IFAC Symposium on Dynamics and Control of Process Systems, June 8-10, Corfu, Greece. 1998: p. 322-330.

279. Van Overschee, P and De Moor B. Subspace identification for linear systems: Theory, implemetnation, applications. 1996: Kluwer Academic Publishers.

280. Van Overschee, P, De Moor B, and Favoreel W. Numerical algorithms for subspace state space system identification (n4sid). in ASME Design Engineering Technical Conferences. 1997.

281. Eykhof, P, Identification: History., in Systems and control encyclopedia, Singh M, Editor. 1987, Pergamon Press: Oxford. p. 2270-2273.

282. Verhaegen, M and Dewilde P. Subspace identification, part i: The output-error state space model identification class of algorithms. Internat. J. Control. 1992. 56: p. 11871210.

283. Larimore, W. Canonical variate analysis in identification, filtering and adaptive control. in 29th Conference on Decision and Control, CDC 90. 1990. Hawaii.

284. Bolz, SS, Pieperhoff S, De Wit C, and Pohl U. Intact endothelial and smooth muscle function in small resistance arteries after $48 \mathrm{~h}$ in vessel culture. Am J Physiol Heart Circ Physiol. 2000. 279(3): p. H1434-9.

285. Chesler, NC, Conklin BS, Han HC, and Ku DN. Simplified ex vivo culture techniques for porcine arteries. J Vasc Invest. 1998. 4: p. 123-127.

286. Peng, X, Recchia FA, Byrne BJ, Wittstein IS, Ziegelstein RC, and Kass DA. In vitro system to study realistic pulsatile flow and stretch signaling in cultured vascular cells. Am J Physiol Cell Physiol. 2000. 279(3): p. C797-805.

287. Cho, KH, Johansson KH, and Wolkenhauer O. A hybrid systems framework for cellular processes. Biosystems. 2005. 80(3): p. 273-82. 
288. Xiao, X, Mullen TJ, and Mukkamala R. System identification: A multi-signal approach for probing neural cardiovascular regulation. Physiol Meas. 2005. 26(3): p. R41-71.

289. Xiao, X, Mukkamala R, and Cohen RJ. A weighted-principal component regression method for the identification of physiologic systems. IEEE Trans Biomed Eng. 2006. 53(8): p. 1521-1530.

290. Shaw, JA. The pid control algorithm how it works, how to tune it, and how to use it. 2nd ed. 2005.

291. Chandran, KB. Cardiovascular biomechanics. 1992, New York: University Press. 544.

292. Nichols, WW and O'Rourke MF. Mcdonald's blood flow in arteries: Theoretical, experimental and clinical principles. Third ed. 1990, Kent: Edward Arnold. 456.

293. Schepers, A, Pires NM, Eefting D, de Vries MR, van Bockel JH, and Quax PH. Shortterm dexamethasone treatment inhibits vein graft thickening in hypercholesterolemic apoe3leiden transgenic mice. J Vasc Surg. 2006. 43(4): p. 809-15.

294. Angelini, GD, Bryan AJ, Williams HM, Soyombo AA, Williams A, Tovey J, and Newby AC. Time-course of medial and intimal thickening in pig venous arterial grafts: Relationship to endothelial injury and cholesterol accumulation. J Thorac Cardiovasc Surg. 1992. 103(6): p. 1093-103.

295. Jeremy, JY, Dashwood MR, Timm M, Izzat MB, Mehta D, Bryan AJ, and Angelini GD. Nitric oxide synthase and adenylyl and guanylyl cyclase activity in porcine interposition vein grafts. Ann Thorac Surg. 1997. 63(2): p. 470-6.

296. Kibbe, MR, Tzeng E, Gleixner SL, Watkins SC, Kovesdi I, Lizonova A, Makaroun MS, Billiar TR, and Rhee RY. Adenovirus-mediated gene transfer of human inducible nitric oxide synthase in porcine vein grafts inhibits intimal hyperplasia. J Vasc Surg. 2001. 34: p. $156-165$.

297. Guan, J, Sacks MS, Beckman EJ, and Wagner WR. Synthesis, characterization, and cytocompatibility of elastomeric, biodegradable poly(ester-urethane)ureas based on poly(caprolactone) and putrescine. J Biomed Mater Res. 2002. 61(3): p. 493-503.

298. Fujimoto, KL, Guan J, Oshima H, Sakai T, and Wagner WR. In vivo evaluation of a porous, elastic, biodegradable patch for reconstructive cardiac procedures. Ann Thorac Surg. 2007. 83(2): p. 648-54.

299. Fujimoto, KL, Tobita K, Merryman WD, Guan J, Momoi N, Stolz DB, Sacks MS, Keller BB, and Wagner WR. An elastic, biodegradable cardiac patch induces contractile smooth muscle and improves cardiac remodeling and function in subacute myocardial infarction. J Am Coll Cardiol. 2007. 49(23): p. 2292-300. 
300. Kawatsu, S, Oda K, Saiki Y, Tabata Y, and Tabayashi K. External application of rapamycin-eluting film at anastomotic sites inhibits neointimal hyperplasia in a canine model. Ann Thorac Surg. 2007. 84(2): p. 560-7; discussion 567.

301. Vorp, DA, Raghavan ML, Borovetz HS, Greisler HP, and Webster MW. Modeling the transmural stress distribution during healing of bioresorbable vascular prostheses. Ann Biomed Eng. 1995. 23(2): p. 178-88.

302. Hayashi, K. Experimental approaches on measuring the mechanical properties and constitutive laws of arterial walls. J Biomech Eng. 1993. 115(4B): p. 481-8.

303. Stankus, JJ, Soletti L, Fujimoto K, Hong Y, Vorp DA, Wagner WR. Fabrication of cell microintegrated blood vessel constructs through electrohydrodynamic atomization. Biomaterials. 2007. 28(17):p. 2738-46.

304. Guan, J, Fujimoto KL, Sacks MS, and Wagner WR. Preparation and characterization of highly porous, biodegradable polyurethane scaffolds for soft tissue applications. Biomaterials. 2005. 26(18): p. 3961-71.

305. Brami, M, Cote G, and Bonan R. [clinical, experimental and physiopathological aspects of restenosis after coronary angioplasty]. Arch Mal Coeur Vaiss. 1997. 90(3): p. 385-91.

306. Wan, S, Shukla N, Yim AP, Johnson JL, Angelini GD, and Jeremy JY. Orally administered penicillamine is a potent inhibitor of neointimal and medial thickening in porcine saphenous vein-carotid artery interposition grafts. J Thorac Cardiovasc Surg. 2007. 133(2): p. 494-500. 